\title{
Transport in nicht-hermiteschen niedrigdimensionalen Systemen
}

\author{
Dissertation
}

zur Erlangung des Doktorgrades

der Mathematisch-Naturwissenschaftlichen Fakultäten der Georg-August-Universität zu Göttingen

vorgelegt von

\section{Oliver Bendix}

aus Wernigerode 
Referent: $\quad$ Prof. Dr. Theo Geisel

Korreferent: Prof. Dr. Ulrich Parlitz

Tag der mündlichen Prüfung: 20. September 2011 


\begin{abstract}
In this work, scientific methods and findings known from mesoscopic physics, primarily used for semiconductor nanostructures, will be transferred to photonic systems, enabled by the analogy of mesoscopic ballistic transport and transport processes in photonics. Therefore a numerical method is developed that is based on Greens functions in combination with the Landauer-Büttiker formalism, and that allows describing coherent transport processes in low-dimensional semiconductor structures. This so-called scattering formalism is tested and elucidated in the framework of the ballistic rectifier. It is furthermore shown to be fruitful in gaining substantial results of comprehensively examined optical systems developed within this work - the chaotic beam splitter with its broad range of applications for photonic devices and the Müller cell, which acts as a biological wave guide in the retina of vertebrates, ensuring light transport through the retina while increasing the optical performance in most parts of the entire eye. The quantum scattering formalism allows investigating novel aspects of transport in optical systems. Based on dynamical tunneling a high effective single-mode laser and a variety of other applications within the context of deformed waveguides are presented and the reduction of scattering light in the surrounding area of the fovea in the human eye is predicted via theoretical analysis of the properties of human Müller cells.

In addition to the intense study of these last two passive optical systems, this work gives special attention to $\mathcal{P} \mathcal{T}$-symmetric active optical systems. Fundamental analytical results for the impact and implication of localization and disorder in such active $\mathcal{P} \mathcal{T}$-symmetric systems are investigated here. Based on this, the possibility of designing optical metamaterials with particular spatial ordered regions of gain and loss are inspected. The study concludes by transferring the scattering formalism to these active optical systems for characterization and classification of transport properties.
\end{abstract}




\section{Kurzfassung}

In der vorliegenden Arbeit wird die Analogie zwischen mesoskopischem ballistischem Transport und Transportvorgängen in der Photonik genutzt, um Methoden und Erkenntnisse aus der Theorie der mesoskopischen Physik, die bisher vornehmlich auf Halbleiter-Nanostrukturen angewandt wurden, auf photonische Systeme zu übertragen. Dazu wird eine numerische Methode entwickelt, die ihren Ursprung in der Theorie der Greenschen Funktionen in Verbindung mit dem Landauer-BüttikerFormalismus zur Beschreibung von kohärenten Transportvorgängen in niedrigdimensionalen Halbleiterstrukturen hat. Am Beispiel des ballistischen Gleichrichters wird diese im Weiteren genutzte numerische Methode des Streuformalismus erläutert und getestet. Sie erweist sich als äußerst effizient, um zu substanziellen Erkenntnissen über hier entwickelte und eingehend untersuchte optische Systeme zu gelangen - den chaotischen Strahlenteiler mit seinen hier abgeleiteten vielfältigen Anwendungen für die Photonik und die Müllerschen Zellen als biologische Wellenleiter der Wirbeltier-Retina, die den Lichttransport in der Netzhaut gewährleisten und dabei zugleich die Leistung des Auges optimieren. Der quantenmechanische Streuformalismus gestattet zudem die Untersuchung neuartiger Aspekte des Transports in optischen Systemen. So werden in dieser Arbeit eine auf dem dynamischen Tunneleffekt basierende Konstruktion eines effektiven Einmodenlasers und vielfältige andere Anwendungen auf der Grundlage deformierter Wellenleiter vorgestellt und die Streulichtreduktion in der Fovea des menschlichen Auges durch Ableitung optischer Eigenschaften der Müllerzelle untersucht.

Neben dem intensiven Studium dieser beiden passiven optischen Systeme widmet sich diese Arbeit dem Studium von $\mathcal{P} \mathcal{T}$-symmetrischen aktiven optischen Systemen. Hierbei werden grundlegende analytische Resultate zur Bedeutung und Auswirkung von Lokalisierung und Unordnung in solchen Systemen hergeleitet. Darauf aufbauend wird die Möglichkeit untersucht, die $\mathcal{P} \mathcal{T}$-Symmetrie ausnutzende quasiverlustfreie optische Metamaterialien zu entwickeln, die aus räumlich speziell angeordneten Bereichen lichtverstärkender und verlustbehafteter Materialien bestehen. Die Übertragung des Streuformalismus auf diese aktiven optischen Systeme zur Beschreibung und Klassifizierung von Transporteigenschaften bildet den Abschluss dieser Untersuchungen. 
Für meine Familie 



\section{Inhaltsverzeichnis}

Tabellenverzeichnis $\quad \mathrm{V}$

Abbildungsverzeichnis vii

Verwendete Größen und Konstanten ix

Einleitung

Gliederung der Arbeit . . . . . . . . . . . . . . . . . . . . 5

1 Der Ballistische Gleichrichter 7

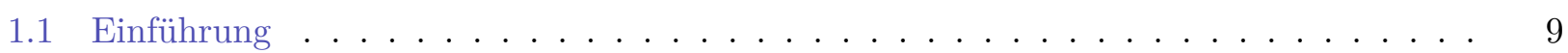

1.1.1 Landauers und Büttikers Ansatz . . . . . . . . . . . . . . . . . . 10

1.1.2 Greensche Funktion und Streumatrix . . . . . . . . . . . . . . . . . . 13

1.1.3 Die lokale Zustandsdichte . . . . . . . . . . . . . . . . . . . . . 18

1.2 Symmetriebrechung und mesoskopischer Transport . . . . . . . . . . . . . . . . . . . . 19

1.2.1 Semiklassische Betrachtung ...................... 19

1.2.2 Quantenmechanische Betrachtung .................... 20

1.3 Zusammenfassung und Ausblick . . . . . . . . . . . . . . . . . . . . . . 24

2 Der Chaotische Strahlenteiler 25

2.1 Einführung . . . . . . . . . . . . . . . . . . . . . . . . . 27

2.1.1 Resonatoren ............................. . . 27

2.1.1.1 Der Fabry-Pérot-Resonator . . . . . . . . . . . . . . . 28

2.1.1.2 Flüstergaleriemodenresonatoren . . . . . . . . . . . . . 30

2.1.1.3 Photonische Kristalle . . . . . . . . . . . . . . . . . . . . . 31

2.1.2 Bauelemente der integrierten Optik . . . . . . . . . . . . . . . . . . . 32

2.1.2.1 Strahlenteiler ....................... 32

2.1.2.2 Strahlenschalter .................... . . . 32

2.1.3 Modifizierte Wellenleiter als Strahlenteiler und Strahlenschalter . . . . . . . . 32

2.2 Das Kosinusbillard-Modell . . . . . . . . . . . . . . . . . . . . . . . 33

2.3 Quantenmechanische Behandlung . . . . . . . . . . . . . . . . 36

2.3.1 Quasi-gebundene Zustände . . . . . . . . . . . . . . . . . 36

2.3.2 Semiklassische Näherung. . . . . . . . . . . . . . . . . . . . . . 37

2.3.3 Experimentelle Untersuchungen mittels Mikrowellenbillard . . . . . . . . . . . 38

2.3.4 Vergleich von klassischem mit quantenmechanischem Phasenraum . . . . . . 39

2.3.5 Auskopplung der quasi-gebundenen Zustände durch Phasenraum-Engineering . 40

2.3.6 Modifikationen des Parametersatzes $(d, a, L)$. . . . . . . . . . . . . 45 
2.4 Weitere Anwendungen . . . . . . . . . . . . . . . . . . . . . . . 46

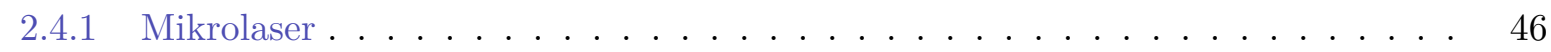

2.4 .2 Multi-Kavitätsresonatoren . . . . . . . . . . . . . . . . 50

2.4.3 Multi-Kavitätsstrahlenteiler . . . . . . . . . . . . . . . 50

2.5 Zusammenfassung und Ausblick . . . . . . . . . . . . . . . . . . . . . . . . 52

3 Verminderung des Streulichts im Auge durch lichtleitende Müllerzellen 53

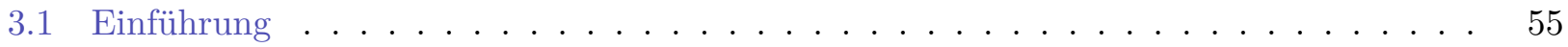

3.1 .1 Das Auge . . . . . . . . . . . . . . . . . . 55

3.1 .2 Die Retina . . . . . . . . . . . . . . . . . . . . 56

3.2 Generische Struktur der Müllerzelle . . . . . . . . . . . . . . . . . . . . . . 60

3.3 Die Müllerzelle als Lichtwellenleiter . . . . . . . . . . . . . . . . . . . . . . . . 62

3.3.1 Modellierung als Quantenkanal . . . . . . . . . . . . . . . . 62

3.3.2 Die Bedeutung der Form des Endfußes für die Lichtleitung . . . . . . . . . . . 64

3.3.3 Modellierung der extrazellulären Umgebung der Müllerzelle . . . . . . . . . . . . 66

3.3.4 Streuwellenfunktionen . . . . . . . . . . . . . . . . . . 68

3.3.5 Vergleich mit dem Experiment . . . . . . . . . . . . . . . . . . 69

3.3.6 Einfluss der Müllerzellenform auf die Lichtleitung . . . . . . . . . . . . . . . . 71

3.3.6.1 Zur Formvariation des Zellstamms . . . . . . . . . . . . . . . 71

3.3.6.2 Zur Position des Zellkörpers . . . . . . . . . . . . . . 73

3.3.7 Lichtleitung zwischen benachbarten Müllerzellen . . . . . . . . . . . . . . . 74

3.4 Modell zur Vorhersage des Streulichts im menschlichen Auge . . . . . . . . . . . . 75

3.4 Das Augenmodell . . . . . . . . . . . . . . . . . . 75

3.4.2 Relative Intensitäten des direkten Lichts und des Streulichts erster Ordnung . . 78

3.4.3 Bestimmung der Grenzwinkel $\phi_{\max }$ und $\delta_{\max } \ldots \ldots \ldots \ldots$. . . . . . . . . . . . . . . 81

3.4.4 Vergleich der relativen Intensitäten . . . . . . . . . . . . . . . . . . . 82

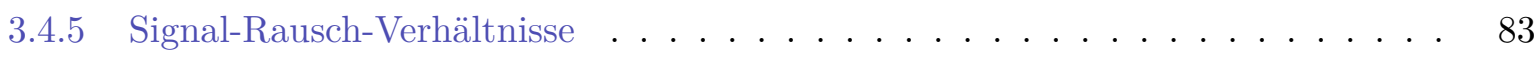

3.5 Zusammenfassung und Ausblick . . . . . . . . . . . . . . . . . . . . 85

$4 \mathcal{P} \mathcal{T}$-Symmetrie in niedrigdimensionalen optischen Systemen $\quad 89$

4.1 Einführung . . . . . . . . . . . . . . . . . . . . . . . . 91

4.1.1 Pseudo-hermitesche Operatoren und $\mathcal{P} \mathcal{T}$-Symmetrie . . . . . . . . . . . . . . . . . . . . . . . . . . . . . .

4.1 .2 Komplexe Brechungsindices . . . . . . . . . . . . . . . . . . . . . 92

4.1 .3 Wellenleiter . . . . . . . . . . . . . . . . . . . . . . . 94

4.2 Lokalisierung als Ursache für spontan gebrochene $\mathcal{P} \mathcal{T}$-Symmetrie in $1 \mathrm{D} \quad \ldots \ldots$. . . . 96

4.2.1 Zwei $\mathcal{P} \mathcal{T}$-symmetrische Fehlstellen . . . . . . . . . . . . . . . . 96

4.2.2 Die endliche $\mathcal{P} \mathcal{T}$-symmetrische Kette mit Unordnung (Eindimensionales $\mathcal{P} \mathcal{T}$ -

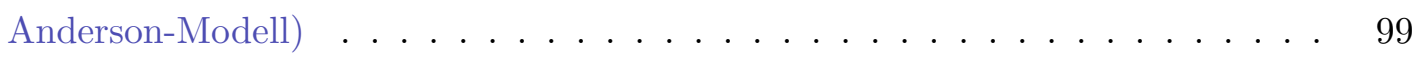

4.2.3 Periodische $\mathcal{P} \mathcal{T}$-symmetrische Potenziale . . . . . . . . . . . . . . . 105

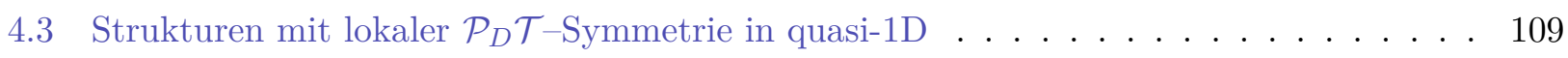

4.3 .1 Der einfache Dimer . . . . . . . . . . . . . . . . . . . . 109

4.3 .2 Zwei gekoppelte Dimere . . . . . . . . . . . . . . . . . . . 110

4.3 .3 Die Quasi 1D-Dimerkette . . . . . . . . . . . . . . . . 113 
4.4 Streuformalismus in lokal $\mathcal{P} \mathcal{T}$-symmetrischen Systemen . . . . . . . . . . . . . 120

4.4.1 Systeme mit abgeschlossenen Rändern . . . . . . . . . . . . . . . 120

4.4 .2 Systeme mit offenen Rändern . . . . . . . . . . . . . . . . . . . . . 124

Zusammenfassung

A Dimensionslose Einheiten 135

B Hamilton-Operatoren stark gebundener Elektronen 137

$\begin{array}{ll}\text { C Transversale Moden } & 139\end{array}$

D Phasenraummethoden 141

D.1 Quantenmechanische Verallgemeinerung der klassischen Phasenraumdichte . . . . . . 141

D.1.1 Zustandsbeschreibungen in der Quantentheorie . . . . . . . . . . . . . . 141

D.1.2 Wellenfunktionen und Dichtefunktionen . . . . . . . . . . . . . . . . . 142

D.1.3 Verteilungsfunktionen des Phasenraums . . . . . . . . . . . . . . . . . . 142

D.1.3.1 Die Wigner-Funktion . . . . . . . . . . . . . . . . . 142

D.1.3.2 Die Husimi-Funktion . . . . . . . . . . . . . . . . . . . 143

D.1.3.3 Andere Verteilungsfunktionen . . . . . . . . . . . . . . . . 143

E Zur Dichteverteilung der Photorezeptoren im menschlichen Auge 145

E.1 Zapfen-Photorezeptordichte . . . . . . . . . . . . . . . . . . 145

E.2 Stäbchen-Photorezeptordichte . . . . . . . . . . . . . . . . . 145

$\begin{array}{lll}\text { F } & \text { Retinale Verschaltung } & 147\end{array}$

G Einfache Fehlstelle in perfekter unendlicher Kette 149

H Der einfache Dimer 151

I Unitäre Transformationen von Hamiltonmatrizen linear gekoppelter Dimere 153

I.1 Der allgemeine Fall $N$ linear gekoppelter verschiedener komplexer Dimere . . . . . . 153

I.2 Zwei gekoppelte Dimere mit komplexen Kopplungskonstanten . . . . . . . . . . . . . . 155

I.3 Zwei gekoppelte identische Dimere mit komplexen Kopplungskonstanten . . . . . . . 156

$\begin{array}{lll}\text { J Eigenwerte und Eigenvektoren nicht-hermitescher Matrizen } & 159\end{array}$

$\begin{array}{ll}\text { Literaturverzeichnis } & 161\end{array}$

$\begin{array}{ll}\text { Lebenslauf } & 179\end{array}$ 



\section{Tabellenverzeichnis}

0.1 Größen und Konstanten . . . . . . . . . . . . . . . . . . . . xii

0.2 Naturkonstanten . . . . . . . . . . . . . . . . . . . xiii

3.1 Brechungsindices von Müllerzellen verschiedener Wirbeltiere . . . . . . . . . . . . 63

3.2 Regressionsparameter aus der Approximation der Reflexionsfunktion . . . . . . . . . 80

E.1 Regressionsparameter aus der Approximation der Stäbchen-Dichteverteilung . . . . . 146 



\section{Abbildungsverzeichnis}

$0.1 \mu-\varepsilon$-Phasendiagramm zur Klassifizierung von Metamaterialien . . . . . . . . . 4

1.1 Das zweidimensionale Elektronengas . . . . . . . . . . . . . . . . . . 9

1.2 Schematische Darstellung zum Landauer Büttiker Ansatz . . . . . . . . . . . . . . 11

1.3 Dispersionsrelation des diskreten und kontinuierlichen 2D-Systems . . . . . . . . . 14

1.4 Gitter-Diskretisierung und Kopplung der Probe mit Anschlussleitern . . . . . . . . . 16

1.5 Aufbau des Ballistischen Gleichrichters . . . . . . . . . . . . . . . . . . . . . . 19

1.6 Streuwellenfunktionen des ballistischen Gleichrichters ～. . . . . . . . . . . . . 20

1.7 Transportkoeffizienten des ballistischen Gleichrichters . . . . . . . . . . . . . . 22

1.8 Vorzeichenwechsel der Gleichrichtungsspannung $V_{\mathrm{UO}} \ldots \ldots \ldots \ldots \ldots$

2.1 Beispiele für 1D-, 2D- und 3D-Mikroresonatoren $\ldots \ldots \ldots \ldots$

2.2 Vereinfachte schematische Darstellung des Aufbaus photonischer Kristalle . . . . . . . 31

2.3 Geometrie des Kosinusbillards im Profil . . . . . . . . . . . . . . . . . . . . 34

2.4 Hufeisen- und Poincaré-Abbildung des Kosinusbillards . . . . . . . . . . . . . . . . . 35

2.5 Quasi-gebundene Zustände des Kosinusbillards $(d, a, L)=(1.0,0.305,5.55) \quad \ldots \quad \ldots \quad 37$

2.6 Mikrowellenexperiment eines Billards mit Reflexionsmessung der Wellenfunktion . . . 39

2.7 Transiente Poincaré-Abbildungen und Husimi-Verteilungen quasi-gebundener Zustände 40

2.8 Konfigurationsbeispiele für Schalter und Strahlenteiler des Kosinusbillards . . . . . . . 41

2.9 Transiente Poincaré-Abbildungen für transversale Anschlüsse unterschiedlicher Breite . 42

2.10 Dimensionslose Leitfähigkeiten des Kosinusbillards ． . . . . . . . . . . . . . . . . . 43

2.11 Aufenthaltswahrscheinlichkeitsdichte eines finiten Strahlenteilers . . . . . . . . . . 44

2.12 Zoo quasi-gebundener Zustände verschiedener Kosinusbillards . . . . . . . . . . . . . 45

2.13 Strahlendynamische Simulation der Laseremission eines Kosinusbillards . . . . . . . 46

2.14 Winkelnomenklatur der Strahlendynamik . . . . . . . . . . . . . . . . . 47

2.15 Bestimmung des maximalen Streuwinkels im Einmodenlaser . . . . . . . . . . . . . . 48

2.16 Transmissionswahrscheinlichkeit $T_{\mathrm{OL}}$ des Einmodenlasers $\ldots \ldots \ldots$. . . . . . . . 49

2.17 Geometrie und Zustandsdichte eines Multi-Kavitätsresonators . . . . . . . . . . . . 50

2.18 Konfigurationsbeispiele für "I"-, "M"- und "V"-förmige quasi-gebundene Zustände . . 51

3.1 Struktureller Aufbau des Auges . . . . . . . . . . . . . . . . . . . . 55

3.2 Aufbau der Retina . . . . . . . . . . . . . . . . . . . . . . . 57

3.3 Zapfen- und Stäbchendichte der menschlichen Retina . . . . . . . . . . . . . . . 58

3.4 Hellempfindlichkeitskurve und normalisierte Absorption der Photorezeptoren . . . . . 59

3.5 Übersicht über Aufbau und Form von Müllerzellen einiger Wirbeltiere . . . . . . . . . 61

3.6 Die Müllerzelle als Quantenkanal . . . . . . . . . . . . . . . . . . . . . . . 64

3.7 Kollimationseffekte des Müllerzellenendfußes im Vergleich mit Quantenpunktkontakt . 65

3.8 Modellierung der extrazellulären Umgebung der Müllerzelle . . . . . . . . . . . . . . 67 
3.9 Streuwellenfunktionen einer Müllerzelle im Zufallspotenzial . . . . . . . . . . . . . . 68

3.10 Vergleich der Simulationsergebnisse zur optischen Leistung mit dem Experiment 69

3.11 Formvariation der Müllerzellen mit Einfluss auf winkelabhängige Transmission 72

3.12 Einfluss der Lage des Zellkörpers einer Müllerzelle auf die optische Leistung . . 73

3.13 Interzellulärer Lichteinfall . . . . . . . . . . . . . . . . . . . . . . . . . . . . . . 74

3.14 Skizze des vereinfachten Augenmodells . . . . . . . . . . . . . . . . . . . 77

3.15 Bezeichnung der Winkel $\alpha$ und $\beta$. . . . . . . . . . . . . . . . . . . . . . . . . 79

3.16 Transmissions- und Reflexionsfunktion . . . . . . . . . . . . . . . . 80

3.17 Direktes Licht und Streulicht auf der Retina . . . . . . . . . . . . . . . . . 83

3.18 Signal-Rausch-Verhältnis des menschlichen Auges . . . . . . . . . . . . . . . . . . . . . 84

4.1 Beispiele elektrischer und optischer Wellenleiter . . . . . . . . . . . . . . . . 95

4.2 Lineare Kette mit zwei komplexwertigen Fehlstellen ............ 96

4.3 Energiespektrum einer perfekten $\mathcal{P} \mathcal{T}$-symmetrischen Kette mit $N=80$ Fehlstellen. . . 100

4.4 Lokalisierte Zustände einer $\mathcal{P} \mathcal{T}$-symmetrischen Kette mit reeller Unordnung . . . . . 101

4.5 Bifurkationsszenario des Dipolmoments und Verteilungsfunktion der kleinsten Energieaufspaltung im Fall starker Unordnung . . . . . . . . . . . . . . . . . . . . . . . . . 102

4.6 Dispersionsrelation und Energieniveauabstände einer linearen Kette . . . . . . . . . . 103

4.7 Bifurkationsszenario des Dipolmoments und Verteilungsfunktion der kleinsten Energieaufspaltung im Fall schwacher Unordnung . . . . . . . . . . . . . . . . . . . . . . . 104

4.8 Periodische lineare Kette . . . . . . . . . . . . . . . . . . . . . 105

4.9 Oberflächenzustände und Energiespektrum für halb- $\infty$ periodische lineare Kette . . 107

4.10 Der Dimer . . . . . . . . . . . . . . . . . . . . 109

4.11 Vorschlag einer experimentellen optischen Realisierung der Dimerkette . . . . . . . . . 110

4.12 Zwei gekoppelte Dimere . . . . . . . . . . . . . . . . . . . . . 110

4.13 Phasendiagramm für zwei gekoppelte Dimere . . . . . . . . . . . . . . . . . . . . . . . 112

4.14 Dimerkette mit $N$ gekoppelten Dimeren . . . . . . . . . . . . . . . . . . . . . . . . . 113

4.15 Phasendiagramm für Dimerkette mit $N=40$ gekoppelten Dimeren . . . . . . . . . . . 117

4.16 Veranschaulichung zur Abnahme der Bandlücke $\Delta$ mit wachsender Unordnung $\beta$. . . 118

4.17 Variationen der Unordnungsparameter der Dimerkette mit $N=40$ Dimeren . . . . . . 119

4.18 Stützstellenapproximation periodischer Potenziale auf einem Quadratgitter . . . . . 121

4.19 Eigenwerte eines Systems mit $\mathcal{P} \mathcal{T}$-symmetrischem Potenzial mit und ohne Unordnung 123

4.20 Vergleich der kritischen Parameter des Phasenübergangs . . . . . . . . . . . . . . . . 124

4.21 Offenes ausgedehntes diskretes System mit zwei externen Anschlüssen . . . . . . . . 125

4.22 Eigenwerte eines offenen Systems mit $\mathcal{P} \mathcal{T}$-symmetrischem Potenzial . . . . . . . . . . 126

4.23 Vergleich des kritischen Parameters $B_{\mathrm{c}}$ mit der Transmissionswahrscheinlichkeit $\widetilde{T} \quad$. 127

C.1 Eigenfunktionen und Eigenenergien des idealen Leiters in harmonischer Näherung . . 140

G.1 Lineare Kette mit reellwertiger Fehlstelle . . . . . . . . . . . . . . . . . . . . . . . . . . 149

G.2 Lokalisierte Wellenfunktionen einer lineare Kette mit reellwertiger Fehlstelle . . . . . . 150

H.1 Eigenwertspektrum und Eigenfunktionen des Dimers . . . . . . . . . . . . . . . . . . 152

I.1 Dimerkette $N=2$ mit komplexwertigen Kopplungskonstanten . . . . . . . . . . . . . . 156 


\section{Verwendete Größen und Konstanten}

Die in dieser Arbeit auftretenden Größen und Konstanten sind in folgender Tabelle vollständig aufgelistet. Fett gedruckte Symbole bedeuten Vektoren, Operatoren sind durch ein Dach " $\hat{\square}$ " gekennzeichnet. Größen mit natürlichen Einheiten sind in SI-Darstellung ${ }^{1}$ angegeben, dimensionslose Größen sind durch einen Geviertstrich “-_" gekennzeichnet.

\begin{tabular}{lll}
\hline Symbol & Beschreibung & Einheiten \\
\hline & & \\
$\mathbf{B}$ & magnetische Flußdichte & $\mathrm{T}$ \\
$\mathbf{D}$ & elektrische Flußdichte & $\mathrm{C} \cdot \mathrm{m}^{-2}$ \\
$\mathbf{E}$ & elektrische Feldstärke & $\mathrm{V} \cdot \mathrm{m}^{-1}$ \\
$\mathbf{H}$ & magnetische Feldstärke & $\mathrm{A} \cdot \mathrm{m}^{-1}$ \\
$\mathbf{M}$ & Magnetisierung & $\mathrm{A} \cdot \mathrm{m}^{-1}$ \\
$\mathbf{P}$ & Polarisation & $\mathrm{C} \cdot \mathrm{m}^{-2}$ \\
$\varepsilon_{0}$ & elektrische Feldkonstante & $\mathrm{F} \cdot \mathrm{m}^{-1}$ \\
$\varepsilon_{\mathrm{r}}$ & relative Permittivität & - \\
$\mu_{0}$ & magnetische Feldkonstante & $\mathrm{H} \cdot \mathrm{m}^{-1}$ \\
$\mu_{\mathrm{r}}$ & relative Permeabilität & - \\
$\chi_{\mathrm{e}}$ & elektrische Suszeptibilität & - \\
$\chi_{\mathrm{m}}$ & magnetische Suszeptibilität & -
\end{tabular}

\section{Kapitel 1}

$\begin{array}{lll}a & \text { Gitterkonstante } & \mathrm{m} \\ \mathbf{A} & \text { Vektorpotenzial } & \mathrm{V} \cdot \mathrm{s}^{\cdot} \mathrm{m}^{-1} \\ A\left(\mathbf{r}, \mathbf{r}^{\prime}\right) & \text { Spektrale Dichte } & \mathrm{eV} \\ d_{\mathrm{s}} & \text { Spärlichkeitsdichte } & - \\ D & \text { Gitterdimension } & - \\ E & \text { Energie } & \mathrm{eV} \\ E_{n} & n \text {-ter reeller Eigenwert } & \mathrm{eV} \\ f(E) & \text { Fermi-Verteilungsfunktion } & - \\ F_{\mathrm{F}}(E) & \text { Funktion der thermischen Linienverbreiterung } & \mathrm{eV}^{-1} \\ g_{\mathrm{L}}(E) & \text { retardierte Greensche Funktion eines Anschlussleiters } & \mathrm{eV}^{-1} \\ G(E), G^{\mathrm{R}}(E) & \text { retardierte Greensche Funktion } & \mathrm{eV}^{-1} \\ G^{\mathrm{A}}(E) & \text { avancierte Greensche Funktion } & \mathrm{eV}^{-1} \\ \mathbb{G} & \text { Greensche Funktion in Matrixdarstellung } & \mathrm{eV}^{-1}\end{array}$

1 In dieser Arbeit werden ausschließlich SI Einheiten nach Richtlinie 80/181/EWG verwendet. 


\begin{tabular}{|c|c|c|}
\hline Symbol & Beschreibung & Einheiten \\
\hline$G^{-1}(E)$ & inverse retardierte Greensche Funktion & $\mathrm{eV}$ \\
\hline$G_{p q}(E)$ & Leitfähigkeit von Kanal $q \rightarrow p$ & $\mathrm{~S}$ \\
\hline $\mathcal{H}$ & Hamilton-Operator & - \\
\hline$I^{ \pm}$ & einlaufender $(+)$und auslaufender $(-)$Strom & $\mu \mathrm{A}$ \\
\hline$I_{p q}$ & Nettostrom von Kanal $q \rightarrow p$ & $\mu \mathrm{A}$ \\
\hline k & Wellenvektor & $\mathrm{m}^{-1}$ \\
\hline$l_{\mathrm{m}}$ & mittlere freie Weglänge & $\mu \mathrm{m}$ \\
\hline$l_{\varphi}$ & Phasenkohärenzlänge & $\mu \mathrm{m}$ \\
\hline$L$ & Systemlänge & $\mu \mathrm{m}$ \\
\hline $\mathcal{M}, M(E)$ & Modenzahl & - \\
\hline$n_{\mathrm{e}}$ & Elektronendichte & $\mathrm{m}^{-3}$ \\
\hline$\hat{p}$ & Impulsoperator & - \\
\hline $\bar{R}(E)$ & Reflexionskoeffizient & - \\
\hline$R(E)$ & Reflexionswahrscheinlichkeit & - \\
\hline$R_{i j, k l}$ & Widerstand der Vierpunktmessung $V_{k l}$ und $I_{i j}$ & $\mathrm{k} \Omega$ \\
\hline$s_{n m}^{p q}$ & Matrixelemente der Streumatrix & - \\
\hline$S$ & Streumatrix & - \\
\hline$t$ & Austauschenergie & $\mathrm{eV}$ \\
\hline$T$ & Temperatur & $\mathrm{K}$ \\
\hline $\bar{T}(E)$ & Transmissionskoeffizient & - \\
\hline$T(E)$ & Transmissionswahrscheinlichkeit & - \\
\hline$V_{p q}$ & Spannung zwischen Kontakt $q$ und $p$ & $\mathrm{mV}$ \\
\hline$V(\mathbf{r})$ & elektrisches Potenzial & $\mathrm{eV}$ \\
\hline$w_{p q}$ & minimale Breite des Transportkanals von Kontakt $q \rightarrow q$ & $\mu \mathrm{m}$ \\
\hline$W$ & Systembreite & $\mu \mathrm{m}$ \\
\hline$\hat{x}$ & Ortsoperator & - \\
\hline$\varepsilon_{n}$ & $n$-ter komplexer Eigenwert & $\mathrm{eV}$ \\
\hline$\lambda_{\mathrm{F}}$ & Fermi-Wellenlänge & $\mathrm{nm}$ \\
\hline$\mu_{p}$ & chemisches Potnezial des Reservoirs $p$ & $\mathrm{eV}$ \\
\hline$\nu_{n}$ & Geschwindigkeit der $n$-ten transversalen Eigenmode & $\mathrm{m} \cdot \mathrm{s}^{-1}$ \\
\hline$\rho_{E}(\mathbf{r})$ & lokale Zustandsdichte & $\mathrm{eV}$ \\
\hline$\sigma$ & elektrische Leitfähigkeit & $\mathrm{S}$ \\
\hline$\Sigma^{\mathrm{R}}(E)$ & retardierte Selbstenergie & $\mathrm{eV}$ \\
\hline$\tau_{\mathrm{L}}^{+}$ & Kopplungsmatrix & $\mathrm{eV}$ \\
\hline$\varphi(\mathbf{r})$ & linker Eigenvektor des Hamilton-Operators & - \\
\hline$\chi(\mathbf{r})$ & transversale Eigenmoden in Ortsdarstellung & - \\
\hline$\psi(\mathbf{r})$ & rechter Eigenvektor des Hamilton-Operators (Streuwellenfunktion) & - \\
\hline$\omega_{0}$ & Stärke des harmonischen Potenzials & $\mathrm{m}^{-1}$ \\
\hline
\end{tabular}




\begin{tabular}{lll}
\hline Symbol & Beschreibung & Einheiten \\
\hline
\end{tabular}

\section{Kapitel 2}

a Breite der Ausbuchtung des Wellenleiters

d Resonatorbreite

$d_{\mathrm{T}} \quad$ Breite der transversalen Anschlüsse

$f_{\mathrm{p}} \quad$ Purcell-Faktor

F $\quad$ Finesse

h Resonatorhöhe m

$H(x, k) \quad$ Husimi-Verteilung -

$L \quad$ Länge des Resonatorbereichs

$n \quad$ Brechungsindex

$n_{\text {eff }} \quad$ effektiver Brechungsindex

Q Gütefaktor

$\begin{array}{ll}V_{\mathrm{m}} & \text { Modenvolumen } \\ w(r) & \text { elektromagnetische Energiedichte }\end{array}$

$\Theta, \Theta_{0} \quad$ Einfallswinkel auf die Poincaréebene

$\Theta$

kritischer Einfallswinkel, Winkel der Totalreflexion

maximaler Einfallswinkel

$\mathrm{rad}$

rad

$\operatorname{rad}$

Wellenlänge

$\mathrm{nm}$

Frequenz, Resonanzfrequenz

$\mathrm{s}^{-1}$

$\nu$

Zustandsdichte der Kavität

Zustandsdichte des freien Raums

\section{Kapitel 3}

A

$A_{\mathrm{N}}$

$A_{\text {Retina }}$

$d_{\max }$

$D_{0}$

$D_{\mathrm{M}}$

$f$

$I_{0}$

$l_{\mathrm{c}}$

NA

$p(\alpha)$

$P(\alpha)$

$S$

$S_{0}$ von der Pupille eingeschlossene Fläche

normalisierte Absorption

Retinaoberfläche

maximaler Pupillendurchmesser

$\mathrm{mm}$

relative Intensität des direkten Lichts ohne Müllerzellen

relative Intensität des direkten Lichts mit Müllerzellen

Kollimationsfaktor

Gesamtintensität des Lichts hinter der Linse

Korrelationslänge der Dichtefunktion

$\mu \mathrm{m}$

numerische Apertur

optische Leistung

Winkelverteilung

Wirkung

$\mathrm{J} \cdot \mathrm{s}$ 


\begin{tabular}{llc}
\hline Symbol & Beschreibung & Einh \\
\hline & & \\
$S_{\mathrm{M}}$ & relative Intensität des Streulichts mit Müllerzellen & - \\
$V$ & Wellenleiter-Parameter & - \\
$V(\lambda)$ & skotopischer Visus & - \\
$w$ & Kanalbreite & $\mu \mathrm{m}$ \\
$Y(\lambda)$ & photopischer Visus & - \\
$\alpha$ & Einfallswinkel & $\mathrm{rad}$ \\
$\beta$ & Ausfallswinkel & $\mathrm{rad}$ \\
$\delta_{\max }$ & maximaler Öffungswinkel der Pupille & $\mathrm{rad}$ \\
$\eta$ & relative Lichtleiteffizienz & - \\
$\sigma_{\mathrm{g}}$ & Varianz des Gaußprozesses & $\mu \mathrm{m}$ \\
$\phi_{\mathrm{G}}$ & retinaler Azimut & $\mathrm{rad}$ \\
$\phi_{\max }$ & maximaler retinaler Azimut & $\mathrm{rad}$
\end{tabular}

\section{Kapitel 4}

$\begin{array}{ll}D, D_{\mathrm{m}} & \text { Dipolmoment } \\ h_{n} & \text { Blockmatrix des } n \text {-ten Dimers } \\ \mathcal{H}_{2} & \text { Hamilton-Operator des Dimers } \\ \bar{K} & \text { diagonaler Petermann-Faktor } \\ \mathcal{P} & \text { Paritätsoperator } \\ q_{\mathrm{c}}(E) & \text { Ordnungsparameter der } \mathcal{P} \mathcal{T} \text {-symmetrischen Phase } \\ t & \text { Inter-Dimer Kopplungskonstante } \\ T & \text { Kopplungsmatrix } \\ \mathcal{T} & \text { Zeitumkehroperator } \\ u & \text { diagonale Inter-Dimer Kopplungskonstante } \\ v & \text { Intra-Dimer Kopplungskonstante } \\ \beta & \text { Realteil des Potenzials } \\ \gamma & \text { Imaginärteil des Potenzials } \\ \gamma_{\mathrm{c}} & \text { kritischer Parameter der } \mathcal{P} \mathcal{T} \text {-Symmetriebrechung } \\ \delta_{n} & \text { Energieaufspaltung } \\ \Delta_{n} & \text { Energieniveauabstand } \\ \varepsilon_{n} & n \text {-tes Dimerpotenzial } \\ \kappa_{k l} & \text { Petermann-Faktoren } \\ \lambda & \text { Eigenwert }\end{array}$

$\mathrm{rad}$

$\mathrm{rad}$

$\mathrm{rad}$

$\mu \mathrm{m}$

$\mathrm{rad}$

$\mathrm{rad}$ 
In dieser Arbeit werden, falls nicht ausdrücklich anders angegeben, dimensionslose Einheiten für Naturkonstanten verwendet. Bei diesem Basiswechsel zu $e=\hbar=m_{e}=1$ treten Umrechnungsfaktoren auf. Dazu befindet sich ein Kapitel im Anhang A auf Seite 135. Der großen Bedeutung wegen sind diese Konstanten in ihren natürlichen Einheiten in der folgenden Tabelle angegeben.

\begin{tabular}{lll}
\hline Symbol & Bedeutung & Einheiten \\
\hline & & \\
$h$ & Elementarladung des Elektrons & $1.602 \cdot 10^{-19} \mathrm{Js}$ \\
$h$ & Plancksches Wirkungsquantum & $6.626 \cdot 10^{-34} \mathrm{Js}$ \\
$\hbar$ & reduziertes Plancksches Wirkungsquantum & $1.055 \cdot 10^{-34} \mathrm{Js}$ \\
$m_{\mathrm{e}}$ & Ruhemasse des Elektrons & $9.109 \cdot 10^{-31} \mathrm{~kg}$
\end{tabular}

Tabelle 0.2.: Naturkonstanten in ihren natürlichen Einheiten. 



\section{Einleitung}

In dieser Arbeit wird die Analogie zwischen mesoskopischem ballistischem Transport und Transportvorgängen in der Photonik genutzt, um Methoden und Erkenntnisse aus der Theorie der mesoskopischen Physik, die bisher vornehmlich auf Halbleiter-Nanostrukturen angewandt wurden, auf photonische Systeme zu übertragen. Es wird eine numerische Methode entwickelt, die ihren Ursprung in der Theorie der Greenschen Funktionen in Verbindung mit dem Landauer-Büttiker-Formalismus zur Beschreibung von kohärenten Transportvorgängen in niedrigdimensionalen Halbleiterstrukturen hat. Am Beispiel des ballistischen Gleichrichters wird diese im Weiteren genutzte numerische Methode des Streuformalismus erläutert und getestet. Wie sich herausstellt, erweist sie sich als äußerst effizient, um zu aufschlussreichen Ergebnissen hinsichtlich der Transporteigenschaften optischer Systeme zu gelangen. Wir gewinnen substanzielle Erkenntnisse über hier entwickelte und eingehend untersuchte optische Systeme - den chaotischen Strahlenteiler mit seinen hier abgeleiteten vielfältigen Anwendungen für die Photonik und die Müllerschen Zellen als biologische Wellenleiter der WirbeltierRetina, die den Lichttransport in der Netzhaut gewährleisten und dabei zugleich die Leistung des Auges optimieren. Der quantenmechanische Streuformalismus gestattet die Untersuchung neuartiger Aspekte des Transports in optischen Systemen. So wird in dieser Arbeit eine auf dem dynamischen Tunneleffekt basierende Konstruktion eines effektiven Einmodenlasers und vielfältiger anderer Anwendungen aus deformierten Wellenleitern vorgestellt und die Streulichtreduktion in der Fovea des menschlichen Auges durch Ableitung optischer Eigenschaften der Müllerzelle untersucht. Neben dem intensiven Studium dieser beiden passiven optischen Systeme widmet sich diese Arbeit dem Studium von $\mathcal{P} \mathcal{T}$-symmetrischen aktiven optischen Systemen. Es werden grundlegende analytische Resultate zur Bedeutung und Auswirkung von Lokalisierung und Unordnung in solchen Systemen hergeleitet. Darauf aufbauend wird die Möglichkeit untersucht, die $\mathcal{P} \mathcal{T}$-Symmetrie ausnutzende quasi-verlustfreie optische Metamaterialien zu entwickeln, die aus räumlich speziell angeordneten Bereichen lichtverstärkender und verlustbehafteter Materialien bestehen. Die Übertragung des Streuformalismus auf diese aktiven optischen Systeme zur Beschreibung und Klassifizierung von Transporteigenschaften bildet den Abschluss dieser Untersuchungen.

\section{Ballistischer Transport}

Seit der ersten Realisierung eines zweidimensionalen Elektronengases (2DEG) bzw. Quantenfilms von Fowler et al. [6] sind eine Vielzahl von Arbeiten veröffentlicht worden, in denen Quanteneffekte niedrigdimensionaler Elektronensysteme beobachtet und erklärt werden konnten. Als bedeutende Arbeiten seien die Aharonov-Bohm-Oszillationen der Leitfähigkeit in einem Quantenring [7], der nichtverschwindende Widerstand in ballistischen Wellenleitern [8], die Quantisierung der transversalen Moden in Abhängigkeit von der Kanalbreite [9], der Quanten-Hall-Effekt [10], schwache und starke Lokalisierung [11, 12] und universelle Leitwertfluktuationen [13, 14, 15] genannt, um nur einige aufzuzählen. 
Die Größenskala dieser elektronischen Systeme wird als mesoskopisch bezeichnet, da sie zwischen den makroskopisch und damit klassisch beschreibbaren Systemen und den mikroskopischen, allein durch die Quantenmechanik beschriebenen Systemen liegen. Die quantenmechanische Beschreibung mesoskopischer Systeme ist aufgrund ihrer Größe zwingend notwendig, häufig erlauben jedoch gerade semiklassische Überlegungen einen tieferen Einblick in physikalische Phänomene. Moderne 2DEG zeichnen sich durch hohe Mobilität und große mittlere freie Weglängen aus, wobei letztere deutlich größer als die Systemlänge der mikro- und nanostrukturierten Bauelemente selbst sind. Die Elektronen können sich somit bei tiefen Temperaturen fast ohne Streuung an den Fehlstellen durch den Quantenfilm bewegen und werden nur noch von elastischen Stößen mit den Systemgrenzen und durch äußere Felder beeinflusst. Dieser Transport wird daher ballistisch genannt. Auf der theoretischen Seite sind viele Konzepte zur Beschreibung von Transporteigenschaften in mesoskopischen Systemen entwickelt worden [16]. Als eine wichtige Stütze mikroskopischer Theorien hat sich die Formulierung des Streuproblems mittels der Methode der Greenschen Funktionen in ihrer zeitabhängigen und -unabhängigen Darstellung [17] bewährt. Die Verknüpfung mesoskopischer Streu- und makroskopischer Transportgrößen gelingt mit dem Landauer-Büttiker-Formalismus [18, 19]. In dieser Arbeit wird eine stabile und effiziente numerische Methode vorgestellt, die mithilfe des Landauer-Büttiker-Formalismus und den Greenschen Funktionen als Lösung der zeitunabhängigen Schrödinger-Gleichung zu beliebig gearteten zweidimensionalen Potenzialen das Verhalten makroskopischer Transportgrößen in der Nähe des thermodynamischen Gleichgewichts vorhersagt und darüber hinaus einen komfortablen Zugriff auf die quantenmechanischen Streuwellenfunktionen ermöglicht.

Als Anwendungsbeispiel soll hier ein Bauelement mit räumlicher Symmetriebrechung dienen, das ballistischer Gleichrichter genannt wird. Das von Song et al. [20] realisierte elektronische Bauteil besteht aus einem Kreuzkontakt (cross junction), dessen räumliche Symmetrie durch einen dreiecksförmigen Antidot gebrochen ist. Im Experiment zeigte sich ein Gleichrichtungseffekt, der bei einer naiven Anwendung des Landauer-Büttiker-Ansatzes zu einem Widerspruch zwischen Theorie und experimentellem Befund führte. Erst die von Fleischmann \& Geisel [21] aufgestellte semiklassische Beschreibung war in der Lage, diesen Widerspruch aufzulösen, indem sie den Gleichrichtungseffekt als eine Konsequenz aus dem Zusammenwirken von semiklassischen und quantisierten Transportkanälen erklärten. Ergebnisse der semiklassischen Betrachtung werden hier mit dem quantenmechanischen Streuformalismus hinterfragt.

\section{Resonatoren, Billards und Lichtwellenleiter}

Optische Resonatoren erfüllen den Zweck, Licht in möglichst wenigen Moden zu konzentrieren und die Strahlungsdichte auf kleinste Raumvolumina zu beschränken, wobei dies durch mehrfache Reflexion in einem optisch aktiven Medium realisiert werden kann, so dass sich die Laufwege verlängern und damit optisch-verstärkende Eigenschaften - beispielsweise zur Realisierung von Lasern - nutzbar werden. Ein Resonator kann auch als optisches Übertragungssystem mit Rückkopplung verstanden werden, ähnlich einem elektronischen $L C$-Schwingkreis. Daher besetzen Resonatoren eine Schlüsselposition in der optischen Informationsverarbeitung. Die Integration opto-elektronischer Bauteile in kompakten Chips analog zur Halbleitertechnologie stellt dabei ein wünschenswertes Ziel dar. Das intensive Interesse an Quantenbillards in diesem Zusammenhang erwuchs aus der Erkenntnis, dass Mikroresonatoren mit chaotischer Dynamik [22, 23] oder mit gemischtem Phasenraum [24, 25, 26] dazu verwendet werden 
können, da sie es rein durch die Wahl der äußeren Form des Resonators erlauben, hohe Gütefaktoren zu realisieren, ohne gleichzeitig auf stark gerichtete Emission verzichten zu müssen. Wir zeigen hier, dass sogar der Wellenleiter selbst als Resonator verwendet werden kann, um interessante technische Anwendungen wie Strahlenteiler oder Strahlenschalter daraus abzuleiten [1, 2]. In diesen Betrachtungen nutzen wir die Formäquivalenz zwischen zeitunabhängiger Schrödinger-Gleichung und optischer Wellengleichung (Helmholtz-Gleichung), um die zuvor entwickelte quantenmechanische Methode anwenden zu können.

Lichtwellenleiter erlauben die verlustarme und unterbrechungsfreie Übertragung von optischen Signalen. Dabei wird der physikalische Effekt der Totalreflexion ausgenutzt, indem optische Fasern mit großem Brechungsindex als Kern von einem Material mit kleinerem Brechungsindex ummantelt werden. Diese künstlich gefertigten Fasern sind seit den 70er Jahren kommerziell erhältlich. Es ist erst seit einigen Jahren bekannt, dass solche Fasern auch in der Natur vorkommen. Als Beispiel sei die intensive Untersuchung der Wellenleiternatur von Photorezeptoren angegeben [27]. In diesem Zusammenhang wurden auch erste theoretische Versuche zur Erklärung des Stiles-Crawford-Effektes 1. Art (SCE1) unternommen [28], wobei der SCE1 einen Wahrnehmungseffekt der Lichtintensität eines senkrecht einfallenden Lichtstrahls in Abhängigkeit von der Position auf der Pupille beschreibt [29] - ein seit langer Zeit theoretisch unbefriedigend beschriebenes Phänomen. Das Studium der Lichtleitung im Auge und speziell in der Retina ist ein faszinierender Forschungszweig, in dem diverse Modelle seither kontrovers diskutiert werden. Zur großen Überraschung fanden Franze et al. [30] kürzlich in der Wirbeltierretina weitere Indizien der Lichtwellenleitung auf viel größeren Skalen als denen der Photorezeptoren: Sie entdeckten die lichtwellenleitende Eigenschaft der Müllerschen Zellen, den Gliazellen der Retina. Sowohl die Wirbeltierretina als auch die Retina vieler Weichtiere besitzt einen invertierten Aufbau. Das Licht muss erst diverse zelluläre Schichten passieren, ehe es von den Photorezeptoren auf der Rückseite der Retina detektiert werden kann. Dieser Lichttransport wäre mit zahlreichen Streuprozessen und Lichtbrechung verbunden, wodurch die Bildschärfe stark reduziert erscheinen müsste. Durch Transmissions- und Reflexionskonfokalmikroskopie in Meerschweinretina wiesen Franze et al. die Lichtwellenleiternatur der Müllerzellen nach. Sie fanden zusätzlich heraus, dass die nahezu parallele Anordnung der Müllerzellen - die die gesamte Retina durchspannen - an die Konfiguration von hocheffizienten faseroptischen Systemen erinnert. Schließlich bewies die experimentelle Untersuchung optischer Eigenschaften einzelner extrahierter Müllerzellen im optischen Strecker ihre Lichtleitfunktion in einem homogenen Medium. Die lichtleitende Eigenschaft der Müllerzelle als biologischer Wellenleiter in seiner natürlichen, nicht-homogenen Umgebung wird in dieser Arbeit theoretisch untersucht. Ein essenzielles formspezifisches Detail der Zelle ist die Ähnlichkeit des Müllerzellenfußes mit einem Trichter. Diese Trichterform findet auch beim Design von Quantenpunktkontakten in der Halbleiterphysik Verwendung und führt zu Kleinwinkelfokussierung. Durch die Untersuchung der winkelabhängigen Reflexions- und Transmissionseigenschaften der Müllerzellen können wir zeigen, dass diese ausgeprägte Eigenschaft in der Nähe der Fovea, dem Zentrum des Scharfsehens, zu einer deutlichen Reduktion des Streulichts führt [5].

\section{Optische Metamaterialien}

Neben den biologischen Wellenleitern erregten in den letzten Jahren Materialeigenschaften von neuen künstlich geschaffenen Metamaterialien besondere Aufmerksamkeit, da ihre physikalischen Eigenschaf- 
ten in der Natur und in herkömmlichen Materialien bisher nicht anzutreffen sind. Diesen artifiziellen Strukturen ist gemein, dass sie Inhomogenitäten auf der mikroskopischen Skala ausnutzen, um das makroskopische optische Verhalten zu beeinflussen. Dies wird durch Modifizierung der zwei Materialparameter, der relativen magnetischen Permeabilität $\mu_{\mathrm{r}}$ und der elektrischen Permittivität $\varepsilon_{\mathrm{r}}$ möglich. Die Größe $\mu=\mu_{0} \mu_{\mathrm{r}}$ verknüpft die magnetische Flussdichte $\mathbf{B}$ mit der magnetischen Feldstärke $\mathbf{H}$,

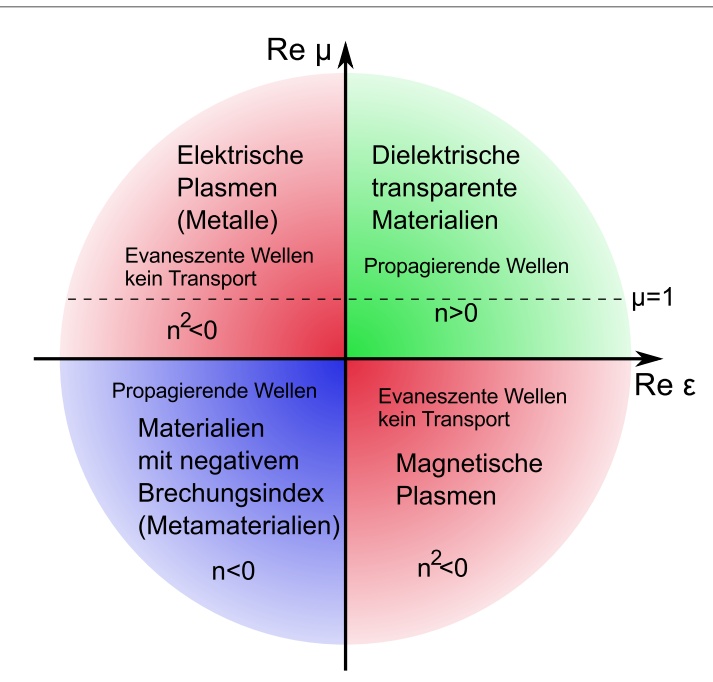

Abbildung 0.1.: Phasendiagramm (PD) für Metamaterialien im visuellen Bereich nach J. B. Pendry [31]. In den Quadranten sind die vier möglichen Materialklassen eingeordnet. Oberhalb der $\varepsilon$-Achse sind natürliche Materialien angesiedelt, während unterhalb die künstlichen Materialien erfasst sind. Die gestrichelte Linie bei $\mu=1$ kennzeichnet die Position nichtmagnetischer Materialien im PD. während $\varepsilon=\varepsilon_{0} \varepsilon_{\mathrm{r}}$ die elektrische Flussdichte $\mathbf{D}$ mit der elektrischen Feldstärke $\mathbf{E}$ verbindet. Dies wird im Allgemeinen durch die zwei Materialgleichungen der Maxwell-Gleichungen

$$
\begin{aligned}
& \mathbf{B}=\mu_{0}(\mathbf{H}+\mathbf{M})=\mu_{0}\left(1+\chi_{\mathrm{m}}\right) \mathbf{H}=\mu_{0} \mu_{\mathrm{r}} \mathbf{H} \\
& \mathbf{D}=\varepsilon_{0} \mathbf{E}+\mathbf{P}=\varepsilon_{0}\left(1+\chi_{\mathrm{e}}\right) \mathbf{E}=\varepsilon_{0} \varepsilon_{\mathrm{r}} \mathbf{E}
\end{aligned}
$$

mit der Magnetisierung $\mathbf{M}$ bzw. der Polarisation $\mathbf{P}$ und der magnetischen Suszeptibilität $\chi_{\mathrm{m}}$ bzw. der elektrischen Suszeptibilität $\chi_{\mathrm{e}}$ ausgedrückt. Der Brechungsindex hängt über $n^{2}=\mu_{\mathrm{r}} \varepsilon_{\mathrm{r}}$ mit den komplexwertigen Materialkonstanten zusammen. Als berühmtes Beispiel ergeben sich - bei entsprechender Wahl von $\mu$ und $\varepsilon$ - Materialien mit negativen Brechungsindices, die bereits 1968 von V.G. Veselago [32] theoretisch vorausgesagt, aber erst vor einigen Jahren von Smith et al. [33] erstmals realisiert wurden. Eine Klassifizierung möglicher Materialien kann nebenstehender Abbildung entnommen werden.

Metamaterialien können beispielsweise durch eine periodische Anordnung von Leiterelementen realisiert werden, die aus sehr kleinen $L C$-Kreisen mit Ausdehnungen unterhalb der verwendeten optischen Wellenlänge bestehen. Durch Resonanzeffekte entsteht dann die gewünschte Eigenschaft als makroskopischer Effekt. Diese Materialien besitzen erstaunliche Merkmale, die Anwendungen wie optische Tarnung [34] und neue bildgebende Verfahren auf Subwellenlängenebene [35, 36] ermöglichen.

Um die in diesen Metamaterialien auftretenden, meist starken Intensitätsverluste auszugleichen, wird versucht, Bereiche optisch verstärkender Strukturen in das Ausgangsmaterial zu integrieren. Dies führt jedoch in der Regel zu Instabilitäten. Hier versprechen so genannte $\mathcal{P} \mathcal{T}$-symmetrische Systeme mit ihrer pseudo-hermiteschen Phase einen Ausweg. Dieses Konzept aus der Theorie nicht-hermitescher Quantensysteme lässt sich beispielsweise vermittels der paraxialen Näherung von der SchrödingerGleichung auf optische Systeme übertragen, wobei ein komplexer ortsabhängiger Brechungsindex $n(\mathbf{r})$ die Rolle des komplexen Potenzials $V(\mathbf{r})$ übernimmt.

Bender \& Böttcher [37] konnten zeigen, dass eine große Klasse von nicht-hermiteschen Quantensystemen trotz ihrer Nicht-Hermitizität ein reelles Energieeigenwertspektrum besitzen können. In diesen Systemen vertauscht der Hamilton-Operator $\mathcal{H}$ mit dem $\mathcal{P} \mathcal{T}$-Operator, wobei $\mathcal{P}$ den Paritätsoperator darstellt, der in seiner Wirkung auf Orts- und Impulsoperator Reflexion beinhaltet und $\mathcal{T}$ den Zeitumkehroperator repräsentiert, der nur auf den Impulsoperator reflektierend wirkt und zusätzlich als 
anti-lineare Operation komplexe Konjugation nach sich zieht. Man spricht von $\mathcal{P} \mathcal{T}$-Symmetrie, wenn die Eigenfunktionen des $\mathcal{P} \mathcal{T}$-Operators den Eigenfunktionen des Hamilton-Operators $\mathcal{H}$ entsprechen. In diesem Fall sind auch die Eigenwerte reell. In Anlehnung an hermitesche Operatoren nennt man die zugehörigen Operatoren auch pseudo-hermitesch. Die von pseudo-hermiteschen Hamilton-Operatoren erzeugte Dynamik kann durch eine Ähnlichkeitstransformation auf eine unitäre Dynamik abgebildet werden, was zum Beispiel in der Optik die Beschränktheit der Intensität garantiert.

Aus der Wirkung des $\mathcal{P} \mathcal{T}$-Operators auf das Potenzial $V(r)$ des Hamilton-Operators lässt sich als notwendige Bedingung für $\mathcal{P} \mathcal{T}$-symmetrisch formulierte Probleme die Bedingung $V(\mathbf{r})=V^{*}(-\mathbf{r})$ ableiten, die jedoch keine hinreichende Bedingung darstellt. Als Konsequenz aus der Anti-Linearität des $\mathcal{P} \mathcal{T}$-Operators ergibt sich eine spontane Symmetriebrechung in Abhängigkeit von der Stärke des Imaginärteils des Potenzials [38, 39, 40]. In dieser Arbeit wird gezeigt, dass sowohl schwächste Unordnung als auch Systemgrenzen Ursachen für das Zusammenbrechen der $\mathcal{P} \mathcal{T}$-symmetrischen Phase darstellen, die jedwede physikalische Realisierung zu verhindern oder zumindest zu erschweren scheinen [3]. Dazu werden lokalisierte Zustände mit exponentiell kleiner Energieaufspaltung in den zugehörigen Eigenwerten untersucht und als Ursache der Symmetriebrechung identifiziert. Diese Erkenntnisse konnten wir jedoch nutzen, um Konfigurationen zu entwickeln, die selbst im Falle von Unordnung über eine stabile pseudo-hermitesche Phase verfügen [4]. Darüber hinaus werden Grundlagen zur Untersuchung von Transporteigenschaften mithilfe des Streuformalismus jenseits der paraxialen Näherung geschaffen und erste Ergebnisse diskutiert.

Im Detail gliedert sich der Aufbau der Arbeit wie folgt:

In Kapitel 1 wird eine stabile und effiziente numerische Methode entwickelt, die es ermöglicht, ausgehend von nicht-hermiteschen Hamiltonmatrizen zu Aussagen über makroskopische Transportgrößen zu gelangen. Hierzu wird gezeigt, wie die Greensche Funktion als Lösung der zeitunabhängigen Schrödinger-Gleichung zusammen mit dem Landauer-Büttiker-Formalismus genutzt werden kann, um eine effektive Implementation zur Berechnung der Transportkoeffizienten zu erhalten. Die hier dargestellte Methode beinhaltet die Berechnung von Streufunktionen, mit denen auch ein Zugriff auf Größen wie die lokale Zustandsdichte möglich wird. Anschließend wird die Methode zur Untersuchung des so genannten ballistischen Gleichrichters exemplarisch genutzt. Neben der hier gegebenen ausführlichen theoretischen Darstellung findet diese numerische Methode in der gesamten Arbeit breite Anwendung.

In Kapitel 2 wird ein neuartiges Konzept vorgestellt, aus den quantenmechanischen quasi-gebundenen Zuständen eines offenen Kosinus-Billards mit gemischtem Phasenraum unter Ausnutzung der zugrunde liegenden klassischen Phasenraumstruktur und mithilfe des dynamischen Tunneleffekts optische und elektronische Kavitäten wie Strahlenteiler, Schalter, Mikrolaser, Multi-Resonatoren, MultiStrahlenteiler und Kombinationen daraus zu konstruieren [1,2]. Der Streuformalismus stellt hier eine Möglichkeit dar, die benötigten Leitfähigkeiten und Verteilungsfunktionen des Phasenraums effektiv berechnen zu können. Insbesondere wird eine einfache Möglichkeit präsentiert, aus einem deformierten Wellenleiter einen leicht zu realisierenden Einmodenlaser hoher Güte zu konstruieren. 
Die Trichterform der Füße von Retina-Müllerzellen erinnert stark an die Form fokussierender Quantenpunktkontakte. Mit der Möglichkeit, den Lichttransport von Retina-Müllerzellen mittels des Streuformalismus zu untersuchen, wurde in Kapitel 3 der Fragestellung gefolgt, warum Photorezeptoren von Wirbeltieren auf der Rückseite der Retina angeordnet sind und das Licht somit zusätzliche, mit Streuverlusten verbundene, dichte Zellschichten durchlaufen muss. Dazu wird die Müllerzelle und ihre natürliche, im Brechungsindex fluktuierende Umgebung durch eine Kanalstruktur modelliert, die in ein ungeordnetes System eingebettet ist. In einem vereinfachten optischen Modell der Geometrie des Auges wird gezeigt, dass die durch die Müllerzellen verursachte Kleinwinkelfokussierung zu einer Reduktion des Streulichts in der näheren Umgebung der Fovea führt [5].

Mit möglichen neuartigen Anwendungen und ersten Experimenten in der Optik und Photonik wuchs in den letzten Jahren erheblich das Interesse an pseudo-hermiteschen und $\mathcal{P} \mathcal{T}$-symmetrischen Operatoren. Die dadurch ermöglichte Realisierung neuer optischer Metamaterialien macht die Entwicklung mathematischer und physikalischer Grundlagen zum Verständnis ihrer optischen Eigenschaften erforderlich. In Kapitel 4 werden fundamentale Ursachen der $\mathcal{P} \mathcal{T}$-Symmetriebrechung in ausgedehnten eindimensionalen und quasi-eindimensionalen Systemen untersucht und analytische Resultate abgeleitet [3]. Besondere Aufmerksamkeit wird auf Unordnung in $\mathcal{P} \mathcal{T}$-symmetrischen Systemen gelenkt, die in jedweder physikalischen Realisierung unvermeidbar ist. Dazu werden auch numerische Diagonalisierungen durchgeführt und gewonnene Erkenntnisse dargestellt und diskutiert. Die abgeleiteten elementaren Resultate werden genutzt, um Konfigurationen zu entwickeln, die sogar in Gegenwart von Unordnung eine stabile $\mathcal{P} \mathcal{T}$-symmetrische Phase aufweisen [4].

Ein Überblick über die wesentlichen in dieser Arbeit vorgestellten Ergebnisse ist in der Zusammenfassung gegeben.

Um den Lesefluss zu verbessern, befinden sich sowohl numerische und aufwendigere mathematische Erläuterungen, als auch technische Ergänzungen und andere detaillierte Ausführungen im Anhang dieser Dissertation. 


\section{Der Ballistische Gleichrichter}

Jetzt benützen die verdammten Göttinger meine schöne Wellenmechanik zur Ausrechnung ihrer Sch...-Matrixelemente!

Erwin Schrödinger [41]

Was Schrödinger über die Anschaulichkeit seiner Theorie schreibt... ich finde es Mist. Die grosse Leistung der Schrödingerschen Theorie ist die Berechnung der Matrixelemente.

Werner Heisenberg [41]

1.1 Einführung . . . . . . . . . . . . . . . . . . . . . . 9

1.1.1 Landauers und Büttikers Ansatz . . . . . . . . . . . . . . . . . 10

1.1.2 Greensche Funktion und Streumatrix . . . . . . . . . . . . . . . . . 13

1.1.3 Die lokale Zustandsdichte . . . . . . . . . . . . . . . . . 18

1.2 Symmetriebrechung und mesoskopischer Transport . . . . . . . . . . . . . . . 19

1.2.1 Semiklassische Betrachtung . . . . . . . . . . . . . . . . . . 19

1.2.2 Quantenmechanische Betrachtung . . . . . . . . . . . . 20

1.3 Zusammenfassung und Ausblick . . . . . . . . . . . . . . . . . . . . 24 


\section{Motivation}

Verbesserte Methoden der Epitaxie ermöglichen die Fertigung von Halbleiter-Heterostrukturen mit extrem hohen Beweglichkeiten bzw. großen mittleren freien Weglängen, während zugleich die hochauflösenden Lithographieverfahren zu ihrer fortschreitenden Miniaturisierung führt. Diese Bauelemente erlauben die experimentelle Untersuchung von Quanteneffekten, die in mesoskopischen ballistischen Systemen auftreten. Auf der theoretischen Seite ist die Entwicklung von Quantenratschen (quantum ratchets) [42] zu erwähnen, die z.B. von Linke et al. [43, 44] in Halbleiter-Heterostrukturen realisiert worden sind. Die Einfachheit, mit der beliebige Streugeometrien in Kreuzkontaktstrukturen (crossbar junction) realisiert werden können, ist dabei sicherlich eine treibende Kraft, die zum Verständnis von Phänomenen des Quantentransports in Systemen mit räumlich gebrochener Symmetrie beiträgt. Weiterhin sind die Arbeiten zum nichtlinearen Verhalten des $Y$-Verzweigers [45, 46] und die Untersuchung der Transporteigenschaften des ballistischen Gleichrichters [20, 47, 48, 49] zu nennen.

Der hier vorgestellte ballistische Gleichrichter in seiner recht unproblematischen experimentellen Realisierung nach Song et al. [20] ist ein häufig zitiertes Standardbeispiel einer Struktur mit räumlich gebrochener Symmetrie. Der Gleichrichter erregte starke Aufmerksamkeit, da die naive Anwendung des Landauer-Büttiker-Formalismus in diesem symmetriegebrochenen System zu einem scheinbaren Widerspruch zwischen Theorie und Experiment führte, den Fleischmann \& Geisel [21] erfolgreich zu lösen vermochten, indem sie eine mikroskopische Theorie aufstellten, die die Energieabhängigkeit der Modenzahl berücksichtigte. Der Gleichrichtungseffekt kann in dieser Weise durch ein Zusammenwirken von quasiklassischen (breiten) und quantisierten (schmalen) Transportkanälen erklärt werden, die sich unterschiedlich auf die chemischen Potenziale der angeschlossenen Reservoire auswirken. Des Weiteren schlugen sie einen optimierten Aufbau des Gleichrichters vor, der die Gleichrichtungsspannung um ein Vielfaches erhöhen würde und sagten einen möglichen Vorzeichenwechsel dieser Spannung voraus. Im Zuge einer angeregten Diskussion [50, 51] wurden auch Experimente zum "optimierten Gleichrichter" durchgeführt [52, 53], die vorausgesagte Eigenschaften bestätigten und die semiklassische Theorie des Transports in Systemen mit schmalen und breiten Kanälen erhärtete.

Die Berücksichtigung der exakten Geometrie der 2DEG Halbleiter-Heterostruktur gestaltete sich bisher als schwierig. Wir stellen hier eine effiziente und numerisch stabile Methode vor, mit der es möglich ist, zu einer beliebigen Geometrie einer Halbleiter-Heterostruktur - mithilfe der Greenschen Funktionen und durch Anwendung des Landauer-Büttiker-Formalismus - auf klassisch messbare Größen, wie Strom und Spannung zu schließen. Die Entwicklung der Methode folgt in den Grundzügen der recht allgemein gefassten Abhandlung zum mesoskopischen Transport von S. Datta [54], die entsprechend erweitert wird, um eine effektive numerische Implementation zu erlauben. Die in diesem Kapitel vorgestellte Methode kann auch als quantenmechanische Weiterentwicklung des semiklassischen Modells aus [21] verstanden werden. In diesem Kapitel wird sie auf den ballistischen Gleichrichter appliziert und findet darüber hinaus in der gesamten Arbeit breite Anwendung. 


\subsection{Einführung}

In herkömmlichen zweidimensionalen elektronischen Systemen finden wir mittlere freie Weglängen $l_{\mathrm{m}}$ vor, die viel kleiner als ihre Länge sind $\left(l_{\mathrm{m}} \ll L\right)$. Die Lösung der als Diffusionsgesetz formulierten Bewegungsgleichung des Drude-Sommerfeld-Modells liefert das bekannte Ohmsche Gesetz für den Leitwert

$$
G=\frac{I}{U}=\sigma \frac{W}{L}=e^{2} \frac{\tau n W}{L m}
$$

mit der Breite $W$ des Leiters und der elektrischen Leitfähigkeit $\sigma$, mittlerer Stoßzeit $\tau$, Ladungsträgerdichte $n$ und Elektronenmasse $m$. Was aber ist Ursache für den endlichen Leitwert in elektronischen Systemen, deren Abmessungen kleiner als die mittlere freie Weglänge sind $\left(L<l_{\mathrm{m}}\right)$ ? Die zum Transport beitragenden Elektronen erfahren keinerlei inelastische Streuung an Defekten oder Phononen und verhalten sich daher phasenkohärent. Die Ursache ist also in der elastischen Streuung zu suchen. Man findet eine Quantisierung des Stroms in den Zuleitungen in transversale Moden, wobei die Modenanzahl von der Breite abhängt. Auf den genauen Zusammenhang und damit auf die Beantwortung der oben gestellten Frage gehen wir später im Abschnitt über den Landauer-BüttikerFormalismus ausführlich ein. In der Halbleiterphysik nutzt man den Fortschritt der epitaktischen Verfahren zur Generierung zweidimensionaler Elektronengase (2DEG), indem man auf intrinsisches GaAs (kleine Bandlücke und niedrige Fermi-Energie ${ }^{1}$ ) mehrere Lagen $n$-dotiertes AlGaAs (große Bandlücke und höhere Fermi-Energie) aufwächst, z.B. mittels Molekularstrahlepitaxie. An ihrer gemeinsamen Kontaktstelle findet ein Ladungsträgeraustausch statt, der aufgrund des sich einstellenden Ladungsträgergleichgewichts zur Akkommodation der Fermi-Niveaus führt. Die zugehörigen elektrostatischen Potenziale, definiert durch feststehende Banddiskontinuitäten $\Delta E_{\mathrm{L}}$ und $\Delta E_{\mathrm{V}}$, deformieren die Energiebänder und verursachen eine scharfe Absenkung des gemeinsamen Leitungsbandes in der Nähe der Grenzfläche, das energetisch teilweise unterhalb der Fermi-Energie liegt (siehe Abb. 1.1). Die hohe Beweglichkeit der Ladungsträger kann mittels einer Modulationsdotierung (modulation doping) erreicht werden, also durch räumliche Trennung der Dotieratome von dem 2DEG, wodurch die Elektro-

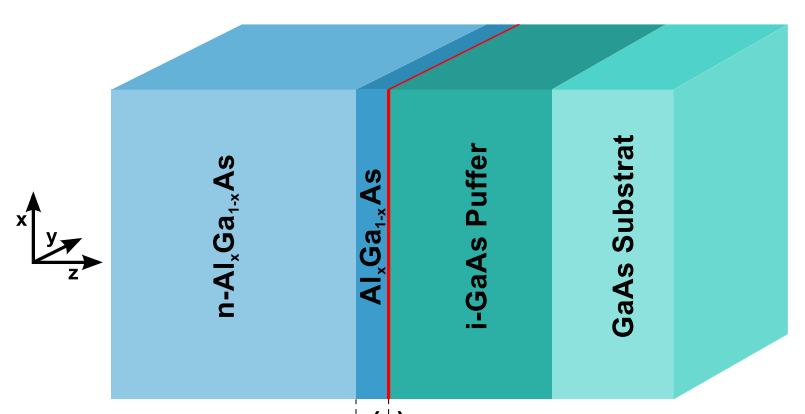

(a)

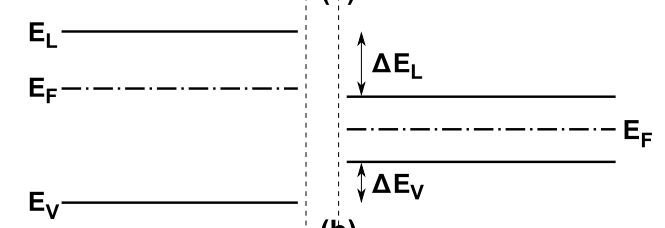

(b)

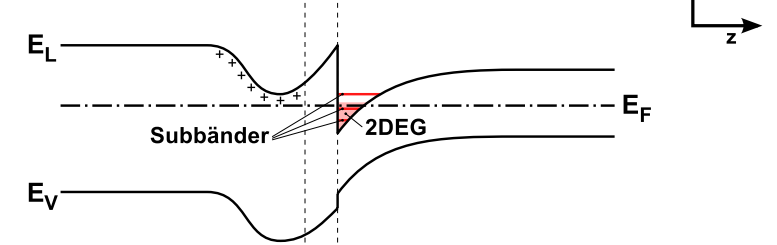

(c)

Abbildung 1.1.: Zur Entstehung des 2DEG. (a) 3D Abbildung einer Heterostruktur bestehend aus einigen $\mathrm{nm}$ dicken undotierten Schicht und einer $n$-dotierten Schicht $\mathrm{Al}_{\mathrm{x}} \mathrm{Ga}_{1-\mathrm{x}} \mathrm{As}$ auf einem intrinsischen GaAs-Puffer und GaAs-Substrat. (b) Energieniveaus des Leitungsbandes mit Energie $E_{\mathrm{L}}$ und des Valenzbandes $E_{\mathrm{V}}$, sowie verschiedenen Fermi-Energien $E_{\mathrm{F}}$ vor und (c) nach dem Ladungsträgeraustausch. Das 2DEG existiert nur in der $x$-y-Ebene an der Kontaktfläche der beiden Halbleiterkristalle.

1 Als Fermi-Energie wird das elektrochemische Potenzial $\mu$ bei $T=0 \mathrm{~K}$ bezeichnet. 
nenstreuung an den ionisierten Dotieratomen stark vermindert wird [55]. Damit ergeben sich mittlere freie Weglängen, die viel größer (Größenordnung $\mu \mathrm{m}$ ) als die Ausdehnung des Bauelements sein können. Diese quasi-freien Ladungsträger bewegen sich damit nicht länger diffusiv, sondern können sich im Fall $\left(l_{\mathrm{m}} \gg \mathrm{L}\right)$ ballistisch im 2DEG bewegen. Mithilfe von verschiedenen Verfahren, z.B. Lithographie, können metallische Kontakte auf die Heterostrukturen aufgebracht werden, wobei die Elektronen durch an diese Kontakte angelegte Spannungen auf bestimmte Gebiete beschränkt werden, an deren Rändern die Elektronen elastisch stoßen (siehe auch Abschnitt 2.1 auf Seite 27). Auf diese Weise ist es möglich, nahezu beliebige zweidimensionale berandete Gebiete (Quantenbillards) herzustellen und die Dynamik der Elektronen darin experimentell zu untersuchen. Das Fehlen inelastischer Stöße zieht eine, in Abhängigkeit von der Beschaffenheit des 2DEG und der Ladungsträgerdichte, sehr hohe Beweglichkeit nach sich, was kleine Schaltzeiten zulässt und hohe Strombelastbarkeiten durch die geringen Leistungsverluste ermöglicht. Moderne GaAs/AlGaAs-Heterostrukturen können Beweglichkeiten bis $10^{7} \mathrm{~cm}^{2} \mathrm{~V}^{-1} \mathrm{~s}^{-1}$ bei Elektronendichten bis $10^{12} \mathrm{~cm}^{-2}$, nahe dem theoretisch berechneten Maximum, erreichen $[56,57]$. Die Entwicklung neuer Heterostrukturen und neue Herstellungverfahren tragen überdies zur Miniaturisierung bei. Die Gesamtheit der aufgezählten Eigenschaften eröffnet also interessante Anwendungen in der Halbleitertechnologie.

Eine weitere Größe zur Klassifikation von Systemen ist die Phasenkohärenzlänge $l_{\varphi}$, die eine weitere Längenskala definiert. Ist sie kleiner als die Systemlänge $\left(l_{\varphi}<L\right)$ so spricht man von mesoskopischen Systemen. Die Eigenschaften dieser Systeme sind bereits seit den 80er Jahren Gegenstand der Forschung. Seitdem sind bemerkenswerte Phänomene, wie der nichtverschwindende Widerstand in ballistischen Wellenleitern [8] oder die Quantisierung der transversalen Moden in Abhängigkeit von der Kanalbreite $W$ [9], auf die wir später noch genauer eingehen werden, der Quanten-Hall-Effekt [10], schwache Lokalisierung [11, 12], universelle Leitwertfluktuationen [13, 14, 15] u.v.a. entdeckt worden. Diese Beispiele zeugen vom großen Interesse für diese Systemklasse.

\subsubsection{Landauers und Büttikers Ansatz}

Wie eingangs erwähnt folgt der Leitwert auf kleinen Skalen nicht mehr dem Ohmschen Gesetz (1.1). Stattdessen findet man zwei Besonderheiten: Erstens wird der Grenzflächenwiderstand unabhängig von der Länge $L$ der Probe. Zweitens skaliert der Leitwert mit der Probenbreite $W$ nicht mehr linear. R. Landauer [18] und - einige Jahre später - M. Büttiker [19] entwickelten einen Ansatz zur Berechnung von Strömen und Spannungen zwischen Kontakten, indem sie annahmen, dass zwar in mesoskopisch aufzufassenden Systemen selbst keine inelastischen Stöße stattfinden, jedoch sehr wohl in den Zuleitungen Dissipation herrscht, und dass der elektrische Widerstand solcher Systeme eine Konsequenz der elastischen Streuung der Elektronen innerhalb der Probe ist. In diesem Formalismus werden die makroskopischen Zuleitungen als eine Kombination aus idealem rückstreuungsfreiem Wellenleiter und Reservoir angenommen, wobei ein Teilchenreservoir $p$ durch sein chemisches Potenzial $\mu_{p}$ und seine Temperatur $T_{p}$ charakterisiert ist (siehe Abb. 1.2 auf der nächsten Seite). Eine zwischen zwei Kontakten $p$ und $q$ angelegte Spannung entspricht damit der Differenz der chemischen Potenziale $\mu_{p}-\mu_{q}$ multipliziert mit der Elementarladung $e$. Die eigentliche Verbindung zwischen Reservoir $p$ und Probe besteht aus einem idealen Elektronenwellenleiter der Breite $W_{p}$, indem keinerlei inelastische Streuung auftritt. Aus den Reservoirs werden nun Elektronenwellen mit einer gewissen Wahrscheinlichkeit in alle Anschlussleitungen gestreut, wobei auch Rückreflexionen in die Anschlussleiter zugelassen werden. 
Die Elektronen erfahren in den Reservoirs inelastische Streuung und verlieren vollständig ihre Phasenkohärenz, wobei sie nach der Relaxation einem erneuten Transportprozess zur Verfügung stehen.

Beginnen wir mit dem einfachen Fall von zwei Anschlussleitern, die an einer Probe angebracht sind. Bei tiefen Temperaturen $T \rightarrow 0$ ist der einlaufende Strom bei einer zwischen Kontakt 1 und 2 angelegten Spannung $V=e\left(\mu_{1}-\mu_{2}\right)$ aus Leiter 1 in die Probe

$$
I_{1}^{+}=\frac{2 e}{h} M\left(\mu_{1}-\mu_{2}\right)
$$

nur abhängig von der Spannung und der Modenzahl $M$ des Leiters 1, die als konstant angenommen werden kann. Für den aus der Probe in Leiter 2 auslaufenden Strom gilt $I_{2}^{-}=I_{1}^{+} T$, wobei $T_{21}$ die Wahrscheinlichkeit angibt, dass Elektronen von Leiter 1 nach Leiter 2 gelangen. Wegen der Energieerhaltung ist der in den Leiter 1 zurückgestreute An-

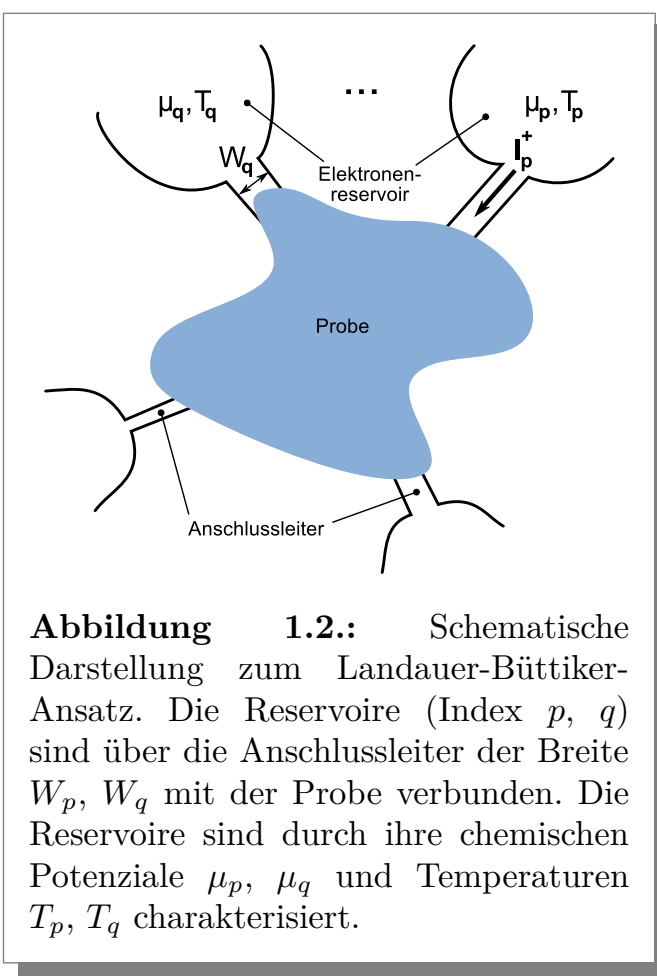
teil $R=1-T$, daher gilt $I_{1}^{-}=I_{1}^{+}(1-T)$. Aus der Berechnung des Nettostroms $I=I_{1}^{+}-I_{1}^{-}$folgt damit

$$
I=\frac{2 e}{h} M T\left(\mu_{1}-\mu_{2}\right)
$$

und wegen $G=\frac{I}{V}=e \frac{I}{\mu_{1}-\mu_{2}}$ gilt

$$
G=\frac{2 e^{2}}{h} M T
$$

Die als Landauer Formel bekannte Gleichung (1.3) verknüpft also die quantenmechanische Transmissionswahrscheinlichkeit $T$ mit der makroskopischen Größe der Leitfähigkeit.

Büttiker hat diese Betrachtungsweise auf Mehrfachanschlüsse an einer Probe erweitert (siehe Abb. 1.2). Für Temperaturen $T \rightarrow 0$ ergibt sich für den Nettostrom in Kontakt $p$

$$
I_{p}=\frac{2 e}{h} \sum_{q} \bar{T}_{q p} \mu_{p}-\bar{T}_{p q} \mu_{q}=\sum_{q} G_{q p} V_{p}-G_{p q} V_{q}
$$

Damit sind Strom und Spannung über die Koeffizienten der Leitfähigkeit

$$
G_{p q}=\frac{2 e^{2}}{h} \bar{T}_{p q}
$$

verknüpft, wobei $\bar{T}_{p q}$ die Transportkoeffizienten von Leiter $q$ nach Leiter $p$ sind, die aus dem Produkt von Modenzahl und Transmissionswahrscheinlichkeit pro Mode entstehen. Die Transmissionskoeffizienten

$$
\bar{T}_{p q}=\sum_{m, n}\left|s_{n m}^{p q}\right|^{2}
$$

lassen sich aus den Matrixelementen der Streumatrix $S=\left\{s_{m n}^{p q}\right\}$ bestimmen, die als im Allgemeinen komplexe Transmissions- und Reflexionsamplituden der Moden $m, n$ der Leiter $p, q$ berechnet werden können. 
Zusätzlich gelten folgende Symmetriebeziehungen und Summenregeln. Die Energieerhaltung (1. Kirchhoffsche Regel) $\sum_{p} I_{p}=0$ findet sich in der Unitarität von $S$ wieder, so dass als Bedingung an die Transmissionskoeffizienten

$$
\sum_{p} \bar{T}_{p q}(E)=\sum_{p} \bar{T}_{q p}(E)=M_{q}(E)
$$

gilt. Somit kann Gleichung (1.4) als lineare Gleichung

$$
I_{p}=\frac{2 e}{h} \sum_{q} \bar{T}_{p q}\left(\mu_{p}-\mu_{q}\right)
$$

zwischen Transmissionskoeffizienten und chemischen Potenzialen geschrieben werden.

Der Formalismus definiert weiterhin zwei Arten von Kontakten. Stromkontakte, zwischen denen entweder ein konstanter Strom fließt oder ein $\Delta \mu$ festgelegt wird - und Spannungskontakte, zwischen denen eine Spannung gemessen wird, wobei kein Strom zwischen den Spannungskontakten fließt und die chemischen Potenziale selbstkonsistent bleiben.

Die Beziehungen zwischen dem aus Anschlussleiter $p$ in die Probe einlaufenden Strom $I_{p}^{+}$bzw. dem aus der Probe in die Anschlussleiter $p$ auslaufenden Strom $I_{p}^{-}$und den chemischen Potenzialen der Reservoire im thermischen Gleichgewicht lautet in einer allgemeineren Darstellung für Temperaturen $T \neq 0[54]$

$$
\begin{aligned}
& I_{p}^{+}=\frac{2 e}{h} \int M_{p}(E) f_{p}(E) d E \\
& I_{p}^{-}=\frac{2 e}{h} \int \sum_{q} \bar{T}_{p q}(E) f_{q}(E) d E
\end{aligned}
$$

wobei die Indices $p, q$ die Anschlussleiter und $\bar{T}_{p q}(E)$ die Transmissionskoeffizienten vom Leiter $q$ in Leiter $p$ bezeichnen und

$$
M_{p}=\sum_{n} \Theta\left(E-\varepsilon_{p, n}\right)
$$

die Anzahl der zum Stromfluss beitragenden transversalen Moden mit den Eigenenergien $\varepsilon_{p, n}$ des idealen Leiters $p$ bezeichnet. Die Funktion $f_{p}(E)$ ist die Fermi-Verteilungsfunktion

$$
f_{p}(E)=\frac{1}{\exp \left(\frac{E-\mu_{p}}{k_{\mathrm{B}} T}\right)+1}
$$

die in Abhängigkeit der Temperatur $T$ und des chemischen Potenzials $\mu_{p}$ des Reservoirs die Wahrscheinlichkeit angibt, Elektronen zur Energie $E$ auszuwählen. Sind die Temperaturen klein gegenüber der Fermi-Energie $\left(k_{\mathrm{B}} T \ll E_{\mathrm{F}}\right)$, so geht die Fermi-Funktion in die Stufenfunktion $\Theta\left(\mu_{p}-E\right)$ über, so dass sich für eine Stromquelle der Nettostrom

$$
I_{p, \text { Quelle }}=I_{p}^{+}-I_{p}^{-}=\frac{2 e^{2}}{h}\left(\left[M_{p}\left(E_{\mathrm{F}}\right)-\bar{R}_{p}\left(E_{\mathrm{F}}\right)\right] V_{p}-\sum_{q \neq p} \bar{T}_{p q}\left(E_{\mathrm{F}}\right) V_{q}\right)
$$

ergibt, wobei $\bar{R}_{p}=\bar{T}_{p p}$ den Reflexionskoeffizienten des Leiters und $V_{p}=\frac{\mu_{p}}{e}$ die elektrische Spannung angibt. $^{2}$ Diese Gleichung ist auch unter dem Namen Landauer-Büttiker-Formel bekannt. Für eine Stromsenke gilt der Energieerhaltung entsprechend $I_{p, \text { Senke }}=-I_{p, \text { Quelle. Für eine Spannungsmessung }}$ gilt entsprechend, dass der Nettostrom wegen $I_{p}^{+}=I_{p}^{-}$verschwinden muss.

2 Unter der Annahme, dass die Transmissionswahrscheinlichkeiten $T_{p q}$ energie- und modenunabhängig sind, können wir die Koeffizienten als $\bar{T}_{p q}(E)=T_{p q} M_{q}(E)$ ausdrücken, so dass $I_{p}^{-}=\sum_{q} T_{p q} I_{p}^{+}$gilt. 
Eine weitere wichtige Eigenschaft, die sich aus dem Landauer-Büttiker-Formalismus ableiten lässt, ist die Onsager-Casimir-Reziprozitätsbeziehung [58]

$$
R_{i j, k l}(B)=R_{k l, i j}(-B)
$$

die als Folge der Zeitumkehrinvarianz gelten muss. Hierbei ist $R_{i j, k l}=\frac{V_{k l}}{I_{i j}}$ eine abkürzende Schreibweise für eine Vierpunktmessung des Widerstands, bei der der Strom vom Kontakt $j \rightarrow i$ fließt und die Spannung zwischen $l \rightarrow k$ gemessen wird. Im magnetfeldfreien Fall $(B=0)$ gilt dann die Symmetriebeziehung

$$
R_{i j, k l}=R_{k l, i j},
$$

was der Invarianz gegenüber Vertauschung von Stromzufuhr und Spannungsmessung über je zwei Anschlussleiter der Probe entspricht.

\subsubsection{Greensche Funktion und Streumatrix}

Wir wissen nun, wie man die makroskopischen Transportgrößen (Strom und Spannung) aus den mikroskopischen Streugrößen (Transmissions- und Reflexionskoeffizienten) berechnet. Diese Koeffizienten sind nach Gleichung (1.6) als modenbezogene Summen über die Elemente der S-Matrix darstellbar. Die Streumatrix $S$ enthält alle Informationen über Amplituden- und Phasenbeziehungen der ein- und auslaufenden Wellen als Folge des sich einstellenden Gleichgewichtszustands, der in den Anschlussleitern der Probe zu erwarten ist, wenn eine Anregung eines beliebigen Anschlussleiters der Probe erfolgt. In diesem Unterabschnitt zeigen wir, dass die Greenschen Funktionen eine geeignete Möglichkeit bieten, die benötigten Matrixelemente von $S$ mathematisch zu formulieren. Der Hamilton-Operator $\mathcal{H}$ wird dazu in eine diskrete Darstellung überführt. Außerdem bietet diese Methode Zugriff auf die quantenmechanischen Streuwellenfunktionen, also auf die Wellenfunktionen an beliebiger Stelle innerhalb oder außerhalb der Probe, mit denen sich beispielsweise die Zustandsdichte berechnen lässt. Diese Ausführungen zeigen wir im nächsten Unterabschnitt.

Auch hier wollen wir uns auf wechselwirkungsfreien Transport beschränken. Daher sind kleine Ströme und tiefe Temperaturen vorausgesetzt. Schauen wir uns zuerst das Problem der Probe ohne Anschlussleiter an. In diesem Fall kann der Hamilton-Operator einer Probe als

$$
\mathcal{H}_{\mathrm{P}}(\mathbf{r})=\frac{(i \nabla+\mathbf{A})^{2}}{2}+V_{\mathrm{el}}(\mathbf{r})
$$

mit dem allgemeinen Vektorpotenzial $\mathbf{A}$ mit $\mathbf{B}=\nabla \times \mathbf{A}$ und beliebigem elektrischen Potenzial $V_{\mathrm{el}}(\mathbf{r})$ geschrieben werden (siehe Anhang B). Die Lösung der Differenzialgleichung

$$
\left[E-\mathcal{H}_{\mathrm{P}}(\mathbf{r})+i \eta\right] G^{\mathrm{R}}\left(r, r^{\prime}\right)=\delta\left(r, r^{\prime}\right)
$$

liefert die zugehörige retardierte Greensche Funktion, die als kontinuierliche Antwortfunktion auf eine punktförmige Anregung angesehen werden kann. ${ }^{3}$ In einer diskreten Darstellung geht Gleichung (1.17) in eine Matrixgleichung über

$$
\left[(E+i \eta) \mathbb{1}-\mathbb{H}_{\mathrm{P}}\right] \mathbb{G}^{\mathrm{R}}=\mathbb{1}
$$

3 Die zugehörige avancierte Greensche Funktion $G^{\mathrm{A}}=\left[E-\mathcal{H}_{\mathrm{P}}(\mathbf{r})-i \eta\right]^{-1}$ als formale Lösung propagiert die Inhomogenität als Empfänger, weshalb hier nur die retardierte Greensche Funktion vorgestellt wird. 
Eine Diskretisierung des Raumes verlangt natürlich auch nach einer diskreten Darstellung des HamiltonOperators $\mathcal{H}_{\mathrm{P}}$. Als Matrixdarstellung des Hamilton-Operators in 1D oder 2D wählen wir die Methode der finiten Differenzen auf einem Quadratgitter, so dass in kompakter Darstellung gilt

$$
\mathbb{H}_{i j}=\left(V_{i}+2 D t\right) \delta_{i, j}-t_{i j}\left(\delta_{i+1, j}+\delta_{i-1, j}+(D-1)\left[\delta_{i, j+1}+\delta_{i, j-1}\right]\right),
$$

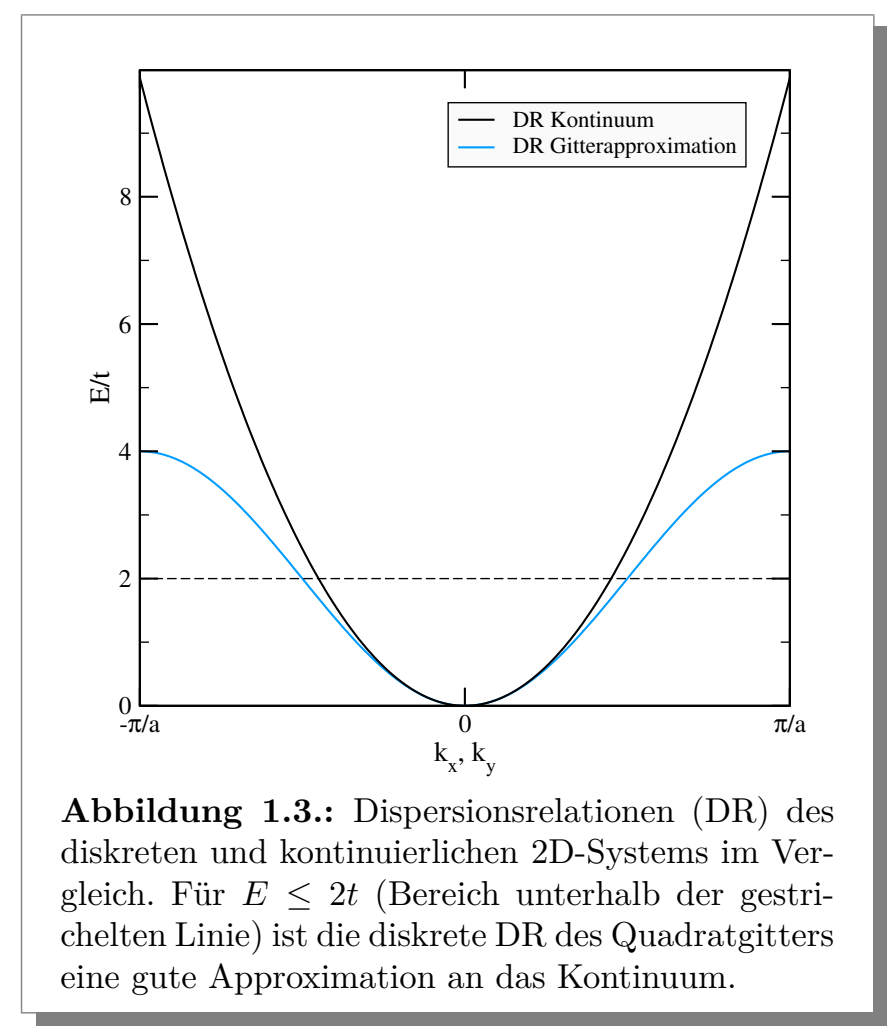

wobei $D$ die Dimension des Gitters bestimmt, $V_{i} \equiv V_{\mathrm{el}}\left(\mathbf{r}_{i}\right)$ das Potenzial am Gitterplatz $i$ definiert und

$$
t_{i j}=t \exp \left(i \mathbf{A} \cdot\left[\mathbf{r}_{i}-\mathbf{r}_{j}\right]\right)
$$

mit $t=\frac{1}{2 a^{2}}$ die Austauschenergie (Hüpfenergie) zweier benachbarter Gitterplätze unter dem Einfluss des Vektorpotenzials A ist, dass an der Stelle $\frac{\mathbf{r}_{i}+\mathbf{r}_{j}}{2}$ ausgewertet werden muss [59]. Die Gitterkonstante $a$ ist definiert als der Abstand der Gitterplätze der linearen Kette $(D=1)$ bzw. der Abstand zum nächsten Nachbarn auf dem Quadratgitter $(D=2)$. Unter der Voraussetzung $B=0$ ist die Austauschenergie $t_{i j}=t$ konstant. Für ein freies Teilchen $\left(V_{\mathrm{el}}\left(r_{i}\right)=\right.$ const $)$ ergibt sich dann als Konsequenz der Diskretisierung eine modifizierte Dispersionsrelation

$$
E=\left\{\begin{array}{ll}
V_{0}+2 t(1-\cos (k a)) & D=1 \\
V_{0}+2 t\left(2-\cos \left(k_{x} a\right)-\cos \left(k_{y} a\right)\right) & D=2
\end{array},\right.
$$

die im Vergleich mit der Dispersionsrelation freier Teilchen im Kontinuum $E(\mathbf{k})=\frac{\mathbf{k}^{2}}{2}$ für $E \leq D t$ eine gute Näherung darstellt (siehe Abbildung 1.3). Wir wollen das geschlossene System erweitern und mit Anschlussleitern versehen, die durch den Hamilton-Operator $\mathcal{H}_{\mathrm{L}}$ verkörpert werden. Der HamiltonOperator des offenen Gesamtsystems lautet

$$
\mathcal{H}=\mathcal{H}_{\mathrm{P}} \oplus \mathcal{H}_{\mathrm{L}}
$$

mit der Greenschen Funktion in Matrixdarstellung als Lösung

$$
\mathbb{G}^{\mathrm{R}}=[(E+i \eta) \mathbb{1}-\mathbb{H}]^{-1} .
$$

Hier ergibt sich vorerst ein Widerspruch, denn in der Matrixdarstellung der allgemeinen Lösung sind wir auf endliche Systeme beschränkt. Der Hamilton-Operator der Probe $\mathcal{H}_{\mathrm{P}}$ ist zwar endlich, jedoch ist der Hamilton-Operator $\mathcal{H}_{\mathrm{L}}$ der halb-unendlichen Anschlussleiter unendlichdimensional. Eine künstliche Beschränkung des Systems durch "Abschneiden" der Leiter weit entfernt von der Probe stellt leider keine geeignete Möglichkeit dar, denn es ergeben sich falsche Lösungen aufgrund der Randeffekte, da wir in diesem Näherungsfall wieder nur ein geschlossenes System mit perfekt reflektierenden Wänden beschreiben würden. Um das offene, unendlich formulierte Problem der zusammengesetzten Hamiltonians [60] aus Gleichung (1.22) zu lösen, müssen wir die Matrix 


$$
\mathbb{G} \equiv\left(\begin{array}{cc}
\mathbb{G}_{\mathrm{L}} & \mathbb{G}_{\mathrm{LP}} \\
\mathbb{G}_{\mathrm{PL}} & \mathbb{G}_{P}
\end{array}\right)=\left(\begin{array}{cc}
(E+i \eta) \mathbb{1}-\mathbb{H}_{\mathrm{L}} & \tau_{\mathrm{L}} \\
\tau_{\mathrm{L}}^{+} & (E+i \eta) \mathbb{1}-\mathbb{H}_{\mathrm{P}}
\end{array}\right)^{-1}
$$

bestimmen, wobei $\mathbb{G}_{\mathrm{L}}$ die Matrixdarstellung der retardierten Greenschen Funktion der Anschlussleiter und $\mathbb{G}_{P}$ die der Probe ist. Die Matrizen $\mathbb{G}_{P L}, \mathbb{G}_{\mathrm{LP}}$ ergeben sich durch die Übergänge von der Probe zum Leiter und umgekehrt. Die Kopplungsmatrizen

$$
\tau_{\mathrm{L}}^{(+)}\left(p_{i}, i\right)=t
$$

sind Hilfsmatrizen, die an der Übergangsstelle zwischen Leiter und Probe den Beitrag $t$ liefern und sonst Null sind (siehe Abbildung 1.4). Aus $\mathbb{G}^{-1} \mathbb{G}=\mathbb{1}$ können wir mittels zwei der vier Gleichungen

$$
\begin{aligned}
& {\left[(E+i \eta) \mathbb{1}-\mathbb{H}_{\mathrm{L}}\right] \mathbb{G}_{\mathrm{LP}}+\left[\tau_{\mathrm{L}}\right] \mathbb{G}_{P}=0} \\
& {\left[(E+i \eta) \mathbb{1}-\mathbb{H}_{\mathrm{P}}\right] \mathbb{G}_{\mathrm{P}}+\left[\tau_{\mathrm{L}}^{+}\right] \mathbb{G}_{L P}=\mathbb{1}}
\end{aligned}
$$

die Größe $\mathbb{G}_{\mathrm{LP}}=-g_{\mathrm{L}} \tau_{\mathrm{L}} \mathbb{G}_{\mathrm{P}}$ eliminieren, wobei $g_{\mathrm{L}}=\left[(E+i \eta) \mathbb{1}-\mathbb{H}_{\mathrm{L}}\right]^{-1}$ die Matrixdarstellung der Greenschen Funktion eines einzelnen halb-unendlichen Leiters definiert. Durch Einsetzen erhalten wir die Gleichung

$$
\mathbb{G}_{\mathrm{P}}=\left[E \mathbb{1}-\mathbb{H}_{\mathrm{P}}-\tau_{\mathrm{L}}^{+} g_{\mathrm{L}} \tau_{\mathrm{L}}\right]^{-1}
$$

als Matrixdarstellung der Greenschen Funktion der Probe und können die analytische Darstellung der Greenschen Funktion eines halb-unendlichen Anschlusses

$$
g_{\mathrm{L}}\left(p_{i}, p_{j}\right)=-\frac{1}{t} \sum_{n} \chi_{n}\left(p_{i}\right) \exp \left(i k_{n} a\right) \chi_{n}\left(p_{j}\right)
$$

mit den transversalen Moden $\chi_{n}\left(p_{i}\right)$ für den magnetfeldfreien Fall $(B=0)$ angeben, die z.B. aus der numerischen Lösung von Gleichung (1.19) für $D=1 \mathrm{zu}$ einem beliebigen Leiterpotenzial $V_{i}$ gewonnen werden können. Eine häufig benutzte Approximation für transversale Moden von HalbleiterAnschlussleitungen ist in Anhang $\mathrm{C}$ angegeben. Unter der Annahme, dass die Anschlussleiter unabhängig voneinander sind, können wir ihre einzelnen Beiträge aufaddieren

$$
\Sigma^{\mathrm{R}}=\sum_{l}\left[\tau_{l} g_{1} \tau_{l}^{+}\right]=t^{2} \sum_{l} g_{l}\left(p_{i}, p_{j}\right)
$$

und erhalten insgesamt

$$
\mathbb{G}^{\mathrm{R}}=\left[E \mathbb{1}-\mathbb{H}_{\mathrm{C}}-\Sigma^{\mathrm{R}}\right]^{-1}
$$

Der effektive Hamiltionian $\Sigma^{\mathrm{R}}$ enthält nun die Wechselwirkungsterme der Probe mit den Anschlussleitern und wird daher als Selbstenergie bezeichnet.

Der noch ausstehende funktionale Zusammenhang zwischen Streumatrix und Greenscher Funktion kann aus [61] übernommen werden. Für die Greensche Funktion in kontinuierlicher Darstellung können wir demnach schreiben

$$
G_{q p}^{\mathrm{R}}\left(y_{q} ; y_{p}\right)=\sum_{m \in p} \sum_{n \in q}\left(\delta_{n m} A_{m}^{<}+\sqrt{\frac{\nu_{m}}{\nu_{n}}} s_{n m} A_{m}^{>}\right) \chi_{n}\left(y_{q}\right)
$$

wobei $G_{q p}^{\mathrm{R}}\left(y_{q} ; y_{p}\right) \equiv G^{\mathrm{R}}\left(x_{q}=0, y_{q} ; x_{p}=0, y_{p}\right)$ die Greensche Funktion zwischen einem Punkt der Ebene $x_{p}=0$ und einem anderen Punkt der Ebene $x_{q}=0$ bezeichnet (siehe Abbildung 1.4) und $A_{m}^{<}=A_{m}^{>}=-\frac{i}{\nu_{m}} \chi_{m}\left(y_{p}\right)$ die komplexen Amplituden der retardierten Greenschen Funktionen für einen 


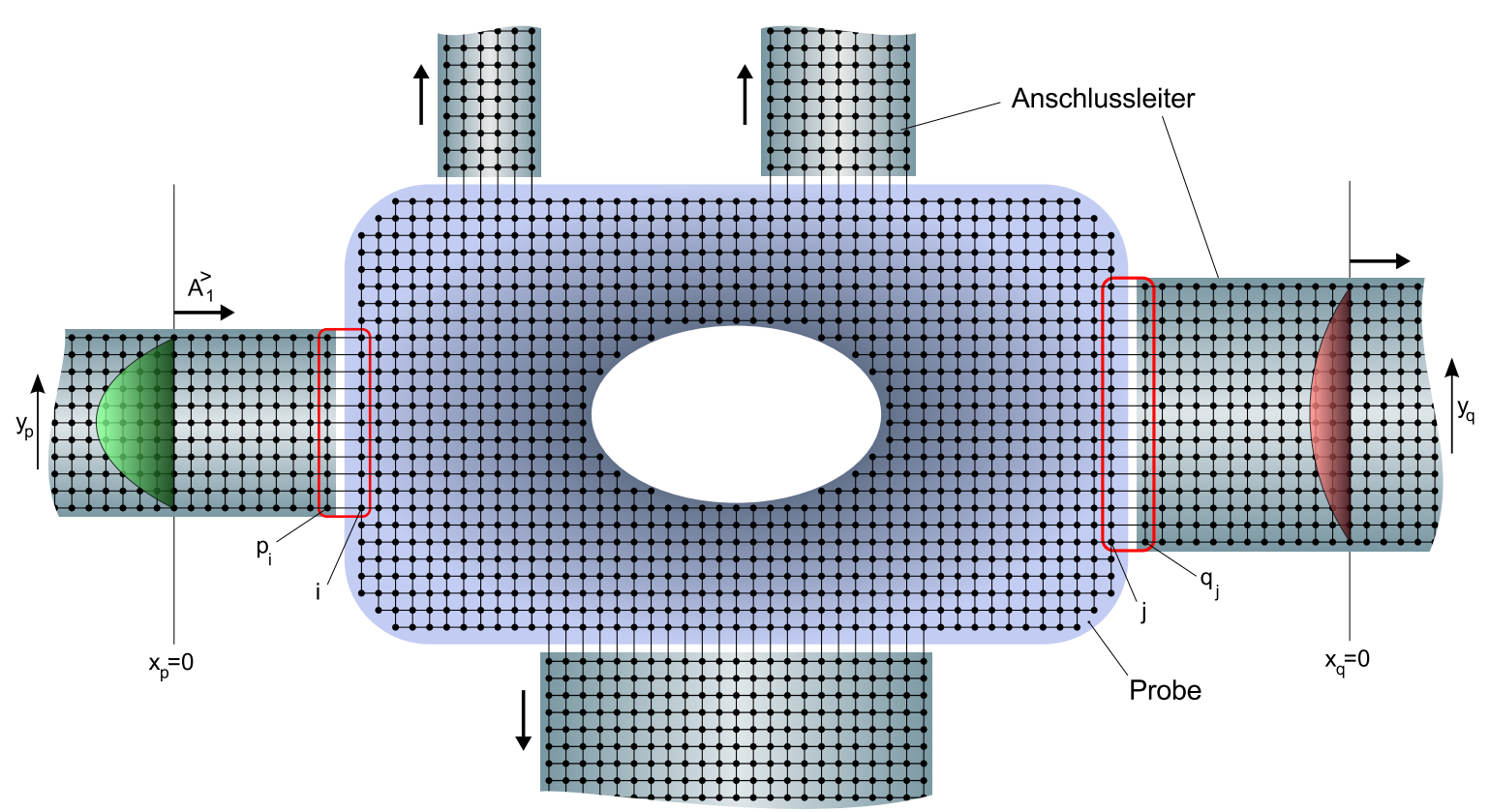

Abbildung 1.4.: Schematische Darstellung zur Diskretisierung einer Probe (mit Loch) und mehreren Anschlussleitern. Die Wahl einer kleineren Gitterkonstante approximiert die Form der Probe und ihr elektrisches Potenzial $V(x, y)$ (dargestellt durch den hell- bis dunkelblauen Farbverlauf) zwar besser, jedoch steigt die Matrixgröße $\left[\mathbb{G}_{q p}^{\mathrm{R}}\right]^{-1}$ und damit die Rechenzeit $\propto \mathcal{O}\left[W^{2} L^{2}\right]$. Die Kopplungen für zwei der Anschlussleiter sind durch rote Rechtecke kenntlich gemacht, wobei immer Gitterplatz $i$ der Probe mit Gitterplatz $p_{i}$ des Leiters $p$ und Gitterplatz $j$ der Probe mit Gitterplatz $p_{j}$ des Leiters $j$ verbunden ist. Die Kopplung ist schwach, wenn entweder der Anschlussleiter viel schmaler im Vergleich zur Dimension der Probe ist, oder das Potenzial des Anschlussleiters viel kleiner als das der Probe ist (hohe Potenzialstufe). Eine einfallende Welle (der transversale Anteil der 1 . Mode ist schematisch in grün dargestellt) mit der komplexen Amplitude $A_{m}^{>}$wird durch Anschlussleiter $p$ mit harmonischem Potenzial (Farbverlauf) in die Probe geleitet, wobei Anteile der Welle mit einer von Form und Potenzial der Probe bestimmten Wahrscheinlichkeit in alle erlaubten Moden aller Anschlussleiter streuen (hier schematisch durch den transversalen Anteil der 1.Mode in Leiter $q$ in rot dargestellt). Die Greensche Funktion (GF) von Leiter $p$ nach $q$ ist dann für alle $y_{p}, y_{q}$ zwischen den Ebenen $x_{p}=0$ und $x_{q}=0$ definiert. Im Formalismus geht die GF der Anschlussleiter als Selbstenergie in die GF der Probe ein (siehe Text).

idealen Mehrfachmoden-Anschlussleiter als Reaktion auf eine Anregung bei $\mathbf{r}=\mathbf{r}^{\prime}$ darstellen. Die Größe $A_{m}^{<}$bezeichnet die Amplitude der propagierenden Antwortfunktion in Ausbreitungsrichtung $x<x^{\prime}$, entsprechend gilt die Bezeichnung $A_{m}^{>}$für $x>x^{\prime}$. Der Korrekturfaktor $\sqrt{\frac{\nu_{m}}{\nu_{n}}}$ ergibt sich aus dem Umstand, dass der Strom als Quadrat der Wellenfunktion multipliziert mit der Geschwindigkeit der zugehörigen Welle definiert ist. Die Amplitude des Stroms muss daher mit der Wurzel der Geschwindigkeit multipliziert werden (siehe z.B. §144 Die Streumatrix bei Reaktionen [62]). Die Geschwindigkeiten der einzelnen Moden in Ausbreitungsrichtung der Welle ergeben sich aus der Ableitung der Dispersionsrelation für das Kontinuum

$$
\nu_{n}=\frac{\partial E_{n}}{\partial k}=k_{n}
$$

Für den diskreten Fall der linearen Kette mit Gitterkonstante $a$ ergibt sich durch partielle Ableitung von Gleichung (1.21) nach $k$

$$
\nu_{n}=\frac{1}{a} \sin \left(k_{n} a\right)
$$

Unter Benutzung der Orthogonalitätsrelation für die transversalen Moden (siehe Anhang Glg. (C.10)) finden wir durch Multiplikation mit $\chi_{m}\left(y_{p}\right)$ von rechts und $\chi_{n}\left(y_{q}\right)$ von links und Integration über $y_{p}$ 
und $y_{q}$ die Fisher-Lee-Relation [61]

$$
s_{n m}^{p q}=-\delta_{n m} \delta_{p q}+i \sqrt{\nu_{n} \nu_{m}} \iint \chi_{n}\left(y_{q}\right) G_{q p}^{\mathrm{R}}\left(y_{q} ; y_{p}\right) \chi_{m}\left(y_{p}\right) d y_{q} d y_{p}
$$

Für die numerische Lösung auf dem Quadratgitter muss also $\mathbb{G}_{q p}^{\mathrm{R}}$ durch Invertierung der Matrix $\left[E \mathbb{1}-\mathbb{H}_{\mathrm{P}}-\Sigma^{\mathrm{R}}\right]$ bestimmt werden. Das bedeutet für ein System mit $W \cdot L$ Gitterplätzen die Invertierung einer $(W \cdot L) \times(W \cdot L)$-Matrix! Dieser Aufwand lässt sich durch folgende mathematische Transformation erheblich vereinfachen. Anstatt die Streuwellenfunktion

$$
\psi_{m}=\mathbb{G}_{q p}^{\mathrm{R}} \chi_{m}
$$

aus dem rechten Teil des Integranden von Gleichung (1.33) direkt zu berechnen, multiplizieren wir Glg. (1.34) von links mit $\left[G^{\mathrm{R}}\right]^{-1}$

$$
\left[\mathbb{G}_{q p}^{\mathrm{R}}\right]^{-1} \psi_{m}=\chi_{m}
$$

wobei wir die Kenntnis der inversen Matrix $\left[\mathbb{G}_{q p}^{\mathrm{R}}\right]^{-1}$ und der transversalen Moden $\chi_{m}$ ausnutzen können und das Problem der Matrixinvertierung auf die Lösung eines linearen Gleichungssystems der Form $A x=y$ reduziert haben. Der große Vorteil bei der numerischen Berechnung liegt im Besonderen darin begründet, dass nur Vektoren $\psi_{m}$ der Länge $W \cdot L$ gespeichert werden müssen und nicht die gesamte invertierte Matrix $\mathbb{G}_{q p}^{\mathrm{R}}$. Zusätzlich ist die Matrix $\left[\mathbb{G}_{q p}^{\mathrm{R}}\right]^{-1}$ eine spärlich besetzte Matrix. Die maximale Anzahl der Matrixelemente von $\left[\mathbb{G}_{q p}^{\mathrm{R}}\right]^{-1}$ ist mit den Gleichungen (1.19) und (1.28) abschätzbar, wobei der Ausdruck für die Spärlichkeitsdichte

$$
d_{\mathrm{s}}(M)=\frac{\text { Anzahl der Elemente von } \mathrm{M} \neq 0}{\text { Anzahl aller Elemente von } \mathrm{M}} \Longrightarrow d_{\mathrm{s}}\left(\left[\mathbb{G}_{q p}^{\mathrm{R}}\right]^{-1}\right) \approx \frac{2}{W^{2}}+\frac{2}{L^{2}}+\frac{5}{W L}
$$

für $W, L>3$ eine obere Grenze für die Anzahl der Matrixelemente von $\left[\mathbb{G}_{q p}^{\mathrm{R}}\right]^{-1}$ festlegt und für $d_{\mathrm{s}} \ll 1$ eine Behandlung mit spärlich besetzten Matrizen gerechtfertigt ist. ${ }^{4}$

In dieser Arbeit wurde das UMFPACK-Paket [63] zur Berechnung der linearen komplexen Systeme gewählt, da es für die Berechnung nicht-symmetrischer spärlich besetzter Gleichungssysteme optimiert wurde. Zur Auswertung der Selbstenergieterme $\Sigma^{\mathrm{R}}$ müssen die Eigenfunktionen $\chi_{m}$ bestimmt werden. Diese können aufgrund der Dimension des Problems $\left(\propto W^{2}\right.$ bzw. $\left.\propto L^{2}\right)$ aus der direkten Diagonalisierung von $\mathbb{H}_{\mathrm{L}}$ bestimmt werden. Diese Berechnung wurde mit der LAPACK-Routine zgeev realisiert [64]. Die hier abgeleitete Matrixdarstellung der inversen Greenschen Funktion erlaubt Lösungen von Problemen, die auf einem äquidistanten Gitter formuliert werden können. Eine allgemeine Übersicht mathematischer und numerischer Methoden zur Lösung spärlich besetzter Gleichungssysteme ist in Davis [65] zu finden.

Häufig stellt eine Gitterrotation des eigentlichen Aufbaus eine geeignete Möglichkeit dar, die Problemstellung auf die Geometrie eines Quadratgitters anzupassen. Für Probleme, die mithilfe dieser geometrischen Operation nicht gelöst werden können, sei auf die von Rotter et al. [66] entwickelte modulare Methode rekursiven Berechnung von Greenschen Funktionen mit verknüpften Dyson-Gleichungen verwiesen.

4 Die exakte Berechnung liefert

$$
d_{\mathrm{s}}=\frac{5}{W L}+\frac{1}{W^{2} L^{2}}\left[\sum_{l} w_{l}^{2}-4 \min \{W, L\}-2\right]
$$

mit $w_{l}$ als Anzahl der mit der Probe verbundenen Gitterpunkte des Anschlussleiters $l$. 


\subsubsection{Die lokale Zustandsdichte}

Durch die Einführung einer Selbstenergie wird der effektive Hamilton-Operator $\mathcal{H}$ nicht-hermitesch, so dass sich für die Schrödinger-Gleichung voneinander verschiedene linke und rechte Eigenvektoren ergeben (siehe Anhang J)

$$
\mathcal{H}|\psi\rangle=\varepsilon|\psi\rangle \quad \wedge \quad\langle\varphi| \mathcal{H}=\langle\varphi| \varepsilon
$$

Mit diesen Funktionen lässt sich die Greensche Funktion in Ortsdarstellung schreiben als

$$
G^{\mathrm{R}}\left(\mathbf{r}, \mathbf{r}^{\prime}\right)=\sum_{n} \frac{\psi_{n}(\mathbf{r}) \varphi_{n}^{*}\left(\mathbf{r}^{\prime}\right)}{E-\varepsilon_{n}}
$$

wobei die Eigenwerte im Allgemeinen komplex sind und als

$$
\varepsilon_{n}=E_{n}-i \frac{\gamma_{n}}{2}
$$

mit $E_{n}=E_{n}^{(0)}+\Delta_{n}$ zu den Eigenenergien $E_{n}^{(0)}$ der isolierten Probe und durch die Kopplung bedingte Bandverschiebungen $\Delta_{n}$ geschrieben werden können. Entsprechendes gilt für die avancierte Greensche Funktion $G^{\mathrm{A}}\left(\mathbf{r}, \mathbf{r}^{\prime}\right)$. Die Spektralfunktion ist definiert als

$$
\begin{aligned}
A\left(\mathbf{r}, \mathbf{r}^{\prime}\right) & \equiv i\left[G^{\mathrm{R}}\left(\mathbf{r}, \mathbf{r}^{\prime}\right)-G^{\mathrm{A}}\left(\mathbf{r}, \mathbf{r}^{\prime}\right)\right] \\
& =\sum_{n} \psi_{n}(\mathbf{r}) \varphi_{n}^{*}\left(\mathbf{r}^{\prime}\right) \frac{\gamma_{n}}{\left(E-E_{n}\right)^{2}+\left(\frac{\gamma_{n}}{2}\right)^{2}}
\end{aligned}
$$

und enthält Summanden über Lorentz-Funktionen mit Verschiebung $E_{n}$ und Linienverbreiterung $\gamma_{n}$. Im Fall schwacher Kopplung $\gamma_{n} \ll 1$ der Leiter ergeben sich scharfe Resonanzen, während für den Fall starker Kopplung $\gamma_{n} \gg 1$ die Resonanzen vermischen und ununterscheidbar werden. Damit ist die Spektralfunktion auch ein Maß für die Art der Kopplung im System. Des Weiteren gilt der Zusammenhang

$$
A\left(\mathbf{r}, \mathbf{r}^{\prime}\right)=-2 \operatorname{Im}\left[G^{\mathrm{R}}\left(\mathbf{r}, \mathbf{r}^{\prime}\right)\right]
$$

und da die Zustandsdichte [67] über

$$
\rho_{E}(\mathbf{r})=\frac{1}{2 \pi} A(\mathbf{r}, \mathbf{r})=-\frac{1}{\pi} \operatorname{Im}\left[G^{\mathrm{R}}\left(\mathbf{r}, \mathbf{r}^{\prime}\right)\right]
$$

mit den Diagonalelementen der Spektralfunktion verknüpft ist, kann im Fall schwacher Kopplung, d.h. für $\gamma_{n} \ll 1$ wegen $\varphi_{n} \rightarrow \psi_{n}$ und $\Delta_{n} \rightarrow 0$ die Zustandsdichte als

$$
\rho_{E}(\mathbf{r})=\sum_{n} \delta\left(E-E_{n}^{(0)}\right)\left|\psi_{n}(\mathbf{r})\right|^{2} \quad, \quad \text { für } \quad \gamma_{n} \rightarrow 0
$$

ausgedrückt werden. ${ }^{5}$ Also liefert eine Summation über alle Absolutwertquadrate der Streuwellenfunktionen $\psi_{n}(\mathbf{r})$ zur Energie $E$ ein Abbild der Zustandsdichte des betrachteten Systems, die wir wegen ihrer physikalischen Bedeutung auch als Aufenthaltswahrscheinlichkeitsdichte der Moden bezeichnen wollen.

5 Für $\gamma_{n} \rightarrow 0$ gehen Lorentz-Funktionen in $\delta$-Distributionen über $\delta(x)=\lim _{\gamma \rightarrow 0^{+}} \frac{1}{\pi} \frac{\gamma}{x^{2}+\gamma^{2}}$, siehe z.B. Anhang A in [68]. 


\subsection{Symmetriebrechung und mesoskopischer Transport}

Ballistischer Transport in mesoskopischen Systemen basiert auf der idealisierten Annahme eines streuungsfreien Transports der Elektronen, wenn die Systemlänge kleiner als die mittlere freie Weglänge ist. Die Ausnahme bildet natürlich die Streuung der Elektronen an den Systemgrenzen, welche durch unendlich hohe Potenzialwände repräsentiert werden. Häufig ist eine semiklassische Beschreibung des Transports in solchen Systemen ausreichend [69]. Durch Untersuchungen des Transportverhaltens im linearen [16] und nicht-linearen [70] Regime gelangte man zu der Feststellung, dass die Probengeometrie einen großen Einfluss auf die nicht-linearen Transporteigenschaften des Systems haben kann. Dies führte zu einer großen Anzahl experimenteller und theoretischer Untersuchungen von Systemen mit räumlicher Symmetriebrechung (z.B.[20, 21, 42, 43, 44, 45, 46, 47, 48, 49, 52]), die bis heute anhalten $[71,72]$.

\subsubsection{Semiklassische Betrachtung}

Wir wollen uns hier auf die Untersuchung des (temperaturabhängigen) Gleichrichtungseffektes eines Bauteils beschränken, das erstmals von Song et al. [20] durch Potenzialdeformierung mittels einer Anordnung eines Antidots in Dreiecksform auf einem Kreuzkontakt (cross junction) hergestellt wurde (siehe Abb. 1.5). Wie in [21] gezeigt wurde, ist das naive Bild des Billards mit "mehr nach unten gestreuten Elektronen" bei einem Stromfluss $I_{\mathrm{QS}}$, der aus einer zwischen den Anschlüssen Q und S (Quelle und Senke) angelegten Spannung resultiert falsch, da die OnsagerCasimir-Reziprozitätsbeziehung

$$
R_{\mathrm{QS}, \mathrm{UO}}=R_{\mathrm{UO}, \mathrm{QS}}
$$

nach Gleichung (1.15) zusammen mit der Symmetrieeigenschaft bei einem Stromfluss $I_{\mathrm{UO}}$ zwischen oberem und unterem Kontakt ergibt, dass für die sich einstellenden Potenziale $\mu_{\mathrm{Q}}=\mu_{\mathrm{S}}$, d.h. $V_{\mathrm{QS}}=0$ gelten muss und damit auch die Widerstände identisch verschwinden müssten. Die experimentellen Befunde scheinen aber genau die naive Herangehensweise zu stützen. Der Widerspruch wurde durch die in [21] aufgestellte mikroskopische Theorie aufgelöst, indem die Energieabhän-

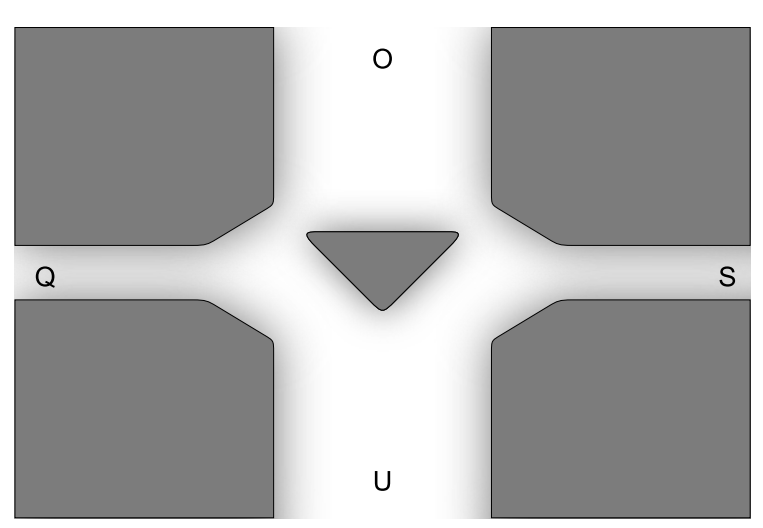

Abbildung 1.5.: Schematischer Aufbau des ballistischen Gleichrichters nach Song et al. [20]. Die räumliche Symmetrie ist durch die Dreiecksstruktur in der Mitte der Kreuzkopplung gebrochen. Die Vierpunktmessung des Widerstands ist $R_{\mathrm{QS}, \mathrm{UO}}$, dass heißt der Strom $I_{\mathrm{QS}}$ Fließt zwischen (Q)uelle und (S)enke, während die Spannung $V_{\mathrm{UO}}$ über die Kontakte (U)nten und $(\mathrm{O})$ ben abfällt. Der Verlauf des in Kantennähe als harmonisch angenommenen Potenzials in den Quer- und Längsstücken und um den Symmetriebrecher ist durch einen Farbverlauf gekennzeichnet.

gigkeit der Modenzahl $M(E)$ berücksichtigt wurde, wobei der Gleichrichtungseffekt durch ein Zusammenwirken von quasiklassischen (breiten) und quantisierten (schmalen) Transportkanälen verursacht wird. Die Anzahl der Moden in quantisierten Kanälen ist über einen großen Energiebereich konstant, während in quasiklassischen Kanälen $M(E) \propto \sqrt{E}$ gilt. Eine Betrachtung der sich einstellenden chemischen Potenziale im thermodynamischen Gleichgewicht unter Beachtung der Energieerhaltung liefert für breitere Kanäle dann erhöhte chemische Potenziale $\mu_{p}>\frac{\mu_{\mathrm{Q}}+\mu_{\mathrm{S}}}{2}$, die den Gleichrichtungseffekt 


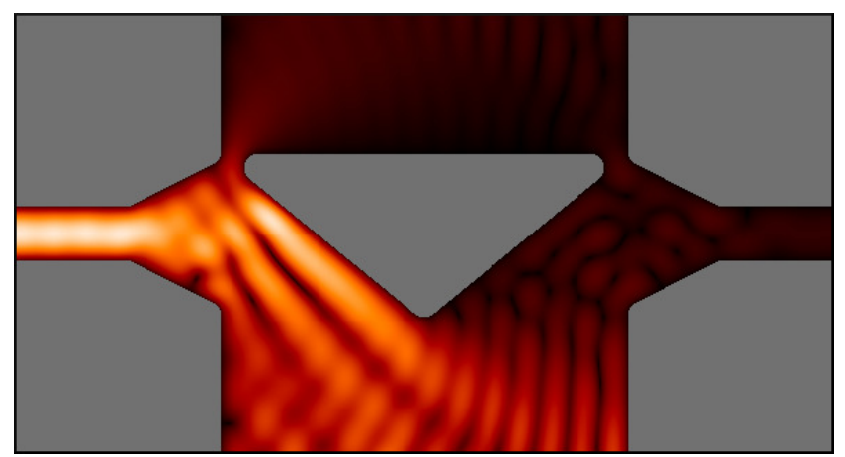

(a)

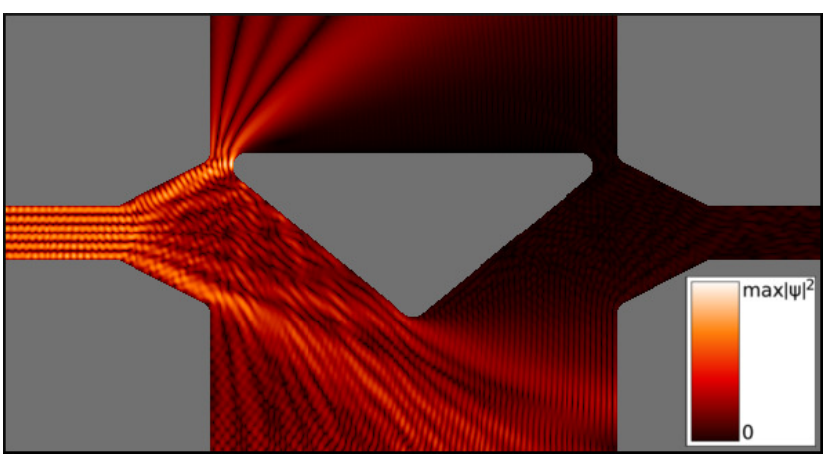

(b)

Abbildung 1.6.: Maximumsnormierte Aufenthaltswahrscheinlichkeitsdichte der Streuwellenfunktionen des ballistischen Gleichrichters für einfallende ebene Wellen zur Energie (a) $E=18.5$ für die 1. Mode und (b) $E=112$ für die 6. Mode. Für kleine Energien (Fall (a)) ist im Eingangskanal (links) nur eine Mode aktiviert. Es wird nur ein kleiner Teil dieser Mode an der schmalen Seite des Symmetriebrechers nach oben durchgelassen, da die Energie hier zu niedrig ist, um eine vollständige Mode hindurchzuleiten. Der Großteil der Welle wird in den breiten unteren Kanal und zurück in den Eingangskanal gestreut. Bei höheren Energien sind mehrere Moden des Eingangskanals (im Fall (b) sind es 10) aktiviert und deshalb kann mit größerer Wahrscheinlichkeit an der schmalsten Stelle des Symmetriebrechers vorbeigestreut werden, da nun auch hier - energetisch gesehen - mehrere Moden aktiviert sind (im Bild sind es 4). Die grauen Flächen stellen die Systemgrenzen der Halbleiter-Nanostruktur dar.

erklären können. ${ }^{6}$ Des Weiteren ist, da jede Mode ein und desselben Anschlussleiters den gleichen Energieanteil trägt, der Beitrag zum Nettostrom, den eine neu hinzugekommene Mode eines schmalen Transportkanals auf die Veränderung der ein- und auslaufenden Ströme hat größer, als dies bei einem quasiklassischen Transportkanal der Fall ist. Damit ist es möglich, dass sich für den Spannungsabfall $V_{\mathrm{UO}}$ auch Vorzeichenwechsel ergeben. Bezüglich der Temperaturabhängigkeit des Gleichrichtungseffektes durchgeführte Untersuchungen von Löfgren et al. [49] und de Haan et al. [52, 53] konnten den in [21] vorhergesagten Vorzeichenwechsel bei der Messung der Gleichrichtungsspannung experimentell bestätigen. Eine semiklassische Betrachtung unter Berücksichtigung der Temperaturabhängigkeit ist ebenfalls möglich, da die thermischen Effekte durch die Fermi-Verteilung miteinbezogen werden. Allerdings darf die gewählte Temperatur nicht zu groß sein, da man anderenfalls das ballistische Regime verlässt. In der semiklassischen Näherung ergeben sich Integrale über Produkte aus Fluktuationen der Transmissionskoeffizienten $\bar{T}_{p q}(E)=T_{p q} \cdot M_{q}(E)$ mit der Fermi-Verteilung $f_{p}(E)$, die die analytischen Lösungen für ein- und auslaufende Ströme aus den Gleichungen (1.9) und (1.10)

$$
\begin{aligned}
& I_{p}^{+}\left(\mu_{p}, T\right)=\frac{2 e}{h} \sum_{n}\left[k_{\mathrm{B}} T \ln \left(\exp \left[\frac{\varepsilon_{p, n}-\mu_{p}}{k_{B} T}\right]+1\right)\right]+\mu_{p}+\varepsilon_{p, n} \\
& I_{p}^{-}\left(\mu_{p}, T\right)=\sum_{q} T_{p q} I_{q}^{+}\left(\mu_{p}, T\right)
\end{aligned}
$$

mit Temperatur $T \equiv T_{p}$ besitzen. Dazu werden auch die als energieunabhängig angenommenen Transportkoeffizienten $T_{p q}$ benötigt, die z.B. aus klassischen Simulationen gewonnen werden können.

\subsubsection{Quantenmechanische Betrachtung}

In Abbildung 1.6 sind beispielhaft zwei Streuwellenfunktionen der quantenmechanischen Simulation des ballistischen Gleichrichters nach der in Unterkapitel 1.1 vorgestellten Methode für einfallende ebe-

6 Für eine detaillierte technische Beschreibung der sich einstellenden Potenziale sei auf [21] verwiesen. 
ne Wellen einer niedrigen und einer höheren Energie dargestellt, die sich durch Lösung von Gleichung (1.35) ergeben. Anhand der Streuwellenfunktionen lässt sich bereits das modenbezogene Verhalten des Gleichrichters voraussagen (siehe Bildunterschrift in Abb. 1.6). Die energieabhängigen Streuwellenfunktionen werden des Weiteren benötigt, um die Transmissionskoeffizienten $\bar{T}_{p q}$ des ballistischen Gleichrichters mittels der Gleichungen (1.33) und (1.6) zu berechnen. Zuvor wollen wir nochmals kurz auf die semiklassische Betrachtung zurückblicken, um die Notwendigkeit quantenmechanischer Berechnungen herauszustellen.

Die Transmissionswahrscheinlichkeiten als energieunabhängige Größen zu betrachten ist nur bei einfachen Geometrien mit ausgeprägten Transportkanälen eine gute Näherung. Bei der vollständigen Berücksichtigung der Geometrie der Probe jedoch gilt $T_{p q}=T_{p q}(E) \neq$ const und die semiklassische Beschreibung bricht zusammen, da die Energiebilanzen nicht aufgestellt werden können. An dieser Stelle wollen wir ansetzen und die energieabhängigen Transmissionskoeffizienten mithilfe des quantenmechanischen Streumatrixformalismus bestimmen. Temperatureffekte werden hierbei durch die in der Energiebilanz auftretenden Fermi-Verteilungen berücksichtigt. Wie wir sehen werden sind wir damit in der Lage, den Vorzeichenwechsel des Spannungsabfalls $V_{\mathrm{UO}}$ bei der temperaturabhängigen Vierpunktmessung des Gleichrichters zu verifizieren.

Die aus der Simulation erhaltenen Transportkoeffizienten $\bar{T}_{U Q}(E)$ und $\bar{T}_{O Q}(E)$ sind zum Vergleich zusammen mit den Funktionen der semiklassischen Näherung in Abbildung 1.7 dargestellt. Im Fall des nach unten gerichteten Ausgangs ergibt sich eine gute Übereinstimmung mit der semiklassischen Näherung, da die Breite des Transportkanals bezüglich der Fermi-Wellenlänge groß ist $\left(w_{\mathrm{UQ}} \gg \lambda_{\mathrm{F}}\right)$ und wir uns im klassischen Regime befinden. Für den nach oben gerichteten Transport gilt diese Näherung nicht, denn für die schmalste Stelle des Transportkanals gilt $w_{\mathrm{OQ}} \approx \lambda_{\mathrm{F}}$, zu erkennen an den starken Fluktuationen verursacht durch quantenmechanische Streueffekte. Im Allgemeinen gibt - bei einfachen Geometrien - die schmalste Stelle des zu betrachtenden Bauteils zwischen zwei Anschlussleitern die maximale Anzahl der zum Transport beitragenden Moden vor, wobei der semiklassische Ausdruck für die Transmissionskoeffizienten

$$
\bar{T}_{p q}(E) \approx \frac{w_{p q}}{\pi} \sqrt{2 E}
$$

eine Näherung mit $w_{p q}=\min \left\{W_{p}, W_{q}\right\}$ als minimaler Abstand des Transportkanals zwischen Leiter $p$ und $q$-inklusive der Leiter selbst - angibt. Diese Näherung besitzt Gültigkeit für den potenzialfreien Anschlussleiter $V(y)=0$, wobei diese Abschätzung aus den Eigenwerten der transversalen Moden ohne Potenzial

$$
E_{n, p}=\frac{1}{2}\left(\frac{\pi n}{W_{p}}\right)^{2}
$$

resultiert. Im Falle von weichen Wänden ${ }^{7}$, die mithilfe eines harmonischen Potenzials $V(y)=\frac{1}{2} \omega_{0}^{2} y^{2}$ an den Kanten des Anschlussleiters modelliert werden, gilt für schmale Anschlussleiter in der harmonischen Näherung ein linearer Zusammenhang der Transportkoeffizienten in Abhängigkeit der Stärke des harmonischen Potenzials $\omega_{0}$

$$
\bar{T}_{p q}(E) \propto \frac{E}{\omega_{0}}
$$

(siehe Gleichung (C.11) im Anhang). Aufgrund der Symmetriebedingung an die Transportkoeffizienten

7 Dieser Begriff bezieht sich auf die Beschaffenheit des Potenzials. Bei harten Wänden erfolgt ein sprunghafter Übergang an den Systemgrenzen, dargestellt durch unendlich hohe Potenzialwände. Weiche Wände hingegen beschreiben eine kontinuierliche Erhöhung des wandnahen Potenzials, beispielsweise parabolisch. 


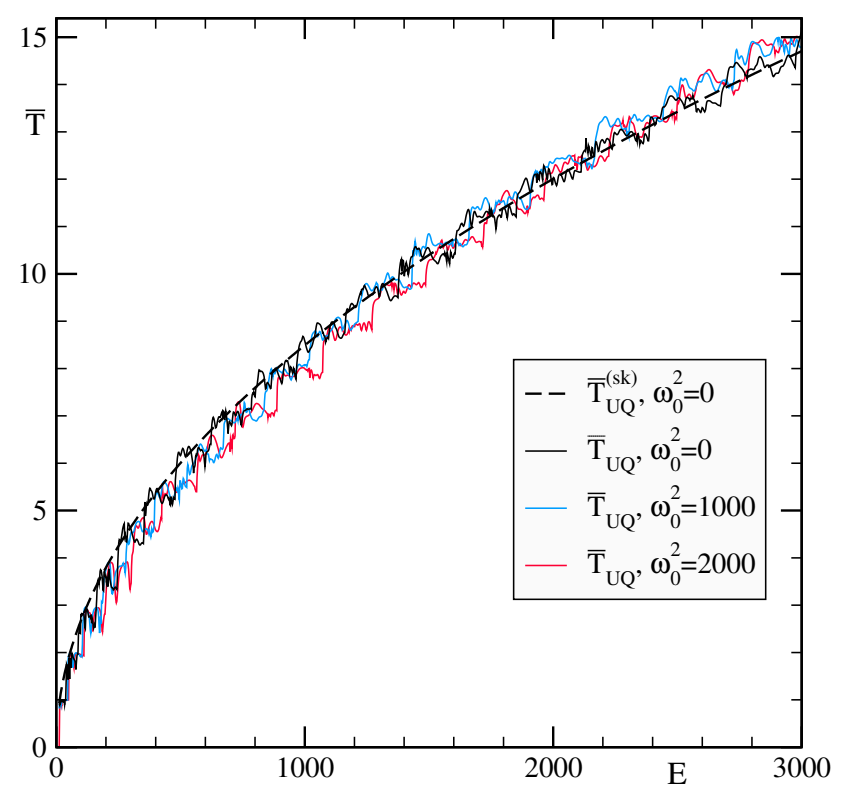

(a)

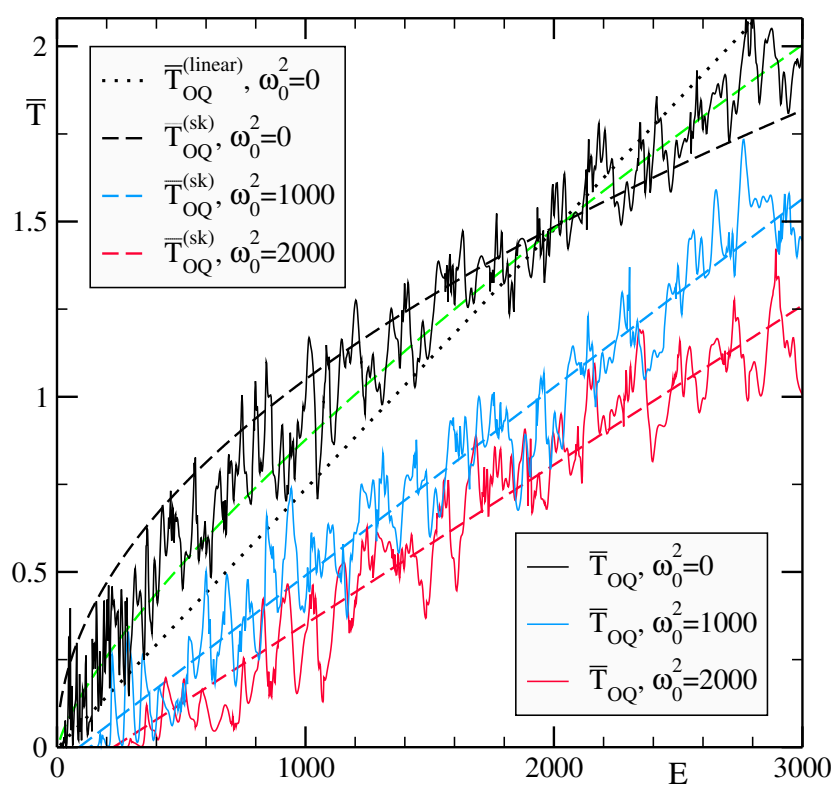

(b)

Abbildung 1.7.: Transportkoeffizienten des ballistischen Gleichrichters [20] für verschiedene Stärken des harmonischen Potenzials $\omega_{0}$ berechnet mit Streumatrixformalismus für (a) $\bar{T}_{U Q}(E)$ und (b) $\bar{T}_{O Q}(E)$. Zum Vergleich sind die semiklassischen Näherungen für $\bar{T}_{p q}^{(\mathrm{sk})}(E)$ nach Gleichungen (1.46) (gestrichelte schwarze Linien: $\left.\omega_{0}=0\right)$ und (1.48) (gestrichelte blaue Linien: $\omega_{0}^{2}=1000$, gestrichelte rote Linien: $\omega_{0}^{2}=2000$ ) ebenfalls eingezeichnet. Für die Bezeichnung der Kontakte siehe Abb. 1.5. Für (a) ergibt sich eine gute Übereinstimmung mit der semiklassischen Näherung, während sich für den Fall (b) stärkere Abweichungen durch Fluktuationen ergeben, da quantenmechanische Effekte wirksam werden. Das harmonische Potenzial in den Randbereichen der Leiter bewirkt für schmale Leiter einen linearen Zusammenhang zwischen Energie und Transportkoeffizienten. Für den Fall $\omega_{0}=0$ wurde zusätzlich eine lineare Regression für $\bar{T}_{\mathrm{OQ}}$ durchgeführt (Bezeichnung $\bar{T}_{\mathrm{OQ}}^{(\text {linear) }}$ ), um zu verdeutlichen, dass das tendenzielle Verhalten von $\bar{T}_{\mathrm{OQ}}$ zwischen linearem und semiklassischem Verlauf liegt. Eine Regression für $\bar{T}_{\mathrm{OQ}}\left(\omega_{0}=0\right) \propto E^{\alpha}$ liefert $\alpha=\frac{3}{4}$ (grüne gestrichelte Linie).

$\bar{T}_{p q}(E)=\bar{T}_{q p}(E)$ und wegen $\bar{T}_{p p}(E)=M_{p}(E)-\sum_{q \neq p} \bar{T}_{p q}(E)$ müssen nur 6 der insgesamt 16 Transportkoeffizienten zur vollständigen Beschreibung des Systems bestimmt werden ${ }^{8}$, wobei hier aus Gründen der Übersichtlichkeit in Abbildung 1.7 nur zwei Transportkoeffizienten, $\bar{T}_{U Q}$ und $\bar{T}_{O Q}$, dargestellt sind.

Für die Berechnung der chemischen Potenziale zum angelegten Strom benötigen wir die Fermi-Energie. Die Ladungsträgerdichte für den experimentellen Aufbau nach [20] ist mit $n_{2 \mathrm{DEG}} \approx 5 \cdot 10^{11} \mathrm{~cm}^{-2}$ angegeben. Damit berechnet sich die Fermi-Energie näherungsweise zu

$$
\mu_{\mathrm{F}}=\frac{\hbar^{2} k_{\mathrm{F}}^{2}}{2 m^{*}}=\frac{n_{2 \mathrm{DEG}} \pi \hbar^{2}}{m^{*}} \approx 18 \mathrm{meV}
$$

mit $m^{*}=0.065 m_{\mathrm{e}}$ als effektive Masse der Elektronen in GaAs. Der Strom kann nun über die Transmissionskoeffizienten

$$
I_{\mathrm{SQ}}=\frac{2 e^{2}}{h} \int_{0}^{\infty} \sum_{p} \bar{T}_{\mathrm{S} \leftarrow p}(E)\left[f_{p}(E)-f_{\mathrm{S}}(E)\right] d E
$$

8 Die $4 \times 4$-Matrix $\mathbb{T}$ der Transportkoeffizienten $T_{p q}$ ist symmetrisch, d.h. die 6 Elemente der unteren Dreiecksmatrix sind bekannt. Die 4 Diagonalelemente sind über die bekannte Modenzahl $M_{p}(E)$ in den Anschlussleitern bestimmt. 


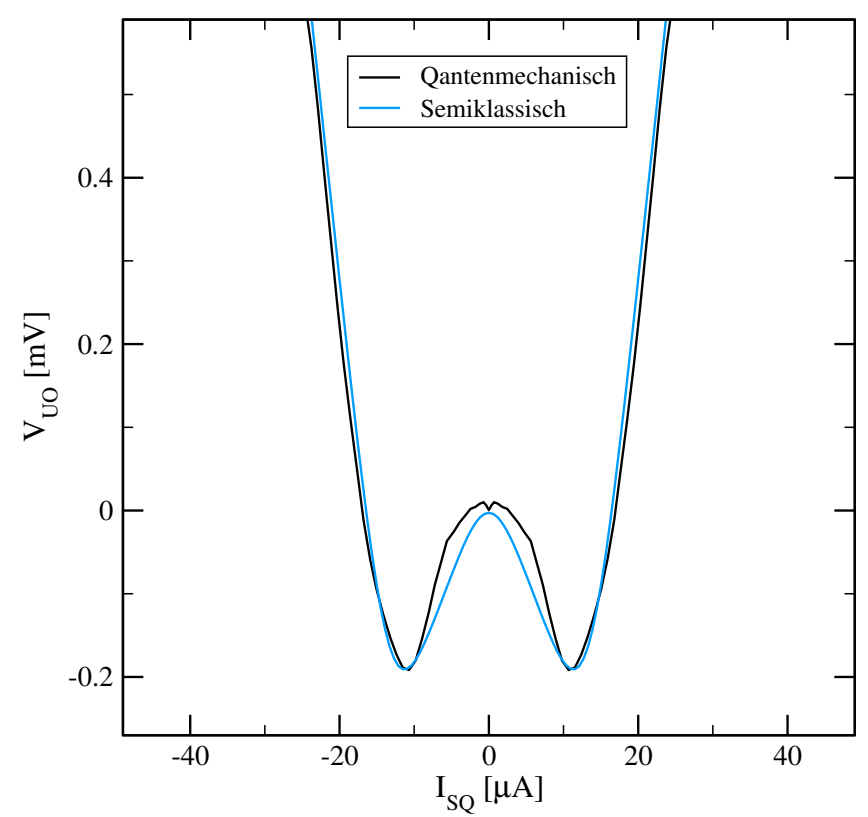

Abbildung 1.8.: Gleichrichtungseffekt mit Vorzeichenwechsel der Spannung $V_{\mathrm{UO}}$ im ballistischen Gleichrichter für $\omega_{0}=0$ bei $T=0.02 \cdot T_{\mathrm{F}}=4.2 \mathrm{~K}$ in Abhängigkeit vom Stromfluss $I_{\mathrm{SQ}}$. Gegenübergestellt sind die Ergebnisse der Modelle der semiklassischen (blau) und des quantenmechanischen (schwarz) Ansatzes. Es ist eine gute Übereinstimmung zu beobachten. Die Abweichungen bei kleinen Strömen (kleine Energien) liegen in der schlechteren Approximation des semiklassischen Modells für $\bar{T}_{\mathrm{OQ}}(E)$ in diesem Bereich begründet (vgl. auch Abb. 1.7b).

berechnet werden [73].

Im Fall konstanter Transportkoeffizienten $\bar{T}_{\mathrm{S} \leftarrow q}(E)=$ const wird nur über Differenzen der Fermi-Funktionen integriert, was bei tiefen Temperaturen den Beitrag $\left(\mu_{\mathrm{Q}}-\mu_{\mathrm{S}}\right)$ liefert (hier gilt $\mu_{\mathrm{O}}=\mu_{\mathrm{U}}$ ). Die Integrationsgrenzen in Gleichung (1.49) können dann als $\mu_{\mathrm{S}}$ und $\mu_{\mathrm{Q}}$ gewählt werden, da sich wegen $T \rightarrow 0$ Stufenfunktionen ergeben. Für höhere Temperaturen verschieben sich die Integrationsgrenzen um einige $k_{\mathrm{B}} T$, da die thermische Verbreiterung der Zustandsdichte berücksichtigt werden muss [74]. Dies liefert - wenn auch nicht sofort offensichtlich - den gleichen Beitrag $\left(\mu_{\mathrm{Q}}-\mu_{\mathrm{S}}\right)$ zum Strom [54]. Im Fall energieabhängiger Transportkoeffizienten $\bar{T}_{\mathrm{S} q}(E) \neq$ const, wie in Abbildung 1.7 mit Quantenfluktuationen bedingt durch die komplizierte Geometrie der Probe, bewirkt eine Integration mit der Differenz der Fermifunktionen bei Temperaturen $T>0$ eine Glättung. Dieses Verhalten wird auch in Abbildung 1.8 wiedergegeben. Als wichtiges Ergebnis fanden wir, das aufgrund dieser Bias-Integration daher nur das unterschiedliche tendenzielle Verhalten der Transportkoeffizienten wichtig für den Stromverlauf ist, da scharfe Resonanzen bzw. Fluktuationen durch Multiplikation mit den Fermi-Funktionen und die Bias-Integration ausgeschmiert werden. Für die Geometrie des ballistischen Gleichrichters fanden wir die Abhängigkeiten der Transportkoeffizienten $\bar{T}_{\mathrm{UQ}}(E) \propto \sqrt{E}$ und $\bar{T}_{\mathrm{OQ}}(E) \propto E^{\alpha}$ (Abb. 1.7) mit $\alpha=\frac{3}{4}$ im Fall $\omega_{0}=0$, was dann zur selbstkonsistenten Einstellung der unterschiedlichen effektiven Potenziale $\mu_{p}$ mit $\mu_{\mathrm{Q}} \geq \mu_{p} \geq \mu_{\mathrm{S}}$ und $p=\{\mathrm{O}, \mathrm{U}\}$ in Abhängigkeit vom Stromfluß $I_{\mathrm{SQ}}$ führt.

Die Ergebnisse aus der numerischen Berechnung der einlaufenden Ströme $I_{p}^{+}$und der auslaufenden Ströme $I_{p}^{-}$im Falle der Vierpunktmessung $R_{\mathrm{SQ}, \mathrm{UO}}$ mithilfe der berechneten Transportkoeffizienten $\bar{T}_{p q}(\mathrm{E})$ unter Berücksichtigung der sich einstellenden Potenziale $\mu_{\mathrm{Q}} \geq \mu_{p} \geq \mu_{\mathrm{S}}$ mit $p=\{\mathrm{O}, \mathrm{U}\}$ sind im $V_{\mathrm{UO}}-I_{\mathrm{SQ}}$-Diagramm in Abbildung 1.8 dargestellt. Es ergibt sich eine gute Übereinstimmung mit der semiklassischen Näherung aus [21], wobei auch der vorhergesagte Vorzeichenwechsel von $V_{\mathrm{UL}}$ sehr gut wiedergegeben wird. 


\subsection{Zusammenfassung und Ausblick}

Der Landauer-Büttiker-Formalismus verbindet observable Größen, wie Strom und Spannung, mit den quantenmechanischen Größen der Transmissions- und Reflexionsamplituden, im Allgemeinen ausgedrückt durch die $S$-Matrix. Mithilfe der Greenschen Funktionen kann die $S$-Matrix als Ausdruck von Matrixelementen des Greenschen Operators in Matrixdarstellung $G_{p q}^{\mathrm{R}}$ und den orthogonalen transversalen Zuständen (Moden) $\chi_{p}$ und $\chi_{q}$ geschrieben werden (Fisher-Lee-Relation). Die Matrix $G_{p q}^{\mathrm{R}}$ enthält dabei alle notwendigen Informationen über das betrachtete offene System. Aus einer genaueren Untersuchung des zu berechnenden Produkts $\left\langle\chi_{p}\left|G_{p q}^{\mathrm{R}}\right| \chi_{q}\right\rangle=\left\langle\chi_{p} \mid \psi_{q}\right\rangle$ schlossen wir auf die elegante Möglichkeit der Lösung eines linearen Gleichungssystems $\left[G^{\mathrm{R}}\right]^{-1} \psi=\chi$, anstatt die bekannte inverse Greensche Funktion in Matrixdarstellung $\left[G^{\mathrm{R}}\right]^{-1}$ direkt zu invertieren. Wie wir zeigen konnten, wird der numerische Aufwand dadurch erheblich reduziert. Die zusätzliche Eigenschaft der Spärlichkeit der Matrix $\left[G^{\mathrm{R}}\right]^{-1}$ lieferte eine schnelle und stabile numerische Methode der Berechnung von energieabhängigen Streufunktionen $\psi(E)$ und damit der Zustandsdichte $\rho(E)$, sowie schließlich auch der $S$-Matrix.

Die Anwendung des Landauer-Büttiker-Formalismus und die Berechnung der Transportkoeffizienten $\bar{T}_{p q}$ zur Geometrie des ballistischen Gleichrichters nach [20] konnten sowohl den in [21] theoretisch beschriebenen Gleichrichtungseffekt als auch den dort vorausgesagten Vorzeichenwechsel der Spannung $V_{\mathrm{UO}}$ bei einer Vierpunkt-Widerstandsmessung $R_{\mathrm{SQ}, \mathrm{UO}}$ bestätigen.

Das Modell kann in viele verschiedene Richtungen erweitert werden. Zunächst ist die relativ einfache Implementierung eines Magnetfeldes in $z$-Richtung durch Modifikation der Kopplungskoeffizienten zu nennen (siehe Gleichung (1.20)), womit sich dann die Zeitumkehrinvarianz brechen lässt. Die Probe wird hierzu um einen kleinen Teil der halb-unendlichen Anschlussleiter erweitert, wobei das Magnetfeld $B(r)$ im erweiterten Bereich $\left[r_{0}, r_{1}\right]$ wie

$$
B(r)= \begin{cases}B & r<r_{0} \\ B\left[10 \tilde{r}^{3}-15 \tilde{r}^{4}+6 \tilde{r}^{5}\right] & r \leq r \leq r_{1} \\ 0 & r>r_{1}\end{cases}
$$

mit $\tilde{r}=\frac{r-r_{0}}{\left|r_{1}-r_{0}\right|}$ stetig abfällt. Die Koeffizienten des Polynoms ergeben sich dabei aus Stetigkeitsbedingungen an die erste und zweite Ableitung des Magnetfelds. Der Vorteil dieser Methode ist, dass die Berechnung der Landau-Niveaus in den Anschlussleitern und der zugehörigen Eigenfunktionen entfallen kann - wir können die Eigenwerte und Eigenfunktionen der magnetfeldfreien transversalen Moden weiterhin nutzen.

Eine aufwendiger zu implementierende, wenn auch gleichwohl interessante Erweiterung stellt die quantenmechanische Betrachtung der Elektron-Elektron-Wechselwirkung dar. Hierzu müssen die Greenschen Funktionen des Nicht-Gleichgewichts eingeführt werden und in einem konvergenten iterativen Verfahren Korrelationsfunktionen der Greenschen Funktionen, Selbstenergieterme und Streufunktionen berechnet werden, aus denen sich dann wiederum die Nicht-Gleichgewichtsströme berechnen lassen $[75,76]$. 


\section{Der Chaotische Strahlenteiler}

Das Chaos sei willkommen, denn die Ordnung hat versagt. Karl Kraus (1874-1936), österreichischer Schriftsteller

2.1 Einführung . . . . . . . . . . . . . . . . . . . 27

2.1.1 Resonatoren . . . . . . . . . . . . . . . . . 27

2.1.1.1 Der Fabry-Pérot-Resonator . . . . . . . . . . . . . 28

2.1.1.2 Flüstergaleriemodenresonatoren . . . . . . . . . . . 30

2.1.1.3 Photonische Kristalle . . . . . . . . . . . . . . . . . 31

2.1.2 Bauelemente der integrierten Optik . . . . . . . . . . . . . . . 32

2.1.2.1 Strahlenteiler . . . . . . . . . . . . . . . . 32

2.1.2.2 Strahlenschalter . . . . . . . . . . . . . . 32

2.1.3 Modifizierte Wellenleiter als Strahlenteiler und Strahlenschalter . . . . 32

2.2 Das Kosinusbillard-Modell . . . . . . . . . . . . . . . . . . . . . . . . 33

2.3 Quantenmechanische Behandlung . . . . . . . . . . . . . . . 36

2.3.1 Quasi-gebundene Zustände . . . . . . . . . . . . . . . . . 36

2.3.2 Semiklassische Näherung . . . . . . . . . . . . . . . . . . . . 37

2.3.3 Experimentelle Untersuchungen mittels Mikrowellenbillard . . . . . . . 38

2.3.4 Vergleich von klassischem mit quantenmechanischem Phasenraum . . 39

2.3.5 Auskopplung der quasi-gebundenen Zustände durch Phasenraum-

Engineering . . . . . . . . . . . . . . . . . . 40

2.3.6 Modifikationen des Parametersatzes $(d, a, L) \ldots \ldots . \ldots . \ldots 45$

2.4 Weitere Anwendungen . . . . . . . . . . . . . . . . . . . 46

2.4.1 Mikrolaser . . . . . . . . . . . . . . . . . . . . . 46

2.4.2 Multi-Kavitätsresonatoren . . . . . . . . . . . . . . . 50

2.4.3 Multi-Kavitätsstrahlenteiler . . . . . . . . . . . . . . . . . . 50

2.5 Zusammenfassung und Ausblick . . . . . . . . . . . . . . . . . . 52 


\section{Motivation}

Es ist wohlbekannt, dass Resonatoren eine Schlüsselrolle in der modernen optischen Informationsverarbeitung (OI) einnehmen. Zur OI gehören vor allem optisch abbildende Verfahren, das Gebiet der integrierten Photonik und der Bereich optischer Kommunikationssysteme. Die optische Nachrichtentechnik ist der Grundpfeiler heutiger Datenübertragung und -verarbeitung. Der rasante Anstieg der benötigten Übertragungsbandbreiten und der damit verbundene Bedarf an ultra-schneller und präziser opto-elektronischer Informationsverarbeitung stellt immer größere Herausforderungen an die Weiterentwicklung moderner Kommunikationssysteme. Neuartige Experimente reichen von Ein-Photonen Manipulation (Emission, Übertragung und Detektierung) über Silizium-basierte photonische Systeme, optisch aktive integrierte Bauteile und photonische Kristalle bis hin zu Quantenroutern, Metamaterialien und nanophotonischen Realisierungen [77]. Die Fertigung effektiver photonischer Komponenten beschäftigt Labore weltweit, wobei vor allem das Ziel verfolgt wird, die Photonik in ähnlicher Art und Weise kompakt in Chips integrieren zu können, wie es heute bereits in der elektronischen Halbleitertechnologie gelingt. Das gestiegene Interesse an Billards erwuchs in diesem Zusammenhang aus der Option, chaotische Kavitäten als Resonatoren zu verwenden $[22,23]$ und so beispielsweise auch im Wellenleiter selbst eine Verarbeitung optischer Signale zu ermöglichen. Ein wünschenswertes Ziel hierbei ist es, den Gütefaktor, auch $Q$-Faktor genannt, deutlich modifizieren zu können und überdies nicht auf eine stark gerichtete Emission als charakteristisches Merkmal verzichten zu müssen [24, 25, 26]. Darüber hinaus, und das wird das zentrale Element dieses Kapitels auszeichnen, können Billards mit gemischter, d.h. mit regulärer und chaotischer Dynamik eine Konstruktionsgrundlage bilden, optische und elektronische Kavitäten als vielversprechende Kandidaten opto-elektronischer Schalter und/oder Strahlenteiler zu realisieren [1]. Weitere optische und elektronische Anwendungen, wie Mikrolaser, Multi-Kavitätsresonatoren und dergleichen, lassen sich aus diesen Systemen ableiten [2]. Ein bereits durchgeführtes und in dieser Arbeit vorgestelltes Mikrowellen-Experiment signalisiert die vielversprechende Realisierung der in diesem Kapitel vorgeschlagenen Strukturen.

Die Untersuchung der komplexen Dynamik eines Billards mit gemischtem Phasenraum wird hier stellvertretend an einem Kosinusbillard durchgeführt. Dazu werden sowohl klassische Phasenraummethoden als auch quantenmechanische Betrachtungen der quasi-gebundenen Zustände und des Phasenraums in Form der Husimi-Verteilung im semiklassischen Bereich unter Zuhilfenahme des Streumatrixformalismus für nicht-hermitesche Hamilton-Matrizen genutzt. 


\subsection{Einführung}

In diesem Kapitel wollen wir uns der Konstruktion von optischen bzw. elektronischen Schaltern und Strahlenteilern widmen, die auf dem Prinzip chaotischer zweidimensionaler Wellenleiter bzw. Resonatoren (mit Mehrfachanschlüssen) beruhen. Der vorliegende einführende Abschnitt dient zunächst der Klärung von Grundbegriffen der Mikrooptik im Bezug auf Resonatoren. Anschließend werden die beiden Bauelemente im Kontext zur integrierten Optik vorgestellt.

\subsubsection{Resonatoren}

Resonatoren stellen das optische Äquivalent zum elektronischen $L C$-Schwingkreis dar. Sie bündeln und speichern Licht bei bestimmten Resonanzfrequenzen, die wiederum von Aufbau und Form der Resonatoren abhängig sind. Ein optischer Resonator kann als Lichtübertragungssystem mit Rückkopplung verstanden werden, wobei das Licht entweder wiederholt im Resonator reflektiert wird oder darin zirkuliert. Zwei wichtige Größen zur Charakterisierung von Resonatoren sind das Modenvolumen $V_{\mathrm{m}}$ und der Gütefaktor $Q$. Das Modenvolumen quantifiziert das von der resonanten Mode eingenommene Volumen. Es ist definiert als (siehe z.B. [78])

$$
V_{\mathrm{m}}=\frac{1}{w_{\max }} \int w(r) d^{N} r
$$

wobei $w(r)$ die elektromagnetische Energiedichte

$$
w(r)=\frac{1}{2}(\mathbf{E} \cdot \mathbf{D}+\mathbf{H} \cdot \mathbf{B})
$$

definiert und $\mathbf{E}, \mathbf{D}, \mathbf{H}, \mathbf{B}$ die gleichen Größen wie in der Einleitung bezeichnen. Die Normierung $w_{\max }$ ist definiert als die maximale Energiedichte der resonanten Mode, $N$ ist durch die Dimension des Resonators festgelegt. Die andere Kenngröße ist der Gütefaktor - auch $Q$-Faktor oder Güte genannt - und wird definiert als

$$
Q=\left.2 \pi \frac{E}{\Delta E}\right|_{t_{0}},
$$

mit $E$ als Gesamtenergie der resonanten Mode und $\Delta E$ als Verlustenergie, die durch Absorption und Streuung innerhalb des Resonators verursacht wird. Der $Q$-Faktor kann also als Qualitätsmaß für die Funktionsweise eines Resonators angesehen werden. Ein idealer Resonator hätte demnach einen unendlich großen Gütefaktor. In der Praxis wird daher versucht, möglichst große Gütefaktoren zu realisieren. Der Gütefaktor kann auch als Quotient aus Resonanzfrequenz $\nu_{0}$ und der Halbwertsbreite $\Delta \nu_{0}$ dieser Resonanz dargestellt werden

$$
Q=\frac{\nu_{0}}{\Delta \nu_{0}}
$$

Der Versuch, hohe Lichtspeicherzeiten bzw. kleine Verluste zu erzielen und gleichzeitig hohe Energiedichten bei hohen Frequenzen zu erhalten führt zu kleinsten räumlichen Abmessungen der Bauteile in der Größenordnung der verwendeten Wellenlänge. ${ }^{1}$ Der Name Mikroresonator bezieht sich auf die in ein oder mehreren Dimensionen durchgeführte Miniaturisierung. Sind alle Dimensionen des Mikroreso-

1 Der Sachverhalt der Maximierung von $Q$ und der Minimierung von $V_{\mathrm{m}}$ und Vergrößerung der Modendichte wird oft in der Maximierung des Quotienten $Q / V_{\mathrm{m}}$ zusammengefasst, auch im Hinblick auf die Maximierung des Purcell-Faktors in Gleichung (2.5). 
nators von derselben Größenordnung, so bezeichnet man diese als Mikrokavitätsresonatoren oder kurz Mikrokavitäten. Mikrolaser zu konstruieren, also Laser, die auf Grundlage von Mikroresonatoren arbeiten, ist wegen ihrer Kompaktheit, ihrer geringeren Leistungsverluste und ihrer ultra-schnellen Modulationsgeschwindigkeit von erhöhtem Interesse [79]. In diesem Zusammenhang sei auch der Purcell-Effekt erwähnt, der eine Verstärkung bzw. Abschwächung der spontanen Emission durch stark gebundene optische Moden oder starke Licht-Materie-Kopplung darstellt [80, 81]. Diese Abhängigkeit wird durch den Purcell-Faktor $f_{\mathrm{p}}$ beschrieben, der durch den Quotienten aus der Zustandsdichte der Kavität $\rho(\nu)$ und der Zustandsdichte des freien Raumes $\rho_{\mathrm{f}}(\nu)$ definiert ist. Für eine Kavität mit konstantem Brechungsindex $n$ und nur einer resonanten Mode der Wellenlänge $\lambda$ gilt [82]

$$
f_{\mathrm{p}}=\frac{3}{4 \pi^{2}}\left(\frac{\lambda}{n}\right)^{3} \frac{Q}{V_{\mathrm{M}}}
$$

Die Proportionalität der Emissionsverstärkung mit $Q / V_{\mathrm{m}}$ erklärt daher den Versuch, hohe PurcellFaktoren für Mikrolaser zu erzielen (siehe dazu Abb. 2.1a). Ist die Dichte der zu betrachtenden photonischen Zustände kleiner als die Zustandsdichte des freien Raumes - dies ist beispielsweise in der Bandlücke eines photonischen Kristalls der Fall - kann die spontane Emission wegen $f_{\mathrm{p}}<1$ auch abgeschwächt werden. Damit wird es möglich, die spontane Emission kontrolliert zu beeinflussen. Die genaue quantenmechanische Untersuchung der Wechselwirkungseffekte zwischen dem ResonatorLichtfeld und Dipolemittern bzw. Atomen ist Aufgabe der Resonator-Quantenelekrodynamik (auch Mikroresonator-QED), deren Begrifflichkeit jedoch nicht weiter vertieft werden soll.

Im Folgenden wird die Charakterisierung von optischen Mikrokavitäten skizziert. Das Gebiet der Mikroresonatoren ist jedoch so umfangreich, dass hier nur ein kleiner Überblick über die drei Klassen gegeben werden soll, die am häufigsten Erwähnung finden. Zusätzlich werden Beispiele für die angesprochenen Resonatorklassen in Abbildung 2.1 vorgestellt. Für ein tieferes Verständnis sind der Übersichtsartikel von K. J. Vahala [81] und zwei Spezialausgaben des Journal of Selected Topics in Quantum Electronics [83, 84] lesenswert.

\subsubsection{Der Fabry-Pérot-Resonator}

Der Fabry-Pérot-Resonator [85, 86], auch Fabry-Pérot-Interferometer genannt, stellt den einfachsten und zugleich wichtigsten Vertreter der Resonatoren dar. Dieser besteht im Wesentlichen aus zwei teildurchlässigen Spiegeln im Abstand $L$, welche ein lichtleitendes bzw. optisch aktives Medium mit dem Brechungsindex $n$ einschließen. Zwischen den Spiegeln wird das Licht wiederholt reflektiert. Die Bedingung für die Ausbildung von stehenden Wellen lautet

$$
m \lambda \equiv 2 n L \quad,
$$

mit $m \in \mathbb{N}$, d.h. für alle von Null verschiedenen natürlichen Vielfachen von $\frac{\lambda}{2}$ finden wir Resonanz. Damit können die Resonanzfrequenzen relativ bequem über den Abstand $L$ der Spiegel eingestellt werden. In Abbildung 2.1a ist ein Beispiel für einen modernen Resonator dieser Bauart aufgeführt. Die dort vorgestellte Mikrosäule ist ein 1D photonischer Kristall und kann zu der Gruppe der FabryPérot-Resonatoren gezählt werden. Die Qualität eines Fabry-Pérot-Resonators wird meistens durch die Finesse

$$
F=\pi \frac{\sqrt{R}}{1-R}
$$



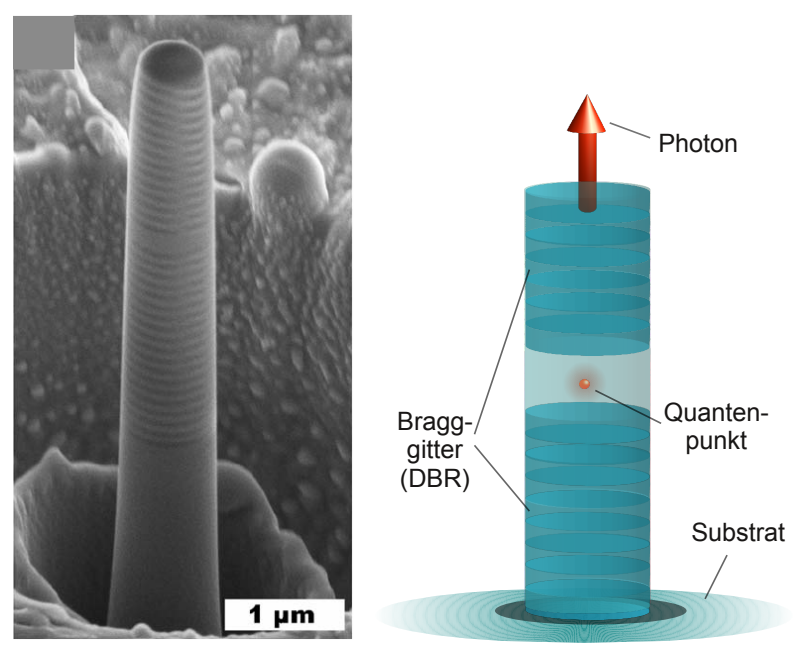

(a)
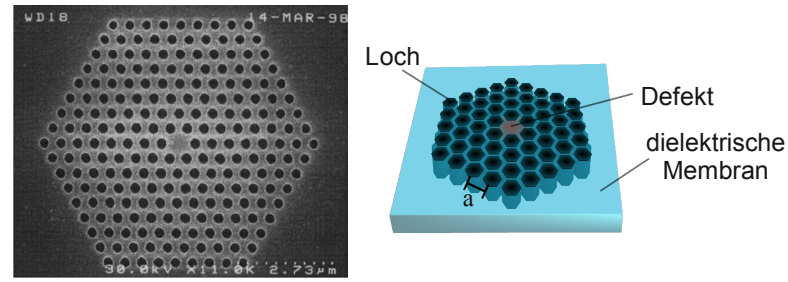

(d)
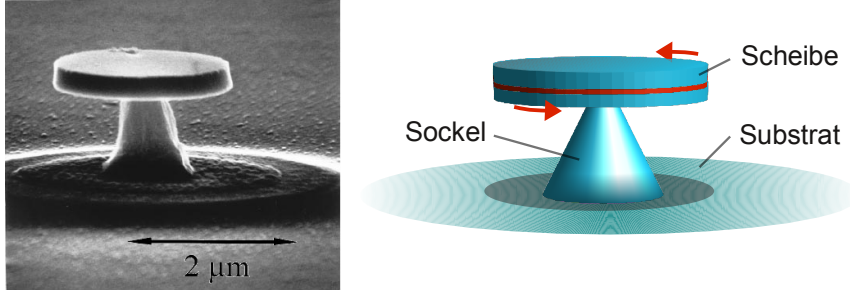

(b)
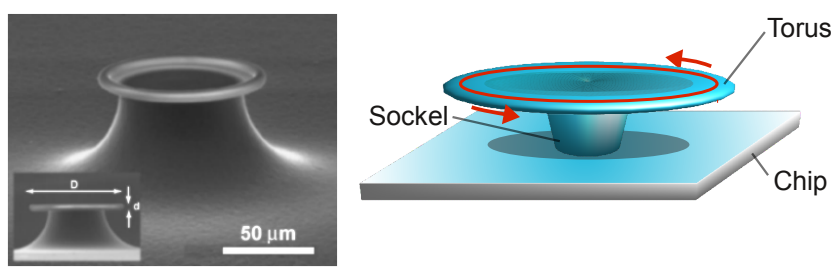

(c)

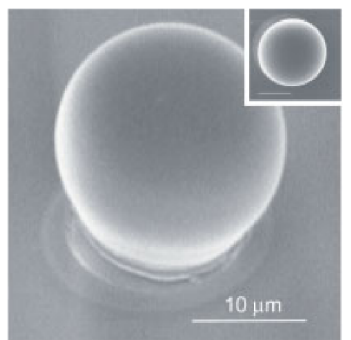

Emissionsstrahl

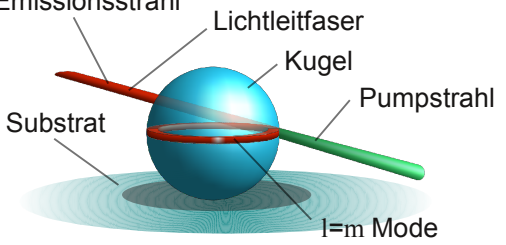

(e)

Abbildung 2.1.: Beispiele für 1D-, 2D- und 3D-Mikroresonatoren:

(a) Mikrosäule auf GaAs (Substrat), bestehend aus MgS-ZnCdSe Bragg-Gittern (Distributed Bragg-grating Reflector) und 3 eingebetteten ZnCdSSe Quantentöpfen für Einzelphotonenemission. Rasterelektronenmikroskop(REM)-Aufnahme aus [87] entnommen. Die Photonen werden oben aus der Spitze emittiert (siehe Skizze). Der Q-Faktor ist mit 3500 angegeben. Eine weiterführende Übersicht über Mikrosäulen ist in [88, 89] zu finden. (b) Mikroscheibe aus GaAs. Die Struktur wurde mittels Molekularstrahl-Epitaxie und Nassätzverfahren hergestellt. Der $Q$-Faktor beträgt 12000. Die umlaufende Welle (Flüstergaleriemode) ist in der Skizze rot dargestellt. REM-Aufnahme aus [90]. (c) Mikrotorus auf Siliziumbasis. Der Ring mit Durchmesser $D$ und Dicke $d$ selbst besteht aus Siliziumoxid, der durch einen vierstufigen Prozess aus Photolithographie, $\mathrm{XeF}_{2}$-isotropem Ätzen und $\mathrm{CO}_{2}$-lasergestütztem Umschmelzen auf einem Standard-Siliziumchip hergestellt wurde. Der $Q$-Faktor beträgt $4 \times 10^{8}\left(\right.$ !), Gütefaktoren mit $Q>10^{8}$ werden gemeinhin als ultrahoch bezeichnet. REM-Aufnahme entstammt [91]. (d) 2D-Photonischer Kristall. Die $180 \mathrm{~nm}$ dünne dielektrische Membran besteht aus InGaAsP-InP, in die Löcher geätzt wurden. Die Gitterkonstante (Inter-Loch-Abstand) des hexagonalen Gitters beträgt $a=500 \mathrm{~nm}$, die Löcher selbst betragen ca. $150 \mathrm{~nm}$ im Durchmesser. Der Defekt, der als Mikrokavität dient, wurde durch ein fehlendes Loch generiert, das dann Paare aus horizontalen und vertikalen Dipolmoden erzeugt (rote Fläche in der Skizze). Durch zusätzliche Symmetriebrechung (Vergrößerung der zwei direkt an den Defekt angrenzenden Löcher links und rechts) kann die Entartung der Dipolmoden aufgehoben werden, und man erhält einen echten Einmodenlaser. Der theoretische $Q$-Faktor ist durch Strahlungsverluste auf 20000 begrenzt. REM-Aufnahme aus [92]. (e) Mikrokugel aus CdSe-CdZnS. Optisches Pumpen durch eine im evaneszenten Außenbereich des Resonators geführte Lichtleitfaser bewirkt die Ausbildung von Flüstergaleriemoden durch zyklisch bedingte Totalreflexion. Der Q-Faktor beträgt 2000. REM-Aufnahme aus [93]. 
beschrieben, wobei die Größe $R \equiv|r|^{2}<1$ die Reflektivität des Resonators und $|r|^{2}$ den Amplitudendämpfungsfaktor für eine Doppelreflexion (Resonatorumlauf) bezeichnen. Finesse $F$ und $Q$-Faktor können über die Beziehung

$$
Q=F m=F \frac{2 n L}{\lambda}
$$

ineinander überführt werden. Da die Resonatorlänge $L$ praktisch extrem groß gewählt werden kann, wird für die Gütecharakterisierung eines Fabry-Pérot-Resonators oftmals die Finesse $F$ anstatt des $Q$-Faktors verwendet. Auch der in diesem Kapitel verwendete chaotische Strahlenteiler kann, sofern er als Laser konstruiert wird, in diese Kategorie eingeordnet werden.

\subsubsection{Flüstergaleriemodenresonatoren}

Als Flüstergaleriemodenresonatoren (whispering gallery modes resonators) werden Resonatoren bezeichnet, die auf dem Prinzip der kontinuierlichen Totalreflexion beruhen, wobei das Licht im Inneren des Resonators nahe seiner Oberfläche bzw. Systemgrenze zirkuliert.

Für die Entstehung von umlaufenden Wellen in quasi-2D Mikroscheiben (siehe Abb. 2.1b) oder Mikrotori (siehe Abb. 2.1c) gilt, ähnlich wie in Unterabschnitt 2.1.1.1 nach Gleichung (2.6), dass die Bedingung

$$
m \lambda=n L
$$

mit $m \in \mathbb{N}$ und Pfadlänge $L=N \cdot 2 R \sin \left(\frac{\pi}{N}\right)$ für $N$ Reflexionen im Resonator mit Radius $R$ erfüllt sein muss. Diese Moden werden auch Flüstergaleriemoden genannt. ${ }^{2}$ Im Limes $N \rightarrow \infty$ geht $L$ in den Umfang $2 \pi R$ des Resonators über. Damit ergibt sich als minimaler Abstand zwischen benachbarten Frequenzen $\nu_{F}=\frac{c_{0}}{2 \pi n R}$. Die Wellennatur des Lichts bedingt eine bisher vernachlässigte Phasenanpassung, die zur Ausbildung eines evaneszenten Feldes im Außenbereich des Resonators führt. Daher müssen die Resonanzfrequenzen

$$
\nu=m \frac{c_{0}}{n_{\mathrm{eff}} L}
$$

durch die Einführung eines effektiven Brechungsindexes $n_{\mathrm{eff}}$, der zwischen dem Brechungsindex des Resonators und dem Brechungsindex seines Außenbereichs liegt, berücksichtigt werden. Die evaneszenten Felder ermöglichen die Einkopplung von Pumpwellen mittels Lichtleitfasern. So können z.B. bei Mikrotori (vgl. Abb. 2.1c) ultra-hohe $Q$-Faktoren $\left(>10^{8}\right)$ erreicht werden [95]. Durch eine Verformung des Kreises, z.B. zu einer Ellipse, kann die Ein- bzw. Auskopplung der Wellen im evaneszenten Bereich gerichtet erfolgen [96]. Dies ist im Hinblick auf Mikrolaser eine interessante Anwendung.

Für 3D-Objekte, wie z.B. Mikrokugeln, ist eine Betrachtung analog zu den 2D-Objekten nicht ausreichend. Stattdessen muss die Helmholtz-Gleichung für eine dielektrische Kugel mit Radius $R$ gelöst werden (siehe z.B. [94]). Die skalare Funktion im Kugelkoordinatensystem $(r, \varphi, \vartheta)$ hat die Lösung

$$
U_{l m}(r, \varphi, \vartheta)=\left\{\begin{array}{ll}
c_{\mathrm{i}} \sqrt{n r k_{0}} P_{l}^{m}(\cos \vartheta) J_{l+\frac{1}{2}}\left(n r k_{0}\right) e^{ \pm i m \varphi} & r \leq R \\
c_{\mathrm{a}} \sqrt{n r k_{0}} P_{l}^{m}(\cos \vartheta) H_{l+\frac{1}{2}}^{(1)}\left(r k_{0}\right) e^{ \pm i m \varphi} & r>R
\end{array},\right.
$$

2 Die resonanten Zustände mit hoher Lebensdauer verdanken ihren Namen den akustischen Moden, die sich an konvexen Oberflächen über große Entfernungen ausbreiten können. Ein prominentes Beispiel ist die Saint Paul's Kathedrale, wo ein Flüstern an der $32 \mathrm{~m}$ durchmessenden Kirchenkuppel auf der gegenüberliegenden Seite wahrnehmbar ist [94]. 
mit $k_{0}=\frac{\omega}{c_{0}}$ und den dimensionslosen Konstanten $c_{\mathrm{i}}$ für den Innen- und $c_{\mathrm{a}}$ für den Außenbereich, den adjungierten Legendre-Funktionen $P_{l}^{m}$, den Bessel-Funktionen $J_{l}$ der ersten Art der Ordnung $l$ und den Hankel-Funktionen $H_{l}^{(1)}$ der ersten Art der Ordnung $l$ mit $l, m \in \mathbb{N}$. In Abbildung 2.1e rechts ist eine einfache Flüstergaleriemode für $l=m$ skizziert. Flüstergaleriemodenresonatoren ${ }^{3}$ sind aufgrund ihrer monolithischen Bauweise meist einfach herzustellen, da hier viele Verfahren aus der Mikroprozessortechnik zur Verfügung stehen. Dafür sind sie aber im Allgemeinen auf bestimmte Resonanzfrequenzen festgelegt. Es gibt jedoch Möglichkeiten, die Resonanzfrequenz indirekt abzustimmen, indem beispielsweise die Temperaturabhängigkeit des Brechungsindexes ausgenutzt wird [99].

\subsubsection{Photonische Kristalle}

Photonische Kristalle bestehen aus periodischen Anordnungen dielektrischer Strukturen, wie z.B. Halbleitern, Polymeren oder Gläsern. Die Periodizität der Dielektrika kann dabei in verschiedenen Dimensionen vorliegen (siehe Abb. 2.2). Sie werden meist mit bekannten Methoden der Mikroelektronik, beispielsweise durch Photolithographie, Oxidation, Nass- oder Trockenätzen hergestellt. Photonische Kristalle sind vielseitig einsetzbar, nicht nur wie hier in ihrer Rolle als Resonatoren beschrieben, können sie auch als hochreflektive Spiegel oder Wellenleiter verwendet werden (vgl. auch Abschnitt 4.1). Zur Verwendung der Kristalle als Resonatoren wird ausgenutzt, dass sie - ähnlich wie Halbleiter eine photonische Bandlücke im Bereich der benutzten optischen Frequenzen aufweisen. Damit ist ein Lichttransport prinzipiell nicht möglich. Erst durch gezieltes Dotieren oder anders eingebrachte Defekte wird die periodische Struktur des Kristalls gestört, so dass zusätzliche Energieniveaus innerhalb der Bandlücke entstehen. Licht mit den zugehörigen Resonanzfrequenzen kann so im Festkörperkristall "eingefangen" werden. Infolgedessen ist es möglich, kleinste Modenvolumen in der Größenordnung von $\left(\frac{\lambda}{n}\right)^{3}$ zu erreichen. Der Gütefaktor ist ebenfalls hoch, etwa in der Größenordnung $10^{4}$. Photonische Kristalle existieren als 1D- (z.B. aktive optische Schicht zwischen zwei Mehrschichtfilmen, siehe Abb. 2.1a), 2D- (siehe Abb. 2.1d) oder 3D-Strukturen. Letztere sind besonders schwierig herzustellen, da eine vollständige Bandlücke ${ }^{4}$ eine Translationsinvarianz der Einheitszelle voraussetzt [101].

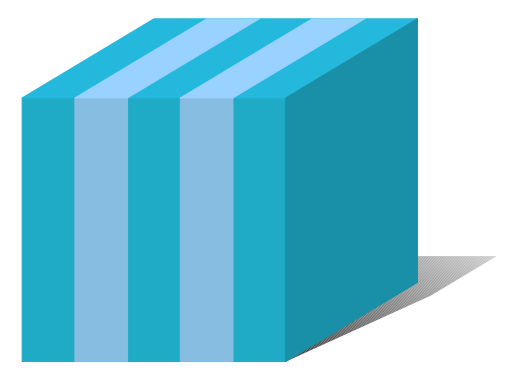

(a) $1 \mathrm{D}$

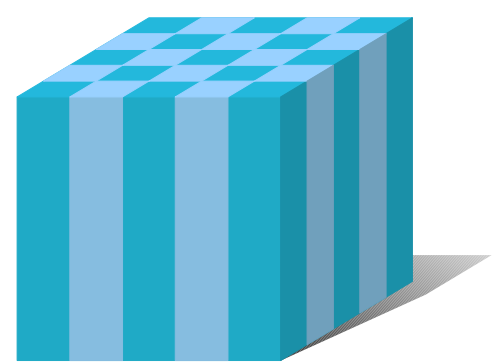

(b) $2 \mathrm{D}$

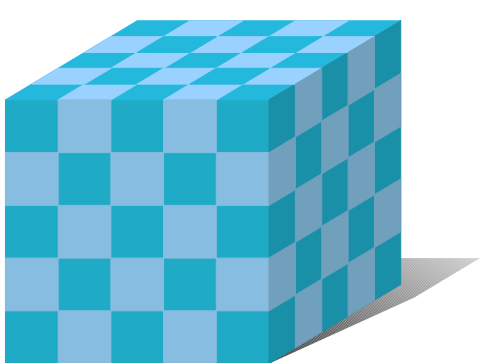

(c) $3 \mathrm{D}$

Abbildung 2.2.: Vereinfachte schematische Darstellung des Aufbaus photonischer Kristalle in verschiedenen Dimensionen. (a) Die periodische Anordnung von Dielektrika im Mehrschichtfilm führt zu einer photonischen 1D-Bandlücke. (b) Die periodische Anordnung dielektrischer Stäbe in Luft oder periodischer Bohrungen in Mehrschichtfilmen führt zu einer 2D-Bandlücke. Eine ebene Anordnung, wie in der Abbildung hier illustriert, führt zur räumlichen Spiegelsymmetrie in der dritten Dimension und damit zur Modenaufspaltung in TEund TM-Wellen. (c) Die periodische Anordnung von dielektrischen Kügelchen oder als inverse Anordnung periodischer Bohrungen oder komplexer Lufteinschlüsse führt zur photonischen 3D-Bandlücke.

3 Flüstergaleriemoden wurden zuerst 1961 von C. G. B. Garrett in von H. Guggenheim hergestellten $\mathrm{CaF}_{2}: \mathrm{Sm}^{++}$ Kugeln unter hohen Anregungsenergien entdeckt [97, 98].

4 Als Erstes vorhergesagt für die inverse Diamantstruktur [100], entspricht der kristallographischen Raumgruppe $F 4_{1} / d \overline{3} 2 / m$, kurz $F d \overline{3} m$. 


\subsubsection{Bauelemente der integrierten Optik}

Unter dem Begriff integrierte Optik oder auch integrierte Photonik soll die Herstellung bzw. Einbettung von optischen Komponenten auf planaren Substraten verstanden werden. Zu typischen Baugruppen zählen optische Verzweiger bzw. Strahlenteiler, Gitter, Koppler, Reflektoren, Polarisatoren, Modulatoren, Interferometer, Strahlenquellen und Detektoren, um die wesentlichen Baugruppen zu nennen. Diese sind nach dem Baukastenprizip beliebig kombinierbar und können in optischen Datenübertragungssystemen in ultra kurzer Zeit sehr komplexe Aufgabenstellungen bewältigen. Das Konzept, Wellenleiter selbst so zu modifizieren, dass sie optische Signale nicht nur weiterleiten, sondern auch verarbeiten können, stammt bereits aus der Pionierzeit der integrierten Optik (siehe z.B. [102]) und wird auch in dieser Arbeit wieder aufgegriffen. Da die Elemente der integrierten Optik, wie oben bereits erwähnt, äußerst zahlreich sind, sollen in diesem Abschnitt nur zwei Komponenten hervorgehoben werden: der optische Verzweiger, der im Folgenden Strahlenteiler genannt wird, sowie der Strahlenschalter. Diese Elemente können, wie weiter unten gezeigt wird, aus Billards konstruiert werden, die über einen gemischten Phasenraum verfügen.

\subsubsection{Strahlenteiler}

Der Strahlenteiler ist ein optisches Bauelement, dass die Leistung eines Eingangssignals auf zwei oder mehrere Ausgänge verteilt. Im Fall des symmetrischen Strahlenteilers geschieht diese Aufteilung gleichmäßig. In seiner einfachsten Ausführung besteht dieser aus einem Eingang und zwei Ausgängen und wird wegen seiner Bauform auch Y-Verzweiger genannt. Y-Verzweiger sind in der Vergangenheit intensiv untersucht worden [103, 104]. Obwohl ihre Funktionsweise relativ fehlertolerant ist, dürfen Krümmungsradien nicht zu groß und Verzweigungen selbst nicht zu ungenau gefertigt sein, um Leistungsverluste durch Absorption und Streuung möglichst gering zu halten, was deren Fertigung etwas aufwendiger gestaltet.

\subsubsection{Strahlenschalter}

Ein Strahlenschalter stellt in gewisser Weise eine Sonderform des Strahlenteilers dar. Die Arbeitsweise erfolgt frequenzselektiv, d.h. der jeweils aktive Ausgang ist von der Frequenz des Eingangssignals abhängig. Der Strahlenschalter kann somit auch zur Aufspaltung eines frequenzgemischten Signals benutzt werden. In dieser Funktion der Signalaufteilung auf verschiedene Ausgänge gehört er einer speziellen Untergruppe der Strahlenteiler an. In einer integrierten elektro-optischen Variante wird die Amplitude des Eingangssignals durch eine angelegte Spannung modifiziert. So wird z.B. durch eine Pockels- oder Kerrzelle destruktive Interferenz in einem integrierten Mach-Zehnder-Interferometer erzwungen [105]. Ein Beispiel eines modernen frequenzselektiven Strahlenschalters in Form eines Rings ist in von $X u$ et al. realisiert worden [106].

\subsubsection{Modifizierte Wellenleiter als Strahlenteiler und Strahlenschalter}

In diesem Kapitel werden wir zeigen, wie durch Ausnutzung dynamischer Eigenschaften des komplexen Phasenraums in chaotischen Systemen eine extreme Vereinfachung der technologischen Fertigung von Strahlenteilern und Strahlenschaltern erreicht werden kann. Dazu benutzen wir einen im Folgenden vorgestellten Wellenleiter-Prototypen, bei dem es sich um einen mit zwei horizontalen externen 
Anschlüssen versehenen 2D-Resonator mit harmonischem Profil $y(x) \propto \cos x$ handelt. Wegen der Proportionalität zu einer Kosinusfunktion in Transportrichtung wird dieser auch Kosinusbillard genannt. Durch zusätzliche externe Anschlüsse wird der Phasenraum des Mikroresonators lokal verformt und bildet einen ternären ${ }^{5}$ unvollständigen Hufeisenphasenraum aus. Charakteristisch für klassische gemischte Phasenräume ist dabei die klare Abgrenzung zwischen Inseln mit stabiler regulärer Dynamik und der chaotischen See, die diese Inseln umgibt und - daher der Name - eine chaotische Dynamik aufweist. Die Begrenzung der regulären von den chaotischen Bereichen des klassischen Phasenraums kann mit den invarianten KAM-Tori erklärt werden, die wie Barrieren wirken [107, 108]. Aufgrund des quantenmechanischen Effekts des dynamischen Tunnelns gibt es aber Übergänge aus den chaotischen Regionen in die stabilen Inseln des Phasenraums. Diese stellen, rein klassisch gesehen, jedoch verbotene Übergänge dar. Angelehnt an die Quantenmechanik werden diese emergenten Zustände aufgrund ihrer Ähnlichkeit mit den Eigenzuständen des geschlossenen Systems als quasi-gebundene Zustände bezeichnet. Durch weitere bzw. zusätzliche transversal zum Wellenleiter ausgerichtete und entsprechend dimensionierte externe Anschlüsse - in den entsprechenden Ortsregionen der quasi-gebundenen Zustände angesiedelt - wird der Phasenraum des Wellenleiters, d.h. des Resonators ohne zusätzliche transversale Anschlüsse, nur leicht gestört. Wir werden zeigen, dass diese quasi-gebundenen Zustände aus dem Wellenleiter durch diese zusätzlichen Anschlüsse herausgeführt werden können und die Realisierung von Schaltern und Strahlenteilern ermöglicht [1]. Die energie- bzw. frequenzabhängige Aufspaltung der quasi-gebundenen Zustände für verschiedene Geometrien des Kosinusbillards erlaubt die Einteilung in Klassen von Moden. Wir zeigen schließlich die Realisierung eines effektiven Einmodenlasers und schlagen die Konstruktion von Multi-Kavitätsgeometrien zur Nutzung als Resonatoren, Schalter und Strahlenteiler vor [2].

\subsection{Das Kosinusbillard-Modell}

Wie in Abschnitt 2.1 ausgeführt, besetzen Schalter und Strahlenteiler eine Schlüsselposition in der optischen und elektronischen Informationsverarbeitung. Wir wollen hier eine neuartige alternative Möglichkeit vorstellen, solche Schalter bzw. Strahlenteiler aus deformierten 2D-Wellenleitern bzw. Resonatoren mit chaotischer Dynamik zu konstruieren. ${ }^{6}$ Der Prototyp eines solchen Wellenleiters besteht aus einem Resonator, der mit zwei externen und kollinear gradlinig verlaufenden, halb-unendlichen Anschlüssen der Breite $d$, welche in $x$-Richtung ausgedehnt sind, verbunden ist. Der Resonator selbst besitzt die Geometrie eines Kosinusbillards [24, 109, 110, 111]. Er besteht aus einer geraden bzw. planen Wand bei $y=0$ und einer ihr gegenüber liegenden verformten Wand, welche der Funktion

$$
y(x)=d+a\left[1-\cos \left(\frac{2 \pi x}{L}\right)\right]
$$

genügt, wobei $a$ die Amplitude und $L$ die Länge der Deformation definiert. Mit Angabe des Parametersatzes $(d, a, L)$ ist das Kosinusbillard eindeutig bestimmt. In Abbildung 2.3 ist das Profil des in den

5 Ternär bezieht sich hier auf die Anzahl der Fixpunkte $N=3$.

6 Durch die Äquivalenz von TM-Wellen eines 2D-Resonators mit Dirichlet-Randbedingungen, beschrieben durch die Lösungen der Helmholtz-Gleichung, und Quantenwellen eines 2D-Billards, beschrieben durch die Lösungen der Schrödinger-Gleichung, gelten alle noch folgenden Aussagen nicht nur für optische, sondern auch für elektronische Systeme. Daher sind in diesem Kapitel mit Wellenleitern bzw. Resonatoren auch immer elektrische Leiter gemeint. Siehe dazu auch Abschnitt 4.1. 


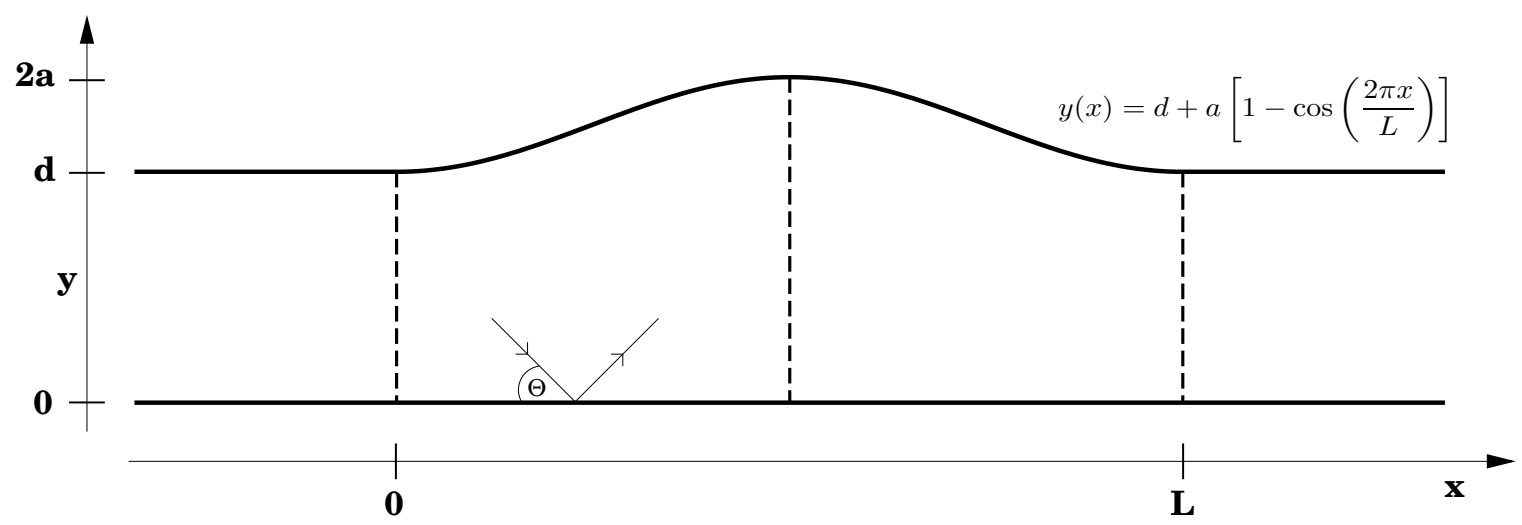

Abbildung 2.3.: Geometrie des modifizierten Wellenleiters (Kosinusbillard) im Profil. Die Verformung der oberen Begrenzung genügt der dort angegebenen Funktion. Fundamentale Orbits: Die zentrale gestrichelte Linie bei $x=\frac{L}{2}$ bezeichnet den einzigen stabilen periodischen Orbit, während die beiden äußeren gestrichelten Linien bei $x=0$ bzw. $x=L$ zwei instabile periodische Orbits kennzeichnen. Die Größe $\Theta$ bezeichnet den von der einfallenden Trajektorie und der unteren Wand aufgespannten Winkel. Die Begrenzung des Billards bei $y=0$ wird als Poincaréebene gewählt.

Berechnungen und Simulationen verwendeten Wellenleiter-Prototypen dargestellt.

Um einen Überblick über die klassische Strahlendynamik des Wellenleiters zu erhalten, konstruieren wir die Smale-Hufeisenabbildung [112, 113]. Die Hufeisenabbildung liefert die Topologie des homoklinen Netzwerkes, welches die gesamte Streudynamik wiedergibt und die wechselwirkenden Gebiete mit den asymptotischen Gebieten zu verknüpfen vermag. Für den zu betrachtenden Wellenleiter besteht die Domäne des Wechselwirkungsgebiets aus dem Wellenleiter selbst. Die asymptotischen Bereiche werden aus den externen halb-unendlichen Anschlüssen gebildet. Die Anzahl der fundamentalen Orbits $^{7}$ legt die Ordnung der Hufeisenabbildung fest. Für $a>0$ besitzt der Resonator drei solcher Orbits (siehe gestrichelte Linien in Abb. 2.3), d.h. es handelt sich um eine ternäre Hufeisenabbildung. Die Hufeisenabbildung wird aus invarianten Mannigfaltigkeiten (stabile und instabile) der hyperbolischen Fixpunkte des Resonators gebildet (die stabile ist im Zentrum, die instabilen an den Kanten des Resonators lokalisiert). Die Darstellung der Hufeisenabbildung geschieht mithilfe einer Poincaré-Abbildung [114]. Wir verfolgen die Orbits zu gegebenen Anfangsbedingungen entlang der Mannigfaltigkeiten. Jedes Mal, wenn die Trajektorie auf die plane, unverformte Seite der Wand trifft - damit definiert $y=0$ die Poincaréebene - zeichnen wir einen Punkt des Phasenraums an der Stelle $x$ mit dem Winkel $\Theta$ ein, wobei $\Theta$ den Winkel bezeichnet, der von der links einfallenden Trajektorie und der Poincaréebene gebildet wird. In Abbildung 2.4a ist die Hufeisenabbildung mit dem Parametersatz $(d, a, L)=(1.0,0.305,5.55)$ aus Gleichung (2.12) dargestellt. Aus Gründen der Übersichtlichkeit sind nur die charakteristischen Ranken [113] bis zur Ordnung 3 abgebildet. Insbesondere ist für den oben genannten Satz von Parametern die Hufeisenabbildung unvollständig. Das bedeutet, dass die ein- und auslaufenden Komponenten des homoklinen Knäuels (homoclinic tangle) im Poincaréschnitt nicht vollständig überlappen. Diese Situation ist typisch für Systeme mit gemischtem Phasenraum [115].

Für den hier benutzten Satz von Parametern besitzt der Wellenleiter resonante Stabilitätsinseln mit Periode eins und vier (die Ränder dieser Inseln sind in Abbildung 2.4a durch dicke schwarze Linien gekennzeichnet). Wie eingangs erwähnt, kann die Abgrenzung der regulären Bereiche gegenüber der chaotischen See mithilfe der invarianten KAM-Tori verstanden werden [107, 108]. Eine sehr lesenswerte Darstellung zur Entstehung der KAM-Barrieren findet man in [116].

7 Es ist üblich, Orbits mit Periode 1 als fundamentale Orbits zu bezeichnen. 


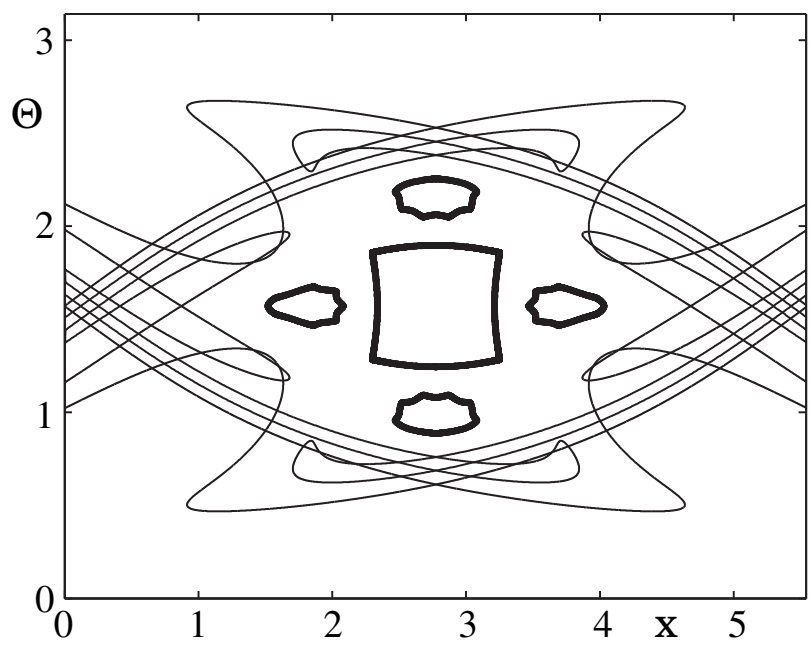

(a)

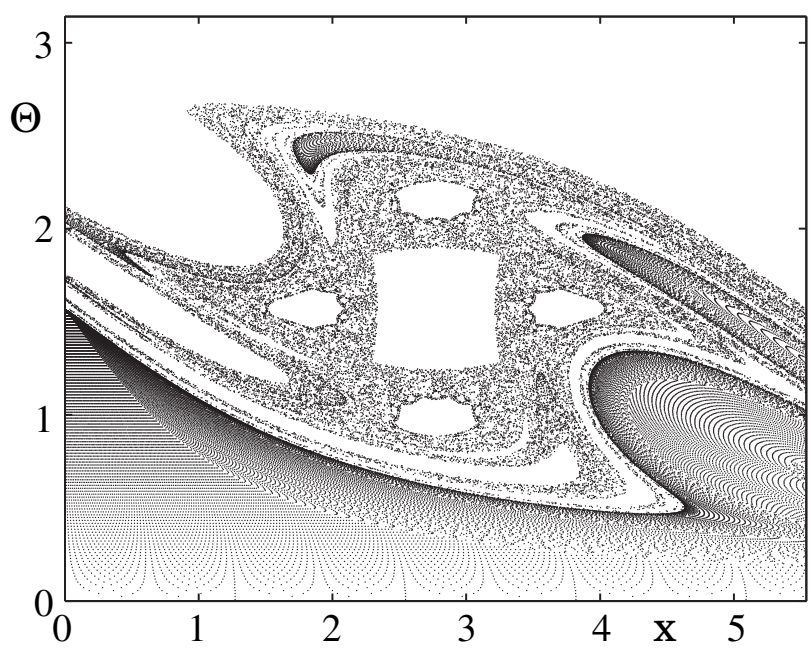

(b)

Abbildung 2.4.: Klassische Phasenraumportraits des Kosinusbillards $(d, a, L)=(1.0,0.305,5.55)$. Die Poincaréebene ist bei $y=0$ definiert. (a) Hufeisenabbildung, deren Ranken bis zur Ordnung 3 dargestellt sind. Die dicken schwarzen Linien im Zentrum der Abbildung begrenzen die Stabilitätsinseln. (b) Transiente PoincaréAbbildung, wobei die Lichtstrahlen/Teilchen von links (siehe Abb. 2.3) starten.

Die Inseln werden von den Trajektorien gebildet, die in der Nähe stabiler Orbits angesiedelt sind. Insbesondere wird die zentrale Insel durch diejenigen Trajektorien gebildet, die nahezu senkrecht bei $x \approx \frac{L}{2}$ zur Poincaréebene, hier die $(y=0)$-Achse, orientiert sind. Hier gilt es zu bemerken, dass diese Orbits von den transienten (von links oder rechts einfallenden) Strahlen nicht erreicht werden können, also klassisch verbotene Bereiche darstellen. Um den Wellenleiter weiter analysieren zu können, benötigen wir überdies die transiente Poincaré-Abbildung [24], also eine Poincaré-Abbildung mit Anfangsbedingungen, die außerhalb des Resonatorgebiets starten. Die transiente Poincaré-Abbildung (siehe Abb. 2.4b) wird von denjenigen Trajektorien generiert, die am linken Eingang des Resonators starten, entsprechend $x<0$ nach Abbildung 2.3. Ein Vergleich der Abbildungen 2.4a und 2.4b zeigt, wie erwartet, dass die Stabilitätsinseln, die von der eingeschränkten Bewegung der Trajektorien herrühren, verbotene Bereiche des Phasenraums für transiente Trajektorien darstellen. Zusätzlich sei bemerkt, dass die Struktur der transienten Poincaré-Abbildung die instabilen Mannigfaltigkeiten der korrespondierenden Hufeisenabbildung widerspiegelt. Startet man beispielsweise mit den Anfangsbedingungen von der rechten Seite, entsprechend den Anfangsbedingungen $\left(x_{0}, \Theta_{0}\right)$ mit $x_{0}>L, \frac{\pi}{2}<\Theta_{0}<\frac{3 \pi}{2}$ nach Abbildung 2.3, so erhalten wir eine transiente Poincaré-Abbildung, die zur Abbildung 2.4b spiegelsymmetrisch bezüglich der $\Theta$-Achse ist.

Die Ergebnisse dieser klassischen Betrachtung werden später benötigt, um sie mit den Resultaten der quantenmechanischen Betrachtungen vergleichen zu können. Darüber hinaus können aus dem klassischen Phasenraum die benötigten Parameter des chaotischen Strahlenteiles bestimmt werden. Diese Aspekte werden Gegenstand des nächsten Abschnitts sein. 


\subsection{Quantenmechanische Behandlung}

Um die quantenmechanischen Streuphänomene zu untersuchen, wird die Schrödinger-Gleichung wie in Kapitel 1 beschrieben - numerisch gelöst. Aus den numerisch bestimmten Wellenfunktionen wird mittels der Fischer-Lee-Relation [61] die S-Matrix bestimmt. Mithilfe des Landauer-BüttikerFormalismus[117] kann die energieabhängige Leitfähigkeit $G_{q \leftarrow p}\left(\right.$ kurz $\left.G_{q p}\right)$ als observable Größe für ein ballistisches elektrisches System

$$
G_{q p}=\frac{2 e^{2}}{h} \bar{T}_{q p}=\frac{1}{\pi} \sum_{m \in p} \sum_{n \in q}\left|s_{q p}^{n m}\right|^{2},
$$

(hier für die Temperatur $T \rightarrow 0$ ) aus der Streumatrix bestimmt werden. Dabei ist $\bar{T}_{q p}$ als Transmissionskoeffizient (Transmissionswahrscheinlichkeit $T_{q p}$ pro Mode multipliziert mit der Anzahl der Moden) von Leiter $p$ zu Leiter $q$ definiert. Es wird über alle Moden $m \in p$ und $n \in q$ der $S$-Matrixelemente $s_{q p}^{n m}$ summiert. ${ }^{8}$ An dieser Stelle sei nochmals auf die Äquivalenz der Lösungen der Schrödinger-Gleichung eines quantenmechanischen 2D-Wellenleiters und den Lösungen der TM-Wellen aus der HelmholtzGleichung eines 2D-Wellenleiters mit Dirichlet-Randbedingungen hingewiesen (siehe Abschnitt 4.1). Alle hier aufgeführten Berechnungen und Simulationen besitzen daher sowohl für optische, als auch für elektronische Systeme Gültigkeit. Für die optischen Systeme stellen die Transmissionskoeffizienten $\bar{T}_{p q}$ experimentell zugängliche Observablen dar.

\subsubsection{Quasi-gebundene Zustände}

Um die quantenmechanischen Streuvorgänge mit der klassischen Dynamik vergleichen zu können, nutzen wir die Methode der Husimi-Verteilung [118], welche die Projektion eines gegebenen Zustands auf einen kohärenten Zustand mit minimaler Unschärfe - in diesem Fall ein Gaußsches Wellenpaket - darstellt. Eine theoretische Ableitung der Verteilung befindet sich im Anhang D.1.3.2. Die HusimiVerteilung nach Gleichung (D.7) gibt demnach die Wahrscheinlichkeitsdichte im Phasenraum an, die dann direkt mit der klassischen Phasenraumdichte verglichen werden kann.

Es wurde bereits in $[24,110]$ gezeigt, dass der Resonator des Wellenleiters nach Abbildung 2.3 durch eine unvollständige Hufeisenabbildung charakterisiert ist. Damit unterliegt auch die Leitfähigkeit $G_{\mathrm{LR}}=G_{\mathrm{RL}}$ starken Fluktuationen mit scharfen Resonanzen. Die Wellenfunktion, die der schärfsten Resonanz zugeordnet ist, kann als Eigenzustand identifiziert werden, der in den Stabilitätsinseln des Phasenraums angesiedelt ist. Im Besonderen fanden wir Wellenfunktionen, die bei entsprechenden Resonanzfrequenzen "I"-, "V"- und "M"-förmige Profile aufweisen, welche den Trajektorien der periodischen Orbits mit Periode 1, 2 und 4 entsprechen (siehe dazu auch [25]). Die Ursache hierfür ist der quantenmechanische Effekt des dynamischen Tunnelns, welcher den Streuwellenfunktionen ermöglicht, durch die KAM-Barrieren zu dringen [119]. Da diese Streuwellenfunktionen den Eigenfunktionen des geschlossenen Systems ähnlich sehen, nennen wir sie quasi-gebundene Zustände. Für den hier gewählten Satz von Parametern $(d, a, L)=(1.0,0.305,5.55)$ finden wir zwei quasi-gebundene Zustände mit "I"- und "M"-Form. In Abbildung 2.5 sind die zwei Wellenfunktionen dargestellt, die in $G_{\mathrm{RL}}$ ein lokales Minimum aufweisen. Man beachte, dass unter diesen Bedingungen der Resonator auch wie ein "echter Resonator" funktioniert. Am Ende dieses und im folgenden Abschnitt werden noch Kosinusbillards mit anderer Amplitude $a$ vorgestellt, die dann auch andere quasi-gebundene Zustände

8 Der Vorfaktor $\frac{1}{\pi}$ ergibt sich als Konsequenz aus der Wahl des Einheitensystems $\hbar=e=m_{e}=1$ (Anhang A). 


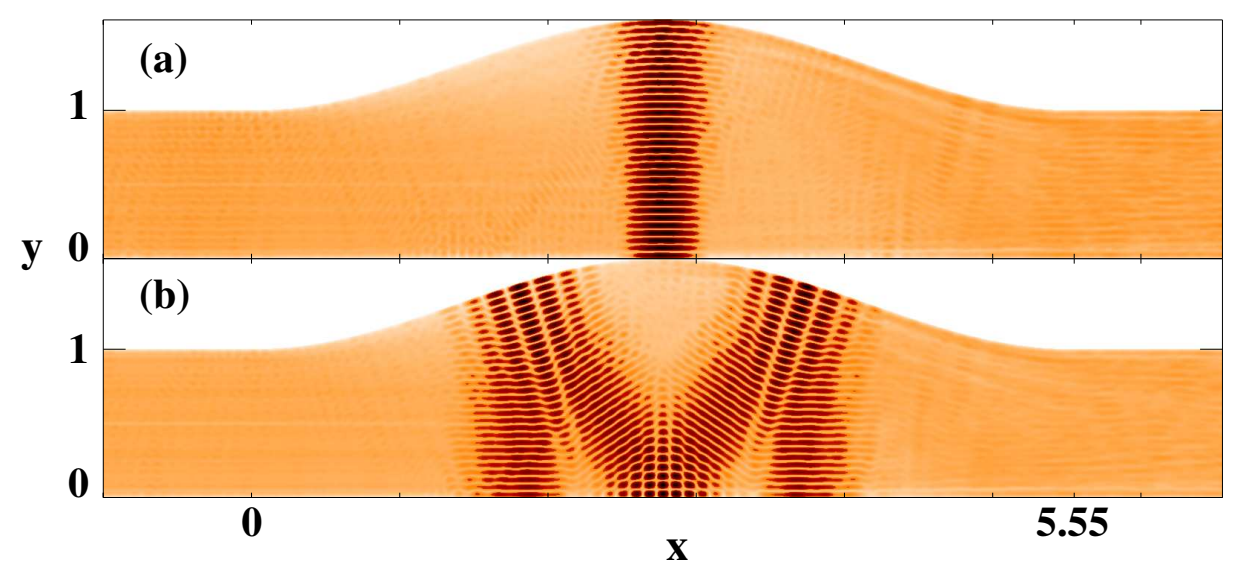

Abbildung 2.5.: Für zwei Resonanzenergien berechnete Aufenthaltswahrscheinlichkeitsdichte der Streuwellenfunktionen (quasi-gebundene Zustände) für das Kosinusbillard mit Parametern $(d, a, L)=(1.0,0.305,5.55)$ für (a) $E=4008.8583$ und (b) $E=4027.0597$. Helle Bereiche bedeuten niedrige, dunkle Bereiche hohe Aufenthaltswahrscheinlichkeitsdichte. Diese quasi-gebundenen Zustände wurden konstruiert, indem über alle $\mathcal{M}=20$ Moden der von links nach rechts eingestrahlten ebenen Wellen $\chi_{m}$ gemittelt worden ist. Die Energie $E=2 d^{2} E_{\mathrm{W}} \sim \mathcal{M}^{2} \pi^{2}$, wobei $E_{\mathrm{W}}$ die Energie der Welle im Eingangskanal darstellt und $\mathcal{M}$ die Nummer $m$ der größtmöglichen transversalen Mode $\chi_{m}$ darstellt, hinter der der longitudinale Wellenvektor $k_{m}=\sqrt{2\left(E_{\mathrm{W}}-E_{\mathrm{T}}\right)}$ komplexe Werte annimmt. Die Energie $E_{\mathrm{T}}=m^{2} \pi^{2} /\left(2 d^{2}\right)$ ist der Transversalanteil der Gesamtenergie der Welle im Eingangskanal $E_{\mathrm{W}}=E_{\mathrm{L}}+E_{\mathrm{T}}$. Zur Wahl von $\mathcal{M}$ siehe Unterabschnitt 2.3.2.

ausbilden, beispielsweise mit "V"- oder "W"-Form. Im nachfolgenden Unterabschnitt wird ein bereits durchgeführtes Experiment mit Mikrowellen zur Generierung eines "I"-förmigen Zustands vorgestellt und diskutiert.

\subsubsection{Semiklassische Näherung}

Bevor wir die Ergebnisse der quantenmechanischen Simulationen mit den bereits vorgestellten klassischen und den noch folgenden strahlenoptischen Betrachtungen vergleichen können, müssen wir sicherstellen, dass wir uns im semiklassischen Bereich befinden, d.h. die de Broglie Wellenlänge $\lambda_{\mathrm{B}}$ muss deutlich kleiner als die Dimension des Wellenleiters sein. Im Falle eines beliebigen zweidimensionalen Wellenleiters mit linkem und rechtem halbunendlichen Anschluss der Breite $d$ kann die Gesamtenergie der eingestreuten Welle als

$$
E_{\mathrm{W}}=E_{\mathrm{L}}+E_{\mathrm{T}}=\frac{k_{m}}{2}+\frac{1}{2}\left(\frac{m \pi}{d}\right)^{2}
$$

mit $k_{m}$ als longitudinalem Wellenvektor der Mode $m$ ausgedrückt werden. In der semiklassischen Näherung $\lambda_{\mathrm{B}} \ll a$ kann der longitudinale Anteil an der Gesamtenergie vernachlässigt werden. Ein Vergleich der zur de Broglie Wellenlänge zugehörigen Energie mit der Gesamtenergie der Welle liefert

$$
E_{\mathrm{W}}=\frac{1}{2}\left(\frac{2 \pi}{\lambda_{\mathrm{B}}}\right)^{2} \approx \frac{1}{2}\left(\frac{\mathcal{M} \pi}{d}\right)^{2},
$$

wobei $\mathcal{M}=\frac{2 d}{\lambda_{\mathrm{B}}}$ die maximale Modenzahl definiert. Mit $\lambda_{\mathrm{B}} \ll a$ gilt auch $\mathcal{M} \gg \frac{2 d}{a}$ als Bedingung für den semiklassischen Bereich. Eine genauere Untersuchung [24, 120] liefert die Ungleichung

$$
\mathcal{M} \geq 6 \frac{d}{a}
$$

Für den hier gewählten Parametersatz $(d, a, L)=(1.0,0.305,5.55)$ ist daher $\mathcal{M} \geq 18$ zu wählen. In den durchgeführten Simulationen wurde $\mathcal{M}=20$ gewählt und Vergleiche der quantenmechanischen Simulationen mit klassischen und strahlenoptischen Untersuchungen sind gerechtfertigt. 


\subsubsection{Experimentelle Untersuchungen mittels Mikrowellenbillard}

Experimentelle Untersuchungen der quantenmechanischen Eigenschaften von klassischen chaotischen Systemen mithilfe von normalleitenden [121] oder supraleitenden [122] Mikrowellenbillards haben sich in der Vergangenheit als leistungsfähiges und zuverlässiges Instrument herausgestellt. Ermöglicht wird dies durch die mathematische Äquivalenz der zeitunabhängigen Schrödinger-Gleichung

$$
\left(\Delta+k_{n}^{2}\right) \psi_{n}=0, \text { mit } k_{n}=\sqrt{2 E_{n}}
$$

mit den Eigenwerten $k_{n}$ eines Punktteilchens im 2D-Potenzialtopf mit unendlich hohen Wänden auf der einen und der Helmholtz-Gleichung

$$
\left(\Delta+k_{n}^{2}\right) \mathbf{E}_{n}(\mathbf{r})=0, \text { mit } k_{n}=\frac{2 \pi \nu_{n}}{c}
$$

auf der anderen Seite. In flachen bzw. quasi-zweidimensionalen Systemen gilt diese Äquivalenz sogar im Bezug auf die Randbedingungen. Ein flacher Resonator bedeutet hierbei, dass die Resonatorhöhe $h_{\mathrm{R}}$ eine Bedingung an die maximale Anregungsfrequenz $\nu_{\max }$ stellt, wobei

$$
\nu_{\max }=\frac{c}{2 h_{\mathrm{R}}}
$$

mit $c$ als Lichtgeschwindigkeit im Resonatorinneren gilt. Für Anregungsfrequenzen $\nu<\nu_{\max }$ existieren nur die TM-Moden, also die Eigenfunktionen, bei denen das elektrische Feld senkrecht auf Boden- und Abdeckplatte steht. In Folge dessen kann die Amplitude des elektrischen Feldes durch eine skalare Funktion $\Phi(\mathbf{r})$ beschrieben werden, also $\mathbf{E}(\mathbf{r})=\Phi(\mathbf{r}) \mathbf{e}_{z}$, und die Äquivalenz zwischen den Lösungen der Helmholtz-Gleichung und den quantenmechanischen Wellenfunktionen als Lösung der SchrödingerGleichung wird ersichtlich (siehe auch [123]).

Im Folgenden berichten wir über eine experimentelle Realisierung des Kosinusbillards $\left(d_{0}, 0.305,5.55\right)$ mit $d_{0}$ als variablem Parameter auf der Basis von Mikrowellen mit normalleitenden Kavitäten im Rahmen einer experimentellen Zusammenarbeit [2]. In Abbildung 2.6a ist eine Photographie des experimentellen Aufbaus zu sehen. Die Parameter $L$ und $a$ (Länge und Amplitude) des Kosinusbillards sind hierbei von der oberen, mit Schrauben befestigten Messingplatte fest vorgegeben, während die untere Wand der Apparatur parallel zur oberen verläuft, aber in vertikaler Richtung frei beweglich ist. Somit bleibt die Breite $d$ als variabler Parameter experimentell zugänglich. Die Apparatur wird von einer Messingabdeckplatte (nicht im Bild) abgeschlossen. Der Abstand $h_{\mathrm{R}}$ zwischen der oberen Abdeckplatte und der unten befindlichen, ebenfalls aus Messing gefertigten Grundplatte ist die Resonatorhöhe und beträgt in diesem Experiment $h_{\mathrm{R}}=0.8 \mathrm{~cm}$. Nach Gleichung (2.19) ergibt sich für die Maximalfrequenz $\nu_{\max }=18.75 \mathrm{GHz}$, d.h. für Anregungsfrequenzen $\nu<\nu_{\max }$ kann das System als Ganzes als zweidimensional angenommen werden.

Um das System in einem Frequenzbereich $\left[\nu_{\min }, \nu_{\max }\right]$ anregen und die Wellenfunktionen messen zu können, ist die Apparatur mit drei elektrischen Dipolantennen ausgestattet. Die erste Antenne befindet sich am linken Eingangsbereich, bei den Koordinaten $(x, y)=(-18.0 \mathrm{~cm}, 7.725 \mathrm{~cm})$, die zweite Antenne ist am rechten Eingang bei $(x, y)=(18.0 \mathrm{~cm}, 7.725 \mathrm{~cm})$ fixiert. Eine dritte Antenne ist fest in der Abdeckplatte montiert und befindet sich während des experimentellen Betriebs innerhalb des Resonators. So ist es möglich, durch Verschiebung der Abdeckplatte die Position der dritten Antenne zu verändern und die gesamte Resonatorregion abzurastern. In der experimentellen Durchführung betrug 

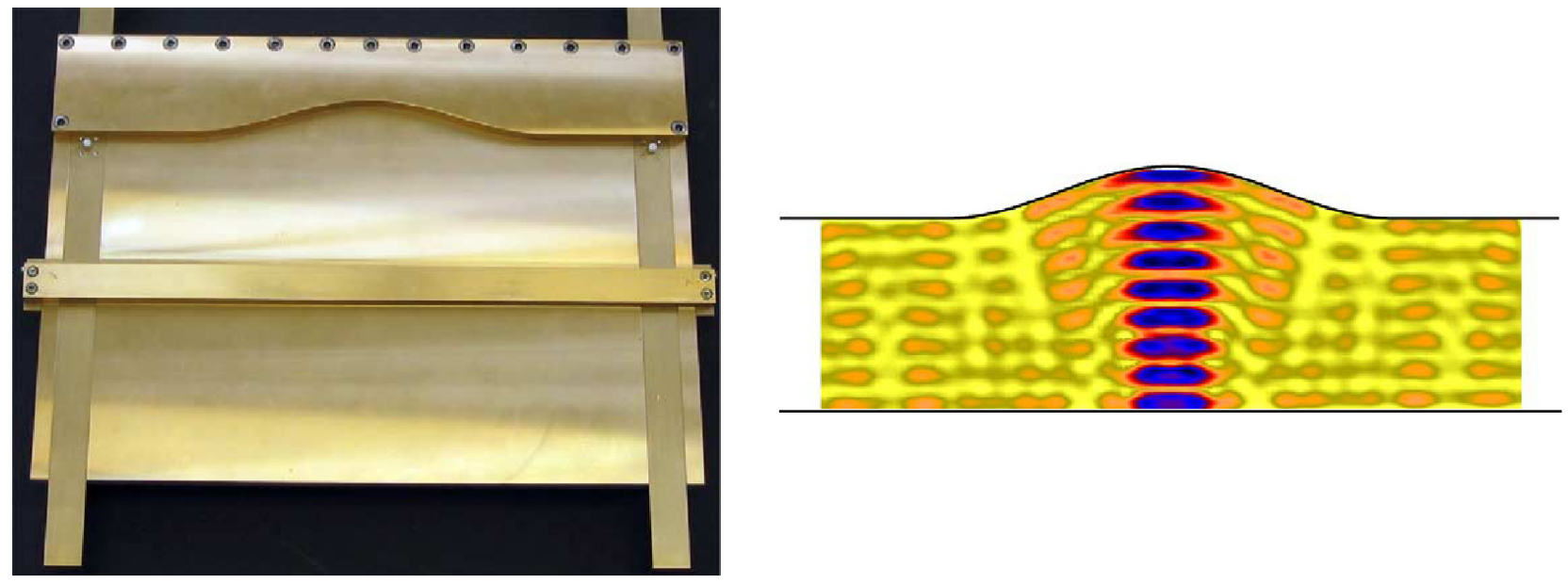

(a)

(b)

Abbildung 2.6.: Mikrowellenapparatur für ein aus Messing gefertigtes Kosinusbillard der Parametermenge $(d, a, L)=\left(d_{0}, 0.305,5.55\right)$ mit $d_{0}$ als experimentell zugänglichem Parameter, d.h. die untere Billardwand kann auf der Grundplatte in vertikaler Richtung versetzt werden. (a) Experimenteller Aufbau der Apparatur (für eine detaillierte Beschreibung siehe Text) und (b) durch eine Reflexionsmessung gewonnener Betrag der Wellenfunktion eines "I"-förmigen quasi-gebundenen Zustands bei $d_{0}=1.0$ und $\nu=13.7708 \mathrm{GHz}$. Die schwarzen Umrandungslinien sind schematische Darstellungen der oberen und unteren Wand aus Bild (a).

die Schrittweite $2.5 \mathrm{~mm}$ für Frequenzen im Bereich $\nu \in[1,18.75]$ GHz. Daraus ergibt sich ein mittleres räumliches Auflösungsvermögen von $L \times(d+a)=144 \times 45$ Punkten. Ein Vektornetzwerkanalysator kann sowohl die Reflexion der Antennen, als auch die Transmission zwischen den Antennen messen. Mit in [124, 125] entwickelten Methoden ist es möglich, sowohl den Betrag als auch das Vorzeichen der Wellenfunktion aus den Transmissionsmessungen abzuleiten. Aus der Reflexion ist allerdings nur die Bestimmung des Betrags möglich. In Abbildung 2.6b ist der Betrag der Wellenfunktion dargestellt, der für $d=1.0$ und $\nu=13.7708 \mathrm{GHz}$ aus einer Reflexionsmessung bestimmt wurde. Dieses Ergebnis lässt auf die Existenz eines quasi-gebundenen Zustands schließen, wobei sich die "I"-Form hier deutlich abzeichnet. Die entsprechende theoretische Vorhersage (vergleiche auch Abb. 2.12b) ist damit in diesem ersten Schritt bestätigt. Experimentelle Untersuchungen des weiter unten vorgeschlagenen Strahlenteilers werden erwartet.

\subsubsection{Vergleich von klassischem mit quantenmechanischem Phasenraum}

Um die klassischen Betrachtungen mit den quantenmechanischen Berechnungen bzw. Simulationen vergleichen zu können, benutzen wir die eingangs erwähnte Husimi-Verteilung (siehe Anhang D.1.3.2)

$$
H(x, k)=\left|\frac{1}{\sqrt[4]{2 \pi \sigma^{2}}} \int \exp \left(-\frac{\left(x-x^{\prime}\right)^{2}}{4 \sigma^{2}}-i k x^{\prime}\right) \psi_{\mathrm{QGZ}}\left(x^{\prime}\right) d x^{\prime}\right|^{2}
$$

für die sich ausbildenden quasi-gebundenen Zustände $\psi_{\mathrm{QGZ}}(\mathbf{x})$ und stellen sie zusammen mit den transienten Poincaré-Abbildungen des klassischen Phasenraums dar. Sowohl Abbildung 2.7a ("I"-Form, Resonanzinsel mit Periode 1), als auch Abbildung 2.7b ("M"-Form, Resonanzinseln mit Periode 4) zeigen eine positive Übereinstimmung der Struktur des klassischen Phasenraums mit der Husimi-Verteilung im Bereich der Stabilitätsinseln. Sogar die Gesamtstruktur des Phasenraums der transienten Trajektorien wird von der Husimi-Verteilung widergespiegelt. 


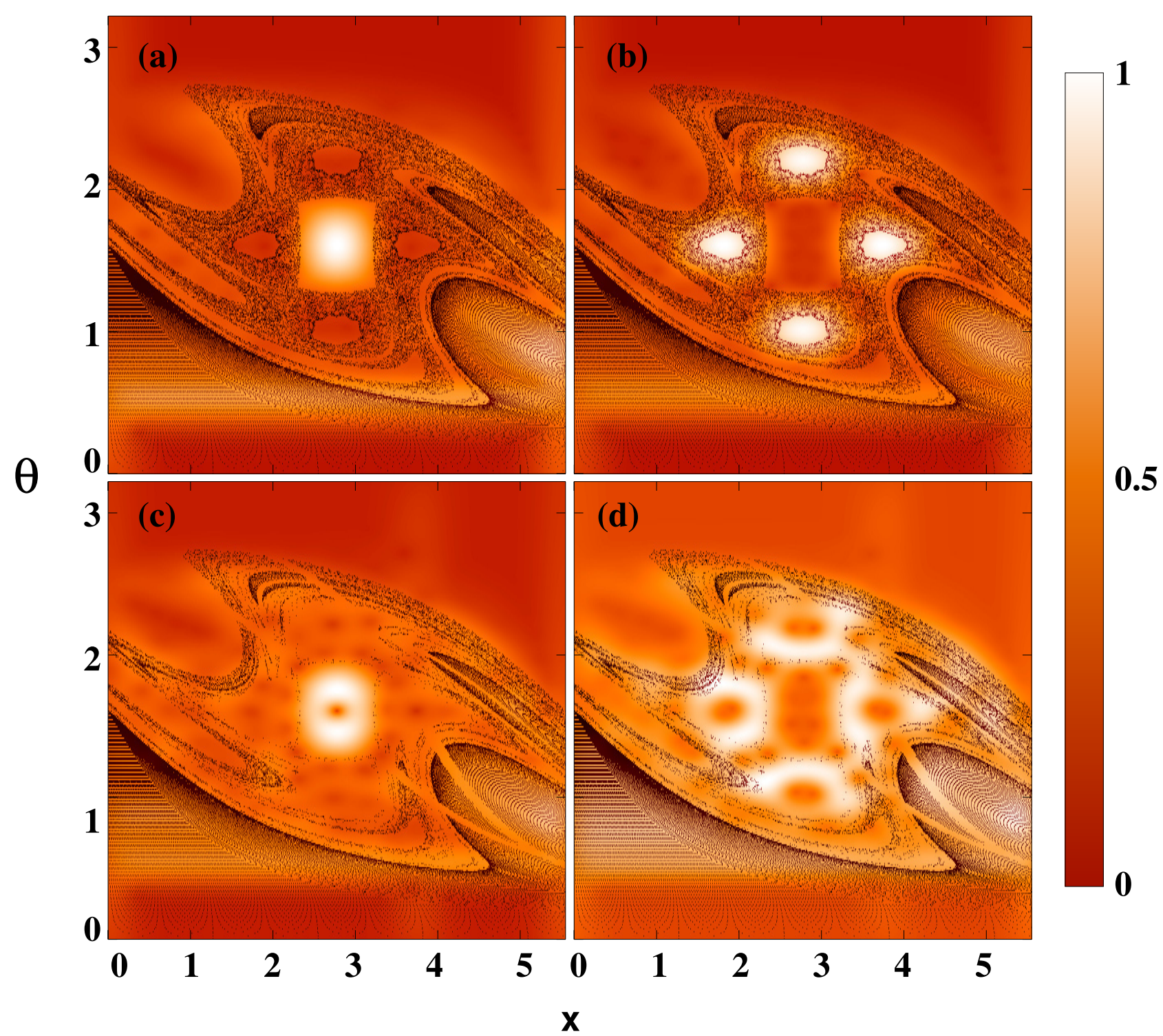

Abbildung 2.7.: Transiente Poincaré-Abbildungen (schwarze Punktwolken) überlagert mit den HusimiVerteilungen (mit Farbkodierung rot-weiß, wobei rot dem Minimum und weiß dem Maximum der maximumsnormierten Aufenthaltswahrscheinlichkeitsdichte entspricht). Die Poincaréebene ist bei $y=0$ definiert und gilt gleichermaßen für Strahlen- und Wellendynamik. Teilchen bzw. ebene Wellen werden von links in den Wellenleiter eingebracht. In der linken Spalte sind die Aufenthaltswahrscheinlichkeitsdichte mit "I"- und in der rechten Spalte die mit der "M"-Form dargestellt. Die Bilder (a) und (b) der oberen Zeile entsprechen der Konfiguration nach Abb. 2.3, die Bilder (c) und (d) in der unteren Zeile entsprechen der Konfiguration nach Abb. 2.8c.

\subsubsection{Auskopplung der quasi-gebundenen Zustände durch Phasenraum-Engineering}

Die Idee ist nun, senkrecht zur Ausbreitungsrichtung ( $x$-Richtung) orientierte, zusätzliche Anschlussleiter im Bereich der quasi-gebundenen Zustände anzubringen, um diese Zustände über jene Anschlüsse aus dem Resonator herausführen zu können, insofern diese bei einer bestimmten Resonanzenergie aktiviert sind. Dazu verbinden wir in $y$-Richtung ausgedehnte (transversale), halbunendliche Anschlussleiter der Breite $d_{\mathrm{T}}$ senkrecht mit dem Resonator. Skizzen dieser und weiterer Anschlussmöglichkeiten sind in Abbildung 2.8 wiedergegeben. Um die "I"-förmigen quasi-gebundenen Zustände aus dem Resonator zu leiten, nutzen wir die Konfiguration nach Abbildung 2.8a mit einem senkrechten Anschluss an der Oberseite bei $x=\frac{L}{2}$, bzw. die Konfiguration nach Abbildung 2.8d mit zwei senkrechten Anschlüs- 

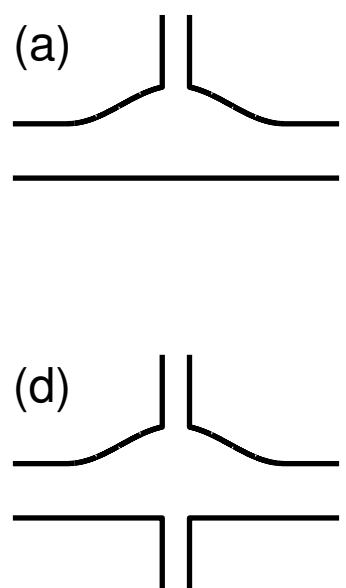

(b)

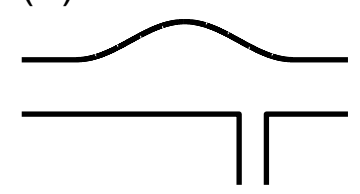

(e)

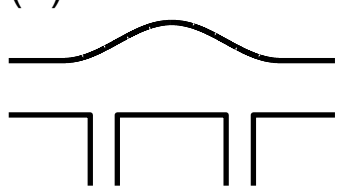

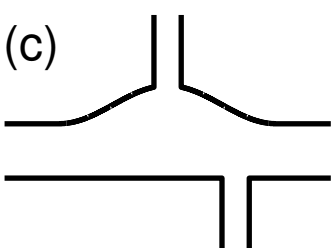

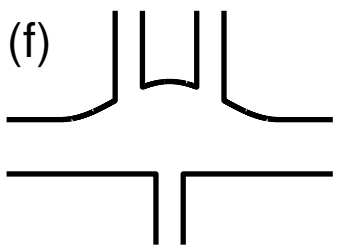

Abbildung 2.8.: Konfigurationsbeispiele des Kosinusbillards mit Parametersatz $(d, a, L)=(1.0,0.305,5.55)$ mit verschiedenen funktionellen Anforderungen. (a)-(c) Schalter, (d)-(f) Strahlenteiler. Im Einzelnen unterstützen (a) und (d) die "I"-Mode; (b), (e) und (f) die "M"-Mode; und (c) sowohl "I"- als auch "M"-Mode.

sen an der Ober- und Unterseite bei $x=\frac{L}{2}$. Für die "M"-förmigen quasi-gebundenen Zustände können Anschlüsse bei $x_{1}=\frac{L}{2}$ und $x_{2,3}=\frac{L}{2} \pm 0.975$ an der Unterseite oder/und $x_{4,5}=\frac{L}{2} \pm 0.975$ an der Oberseite angeschlossen werden (vergleiche dazu Abb. 2.8b, 2.8e und 2.8f). Die Werte $x_{i}$ können dabei direkt aus den Poincaré-Abbildungen abgelesen werden, indem man beispielsweise geometrisch die Zentren der Stabilitätsinseln des Phasenraums ermittelt. Natürlich sind hier noch andere Konfigurationen möglich, worauf aber erst später eingegangen werden soll. Vorerst wird insbesondere die Konfiguration nach Abbildung 2.8c Anwendung finden, da diese sowohl "I"-förmige, als auch "M"-förmige quasi-periodische Zustände unterstützt und diese voneinander trennt.

Augenscheinlich stören jegliche Art von zusätzlichen transversalen Anschlüssen den Phasenraum des Wellenleiters. Da wir jedoch die quasi-gebundenen Zustände aus dem Resonator führen wollen, müssen wir dieses Faktum hinnehmen, können aber die Breite $d_{\mathrm{T}}$ der zusätzlichen transversalen Anschlüsse so schmal halten, dass die globale Phasenraumstruktur erhalten bleibt. Wir haben den Zusammenhang der Strukturveränderung des Phasenraums mit zunehmender Breite $d_{\mathrm{T}}$ für die Konfiguration aus Abbildung 2.8c untersucht und als transiente Poincaré-Abbildungen in der Abbildung 2.9 dargestellt. Mit zunehmender Breite $d_{\mathrm{T}}$ verringert sich auch die Barriere zwischen den stabilen Inseln der Periode 1 und denen der Periode 4, bis diese schließlich völlig verschwindet. In weiteren Untersuchungen verwenden wir $d_{\mathrm{T}}=0.15$, da hier die Phasenraumstruktur noch ausreicht, um die Stabilitätsinsel der Periode 1 von den stabilen Inseln der Periode 4 zu separieren (siehe Abb. 2.9c). Wie eingangs bereits erwähnt, werden die quasi-gebundenen Zustände bei Frequenzen aktiviert, die scharfen lokalen Minima in der Leitfähigkeit $G_{\mathrm{RL}}$ entsprechen (siehe Abb. 2.10a) [126, 127]. Es können nun für die Konfiguration nach Abbildung 2.8c die 16 möglichen Kombinationen der Leitfähigkeiten aller vier Anschlüsse bestimmt werden. Wir beschränken uns auf die Bestimmung der Leitfähigkeiten $G_{\mathrm{OL}}$ vom linken Eingang zum oberen transversalen Ausgang, $G_{\mathrm{UL}}$ vom linken Eingang zum unteren transversalen Ausgang und $G_{\mathrm{RL}}$ vom linken Eingang zum rechten Ausgang (siehe Abb. 2.10b). Aus Gründen der Symmetrie und aufgrund der Summenregel ${ }^{9}$ ist in der hier diskutierten Fragestellung die Bestimmung von drei dieser 16

9 Büttiker formulierte eine Summenregel für die Koeffizienten der Leitfähigkeit aus der linearen Antworttheorie (siehe z.B. [19]), wobei er sicherstellte, dass der Gesamtstrom für den Fall gleicher Potenziale an allen Ein- bzw. Ausgängen unter Berücksichtigung der Kirchhoffschen Regel identisch verschwindet. Unter dieser Voraussetzung ergibt sich folgende Bedingung an die Koeffizienten der Leitfähigkeit 


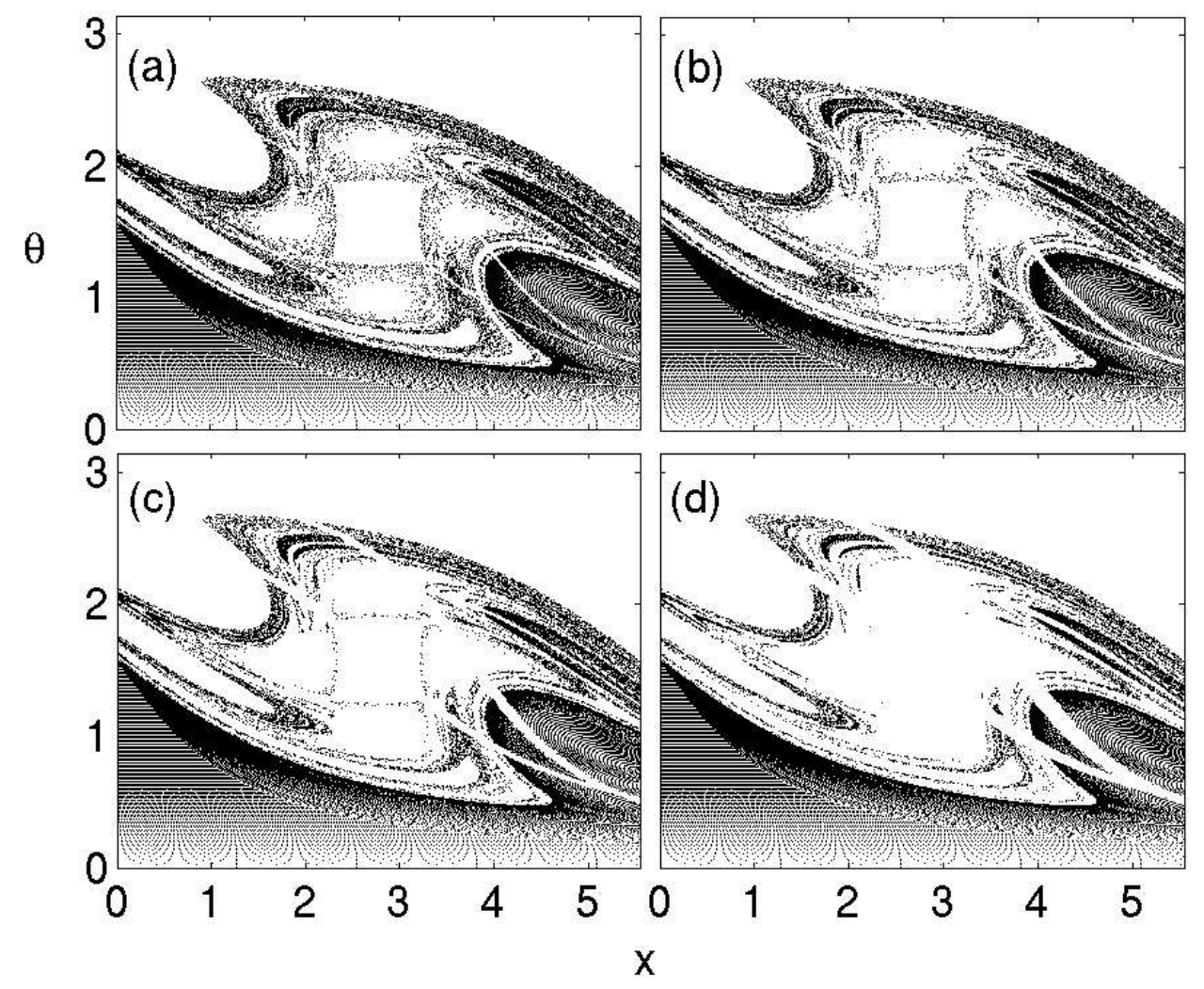

Abbildung 2.9.: Transiente Poincaré-Abbildungen mit Poincaréebene bei $y=0$ für von links einfallende Strahlen für die Kosinusbillardkonfiguration nach Abbildung 2.8c für verschiedene transversale Kanalbreiten $d_{\mathrm{T}}$. (a) $d_{\mathrm{T}}=0.05$, (b) $d_{\mathrm{T}}=0.10$, (c) $d_{\mathrm{T}}=0.15$, und (d) $d_{\mathrm{T}}=0.25$. Die Zerstörung der Phasenraumstruktur für wachsende Breiten $d_{\mathrm{T}}$ ist deutlich zu erkennen (vgl. auch mit $d_{\mathrm{T}}=0$ aus Abb. 2.7a).

Größen ausreichend, da sich dann alle anderen noch benötigten Größen aus diesen drei "gemessenen" berechnen lassen. Ohne äußeres Magnetfeld gelten die Onsager-Casimir-Reziprozitätsbeziehungen nach Gleichung (1.15) auf Seite 13. Diese stellen eine einfache Symmetriebedingung an die Kreuzkoeffizienten der Leitfähigkeit

$$
G_{\mathrm{XY}}=G_{\mathrm{YX}}
$$

Dies spiegelt sich in der weiteren Summenregel

$$
\sum_{\mathrm{X}} G_{\mathrm{XY}}=\mathcal{M}_{\mathrm{Y}}, \quad \text { mit } \mathrm{X}, \mathrm{Y} \in\{\mathrm{L}, \mathrm{R}, \mathrm{O}, \mathrm{U}\}
$$

wider, wobei $\mathcal{M}_{\mathrm{L}}=\mathcal{M}_{\mathrm{R}}=20$ als Anzahl der in den linken bzw. rechten Anschluss eingestrahlten Moden gegeben ist. Die verwendete Frequenz bzw. Energie beschränkt hierbei die Anzahl der maximal möglichen Moden. Aufgrund der Symmetrie ist die Wahl des Einschusskanals (Index L für links bzw. R für rechts) unerheblich. Die benötigte Größe $G_{\mathrm{LL}}$ bzw. $G_{\mathrm{RR}}$ lässt sich aus Gleichung (2.23) bestimmen. Wie erhofft beobachten wir, dass einige der schärfsten Minima in $G_{\mathrm{RL}}$ scharfen Maxima in $G_{\mathrm{OL}}$ bzw. $G_{\mathrm{UL}}$ entsprechen. Das bedeutet, dass bei genau diesen Frequenzen bzw. Energien Wellen durch die chaotische See in die Gebiete mit stabilen Inseln tunneln und in die transversalen Ausgänge (Index $\mathrm{O}$ für oben und U für unten) geleitet werden. Um jenes Verhalten zu demonstrieren, haben wir in

$$
\sum_{p} G_{p q}=\sum_{p} G_{q p}
$$




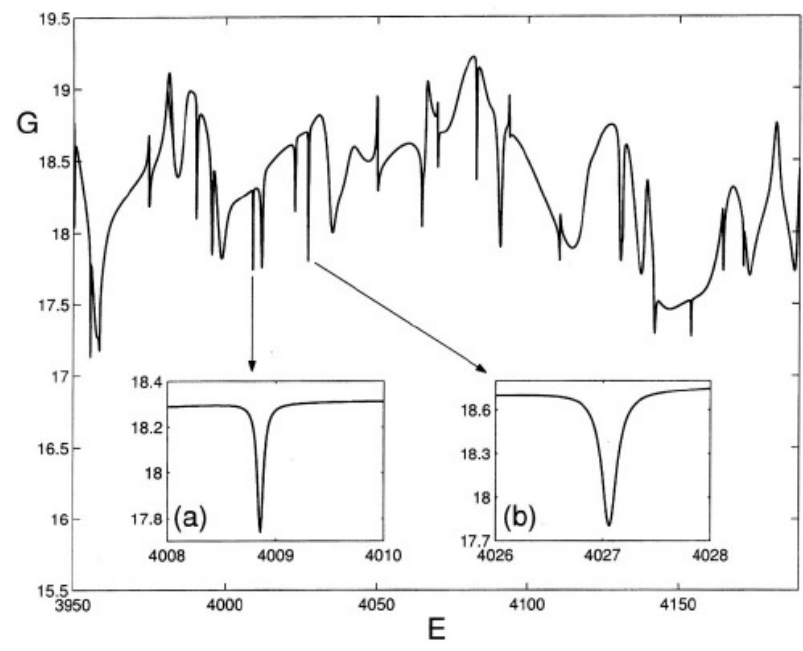

(a)

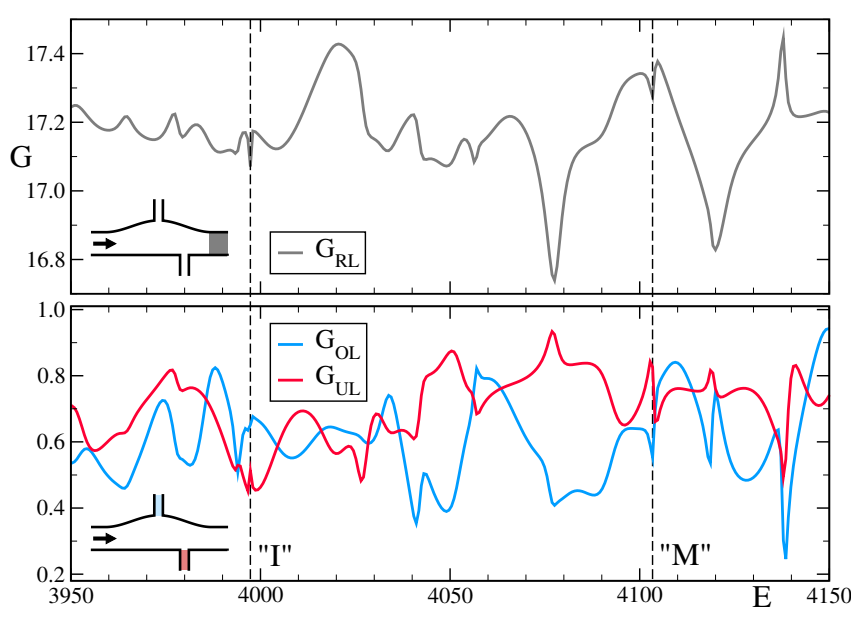

(b)

Abbildung 2.10.: Dimensionslose Leitfähigkeiten $G=\pi G_{p q}$ des Kosinusbillards nach Gleichung (2.13) in Abhängigkeit der Energie für den Parametersatz $(d, a, L)=(1.0,0.305,5.55)$ für $\mathcal{M}=20$ von links eingestrahlte ebene Wellen (a) nach Konfiguration aus Abbildung 2.3: Die Unterabbildungen (a) und (b) zeigen vergrößerte Ausschnitte der lokalen Energieminima, die zur Berechnung der beiden quasi-gebundenen Zustände "I" und "M" aus Abbildung 2.5 benutzt worden sind (aus [25]). Die Q-Faktoren nach Lorentz-Approximationen ergeben $Q_{\mathrm{I}} \approx 50000$ und $Q_{\mathrm{M}} \approx 25000$. (b) nach Konfiguration aus Abbildung 2.8c: Die schematischen Unterabbildungen skizzieren die benutzten Anschlüsse für die jeweilige Leitfähigkeitsberechnung. Die Energien der gestörten quasigebundenen Zustände "I" $(E=3997.21)$ und "M" $(E=4103.33)$ sind durch gestrichelte Linien gekennzeichnet. Die Gütefaktoren wurden zu $Q_{\mathrm{I}} \approx 10000$ und $Q_{\mathrm{M}} \approx 3000$ bestimmt.

den Abbildungen 2.7a,b die Husimi-Darstellung der quasi-gebundenen Zustände "I" und "M" (siehe Abbildung 2.5 des Wellenleiters ohne transversale Anschlüsse) mit den Streuzuständen zu den Resonanzenergien, die den Minima von $G_{\mathrm{RL}}$ entsprechen, verglichen. Analoges gilt für die Abbildungen 2.7c,d mit Konfiguration nach Abbildung 2.8c. Die quantenmechanischen Streuzustände aus Abbildung 2.7c,d entsprechen also den Minima in $G_{\mathrm{RL}}$ und Maxima in $G_{\mathrm{UL}}$ bzw. $G_{\mathrm{OL}}$. Wie erwartet befinden sich die Maxima der Aufenthaltswahrscheinlichkeit dieser Zustände innerhalb der stabilen Regionen des klassischen Phasenraums ${ }^{10}$. Dieser Sachverhalt kann direkt aus den Darstellungen der Husimi-Verteilung abgelesen werden. Im Zentrum der stabilen Inseln (Abb. 2.7c,d) ist die Aufenthaltswahrscheinlichkeit nahe Null, was zusammen mit $G_{\mathrm{OL}}$ maximal bzw. $G_{\mathrm{UL}}$ maximal darauf hindeutet, dass die quasi-gebundenen Zustände aus dem Wellenleiter durch die transversalen Anschlüsse geleitet werden.

Für die Bestimmung der $Q$-Faktoren nach Gleichung (2.4) müssen noch die Resonanzbreiten bestimmt werden. Diese können durch Lorentz-Cauchy-Ausgleichsrechnungen spezifiziert werden. ${ }^{11}$ Für das System ohne transversale Anschlüsse ergeben sich Gütefaktoren von $Q_{\mathrm{I}} \approx 50000$ und $Q_{\mathrm{M}} \approx 25000$ und für das System mit transversalen Anschlüssen finden wir $Q_{\mathrm{I}} \approx 10000$ und $Q_{\mathrm{M}} \approx 3000$ (siehe auch Abb. 2.10). Die Faktoren nehmen so stark ab, da die Frequenzunschärfe $\Delta \nu$ des Leitwerts mit der

10 Zur Erinnerung: Stabile Phasenrauminseln stellen den klassisch verbotenen Bereich für transiente Trajektorien dar.

11 Eine Begründung der Approximation der Zustandsdichte mit der Lorentz-Funktion wird in Unterabschnitt 1.1.3 abgeleitet. Über den quantenmechanischen Zusammenhang der Stromdichte als Produkt aus Zustandsdichte und Geschwindigkeit gilt diese Näherung auch für den Leitwert. 


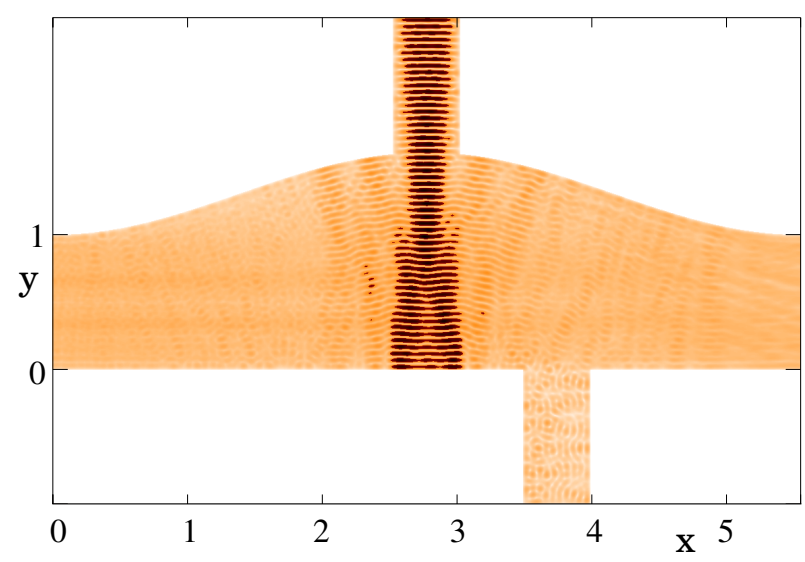

(a)

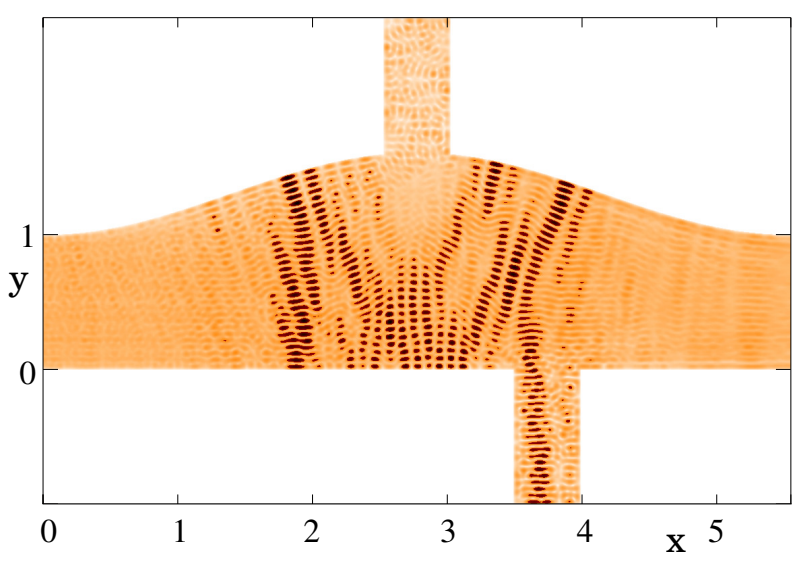

(b)

Abbildung 2.11.: Aufenthaltswahrscheinlichkeitsdichte für zwei Beispiele eines Strahlenteilers mit Parametersatz $(d, a, L)=(1.0,0.305,5.55)$, der mit zwei endlichen transversalen Anschlüssen versehen wurde (entspricht Konfiguration nach Abb. 2.18d). Dargestellt sind der (a) "I"-förmige $(E=4240.71)$ (b) "M"-förmige ( $E=4279.78)$ quasi-gebundene Zustand.

Verweildauer $\tau$ der Photonen bzw. Elektronen nach Gleichung (2.4) über

$$
\Delta \nu=\frac{1}{2 \pi \tau}=\frac{\nu}{Q}
$$

indirekt proportional zusammenhängt. Zusätzliche transversale Anschlüsse verkürzen also die mittlere Verweildauer der Teilchen im Resonator, wodurch auch die Schärfe der Leitwertextrema entsprechend abnimmt. Zum Vergleich mit anderen $Q$-Faktoren sei auf Abb. 2.1 verwiesen.

Die Darstellung der Zustandsdichte der zugehörigen Streuwellenfunktionen für den Fall mit transversalen Anschlussleitern analog zu Abb. 2.5 erweist sich als nicht aufschlussreich, da die Streuwellenfunktionen aus den entsprechenden Gebieten herausgeleitet werden und deshalb nicht in der Zustandsdichte in Erscheinung treten. Zur Veranschaulichung haben wir daher den Fall mit endlichen transversalen Anschlüssen gewählt, um das Strahlenteilerprinzip anhand der Wellenfunktion zu verdeutlichen (siehe Abb. 2.11).

Mit der Konfiguration nach Abbildung 2.8c bzw. 2.8f ist es auch möglich, einen frequenz- bzw. energieabhängigen Schalter zu konstruieren. Je nach verwendeter Energie wird entweder ein "I"- oder ein "M"-förmiger Zustand angeregt, wobei der "I"-förmige Zustand nach oben und der "M"-förmige Zustand nach unten aus dem Wellenleiter geführt wird. In gleicher Weise lassen sich Strahlenteiler konstruieren: Aus der Konfiguration nach Abbildung 2.8d wird beispielsweise der "I"-förmige quasigebundene Zustand in einen unteren und einen oberen Anteil aufgespalten, während aus Konfiguration nach Abbildung 2.8e ersichtlich ist, dass hieraus zwei untere Anteile resultieren.

Die Diskussion der Konfigurationen soll aufgrund der zahlreichen Kombinationsmöglichkeiten nicht vollständig geführt werden, weshalb im Folgenden andere mögliche Modifikationen nur kurz thematisiert werden. 

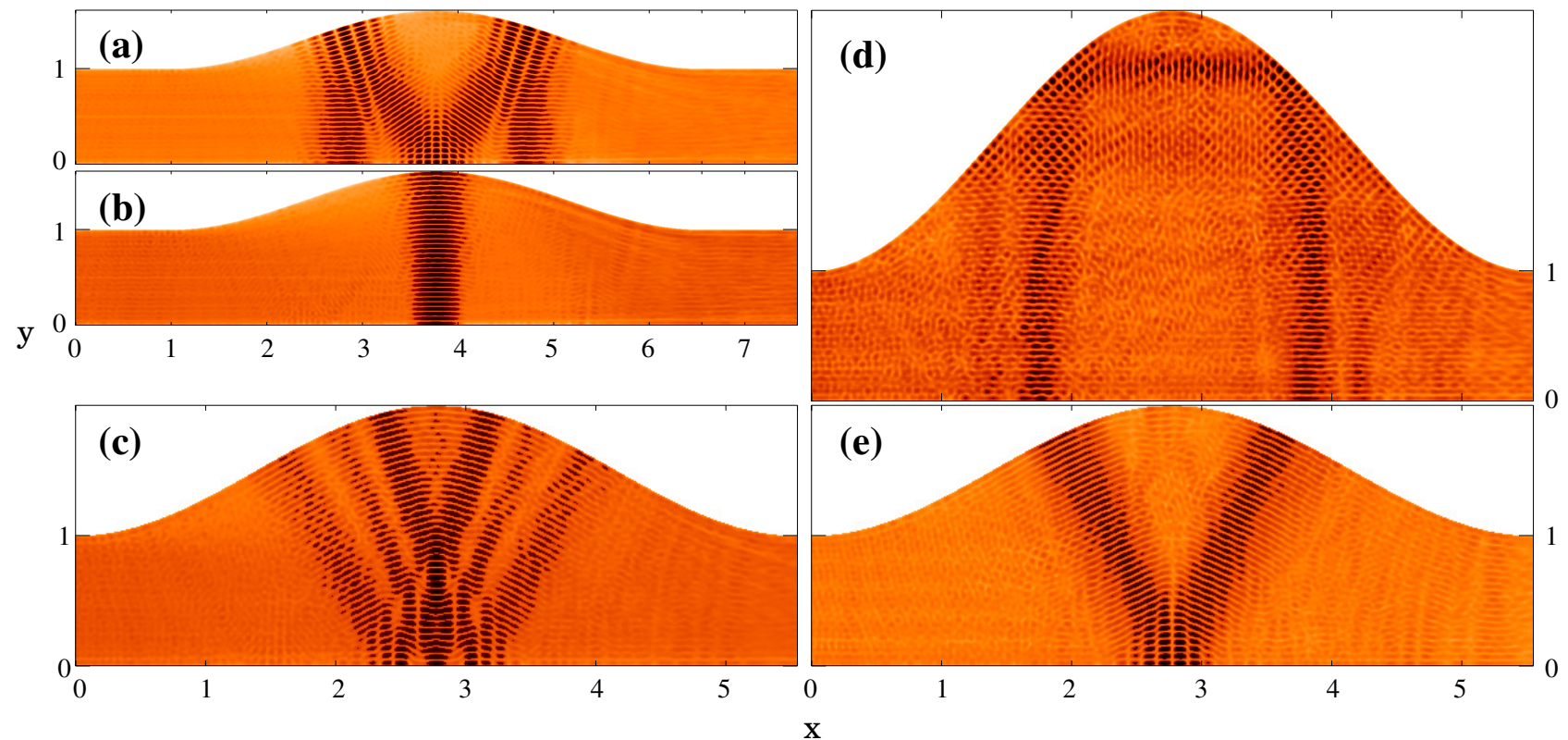

Abbildung 2.12.: Aufenthaltswahrscheinlichkeitsdichte für verschiedene quasi-gebundene Zustände unterschiedlicher Kosinusbillards $(d, a, L)$. Hier wurde nur der Parameter $a$ variiert, die Parameter $d=1.0$ und $L=5.55$ sind für alle Abbildungen gleich. (a) "M"-, (b) "I"-, (c) "W"-, (e) "V"- und (d) "П"-förmige quasi gebundene Zustände. In Unterabbildung (a) und (b) wurde $a=0.305$, in (c) und (e) wurde $a=0.5$ und für (d) wurde $a=1.0$ gewählt. Der quasi-gebundene Zustand in (d) stellt einen narbigen Zustand dar, der in der Nähe von klassisch instabilen periodischen Orbits gebildet wird (siehe Text).

\subsubsection{Modifikationen des Parametersatzes $(d, a, L)$}

Für andere geometrische Parametersätze $(d, a, L)$ können weitere unterschiedlichste Konfigurationen abgeleitet werden. Wie schon erwähnt, entwickeln sich für $(d, a, L)=(1.0,0.305,5.55)$ die beiden "I"und "M"-förmigen Strukturen, während sich für $(d, a, L)=(1.0,0.5,5.55)$ auch anders geformte quasigebundene Zustände mit "V"- und "W"-förmigen Strukturen ausbilden. Die bereits genannte " $\Pi$ "-Form bildet sich bei $(d, a, L)=(1.0,1.0,5.55)$ aus. Eine Übersicht der hier aufgezählten verschiedenen quasigebundenen Zustände ist in Abbildung 2.12 beispielhaft dargestellt. Einige technische Anwendungen sollen im folgenden Abschnitt genauer besprochen werden.

Als zusätzliche Erläuterung sei angeführt, dass im Falle eines Resonators mit vollständiger Hufeisenabbildung die Leitfähigkeit im Allgemeinen eine glatte Funktion der Energie (bzw. Frequenz) ist. Einige nicht-generische vollständige Hufeisenabbildungen weisen auf das Vorhandensein von narbigen $\mathrm{Zu}-$ ständen (scars) hin [128, 129], welche die Leitfähigkeitsresonanzen verursachen und z.B. "П"-förmige quasi-gebundene Zustände hervorbringen. Hierbei ist es die unmittelbar angrenzende Umgebung der klassisch instabilen periodischen Orbits, in welcher die Aufenthaltswahrscheinlichkeit der entsprechenden quantenmechanischen Wellenfunktion deutlich erhöht ist und somit einen Lokalisierungseffekt darstellt. Ein Beispiel eines "П"-förmigen quasi-gebunden Zustands ist in Abbildung 2.12d dargestellt, wobei $(d, a, L)=(1.0,1.0,5.55)$ als Parametersatz gewählt wurde. Klassisch verhält sich dieses System rein chaotisch. Die Konstruktion von Mikrolasern, Multi-Kavitätsresonatoren, Strahlenteilern und dergleichen auf Grundlage von narbigen quasi-gebundenen Zuständen sollte daher ebenfalls möglich sein. 


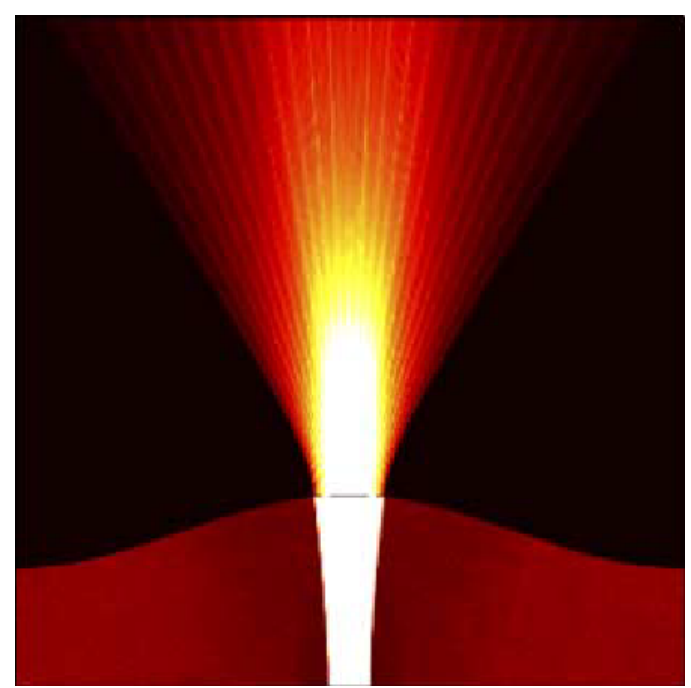

(a)

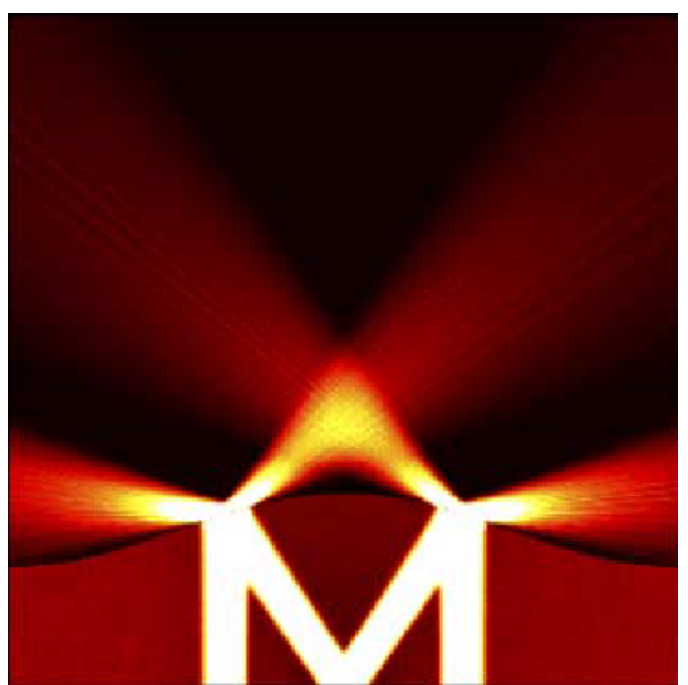

(b)

Abbildung 2.13.: Simulation der Strahlendynamik nach dem Snelliusschem Brechungsgesetz nach Glg. (2.25) und den Fresnelschen Formeln nach Glg. (2.26) für die Laseremission des Kosinusbillards mit Parametersatz $(d, a, L)=(1.0,0.305,5.55)$ mit typischen Brechungsindices $n$ und Übergang in Luft für die quasi-gebundenen Zustände (a) "I"-Form mit $n=6$ und (b) "M"-Form mit $n=3.33$ auf einem $555 \times 555$-Gitter. Zur Wahl der Brechungsindices siehe Text.

\subsection{Weitere Anwendungen}

In diesem Abschnitt werden weitere, den dynamischen Tunneleffekt des Kosinusbillards ausnutzende Anwendungen vorgestellt, die sowohl die Konstruktion von Mikrolasern, als auch von Strahlenteilern beinhalten. Im Besonderen wird ein effektiver Einmodenlaser konstruiert. Die Hintereinanderschaltung von Mikrolasern und Strahlenteilern ermöglicht die Realisierung von Multi-Kavitätsresonatoren und Multi-Kavitätsstrahlenteiler. Dieser Teil bildet den Abschluss der Untersuchungen zu chaotischen Billards.

\subsubsection{Mikrolaser}

Besteht der oben ausführlich beschriebene Wellenleiter aus einem Halbleitermaterial, so besitzt dieser einen konstanten Brechungsindex $n_{1}$. Streut man eine Welle zur Energie $E_{\mathrm{R}}$ durch einen der Anschlüsse des kosinusförmigen Wellenleiters ein, so wird sich dieser genau dann wie ein Laserresonator verhalten, wenn $G\left(E_{\mathrm{R}}\right)$ einer scharfen Leitfähigkeitsresonanz entspricht. Die Elektronen- bzw. Lichtwelle reflektiert in der Billardregion so, dass sie nur unter bestimmten Winkeln $\Theta_{i}$ aus dem Wellenleiter austreten kann. Jeder dieser Winkel $\Theta_{i}$ ist von der Energie $E_{\mathrm{R}}$ der Welle und damit dem zugehörigen quasi-gebundenen Zustand $\psi_{\mathrm{QGZ}}$, vom Brechungsindex $n_{1}$ des Resonators und dem Brechungsindex $n_{2}$ seiner Umgebung abhängig. In Abbildung 2.13 wurde eine Strahlendynamik benutzt, um die Austrittsrichtung für verschiedene Moden ("M" und "I") vorherzusagen. Beide Strahlen weisen eine hohe Qualität und eine hohe Richtcharakteristik auf [25]. Die Berechnung der Richtung der Trajektorien erfolgt mit dem Snelliusschem Brechungsgesetz

$$
\sin \left(\Theta_{\mathrm{t}}^{\prime}\right)=n \sin \left(\Theta^{\prime}\right) \quad,
$$


wobei die gestrichenen Größen die Winkel bezeichnen, die zwischen Ausbreitungsrichtung der Welle und dem Lot auf den reflektierenden Rand des Wellenleiters aufgespannt werden. Es gilt die Winkelbeziehung $\Theta+\Theta^{\prime}=\frac{\pi}{2}$. Der Index $t$ bezeichnet die transmittierte Größe. Im Außenbereich des Wellenleiters soll $n_{2}=1$ gelten. Zur Nomenklatur der auftretenden Winkel und Brechungsindices siehe Abbildung 2.14. Die Intensitäten der einfallenden und auslaufenden Welle sind mit den Fresnelschen Formeln

$$
\begin{aligned}
& R=\left[\frac{\tan \left(\Theta^{\prime}-\Theta_{\mathrm{t}}^{\prime}\right)}{\tan \left(\Theta^{\prime}+\Theta_{\mathrm{t}}^{\prime}\right)}\right]^{2} \\
& T=\left(\frac{\cos \left(\Theta_{\mathrm{t}}^{\prime}\right)}{n \cos \left(\Theta^{\prime}\right)}\right)\left[\frac{2 \sin \left(\Theta_{\mathrm{t}}^{\prime}\right) \cos \left(\Theta^{\prime}\right)}{\sin \left(\Theta^{\prime}+\Theta_{\mathrm{t}}^{\prime}\right) \cos \left(\Theta^{\prime}-\Theta_{\mathrm{t}}^{\prime}\right)}\right]^{2},
\end{aligned}
$$

als quantitative Beschreibung der Reflexions- und der Transmissionseigenschaften der reflektierten und transmittierten TM-Wellen anteilig bestimmbar [130]. Die Simulation wurde auf einem $555 \times 555$-Gitter durchgeführt. Hierbei wurde vermerkt, wie oft jeder Gitterpunkt $P(x, y)$ pro Anfangsbedingung erreicht wird. Die daraus berechnete Punktdichte $\rho_{P}(x, y)$ kann als klassische Entsprechung der quantenmechanischen Aufenthaltswahrscheinlichkeitsdichte $|\psi(x, y)|^{2}$ gedeutet werden (siehe dazu auch Abb. 2.5). Die Anfangsbedingungen $\left(x_{0}, \Theta_{0}\right)$ der Starttrajektorien sind in diesem Fall so gewählt, dass sie innerhalb der stabilen Inseln des

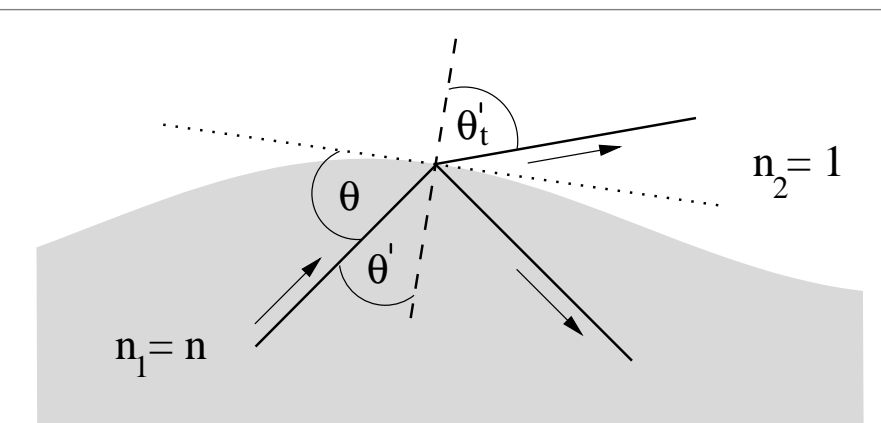

Abbildung 2.14.: Zur Nomenklatur der auftretenden Winkel im Snelliusschen Brechungsgesetz (2.25) und den Fresnelschen Formeln (2.26). Der grau eingefärbte Bereich kennzeichnet einen Ausschnitt des Wellenleiters, die Pfeile bezeichnen die Richtung der einfallenden, der reflektierten und der transmittierten Wellenanteile.

Phasenraums liegen (siehe auch Abb. 2.4b). Der Brechungsindex $n_{1}=n$ (und $n_{2}=1$, da Luft im Außenraum angenommen wurde) ist so gewählt, dass der kritische Winkel $\Theta_{c}^{\prime}$, also der Grenzwinkel der Totalreflexion

$$
\Theta_{\mathrm{c}}^{\prime}=\arcsin \left(\frac{1}{n}\right) \Longleftrightarrow \Theta_{\mathrm{c}}=\arccos \left(\frac{1}{n}\right)
$$

ebenfalls innerhalb der entsprechenden stabilen Inseln des Phasenraums anzutreffen ist. Gilt für den Anfangswert $\Theta_{0}<\Theta_{c}$, so können die Strahlen die Kavität aufgrund des Snelliusschen Brechungsgesetzes verlassen. Im Falle $\Theta_{0} \geq \Theta_{c}$, das heißt für größere Brechungsindices, kann im Fall des "M"-förmigen quasi-gebundenen Zustands sogar Totalreflexion erreicht werden. Damit sind sowohl Richtung als auch Intensität des Lasers über den Brechungsindex steuerbar.

Ein großer Vorteil der hier vorgestellten, auf dem Tunnelprinzip basierenden Version des Mikrolasers gegenüber herkömmlichen Wellenleitern ist, dass sie ohne Sockel oder Optokoppler und ohne optische Pumpen in der Nähe der Kavität auskommt. Dies ist ein neuartiger Aspekt im Vergleich zu bisher entwickelten und untersuchten 3D/2D-Mikrolasern (vergleiche Kap. 2.1).

Eine zusätzlich aufgebrachte, durchlässige Verspiegelung bzw. die Aufbringung einer dünnen Schicht mit hohem Brechungsindex vermindert die Kopplung und kann die Resonanzamplituden der Leitfähigkeit bzw. Transmission verstärken und somit das Auskoppeln zu bestimmten Frequenzen - analog zum Fabry-Pérot-Interferometer - bei gleichzeitiger Verringerung der Peakbreite verbessern (siehe Unterabschnitt 2.1.1.1). In der hierzu mittels Streumatrixformalismus durchgeführten Simulation zum Para- 
metersatz $(d, a, L)=(1.0,0.305,5.55)$ nach Konfiguration 2.8a wird der Wellenleiter mit einer perfekten Verspiegelung - entsprechend einem unendlich hohen Potenzial - versehen, wobei der obere transversale Anschluss mit einer durchlässigen Verspiegelung - entsprechend einer endlichen Potenzialstufe ausgestattet ist. Sowohl Breite als auch Länge der Potenzialstufe bleiben als Parameter frei wählbar. In einem Experiment entspräche dies einer Totalverspiegelung des Wellenleiters und anschließendem teilweisen Wegätzen der Extraschicht in der Region der maximalen Ausdehnung bei $x=\frac{L}{2}$. Anhand der

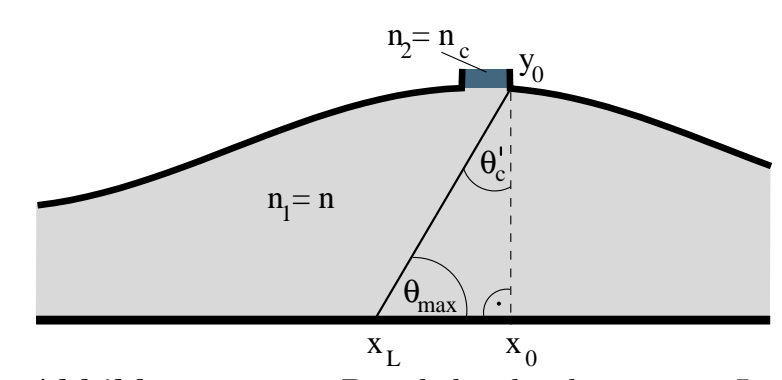

Abbildung 2.15.: Die dicke durchgezogene Linie kennzeichnet die mit perfekter Verspiegelung versehene Billardwand, der dunkelblaue Bereich die Potenzialstufe (teildurchlässige Verspiegelung). Zur Bezeichnung der auftretenden Größen siehe Text.

Abbildung 2.15 wird im Folgenden die Berechnung der kritischen Größe $n_{c}$ sowie die für die Simulation benötigte Höhe der Potenzialstufe $E_{y}$ erläutert. Für Anfangswerte bzw. Einfallswinkel von $\Theta_{0} \leq \Theta_{\max } \equiv \frac{\pi}{2}-\Theta_{\mathrm{c}}^{\prime}$ erfolgt Totalreflexion, für $\Theta_{0}>\Theta_{\max }$ kann die einfallende Welle aus dem Wellenleiter auskoppeln. Der Wert von $\Theta_{\max }$ ist durch die Geometrie des Wellenleiters und den kritischen Brechungsindex $n_{\mathrm{c}}$ festgelegt. Der Punkt $\left(x_{\mathrm{L}}, 0\right)$ lässt sich direkt aus der Poincaré-Abbildung als Begrenzung des linken Rands der zentralen Insel ablesen. Der Punkt $\left(x_{0}, 0\right)$ ist durch die Wahl der Breite der transversalen Öffnung $W$ und der Länge des Resonatorbereichs $L$ mit $x_{0} \equiv \frac{W+L}{2}$ definiert. Der letzte noch zu bestimmende Punkt $\left(x_{0}, y_{0}\right)$ des rechtwinkligen Dreiecks aus Abbildung 2.15 ist mittels des Funktionswertes des Kosinusprofils als $y_{0} \equiv y\left(x_{0}\right)$ zu berechnen. Für den kritischen Winkel der Totalreflexion gilt somit

$$
\Theta_{\mathrm{c}}^{\prime}=\arctan \left(\frac{x_{0}-x_{\mathrm{L}}}{y_{0}}\right)
$$

und aus Gleichung (2.27) bestimmen wir den kritischen Brechungsindex zu

$$
n_{\mathrm{c}}=\frac{1}{\sin \left(\Theta_{\mathrm{c}}^{\prime}\right)}=\frac{\sqrt{\left(x_{0}-x_{\mathrm{L}}\right)^{2}+y_{0}^{2}}}{x_{0}-x_{\mathrm{L}}}
$$

Für die Wellenausbreitung im Resonator unter dem Winkel $\theta_{\text {max }}$ gilt

$$
\tan \left(\Theta_{\max }\right)=\frac{k_{y}}{k_{x}}
$$

für die Wellenzahlen in $x$ - und $y$-Richtung. Mit der Dispersionsrelation $k \propto \sqrt{E}$ zwischen Wellenzahl und Energie ${ }^{12}$ und dem Superpositionsprinzip für longitudinale und transversale Wellen mit der Konsequenz für die Gesamtenergie der Welle $\langle E\rangle=E_{x}+E_{y}$ und der Winkelbeziehung $\Theta_{\mathrm{c}}^{\prime}=\frac{\pi}{2}-\Theta_{\max }$ ergibt

12 Hier wurde die Dispersionsrelation für ein kontinuierliches System angenommen. In einer diskreten Gitterapproximation mit Gitterkonstante $a$ gilt $k \propto \frac{1}{a} \arccos \left(1-a^{2}\langle E\rangle\right)$, wobei sich dann in erster Näherung für die Potenzialstufe

$$
E_{y} \approx \frac{1}{a^{2} p^{2}}\left[\left(1-g^{2}\right)+\sqrt{\left(g^{2}-1\right)\left[\left(g^{2}-1\right)+2 p^{2} \sqrt{g^{2}-1} \operatorname{arccosh}(g)\right]}-p^{2} \sqrt{1-g^{2}} \operatorname{arccosh}(g)\right]
$$

mit $g \equiv 1-a^{2}\langle E\rangle$ und $p \equiv \frac{x_{0}-x_{\mathrm{L}}}{y_{0}}$ ergibt. 

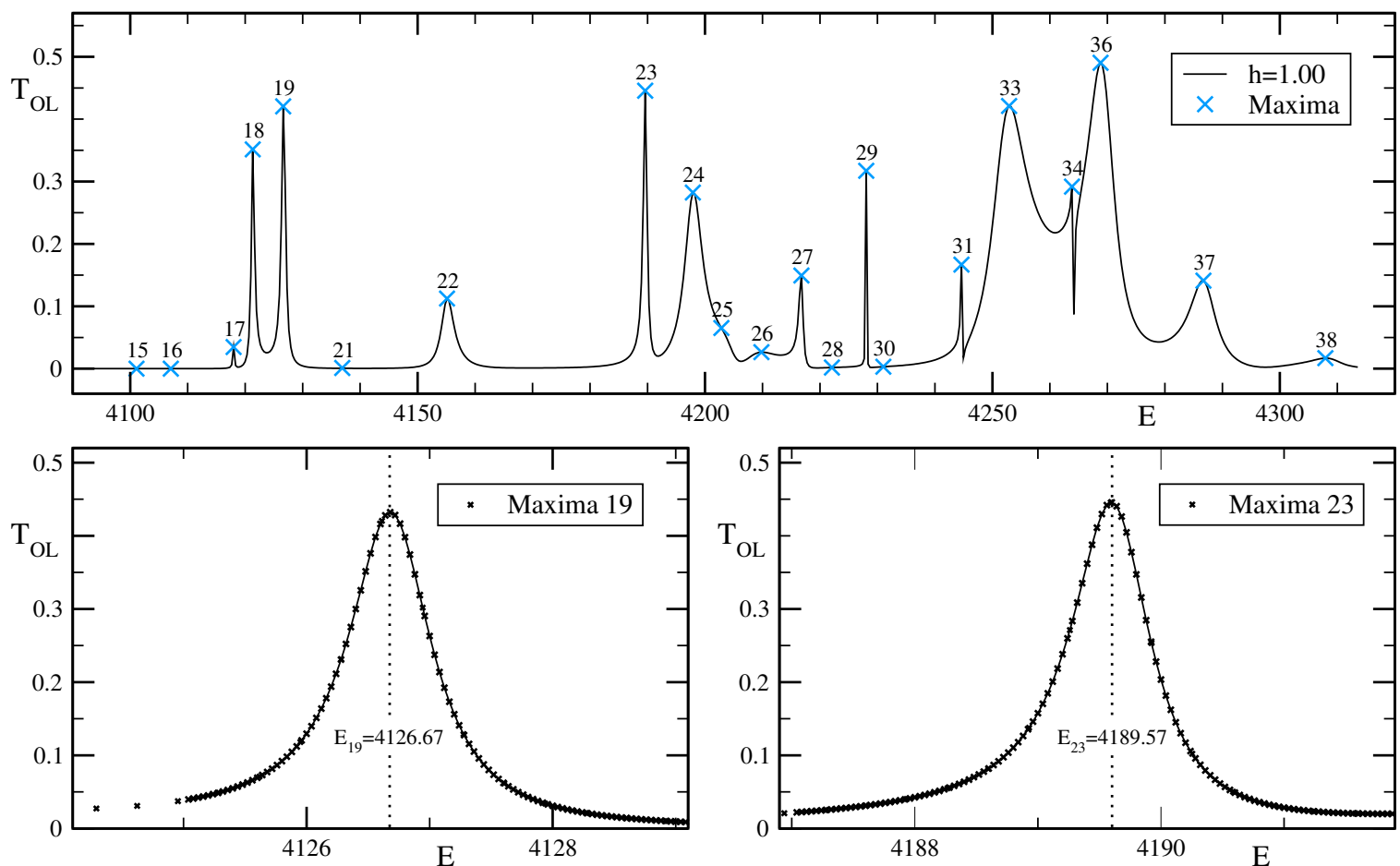

Abbildung 2.16.: Transmissionswahrscheinlichkeit $T_{\mathrm{OL}}$ nach Konfiguration 2.8a (unterstützt "I"-Form) für einen Einmodenlaser. Die verbesserte Laseremission kommt durch die teildurchlässige Verspiegelung des oberen Ausgangs zustande (zusätzliches Potential bei $0.92\langle E\rangle$, siehe Text). Die $Q$-Faktoren der beiden schärfsten Maxima (Nr. 19 und 23) wurden mittels Lorentz-Ausgleichsrechnungen zu $Q_{19} \approx 6000$ und $Q_{23} \approx 6000$ bestimmt (die beiden vergrößerten Ausschnitte unten in der Abbildung).

sich durch Einsetzen und Vergleichen mit Gleichung (2.28) und damit schließlich für die Energie der Potenzialstufe

$$
\frac{\langle E\rangle-E_{y}}{E_{y}}=\frac{\left(x_{0}-x_{\mathrm{L}}\right)^{2}}{y_{0}^{2}} \Longrightarrow E_{y}=\langle E\rangle \frac{y_{0}^{2}}{y_{0}^{2}+\left(x_{0}-x_{\mathrm{L}}\right)^{2}}
$$

mit $x_{0}=\frac{W+L}{2}$ und $y_{0}=d+a\left[1-\cos \left(\pi\left[1+\frac{W}{L}\right]\right)\right] \approx d+2 a$ für $W \ll L$. In Abbildung 2.16 ist die Transmissionswahrscheinlichkeit $T_{\mathrm{OL}}$ nach Konfiguration 2.8a für $W=0.15$ dargestellt. Für das System mit dem Parametersatz $(d, a, L)=(1.0,0.305,5.55)$ finden wir für die zentrale Stabilitätsinsel der Periode 1 die linke Grenze $x_{\mathrm{L}} \approx 2.37$. Der maximale Einfallswinkel auf die Poincaréebene ergibt sich nach Gleichung (2.28) zu $\Theta_{\max } \approx 73.3^{\circ}$. Für den kritischen Brechungsindex der Totalreflexion muss daher nach Gleichung (2.29) $n_{\mathrm{c}}=3.5$ gelten. Die entsprechende Minimalenergie der Potenzialstufe kann nach Gleichung (2.31) berechnet werden und beträgt $E_{y} \approx 0.92\langle E\rangle$.

Durch die Wahl von $W=0.15$ für den transversalen Ausgang und den Energiewert der Potenzialstufe $E_{y}$ ist ein Einmodenlaser konstruiert worden. Die Peaks der Transmissionswahrscheinlichkeit $T_{\mathrm{OL}}$ weisen nun deutlich schärfere Maxima als im Spektrum der Abbildung 2.10b auf. Die Gütefaktoren der beiden schärfsten Maxima betragen $Q_{19} \approx 6000$ und $Q_{23} \approx 6000$. Damit wurde also gezeigt, dass ein effektiver Einmodenlaser hoher Güte relativ einfach durch eine Verspiegelung des Wellenleiters erzeugt werden kann, wobei das verwendete Material - und damit schließlich die Struktur des Phasenraums - die Breite des transversalen Anschlusses vorgibt oder umgekehrt zu gegebener Breite ein Material mit entsprechend gestalteter Verspiegelung konzipiert werden muss. 


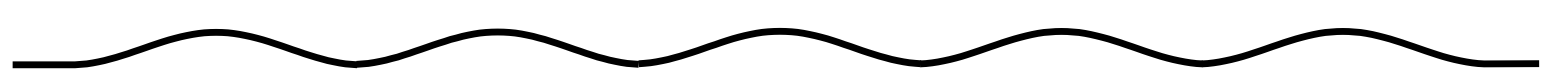

(a)

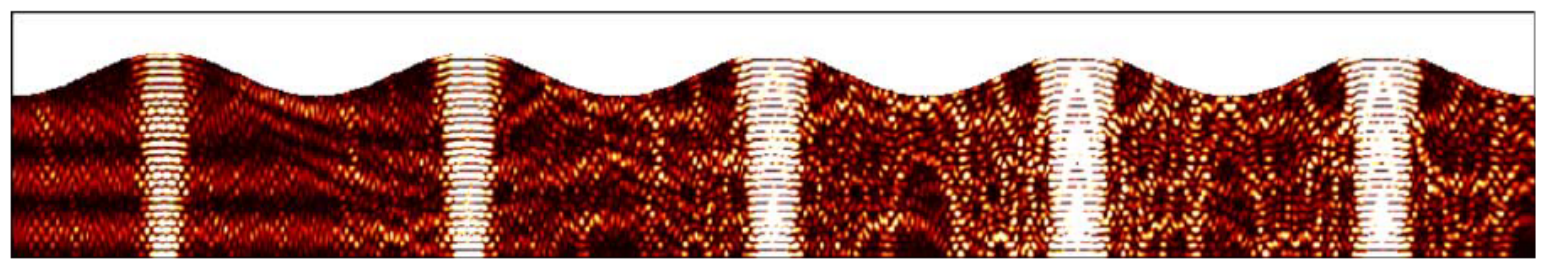

(b)

Abbildung 2.17.: Konfigurationsbeispiel einer Multi-Kavitätsgeometrie. (a) Multi-Kavitätsresonator. (b) Aufenthaltswahrscheinlichkeitsdichte eines Multi-Kavitätsresonators nach Konfiguration nach Abbildung 2.17a mit "I"-förmigem quasi-gebundenen Zustand.

\subsubsection{Multi-Kavitätsresonatoren}

Das vorgestellte Wellenleitermodell kann gleichermaßen benutzt werden, um Multi-Kavitätsresonatoren herzustellen. Denkbar ist z.B. eine kollineare Anordnung gekoppelter zweidimensionaler chaotischer Kavitäten (vergleiche mit Abb. 2.17a). Es ist einsichtig, dass mit wachsender Anzahl der Kavitäten (Resonatoren) ein Großteil der Strahlen bzw. Teilchen eine längere Zeitdauer benötigt, um transmittiert oder reflektiert zu werden. Diese Strahlen bzw. Teilchen streuen irregulär in die Ausbuchtungen der gekoppelten Resonatoren. Die erhöhte Strahlendichte in diesen Randbereichen führt zu erhöhter dynamischer Tunnelwahrscheinlichkeit in die klassisch verbotenen Bereiche. Daher ist "das Einfangen" der Wellenfunktionen entlang der beschränkten Strahlentrajektorien (nahe der periodischen Orbits) ebenfalls erhöht. In Abbildung 2.17b wurde die Wellenfunktion als Dichteplot für einen Multi-Resonator-Wellenleiter eines "I"-förmigen quasi-gebundenen Zustand als Beispiel einer Realisierung dargestellt. Die Konstruktion eines resonanten Multi-Resonatormikrolasers wäre sicherlich eine überaus interessante Anwendung, obwohl der Multi-Kavitätsresonator selbst bereits auf großes Interesse auf dem Gebiet der Optik stoßen dürfte. Ein Multi-Resonatormikrolaser hätte demnach zwei entscheidende Vorteile:

1. Eine Multi-Resonatoranordnung verfügt, im Vergleich zu einem Einzelresonator, über eine deutlich erhöhte Emissionsintensität (siehe z.B. Abb. 2.17b).

2. Der Multi-Resonatormikrolaser emittiert mehrere parallele Strahlen in eine oder mehrere Richtungen gleichzeitig, abhängig von der Anzahl der einzelnen Resonatoren.

Durch die Kombination verschiedener Resonatoren $R_{i}, i \in\{1, \ldots, N\}$ ist es auch möglich, gleichzeitig "I"-, "M"-, "V"-, "W"-, "П"- und andersförmige quasi-gebundene Zustände anzuregen. Je nach Geometrie des einzelnen Resonators $R_{i}$ mit Parametersatz $\left(d_{i}, a_{i}, L_{i}\right)$ müssen dazu nur die entsprechenden Resonanzfrequenzen aktiviert werden (siehe dazu auch [131]).

\subsubsection{Multi-Kavitätsstrahlenteiler}

Die Kenntnis über Form und Position des quasi-gebundenen Zustands, der in einem Resonator der Geometrieparametermenge $(d, a, L)$ angeregt wird, ermöglicht es, optische bzw. elektronische Multi- 


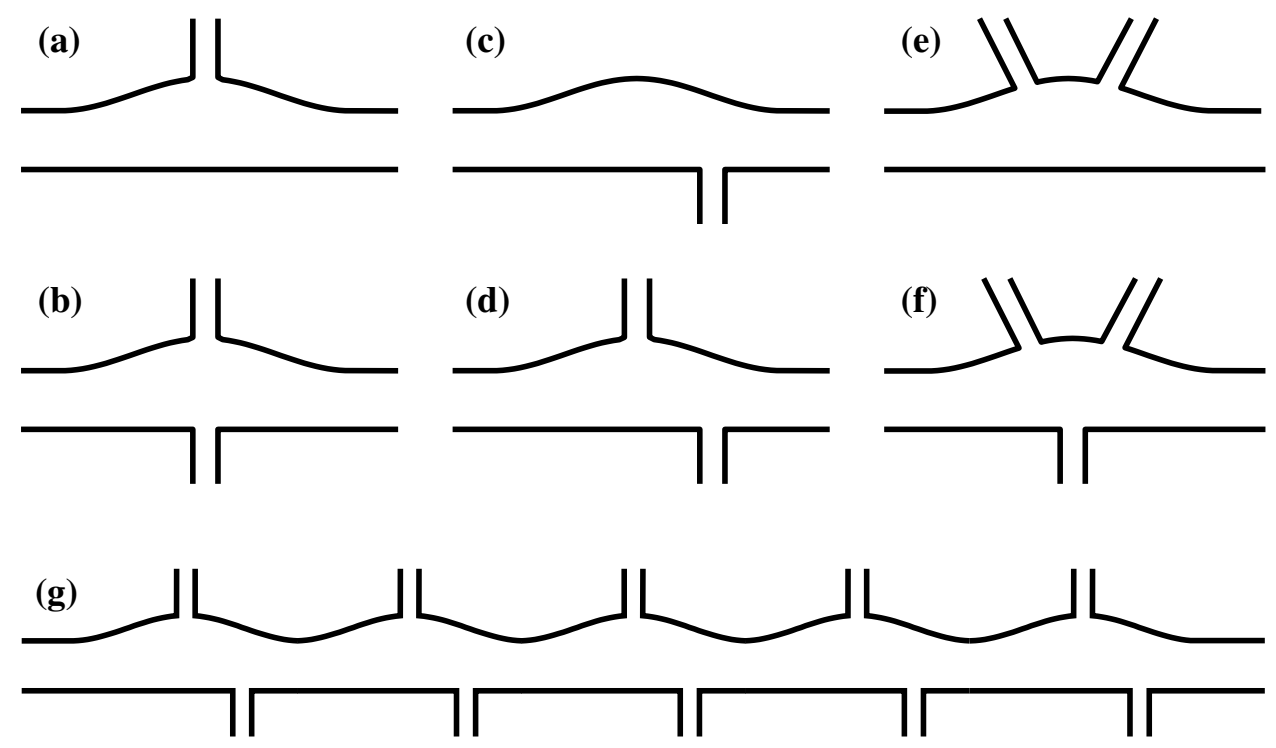

Abbildung 2.18.: Konfigurationsbeispiele mit Unterstützung für (a), (b) "I"-förmige, (c), (d) "M"-förmige und (e), (f) "V"-förmige quasi-gebundene Zustände. Die beiden Konfigurationen der oberen Zeile (a) und (c) stellen Beispiele für einfache, die Konfigurationen (b), (d), (e) und (f) Beispiele für multi-direktionale Strahlenteiler dar. Die schräg verlaufenen Anschlüsse in (e) und (f) berücksichtigen die Austrittsrichtung der auskoppelnden Welle. (g) Beispiel für Multi-Strahlenteiler konstruiert aus Hintereinanderschaltung von 5 Strahlenteilern nach Konfiguration (d).

Strahlenteiler zu konstruieren. Hierzu müssen noch transversal zum Wellenleiter verlaufende Anschlüsse an den Resonator angebracht werden. Die hier vorgeschlagene Anordnung benötigt nur zwei wesentliche Voraussetzungen an diese transversalen Anschlüsse, um die Wellen bzw. Strahlen aus dem Resonator herauszuführen:

1. Die externen Anschlüsse müssen dort angebracht werden, wo sich die stabilen Inseln des klassischen Phasenraums befinden, die die gewünschten quasi-gebundenen Zustände anregen.

2. Die Breite der externen Anschlüsse muss schmal genug bleiben, um die Struktur des Phasenraums nicht zu zerstören (vergleiche hierzu auch Abb. 2.9 des Unterabschnitts 2.3.5).

Als mögliche Geometrien kommen beispielsweise die Konfigurationen aus Abbildungen 2.18a,b für die Unterstützung "I"-förmiger, die Abbildung 2.18c "M"-förmiger und die Abbildungen 2.18e,f "V"förmiger quasi-gebundener Zustände in Frage.

Betrachten wir o.B.d.A. von links einfallende ebene Wellen für die horizontalen Anschlüsse, so tunneln die Streuwellenfunktionen dynamisch in die Regionen mit stabilen Inseln und werden von den transversalen Anschlüssen aus der Resonatorregion geleitet. Abbildung 2.18d stellt ein Beispiel für einen Strahlenteiler für "I"- und "M"-förmige quasi-gebundene Zustände dar, wobei die "I"-förmigen Zustände nach oben und die "M"-förmigen Zustände nach unten aus der Resonatorregion geleitet werden. Die Einstellung der Arbeitsweise, d.h. ob ein "I"-förmiger, ein "M"-förmiger oder anders geformter Zustand unterstützt wird, geschieht allein durch die Wahl der Frequenz bzw. Energie. Durch die Hintereinanderschaltung dieser Konfigurationen entstehen Multi-Kavitätsstrahlenteiler bzw. -schalter (siehe z.B. $2.18 \mathrm{~g})$.

Abschließend weisen wir darauf hin, dass das Konzept des (Multi-)Strahlenteilers mit dem Konzept des Multi-Kavitätsresonators kombinierbar ist. Auf diese Weise wird auch die Konstruktion eines 
Multi-Kavitätsstrahlenteilers ermöglicht, indem beispielsweise nur jede dritte Ausbuchtung in Abbildung 2.17a mit einem Anschlussleiter versehen wird. Damit ließe sich gerichtete parallele Emission mit erhöhter Emissionsintensität vereinbaren.

\subsection{Zusammenfassung und Ausblick}

Wir konnten zeigen, dass Billards mit gemischtem Phasenraum eine Konstruktionsgrundlage bilden, optische und elektronische Kavitäten als vielversprechende Kandidaten opto-elektronischer Schalter und/oder Strahlenteiler zu realisieren [1]. Weitere optische und elektronische Anwendungen, wie Einmoden-Mikrolaser, Multi-Kavitätsresonatoren, Multi-Strahlenteiler und entsprechende Kombinationen, ließen sich aus diesen Systemen ableiten [2]. Ein bereits durchgeführtes und hier vorgestelltes Mikrowellen-Experiment signalisierte bereits vielversprechende Realisierungen der vorgeschlagenen Strukturen. Als Repräsentant für eine Kavität mit gemischtem Phasenraum diente ein Billard mit Kosinusstruktur. Dieses Billard konnte durch eine einfache Deformation des Wellenleiters selbst als Resonatorstruktur dienen.

Durch die unterschiedlichen Betrachtungsweisen von klassischer Strahlen- und quantenmechanischer Wellendynamik konnten wir zeigen, dass sowohl der Schalt- als auch der Strahlenteilmechanismus auf dem dynamischen Tunneleffekt basiert, da es quantenmechanisch gesehen möglich ist, dass Streuzustände in die klassisch verbotenen stabilen Bereiche des Phasenraums gelangen.

Unter Verwendung der Fresnelschen Formeln und dem Snelliusschen Brechungsgesetz ist es uns gelungen, die gerichtete Emission auch mit einer Strahlendynamik zu beschreiben. Ein geometrischer Vergleich der kritischen Winkel der Totalreflexion mit dem als Potenzialstufe ausgedrückten kritischen Brechungsindex ermöglichte die Konstruktion eines effektiven Einmodenlasers hoher Güte durch Aufbringung einer einfachen, aber speziell gearteten Verspiegelung. Der Einmodenlaser gilt somit als direkte realisierbare Anwendung. Der Gütefaktor ist dabei von der Dicke der Verspiegelung abhängig, weshalb dieser funktionale Zusammenhang durch weitere numerische Untersuchungen abgeleitet werden könnte.

Die Wahl eines Kosinusbillards als Prototypen schränkt die vielseitige Anwendbarkeit unserer Resultate nicht ein, da prinzipiell alle Kavitäten benutzt werden können, deren Hufeisenabbildung unvollständig ist, d.h. die über einen gemischten Phasenraum verfügen. Für diesen Typ Wellenleiter ist es immer möglich, ein oben geschildertes Szenario zu konstruieren, damit sich diese in ihrer Funktionsweise als Strahlenteiler bzw. Schalter verwenden lassen.

Weiterhin ist es möglich, für chaotische Systeme mit instabilen Fixpunkten, deren Hufeisenabbildungen vollständig sind, ebenfalls solche Strukturen zu realisieren. In diesen findet man narbige Zustände (scars), die sich um diese instabilen periodischen Orbits ausbilden und auf die gleiche Weise nutzen lassen.

$\mathrm{Zu}$ Optimierungszwecken könnten weitere intensive Untersuchungen des Parameterraums $(d, a, L)$ bzw. klassische Analysen zu anderen Billards mit gemischten Phasenräumen durchgeführt werden, um größere Stabilitätsinseln in den zugehörigen Poincaré-Abbildungen zu identifizieren. Inwieweit diese dann auch über eine höhere Effektivität im Vergleich zu den hier vorgestellten Kosinusbillards aufweisen würden, müsste die volle quantenmechanische Untersuchung erweisen. 


\title{
3. Verminderung des Streulichts im Auge durch lichtleitende Müllerzellen
}

\author{
Wer bei der Verfolgung der Wissenschaften nach unmittelbarem praktischen \\ Nutzen jagt, kann ziemlich sicher sein, dass er vergebens jagen wird.
}

Hermann von Helmholtz, Öffentlicher Vortrag [132]

3.1 Einführung . . . . . . . . . . . . . . . . . . . . 55

3.1 .1 Das Auge ......................... 55

3.1 .2 Die Retina . . . . . . . . . . . . . . . . . . . 56

3.2 Generische Struktur der Müllerzelle . . . . . . . . . . . . . . . . . . . 60

3.3 Die Müllerzelle als Lichtwellenleiter . . . . . . . . . . . . . . . . . . . . . . 62

3.3.1 Modellierung als Quantenkanal . . . . . . . . . . . . . . . 62

3.3.2 Die Bedeutung der Form des Endfußes für die Lichtleitung . . . . . . 64

3.3.3 Modellierung der extrazellulären Umgebung der Müllerzelle . . . . . . 66

3.3.4 Streuwellenfunktionen . . . . . . . . . . . . . . . . . 68

3.3.5 Vergleich mit dem Experiment . . . . . . . . . . . . . . . . . 69

3.3.6 Einfluss der Müllerzellenform auf die Lichtleitung . . . . . . . . . . . . 71

3.3.6.1 Zur Formvariation des Zellstamms . . . . . . . . . . . . . 71

3.3.6.2 Zur Position des Zellkörpers . . . . . . . . . . . . . 73

3.3.7 Lichtleitung zwischen benachbarten Müllerzellen . . . . . . . . . . . . 74

3.4 Modell zur Vorhersage des Streulichts im menschlichen Auge . . . . . . . . . 75

3.4.1 Das Augenmodell . . . . . . . . . . . . . . . . . . . . 75

3.4.2 Relative Intensitäten des direkten Lichts und des Streulichts erster Ordnung . . . . . . . . . . . . . . . . . . 78

3.4.3 Bestimmung der Grenzwinkel $\phi_{\max }$ und $\delta_{\max } \ldots \ldots . \ldots . . . . . .81$

3.4.4 Vergleich der relativen Intensitäten . . . . . . . . . . . . . . . 82

3.4.5 Signal-Rausch-Verhältnisse . . . . . . . . . . . . . . . . 83

3.5 Zusammenfassung und Ausblick . . . . . . . . . . . . . . . . . . . . . . 85 


\section{Motivation}

Es ist eine lang ausstehende und kontrovers diskutierte Frage, warum die Retina von Wirbeltieren invertiert ist, d.h. warum sich die Photorezeptoren auf der lichtabgewandten Seite der Retina befinden. Das einfallende Licht muss in dieser Anordnung erst diverse neuronale Schichten passieren, die wiederum sehr viele Lichtstreuelemente wie beispielsweise Mitochondrien und Zytoskelettbestandteile enthalten. Das Licht wird dort gebrochen, reflektiert und vor allem gestreut, bevor es endlich von den lichtempfindlichen Zapfen und den noch lichtsensitiveren Stäbchen der Netzhaut detektiert werden kann.

Experimentelle Studien haben vor kurzem gezeigt, dass die Müllerzellen der Retina den Glasfasern in der angewandten Optik ähneln [30, 133]. Die weitestgehend parallele Anordnung der Müllerzellen erinnert außerdem an den Aufbau von faseroptischen Systemen, die zur Bildübertragung zwischen zwei räumlich getrennten Gebieten benutzt werden. Diese lang gestreckten Gliazellen verbinden die ventrikuläre Schicht (am Glaskörper) mit der mesenchymalen Grenzschicht (Schicht der Photorezeptoren). Da sie einen etwas höheren Brechungsindex aufweisen als ihre Umgebung im Mittel, könnten diese Zellen als lebende Lichtwellenleiter fungieren. Für extrahierte Zellen in einer homogenen Umgebung konnte dies bereits nachgewiesen werden. Wie aber funktioniert die Lichtleitung der Müllerzelle in ihrer natürlichen Umgebung?

Die Beantwortung dieser Frage wird in diesem Kapitel gegeben. Eine strukturelle Analyse der Retina ergibt, dass sie aus verschiedenen Schichten unterschiedlichster Neurone besteht, die mit neuronalen Verbindungskabeln (Axone und Dendriten) verbunden sind. Hinzu kommen Membranen und eine große Anzahl vaskulärer Gefäße. Die Retina ist also ein sehr komplexes Organ des zentralen Nervensystems, dass - die Lichtausbreitung betreffend - als System von vielen im Brechungsindex fluktuierenden und ungeordneten Einzelstrukturen aufgefasst werden kann. Durch eine Transformation der Brechungsindices in ein Gaußsches Unordnungspotenzial und die Anwendung der in Kapitel 1 entwickelten Methode des Streuformalismus auf die optische Wellengleichung wird es möglich, die Müllerzelle als Wellenleiter in einem Modell ihrer natürlichen Umgebung zu untersuchen und ihre Lichtwellenleitereigenschaft nachzuweisen.

Weiterhin wird in diesem Kapitel mithilfe numerischer Simulationen von Müllerzellen gezeigt, dass der trichterförmig geformte Endfuß der Müllerzellen dass einfallende Licht bündelt und so zu einer fokussierten Lichttransmission in der Retina bei gleichzeitiger Reduktion der Kleinwinkelstreuung führt. Diese Trichterform wird auch bei der Konzeption von Quantenpunktkontakten in der Mikroelektronik genutzt, um durch gerichteten Transport die Effektivität dieser Strukturen zu erhöhen (siehe z.B. $[8,134,135])$. Die reduzierte Kleinwinkelstreuung erweist sich auch als Ursache für das erhöhte Signal-Rausch-Verhältnis der gesamten Retina. Die Geometrie des Auges findet hierbei durch ein vereinfachtes optisches Modell Berücksichtigung. Es stellt sich heraus, dass die kugelähnliche Form des Auges zusammen mit der besonderen Geometrie der Müllerzellen zu einer maximalen Reduktion des Streulichts in der näheren Umgebung der Fovea führt [5].

Prinzipiell sollten sich mit dieser Methode für Spezies mit ähnlichem visuellem System ähnliche Vorhersagen für das Signal-Rausch-Verhältnis der Netzhaut finden lassen. Dies gilt insbesondere für Wirbeltiere, aber auch für viele Wirbellose (Weichtiere), die neben linsenoptischen Systemen und foveaähnlichen Sehzentren ebenfalls über eine invertierte Retina verfügen. 


\subsection{Einführung}

Das Prinzip der invertierten Retina bei Wirbeltieren galt lange Zeit als unverstanden und befindet sich noch immer im Diskussionsstadium (siehe z.B. [136]). Ebenso wie bei der Fragestellung der spezifischen Funktionen von Gliazellen ${ }^{1}$ im zentralen Nervensystem (ZNS) wurde den Müllerzellen der Retina in der Vergangenheit lediglich eine passive Rolle als Stützgewebe zugewiesen (griech. " $\gamma \lambda \iota \alpha$ " heißt "Leim"). Wie wir in Abschnitt 3.2 sehen werden, hat die Müllerzelle allerdings noch viele andere Funktionen. Erstaunlicherweise gab es bis zum Anfang dieses Jahrhunderts kaum wissenschaftliche Analysen der Neuromechanik und Neurooptik von Gliazellen des ZNS. Die Untersuchung der biomechanischen Materialeigenschaften des Hippocampus und der Retina [141] (hierzu zählen z.B. Viskosität und Elastizität), sowie ihre neurooptischen Eigenschaften [30] (Brechungsindices, Transmission, Reflexion und Absorption) - soweit bisher untersucht - lassen diese klassischen Fragen unter neuen Gesichtspunkten erscheinen. In diesem einführenden Kapitel sollen Grundlagen des visuellen Systems von Wirbeltieren vor allem in seiner optischen Funktion vorgestellt und einige wichtige Eigenschaften genauer hervorgehoben werden.

\subsubsection{Das Auge}

Das Auge wird häufig mit einer Kamera verglichen, obwohl bis heute keine Kamera existiert, die den vielfältigen Funktionen des Auges gerecht wird. Die Kamera müsste über einen $\mathrm{Au}$ tofokus, eine Blendenautomatik, ein automatisch nachgeführtes Stativ, eine selbstreinigende und selbstwartende Linse und einen hochperformanten Parallelcomputer zur Bildvorverarbeitung verfügen und eine Empfindlichkeit besitzen, die bereits bei einigen wenigen Photonen Kontrastwahrnehmungen, aber auch bei grellem Tageslicht gestochen scharfe Stereobilder liefert [142]. Dies entspricht einem Helligkeitsbereich, der mehr als 11 Größenordnungen umspannt (für das menschliche Auge entspricht das Leistungsaufnahmen zwischen $10^{-17} \mathrm{~W}$ und $\left.10^{-6} \mathrm{~W}\right)$, und der das technisch Erreichbare immernoch weit hinter sich lässt. Es handelt sich also um ein hochkomplexes Sinnesorgan, dessen mannigfaltige Funktionen ebenfalls noch immer nicht vollständig verstanden sind. Für die Beschreibung optischer Ei-

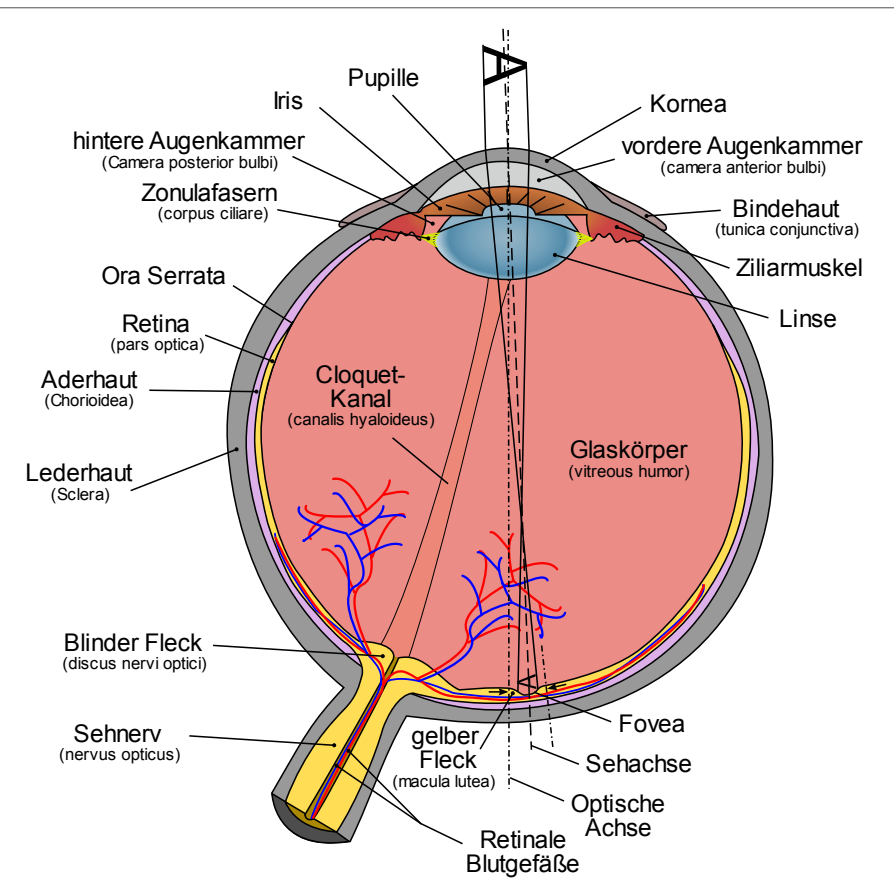

Abbildung 3.1.: Schematische Abbildung des menschlichen Auges. In einem scheibenförmigen Bereich um die Fovea befindet sich das Zentrum des Scharfsehens. Durch entsprechende Ausrichtung und Adaption der Pupille und elastische Modifikation der Linse wird das betrachtete Objekt mit optimaler Schärfe dorthin abgebildet, hier am Beispiel des Buchstabens "A" dargestellt.

1 Der von Virchow 1856 geprägte Begriff "Nervenkitt" oder "Neuroglia" (siehe [137] auf Seite 890) entstammt eigentlich einer früheren Abhandlung aus dem Jahre 1842 [138], wobei die ursprüngliche Entdeckung der damals als nichtneuronale Zellen eingestuften Gewebezellen nach Aussage von Ramón y Cajal angeblich auf von Deiters 1865 [139] geführte Untersuchungen zurückgeht. Eine historische Übersicht kann in [140] nachgelesen werden. 
genschaften hingegen, wie beispielsweise der Weg des Lichts durch das Auge, existieren es aber gute Modelle. Im Normalfall wird die Strahlenoptik als Näherungsmethode gewählt, um den Weg des durch Kornea, Pupille und Linse einfallenden Lichts durch den Glaskörper bis zur Fovea der Retina darzustellen (siehe Abb. 3.1). Die Gesamtbrechkraft des entspannten emmetropen (normalsichtigen) menschlichen Auges beträgt etwa $59 \mathrm{~m}^{-1}$. Die $11 \mathrm{~mm}$ durchmessende Kornea sorgt bereits für zwei Drittel der notwendigen refraktiven Lichtbrechung, die allerdings mit $43.1 \mathrm{~m}^{-1}$ [143] nur über eine feste Brennweite verfügt. Die Iris fungiert als variable Lochblende, die sich bei Lichteinfall reflektorisch rund um die Pupille zusammenzieht und bei schwachen Lichtverhältnissen weitet. ${ }^{2}$ Die Pupillenfläche kann sich so im Verhältnis 1 : 16 ändern. Allerdings wird die Iris auch von psychologischen Faktoren beeinflusst. So erweitert sich die Pupille beispielsweise auch bei geistiger Anstrengung [146] oder bei positiv-emotionaler Stimulierung [147]. Die Linse sorgt für das restliche Drittel der Brechung und invertiert gleichzeitig das Bild. Die Brechkraft der Linse kann durch Akkommodation, d.h. durch Kontraktion des glatten parasympathischen innervierten Ziliarmuskels, Werte von 19-34 $\mathrm{m}^{-1}$ annehmen [148]. Im entspannten Zustand (auf " $\infty$ " adaptiert) nimmt die Brechkraft der Linse das Minimum an. Der Glaskörper nimmt mit etwa 90\% den größten Teil des Augenvolumens ein und ist mit 98-99\% Wasseranteil nahezu perfekt lichtdurchlässig. Ein Stütznetz aus Kollagenfasern $(<0.1 \%)$ sorgt für die Aufrechterhaltung der Form, während ca. 1-2\% Hyaluronsäure die Fasern auseinanderhalten. Der Aufbau aus Kollagenfasern, Hyaluronsäure und Wasser bildet eine Art lichtdurchlässigen Schwamm, der selbst fast nicht zur Lichtbrechung beiträgt. Der Brechungsindex wird in der Literatur mit Werten zwischen 1.336-1.338 angegeben [148, 149]. ${ }^{3}$ Der den Glaskörper enthaltende und $24 \mathrm{~mm}$ durchmessende Augapfel (bulbus oculi) ist in der Lage, durch sechs extraokuläre (äußere) Augenmuskeln, die einer kardanischen Aufhängung ähneln, sehr schnell Objekte zu fokussieren [150], ohne dabei die Ausrichtung des Kopfes ändern zu müssen. Umgekehrt kann aber ebenso bei freier Beweglichkeit des Kopfes ein Objekt fokussiert werden. Kornea, Linse und Glaskörper sind nicht vaskulär, da in ihnen befindliche Blutgefäße zu extremer Lichtstreuung führen würden. ${ }^{4}$ Das direkt in die Fovea abgebildete Objekt und die auf die Retina geleiteten Photonen werden durch die Photorezeptoren in elektronische Signale transformiert, vorverarbeitet und über die Sehbahnen (Leitungsbahnen der Ganglienzellen, Sehnerv, etc.) zur primären Sehrinde weitergeleitet. Obige Abhandlung zum Aufbau des menschlichen Auges kann im wesentlichen auf den Aufbau des Einzelauges aller Wirbeltiere [151, 152] und sogar vieler Weichtiere [153] übertragen werden.

\subsubsection{Die Retina}

Die Wirbeltierretina bzw. Netzhaut entsteht im Embryonalstadium aus einer Ausstülpung des Zwischenhirns und ist somit im eigentlichen Sinne gar keine Haut, sondern anatomisch gesehen ein aus zwei Teilen bestehendes Organ des zentralen Nervensystems. Der vordere Teil (pars caeca) ist lichtunempfindlich und überdeckt Iris und Ziliarkörper von innen, während der hintere (pars optica) den lichtempfindlichen Teil darstellt, der die Photorezeptoren enthält und einen invertierten Aufbau be-

2 In der Vergangenheit wurde angenommen, dass der Regelkreis der Iris allein vom Lichteinfall auf die Photorezeptoren beeinflusst wird. Neuere Studien zeigen, dass dafür eher eine bisher unbekannte Klasse photosensitiver melanopsinhaltiger Ganglienzellen verantwortlich sind [144, 145].

3 Zum Vergleich: Der Brechungsindex von Wasser beträgt 1.33. Für die Kornea ist ein Brechungsindex von 1.376 und für die Linse ca. 1.4 (nicht homogen) angegeben.

4 Die notwendige Nährstoffversorgung erfolgt osmotisch über das Kammerwasser der vorderen und hinteren Augenkammer, sowie von außen über die Tränenflüssigkeit. 


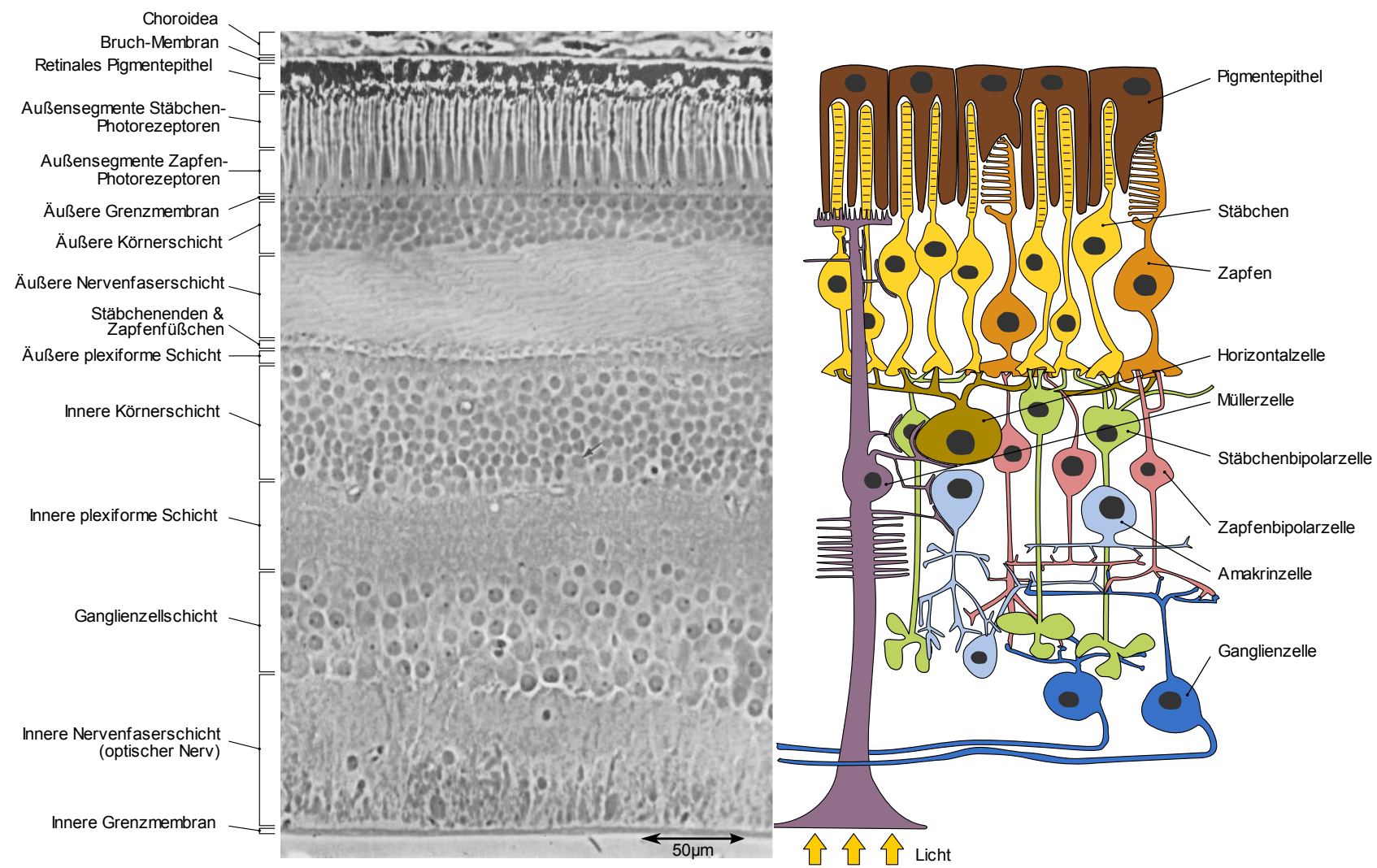

Abbildung 3.2.: Aufbau der und Struktur der Retina. Links: Elektronenmikroskopische Aufnahme eines vertikalen Schnitts durch eine menschliche Retina (Ausschnitt aus plate 32 von Boykott \& Dowling [154], eine etwa $400 \mu \mathrm{m}$ dicke Schicht $1.25 \mathrm{~mm}$ vom Zentrum der Fovea entfernt). Rechts: Schematischer Aufbau mit den fundamentalen Zellen der Gewebeschichten der "entrollten" invertierten Retina. Die skizzierten Zelltypen bilden nach dem Konzept einer säulenförmigen Einheitszelle von Reichenbach $[155,156]$ eine funktionale Einheit, wobei sich die Zellen in einer 3D-Struktur säulenartig um die Müllerzelle herum gruppieren. Beim Menschen findet man etwa $10^{7}$ solcher Einheitszellen, im Durchschnitt bei Wirbeltieren etwas weniger, im Durchschnitt etwa $4 \cdot 10^{6}$. Die schematische Abbildung steht stellvertretend für den prinzipiellen Aufbau aller Wirbeltier-Retinae. Zur Erklärung der einzelnen Zelltypen und Schichten siehe Text.

sitzt. Das bedeutet, dass das Licht erst eine große Anzahl von Zellen, Nervenfasern, Membranen und Blutgefäßen passieren muss, ehe es von den Photorezeptoren detektiert werden kann. In der Abbildung 3.2 links ist eine elektronenmikroskopische Aufnahme des Querschnitts durch eine gesunde menschliche Retina dargestellt. ${ }^{5}$ Die Pars optica ist etwa $1100 \mathrm{~mm}^{2}$ groß ${ }^{6}$ und wird üblicherweise in 10 weitere Schichten unterteilt. Eine Einteilung in Schichten, die die Zellkörper der Zellen enthalten und Schichten, die für die neuronale Verschaltung und die Nährstoffversorgung zuständig sind, bildet eine erste Differenzierung. Die erste, an die Choroidea angrenzende und durch die Bruch-Membran abgegrenzte Schicht ist das retinale Pigmentepithel - die Pigmentzellschicht. Sie ist als einzige retinale Schicht lichtundurchlässig, da sie prismatische, mit Melanin pigmentierte Zellen enthält, die hervorragende Lichtabsorber verkörpern und deshalb natürlich erst hinter den Photorezeptoren angeordnet sind. Die Pigmente absorbieren das Restlicht, das von den Photorezeptoren nicht detektiert wurde, um so unerwünschtes Streulicht und störende Reflexionen möglichst gering zu halten. Die anderen Funktionen des

5 Wie in der Optik und Optometrie üblich, wird die Retina so dargestellt, dass die äußeren Schichten in der Abbildung oben erscheinen. Der Lichtweg ist dann von unten nach oben orientiert. In klinischen Studien findet man im Allgemeinen eine umgekehrte Darstellung vor.

6 Die Größe der Retina-Oberfläche wird später nach Gleichung (3.41) auf Seite 81 berechnet. 


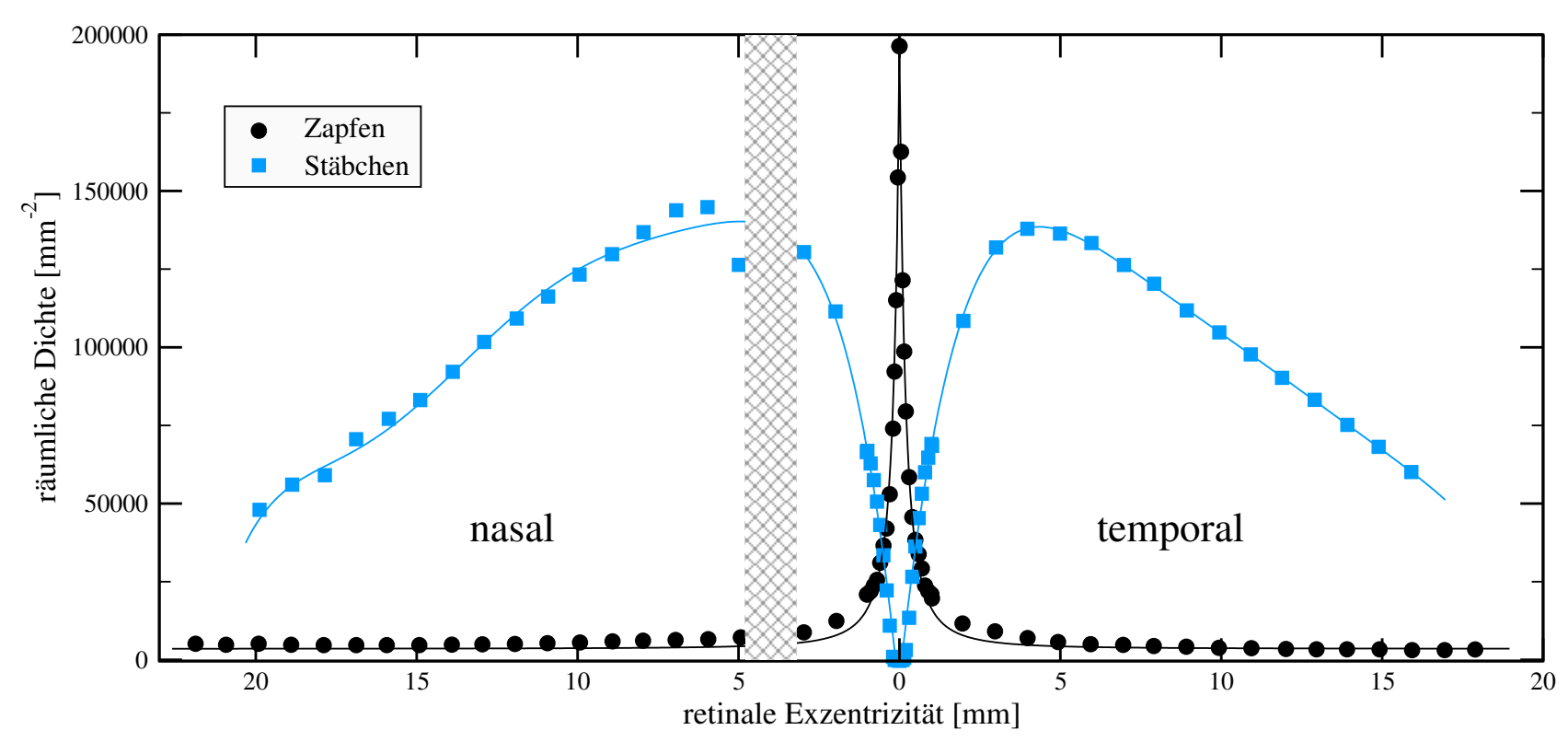

Abbildung 3.3.: Zapfen- und Stäbchendichte als Funktion der retinalen Exzentrizität (medialer Abstand von der Fovea) als horizontaler Querschnitt durch eine gesunde menschliche Retina. Dabei entspricht $1 \mathrm{~mm}$ retinale Exzentrizität in Foveanähe einem Gesichtswinkel von ca. 3.5 $[158]$. Der schraffierte Bereich kennzeichnet den blinden Fleck (Austrittsstelle des Sehnervs), in dem keine Photorezeptoren vorhanden sind. Die Daten wurden aus [159] entnommen. Die durchgezogenen Linien ergeben sich durch Ausgleichsrechnungen der experimentellen Daten (siehe Anhang E). Die stark variierende Anordnung der einzelnen Zapfen-Photorezeptoren (S, M, und L) kann durch eine modifizierte Lorentz-Ausgleichsrechnung approximiert werden [160, 161]. Die Dichteverteilung der Stäbchen-Photorezeptoren wird durch eine polynomiale Regression 6. Ordnung approximiert [162, 163]. Eine zweidimensionale Dichtedarstellung der gesamten Retina für Primaten ist in [164, 165] zu finden.

Pigmentepithels, wie der epitheliale Transport, der visuelle Zyklus (11-cis Retinal), die Phagozytose, die Sekretion und die Gliafunktion können in dem Übersichtsartikel von Strauss [157] nachgelesen werden. Anschließend folgt die Schicht der Photorezeptoren. In den schmalen Außensegmenten sind die Stäbchen und in den breiten Außensegmenten sind die Zapfen untergebracht. Beim Menschen sind es 110-125 Millionen Stäbchen und ca. 7 Millionen Zapfen, die Sehpigmente enthalten. Die Zapfendichte hat ihr Maximum in der ungefähr $1.5 \mathrm{~mm}$ durchmessenden Fovea, wo sich auf ca. $0.2 \%$ der retinalen Gesamtfläche etwa $3 \%$ der über die gesamte Retina verteilten Zapfen-Photorezeptoren befinden. Die Stäbchendichte variiert stark über die gesamte Retina. Hier befindet sich das Dichtemaximum in einem Ring von etwa $5 \mathrm{~mm}$ Radius um die Fovea herum, während sich im Zentrum der Fovea (fovea centralis) - in einem Radius von $500 \mu \mathrm{m}$ - nahezu keine Stäbchen befinden (siehe Abbildung 3.3). In der Regel findet man 5-10\% der Zapfen als $S$-Rezeptoren vor, während sich etwa gleich viele $M$ und $L$-Rezeptoren auf der Retina befinden. ${ }^{7}$ Ihre Anordnung kann durchaus als kompliziert bezeichnet werden, denn sie ist von der Spezies, vom Rezeptortyp und von der retinalen Exzentrizität abhängig, wobei sich oftmals mosaikartige selbstorganisierende Strukturen ausbilden. Aufgrund der enormen Vielfalt sind nicht alle Wirbeltierarten dahingehend untersucht worden. Beim Menschen können diese überlappenden Mosaiken heute zumindest qualitativ beschrieben werden [166]. Eine Theorie zur quantitativen Beschreibung der zweidimensionalen Dichteverteilung steht aber immer noch aus. Die in Abbildung 3.3 dargestellten Kurven stellen Approximationen an die experimentellen Daten dar. Im

7 Die Bezeichnungen $S, M$ und $L$ stehen für short, middle und long, im Bezug auf die Wellenlänge des jeweilig absorbierten Lichts. 


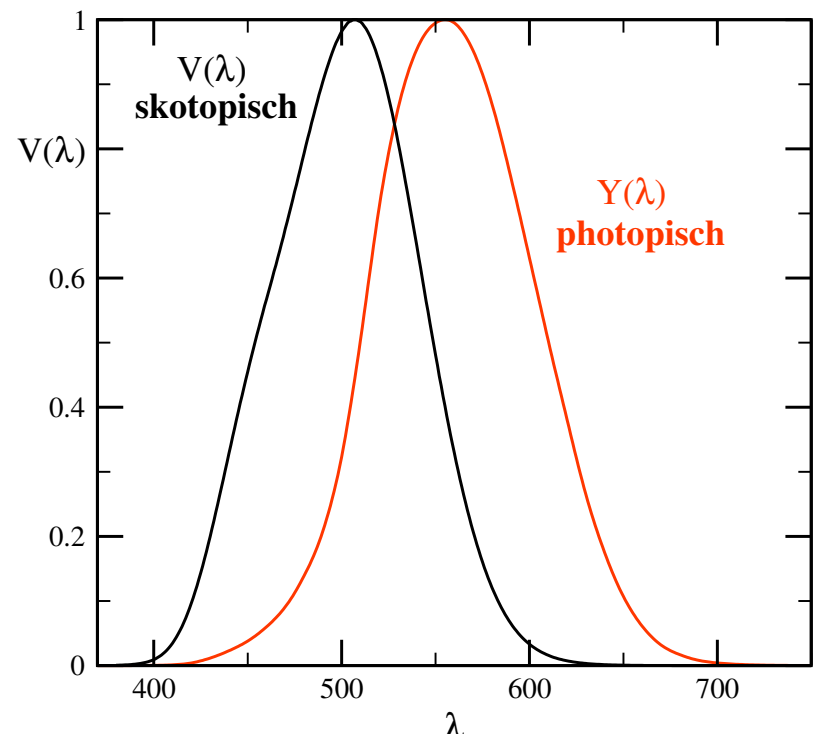

(a)

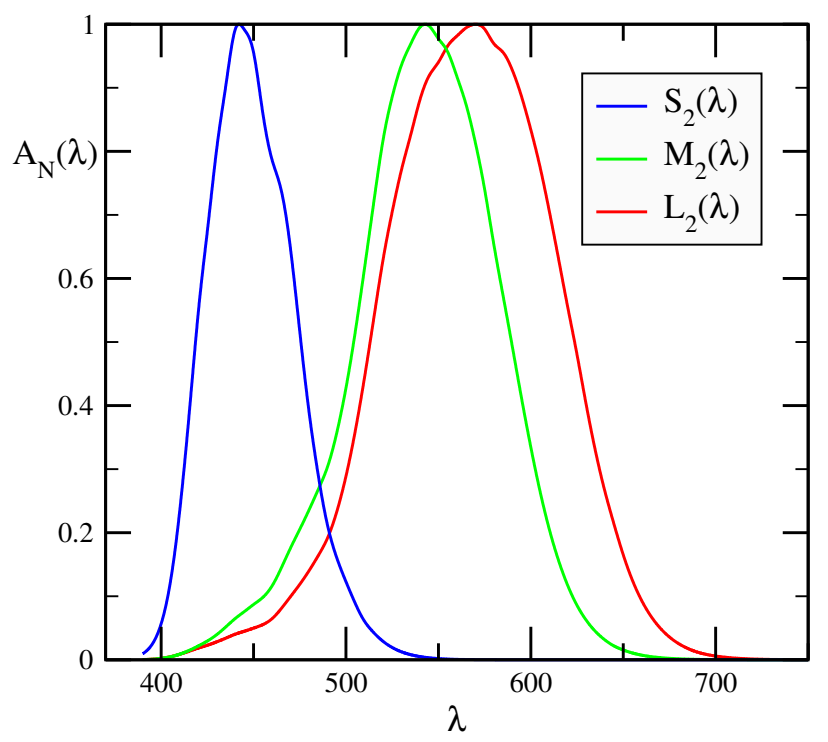

(b)

Abbildung 3.4.: (a) Hellempfindlichkeitskurven des skotopischen Visus $V(\lambda)$ (Nachtsicht) und des photopischen Visus $Y(\lambda)$ (Tagsicht). Die Verschiebung des Maximums $\max \{Y(\lambda)\} \approx 550 \mathrm{~nm}$ ins Blaue zu max $\{V(\lambda)\} \approx$ $500 \mathrm{~nm}$ ist deutlich zu erkennen (Purkinje-Verschiebung). Daten entnommen aus [169],[170] Table I (4.3.2).

(b) Normalisierte Absorption $A_{N}(\lambda)$ der $S_{2}, M_{2}$ und $L_{2}$ Zapfen-Photorezeptoren (siehe Fußnote 7) mit den Absorptionsmaxima $\max \left\{S_{2}\right\} \approx 440 \mathrm{~nm}, \max \left\{M_{2}\right\} \approx 540 \mathrm{~nm}$ und $\max \left\{L_{2}\right\} \approx 570 \mathrm{~nm}$. Die Farben der dargestellten Absorptionsspektren kennzeichnen die Wellenlänge der von den Zapfen bevorzugt absorbierten Photonen. Daten entnommen aus $[171,172]$.

Falle der Zapfen wurde nach Konzeption von [160, 161] eine Lorentz-Ausgleichsrechnung durchgeführt. Für die Dichteverteilung der Stäbchen wurde eine Regression 6. Ordnung bemüht, wie in [163, 167] motiviert. Detaillierte Ausführungen hierzu befinden sich im Anhang E auf Seite 145.

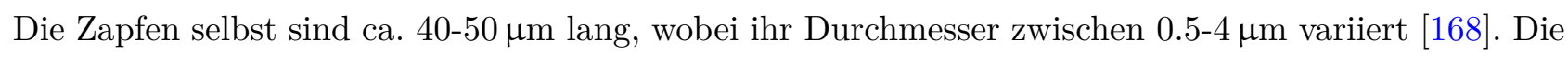
Membran des Außensegments enthält Rhodopsin, wodurch Lichtabsorption zu einer Hyperpolarisation des Membranpotenzials der Stäbchenzelle führt. Das Innensegment enthält Strukturen zur Synthese der Sehfarbstoffe, die kontinuierlich zerfallen und wieder regeneriert werden müssen. Die Stäbchen haben die Aufgabe, Hell-Dunkel Kontraste zu detektieren (skotopischer Visus), während die Zapfen für das Farb- und Scharfsehen (photopischer Visus) zuständig sind. Beim Menschen gibt es drei Arten von Farbsehzellen mit unterschiedlichen Opsinen: rot-, grün- und blau-empfindliches Photopsin (siehe Abbildung 3.4). Die Lichtabsorption ist demnach stark wellenlängenabhängig und hängt überdies von der Art des Visus ab. Bei manchen Tierarten kommen entweder infrarot- oder ultraviolettempfindliche Sehzellen mit Iodopsin hinzu. Für eine ausführliche Darstellung des komplexen Ablaufs der opto-elektrischen Signalwandlung, den Transduktionsprozess, sei auf [173, 174] verwiesen. Auf die Verschaltung der Photorezeptoren mit den verschiedenen Zelltypen und den Zelltypen untereinander wird in Anhang F genauer eingegangen.

Es folgt eine Kurzbeschreibung der 8 Schichten, die das Licht vom Glaskörper zu den Photorezeptoren passiert. Wegen der unterschiedlichen optischen Eigenschaften dieser Schichten muss später bei den numerischen Simulationen des Lichttransports durch die Retina das Brechungsindexprofil besondere Berücksichtigung finden.

An die Photorezeptorschicht schließt die äußere Grenzmembran an. In ihr verlaufen die horizontalen 
Fortsätze der Müllerzellen. Die darauffolgende äußere Körnerschicht enthält die Zellkörper der Zapfen und Stäbchen. Die anschließende äußere Nervenfaserschicht besteht aus den Fasern, die die Zellkörper der Zapfen- und Stäbchen-Photorezeptoren mit den Zapfenfüßen und Stäbchenenden verbindet. Die Synapsen zwischen den Photorezeptorenden und den Bipolarzellen und den Horizontalzellen bilden die äußere plexiforme Schicht. Die Zellkörper der Bipolarzellen, der Horizontalzellen und der Amakrinzellen formen die innere Körnerschicht. Auch die Perikaryen ${ }^{8}$ der Müllerzellen sind in dieser Schicht beheimatet. Die Synapsen und Neuriten der Zellen der inneren Körnerschicht bilden die innere plexiforme Schicht. In der letzten retinalen Schaltstation befinden sich die Ganglienzellen (im menschlichen Auge sind etwas mehr als $10^{6}$ ), deren Perikaryen die Ganglienzellschicht formen. Die rezeptiven Felder werden in Aktionspotenziale umgewandelt und über die Axone der Ganglienzellen, die wiederum eine Schicht, die innere Nervenfaserschicht, bilden und zum optischen Nerv gebündelt werden, ausgeleitet. Den Abschluss der Netzhaut bildet die innere Grenzmembran, die Basalmembran der Müllerzellen, die eine Grenzschicht zum Glaskörper des Auges darstellt.

\subsection{Generische Struktur der Müllerzelle}

Im Anschluss an die Betrachtung des schichtartigen Aufbaus und der neuronalen Funktion der Retina wollen wir uns nun den Müllerzellen zuwenden, denen mehr als ein Jahrhundert lang nur eine passive Rolle als Stützzelle und interzelluläre Füllmasse zuteil wurde (siehe auch Fußnote 1 auf Seite 55). Ihre für Gliazellen besondere Aufgabe als Mitwirkende bei der neuronalen Informationsverarbeitung und ihre Funktion bei der Steuerung des retinalen Blutflusses in den vaskulären Bereichen ist in den letzten 30 Jahren ausgiebig untersucht worden [175, 176, 177, 178]. Außerdem spielen sie eine wichtige Rolle bei der retinalen Wundheilung, da unter bestimmten Voraussetzungen verletzte Bereiche der Retina durch Zellfortsätze, die von der Müllerzelle neu ausgebildet werden, besser erreicht und unterstützt werden können [179, 180]. Es lässt sich zweifellos aussagen, dass die Funktionen der Müllerzelle, soweit überhaupt bekannt, sehr zahlreich sind. Zu den wichtigsten bekannten metabolischen Aufgaben der Müllerzellen zählen dabei im Wesentlichen

1. Die $\mathrm{K}^{+}$-Homeostase

2. Die Transmitter-Regenerierung

3. Der Energiemetabolismus

Bei der $\mathrm{K}^{+}$-Homeostase werden die von den angeregten polarisierten Neuronenzellen freigesetzten $\mathrm{K}^{+}$Ionen aus den extrazellulären Räumen über die feinverästelten Zellfortsätze der Müllerzelle (siehe Abb. 3.5) aufgenommen und über die vertikalen Stammfortsätze zum Glaskörper abgeleitet. Hierzu bildet die Müllerzelle spezielle $\mathrm{K}^{+}$-Ionenkanäle aus (Ultrastruktur). Auch die Membran der Müllerzelle ist für die Leitfähigkeit von $\mathrm{K}^{+}$-Ionen besonders gut angepasst [178, 181].

Von den Zellen der Photorezeptoren wird bei Dunkelheit Glutamat freigesetzt, welches als Neurotransmitter wirkt und deshalb umgehend aus den synaptischen Spalten entfernt werden muss. Die Müllerzelle nimmt dieses freie Glutamat auf und wandelt es mit im Zytoplasma der Müllerzelle befindlichen Enzymen in Glutamin um, welches somit keinen Einfluss mehr auf die Synapsen hat. Dieser als

8 Als Perikaryen werden in der Regel die pyramidalen oder kugelförmigen Zellkörper von Nervenzellen bezeichnet, um sie von den Zellausläufern (Dendriten+Axone=Neuriten) abzugrenzen. 


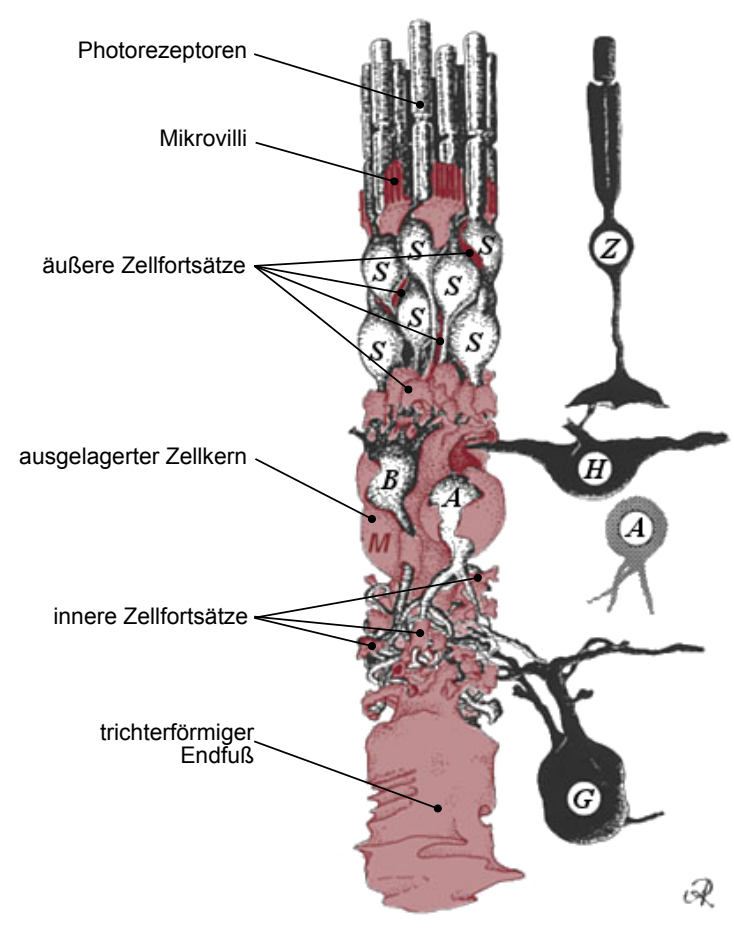

(a)
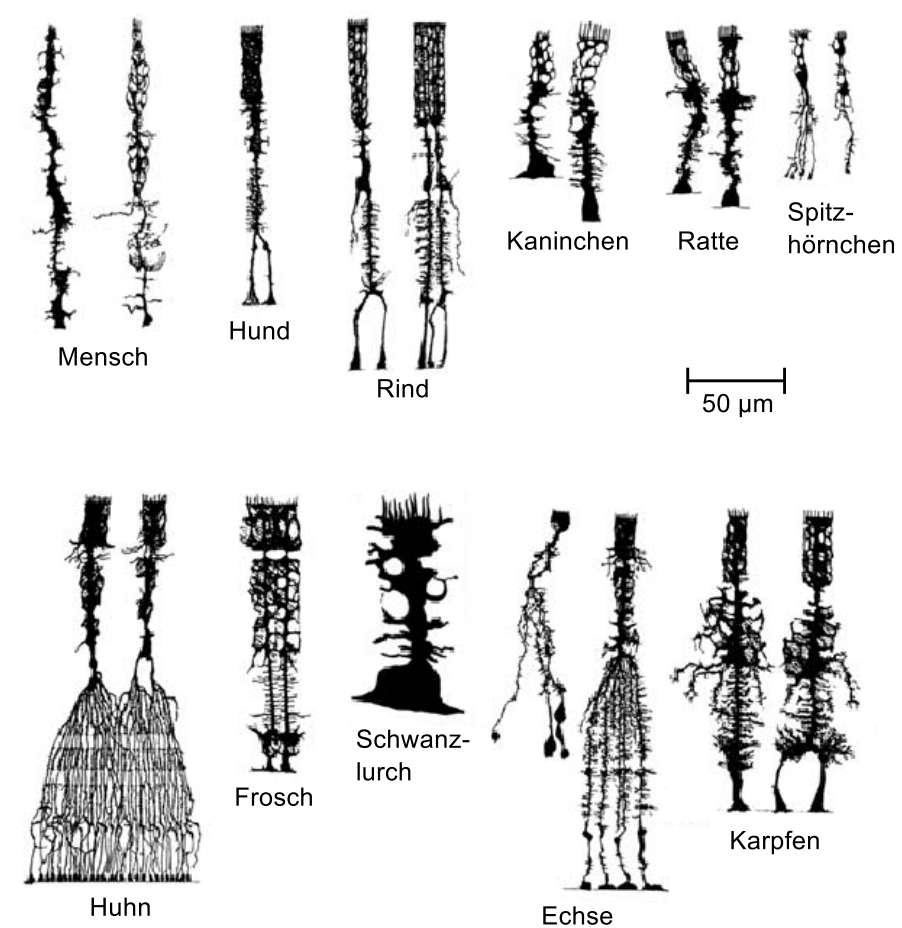

(b)

Abbildung 3.5.: Übersicht über Aufbau und Form von Müllerzellen. (a) Aufbau der Müllerzelle (MZ) als Säuleneinheit (Kaninchen). Die MZ umhüllt die Rezeptorzellen und Neuronen der Retina. Dabei bedeuten S: Stäbchenrezeptor, Z: Zapfenrezeptor, B: Bipolarzelle, H: Horizontalzelle, A: Amakrinzelle, G: Ganglienzelle (nach Reichenbach [186]) (b) Müllerzellformen verschiedener Wirbeltiere, teils als Zeichnungen von Golgi-Färbungen, teils als camera lucida Zeichnungen von eingefärbten Zellen. Die trichterförmigen Ausstülpungen der Endfüße sind deutlich zu erkennen (Rind, Huhn, Frosch, Echse und Karpfen nach Cajal [187, 188], Kaninchen nach Reichenbach \& Robinson [156], andere nach Sarthy \& Ripps [189]).

Transmitter-Regenerierung bekannte Prozess sorgt für die Transmitter-Bereitstellung von Glutamin für die Photorezeptoren, die es bei Bedarf aufnehmen und erneut in Glutamat umsetzen und in den extrazellulären Raum abgeben [182, 183].

Den Müllerzellen selbst wird Energie hauptsächlich in Form von Glykose bereitgestellt. Über einen bisher unbekannten Signalweg (vermutlich sind $\mathrm{K}^{+}$-Ionen involviert) wird die Glykogenolyse eingeleitet und Laktat bzw. Pyruvat produziert, das von den Müllerzellen an die Neuronen abgegeben wird, die diese Stoffe im Citratzyklus metabolisieren [184, 185].

Für diese Aufgaben muss die Müllerzelle die Neuronen der Retina regelrecht einhüllen und über zahlreiche Versorgungskanäle (horizontale Zellfortsätze) verfügen. In Abbildung 3.5a ist diese einhüllende Struktur als die bekannte Säuleneinheit, die bereits in der Einleitung vorgestellt wurde, stellvertretend für alle Wirbeltiere dargestellt. In der Abbildung 3.5b sind die Müllerzellen einiger Wirbeltiere wiedergegeben. Auffällig sind die vielen Zellfortsätze und - soweit bei der Präparation nicht entfernt - der ausgeprägte trichterförmige Endfuß (siehe z.B. Schwanzlurch und Kaninchen). Bei vielen Sauropsiden ${ }^{9}$ scheint sich der Hauptzellfortsatz am Endfuß regelrecht aufzufächern und einen großen filamentartigen Trichter zu bilden (siehe z.B. Huhn und Echse). Form und Größe der Müllerzellen sind stark speziesabhängig. Sie weisen Längen zwischen 50-200 $\mu \mathrm{m}$ auf, abhängig von der exzentrizitätsabhängigen Dicke

9 Zu den Sauropsiden zählen Aves (Vögel) und Reptilia. 
der untersuchten Retina. Die äußeren Zellfortsätze bilden Mikrovilli aus, die tief in die Schicht der Photorezeptoren hineinreichen und diese umschließen (siehe z.B. Rind). Der generelle Aufbau und die optischen Eigenschaften der Müllerzellen werden im Folgenden in einem Modell erfasst und in wesentlichen Strukturen integriert, die die Lichtausbreitung beschreiben.

\subsection{Die Müllerzelle als Lichtwellenleiter}

Die Müllerzelle verbindet die ventrikuläre Schicht von der Bruch-Membran am Glaskörper mit der mesenchymalen Grenzschicht der Photorezeptoren und ist damit der einzige Zelltyp, der die gesamte Retina durchspannt. Dieser Sachverhalt zusammen mit der bislang ungeklärten Tatsache, warum die Retina eine vom Lichtweg aus gesehen invertierte Struktur darstellt, brachte Franze et al. [30] auf die Idee, die opto-physikalischen Eigenschaften von Müllerzellen zu untersuchen und deckte ihre bahnbrechende Funktion als Lichtwellenleiter auf. In früheren Arbeiten von Lakshminarayanan \& Enoch [190] über biologische Lichtwellenleiter lassen sich Theorien zur Wellenleitung und Lichtstreuung in Photorezeptoren finden. ${ }^{10}$ Wir wollen diese Ideen aufgreifen und optimieren, indem wir ein Lichtwellenleitermodell der Müllerzelle und ihrer unmittelbaren natürlichen Umgebung entwickeln, um die lichtleitenden Eigenschaften der Zelle besser untersuchen zu können. Die hier abgeleiteten Transmissions- und Reflexionseigenschaften der Müllerzelle werden im anschließenden Abschnitt genutzt, um ein vereinfachtes Augenmodell zu entwickeln, das den Vergleich des Streulichts mit dem direkt einfallenden Licht zulässt und die Optimierungsfunktion der Müllerzelle bezüglich des verbesserten Sehens erklären kann.

\subsubsection{Modellierung als Quantenkanal}

Um die Müllerzelle als Lichtwellenleiter zu modellieren, gehen wir von der optischen Wellengleichung aus. Eine Separation der Variablen der allgemeinen Wellengleichung

$$
\left(\nabla^{2}-\frac{\partial^{2}}{\partial t^{2}}\right) E(\mathbf{r}, t)=0
$$

in einen orts- und einen zeitabhängigen Term unter der Annahme harmonischer Zeitabhängigkeit

$$
E(\mathbf{r}, t)=E(\mathbf{r}) \exp (i \omega t)
$$

liefert als ortsabhängigen Teil die Helmholtz-Gleichung der klassischen Optik

$$
\left(\Delta+k^{2}\right) E=0
$$

Für den Wellenvektor gilt

$$
k=k_{0} n=k_{0} \sqrt{\varepsilon_{\mathrm{r}}}
$$

für nicht-magnetisierbare Materialien. ${ }^{11}$ Die Größe $k_{0}$ ist der Betrag des Vakuum-Wellenvektors mit $k_{0}=\frac{\omega}{c}$. Der enorme numerischen Aufwand einer realistischen 3D-Simulation, vor allem im Hinblick

10 Als Beispiel sei der "Blütenkorb der Venus" (Euplectella aspergillum) aus der Familie der Tiefseeschwämme aufgeführt, dessen $5-15 \mathrm{~cm}$ lange Fasern ein Skelettbestandteil aus hydriertem amorphem Silizium bilden, die trotz des einfachen Materials über bemerkenswerte Lichtwellenleitereigenschaften bei enormer Stabilität verfügen [191] und deshalb viele Forschergruppen zur Untersuchung und zum Biomimikri dieser lichtleitenden Fasern veranlasste [192, 193]. Insbesondere dienen retinale Strukturen als Vorlage für technische Anwendungen, wie beispielsweise bei der Oberflächenvergütung zur Effizienzsteigerung moderner Solarzellen [194].

11 Eigentlich trifft für den Brechungsindex formal $n=\sqrt{\varepsilon_{\mathrm{r}} \mu_{\mathrm{r}}} \mathrm{zu}$, jedoch verschwindet für organisches Gewebe die magnetische Suszeptibilität $\chi_{\mathrm{m}} \approx 0$, so dass in diesem Fall $\mu_{\mathrm{r}} \approx 1$ gilt. 


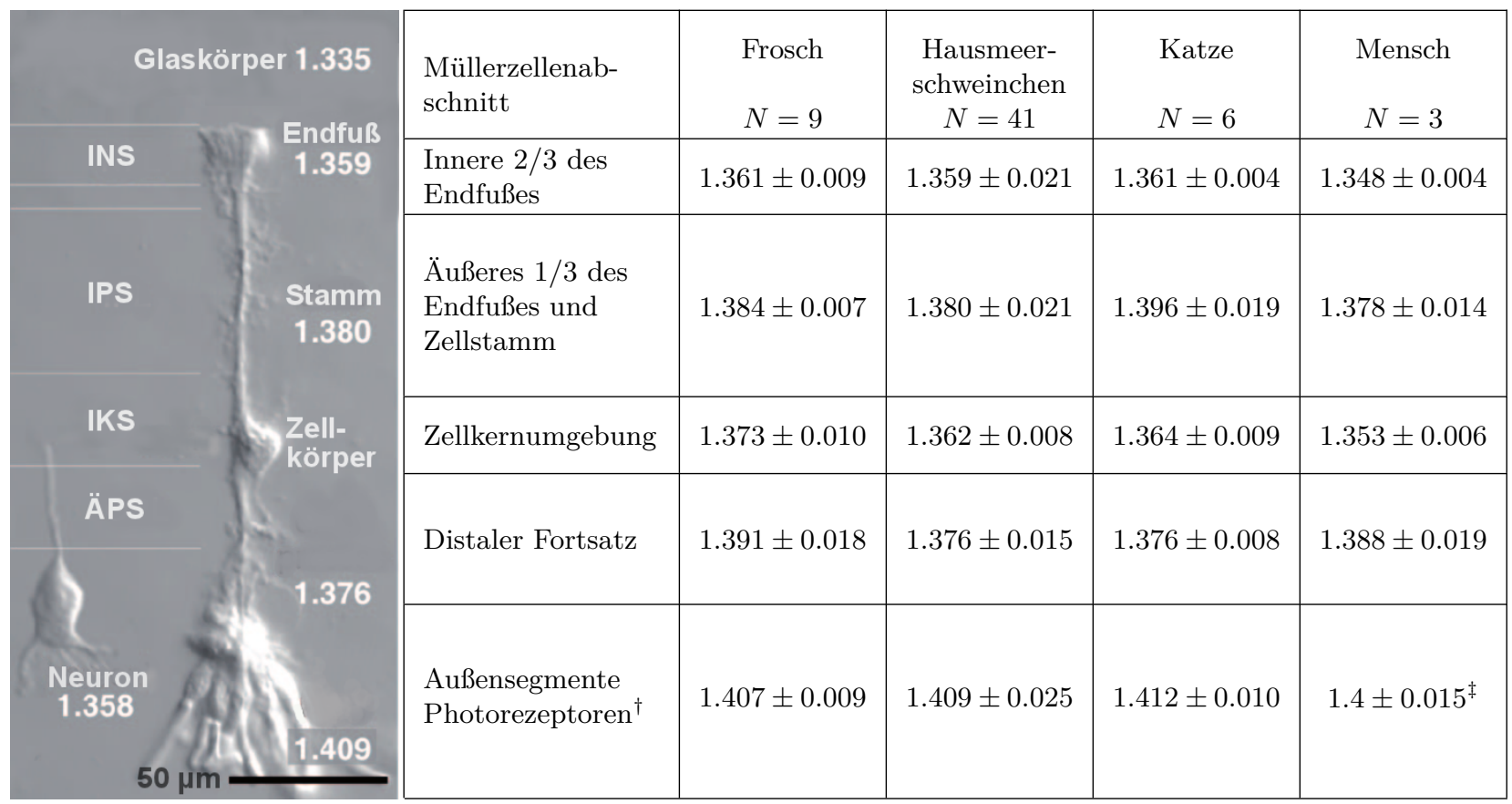

Tabelle 3.1.: Brechungsindices von Müllerzellen verschiedener Wirbeltiere. Links: Die NormaskiKontrastaufnahme (Differential-Interferenz-Kontrast-Mikroskopie) einer dissoziierten HausmeerschweinchenMüllerzelle, einigen dissoziierten Photorezeptorzellen und einer dissoziierten Bipolarzelle (als "Neuron" beschriftet) dient der Orientierung. Die gemessenen Brechungsindices sind an entsprechender Stelle eingetragen (nach Franze et al. [30]). Es bedeuten INS: Innere Nervenfaserschicht, IPS: Innere plexiforme Schicht, IKS: Innere Körnerschicht und ÄPS: Äußere plexiforme Schicht. Rechts: Brechungsindices für Retinae einiger Wirbeltierspezies. $N$ bezeichnet die Anzahl der untersuchten Individuen pro Spezies. Die Daten entstammen Untersuchungen von lebenden enzymatisch dissoziierten Zellen von Retinae, die mittels computergestützter Phasenmikroskopie gewonnen wurden (nach Tychinsky et al. [196] und Beuthan et al. [197]). Die Messungen der Brechungsindices der Photorezeptoraußensegmente stammen aus ${ }^{\dagger}$ Winston [198] und ${ }^{\ddagger}$ Sidman [199].

auf die große Länge der Müllerzelle von mehreren 100 Wellenlängen des betrachteten Lichts, lässt sich unter Verwendung der Rotationssymmetrie der Müllerzelle zu einem zweidimensionalen Wellenmodell vereinfachen, indem ein effektiver 2D-Brechungsindex gewählt wird, der einem Horizontalschnitt durch die Müllerzelle an der Stelle ihrer maximalen Ausdehnung (Zelldurchmesser) entspricht. Zur Rechtfertigung dieser Näherung siehe z.B. [195].

Für die Lösung der zur optischen Wellengleichung (3.3) formal äquivalenten zeitunabhängigen Schrödinger-Gleichung

$$
\left(-\Delta+V-k_{0}^{2}\right) \psi=0
$$

kann die numerische Methode aus Kapitel 1 genutzt werden. Dafür muss das Potenzial $V$ in eine vom Brechungsindex $n$ abhängige Funktion

$$
V(n)=k_{0}^{2}\left(1-n^{2}\right) \quad,
$$

transformiert werden, so dass das Potenzial für optisch dichte Materialien (also $n>1$ ) negative Werte annimmt. $^{12}$

Die Brechungsindices entlang der Müllerzelle sind nicht zuletzt aufgrund der Artenvielfalt der Wirbeltiere von vielen experimentellen Gruppen untersucht worden (vgl. Tabelle 3.1). Da die Müllerzelle größere Brechungsindices als ihre Umgebung aufweist, entsteht nach Gleichung (3.6) im Querschnitt

12 Vergleiche auch mit Unterabschnitt 2.3.3 im Kapitel über Chaotische Strahlenteiler und Unterabschnitt 4.1.2 im Kapitel über $\mathcal{P} \mathcal{T}$-symmetrische niedrigdimensionale Systeme. 


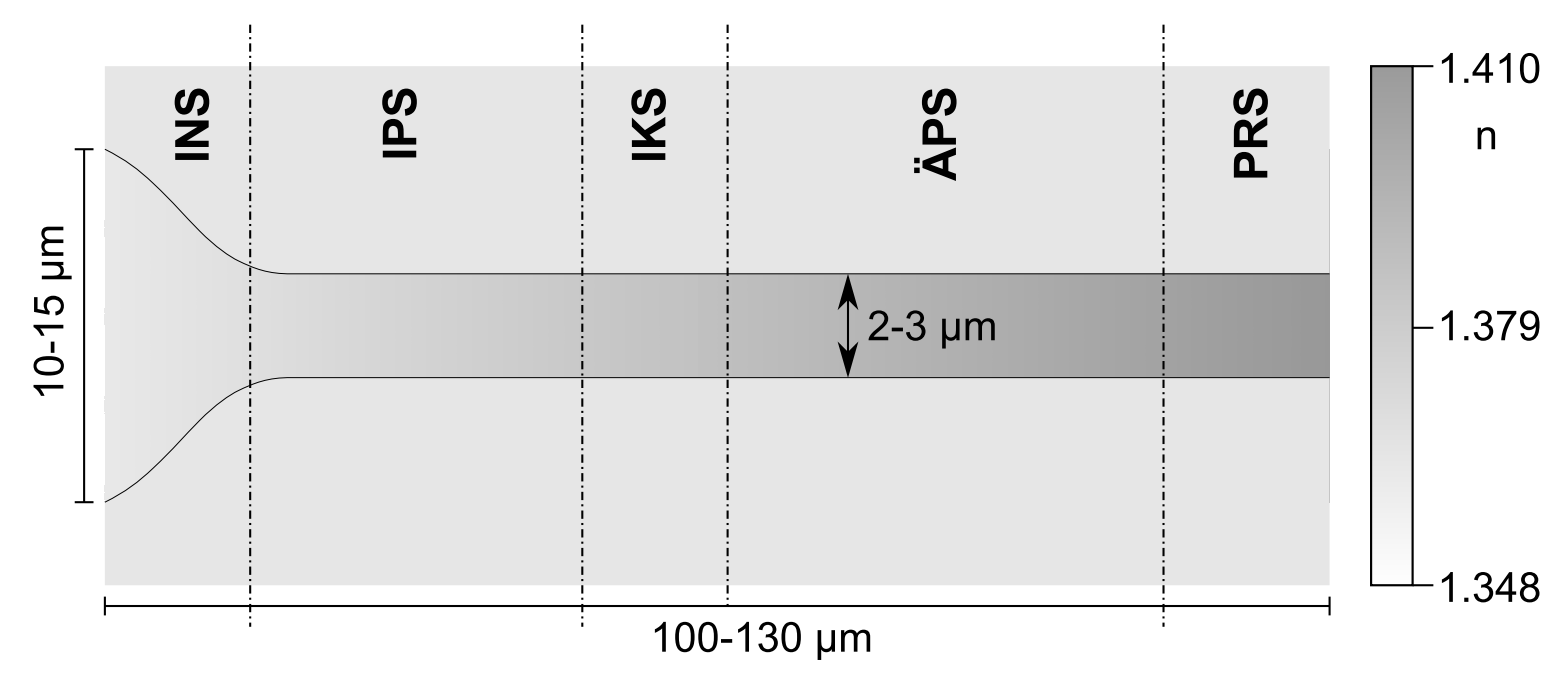

Abbildung 3.6.: Die Müllerzelle (MZ) als Quantenkanal. Dargestellt ist der (von der Zelle aus gesehene) vertikale Potenzialverlauf (Höhenprofil des Brechungsindexes). Es bedeuten INS: Innere Nervenfaserschicht, IPS: Innere plexiforme Schicht, IKS: Innere Körnerschicht, ÄPS: Äußere plexiforme Schicht und PRS: Photorezeptorschicht. Die Schichten sind zur besseren Orientierung angegeben, ebenso wie die durch dünne schwarze Linien angedeuteten Außenmembrane der MZ. Der nahezu rotationssymmetrische Aufbau der MZ rechtfertigt eine Approximation auf ein quasi-zweidimensionales Potenzial. Die Maßangaben der MZ [200] und der graduelle Verlauf ihres Brechungsindexprofils (vgl. auch Tabelle 3.1) sind einer durchschnittlichen MZ des Menschen angepasst.

ein Potenzialtopf. Verfolgt man das Profil des Potenzials entlang der Müllerzelle vom Endfuß bis hin zu den Photorezeptorenden, so ergibt sich ein Kanal, dessen Breite im Endfußbereich der Müllerzelle erst abnimmt und dann um einen konstanten Wert von etwa 2-3 $\mu \mathrm{m}$ nur leicht variiert, und dessen Tiefe über die gesamte Ausdehnung der Zelle vom Endfuß zur Photorezeptorschicht hin zunimmt (vgl. Abb. 3.6). Im Hinblick auf das Modell der Streulichtreduzierung des nächsten Abschnitts wurde in diesem Beispiel eine menschliche Müllerzelle gewählt und die Dimensionen der Zelle in der Abbildung vermerkt. Der Verlauf der retinalen Schichten ist in die Abbildung integriert worden und dient der besseren Orientierung. In Endfußnähe besitzen Zelle und Glaskörper nahezu gleiche Brechungsindices. Die Umgebung der Müllerzelle wurde als Bereich mit konstantem Brechungsindex, äquivalent zum mittleren Brechungsindex der benachbarten Neuronen (gemessen $n=1.358$ in [30]), dargestellt. Auf die notwendige realistische Erweiterung des Müllerzellenmodells und die Fluktuationen des Brechungsindex ihrer extrazellulären Umgebung wird weiter unten eingegangen.

\subsubsection{Die Bedeutung der Form des Endfußes für die Lichtleitung}

Der Fuß der Müllerzelle erinnert stark an die Geometrie von trichterförmigen Punktkontakten, die beim Design von Halbleiter-Nanostrukturen eine wichtige Rolle bei der Optimierung des Elektronentransports spielen. Trichterförmige Geometrien bzw. elektronisch geartete Strukturen (in diesem Fall als Potenzialstufe ausgeprägt) bedingen eine Modifikation der Winkelverteilung, die als Kollimation bezeichnet wird (als Beispiel siehe auch Abb. 1.6 auf Seite 20). Vom Standpunkt der semiklassischen Betrachtung resultiert der Kollimationseffekt solcher Strukturen aus der adiabatischen Invarianz des Produktes aus transversalem Impuls und Kanalbreite [201]. Damit hat dieses Produkt die Einheit einer Wirkung. Der Vergleich der Wirkung einer Potenzialstufe im Kanal (vgl. Abb. 3.7a)

$$
S_{\text {Stufe }}=\sqrt{2\left(E_{\mathrm{F}}-E_{\mathrm{S}}\right)} w_{\min }
$$




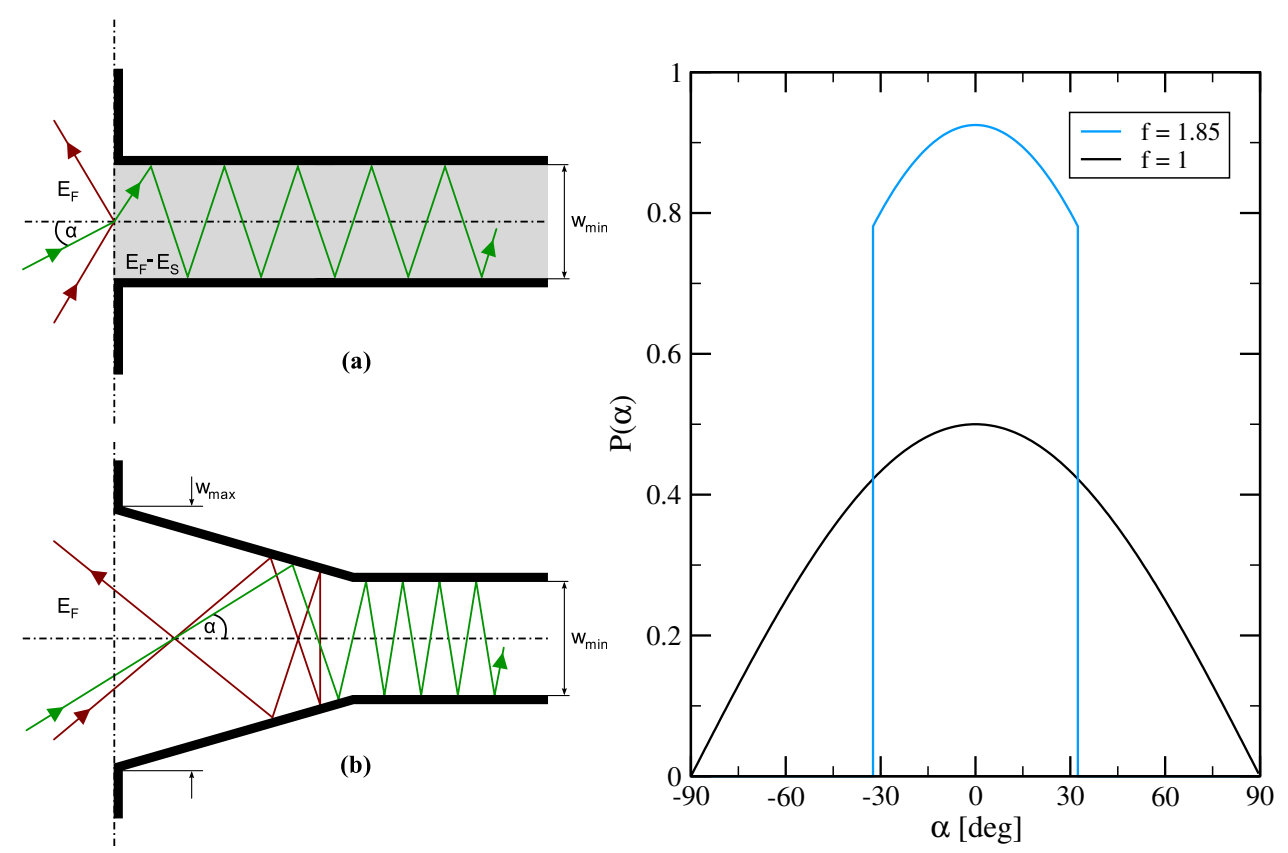

Abbildung 3.7.: Links: Kollimationseffekte verursacht durch (a) eine sprunghafte Verengung auf einen Kanal der Breite $w_{\text {min }}$ mit Potenzialstufe der Höhe $E_{\mathrm{S}}$ und (b) trichterförmige Verengung von Breite $w_{\max }$ auf Breite $w_{\text {min }}$. Die Größe $E_{\mathrm{F}}$ bezeichnet das Fermi-Niveau der zugrunde liegenden 2D-Halbleiter-Nanostruktur (nach [16]). Die grün gezeichneten Linien bezeichnen transiente, die roten Linien reflektierte Trajektorien. Zur Berechnung des Kollimationsfaktors $f$ und des maximalen Einfallswinkels siehe Text. Rechts: Winkelverteilung zur Verdeutlichung des Kollimationseffektes. Die schwarze Linie gibt die analytische Lösung der Winkelverteilung für Realisierung (a) ohne Potenzialstufe an $\left(E_{\mathrm{S}}=0\right)$. Die zyanfarbene Linie ergibt sich für die Realisierung nach (b) als analytische Lösung in der adiabatischen Näherung mit dem Kollimationsfaktor $f=1.85$ (nach Molenkamp et al. [135]). Der "doppelte" Kollimationseffekt der Müllerzelle ergibt sich durch eine Kombination beider hier vorgestellter Effekte, da sich sowohl der Brechungsindex ändert (a), als auch der Endfuß eine deutliche Trichterform aufweist (b).

auf der einen mit der Wirkung eines Trichters (vgl. Abb. 3.7b)

$$
S_{\text {Trichter }}=\sqrt{2 E_{\mathrm{F}}} \sin \left(\alpha_{\max }\right) w_{\max }
$$

auf der anderen Seite liefert unter der Annahme einer stationären Wirkung, also für $S_{\text {Stufe }}=S_{\text {Trichter }}$, die Bedingung für den maximalen Einfallswinkel

$$
\alpha_{\max }=\arcsin \left(\frac{1}{f}\right), \text { mit } f \equiv \sqrt{\frac{E_{\mathrm{F}}}{E_{\mathrm{F}}-E_{\mathrm{S}}}} \frac{w_{\max }}{w_{\min }} .
$$

Der Kollimationsfaktor $f$ ergibt sich folglich als Produkt aus dem Term, der den Kollimationseffekt der Stufe beschreibt und dem Term, der die geometrische Beschaffenheit des Trichters berücksichtigt. In der adiabatischen Näherung lässt sich daraus die Winkelverteilung ableiten

$$
P(\alpha)=\left\{\begin{array}{ll}
\frac{f}{2} \cos (\alpha) & |\alpha|<\alpha_{\max } \\
0 & \text { sonst }
\end{array},\right.
$$

die verdeutlicht, dass kleine Winkel $|\alpha|<\alpha_{\max }$ bevorzugt eingesammelt, während Winkel $|\alpha| \geq \alpha_{\max }$ vollständig reflektiert werden (siehe Abb. 3.7). Diese Eigenschaft der Kollimation kann direkt auf die Müllerzelle übertragen werden, da ihr Endfuß diese Trichterform aufweist und zusätzlich - verursacht durch die Änderung des Brechungsindexes an der Membran zum Endfuß der Müllerzelle - eine Po- 
tenzialstufe besitzt. Der geometrische Anteil lässt sich zu $f=\frac{w_{\max }}{w_{\operatorname{mion}}} \approx 3$ bestimmen. Der Effekt der Potenzialstufe ist klein und soll hier vernachlässigt werden. Damit ergibt sich ein maximaler Winkel von $\alpha_{\text {max }} \approx 20^{\circ}$, der sich auch im Vergleich mit den Simulationen bestätigt (vgl. mit Abb. 3.11). Abweichungen vom Maximalwinkel sind durch Streuverluste aufgrund der kleinen Apertur bedingt, da die Außenmembrane der Müllerzelle endlich hohe Potenzialwände darstellen und die hier abgeleitete Theorie der Winkelverteilung nach Gleichung (3.10) streng betrachtet nur für unendlich hohe Potenzialwände gilt.

\subsubsection{Modellierung der extrazellulären Umgebung der Müllerzelle}

Im Experiment [30] konnte die Lichtwellenleitereigenschaft der Müllerzelle in einer homogenen Lösung, die dem mittleren Brechungsindex der extrazellulären Umgebung der Müllerzelle entsprach, nachgewiesen werden. Wir wollen nun ein Modell entwickeln, dass die Fluktuationen des Brechungsindex mitberücksichtigt, um die Lichtwellenleitereigenschaft der Müllerzelle in ihrer natürlichen Umgebung zu untersuchen. Die Modellbildung des extrazellulären Raums um die Müllerzelle erfordert die Einbeziehung der Brechungsindices von retinalen Blutgefäßen und Nervenfasern bis hin zu den Zellkörpern und Membranen der Retina-Neuronen. Eine 1:1 Nachbildung der in Unterabschnitt 3.1.2 definierten Schichten der Retina gestaltet sich hierbei äußerst schwierig, da der strukturelle Aufbau stark von der Position innerhalb der Retina abhängt und von Müllerzelle zu Müllerzelle stark variiert. Aus diesem Grund ist es zweckdienlich, den extrazellulären Raum um die Müllerzelle durch ein stationäres Zufallspotenzial zu approximieren, das die Fluktuationen des Brechungsindex der Retina berücksichtigt, und in das die Müllerzelle eingebettet werden kann. Ein solches Potenzial ist durch ein zweidimensionales Gaußsches Zufallsfeld gegeben, das wie folgt konstruiert werden kann. Ausgangspunkt ist die Autokorrelationsfunktion des gesuchten Zufallspotentials

$$
C(\mathbf{r})=\left\langle V\left(\mathbf{r}^{\prime}\right) \mid V\left(\mathbf{r}^{\prime}+\mathbf{r}\right)\right\rangle=\sigma_{\mathrm{g}}^{2} g_{\mathrm{c}}\left(\mathbf{r}, l_{\mathrm{c}}\right)
$$

mit $l_{\mathrm{c}}$ als Korrelationslänge der Dichtefunktion $g_{\mathrm{c}}\left(\mathbf{r}, l_{\mathrm{c}}\right)$ und $\sigma_{\mathrm{g}}^{2}$ als Varianz der Fluktuationen. Als zugrundeliegende Korrelationsfunktion wählen wir nun ebenfalls eine Gauß-Funktion mit Korrelationslänge $l_{\mathrm{c}}$

$$
g_{\mathrm{c}}\left(\mathbf{r}, l_{\mathrm{c}}\right)=\frac{1}{\sqrt{2 \pi} l_{\mathrm{c}}} \exp \left(-\frac{\mathbf{r}^{2}}{2 l_{\mathrm{c}}^{2}}\right) .
$$

Das Wiener-Khintchine-Theorem besagt nun, dass für stationäre Prozesse die Fourier-Transformierte der Autokorrelationsfunktion die spektrale Leistungsdichte liefert [202, 203]. So kann das gewünschte Zufallsfeld generiert werden, indem die Quadratwurzel der Fouriertransformierten der Korrelationsfunktion mit einem Feld aus komplexen Phasen multipliziert und das Ergebnis wieder in den Ortsraum zurücktransformiert wird

$$
V(\mathbf{r})=\mathcal{F T}^{-1}[\sqrt{\mathcal{F} \mathcal{T}[C(\mathbf{r})]} \exp (i \Phi(\mathbf{r}))],
$$

wobei $\Phi(\mathbf{r})$ ein Feld mit gleichverteilten zufälligen Phasen ist, dessen Werte zwischen $[0,2 \pi)$ liegen. Der zentrale Grenzwertsatz garantiert dabei, dass ein Gaußsches Zufallsfeld erzeugt wird, denn durch die Multiplikation mit $\exp (i \Phi(\mathbf{r}))$ stellen die Koeffizienten der Fouriertransformation dann unabhängige Zufallsvariablen dar (siehe auch Schema in Abb. 3.8). Der zur Erzeugung der Zufallspotenziale benutzte Quellcode entstammt [204]. Als Korrelationslänge wurden Werte zwischen 1.0-2.0 $\mu \mathrm{m}$ angenommen, 

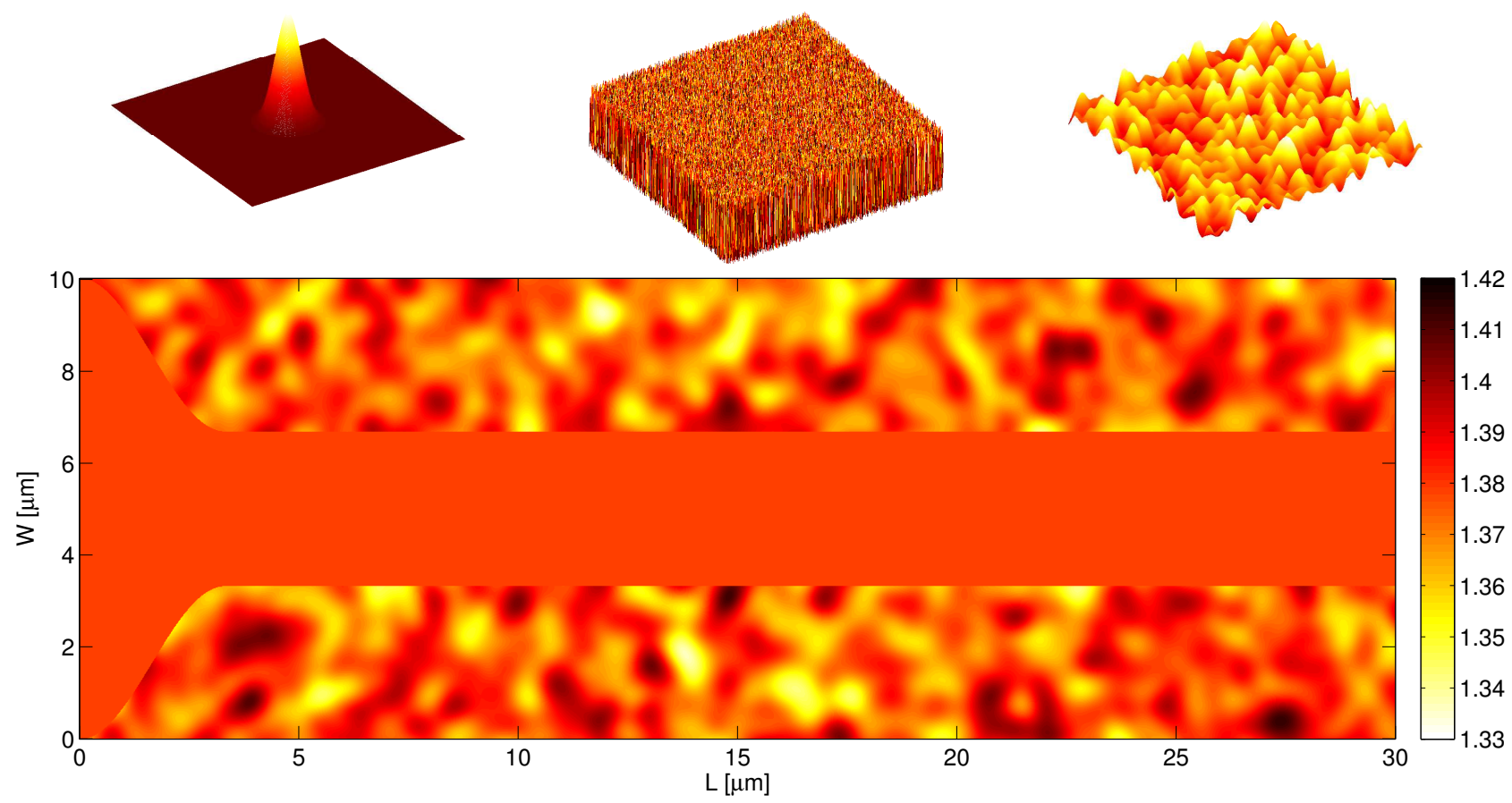

Abbildung 3.8.: Oben: Schema zur Generierung eines Zufallspotenzials. Fouriertransformierte 2D-GaußFunktion (links) wird mit zufälligen Phasen (Mitte) multipliziert und dann in den Ortsraum zurücktransformiert. Das Ergebnis ist ein stationäres 2D-Gaußsches Zufallsfeld (rechts). Zur mathematischen Beschreibung siehe Unterabschnitt 3.3.3. Unten: Ausschnitt einer Müllerzelle, die in ein Zufallspotenzial eingebettet wurde. Der mittlere Brechungsindex der Zelle liegt nur leicht über dem mittleren Brechungsindex des Zufallspotenzials.

die als Mittelwerte aus verschiedenen Retinae-Aufnahmen gemittelt wurden (z.B. [154, 205]). Die Anzahl und der Durchmesser der Nervenfasern (Inter-Faser-Abstände bzw. Durchmesser von Dendriten, Axonen und Zellfortsätzen $\varnothing \leq 0.5 \mu \mathrm{m}$ ), bestimmt hierbei die untere Grenze der Korrelationslänge, während Anzahl und Durchmesser der Neurone $(\varnothing>5 \mu \mathrm{m})$ und extrazelluläre Zwischenräume die obere Grenze beeinflussen. Ebenso wird auch die Amplitude der Korrelationsfunktion durch den maximalen und minimalen Brechungsindex der Retina beeinflusst. Für das Minimum wird der reine extrazelluläre und mit Wasser gefüllte Raum angenommen $\left(n_{\min }=1.33\right)$, wobei für das Maximum des Brechungsindexes der Retina die Fasern der Außensegmente der Photorezeptorschicht mit $n_{\max } \simeq 1.42$ infrage kommen [199, 206]. In Abbildung 3.8 ist ein Ausschnitt einer typischen Generierung eines solchen Potenzials mit eingebetteter Müllerzelle dargestellt. In der Abbildung ist zu erkennen, dass in der Nähe der Müllerzelle befindliche und durch Generierung des Zufallspotenzials entstandene Bereiche einen Brechungsindices aufweisen, die zum Brechungsindex des Zellstammfortsatzes der Müllerzelle äquivalent sind. Diese ausgedehnten und teilweise verbundenen Gebiete approximieren die horizontalen Zellfortsätze der Müllerzelle, die zur eingangs beschriebenen metabolischen Funktion in die einzelnen Schichten der Retina reichen. Die dunklen Bereiche stellen Approximationen von retinalen Blutgefäßen, Filamenten und Neuriten dar, während die gelbfarbenen Bereiche Neuronen und die sehr hellen und vereinzelt vorkommenden weißen Bereiche extrazelluläre, mit Wasser gefüllte Zwischenräume darstellen. Damit bleibt zwar die exakte Schichtenreihenfolge der Retina unberücksichtigt, jedoch ist sowohl die Anzahl der retinalen Neurone, Filamenten und Neuriten, als auch die Beschaffenheit der unmittelbaren Umgebung der Müllerzelle hinreichend gut approximiert. Ein Modell, das ausschließlich schichtartige Fluktuationen berücksichtigt, ist in [207] zu finden. 

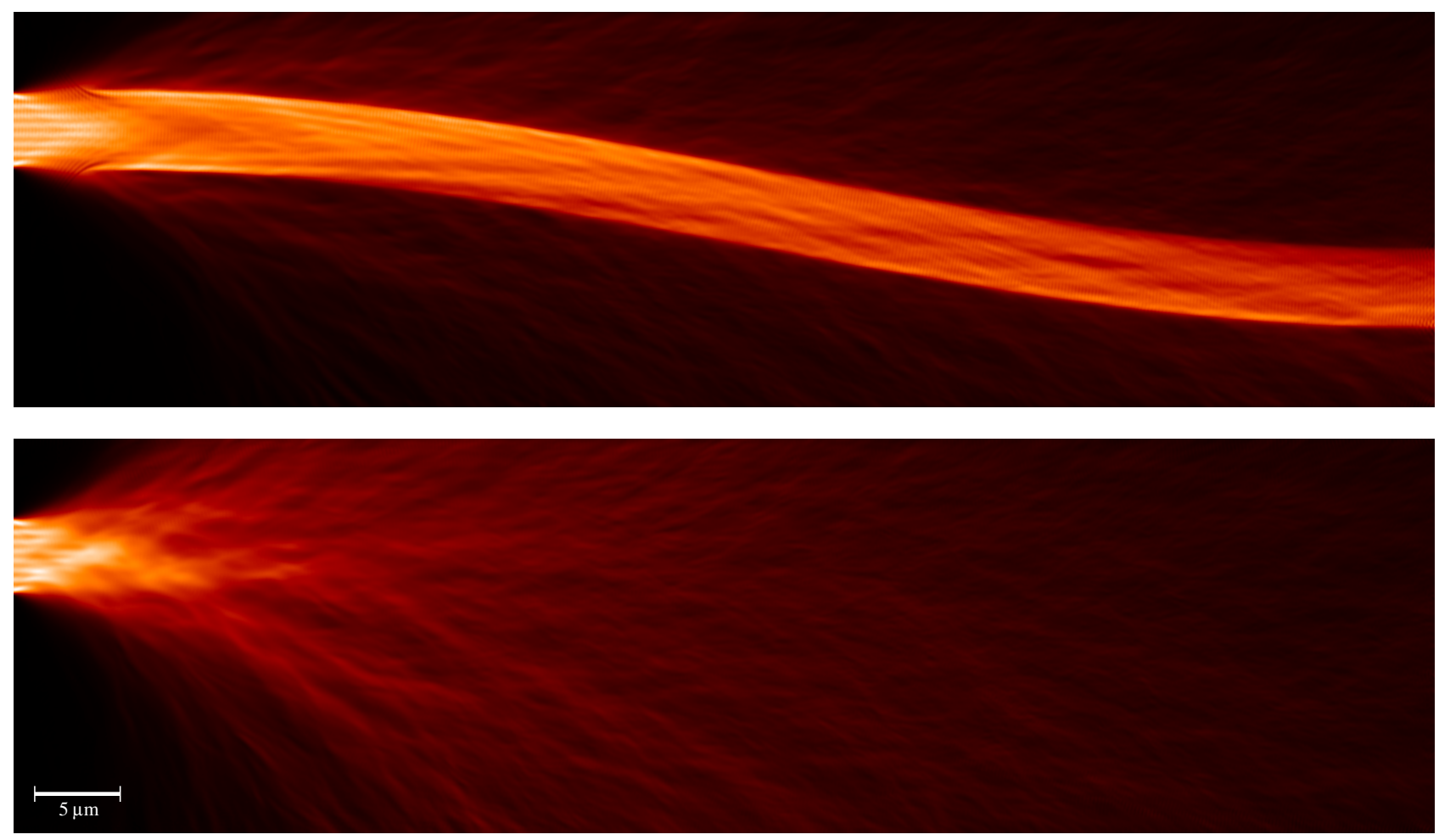

Abbildung 3.9.: Beispiele für Streuwellenfunktionen. Oben: Aufenthaltswahrscheinlichkeitsdichte der Streuwellenfunktion einer doppelt gebogenen Müllerzelle (MZ) mit Krümmungsradien von jeweils $R=120 \mu \mathrm{m}$ im Zufallspotenzial (als Summe über die ersten 10 Moden). Der Verlauf der Welle in der MZ ist klar zu erkennen. Unten: Aufenthaltswahrscheinlichkeitsdichte der Streuwellenfunktion des reines Zufallspotenzials ohne MZ (als Summe der ersten 10 Moden). Das Potenzial erzeugt eine diffuse Streuung des Lichts ohne erkennbare Richtungsabhängigkeit. Beide Abbildungen entsprechen Konfigurationen, in denen von links in einen schmalen Kanal mit einer Breite von ca. 3-4 $\mu$ m ebene Wellen einfallen, die der Wellenlänge $\lambda \simeq 500 \mathrm{~nm}$ entsprechen. Der Maßstab ist im unteren Bild angegeben.

\subsubsection{Streuwellenfunktionen}

Als erste Demonstration der Lichtleitfähigkeit von Müllerzellen in dem oben entwickelten fluktuierenden Potenzial liefern die Streuwellenfunktionen aus der numerischen Simulation nach der in Kapitel 1 vorgestellten Methode. In Abbildung 3.9 ist die normierte Aufenthaltswahrscheinlichkeitsdichte der Streuwellenfunktionen für von links einfallende ebene Wellen in ein offenes Gebiet einmal mit, sowie einmal ohne Müllerzelle im Zufallspotenzial dargestellt. In der Darstellung wurde über die ersten 10 Moden summiert, um auch den Einfluss schräg einfallender Wellen (mit Einfallswinkeln $\alpha \lesssim 25^{\circ}$ ) zu veranschaulichen. Der deutlich erkennbare Verlauf der Welle in der doppelt gekrümmten Müllerzelle (Krümmungsradius jeweils $R \approx 120 \mu \mathrm{m}$ ) ist ein eindeutiger qualitativer Beweis der Lichtleitfunktion. ${ }^{13}$ Der Kollimationseffekt im Endfußbereich der Müllerzelle ist ebenfalls deutlich zu erkennen.

Vergleicht man dies mit der Lichtpropagation durch eine fiktive Retina ohne Müllerzellen (reines Zufallspotenzial), so lässt sich deutlich die starke Streuung des Lichts, vor allem auch in retinale Nachbarbereiche (nach oben und nach unten) erkennen, die einen großen Informationsverlust im Bezug auf die Ortsauflösung des abzubildenden Objektes darstellen. Ein signifikanter Verlust - mit absehbaren Folgen für die Infomationsübertragung - ist bereits nach einigen $10 \mu \mathrm{m}$ deutlich zu erkennen. Die

13 Die schwache Modulation des Zufallsfeldes im Vergleich zur hohen Energie der einfallenden Wellen führt zur Ausbildung von fein verästelten Strukturen (siehe Abb. 3.9) - ein skalenübergreifender Effekt, der mit der Ausbildung von Kaustiken zusammenhängt. Siehe dazu auch [208, 209]. 

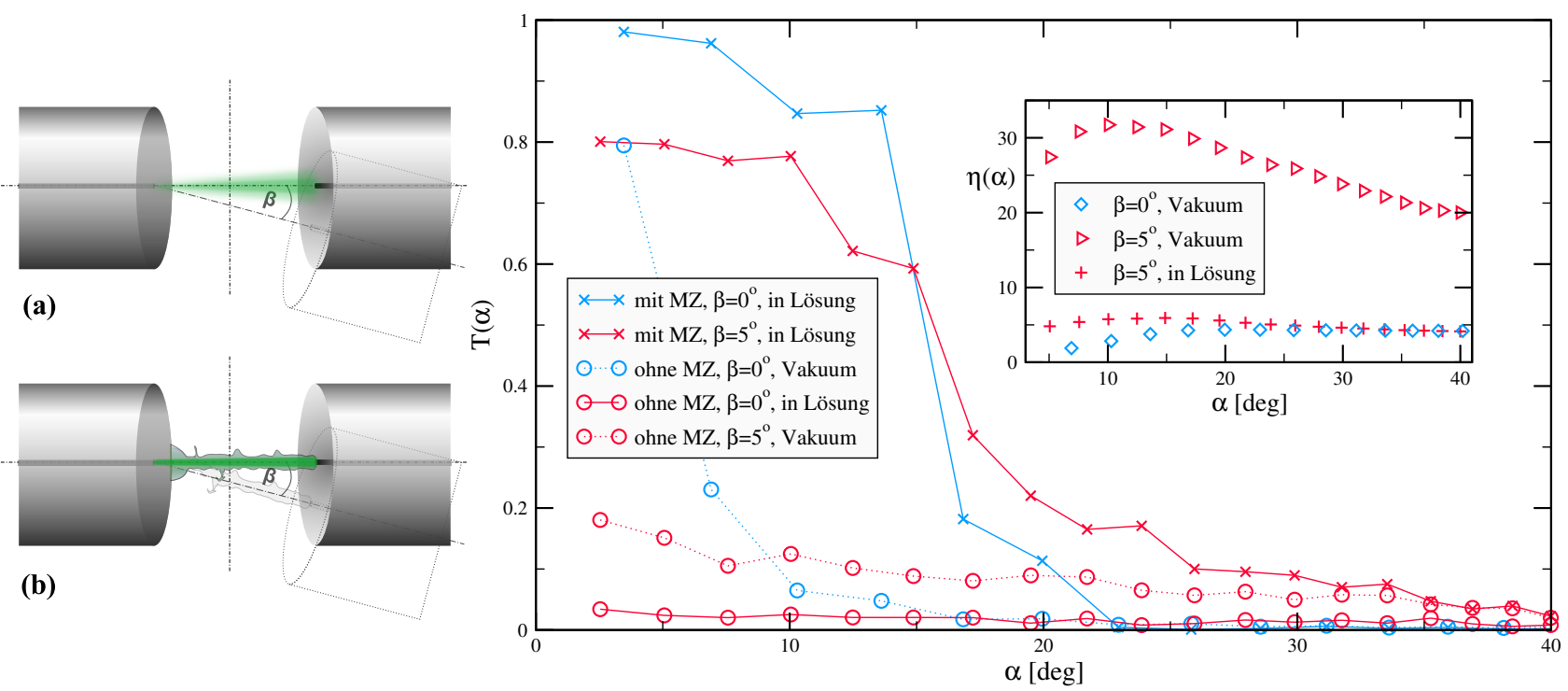

Abbildung 3.10.: Vergleich der Simulationsergebnisse der optischen Leistung der Müllerzelle (MZ) aus der Simulation mit experimentellen Ergebnissen des "optischen Streckers" nach Franze et al. [30] (siehe Text). Links: Schematischer Aufbau des Experiments zwischen zwei Lichtwellenleitern (Moltimodenlichtwellenleiter) (a) Referenzmessung der Lichtleistung ohne MZ. (b) Messung der Lichtleistung mit MZ zur Bestimmung der Lichtleiteffizienz $\eta$. Der Winkel $\alpha$ (nicht eingezeichnet) gibt die Verkippung des linken, der Winkel $\beta$ die Verkippung des rechten Lichtwellenleiters an. In der Simulation wurde nur der zweite Wellenleiter verkippt, allerdings so, dass $\alpha+\beta=5^{\circ}$ wie im Experiment gilt. Rechts: Winkelabhängige Transmission und daraus bestimmte optische Leistung (siehe Unterabbildung) des dem Experiment nachempfundenen Aufbaus. Die Transmission für applizierte MZ (mit " $\mathrm{x}$ " gekennzeichnet) ist im Vergleich zu den Referenzsimulationen ohne MZ (mit "o" gekennzeichnet) deutlich erhöht. Es wurden Simulationen für zwei verschiedene Kippwinkel $\beta=0^{\circ}$ (blaue Kurven) und $\beta \approx 5^{\circ}$ (rote Kurven), dem Experiment nachempfunden, durchgeführt. Die gestrichelten Linien kennzeichnen Simulationen im Vakuum $(n=1)$. Die durchgezogenen Linien kennzeichnen Simulationen für mit einer speziellen Lösung $(n=1.36)$ gefüllten Zwischenraum. Die experimentellen Messungen nach Franze et al. [30] ergaben $\eta_{\beta=0}(\alpha \approx 0)=1.9 \pm 0.1$ (parallele Anordnung) und $\eta_{\beta \approx 2.5}(\alpha \approx 2.5)=7.2 \pm 0.9$ (gekippte Anordnung). In unseren Simulationen finden wir $\eta_{\beta=0}(\alpha \approx 0)=2$ und $\eta_{\beta \approx 5}(\alpha \approx 0)=5$, also eine gute Übereinstimmung mit dem Experiment und eine Bestätigung der Lichtwellenleitertheorie.

Dimensionen der Müllerzelle wurden den menschlichen Müllerzellen nachempfunden.

Die Streuwellenfunktionen stellen einen direkten qualitativen Nachweis der Lichtwellenleitereigenschaft von Müllerzellen dar. Für einen quantitativen Nachweis werden zusätzlich observable Größen benötigt, die in den folgenden zwei Unterabschnitten abgeleitet werden.

\subsubsection{Vergleich mit dem Experiment}

Wie eingangs erwähnt, wurden von Franze et al. [30] infolge von Untersuchen zur Elastizität von Gliazellen auch die opto-physikalische Eigenschaft von Müllerzellen in ihrer Funktion als lebende Lichtwellenleiter entdeckt. Zum experimentellen Nachweis der Lichtleitung wurden einige einzelne Müllerzellen aus der Retina eines Meerschweins extrahiert und nacheinander in einem Optischen Strecker gespannt (siehe schematische Abb. 3.10). Ein optischer Strecker ist eine von Guck et al. [210, 211] entworfene Apparatur, die zwei gegenläufige Laserstrahlen verwendet, um einzelne Zellen bei niedriger Lichtleistung (ca. 10-100 mW pro Strahl) einzufangen. Dabei wird das Zusammenwirken zwei gegeneinander wirkender Kräfte ausgenutzt. Zum einen die Gradientenkraft, die aus der Lorentz-Kraft ableitbar ist und zum anderen die Streukraft, die aus dem klassischen Strahlungsdruck abgeleitet werden kann. Eine Zelle, die sich in einer speziellen Lösung zwischen zwei Lichtleitfasern befindet, kann somit regel- 
recht eingespannt werden [212, 213]. Das Laserlicht muss dabei eine signifikant größere Wellenlänge im Vergleich zur Ausdehnung des zu untersuchenden Objekts besitzen (siehe Rayleigh-Näherung [214]). Bei genügend großer Intensität und passend gewählten Laserprofilen in Ausbreitungs- und Streurichtung (beispielsweise senkrecht zur Zelle) stellt sich ein Gleichgewicht zwischen den erwähnten Kräften ein, und die so ausgerichtete und gespannte Zelle kann beispielsweise mit einem weiteren Laser beliebiger Frequenz auf ihre Lichtleitfähigkeit hin untersucht werden. ${ }^{14} \mathrm{Im}$ Experiment wurden die Zellen mit dem fluoreszierenden Vitalfarbstoff MitoTracker orange eingefärbt und in eine Spüllösung BSA P-0834 Sigma gelegt. Der homogene Brechungsindex der Lösung gilt mit $n=1.36$ als "Nachempfindung natürlicher Umgebung". Der Laser-Fangstrahl mit Wellenlänge $\lambda=1064 \mathrm{~nm}$ wurde in zwei gegenläufig ausgerichtete Einzelmodenfasern (Typ Corning PureMode HI 1060) eingekoppelt und die Müllerzelle gestreckt. Später wurden beide Fasern um jeweils $2-3^{\circ}$ gegeneinander verkippt. Ein zusätzlicher Messstrahl der Wellenlänge $\lambda=514 \mathrm{~nm}$, also im sichtbaren Bereich, wurde in die linke Faser eingekoppelt und die von der rechten Faser aufgenommene Intensität mittels eines Kurzpassfilters vom Trägersignal getrennt und der zeitliche Verlauf der Leistung gemessen. Dieser Versuch wurde mit mehreren Müllerzellen wiederholt, wobei dann auch der zeitliche Verlauf der Leistung nach dem Entfernen der Müllerzelle, also bei Messung der reinen zelllosen Spüllösung festgehalten wurde. Das Verhältnis der gemittelten Leistungen

$$
\eta=\frac{P_{\text {mit Zelle }}}{P_{\text {ohne Zelle }}}
$$

gilt als Maß für die optische Verstärkung des Signals und wurde von den Autoren als relative Lichtleiteffizienz (relative guiding efficiency) bezeichnet.

Der Vergleich des experimentellen Nachweises der Lichtwellenleitereigenschaft der Müllerzelle mit einer Simulation verlangt nach einer ähnlich definierten Größe. Hierzu definieren wir die optische Leistung

$$
p(\alpha)=\int_{0}^{\alpha_{\max }} T(\alpha) d \alpha
$$

als eindimensionales Integral über den Einfallswinkel $\alpha$, was im diskreten Fall eine Summe über alle zur Gesamtleistung anteilig beitragenden Moden liefert. Die Größe $\eta$ kann dann direkt aus Gleichung (3.14) übernommen werden. Die Definition der Größe $T(\alpha)$ ist die normierte Transmission, also ist $p(\alpha)$ proportional zur Anzahl der eingekoppelten Moden. Der Einfallswinkel

$$
\alpha(\mathbf{k})=\arctan \left(\frac{k_{y}}{k_{x}}\right)
$$

kann als Funktion des k-Vektors transformiert werden. Für die Komponenten des Wellenvektors bei der numerischen Berechnung, also im Falle einer diskreten Approximation aus der Dispersionsrelation, gilt

$$
\begin{aligned}
k_{x} & =\frac{1}{a} \arccos \left(1-a^{2}\left[E-E_{m}\right]\right) \\
k_{y} & =\frac{1}{a} \arccos \left(1-a^{2} E_{m}\right)
\end{aligned}
$$

mit der Gitterkonstante $a=\frac{1}{w+1}$ und Kanalbreite $w$ zur eingekoppelten Gesamtenergie E. Die Ener-

14 Häufig wird das langwellige Licht durch zwei Einzelmodenfasern geleitet, die zwar parallel, jedoch gegenläufig ausgerichtet sind. Die dazwischen befindliche, in eine Lösung verbrachte und mit Fluoreszenzproteinen gemarkerte Zelle wird eingefangen und kann beispielsweise mit sichtbarem Licht, das zusätzlich durch eine der beiden Fasern eingebracht wird, auf optische Eigenschaften hin untersucht werden. Das in die andere Faser wieder einkoppelnde Licht muss mit einem Filter vom langwelligen Licht getrennt werden, bevor es weiter analysiert werden kann. 
gien $E_{m}$ sind die Eigenenergien der transversalen Moden des Lichtwellenleiters (Müllerzelle). Die Kanalbreite $w$ der Simulation ist äquivalent zum Modenfelddurchmesser des Experiments. Im Datenblatt der Einzelmodenfaser [215] findet sich allerdings keine Angabe zum Modenfelddurchmesser bei sichtbaren Wellenlängen. Eine nach [216] motivierte lineare Regression für die ebenda angegebenen Werte liefert

$$
w\left(\lambda-\lambda_{0}\right)=w(0)+0.00705 \cdot\left(\lambda-\lambda_{0}\right)
$$

mit $w\left(\lambda_{0}=1060 \mathrm{~nm}\right)=6.2 \mu \mathrm{m}$, so dass für $w(\lambda=514 \mathrm{~nm}) \approx 2.4 \mu \mathrm{m}$ angenommen wurde. In der Simulation wurde $w \simeq 3 \mu \mathrm{m}$ gewählt. Etwaige Abweichungen zwischen Simulation und Experiment sind unter anderem auf diese Abschätzung zurückzuführen. Des Weiteren wurde der Kippwinkel im Experiment als $2.5^{\circ} \pm 0.5^{\circ}$, also mit einer absoluten Toleranz von $1^{\circ}$ angegeben, so dass auch die Unbestimmtheit dieser Größe zu Abweichungen führt.

Die Simulationen wurden auf einem Gitter der Größe $1001 \times 3601$ durchgeführt. Die Dimension und Form der Müllerzelle können Abb. 3.9 oben entnommen werden. Die ermittelten winkelabhängigen Transmissionen wurden nach Gleichung (3.15) integriert und die relative Lichtleiteffizienz bestimmt. Anders als im Experiment, wo beide Lichtleitfasern jeweils um den Winkel $\alpha \approx \beta \approx 2.5^{\circ}$ verkippt wurden, ist in den Simulationen nur der rechte Lichtwellenleiter verkippt, also $\alpha=0^{\circ}$ und $\beta \simeq 5^{\circ}$, da wir ebene Wellen einkoppeln. Jedoch gilt in beiden Fällen $\alpha+\beta \approx 5^{\circ}$, damit sich die berechneten Effektivitäten vergleichen lassen. Im experimentellen Fall ergeben sich $\eta_{\beta=0}(\alpha \approx 0)=1.9 \pm 0.1$ in der parallelen Anordnung der Lichtleitfasern und $\eta_{\beta \approx 2.5}(\alpha \approx 2.5)=7.2 \pm 0.9$ für die gekippte Anordnung. In unseren Simulationen finden wir $\eta_{\beta=0}(\alpha \approx 0)=2$ und $\eta_{\beta \approx 5}(\alpha \approx 0)=5$. Dieses Ergebnis kann als gute Übereinstimmung mit dem Experiment bezeichnet werden und bestätigt die Theorie der Lichtwellenleiternatur der Müllerschen Gliazellen. Zusätzlich durchgeführte Simulationen im Vakuum (experimentell natürlich unzugänglich) bekräftigen ebenfalls die Lichtwellenleitertheorie der Müllerzelle, da wie beim herkömmlichen Wellenleiter die relative Effizienz vom Verhältnis der Brechungsindices $\frac{n_{\text {außen }}}{n_{\text {innen }}}$ abhängt. Die relative Effizienz steigt in diesem Fall auf den maximalen Wert $\eta \approx 30$ an.

\subsubsection{Einfluss der Müllerzellenform auf die Lichtleitung}

Das hier gewählte Modell der Müllerzelle hatte bisher die Form eines "stumpfen Nagels" mit trichterförmig zulaufendem Endfuß und geradem Zellstamm konstanter Dicke. Um auch den Fall von realistisch geformten Müllerzellen analysieren und damit den Einfluss auf die Lichtwellenleiterfunktion untersuchen zu können, haben wir die Form des Zellstamms variiert. Anschließend untersuchen wir den Einfluss der Lage des Zellkerns der Müllerzelle auf ihre optische Leistung.

\subsubsection{Zur Formvariation des Zellstamms}

In Abbildung 3.11 sind verschiedene Formveränderungen und ihre Auswirkungen auf die winkelabhängige Transmission dargestellt. Die Grundform (a) repräsentiert die idealisierte Müllerzelle. Als Formvariationen wurden (b)-(d) gewählt. Eine einfache Auslenkung aus der zentrierten Lage des Zellstamms (b) hat demnach wenig Einfluss auf die Transmission. Auch eine Defornation der Zelle durch Variation der Zellstammdicke (d) zur Simulation angrenzender Neurone zeigen für Licht, das unter kleinen Winkeln einfällt, kaum eine Reduktion der Transmissionseigenschaften. Wie erwartet liefern Verbiegungen des Zellstamms mit kleinen Krümmungsradien - in Analogie zu stark gekrümmten Fa- 

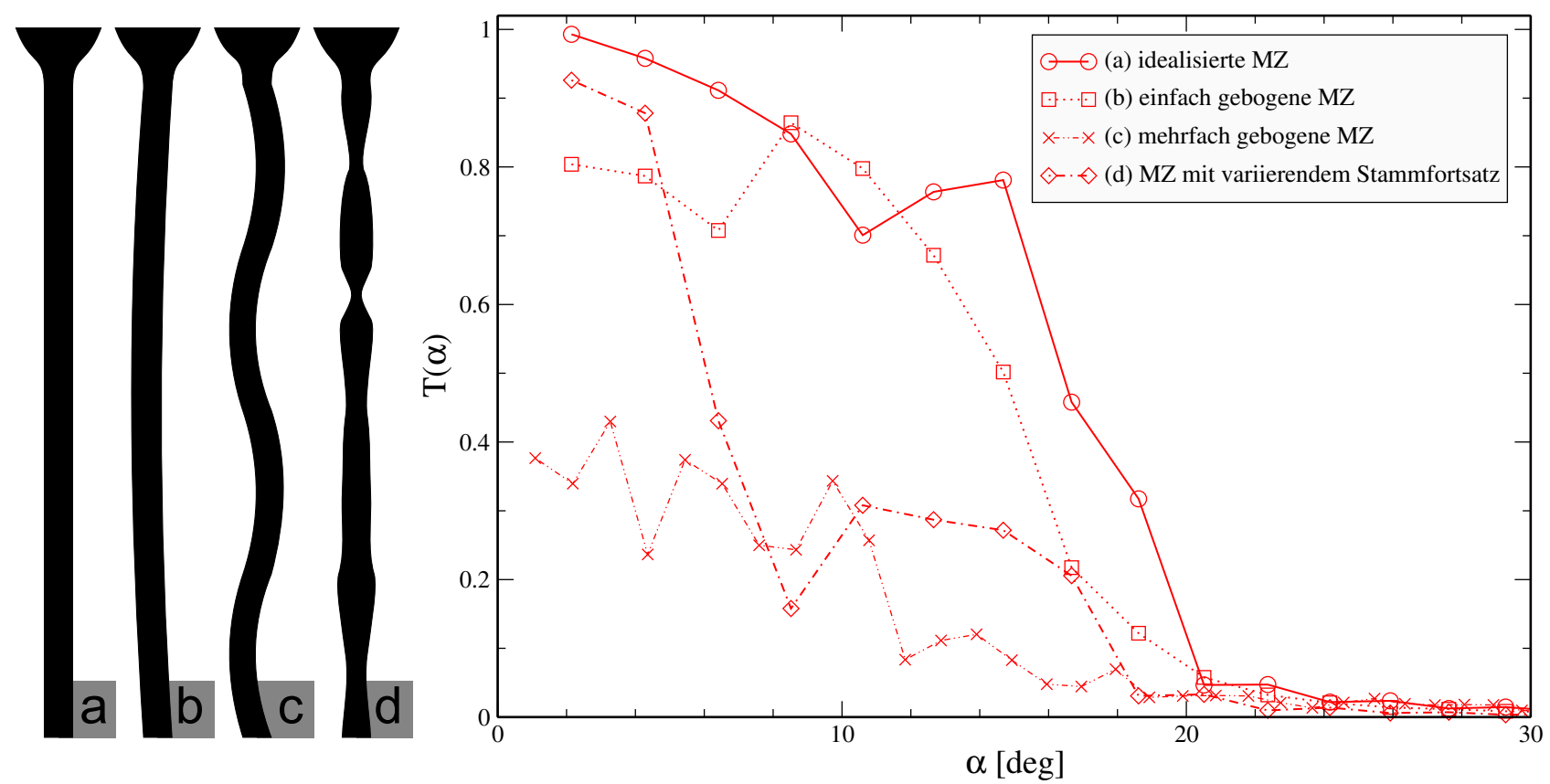

Abbildung 3.11.: Formvariation der Müllerzellen (MZ) mit Einfluss auf winkelabhängige Transmission. Links: Auswahl an untersuchten Potenzialrealisierungen. (a) idealisierte MZ (b) Krümmung der MZ mit großem Radius $R \approx 800 \mu \mathrm{m}$. (c) mehrfache Krümmung der MZ mit kleinem Radius $R \approx 200 \mu \mathrm{m}$ (d) Variation der Zellstammdicke $w$ zwischen 1 und $4 \mu \mathrm{m}$. Rechts: Winkelabhängige Transmissionsfunktionen der untersuchten Realisierungen von idealisierter MZ und deformierten MZ (b)-(d) in generierten Zufallspotenzialen.

sern - eine entsprechen ausgeprägte Verminderung der Transmission. Als vergleichende Größe für eine idealisierte zylindrische Faser kann hier der so genannte $V$-Parameter des Wellenleiters

$$
V=\frac{\pi w}{\lambda} \mathrm{NA}=\frac{\pi w}{\lambda} \sqrt{n_{\text {innen }}^{2}-n_{\text {außen }}^{2}}
$$

genutzt werden, der eine Abschätzung der maximal geführten Moden darstellt. Die Größe $\lambda$ bezeichnet die Vakuumwellenlänge. Die effektive Wellenlänge in einem Medium mit Brechungsindex $n$ kann über

$$
\lambda_{\text {eff }}=\frac{\lambda}{n}
$$

ermittelt werden. Die im Experiment bestimmten $V$-Parameter liegen für grünes Licht $(\lambda=500 \mathrm{~nm})$ im Bereich 2.6 .2.9 und für rotes Licht $(\lambda=700 \mathrm{~nm})$ im Bereich zwischen 3.6 ..4.0. Durch eine Krümmung mit dem Radius $R$ ergibt sich als Korrektur der Apertur NA eine effektive Apertur von

$$
\mathrm{NA}_{\text {eff }}=\sqrt{n_{\text {innen }}^{2}-n_{\text {außen }}^{2}\left(1+\frac{w}{2 R}\right)^{2}},
$$

wobei die Moden jedoch durch Rayleigh- oder Mie-Streuungen innerhalb der Müllerzelle teilweise wieder "aufgefüllt" werden können [217, 218]. Bei der Wahl von Konfiguration nach Abb. 3.11c mit den Krümmungsradien $R \approx 200 \mu \mathrm{m}$ ergibt sich etwa eine Halbierung des $V$-Parameters, was sich auch in der Halbierung der Transmissionswerte widerspiegelt. Bei Konfiguration nach Abb. 3.11b ergeben sich Korrekturen zwischen 5-10\%, was sich in der gleichwertigen Abnahme der Transmissionswerte zeigt. Für Konfigurationen nach Abbildung 3.11d gilt für harte Wände $T \propto \frac{w_{\text {klein }}^{2}}{w_{\text {groß }}^{2}}$, jedoch ist die verwandte Wellenlänge $\lambda$ in der Größenordnung der Verengung und die Müllerzelle im Milieu mit einem nur etwas kleinerem Brechungsindex, so dass - anders als bei den radialen Krümmungen - der Tunneleffekt 

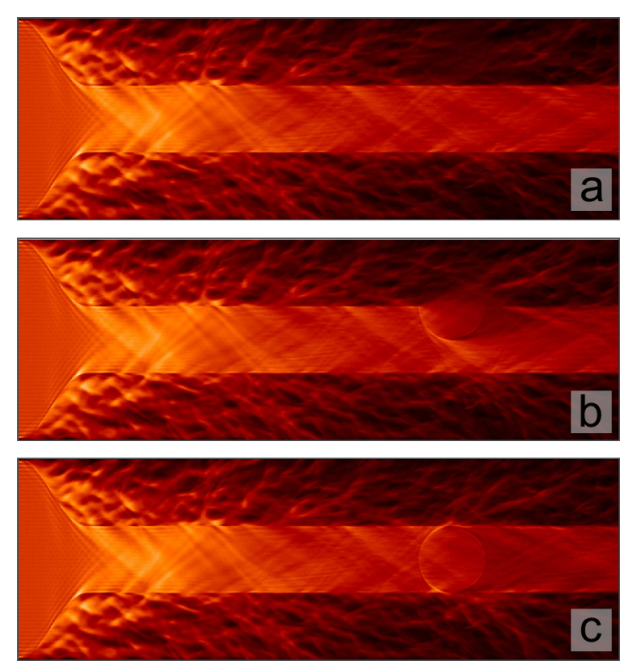

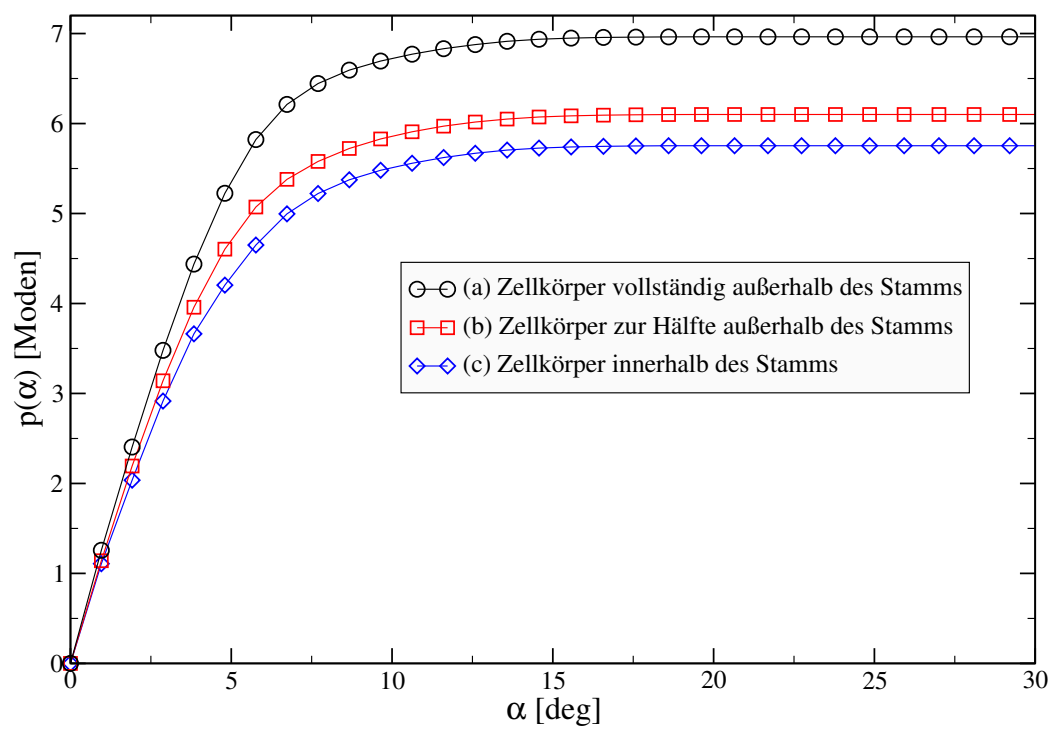

Abbildung 3.12.: Untersuchung des Einflusses der Lage des Zellkörpers (ZK) einer Müllerzelle (MZ) auf die optische Leistung. Der ZK hat einen kleineren Brechungsindex als der Stamm der Zelle und stellt mit seiner ausgedehnten Größe einen starken Streuer inmitten des Lichtwegs dar. Links: Streuwellenfunktionen der MZ mit ZK im Zufallspotenzial. Die Simulationen wurden für die drei Konfigurationen (a) ZK außerhalb der Zelle, (b) ZK halb in der Zelle und (c) ZK vollständig innerhalb der MZ durchgeführt. Rechts: Optische Leistung $p(\alpha)$ der Konfigurationen (a)-(c) im Vergleich. Im Vergleich zur Konfiguration (c) mit ZK innerhalb der Zelle steigt die optische Leistung für Konfiguration (b) um 6\% - 10\% und im Fall (a) sogar um 20\% - 25\%. Es wurde ein homogener ZK angenommen. Die realistische Annahme eines nicht transparenten Zellkerns würde die optische Verstärkung noch weiter vergrößern, da Anteile des Lichts von ihm absorbiert würden.

einen wesentlich größeren Beitrag liefert und deshalb die Transmission in Ausbreitungsrichtung der Welle nicht so stark abnimmt.

Es sei noch einmal darauf verwiesen, dass nach der adiabatischen Näherung ein maximaler Einfallswinkel nach Gleichung (3.9) von $\alpha_{\max } \approx 20^{\circ}$ berechnet wurde, der auch hier beobachtet werden kann. Auf Variationen der Länge wurde hier verzichtet, da sich je nach Wahl des Zellstammdurchmessers der Müllerzelle von 2-4 $\mu \mathrm{m}$ entsprechende Längen der Zelle zwischen 70-100 $\mu \mathrm{m}$ ergeben, da die Gittergröße für die durchgeführten Simulationen als konstant angenommen wurde.

\subsubsection{Zur Position des Zellkörpers}

Ein weiterer Aspekt zur Unterstreichung der Lichtleitfunktion der Müllerzellen ist die Tatsache, dass sich die Zellkörper (soma) der Müllerzellen vieler Wirbeltiere außen an ihren Hauptfortsätzen ausgelagert befinden $[178,189]$. Innerhalb der Hauptfortsätze würden sich die Zellkörper direkt im Lichtweg befinden und der interne Aufbau aus Zytoplasma, Golgi-Apparat, Neurofilamenten und Lyosomen große Hindernisse als Lichtstreuer darstellen. Die Auslagerung des annähernd runden Zellkörpers wird durch die Annahme eines zylinderförmigen Potenzials approximiert, wobei mit dem äquidistanten Potenzial-Plateau im Wesentlichen das Zytoplasma approximiert werden soll, das im Allgemeinen den größten Bereich im Zellkörper einnimmt. Der Brechungsindex des Zytoplasmas wird mit $n=1.36$ als homogen angenommen [219]. Für die Müllerzelle wird die idealisierte Form nach Abbildung 3.11a gewählt und über 15-20 verschiedene Zufallspotenziale und jeweils über 10-15 verschiedene Wellenlängen $\lambda$ im Intervall $[490,510] \mathrm{nm}$ zu den entsprechenden Energien gemittelt. Die Aufenthaltswahrscheinlichkeitsdichte der Streuwellenfunktionen bzw. die lokale Zustandsdichte (hier für 25 Moden aufsummiert) 


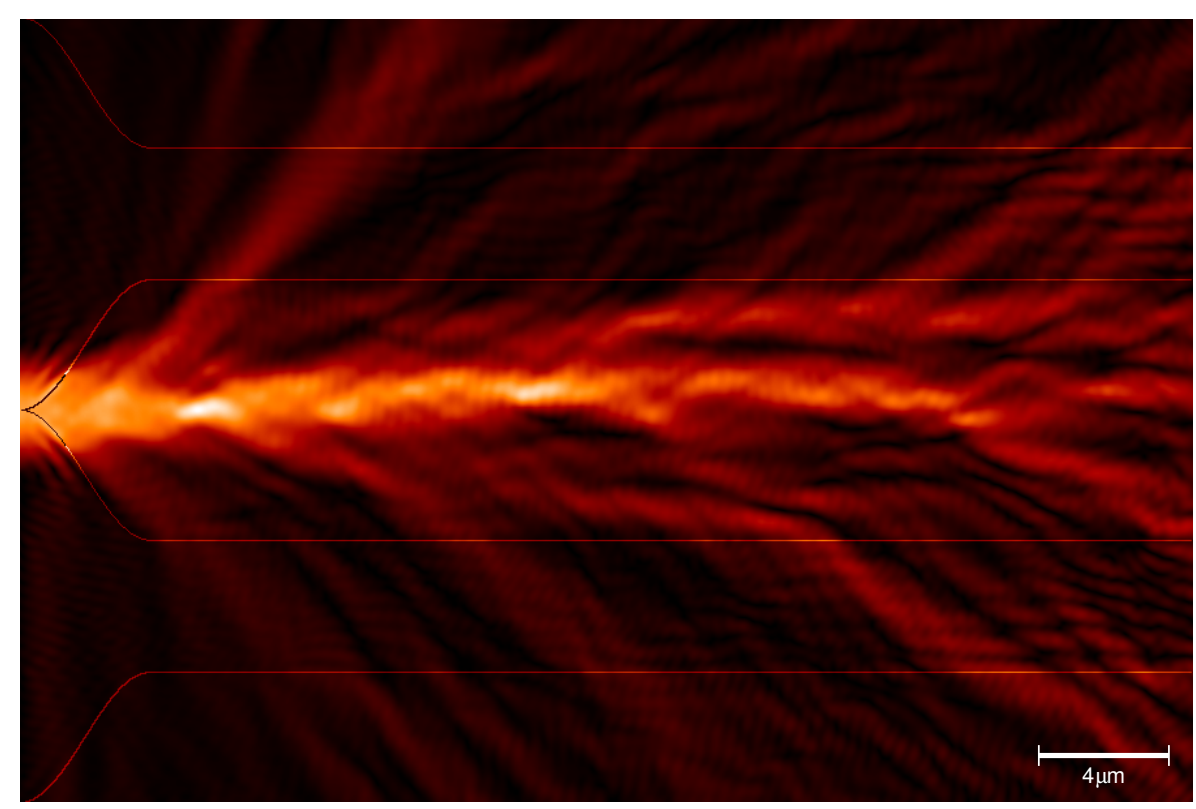

Abbildung 3.13.: Aufenthaltswahrscheinlichkeitsdichte der Streuwellenfunktion der 1. Mode einer einfallenden ebenen Welle (entspricht senkrechtem Lichteinfall auf die Retina) zwischen zwei benachbarten Müllerzellen. In dieser Konfiguration treffen etwa 10\% des Lichts hinten auf die Photorezeptoren. Schematische Begrenzungen durch Zellwände und der Maßstab sind ebenfalls eingezeichnet.

ist in Abbildung 3.12 dargestellt, wobei nur ca. zwei Drittel der gesamten Zelle simuliert wurden. Der Endfuß ist ca. $12 \mu \mathrm{m}$ breit, so dass sich bei Wellenlängen $\lambda \approx 500 \mathrm{~nm}$ etwa 25 Moden ausbilden. In Unterabbildung 3.12c ist die Aufenthaltswahrscheinlichkeitsdichte der Streuwellenfunktion für den Fall gezeigt, dass sich der Zellkörper vollständig im Zellstamm befindet. In 3.12b ist der Zellkörper halb innen und halb außen, in 3.12a vollständig außerhalb der Müllerzelle angeordnet. Die optische Leistung ist zum Vergleich wiedergegeben. Im Vergleich mit dem im Zellstamm befindlichen Zellkern ergibt sich im Fall der Konfiguration nach 3.12b eine relativen Lichtleiteffizienz von $\eta=1.03 \ldots 1.08$, im Fall der Konfiguration nach 3.12c sogar $\eta=1.13 \ldots 1.23$. Die Annahme eines nicht homogenen Zellkörpers und die realistische Einbeziehung eines nicht transparenten Zellkerns würde die relative Lichtleiteffizienz noch weiter erhöhen. Die Erhöhung der Lichtleiteffizienz durch Homogenisierung des Lichtwegs ist ein deutlicher Hinweis auf eine evolutionäre Ausbildung eines ausgelagerten Zellkörpers für retinale Gliazellen.

\subsubsection{Lichtleitung zwischen benachbarten Müllerzellen}

Die Müllerzellen sind zwar sehr dicht in der Retina angeordnet, jedoch gibt es einen minimalen Abstand der Zellstämme zwischen zwei benachbarten Müllerzellen, bedingt durch den häufig mehr als doppelt so großen Durchmesser der Endfüße. In einer vereinfacht angenommenen hexagonal dichten Packung ergibt sich eine untere Schranke für den Abstand der Zellstämme $d_{\min }=\min \left\{d_{\text {Endfuß }}\right\}-\max \left\{d_{\text {Zellstamm }}\right\}$ bzw. für die gesamte Retina ein mittlerer Abstand $\langle d\rangle=\left\langle d_{\text {Endfuß }}\right\rangle-\left\langle d_{\text {Zellstamm }}\right\rangle$. In [30] wurde ein Experiment mit Meerschweinretina zum Zwecke des Vergleichs mit einem artifiziellen faseroptischen System durchgeführt, indem der Buchstabe "t" eines Mikrofiche auf einen von Photorezeptoren befreiten Teil der Retina projiziert wurde und auf der anderen Seite der Retina in eine vom Abstand der Lichtwellenleiter-Müllerzellen abhängige Abbildung überführte. Ein Vergleich beider Verteilungen für die Abstände zwischen den hellen Punkten der Abbildungen durch das Retinagewebe und durch 
das faseroptische System ergab eine gute Übereinstimmung. Die Form der Verteilung blieb dort zwar undiskutiert, weist jedoch Ähnlichkeiten mit einer Gauß-Verteilung auf. Wir finden für die Verteilung der Zellstammabstände für Meerschweinretina dieses Experiments eine Gauß-Verteilung mit Mittelwert $\mu=6 \pm 0.5 \mu \mathrm{m}$ und Standardabweichung $\sigma=1.5 \pm 0.5 \mu \mathrm{m})$. Unter der Annahme, dass menschliche Retina einen ähnlichen Aufbau besitzt, ergibt sich für den mittleren Abstand der Zellstämme ein vergleichbarer Wert. Dies bedeutet für nahezu senkrechten Lichteinfall, dass ein Anteil ${ }^{15}$ von etwa $10 \%$ an den als kreisrund angenommenen Endfüßen vorbei auf die Retina fällt (siehe Abbildung 3.13), wobei davon ca. 10\% durch die Retina gelangen. Der Gesamtanteil des zwischen den Müllerzellen transmittierten Lichts beträgt also etwa $c_{\text {inter }}=0.01$, was im folgenden Modell zur Untersuchung der Streulichtreduktion im menschlichen Auge als Konstante Verwendung findet.

\subsection{Modell zur Vorhersage des Streulichts im menschlichen Auge}

Der weiter oben verifizierte Nachweis der Lichtwellenleitereigenschaft und die Bestätigung des Kollimationseffektes der Müllerzelle müssen als wesentliche Voraussetzungen für die Modellbildung der Streulichtreduzierung im Auge angesehen werden. Die Idee, dass der Kollimationseffekt der Müllerzelle zur Reduktion des Streulichts im Auge führt beruht auf der Tatsache, dass bei konvexer Geometrie (beispielsweise in einer so genannten Ulbricht-Kugel) das gestreute Licht unter größeren Winkeln vom Lot auf die Reflexionsfläche aus gemessen - auf die Reflexionsfläche einfällt als das direkte Licht. Da die Müllerzelle die Eigenschaft der Kleinwinkelfokussierung besitzt, wird das unter großen Winkeln einfallende Licht anteilig stärker reflektiert als dies bei einem diffusen Streuer der Fall ist. Damit würde auch das Signal-Rausch-Verhältnis im Auge verbessert. Dieser Abschnitt dient daher der Ableitung einer Theorie zur quantitativen Vorhersage der Streulichtreduzierung im menschlichen Auge, nicht zuletzt als Beitrag zur Optimierungstheorie evolutionärer Systeme.

\subsubsection{Das Augenmodell}

Unser Modell geht von einem vereinfachten schematischen Auge aus (vereinfachtes Liou-BrennanModell, siehe [220, 221]). Der Aufbau und die Annahmen des Modells werden im Folgenden erläutert. Das Modellauge befindet sich mit dem zu betrachtenden Objekt gemeinsam auf einer optischen Achse. Als Abbildung wählen wir ein helles Objekt auf einem weißen Hintergrund, ähnlich einer gleichförmig und diffus selbstleuchtenden Leinwand. Das ebenso gleichförmige diffuse Licht, das von dem Objekt ausgeht, wird von der Linse auf die Retina projiziert, wobei der im Strahlengang befindliche Glaskörper über einen homogenen Brechungsindex verfügt und daher in dieser Betrachtung vernachlässigt werden kann. In dieser Anordnung wird der Lichteinfall nur vom Öffnungswinkel $\delta$ der Pupille begrenzt, die als perfekte Blende fungiert. Außerdem wird in dieser Betrachtung immer eine gleichgroße scharfe Abbildung des Objekts durch die Linse auf die Retina vorausgesetzt (Autofokus). Die Pupille kann somit als perfekter diffuser Selbstleuchter angenommen werden. Auf diese Weise wird es möglich, die Intensität für direktes Licht und Streulicht auf der gesamten Retina in kompakter Form zu beschreiben und untersuchen zu können.

15 Der Lichtanteil kann einfach durch Vergleich der Flächenanteile der dichtesten Kugelpackung (hier 2D) abgeschätzt werden und beträgt $1-\frac{\pi}{2 \sqrt{3}} \approx 0.093$, also etwa $10 \%$ der vom Lichteinfall getroffenen Gesamtfläche. 
Um quantitative Aussagen über die Intensitäten des auf die Retina einfallenden bzw. gestreuten Lichts treffen zu können, wollen wir von folgenden vereinfachenden Annahmen ausgehen:

1. Wir betrachten ein rotationssymmetrisches Auge mit der optischen Achse (= Sehachse) als Symmetrieachse, auf der sowohl die Linse als auch die Pupille angeordnet sind. Der Öffnungswinkel $\delta$ der Pupille ist ein freier Parameter.

2. Das Linsensystem des Auges kann durch eine $\cos ^{4}$-Funktion approximiert werden.

3. Die Photorezeptordichte in der gesamten Retina wird als konstant angenommen.

4. Die chromatische Aberration des Linsensystems kann vernachlässigt werden (verschwindende Dispersion).

In Abbildung 3.14 ist eine schematische Darstellung des vereinfachten Augenmodells dargestellt. Die Eintrittsöffnung des Auges wird hier durch eine kreisrunde Pupille mit Öffnungswinkel $\delta$ approximiert. Die größte Vereinfachung stellt sicherlich die Zusammenfassung des Linsensystems des Auges, bestehend aus Kornea und Linse, zu einer als $\cos ^{4}$-Funktion wirkenden Sammellinse dar, die optimal auf den Punkt $F$ der optischen Achse fokussiert, wobei dies zusammen mit der vorgezogenen Position der Linse in den Punkt $O$ Näherungen des bereits erwähnten Liou-Brennan-Modells darstellen. Die $\cos ^{4}$ Funktion beschreibt vor allem die Eigenschaft des Randlichtabfalls der Linse, da sich beim Verlassen der optischen Achse verschiedene geometrische Faktoren wie Raumwinkelverkürzung und Reduktion der photometrischen Lichtstärke durch das Lambertsche Gesetz ergeben (siehe [225, 226]).

Ein Punkt $R$ auf der Retina (Kugeloberfläche) ist durch die Kugelkoordinaten $\{\phi, \vartheta, r=$ const $\}$ eindeutig bestimmt. Der Azimut $\phi$ ist auf ein Intervall $\left[-\phi_{\max }, \phi_{\max }\right]$ beschränkt, da die Ora serrata den lichtempfindlichen Teil der Retina begrenzt. Die Rotationssymmetrie des gewählten Augenmodells um die Symmetrieachse $\overline{O F}$ macht eine Betrachtung retinaler Eigenschaften unabhängig vom Höhenwinkel $\vartheta$, der hier als $\vartheta_{\mathrm{G}}=\frac{\pi}{2}$ das horizontale Kreissegment auswählt (siehe Abbildung 3.14). Ein Punkt $R_{\mathrm{G}}$ auf diesem Segment ist durch die Wahl des Azimuts $\phi_{\mathrm{G}}$ eindeutig bestimmt.

Um Vergleiche zwischen direkt einfallendem und gestreutem Licht im Auge anzustellen, wird ein Ausdruck für die Lichtintensität des abzubildenden Objekts benötigt. Dazu betrachten wir die optische Größe der Strahldichte $i_{\mathrm{S}}$, die eine photometrische Größe der Helligkeitswahrnehmung des Auges im Bezug auf eine Lichtquelle als

$$
i_{\mathrm{S}}=\frac{d I}{d A}
$$

definiert, also als Verhältnis von ausgestrahlter Lichtintensität (auch Lichtstärke) I zu scheinbar selbstleuchtender Fläche $A$ zu schreiben ist. Die Fläche $A$ beschreibt hierbei den von der Pupille begrenzten Bereich, der nur vom Öffnungswinkel $\delta$ abhängig ist. Damit lässt sich die Lichtintensität als Integral über die Strahldichte formulieren

$$
I=\int i_{\mathrm{S}} d A
$$

Aus dem Zusammenhang $A=4 r^{2} \delta$ zwischen der homogen beleuchteten Pupillenfläche $A$ und dem Öffnungswinkel $\delta$ analog zu Gleichung (3.42) ergibt sich somit das Flächendifferential $d A=4 r^{2} d \delta$.

Wir interessieren uns jedoch in folgenden Betrachtungen für die Lichtintensität, die auf eine bestimmte Umgebung der Retina einfällt. Hinsichtlich der Rotationssymmetrie des Augenmodells kann diese 


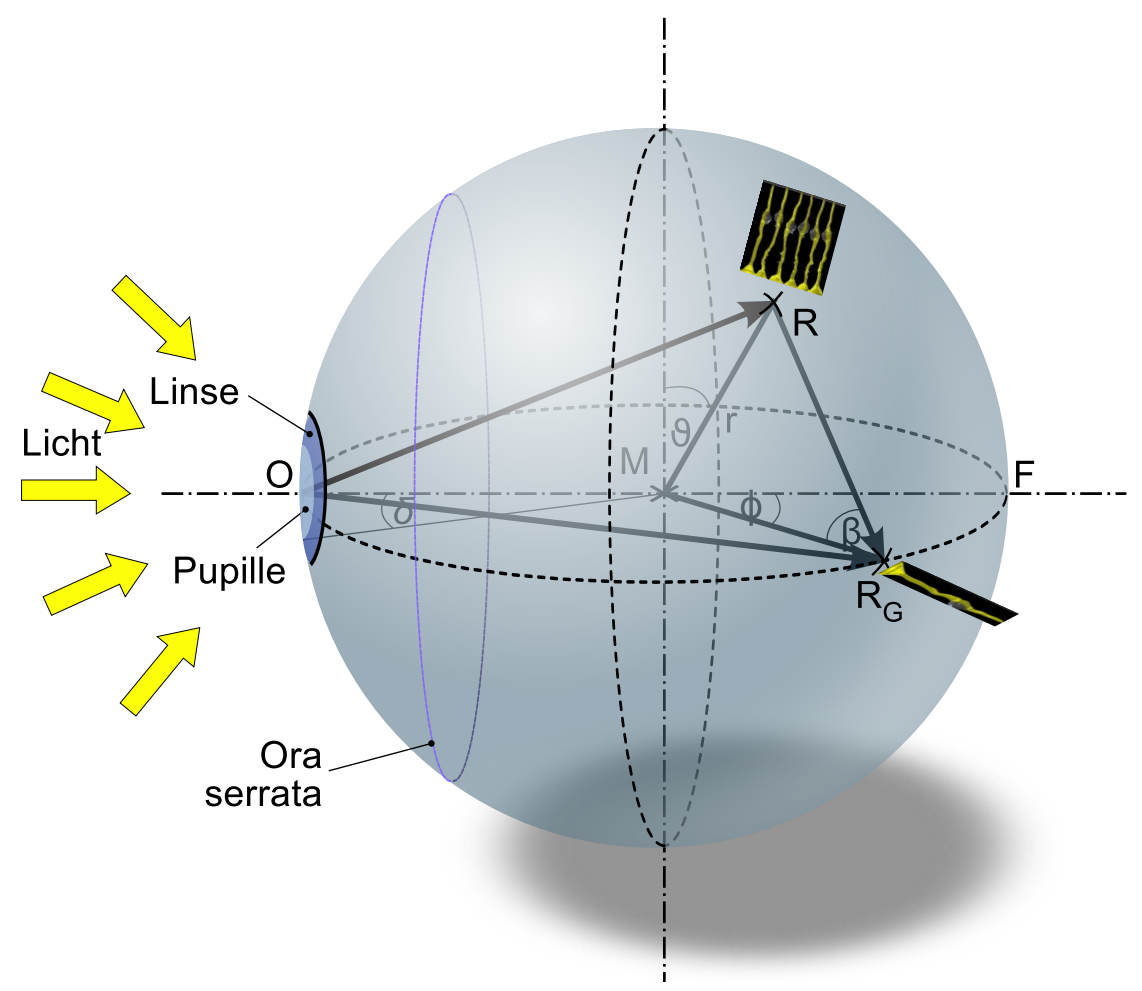

Abbildung 3.14.: Skizze zur Erläuterung der Größen im vereinfachten Augenmodell. Licht tritt aus verschiedenen Richtungen durch die Pupille mit Öffnungswinkel $\delta$ ein, und wird durch die Linse auf die Netzhaut fokussiert. Der Öffnungswinkel kann für ein emmetropes menschliches Auge Werte zwischen $5^{\circ}-15^{\circ}$ annehmen [222]. Der Anteil des direkt einfallenden Lichts - hier stellvertretend durch den Vektor $\overrightarrow{O R}_{\mathrm{G}}$ dargestellt - ist dabei nur vom Azimut $\phi$ und vom Pupillenöffnungswinkel $\delta$ abhängig, wobei der Punkt $R_{\mathrm{G}}$ eindeutig durch den seinen Azimut $\phi_{\mathrm{G}}$ definiert ist, da der Anteil des direkt einfallenden Lichts auf die Retina wegen der angenommenen Rotationssymmetrie um die Achse $\overline{O F}$ unabhängig vom Höhenwinkel $\vartheta$ ist (Lage des Kreises durch die Punkte $O F R_{\mathrm{G}}$ ). Daher ist im Folgenden bei der Untersuchung retinaler Eigenschaften die Wahl von $\vartheta=\frac{\pi}{2}$ (siehe horizontal eingezeichneter Kugelumfang) ausreichend. Für das Streulicht 1.Ordnung im Punkt $R_{\mathrm{G}}$ gilt, dass ein gewisser Anteil des direkt auf den Punkt $R$ einfallenden Lichts - hier durch den Vektor $\overrightarrow{O R}$ dargestellt - in den Punkt $R_{\mathrm{G}}$ gestreut wird, wobei der daraus resultierende Vektor $\overrightarrow{R R}_{\mathrm{G}}$ die Richtung des Streulichtanteils angibt. Der maximale Azimut ist durch die Ora serrata (Übergang der Netzhaut in die stark lichtabsorbierende Pars caeca retinae) begrenzt und beträgt $\phi_{\max }=\max \left\{\phi_{\mathrm{G}}\right\}=130^{\circ}$ (vertikaler blauer Kreis) [223, 224].

Position durch den retinalen Azimut $\phi_{\mathrm{G}}$ ausgedrückt werden, so dass insgesamt

$$
I\left(\phi_{\mathrm{G}}\right)=\frac{4 r^{2}}{I_{0}} \int i_{\mathrm{S}}\left(\phi_{\mathrm{G}}\right) d \delta
$$

ein Maß für die relative Intensität darstellt, den durch die Pupille einfallendes Licht auf einen durch $\phi_{\mathrm{G}}$ bestimmten Bereich der Retina liefert. Die Größe $I_{0}$ ist eine Normierungsgröße, die den Gesamtanteil des einfallenden Lichts in das Auge repräsentiert. Damit können nun die Anteile des direkten Lichts und des Streulichts berechnet werden. 


\subsubsection{Relative Intensitäten des direkten Lichts und des Streulichts erster Ordnung}

Im Folgenden werden wir Ausdrücke für die Intensitäten des direkten und gestreuten Lichts erster Ordnung in unserem rotationssymmetrischen Augenmodell aufstellen und mit einem hypothetischem Auge ohne Müllerzellen vergleichen. Die Größen der relativen Intensitäten des direkten Lichts im Augenmodell

$$
\begin{aligned}
D_{0}\left(\phi_{\mathrm{G}}\right) & =\frac{4 r^{2}}{I_{0}} \int_{-\delta_{\max }}^{\delta_{\max }} i_{\mathrm{L}}\left(\phi_{\mathrm{G}}, \delta\right) \cos \left(\frac{\phi_{\mathrm{G}}+\delta}{2}\right) d \delta \\
D_{\mathrm{M}}\left(\phi_{\mathrm{G}}\right) & =\frac{4 r^{2}}{I_{0}} \int_{-\delta_{\max }}^{\delta_{\max }} i_{\mathrm{L}}\left(\phi_{\mathrm{G}}, \delta\right) T\left(\frac{\phi_{\mathrm{G}}+\delta}{2}\right) d \delta
\end{aligned}
$$

können analog zu Gleichung (3.24) als Integrale der Leuchtdichte $i_{\mathrm{L}}\left(\phi_{\mathrm{G}}, \delta\right)$ der Linse in Abhängigkeit von der Position $\phi_{\mathrm{G}}$ auf der Retina dargestellt werden, wobei über den Öffnungswinkel $\delta$ der ausgedehnten Linse integriert wird. Der Index 0 kennzeichnet dabei Größen des hypothetischen $\mathrm{Au}-$ genmodells ohne Müllerzellen, der Index $M$ Größen des Augenmodells mit Müllerzellen.

Die hier auftretende Größe $I_{0}$ bezeichnet die Intensität des gesamten Lichts, das durch die Pupille in das Auge einfällt und kann als

$$
I_{0}=2 r^{2} \int_{-\phi_{\max }}^{\phi_{\max }} \int_{-\delta_{\max }}^{\delta_{\max }} i_{\mathrm{L}}(\phi, \delta) d \delta d \phi
$$

geschrieben werden. Dieser Normierungsfaktor wird für die Auswertung der Integrale benötigt. Die Linse mit Öffnungswinkel $\delta$ wird in diesem Modell wie bereits erwähnt durch eine $\cos ^{4}$-Funktion approximiert

$$
i_{\mathrm{L}}\left(\phi_{\mathrm{G}}, \delta\right)=\cos ^{4}\left(\frac{\phi_{\mathrm{G}}+\delta}{2}\right)
$$

so dass das Licht für $\phi_{\mathrm{G}} \approx 0$ optimal in die Fovea projiziert wird. Das Argument $\alpha_{\mathrm{G}}=\frac{\phi_{\mathrm{G}}+\delta}{2}$ der Kosinusfunktion ergibt sich aus geometrischen Überlegungen. Die Funktion $T\left(\alpha_{\mathrm{G}}\right)$ ist die Transmissionsfunktion für eine Müllerzelle in der Retina und $\alpha_{\mathrm{G}}$ der zugehörige Einfallswinkel des direkten Lichts. Die zweite Kosinusfunktion in Gleichung (3.25) dient einer geometrischen Korrektur.

Für die Bestimmung der relativen Intensitäten des gestreuten Lichts erster Ordnung

$$
\begin{aligned}
S_{0}\left(\phi_{\mathrm{G}}\right) & =\frac{4 r^{2}}{I_{0}} \int_{0}^{\pi} \int_{\phi_{\max }}^{\phi_{\max }} \int_{\delta_{\max }}^{\delta_{\max }} i_{\mathrm{L}}(\phi, \delta) i_{\mathrm{R}}\left(\phi_{\mathrm{G}}, \phi, \delta, \vartheta\right) d \delta d \phi d \vartheta \\
S_{\mathrm{M}}\left(\phi_{\mathrm{G}}\right) & =\frac{4 r^{2}}{I_{0}} \int_{0}^{\pi} \int_{-\phi_{\max }}^{\phi_{\max }} \int_{\delta_{\max }}^{\delta_{\max }} i_{\mathrm{L}}(\phi, \delta) f\left(\phi_{\mathrm{G}}, \delta, \phi, \vartheta\right) d \delta d \phi d \vartheta
\end{aligned}
$$

bedeutet das jedoch die Berechnung von Dreifachintegralen, da die gesamte Oberfläche des Innenauges zur Reflexion in den Punkt $\phi_{\mathrm{G}}$ beiträgt. Auch hier bezeichnet $i_{\mathrm{L}}\left(\phi_{\mathrm{G}}, \delta\right)$ wieder die Leuchtdichte der Linse. Die beiden Reflexionsfunktionen $i_{\mathrm{R}}\left(\phi_{\mathrm{G}}, \phi, \delta, \vartheta\right)$ und $f\left(\phi_{\mathrm{G}}, \phi, \delta, \vartheta\right)$ dienen der Berücksichtigung des winkelabhängigen Streulichts. Unter der vergleichenden Annahme, dass keine Müllerzellen in der Retina vorhanden wären, kann für die normierte Reflexionsfunktion nach dem Lambertschen Gesetz 


$$
i_{\mathrm{R}}\left(\phi_{\mathrm{G}}, \phi, \delta, \vartheta\right)=\frac{\text { const. }}{2 \pi \phi_{\max }} \cos (\alpha-\beta) \cos (\beta)
$$

perfekte diffuse Streuung angenommen werden. Die erste Kosinusfunktion ergibt sich durch das um den Einfallswinkel $\alpha$ gedrehte Lot auf die Lambertfläche und durch den zugehörigen Ausfallwinkel $\beta$ (zur Berechnung der Winkel $\alpha$ und $\beta$ siehe weiter unten im Text). Damit ist die Reflexion für $\alpha=\beta$ maximal. Die zweite Kosinusfunktion ist ein geometrischer Faktor, der der Intensitätsverminderung des Streulichts, das am Zielort $\phi_{\mathrm{G}}$ auf die Photorezeptoren unter dem Winkel $\beta$ einfällt, geschuldet ist. Die Funktion

$$
f(\alpha, \beta)=R(\alpha, \beta) \cdot T(\beta)
$$

aus Gleichung (3.30) ist eine zusammengesetzte Funktion aus der Reflexionsfunktion $R(\alpha, \beta)$ und der Transmissionsfunktion $T(\beta)$, da sich das Streulicht aus dem Produkt des unter dem Winkel $\alpha$ einfallenden und unter dem Winkel $\beta$ aus der Retina gestreuten und von der Müllerzelle am Ort $\phi_{\mathrm{G}}$ unter dem Winkel $\beta$ wieder einfallenden und transmittierten Anteils zusammensetzt. Um die Streuung des unter dem Einfallswinkel $\alpha$ auf die Retina fallenden Lichts hinreichend zu approximieren, soll auch das schräg in benachbarte Bereiche $\phi+\Delta \phi$ der Müllerzelle gestreute Licht berücksichtigt werden, da dies wiederum anteilig zum Streulicht in Richtung des Ausfallswinkels $\beta$ in der benachbarten Müllerzelle beitragen kann. Gleiches gilt für die am Ort $\phi$ betrachtete Müllerzelle, denn das aus benachbarten Müllerzellen $\phi+\Delta \phi$ stammende Licht kann ebenso in Richtung des Winkels $\beta$ reflektiert werden (siehe Abbildung 3.15). Die Berechnung von $R(\alpha, \beta)$ und $T(\beta)$ als Streugrößen geschieht mit dem in Ka-

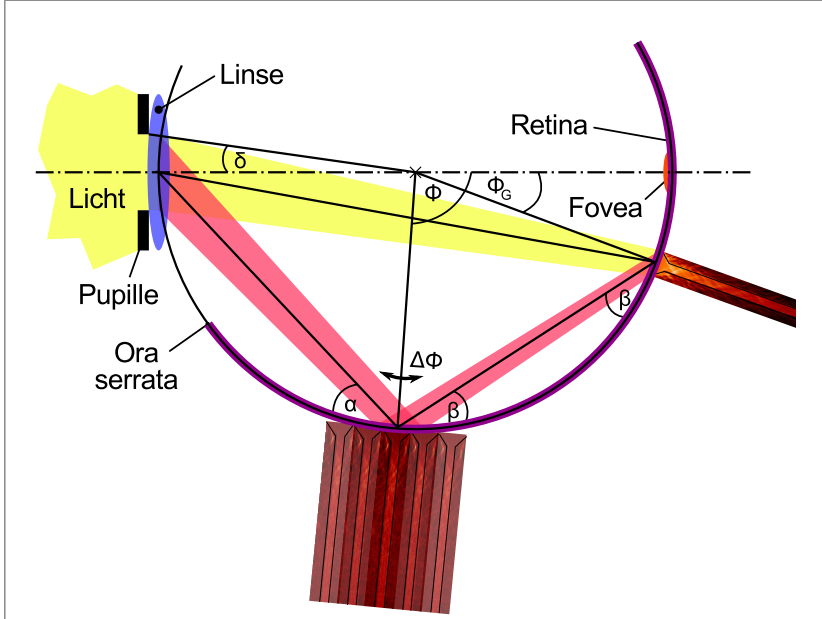

Abbildung 3.15.: Zur Bezeichnung der Winkel $\alpha$ und $\beta$ des Augenmodells für das Streulicht im Zusammenhang mit der Wahl von periodischen Randbedingungen der Reflexionsfunktion $R(\alpha, \beta)$. pitel 1 entwickelten Streuformalismus. Durch die Verwendung von periodischen Randbedingungen senkrecht zur Ausbreitungsrichtung des Lichts wird das Streuverhalten der Müllerzelle in unserem Streuformalismusmodell bei der Berechnung von $R(\alpha, \beta)$ entsprechend berücksichtigt. Für die Berechnung von $T(\beta)$ werden hier ebenso wie bei der Berechnung des direkt einfallenden Lichts offene Ränder verwendet, da hier nur das unter dem Winkel $\beta$ in die Müllerzelle einfallende Streulicht zum richtungsabhängigen Streulicht beiträgt. Einfallswinkel $\alpha$ und Ausfallswinkel $\beta$ lassen sich als geometrische Transformationsfunktionen

$$
\begin{aligned}
\alpha(\phi, \delta) & =\frac{\phi+\delta}{2} \\
\beta\left(\phi_{\mathrm{G}}, \phi, \delta, \vartheta\right) & =\arccos \left(\sqrt{\frac{1-\cos (\phi+\delta) \cos \left(\phi_{\mathrm{G}}\right)-\cos (\vartheta) \sin (\phi+\delta) \sin \left(\phi_{\mathrm{G}}\right)}{2}}\right)
\end{aligned}
$$

der mit $\left\{\phi, \phi_{\mathrm{G}}, \delta, \vartheta\right\}$ bezeichneten Winkel nach Abbildung 3.14 darstellen. Die Reflexionsfunktion $R(\alpha, \beta)$ hängt hierbei vom Einfallswinkel $\alpha$ und Reflexionswinkel $\beta$ ab, während die Transmissionsfunktion $T(\beta)$ nur vom Einfallswinkel $\beta$ der Transmission abhängt, der - wie bereits erwähnt - aus geometrischen Überlegungen resultierend identisch mit dem Reflexionswinkel $\beta$ bei der Streuung ist. 


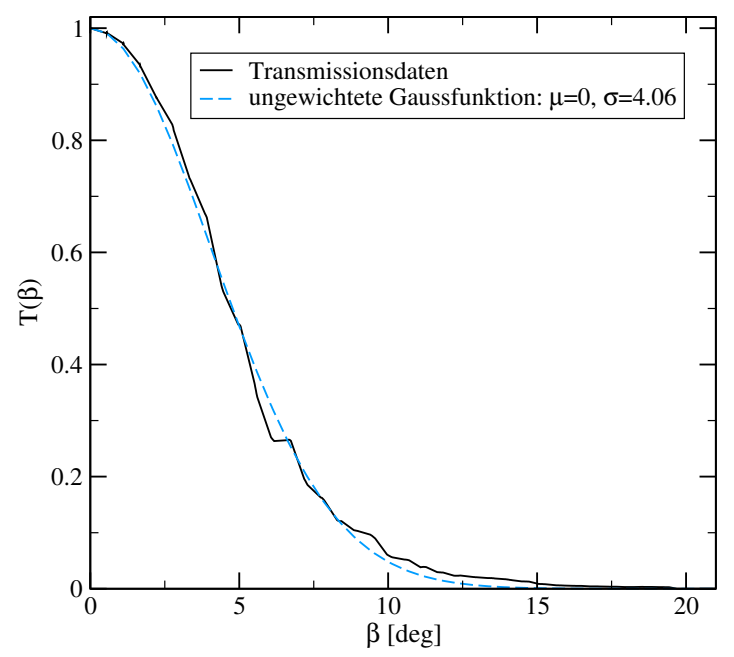

(a)

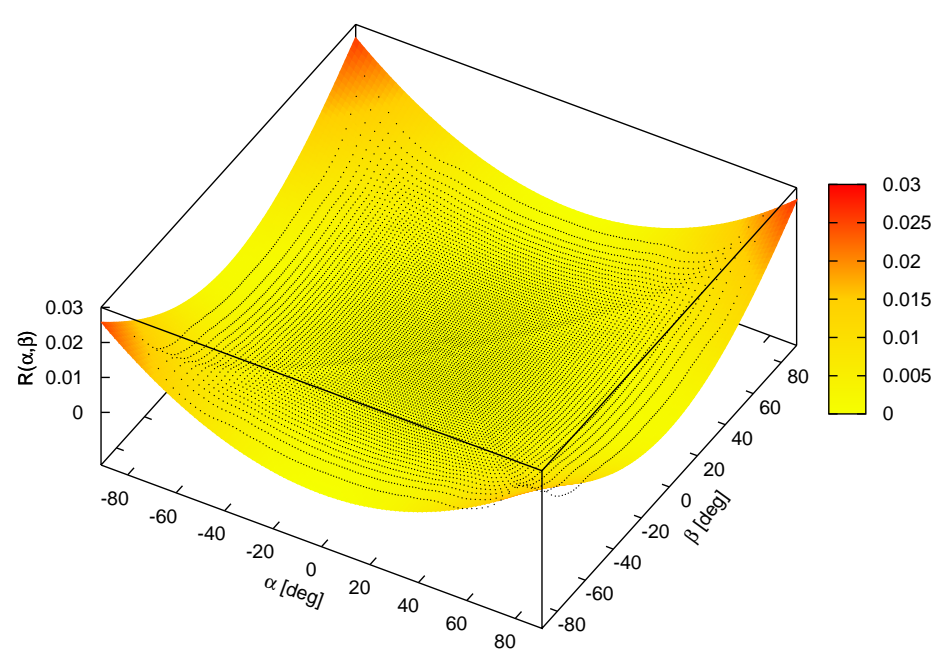

(b)

Abbildung 3.16.: Numerisch bestimmte (a) Transmissionfunktion $T(\beta)$. Die durchgezogene Linie kennzeichnet die Simulationsdaten, die blaue gestrichelte Linie die Ausgleichsrechnung mit einer ungewichteten GaußFunktion zum Mittelwert $\mu=0$ und mit der Standardabweichung $\sigma \approx 4^{\circ}$. Dargestellt ist nur der positive Winkelanteil, da diese Funktion symmetrisch um $\beta=0^{\circ}$ ist. (b) Reflexionsfunktion $R(\alpha, \beta)$. Die blau gekennzeichneten Punkte stellen die numerisch bestimmten Simulationsdaten dar. Die farbige Fläche repräsentiert die zugehörige biquadratisch-stabile Ausgleichsrechnung mit einem Polynom vom Grad $P_{2,4}(\alpha, \beta)$ (siehe Text).

\begin{tabular}{|c|c|c|c|c|c|c|}
\hline$a_{00}$ & $a_{01}$ & $a_{02}$ & $a_{03}$ & \multicolumn{1}{c|}{$a_{04}$} & \multirow{2}{*}{} \\
\cline { 2 - 7 } & 76380 & 16.45 & 34.48 & -0.0033 & -0.0052 & \multicolumn{1}{c}{} \\
\hline$a_{10}$ & $a_{11}$ & $a_{12}$ & $a_{13}$ & $a_{20}$ & $a_{21}$ & $a_{22}$ \\
\hline \hline 5.718 & 0.0607 & 0.0292 & -0.00002 & 10.77 & 0.0266 & 0.0368 \\
\hline
\end{tabular}

Tabelle 3.2.: Parameter der biquadratisch-stabilen Regression mit Polynom 4. Grades zur Approximation der Reflexionsfunktion in simulierter menschlicher Retina mit Müllerzellen in Einheiten von $\frac{10^{-8}}{d e g}$.

Auf die Berechnung der relativen Intensitäten des Streulichts höherer Ordnung soll hier verzichtet werden, da ihr Beitrag klein ist und vernachlässigt werden kann.

Die Bestimmung der Transmissions- und Reflexionsfunktion aus den numerischen Berechnungen mit zugehörigen Ausgleichsrechnungen sind in Abbildung 3.16 dargestellt. Die Daten stammen aus Simulationen von Müllerzellen, die mit 20 verschiedenen Zufallspotenzialen zu je 10 unterschiedlichen Kanalenergien $E$ durchgeführt und gemittelt wurden. Die ebenfalls dargestellten Approximationsfunktionen resultieren aus Ausgleichsrechnungen. Für die Transmission

$$
T(\beta)=\left(1-c_{\text {inter }}\right) \exp \left(-\frac{(\beta-\mu)^{2}}{2 \sigma^{2}}\right)+c_{\text {inter }}
$$

mit $c_{\text {inter }}$ aus Unterabschnitt 3.3.7 fanden wir eine ungewichtete Gauß-Funktion mit dem Mittelwert $\mu=0^{\circ}$ und der Standardabweichung $\sigma \approx 4^{\circ}$. Die Reflexionsfunktion wurde mit einem Polynom 4. Grades in $\alpha$ und $\beta$ genähert, wobei die Koeffizienten des Polynoms

$$
P_{2,4}(\alpha, \beta)=\sum_{i=0}^{2} \alpha^{i}\left[\sum_{j=0}^{4-i} a_{i j} \beta^{j}\right]
$$

in der Tabelle 3.2 angegeben sind. 
Wir können nun für die Gesamtintensität und die relativen Intensitäten des direkten Lichts analytische Ausdrücke angeben. Die Gesamtintensität des durch die Linse einfallenden Lichts berechnet sich nach Gleichung (3.27) zu

$$
I_{0}=r^{2}\left(3 \delta_{\max } \phi_{\max }+\sin \left(\delta_{\max }\right) \sin \left(\phi_{\max }\right)\left[4+\cos \left(\delta_{\max }\right) \cos \left(\phi_{\max }\right)\right]\right) \quad .
$$

Es ergeben sich damit folgende analytische Teillösungen für die Integrale des direkten Lichtsanteils ${ }^{16}$

$$
\begin{aligned}
& D_{0}\left(\phi_{\mathrm{G}}\right)=\frac{4 r^{2}}{I_{0}} \frac{1}{60}\left[150 \cos \left(\frac{\phi_{\mathrm{G}}}{2}\right) \sin \left(\frac{\delta_{\max }}{2}\right)+25 \cos \left(\frac{3 \phi_{\mathrm{G}}}{2}\right) \sin \left(\frac{3 \delta_{\max }}{2}\right)+\right. \\
& \left.+3 \cos \left(\frac{5 \phi_{\mathrm{G}}}{2}\right) \sin \left(\frac{5 \delta_{\max }}{2}\right)\right] \\
& D_{\mathrm{M}}\left(\phi_{\mathrm{G}}\right)=\frac{4 r^{2}}{I_{0}} \sqrt{\frac{\pi}{128}} \sigma\left(6\left[\operatorname{erf}\left(\Phi_{0}^{+}\right)-\operatorname{erf}\left(\Phi_{0}^{-}\right)\right]+\right. \\
& +4 e^{-2 \sigma^{2}}\left(\left[\operatorname{erf}\left(\Phi_{4}^{+}\right)-\operatorname{erf}\left(\Phi_{4}^{-}\right)\right]+\left[\operatorname{erf}\left(\Phi_{4}^{+}\right)^{*}-\operatorname{erf}\left(\Phi_{4}^{-}\right)^{*}\right]\right)+ \\
& \left.+e^{-8 \sigma^{2}}\left(\left[\operatorname{erf}\left(\Phi_{8}^{+}\right)-\operatorname{erf}\left(\Phi_{8}^{-}\right)\right]+\left[\operatorname{erf}\left(\Phi_{8}^{+}\right)^{*}-\operatorname{erf}\left(\Phi_{8}^{-}\right)^{*}\right]\right)\right) \quad,
\end{aligned}
$$

wobei die Argumente der Fehlerfunktion

$$
\operatorname{erf}\left(\Phi_{n}^{ \pm}\right)=\frac{2}{\sqrt{\pi}} \int_{0}^{\Phi_{n}^{ \pm}} \exp \left(-t^{2}\right) d t
$$

mit den komplexen Integralgrenzen

$$
\Phi_{n}^{ \pm}=\frac{\phi_{\mathrm{G}} \pm \delta_{\max } \pm n i \sigma}{2 \sqrt{2}}
$$

und der Standardabweichung $\sigma \approx 4^{\circ}$ aus oben bestimmter Transmissionsfunktion definiert sind.

Analytische Lösungen für die Intensitäten des gestreuten Lichts sind nicht zugänglich, da sich aus der Integration über $\vartheta$ wegen der Transformationsfunktion $\beta$ aus Gleichung (3.33) Summen aus elliptischen Integralen 2. Art und hypergeometrischen Funktionen ergeben, die sich nicht weiter vereinfachen lassen. Diese Integrale müssen daher numerisch bestimmt werden.

\subsubsection{Bestimmung der Grenzwinkel $\phi_{\max }$ und $\delta_{\max }$}

Für die Winkel $\phi_{\max }$ und $\delta_{\max }$ liegen leider keine exakten experimentellen Daten vor. Bei der approximativen Bestimmung von $\phi_{\max }$ können wir auf die von Taylor \& Jennings [223] bestimmte Retinaoberfläche von $A_{\text {Retina }}=1100 \mathrm{~mm}^{2}$ und den angegebenen mittleren Augenradius von $r=11 \mathrm{~mm}$ zurückgreifen. Für die Retinaoberfläche gilt

$$
A_{\text {Retina }}=\iint_{\mathcal{S}} d A=r^{2} \int_{-\phi_{\max }}^{\phi_{\max }} d \phi \int_{0}^{\pi} \sin (\vartheta) d \vartheta .
$$

Damit ergibt sich für die Position der Ora serrata als Begrenzung des lichtsensitiven Teils der Retina

$$
\phi_{\max }=\frac{A_{\text {Retina }}}{4 r^{2}},
$$

16 Der Übersichtlichkeit halber wurde $c_{\text {inter }}=0$ gesetzt. Mit $c_{\text {inter }} \neq 0$ ergibt sich für das Integral $D_{\mathrm{M}}+c_{\text {inter }}\left(1-D_{\mathrm{M}}\right)$. 
bzw. mit oben angegebenen Werten $\phi_{\max } \approx 130^{\circ}$ im Bogenmaß. In einer aktuelleren Messung ist auch die Bogenlänge der Retina mit $B_{\text {Retina }} \approx 22.4 \mathrm{~mm}$ bestimmt worden [227]. Hier ergibt sich durch Umrechnung in das Bogenmaß

$$
\phi_{\text {max }}=180 \frac{B_{\text {Retina }}}{\pi r} \approx 130^{\circ},
$$

so dass der Begrenzungswinkel der Ora serrata als gute Abschätzung angenommen werden kann.

Für den maximalen Pupillendurchmesser $d_{\max }$ finden wir eine stark altersabhängige Funktion [222, 228]. Eine lineare Regression der Daten aus [228] liefert als Approximation

$$
d_{\max }(a) \approx 9.85 \mathrm{~mm}-0.092 \frac{\mathrm{mm}}{\mathrm{Jahr}} a,
$$

wobei $a$ das menschliche Alter im Intervall zwischen 20-80 Jahren angibt. Für das emmetrope Auge eines Menschen im Alter zwischen 30-50 Jahren beträgt der Durchmesser etwa $d_{\max } \approx 6 \mathrm{~mm}$ und es ergibt sich für den maximalen Pupillenöffnungswinkel

$$
\delta_{\max }=\arctan \left(\frac{d_{\max }}{2 r}\right) \approx 15^{\circ}
$$

wobei für den minimalen Pupillendurchmesser $1 \mathrm{~mm}$ angegeben ist, was einem Öffnungswinkel von $\delta_{\text {min }} \approx 5^{\circ}$ entspricht $^{17}$. Die hier bestimmten Grenzwinkel finden bei der Berechnung der relativen Intensitäten und ihrem Vergleich Berücksichtigung.

\subsubsection{Vergleich der relativen Intensitäten}

Wir haben nun alle notwendigen Parameter und Normierungsfaktoren bestimmt, um alle Integrale für die relativen Intensitäten des direkten Lichts nach Gleichung (3.37) und (3.38) bzw. die relativen Intensitäten des gestreuten Lichts nach Gleichung (3.29) und (3.30) auszuwerten. In Abbildung 3.17 sind die normierten Intensitäten des direkt einfallenden Lichts und des Streulichts als Funktion vom Retinaazimut $\phi_{\mathrm{G}}$ für den Fall mit Müllerzellen $\left(D_{\mathrm{M}}\right)$ und ohne Müllerzellen $\left(D_{0}\right)$ dargestellt. Für den Anteil des direkten Lichts wäre es demnach wie erwartet von Vorteil, die Photorezeptoren direkt auf der Retina anzuordnen. Diese Konfiguration entspricht in unserem Modell gleichzeitig dem oberen Maximum für die erreichbare relative Intensität des direkt einfallenden Lichts. Das globale Maximum der relativen Intensität ist in beiden Konfigurationen in der Fovea centralis $\left(\phi_{\mathrm{G}}=0^{\circ}\right)$ lokalisiert. In Ergänzung dazu finden wir für die relative Intensität der Konfiguration ohne Müllerzellen eine schwache Abhängigkeit vom Pupillenöffnungswinkel $\delta_{\max }$. Dies rührt von der Normierung mit $I_{0}$ her, da die Gesamtintensität nach Gleichung (3.36) natürlich eine deutliche Abhängigkeit vom Pupillenöffnungswinkel $\delta$ aufweist. Im Gegensatz dazu ist die relative Intensität des direkt einfallenden Lichts für die Retina mit Müllerzellen stark abhängig vom Pupillenöffnungswinkel $\delta_{\max }$. Eine Reihenentwicklung bis erster Ordnung in $\delta_{\max }$ in Foveanähe liefert für Abhängigkeit der relativen Intensität des direkten Lichts $\propto 1-$ const $\delta_{\max }$ mit const $<\frac{\pi}{180}$. Diese lineare Abhängigkeit findet sich in der abgebildeten Funktion bestätigt.

Der Vergleich der relativen Intensitäten der Streulichtanteile 1. Ordnung fällt eindeutig zu Gunsten des natürlichen Aufbaus der invertierten Retina aus, da der Anteil des auf die Photorezeptoren auftreffenden Streulichts mit Müllerzellen im Mittel um einen Faktor 50 kleiner ist als Retina ohne Müllerzellen,

17 Die Miosis (Pupillenverkleinerung) ist nicht so stark altersabhängig, da der zugehörige Muskel (Musculus sphincter pupillae) ein parasympathisch gesteuerter Muskel ist, der zum Naheinstellungstrias gehört. 


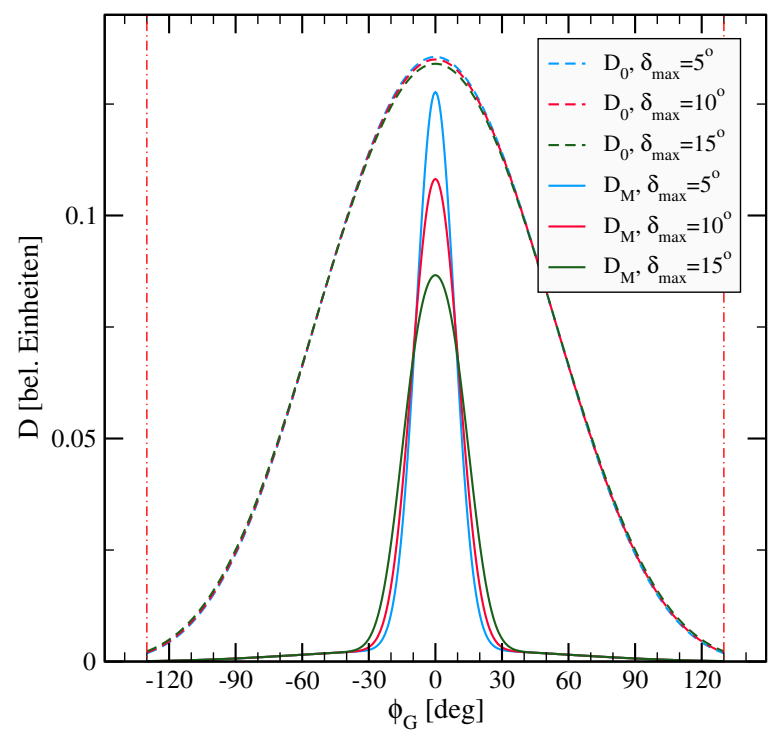

(a)

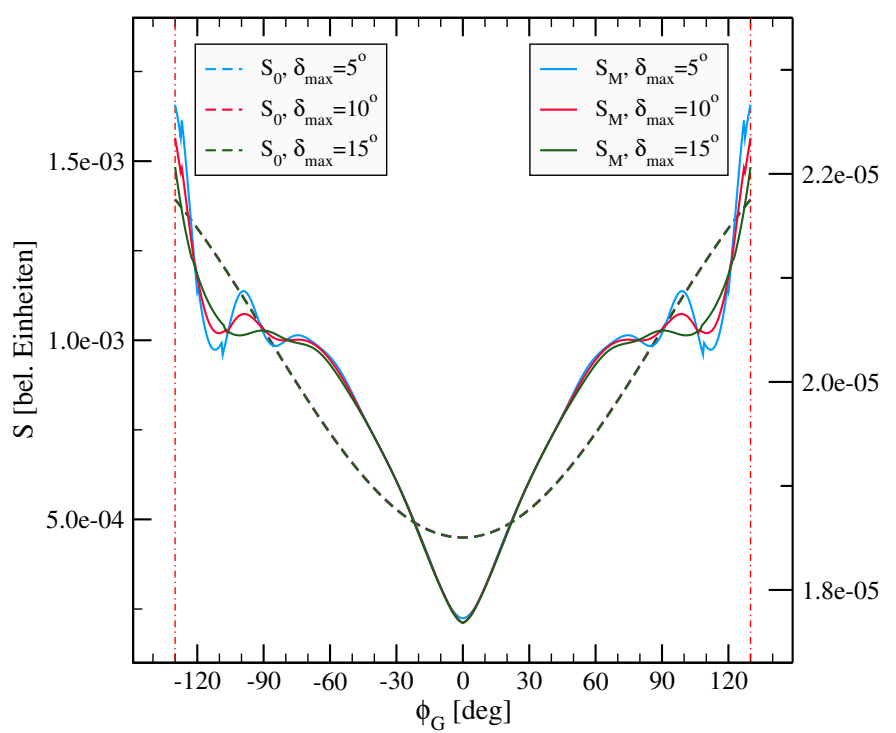

(b)

Abbildung 3.17.: Vergleich der relativen Intensitäten des (a) direkt einfallenden Lichts und (b) gestreuten Lichts erster Ordnung auf der Retina jeweils mit und ohne Müllerzellen zu verschiedenen Öffnungswinkeln der Pupille $\delta_{\max }$. Die vertikalen roten Linien kennzeichnen den Verlauf der Ora serrata. Demnach wäre es besonders für die Bereiche außerhalb der Fovea von Vorteil, wenn das direkt einfallende Licht auch direkt auf die Photorezeptoren träfe. Jedoch muss auch der Anteil des Streulichts berücksichtigt werden. In Unterabbildung (b) ist der Streulichtanteil in Abhängigkeit von der Retinaposition auf verschiedenen Skalen dargestellt (linke Skala: Streulicht ohne Müllerzellen, rechte Skala: Streulicht mit Müllerzellen). Hier wird deutlich, dass das Streulicht im Falle von Retina mit Müllerzellen etwa um einen Faktor 50 kleiner ist! Die Ursache für diesen Größenunterschied liegt in der Kleinwinkelfokussierung der Müllerzellen begründet.

d.h. mit Photorezeptoren an der Retinaoberfläche (vgl. Abb. 3.17b). Augenscheinlich besitzt die relative Intensität des Streulichtanteils für beide Konfigurationen ein globales Minimum in der Fovea $\left(\phi_{\mathrm{G}}=0^{\circ}\right)$. Eine Taylorentwicklung für $\phi_{\mathrm{G}}=0^{\circ}$ liefert für den Streulichtanteil beider Konfigurationen einen von $\delta_{\max }$ unabhängigen führenden Term und einen ähnlichen Korrekturterm wie oben angegeben und daher nur eine schwache Abhängigkeit vom Pupillenöffnungswinkel.

\subsubsection{Signal-Rausch-Verhältnisse}

Als vergleichende Größen lassen sich sowohl das Signal-Rausch-Verhältnis ohne Müllerzellen

$$
\operatorname{SNR}_{0}\left(\phi_{\mathrm{G}}\right)=\frac{D_{0}\left(\phi_{\mathrm{G}}\right)}{S_{0}\left(\phi_{\mathrm{G}}\right)}
$$

als auch das Signal-Rausch-Verhältnis mit Müllerzellen

$$
\operatorname{SNR}_{\mathrm{M}}\left(\phi_{\mathrm{G}}\right)=\frac{D_{\mathrm{M}}\left(\phi_{\mathrm{G}}\right)}{S_{\mathrm{M}}\left(\phi_{\mathrm{G}}\right)}
$$

definieren. Beide Größen sind in Abbildung 3.18a gegenübergestellt. Wie der Abbildung erwartungsgemäß zu entnehmen ist, übersteigt das Signal-Rausch-Verhältnis der natürlichen Konfiguration mit Müllerzellen deutlich die der Konfiguration ohne Müllerzellen in Foveanähe. Für Positionen auf der Retina in foveafernen Bereichen $\phi_{\mathrm{G}}>30^{\circ}$ liefert Abbildung 3.18a keine signifikanten Aussagen. Eine aussagekräftige Analyse wird erst durch die Definition des Verhältnisses der beiden Größen $\operatorname{SNR}_{\mathrm{M}}\left(\phi_{\mathrm{G}}\right)$ zu $\operatorname{SNR}_{0}\left(\phi_{\mathrm{G}}\right)$ ermöglicht. 


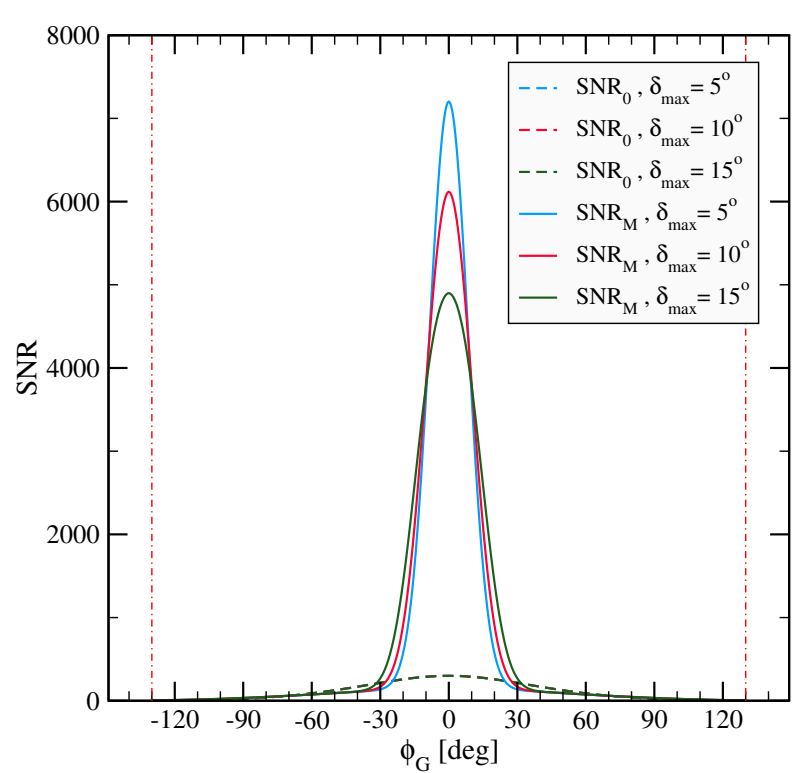

(a)

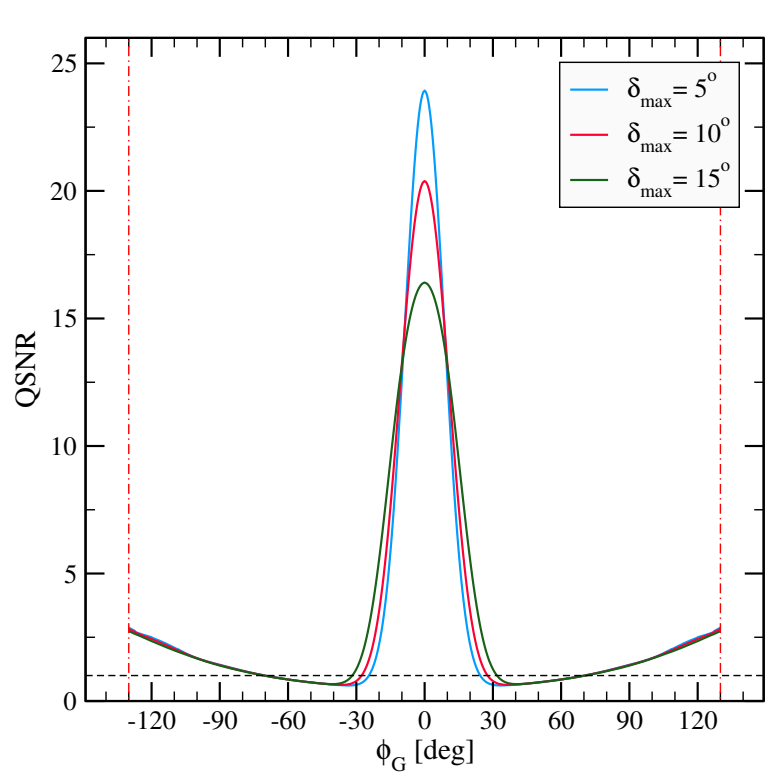

(b)

Abbildung 3.18.: (a) Signal-Rausch-Verhältnis mit Müllerzellen $\left(\mathrm{SNR}_{\mathrm{M}}\right)$ und ohne Müllerzellen $\left(\mathrm{SNR}_{0}\right)$ der Retina des menschlichen Auges (b) Quotient QSNR $=\frac{\mathrm{SNR}_{\mathrm{M}}}{\mathrm{SNR}_{0}}$ der Signal-Rausch-Verhältnisse für das menschlichen Auge, der auch als Verstärkungsfaktor angesehen werden kann. Die vertikalen roten Linien kennzeichnen den Verlauf der Ora serrata. Die horizontale schwarze Linie separiert den verstärkten Bereich $($ QSNR $>1)$ vom abgeschwächten Bereich (QSNR < 1). Die Dominanz der natürlichen Konfiguration mit Müllerzellen in weiten Teilen der Retina ist deutlich sichtbar. In der Fovea centralis erreicht sie Werte bis ca. 25. Im Bereich von etwa $30-65^{\circ}$ ist der Quotient in unserem Modell bis um einen Faktor 0.6 abgesenkt (Minimum QSNR bei $\left|\phi_{\mathrm{G}}\right| \approx 33.5^{\circ}$ ).

Der Quotient der beiden ermittelten Signal-Rausch-Verhältnisse

$$
\operatorname{QSNR}\left(\phi_{\mathrm{G}}\right)=\frac{\operatorname{SNR}_{\mathrm{M}}\left(\phi_{\mathrm{G}}\right)}{\operatorname{SNR}_{0}\left(\phi_{\mathrm{G}}\right)}=\frac{D_{\mathrm{M}}\left(\phi_{\mathrm{G}}\right)}{S_{\mathrm{M}}\left(\phi_{\mathrm{G}}\right)} \frac{S_{0}\left(\phi_{\mathrm{G}}\right)}{D_{0}\left(\phi_{\mathrm{G}}\right)}
$$

ist ein Maß für die positionsabhängige Verstärkung des Lichtsignals gegenüber ebenfalls detektiertem Streulicht der Retina. Wie Abbildung 3.18b zu entnehmen ist, finden wir in Foveanähe ein um den Faktor 25 verbessertes Signal-Rausch-Verhältnis für die Konfiguration mit Müllerzellen vor, wobei sich der Quotient der beiden ermittelten Signal-Rausch-Verhältnisse QSNR $\left(\phi_{\mathrm{G}}\right)$ mit zunehmendem Pupillenöffnungswinkel $\delta_{\max }$ verbreitert. In foveafernen Bereichen zwischen ca. 30-60 ist der Quotient der Signal-Rausch-Verhältnisse bis zu einem Faktor 0.6 reduziert. In den retinalen Randbereichen $\phi_{\mathrm{G}}>60^{\circ}$ dominiert das Signal-Rausch-Verhältnis der Konfiguration mit Müllerzellen wieder. Dieses Verhalten kann wie folgt interpretiert werden: Bei starkem Lichteinfall ist die Pupille klein und das Intensitätsmaximum für die Fovea optimiert, während bei schwachem Lichteinfall die Pupille geweitet ist und die Fovea-Randbereiche auf Kosten der Intensität in der Fovea centralis durch Verbreiterung des Intensitätsprofils optimiert werden. Obwohl in unserem Modell eine konstante Photorezeptordichte angenommen wurde, spricht die Physik des Auges zusammen mit dem Vorhandensein von Müllerzellen für eine Konzentration der Zapfen-Photorezeptoren im Bereich der Fovea bei photopischer Sicht (scharfes Sehen), während sich bei skotopischer Sicht (Kontraste) eine ausgedehnte Anordnung der Stäbchen-Photorezeptoren in den Außenbereichen der Fovea als vorteilhaft erweist (vgl. auch Abb. 3.3). Alle beobachteten Phänomene, insbesondere die Lokalisierung der Maxima der Intensität des 
direkten Lichts und die Lokalisierung der Minima der Streulichtintensität bei $\phi_{\mathrm{G}}=0^{\circ}$ unabhängig von der gewählten Konfiguration, sprechen daher für eine Konzentration der Photorezeptoren im fovealen Bereich und somit auch für die evolutionäre Herausbildung einer Fovea. Die invertierte Retina mit einer dichten Anordnung von Müllerzellen stellt insbesondere eine starke Optimierung für das Scharfsehen in Foveanähe und Reduktion des Streulichts in den retinalen Randgebieten dar.

\subsection{Zusammenfassung und Ausblick}

Die Analyse der Form von Müllerzellen erlaubte uns die Zerlegung in zwei für die Optik der Müllerzelle relevante Teile: den Endfuß mit seiner markanten Trichterform und den länglichen Zellstamm. Wir konnten zeigen, dass der Endfuß die optische Funktion eines Kollimators erfüllt und leiteten die Winkelverteilung der Müllerzelle ab. Da bereits experimentelle Refraktionsmessungen an Müllerzellen verschiedener Spezies durchgeführt wurden, konnten wir die unterschiedlichen Brechungsindices für die einzelnen Abschnitte der Zelle in Profile des Potenzials eines Hamilton-Operators überführen und so mithilfe der in Kapitel 1 entwickelten Methode des Streuformalismus die Transmissions- und Reflexionsspektren in Abhängigkeit vom Einfallswinkel bestimmen. Die in experimentellen Untersuchungen gemessenen optischen Leistungen der Müllerzelle, die sich in einer homogenen Lösung befand, konnten quantitativ bestätigt werden.

Wir gingen dann erstmalig der Frage nach, inwiefern die natürliche nicht-homogene Umgebung der Müllerzelle (innerhalb der Retina) die Lichtwellenleitung der Müllerzelle beeinflusst. Diese Frage ist essenziell, da der Brechungsindex außerhalb der Zelle stärker fluktuiert als die Differenz der mittleren Brechungsindices von Zelle und Umgebung. Zur weiteren Untersuchung wurde die extrazelluläre Umgebung der Müllerzelle mit korrelierten Gaußschen Zufallspotenzialen approximiert und die Zelle darin eingebettet. Wie sich zeigte, bleibt der lichtwellenleitende Charakter der Müllerzelle in der natürlichen Umgebung erhalten.

Es wurden ebenfalls zellstrukturbedingte Transmissions- und Reflexionsspektren in Abhängigkeit vom Einfallswinkel bestimmt und unter Zuhilfenahme von Größen wie $V$-Parameter bzw. numerischer Apertur ausgewertet. Dabei fanden auch die Form der Müllerzelle und die Lage ihres Zellkörpers Berücksichtigung. Mit den so bestimmten Spektren waren wir in der Lage, ein vereinfachtes Augenmodell zu entwickeln, um die Anteile des direkt auf die Retina einfallenden Lichts mit den Streulichtanteilen vergleichen zu können. Zum Vergleich simulierten wir eine artifizielle Retina ohne Müllerzellen (nicht-invertierte Retina), bei der sich die Photorezeptoren an der Oberfläche des zum Glaskörper zugewandten Teils der Retina befinden. Ohne die Photorezeptordichte zu berücksichtigen, sprachen die Ergebnisse beider Konfigurationen dennoch für die Ausbildung einer fovea-ähnlichen Struktur, denn das berechnete positionsabhängige Signal-Rausch-Verhältnis der Retina ist in der Nähe der optischen Achse deutlich erhöht. Ein Vergleich der Signal-Rausch-Verhältnisse beider Konfigurationen, also der Retina mit und ohne Müllerzellen, fiel deutlich zu Gunsten des Vorhandenseins von Müllerzellen aus, da diese das Signal-Rausch-Verhältnis in der Foveanähe in unserem Modell bis zu einem Faktor 25 verbessert und auch in den weiter entfernt liegenden Außenbereichen der Retina erhöht erscheint. Dies lässt den eventuellen Schluss zu, dass die Ausbildung einer invertierten Retina nicht nur evolutionären Zwangsbedingungen geschuldet ist, wie oft angenommen wird, sondern sogar einen Vorteil für die Wahrnehmung birgt, da die Kugelform des Auges zusammen mit der Kleinwinkelfokussierung 
der Müllerzellen zu einer optimierten und in weiten Teilen rauschunterdrückten Abbildung sowohl bei photopischer als auch bei skotopischer Sicht führt.

Ausblickend kann gesagt werden, dass obwohl unser Modell auf die Architektur des menschlichen Auges und auf die Struktur menschlicher Retina angepasst wurde, diese Wahl die Anwendbarkeit auf visuelle Systeme anderer Spezies mit Linsenaugen nicht einschränkt. Die Anpassung der Parameter des Auges, wie Augenform, Linsenöffnungswinkel und Lage der Linse und die Neuauswertung der Transmissionsund Reflexionsspektren, bedingt durch die veränderte Form des Endfußes der Müllerzellen und die Länge der Müllerzellen, lassen eine Untersuchung der Signal-Rausch-Verhältnisse für andere Spezies ohne Weiteres zu.

Unser Modell kann weiter ausgebaut werden, um die Ergebnisse mit bekannten Phänomenen, wie dem Stiles-Crawford-Effekt 1.Art (SCE1) [29] vergleichen zu können. Eine theoretische Erklärung und Neuformulierung des SCE1 mithilfe der Transporteigenschaften der Müllerzellen wird damit diskussionsfähig.

Ein erster Schritt einer naturgetreueren Anpassung an die Optik des Auges stellt die Verbesserung der Näherung des Linsensystems als System bestehend aus Kornea und nach innen verschobener Linse dar (Liou-Brennan-Modell [220, 221]). Weiterhin kann die optische Achse zur Sehachse um die natürlichen $5^{\circ}$ verkippt und der Glaskörper zum Modell hinzugefügt werden.

Die Photorezeptordichte und damit auch die Dichteverteilung der Müllerzellen blieb bisher unberücksichtigt, ebenso wie die exzentrizitätsabhängige Dicke der Retina und damit auch die Länge der Müllerzellen. Beispielsweise befinden sich genau im Zentrum der fovea centralis, einem kleinen inneren kreisförmigen Bereich der Fovea im menschlichen Auge fast keine Müllerzellen. Durch die dichte hexagonale Anordnung der in der Fovea fast ausschließlich vorherrschenden Zapfen-Photorezeptoren des photopischen Sehens und die für die Signaltransduktion bei hoher Bildauflösung notwendige 1:1 Verschaltung der Photorezeptoren mit den Ganglienzellen sind diese Zelltypen, zusammen mit den Müllerzellen, in die Peripherie der Fovea (Peri- und Parafovea) ausgelagert und so die Dicke der Retina in der direkten ringförmigen Umgebung der Fovea in etwa verdoppelt. Infolge der (beim Menschen im Wesentlichen postnatalen [229]) Ausbildung der Fovea, entwickeln die Müllerzellen längere "Z"förmige Nervenfasern, um die Verbindungen der Photorezeptorzellen der Fovea mit den Bipolar- und Ganglienzellen der Parafovea zu gewährleisten. Durch die damit verbundene Auslagerung der Zellkörper ist die Retina im Zentrum der Fovea demnach viel dünner, so dass die in der Fovea ansässigen Photorezeptoren sowohl dem Lichtsignal der optischen Abbildung, als auch dem Streulicht nahezu direkt ausgesetzt sind, während in der Parafovea eine besonders dicke Schicht vom einfallenden Licht durchquert werden muss. Eine Berücksichtigung der Photorezeptordichte und der exzentrizitätsabhängigen Dicke kann entsprechend implementiert werden, wobei dann bei der Intensitätsberechnung des direkten Lichts und des Streulichts fourieroptische Methoden [230] zum Einsatz kommen könnten, die dann mit so genannten BRDF-Methoden (Bidirectional Reflectance Distribution Function) [231] verglichen werden könnten. Eine Modelloptimierung der "artifiziellen Retina" (Retina ohne Müllerzellen) als Alternative bei der Berechnung der Reflexionsfunktionen stellt die von Pokrowski [232] aufgestellte Theorie über Reflexion an diffusen Flächen dar, die mangels experimenteller Daten zu Reflexionskoeffizienten von Photorezeptoren zumindest theoretisch angepasst werden könnte.

Eine bisher gänzlich unbeantwortete Frage ist die nach der Auskopplung des Lichts aus den Müllerzellen in das Gebiet der Photorezeptoren. Die Erweiterung des Modells durch verzweigende Filamente 
und Mikrovilli der Müllerzelle in den Außensegmenten der Retina und der Implementation von - nach neuen Erkenntnissen von Kreysing et al. [233] - invers aufgebauten Photorezeptorkernen (Euchromatin außen und dichteres Heterochromatin innen) könnte diesbezüglich helfen, zu wegweisenden theoretischen Erkenntnissen hinsichtlich des Lichttransports durch die gesamte Retina zu gelangen.

Eine theoretisch aufschlussreiche Erweiterung des Modells stellt eine Berücksichtigung der Wellenlängenabhängigkeit der Transmissions- und Reflexionsgrößen dar, wobei dann auch die chromatische Aberration der Linse Beachtung finden müsste. Auch hier könnten die Ergebnisse mit den Beobachtungen des Stiles-Crawford-Effektes 2. Art [234] verglichen werden, was hinsichtlich einer in weiten Teilen fehlenden theoretischen Beschreibung sicherlich eine überaus interessante Erweiterung darstellt [235]. 



\section{4. $\mathcal{P} \mathcal{T}$-Symmetrie in niedrigdimensionalen optischen Systemen}

Was wir mathematisch festlegen, ist nur zum kleinen Teil ein objektives Faktum, zum größeren Teil eine Übersicht über Möglichkeiten.

Werner Heisenberg [236]

4.1 Einführung . . . . . . . . . . . . . . . . . . . . . . . 91

4.1.1 Pseudo-hermitesche Operatoren und $\mathcal{P} \mathcal{T}$-Symmetrie . . . . . . . . . . 91

4.1.2 Komplexe Brechungsindices . . . . . . . . . . . . . . . . . . . . . 92

4.1 .3 Wellenleiter . . . . . . . . . . . . . . . . . . . 94

4.2 Lokalisierung als Ursache für spontan gebrochene $\mathcal{P} \mathcal{T}$-Symmetrie in 1D . . . 96

4.2.1 Zwei $\mathcal{P} \mathcal{T}$-symmetrische Fehlstellen . . . . . . . . . . . . . . . . 96

4.2.2 Die endliche $\mathcal{P} \mathcal{T}$-symmetrische Kette mit Unordnung (Eindimensionales $\mathcal{P} \mathcal{T}$-Anderson-Modell $\quad \ldots \ldots$. . . . . . . . . . . . . . . . 99

4.2.3 Periodische $\mathcal{P} \mathcal{T}$-symmetrische Potenziale . . . . . . . . . . . . 105

4.3 Strukturen mit lokaler $\mathcal{P}_{D} \mathcal{T}$-Symmetrie in quasi-1D . . . . . . . . . . . . . 109

4.3.1 Der einfache Dimer . . . . . . . . . . . . . . . . . . . . . 109

4.3.2 Zwei gekoppelte Dimere . . . . . . . . . . . . . . . . . . 110

4.3.3 Die Quasi 1D-Dimerkette . . . . . . . . . . . . . . . . . . 113

4.4 Streuformalismus in lokal $\mathcal{P} \mathcal{T}$-symmetrischen Systemen . . . . . . . . . . . 120

4.4.1 Systeme mit abgeschlossenen Rändern . . . . . . . . . . . . . . . . 120

4.4.2 Systeme mit offenen Rändern . . . . . . . . . . . . . . . . . . . . . . 124 


\section{Motivation}

Lichttransport in optischen Materialien ist stets mit Intensitätsverlusten verbunden. Besonders gravierend wirkt sich diese Tatsache auf künstlich hergestellte, aktive optische Materialien - die in der Einleitung vorgestellten Metamaterialien - aus. Um diese starken Verluste auszugleichen, werden bei der experimentellen Realisierung dieser Systeme Bereiche optisch verstärkender Strukturen in diese Metamaterialien integriert, was in der Regel jedoch zu instabilem Transportverhalten führt.

Einen möglichen Ausweg aus diesem Dilemma bieten so genannte $\mathcal{P} \mathcal{T}$-symmetrische Systeme mit ihrer pseudo-hermiteschen Phase. Das aus der Quantenmechanik stammende Konzept der $\mathcal{P} \mathcal{T}$-symmetrischen Operatoren kann, wie wir zeigen werden, auch auf optische Systeme übertragen werden. In der pseudo-hermiteschen Phase besitzt der Hamilton-Operator reelle Eigenwerte (daher rührt die Bezeichnung pseudo-hermitesch). Die von diesem Operator erzeugte Dynamik kann durch eine Ähnlichkeitstransformation auf eine unitäre Dynamik abgebildet werden. Daraus resultieren stabile Observablen, wie beispielsweise eine beschränkte Intensität als optische Größe. Es erweist sich aber, dass das Problem damit vorerst nicht gelöst ist, denn die pseudo-hermitesche Phase kann schon durch kleinste räumliche und energetische Asymmetrien gebrochen werden.

Wir zeigen hier, dass bereits schwache Unordnung, die in jedweder physikalischen Realisierung eine unvermeidbare Tatsache darstellt, im Allgemeinen zu einer gebrochenen $\mathcal{P} \mathcal{T}$-Symmetrie führt. Dazu untersuchen wir fundamentale Ursachen der $\mathcal{P} \mathcal{T}$-Symmetriebrechung in ein- und quasi-eindimensionalen Systemen und leiten analytische Resultate ab [3]. Es zeigt sich, dass in diesem Fall dann paarweise komplexe Eigenwerte auftreten, die die Dynamik beeinflussen und eine ungehinderte Wellenausbreitung unmöglich machen. Daher ist man im Hinblick auf realistische Anwendungen an einer ausgedehnten pseudo-hermiteschen Phase interessiert. Unter Ausnutzung der hier gewonnenen elementaren Resultate sind wir in der Lage, Konfigurationen von speziellen $\mathcal{P} \mathcal{T}$-symmetrischen Strukturen zu entwickeln, die auch in der Gegenwart von Unordnung über eine stabile pseudo-hermitesche Phase verfügen [4]. Der letzte Abschnitt dieses Kapitels beschäftigt sich mit dem Transport in $\mathcal{P} \mathcal{T}$-symmetrischen Streusystemen. Dazu wird der in Kapitel 1 entwickelte Streuformalismus, der bereits in den vorausgehenden Kapiteln eine zentrale Rolle einnahm, auf diese Systeme übertragen und ein Konzept zur theoretischen Untersuchung von Transporteigenschaften in geschlossenen und offenen $\mathcal{P} \mathcal{T}$-symmetrischen Systemen erarbeitet. 


\subsection{Einführung}

In der klassischen Mechanik wird ein Massenpunktsystem durch eine Punktwolke im $N$-dimensionalen Phasenraum beschrieben. Jede klassische Trajektorie ist als Lösung einer Hamilton- bzw. EulerLagrange-Gleichung und zugehörigen Anfangsbedingungen eindeutig bestimmt. Alle messbaren Größen des klassischen Systems sind durch Funktionen gegeben, die im Phasenraum operieren.

Die Quantenmechanik beschreibt ein gegebenes System auf andere Weise: Hier geht man von einem unendlichdimensionalen Hilbertraum aus, welcher einen vollständigen Vektorraum bildet. Der Begriff der Vollständigkeit bezieht sich auf die Norm, üblicherweise dargestellt durch das wohldefinierte Skalarprodukt. Jedes quantenmechanische System ist dann durch einen Vektor dieses Hilbertraums darstellbar. Ein weiteres Postulat der Kopenhagener Interpretation besagt, dass physikalisch messbare Größen durch das reelle Eigenwertspektrum der im Zustandsraum wirkenden Operatoren, der Observablen, beschrieben werden. Da ein reelles Spektrum voraus gesetzt wird, beschränkte man sich in der Vergangenheit auf hermitesche (selbst-adjungierte) Operatoren als physikalisch sinnvolle Observablen.

\subsubsection{Pseudo-hermitesche Operatoren und $\mathcal{P} \mathcal{T}$-Symmetrie}

Seit einigen Jahren sind so genannte $\mathcal{P} \mathcal{T}$-symmetrische Hamilton-Operatoren Gegenstand der Forschung, da diese unter bestimmten Umständen ein reelles Spektrum aufweisen können. Diese Operatoren sind dadurch gekennzeichnet, dass sie mit dem Hamilton-Operator vertauschen $([\mathcal{P} \mathcal{T}, \mathcal{H}]=0)$. Dabei bezeichnet $\mathcal{P}$ den Paritäts- und $\mathcal{T}$ den Zeitumkehroperator. Die Definition des $\mathcal{P} \mathcal{T}$-Operators lässt sich einfach anhand der Wirkung auf den Ortsoperator $\hat{x}$ und den Impulsoperator $\hat{p}$ angeben

$$
\begin{aligned}
& \mathcal{P}: \hat{x} \longrightarrow-\hat{x}, \hat{p} \longrightarrow-\hat{p} \\
& \mathcal{T}: \hat{x} \longrightarrow \hat{x}, \hat{p} \longrightarrow-\hat{p}, i \longrightarrow-i .
\end{aligned}
$$

Die Klasse der Operatoren mit $\mathcal{P} \mathcal{T}$-Symmetrie ist eine Unterklasse der allgemeineren Klasse der pseudo-hermiteschen Operatoren [237]. Letztgenannte sind dadurch definiert, dass sie der Bedingung

$$
\mathcal{H}^{\dagger}=\eta \mathcal{H} \eta^{-1}
$$

genügen, wobei $\eta$ einen nicht eindeutigen, positiv definiten Operator bezeichnet. ${ }^{1}$ Pseudo-hermitesche Operatoren $^{2}$ besitzen also ein reelles Spektrum, solange die Bedingung (4.2) erfüllt ist [39]. In [38, 39] wurde gezeigt, dass pseudo-Hermitizität von $\mathcal{H}$ die Präsenz einer anti-linearen Symmetrie des Operators beinhaltet. Ein Operator $\mathcal{A}: \mathcal{H} \rightarrow \mathcal{Y}$ heißt anti-linear, wenn für alle $a, b \in \mathcal{H}, \lambda \in \mathbb{C}$ gilt:

1. $\mathcal{A}(\lambda a)=\lambda^{*} \mathcal{A}(a)(\mathcal{A}$ ist anti-homogen $)$

2. $\mathcal{A}(a+b)=\mathcal{A}(a)+\mathcal{A}(b)(\mathcal{A}$ ist additiv $)$.

Der $\mathcal{P} \mathcal{T}$-Operator stellt mathematisch gesehen nur einen speziellen anti-linearen Operator dar [40, 238, 239]. Als Verallgemeinerung des $\mathcal{P} \mathcal{T}$-Operators sei hier auf anti-lineare Operatoren $\mathcal{S} \mathcal{T}$ verwiesen, wobei $\mathcal{T}$ der bereits erwähnte anti-lineare Zeitumkehroperator ist und $\mathcal{S}$ einen beliebigen linearen Operator repräsentiert. Die Hauptcharakteristik besteht allerdings auch hier im Vertauschen von $\mathcal{H}$

1 Ein Operator $\eta$ heißt positiv definit, wenn er hermitesch ist und ein rein positives reelles Spektrum aufweist. In einer äquivalenten Formulierung von Pseudo-Hermitizität wird $\eta$ als einen hermiteschen, invertierbaren und linearen Operator definiert.

2 Für pseudo-hermitesche Operatoren $\mathcal{H}$ wird häufig die Schreibweise $\mathcal{H}=\mathcal{H}^{\ddagger}$ verwendet. 
und $\mathcal{S T}([\mathcal{S T}, \mathcal{H}]=0)$. Beispiele solcher Operatoren findet man in vielen Bereichen der Physik, von der Quantenfeldtheorie bis hin zur Festkörperphysik und Optik [237, 240, 241, 242, 243, 244, 245, 246, 247, 248, 249, 250, 251, 252, 253, 254, 255, 256, 3, 4]. Aufgrund der Anti-Linearität folgt aus dem Verschwinden des Kommutators $[\mathcal{S T}, \mathcal{H}]=0$ nicht notwendigerweise, dass $\mathcal{H}$ und $\mathcal{S} \mathcal{T}$ eine gemeinsame Basis von Eigenfunktionen besitzen. Im Falle gleicher Eigenzustände von $\mathcal{H}$ und $\mathcal{S} \mathcal{T}$ sind alle Eigenwerte von $\mathcal{H}$ reell. Diese Phase wird exakte $\mathcal{S} \mathcal{T}$-symmetrische Phase genannt. Im anderen Fall spricht man von spontan gebrochener $\mathcal{S} \mathcal{T}$-Symmetrie. In vielen physikalischen Realisierungen wird der Übergang von exakter zu spontan gebrochener $\mathcal{S T}$-Symmetrie durch einen oder mehrere Parameter von $\mathcal{H}$ gesteuert. Wir wollen uns jedoch im Hinblick auf die Realisierung von Metamaterialien auf den Paritätsoperator $\mathcal{P}$ beschränken.

Eine viel versprechenden Realisierung aus dem Bereich der Optik wurde in [249] vorgeschlagen und erste experimentelle Ergebnisse mit Bezug auf optische Systeme kürzlich in [255] vorgestellt. Weitere vielschichtige Anwendungen pseudo-hermitescher Hamilton-Operatoren und die Untersuchung ihrer Eigensysteme, also ihrer Eigenspektren und Eigenvektoren, reichen von der Dynamik neuronaler Netze [257, 258] bis zu Problemen des biologischen Wachstums [259], von klassischer Diffusion in zufallsverteilten Medien [260] bis zur statistischen Mechanik von Feldliniendichten von Supraleitern mit säulenförmigen Defekten [261], um nur einige zu nennen. Es ist hierbei die Quantenmechanik bzw. ihre mathematische Beschaffenheit, die das größte Interesse auf sich zieht [262]. Pseudo-hermitesche Hamilton-Operatoren treten bei der Reduktion von externen Freiheitsgraden auf und ermöglichen so erst die Berechnung bzw. Simulation des zu betrachtenden Systems und damit die konzeptionelle Komplexität des Problems zu berücksichtigen. Ein weiterer Zugang der Behandlung dieser komplexen Systeme liegt in der Annahme begründet, dass die statistischen Eigenschaften durch nicht-hermitesche Zufallsmatrizen getreu dargestellt werden können [123, 263, 264, 265, 266, 267]. Dieser Forschungszweig erlangte seitens der mathematischen Physik viel Aufmerksamkeit, manifestiert in der ausführlichen Untersuchung nicht-selbstadjungierter Matrizen (siehe [267] und dort angegebene Referenzen).

\subsubsection{Komplexe Brechungsindices}

Im Folgenden soll zuerst der Fragestellung gefolgt werden, wann ein Hamilton-Operator der $\mathcal{P} \mathcal{T}$ Symmetrie genügt. Danach zeigen wir, wie die paraxiale Näherung dazu verwendet werden kann, ein $\mathcal{P} \mathcal{T}$-symmetrisches Potenzial $V(\mathbf{r})$ in einen komplexen Brechungsindex $n(\mathbf{r})$ zu überführen, um fundamentale Fragen auch in optischen Systemen klären zu können.

Sehen wir uns zuerst die Wirkung des $\mathcal{P} \mathcal{T}$-Operators auf den in dieser Arbeit verwendeten stark gebundenen Hamilton-Operator (tight-binding hamiltonian)

$$
\mathcal{H}=\frac{p^{2}}{2}+V(r)
$$

an. Mit Gleichung (4.1) ist leicht zu sehen, dass der Hamilton-Operator $\mathcal{P} \mathcal{T}$-symmetrisch formuliert ist, wenn das Potenzial die notwendige Bedingung

$$
V(r)=V^{*}(-r)
$$

erfüllt. Wie zu Beginn erwähnt, reichen die Beispiele für Systeme mit $\mathcal{P} \mathcal{T}$-Symmetrie von der Quantenfeldtheorie bis zur Festkörperphysik und Optik. Ein optisches System kann durch ein Medium 
bestehend aus alternierenden Regionen mit Verstärkung (gain) und Absorption (loss) geschaffen werden, was sich in einem komplexem Brechungsindex $n(x) \in \mathbb{C}$ widerspiegelt $[251,252,253$, 268]. Die Überführung von einem Brechungsindex der Form

$$
n(x)=n_{0}+\beta+i \gamma
$$

in ein Potenzial $V(x)$, wobei $n_{0}$ den Brechungsindex des Hintergrundmaterials ${ }^{3}, \beta$ das reelle periodische und $\gamma$ das verstärkende $(\gamma>0)$ bzw. abschwächende $(\gamma<0)$ periodische Brechungsindexprofil bezeichnen, ist im Folgenden dargestellt (als Beispiel eines komplexen Brechungsindexprofils siehe Abbildung 4.1a). In zwei Dimensionen genügt die Wellenfunktion in der $(x, z)$-Ebene der skalaren Helmholtz-Gleichung

$$
\partial_{z}^{2} \psi(x, z)+\partial_{x}^{2} \psi(x, z)+k^{2} n(x)^{2} \psi(x, z)=0
$$

mit Wellenvektor $k=\frac{2 \pi}{\lambda}$, wobei $\lambda$ die Wellenlänge des einfallenden Lichts repräsentieren soll. Im Hinblick auf Wellenleiter interessieren wir uns nur für Lösungen mit Ausbreitung in $z$-Richtung

$$
\psi(x, z)=e^{i k n_{0} z} \psi(\xi, \zeta)
$$

mit den dimensionslosen Größen

$$
\xi \equiv \frac{x}{l} \text { und } \zeta \equiv \frac{z}{2 k l^{2} n_{0}}
$$

und der Gitterkonstante $l$. Wir erhalten nach Einsetzen der Lösung (4.7) in Gleichung (4.6) für die partiellen Ableitungen

$$
\begin{gathered}
\partial_{z}^{2} \psi(x, z)=\partial_{z}\left(i k n_{0} e^{i k n_{0} z} \psi(\xi, \zeta)+e^{i k n_{0} z} \partial_{z} \psi(\xi, \zeta)\right) \\
=-k^{2} n_{0}^{2} \psi(x, z)+2 i k n_{0} e^{i k n_{0} z} \partial_{z} \psi(\xi, \zeta)+e^{i k n_{0} z} \partial_{z}^{2} \psi(\xi, \zeta) \\
\partial_{x}^{2} \psi(x, z)=e^{i k n_{0} z} \partial_{x}^{2} \psi(\xi, \zeta)
\end{gathered}
$$

wobei die zweite Ableitung der Wellenfunktion nach der Ausbreitungsrichtung $z$ der Gleichung (4.9) in der so genannten paraxialen Näherung

$$
\left|\partial_{z}^{2} \psi(x, z)\right| \ll\left|k^{2} \psi(x, z)\right|
$$

vernachlässigt werden kann. Mit den partiellen Ableitungen der dimensionslosen Größen aus Gleichung (4.8), also $\partial_{\xi} x=l$ und $\partial_{\zeta} z=2 k l^{2} n_{0}$, ergibt sich insgesamt

$$
\begin{gathered}
i \partial_{\zeta} \psi(\xi, \zeta)+\partial_{\xi} \psi(\xi, \zeta)-\mu \psi(\xi, \zeta)=0, \text { mit } \\
\mu=k^{2} l^{2}\left[n(x)^{2}-n_{0}^{2}\right]
\end{gathered}
$$

die Helmholtz-Gleichung in paraxialer Näherung. Diese kann nun in eine Schrödinger-Gleichung mit fiktiver Zeit, welche in Beziehung zur Ausbreitungsrichtung steht, überführt werden, wobei der dimen-

3 In experimentellen Realisierungen gilt für den Brechungsindex des Hintergrundmaterials $n_{0} \gg \beta, \gamma$, siehe [250]. 
sionslose reduzierte Brechungsindex $\mu$ die Rolle des negativen Potenzials $-V(x)$ übernimmt, so dass wir für

$$
n(x)^{2}-n_{0}^{2}=\neq \beta^{2^{0}}-\gamma^{\varpi^{0}}+2 i \beta \gamma^{0}+2 n_{0}(\beta+i \gamma)
$$

die drei ersten Terme wegen der Voraussetzung $n_{0} \gg \beta, \gamma$ vernachlässigen können. Für das Potenzial ergibt sich somit

$$
\begin{aligned}
V(x) & \simeq 2 k^{2} l^{2} n_{0}(\beta+i \gamma) \\
& =\widetilde{n}_{0}(\beta+i \gamma)=\widetilde{n}_{0} n(x)
\end{aligned}
$$

Wir können also die Helmholtz-Gleichung in paraxialer Näherung durch einfache Skalierung in eine Schrödinger-Gleichung überführen. ${ }^{4}$ Wegen Gleichung (4.13) ist die Bedingung (4.4) auch für den Brechungsindex gültig

$$
\begin{aligned}
& n(x)=n^{*}(-x) \\
& \Longleftrightarrow \begin{cases}\operatorname{Re} n(x) & =\operatorname{Re} n(-x) \\
\operatorname{Im} n(x) & =-\operatorname{Im} n(-x)\end{cases}
\end{aligned}
$$

Diese Bedingung impliziert, dass Emission und Absorption von Photonen einander ausgleichen, so dass der Nettogewinn bzw. der Nettoverlust Null ist.

\subsubsection{Wellenleiter}

Die experimentelle Untersuchung der Lichtausbreitung in linearen und nicht-linearen gekoppelten Wellenleitern weist das typische charakteristische Verhalten von diskreten Systemen auf. Die Erforschung der Beugungseigenschaften dieser Art von synthetischen Materialien bringt Phänomene wie Doppelbrechung oder nicht-reziproke Beugungsbilder hervor und lässt sie als mögliche Kandidaten für uni-direktionale optische Koppler oder als Sensoren für gerichteten Lichttransport in Frage kommen. Die Fähigkeit, den Lichtfluss in photonischen periodischen Strukturen zu modifizieren, ist daher von immenser wissenschaftlicher und praktischer Bedeutung. Einige Beispiele elektrischer und optischer Wellenleiter findet man in Abbildung 4.1. Die Unterabbildungen (a) und (b) sind Beispiele für Wellenleiterarrays, die ein laterales Übergitter (superlattice) als strukturbildende Komponente nutzen [280], während (c) und (d) moderne Wellenleiterbeispiele auf Faserkernbasis im optisch linearen und nichtlinearen Regime darstellen und (e) einen neuartigen, alternativ gefertigten Wellenleiter aus synthetischem Quarzglas repräsentiert.

Eine Übersicht der historischen Entwicklung von Lichtwellenleitern ist in [281] zu finden. Weiterhin sei auf den Übersichtsartikel von P. Russel zu lichtleitenden kristallinen Faserbündeln [282], sowie auf J. Joannopoulos Textbuch über photonische Kristalle verwiesen [283] (siehe dazu auch Unterabschnitt 2.1.1.3). Weitere umfangreiche moderne Applikationen nichtlinearer optischer Wellenleiter können in [284] studiert werden.

An dieser Stelle soll, wie in der Einleitung bereits geschehen, nochmals auf die wachsende Bedeutung der optischen Metamaterialien hingewiesen werden. Eine Übersicht über moderne Metamaterialien und ihre Verwendung als Wellenleiter findet sich in [285].

4 Weitere Ausführungen findet man z.B. in [250, 269]. Eine allgemeine und ausführliche Darstellung inklusive Herleitung der Helmholtz-Gleichung aus den Maxwell-Gleichungen findet man in [270]. 

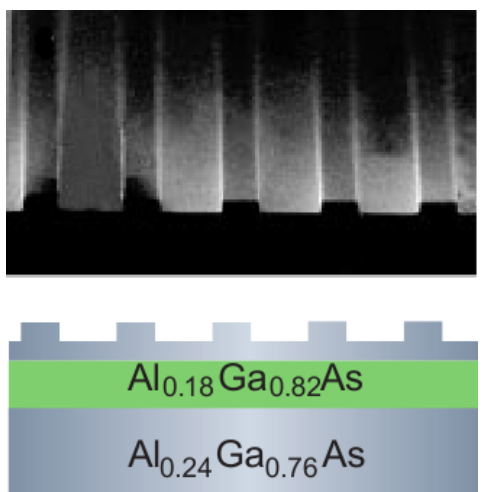

(a)

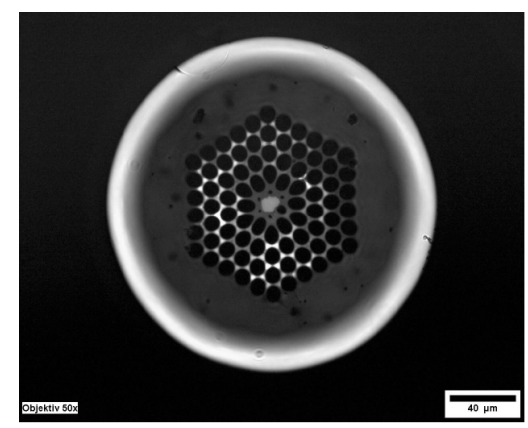

(d)

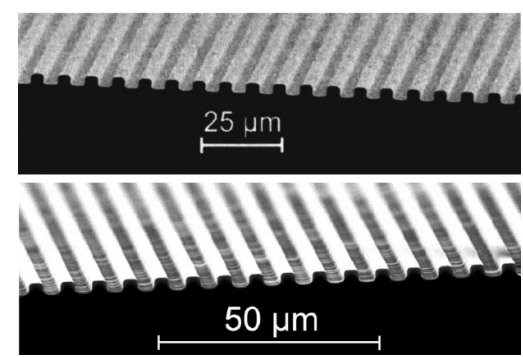

(b)
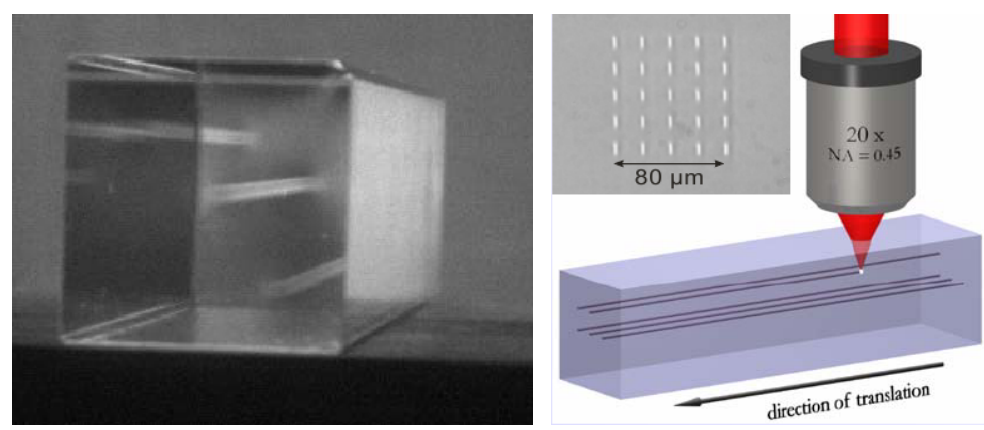

(e)

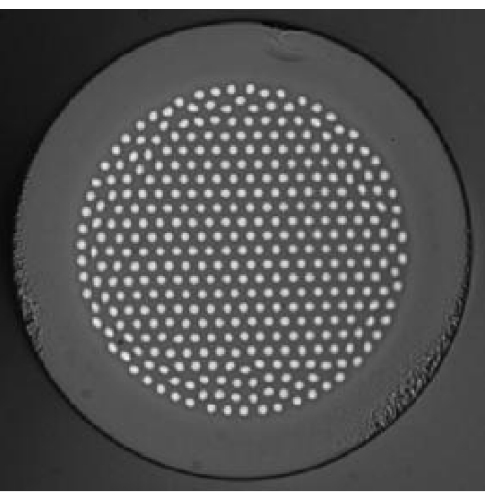

(c)

Abbildung 4.1.: Beispiele einiger elektrischer und optischer Wellenleiter. (a) Oben: Wellenleiterarray bzw. quasi-eindimensionales Übergitter (superlattice) bestehend aus einer $A l_{0.18} G a_{0.82} A s$-Kernschicht und einer $A l_{0.24} G a_{0.76} A s$-Mantelschicht auf einem $\mathrm{GaAs}$-Substrat. Der Abstand zwischen den 41 geätzten Wellenleiterprofilen beträgt etwa $6 \mu \mathrm{m}$ [271, 272]. Unten: schematisches Profil des Wellenleiterarrays. Durch abwechselnde (periodische) Anregung und Absorption mittels gleich- und gegenläufiger Laser in der aktiven Schicht (grün) entsteht so ein komplexes $(\mathcal{P} \mathcal{T}$-symmetrisches) Brechungsindexprofil. Diese Strukturen zählen zur Klasse der DFB-Laser (Distributed-Feedback-Laser) [273]. (b) Polymer-Wellenleiterarray bestehend aus 75 EinzelmodenWellenleitern (vor der Verbindung mit der Polymer-Mantelschicht) auf einem Glassubstrat [274, 275]. (c) Mikroskopische Aufnahme eines Wellenleiterarrays von 400 identischen Faserkernen ( $\varnothing 6.9 \mu \mathrm{m}$, mittlerer Kern-zu-KernAbstand $12.1 \mu \mathrm{m}$, Gesamtdurchmesser $\approx 250 \mu \mathrm{m}$ ) [276, 277]. (d) Mikrophotographie einer Photonenkristallglasfaser mit Glaskern. Der Glaskern im Zentrum ist Ursache für die hohe Nichtlinearität beim Lichttransport [278]. (e) Links: Polariskopabbildung eines synthetischen Quarzglasquaders (Lithosil ${ }^{\circledR}$ ). Die in Transmissionsrichtung sichtbaren Mikrokanäle wurden mit einem Excimerlaser direkt in die 10-20 cm langen Proben geschrieben (siehe schematische Abb. rechts) [279]. 


\subsection{Lokalisierung als Ursache für spontan gebrochene $\mathcal{P} \mathcal{T}$-Symmetrie in 1D}

Wie in der Einführung 4.1 bereits erwähnt ist es oft möglich, Systeme mit $\mathcal{P} \mathcal{T}$-Symmetrie durch pseudo-hermitesche Hamilton-Operatoren $\mathcal{H}$ mathematisch darzustellen. Diese Hamilton-Operatoren weisen dann interessanterweise eine unitäre Zeitentwicklung auf, obwohl sie selbst im Allgemeinen nicht hermitesch sind. Des Weiteren kann nicht selten durch Veränderung eines oder mehrerer Parameter von $\mathcal{H}$ eine spontane $\mathcal{P} \mathcal{T}$-Symmetriebrechung herbeigeführt werden. An diesem Phasenübergang sind die Eigenfunktionen von $\mathcal{H}$ nicht länger Eigenfunktionen des $\mathcal{P} \mathcal{T}$-Operators, obwohl $\mathcal{H}$ und $\mathcal{P} \mathcal{T}$ miteinander kommutieren [286]. Grund hierfür ist die Anti-Linearität des $\mathcal{P} \mathcal{T}$-Operators. Als Resultat erhalten wir ein teilweise oder auch völlig komplexes Eigenspektrum in der gebrochenen $\mathcal{P} \mathcal{T}$-symmetrischen Phase. Im anderen Fall, in dem sowohl $\mathcal{H}$-als auch $\mathcal{P} \mathcal{T}$-Operator die gleiche Basis von Eigenvektoren besitzen, ist das Spektrum reell (exakt $\mathcal{P} \mathcal{T}$-symmetrische Phase).

Im Folgenden wollen wir uns der großen Klasse von Systemen mit lokalisierten Eigenzuständen zuwenden und die spontane $\mathcal{P} \mathcal{T}$-Symmetriebrechung in ihnen untersuchen. Lokalisierte Zustände sind universell in makroskopischen Systemen, denn sie treten an den Kanten bzw. Enden oder an Fehlstellen von sonst perfekten Gittern bzw. Ketten auf. Darum ist es zwingend notwendig, sich mit Systemen zu befassen, die lokalisierte Zustände ausbilden. Selbst mehr als 50 Jahre nach den bahnbrechenden Arbeiten von Anderson [287] bleibt Lokalisierung im Fokus der Forschung, nicht nur in der Festkörperphysik, sondern auch in den Feldern der Physik ultrakalter Atome, Akustik, Mikrowellen und der klassischen Optik und kann so zum Verständnis der grundlegenden Aspekte moderner Physik beitragen.

Lokalisierung ist in eindimensionalen Systemen besonders stark ausgeprägt und deshalb in der Vergangenheit eingehend untersucht worden (siehe z.B. [288] und dort angegebene Referenzen). Wir zeigen am Beispiel verschiedener eindimensionaler Kettenmodelle, dass Lokalisierung zu einer sehr fragilen exakt $\mathcal{P} \mathcal{T}$-symmetrischen Phase führt. Im Einzelnen betrachten wir in den folgenden Abschnitten eine unendliche Kette mit zwei symmetrischen Fehlstellen, eine endliche Kette mit $\mathcal{P} \mathcal{T}$-symmetrischem reellen Unordnungspotenzial und eine halb-unendliche bzw. unendliche Kette mit einem $\mathcal{P} \mathcal{T}$-symmetrischem periodischem Potenzial und zeigen, dass diese Systeme lokalisierte Moden bzw. Oberflächenzustände aufweisen, die den Grund für den Übergang von reellen zu komplexen Eigenwertspektren darstellen.

\subsubsection{Zwei $\mathcal{P} \mathcal{T}$-symmetrische Fehlstellen}

Ein einfaches Modell, das besonders anschaulich den Einfluss von Lokalisierung auf $\mathcal{P} \mathcal{T}$-Symmetrie zeigt, besteht aus einem Paar $\mathcal{P} \mathcal{T}$-symmetrischer Fehlstellen, das in eine perfekte unendliche Kette eingebettet ist (siehe Abb. 4.2). Dieses System kann durch die rekursive Schrödinger-Gleichung ${ }^{5}$

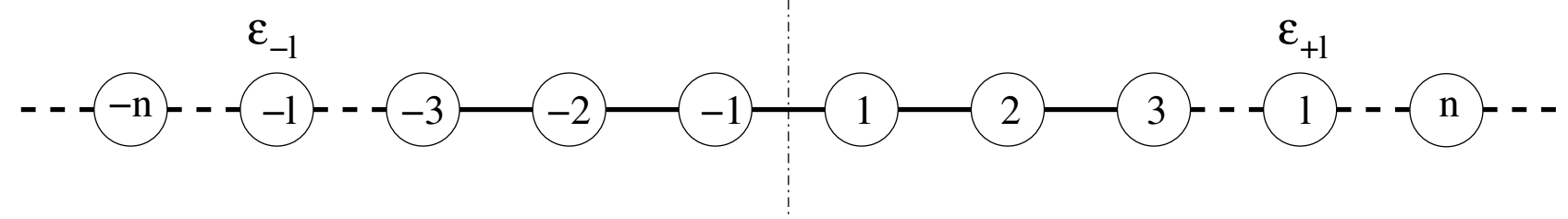

Abbildung 4.2.: Schematische Darstellung einer unendlichen linearen Kette mit zwei komplexwertigen Fehlstellen $\varepsilon_{ \pm l}=\beta_{l} \pm i \gamma_{l}$ am Ort $\pm l$. In der restlichen Kette gilt $\varepsilon_{n}=0$ für $n \neq \pm l$. 


$$
-\psi_{n+1}-\psi_{n-1}=\left(E-\varepsilon_{n}\right) \psi_{n}
$$

wobei $\psi_{n}$ die komplexe Amplitude der Eigenfunktion am Ort $n$ bezeichnet und die Fehlstellen

$$
\varepsilon_{n}= \begin{cases}-\beta+i \gamma & n=+l \\ -\beta-i \gamma & n=-l \\ 0 & \text { sonst }\end{cases}
$$

mit $\beta, \gamma \in \mathbb{R}^{+}$beschrieben werden. Der Ansatz für die lokalisierte Wellenfunktion lautet

$$
\psi_{n}= \begin{cases}A e^{k n} & n \leq-l \\ B e^{k n}+C e^{-k n} & -l \leq n \leq l \\ D e^{-k n} & n \geq l\end{cases}
$$

mit der Bedingung Re $[k]>0$ für exponentiell abklingende Lösungen und der Eigenenergie der lokalisierten Wellenfunktion

$$
E=-2 \cosh (k)
$$

die wir durch Einsetzten von $\psi_{n}=A e^{k n}$ mit $A \neq 0$ in die Schrödinger-Gleichung (4.16) erhalten. Durch die Stetigkeitsforderungen an die Wellenfunktion an den Stellen $n= \pm l$ und Einsetzen der Dispersionsrelation (4.19) in Gleichung (4.16) finden wir diese vier Bedingungen an die komplexwertigen Koeffizienten

$$
\begin{aligned}
& A=B+C e^{2 k l} \\
& B=-e^{-2 k l}\left(1+e^{2 k} C+D e^{k}(E+\beta+i \gamma)\right) \\
& C=-e^{-2 k l}\left(1+e^{2 k} B+A e^{k}(E+\beta-i \gamma)\right) \\
& D=B e^{2 k l}+C
\end{aligned}
$$

Durch Null setzen der Koeffizientendeterminante

$$
\left|\begin{array}{cccc}
1 & -1 & -e^{2 k l} & 0 \\
0 & e^{2 k l} & 1 & -1 \\
-e^{k}\left(e^{k}-\beta-i \gamma\right) & e^{2 k} & e^{2 k l} & 0 \\
0 & e^{2 k l} & e^{2 k} & -e^{k}\left(e^{k}-\beta+i \gamma\right)
\end{array}\right|=0
$$

ergibt sich als Lösung eine transzendente Gleichung für $k$

$$
\begin{array}{cc}
\beta^{2}+\gamma^{2}-4 \beta \sinh (k)+4 \sinh ^{2}(k)=\left(\beta^{2}+\gamma^{2}\right) & e^{-4 k l} \\
& \sinh (k)=\frac{\beta}{2} \pm \frac{1}{2} \sqrt{\left(\beta^{2}+\gamma^{2}\right) e^{-4 k l}-\gamma^{2}}
\end{array} .
$$

5 Wir benutzen die Terminologie der Schrödinger-Gleichung, lassen damit aber Anwendungen der Optik keinesfalls außer Acht. Die Überführung der Schrödinger-Gleichung in eine Helmholtz-Gleichung befindet sich in Kapitel 3, die Verbindung zu optischen Systemen in der Einleitung und in Abschnitt 4.1. 
Im Falle $\gamma=0$ und $\beta l \gg 1$ vereinfacht sich Gleichung (4.22) zu

$$
\beta-2 \sinh (k)= \pm \beta e^{-2 k l} \approx 0
$$

und damit als Lösung 0. Ordnung in $k=k_{0}$

$$
k_{0}=\operatorname{arcsinh}\left(\frac{\beta}{2}\right)
$$

Eine störungstheoretische Betrachtung zweiter Ordnung ${ }^{6}$ in $k$ liefert die Korrekturen $\delta k$

$$
k=k_{0} \pm \delta k \approx \operatorname{arcsinh}\left(\frac{\beta}{2}\right) \pm \frac{\beta}{2} e^{-\beta l}
$$

Unter Zuhilfenahme der Additionstheoreme

$$
\cosh \left(z_{1} \pm z_{2}\right)=\cosh \left(z_{1}\right) \cosh \left(z_{2}\right) \pm \sinh \left(z_{1}\right) \sinh \left(z_{2}\right)
$$

und mit der Dispersionsrelation (4.19) der linearen Kette finden wir für die beiden Energieeigenwerte

$$
\begin{aligned}
E_{ \pm} & =-2 \cosh \left(k_{0} \pm \delta k\right) \\
& =-2\left\{\cosh \left[\operatorname{arcsinh}\left(\frac{\beta}{2}\right)\right] \cosh (\delta k) \pm \frac{\beta}{2} \sinh (\delta k)\right\} \\
& =-\sqrt{4+\beta^{2}} \pm \beta \delta k
\end{aligned}
$$

also zwei gebundene Zustände mit den Energien $E_{ \pm}=E_{0} \pm \frac{1}{2} \delta_{1}$, wobei $E_{0}=-\sqrt{4+\beta^{2}}$ die Energie eines lokalisierten Zustands einer isolierten Fehlstelle in einer unendlichen perfekten linearen Kette ist (siehe Anhang G) und

$$
\delta_{1}=\delta(l)=\beta^{2} e^{-\beta l}
$$

den exponentiell kleinen Energieaufspaltungsterm für das Zwei-Fehlstellen-Problem darstellt. Für $\gamma \neq 0$ und $\beta l \gg 1$ liefert eine analoge Rechnung aus Gleichung (4.22)

$$
\delta k=\frac{1}{2} \sqrt{\beta^{2} e^{-2 \beta l}-\gamma^{2}},
$$

und damit auch die Aussage, dass bereits ein exponentiell kleines $\gamma$ zu komplexen $k$ - bzw. E-Werten führt, was gleichbedeutend mit gebrochener $\mathcal{P} \mathcal{T}$-Symmetrie ist. Die $\mathcal{P} \mathcal{T}$-Symmetrie bricht zusammen, wenn $\gamma$ den kritischen Wert $\gamma_{\mathcal{P} \mathcal{T}} \approx \beta e^{-\beta l}$ erreicht, denn in diesem Fall entarten beide zugehörigen Eigenwerte. Für $\gamma>\gamma_{\mathcal{P} \mathcal{T}}$ verzweigen sich die Eigenwerte in der komplexen Ebene unter dem charakteristischen Verhalten

$$
\operatorname{Im}\left(E_{ \pm}\right) \propto \pm \sqrt{\gamma^{2}-\gamma_{\mathcal{P} \mathcal{T}}^{2}}
$$

Diese Quadratwurzelsingularität scheint eine generische Besonderheit der gebrochenen $\mathcal{P} \mathcal{T}$-Symmetrie zu sein.

6 Eine störungstheoretische Rechnung in erster Ordnung verschwindet aufgrund der $\mathcal{P} \mathcal{T}$-Symmetrie identisch. 
Die Eigenfunktionen erfahren ebenfalls eine charakteristische Änderung. Solange $\gamma<\gamma_{\mathcal{P}} \mathcal{T}$ erfüllt ist, gilt für die Wellenfunktion $\psi_{n}^{*}= \pm \psi_{-n}$. Als messbare Größe für exakte bzw. gebrochene $\mathcal{P} \mathcal{T}$-Symmetrie wollen wir auf das Dipolmoment

$$
D=\sum_{n=-N}^{N} n\left|\psi_{n}\right|^{2}
$$

eines Eigenzustands zurückgreifen. Im Fall $|\gamma| \leq \gamma_{\mathcal{P} \mathcal{T}}$ gilt $D=0$. Für $|\gamma|>\gamma_{\mathcal{P} \mathcal{T}}$ nimmt das Dipolmoment endliche reelle Werte $D \neq 0$ an und stellt somit ein Charakteristikum für die Eigenzustände dar. Genauer ist für $D<0$ ein Eigenzustand $\psi_{n}$ in der linken, für $D>0$ in der rechten Kettenhälfte lokalisiert.

\subsubsection{Die endliche $\mathcal{P} \mathcal{T}$-symmetrische Kette mit Unordnung (Eindimensionales} $\mathcal{P} \mathcal{T}$-Anderson-Modell)

Mit dem Wissen über das $\mathcal{P} \mathcal{T}$-symmetrische Fehlstellenpaar können wir nun zunächst auf die endliche $\mathcal{P} \mathcal{T}$-symmetrische Kette mit Unordnungspotenzial schauen. Hierbei soll die Unordnung so beschaffen sein, dass die $\mathcal{P} \mathcal{T}$-Symmetriebedingung (4.4) weiterhin gilt. Trotzdem wird die $\mathcal{P} \mathcal{T}$-symmetrische Phase mit wachsender Unordnung schrittweise zerstört. Für hinreichend große Unordnung verkleinert sich diese Phase zu einem exponentiell schmalen Bereich, selbst für vergleichsweise kleine Systeme. Das Modell der $\mathcal{P} \mathcal{T}$-symmetrischen linearen Kette mit Zufallspotenzial wird durch die SchrödingerGleichung

$$
-\psi_{n+1}-\psi_{n-1}=\left(E-\varepsilon_{n}\right) \psi_{n}
$$

beschrieben. Die $\varepsilon_{n} \in \mathbb{C}$ sind komplexe Zufallszahlen, wobei die Bedingung (4.4) für das Potenzial, also

$$
\varepsilon_{n}=\varepsilon_{-n}^{*}
$$

für $n \in[-N, N], N \in \mathbb{N}^{+}$gilt, wir somit eine $\mathcal{P} \mathcal{T}$-symmetrische Kette der Länge $2 N+1$ definieren. Für die Unordnung selbst sind verschiedene Realisierungen vorstellbar: rein reelle (in $\beta_{n}$ ) oder rein imaginäre (in $\gamma_{n}$ ) oder komplexe Unordnung (in $\varepsilon_{n}=\beta_{n}+i \gamma_{n}$ ). Wir wollen uns hier vorerst auf Unordnung in $\beta_{n}$ beschränken. ${ }^{7}$ Wir ziehen die $\beta_{n}(n \geq 0)$ aus einem gleichverteilten Intervall $I=$ $\left[-\frac{\beta}{2}, \frac{\beta}{2}\right]$ und setzen $\gamma_{n}=\gamma=$ const. (für $n \geq 1$ ). Gleichung (4.33) beinhaltet folgende Bedingungen an die Potenziale

$$
\begin{aligned}
\beta_{n} & =\beta_{-n} \\
\gamma_{n} & =-\gamma_{-n} \\
\gamma_{0} & =0
\end{aligned}
$$

wobei die $\mathcal{P} \mathcal{T}$-Symmetriebedingungen (4.34a) und (4.34b) langreichweitige Korrelationen implizieren. Um diesen Sachverhalt zu klären, beginnen wir mit dem hermiteschen Problem $\gamma=0$ und setzen eine lange Kette voraus, so dass selbst Eigenzustände im Zentrum des Eigenenergiespektrums lokalisiert sind. Das ist gleichbedeutend mit einer Lokalisierungslänge, die kleiner als die Systemlänge $2 N+1$

7 Am Ende dieses Abschnitts und in Anhang I werden Fluktuationen in den anderen Parametern angesprochen. 


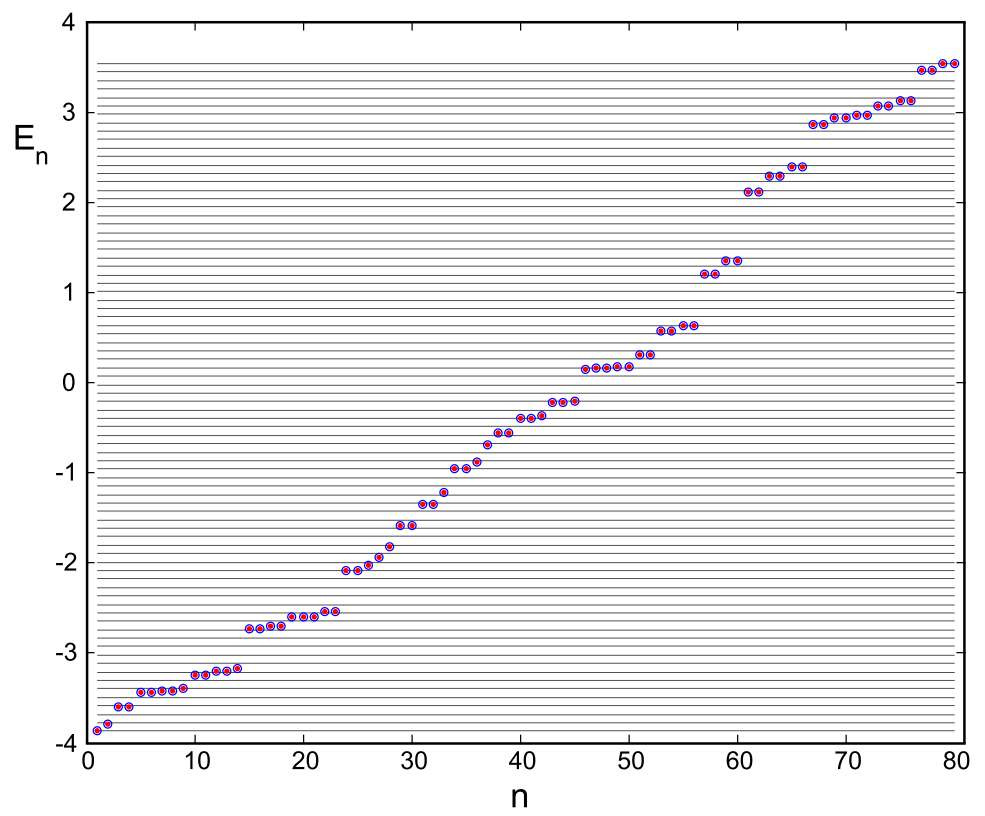

Abbildung 4.3.: Energiespektrum einer Beispielrealisierung für eine perfekte $\mathcal{P} \mathcal{T}$-symmetrische Kette mit $N=80$ Fehlstellen. Dabei nummeriert $n$ die Energieeigenwerte und $E_{n}$ bezeichnet ihren Realteil. Es existiert eine exponentiell kleine Energieaufspaltung $\delta$ zwischen den beiden Eigenwerten eines Doublets (zusammengehörige Eigenwerte eines solchen Doublets sind jeweils durch eine graue waagerecht verlaufenden Linien gekennzeichnet), wobei viel größere Abstände $\Delta$ zwischen benachbarten Doublets zu beobachten sind.

ist. Wenn man für einen Moment annimmt, dass die Kette in ihrer Mitte $n=0$ in zwei Teile getrennt wird, dann ist ein systemtypischer Zustand in der einen Hälfte der Kette lokalisiert, jedoch weit entfernt vom neuen Rand $n=0$, bei $l \gg 1$, mit der Lokalisierungslänge $\xi$. Dieser Zustand hat seinen Gegenspieler wegen der $\mathcal{P} \mathcal{T}$-Symmetriebedingung in der anderen Kettenhälfte, bei $-l$. In der wiedervereinigten Kette hat dieses Paar von lokalisierten Zuständen einen exponentiell schmalen Überlapp im Zentrum der Kette $(n=0)$. Das bedeutet für die Gesamtkette die Existenz von zwei reellen fast entarteten Eigenzuständen. Jeder dieser Eigenzustände (einer symmetrisch, einer antisymmetrisch) hat zwei Scheitelpunkte in der Nähe der Orte $\pm l$. Die Energieaufspaltung zwischen den beiden Eigenwerten ist exponentiell klein, $\delta \simeq e^{-2 l / \xi}$, in Analogie zum Modell der beiden Fehlstellen in einer perfekten Kette aus Unterabschnitt 4.2.1. Demzufolge sind die Eigenzustände in einer $\mathcal{P}$-symmetrischen Kette mit Unordnung in Paare (Doublets) unterteilbar, wobei die Energieaufspaltungen entsprechend

$$
\delta_{1}<\delta_{2}<\delta_{3}<\ldots
$$

angeordnet sind. Die Energieaufspaltung zwischen zwei Zuständen eines Doublets ist exponentiell klein, während die Energiedifferenz zwischen zwei benachbarten Doublets viel größer ist und zwar in der Größenordnung des Abstands der Energieniveaus $\Delta$ der halben (perfekten) Kette (siehe Abbildung 4.3). Das Doublet mit der kleinsten Energieaufspaltung $\delta_{1}$ stammt von Zuständen, die weit entfernt vom Zentrum der Kette $(n=0)$ lokalisiert sind, mit Energien nahe der Energiebandkante (und kleiner Lokalisierungslänge $\xi$ ). Durch Hinzunahme von $\gamma>0$ bleibt anfänglich die $\mathcal{P} \mathcal{T}$-symmetrische Phase erhalten (siehe Abb. 4.4a,b). Bei $\gamma=\gamma_{\mathcal{P} \mathcal{T}} \simeq \delta_{1}$ kreuzen sich die Energieniveaus der beiden lokalisierten Zustände, was die $\mathcal{P} \mathcal{T}$-Symmetrie bricht (siehe Abb.4.5a) Bei weiterer Vergrößerung von $\gamma>\gamma_{\mathcal{P}} \mathcal{T}$ sind die soeben behandelten Zustände keine Eigenzustände des $\mathcal{P} \mathcal{T}$-Operators mehr. Stattdessen verschiebt sich das Gewicht der Zustände weg von den Lokalisierungszentren. Ein Beispiel für ein solches 


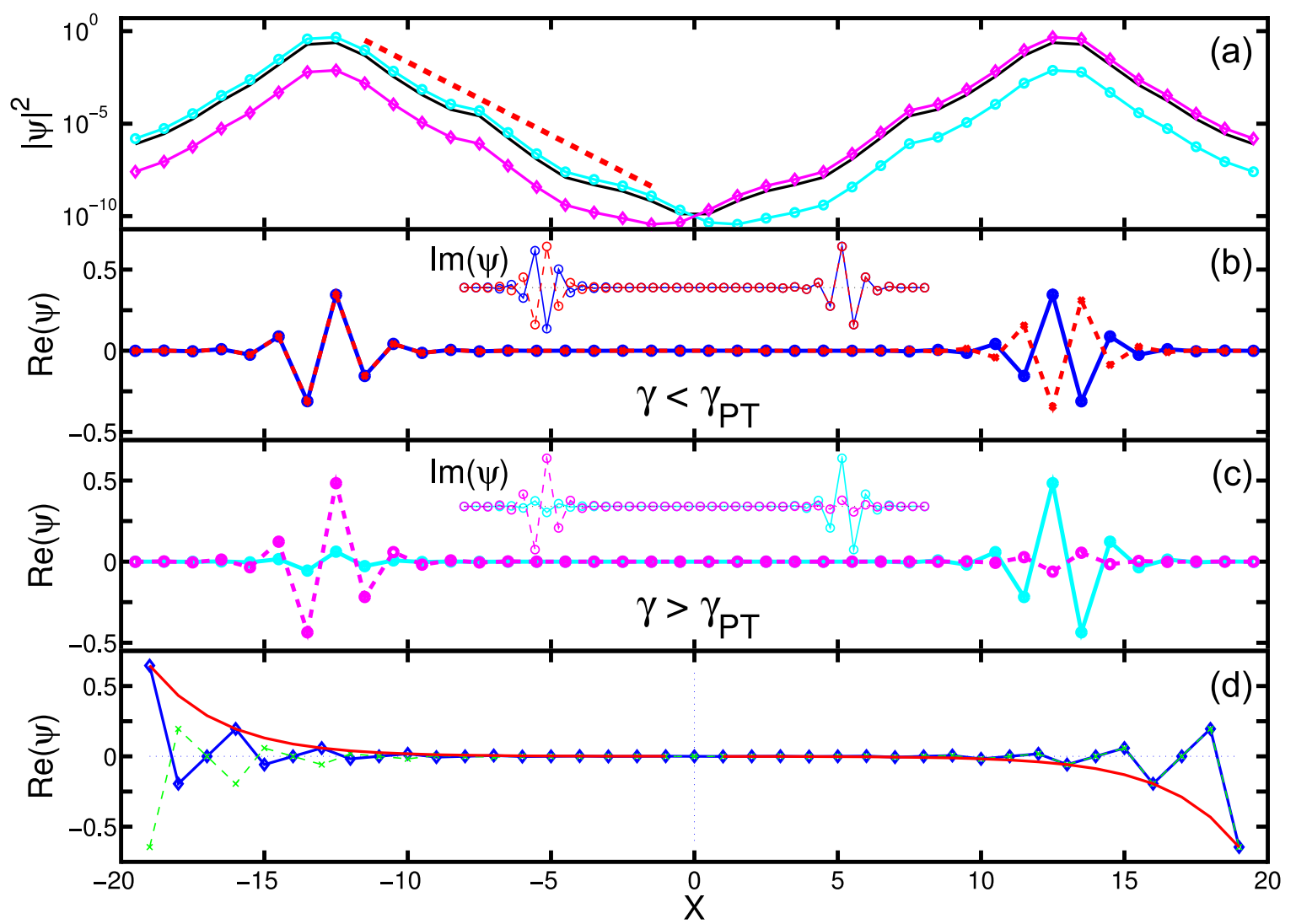

Abbildung 4.4.: Paare von exponentiell lokalisierten Moden in einer $\mathcal{P} \mathcal{T}$-symmetrischen Kette mit reeller Unordnung [(a)-(c)] und Oberflächenzustände einer $\mathcal{P} \mathcal{T}$-symmetrischen Kette mit periodischem Potenzial (d), die jeweils die kleinste Energieaufspaltung $\delta_{1}$ liefern für $\beta=3$. (a) Aufenthaltswahrscheinlichkeit (die rote gestrichelte Linie veranschaulicht den exponentiellen Abfall) und (b) Real- und Imaginärteil von zwei lokalisierten Zuständen für $\gamma<\gamma_{\mathcal{P} \mathcal{T}}$. (c) Für $\gamma>\gamma_{\mathcal{P} \mathcal{T}}$ sind die zwei Eigenzustände nicht länger $\mathcal{P} \mathcal{T}$-symmetrisch. (d) Oberflächenzustände mit exponentieller Lokalisierung. Die durchgezogene rote Linie dient der Veranschaulichung.

Doublet mit kleinster Energieaufspaltung $\delta_{1}$ ist in Abbildung 4.4a,c dargestellt. Für anwachsendes $\gamma$ kreuzen sich die Energieniveaus des nächsten Doublets, verknüpft mit der nächstgrößeren Energieaufspaltung $\delta_{2}$ (siehe Abb. 4.5a). Dieses Szenario kann für alle Eigenwertpaare mit Energieaufspaltung $\delta_{i}$ fortgeführt werden.

Alle qualitativen Aussagen dieses Abschnitts beziehen sich auf eine Realisierung eines Zufallspotenzials. Wir können nun einen Schritt weiter gehen und die Verteilungsfunktion $\mathrm{P}\left(\gamma_{\mathcal{P}}\right)$ zum kritischen Wert $\gamma_{\mathcal{P} \mathcal{T}}$ untersuchen, da dieser von Realisierung zu Realisierung fluktuiert. Wie wir bereits zeigten, gilt $\gamma_{\mathcal{P T}} \propto \delta_{1}$ (siehe Unterabbildung 4.5a). Damit reduziert sich das Problem auf die Untersuchung der Verteilungsfunktion $\mathrm{P}\left(\delta_{1}\right)$.

Es gibt verschiedene Quellen der Fluktuationen in $\delta_{1}$ : Fluktuationen in Ort und Energie der relevanten lokalisierten Zustände, ebenso wie Fluktuationen der Wellenfunktionen selbst. Von den Ausläufern fluktuierender lokalisierter Zustände - weit vom Zentrum der Kette entfernt - weiß man, dass sie starken Lognormal-Schwankungen unterworfen sind [289]. Diese Fluktuationen dominieren, und wir erhalten daraus eine Lognormal-Verteilung für $\delta_{1}$ (siehe Abb. 4.5b), da $\delta_{1}$ proportional zu den Überlappintegralen eines Paares aus weit entfernten und stark lokalisierten Zuständen ist.

Das oben erwähnte Szenario von aufeinanderfolgenden Bifurkationen, d.h. das aufeinanderfolgende Verzweigen von Eigenwertpaaren in die komplexe Ebene, kann ebenfalls quantitativ untersucht wer- 

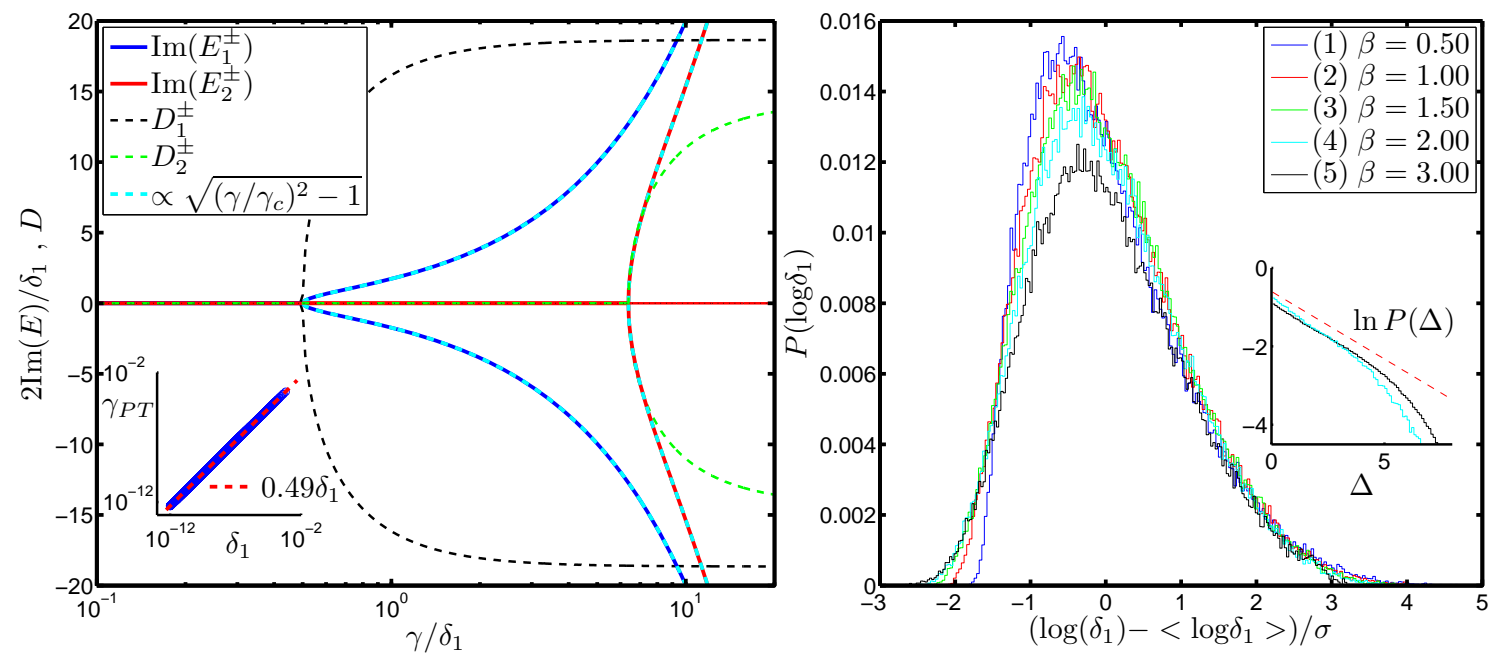

Abbildung 4.5.: Bifurkationsszenario des Dipolmoments und Verteilungsfunktion der kleinsten Energieaufspaltung im Fall starker Unordnung. (a) Bifurkationen für das Dipolmoment $D$ und für den Imaginärteil der Energieniveaus $\operatorname{Im}[E]$ für eine $\mathcal{P} \mathcal{T}$-symmetrische Kette mit $\gamma=$ const. und aus einem Intervall $[-\beta / 2, \beta / 2]$ gleichverteilter Zufallszahlen gezogener $\beta_{n}$ im Fall $N / \xi \gg 1$ (starke Unordnung). Die ersten beiden Bifurkationen (zugehörige Energieaufspaltungen $\delta_{1}$ und $\delta_{2}$ ) sind dargestellt. Die zyanfarbenen gestrichelten Linien geben das Quadratwurzelverhalten der Bifurkationen am jeweiligen Verzweigungspunkt wieder (siehe Text). Die Unterabbildung zeigt das lineare Verhalten von $\gamma_{\mathcal{P} \mathcal{T}}$ mit $\delta_{1}$ über mehr als 10 Größenordnungen. (b) Verteilungsfunktion $\mathrm{P}(x)$ der reskalierten ersten Energieaufspaltung $x \equiv\left(\log \delta_{1}-\left\langle\log \delta_{1}\right\rangle\right) / \sigma$ (mit $\sigma$ als Standardabweichung) für 5 verschiedene Werte von $\beta$ mit jeweils $10^{7}$ Realisierungen. Im Limes großer $\beta$ finden wir eine Lognormalverteilung. Die Unterabbildung zeigt die Verteilungsfunktion $\mathrm{P}(\Delta)$ mit $\Delta \equiv \log \delta_{2}-\log \delta_{1}$ in halb-logarithmischer Darstellung. Hier finden wir eine Poisson-Verteilung für große $\beta$.

den. Betrachtet man den Abstand $\delta \gamma$ zwischen der ersten Bifurkation $\left(\gamma=\gamma_{\mathcal{P} \mathcal{T}}\right)$ und der nächsten entlang der $\gamma$-Achse, so ist dieses $\delta \gamma$ proportional zu $\left(\delta_{2}-\delta_{1}\right)$. Unter der Annahme, dass die lokalisierten Doubletzustände zufällig verteilt sind, können wir eine Poisson-Verteilung für $\Delta \equiv \log \delta_{2}-\log \delta_{1}$ mit mittlerem Abstand $2 / \xi$ auf der $\log (\gamma)$-Skala zwischen den Bifurkationen vorhersagen (siehe Unterabbildung 4.5b).

Unsere Betrachtungen können auf den Fall $N / \xi \ll 1$ (schwache Unordnung) erweitert werden, wenn die mit $\delta_{1}$ verknüpften Zustände über die gesamte Kette ausgedehnt sind. In diesem Fall ist allerdings das Bild von Doublets mit exponentiell kleiner Energieaufspaltung ungültig, weiterhin jedoch gilt die Proportionalität zwischen dem kritischen Wert $\gamma_{\mathcal{P} \mathcal{T}}$ und dem kleinsten Energieniveauabstand $\delta_{1} \equiv \Delta_{\text {min }}$ des hermiteschen Systems. Betrachtet man die Dispersionsrelation für eine ideale lineare Kette (wie in Abb. 4.2 auf Seite 96, jedoch mit Null-Potenzial $\left.\forall n: \varepsilon_{n}=0\right)$ in Hückel-Näherung

$$
E_{n}=2 \cos \left(\frac{[n-N] \pi}{2 N+1}\right)
$$

für $n \in[-N, N], N \in \mathbb{N}^{+}, N \gg 1$ (siehe Abb. 4.6a), so ergibt sich für den Energieniveauabstand der Eigenwerte $n+1$ und $n$

$$
\Delta_{n}=2\left[\cos \left(\frac{[n-N+1] \pi}{2 N+1}\right)-\cos \left(\frac{[n-N] \pi}{2 N+1}\right)\right]
$$

Die Differentiation von $\Delta_{n}$ liefert ein Maximum $\Delta_{\max }$ im Zentrum der Eigenwertspektrums, während 


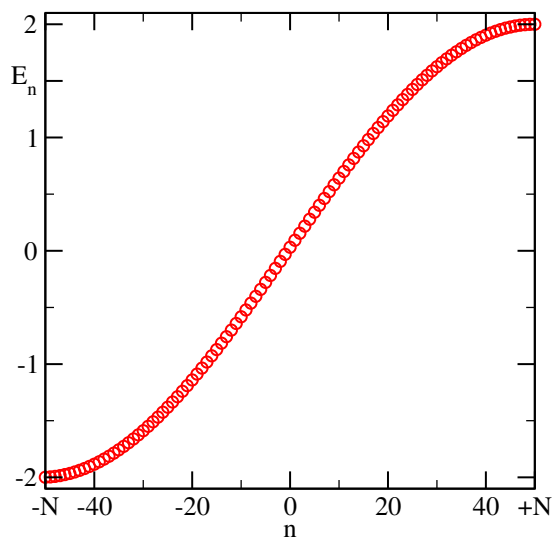

(a)

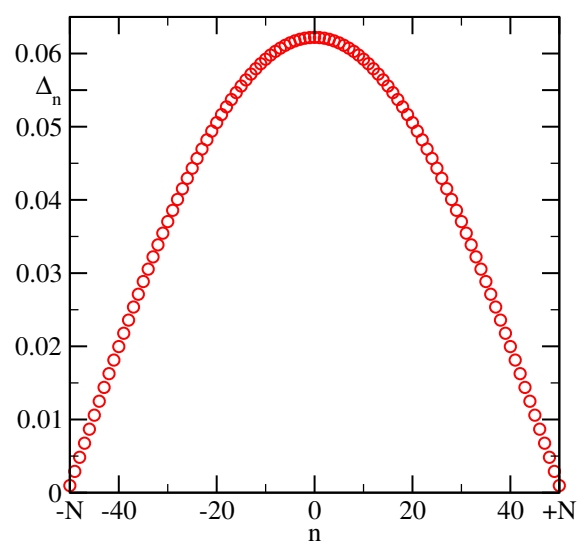

(b)

Abbildung 4.6.: Dispersionsrelation und Energieniveauabstände für eine perfekte lineare Kette mit $M=$ $2 N+1=101$ Gitterpunkten. (a) Dispersionsrelation in Hückel-Näherung (siehe Gleichung (4.36)). (b) Energieniveauabstände $\Delta_{n}$ mit Maximum $\Delta_{\max }$ bei $n=0$ und Minima $\Delta_{\min }$ bei $n= \pm N$.

an den Rändern des Eigenwertspektrums zwei Minima $\Delta_{\text {min }}$ auftreten (siehe Abb. 4.6b). Die Abschätzung der Größenordnungen lauten für das Maximum des Energieniveauabstands im Zentrum des Energiebands

$$
\begin{aligned}
\Delta_{\max } & =2\left[\cos \left(\frac{[1-N] \pi}{2 N+1}\right)-\cos \left(\frac{N \pi}{2 N+1}\right)\right] \\
\left.\cos (x)\right|_{\frac{\pi}{2}} ^{=} \approx \frac{\pi}{2}-x & \frac{2 \pi}{2 N+1} \propto \frac{1}{N}
\end{aligned}
$$

und für die Minima an den Bandkanten

$$
\begin{array}{cll}
\delta_{1} \equiv \Delta_{\min } & = & 2\left[\cos \left(\frac{[1-2 N] \pi}{2 N+1}\right)-\cos \left(\frac{2 N \pi}{2 N+1}\right)\right] \\
& \stackrel{\left.\cos (x)\right|_{0 \approx 1-\frac{x^{2}}{2}}}{=} & 2\left(\frac{\pi}{2 N+1}\right)^{2} \propto \frac{1}{N^{2}}
\end{array}
$$

Für Ketten mit $N \gg 1$ ist also der kleinste Energieniveauabstand $\delta_{1} \equiv \Delta_{\min } \simeq 1 / N^{2}$. Eine Störungstheorie für kleine $\gamma$ liefert Energieverschiebungen proportional zu $\gamma^{2}$ (Korrekturen aus erster Ordnung verschwinden aus Gründen der $\mathcal{P} \mathcal{T}$-Symmetrie). Die Störungstheorie bricht für $\gamma=\gamma_{\mathcal{P} \mathcal{T}} \simeq \delta_{1}$ zusammen, da hier die Energieniveaus kreuzen und somit das erste Eigenenergiewertepaar in die komplexe Ebene verzweigt. Deshalb unterscheidet sich der Schwellwert für den $\mathcal{P} \mathcal{T}$-Phasenübergang für $N / \xi \ll 1$ (schwache Unordnung) mit $\gamma_{\mathcal{P} \mathcal{T}} \propto 1 / N^{2}$ von dem für $N / \xi \gg 1$ (starke Unordnung) mit $\gamma_{\mathcal{P} \mathcal{T}} \propto e^{-2 N / \xi}$, jedoch verschwindet auch hier für große Systeme die $\mathcal{P} \mathcal{T}$-symmetrische Phase.

Die Bifurkationen und ihre Charakteristik - das quadratwurzelartige Aufspalten der komplexen Energieeigenwerte - ist in beiden Fällen gleich, wobei die Energieskalen unterschiedlich sind, wie oben bereits erwähnt. Unsere numerischen Ergebnisse aus Abbildung 4.7a bestätigen unsere theoretischen Ausführungen hervorragend. Wir wollen auch für den Fall $N / \xi \ll 1$ (schwache Unordnung) die Verteilungsfunktion $\mathrm{P}\left(\gamma_{\mathcal{P} \mathcal{T}}\right)$ untersuchen. Hierzu berufen wir uns erneut auf die Störungstheorie hinsichtlich der linearen Kette. Gegeben sei eine perfekte lineare Kette der Länge $2 N+1$ mit reellem Unordnungspotenzial $\beta_{n} \ll \Delta_{\min }$ unter der Bedingung (4.34a) $\left(\beta_{n}=\beta_{-n}\right)$ und $\gamma_{n}=0$, wobei wir die Störung 

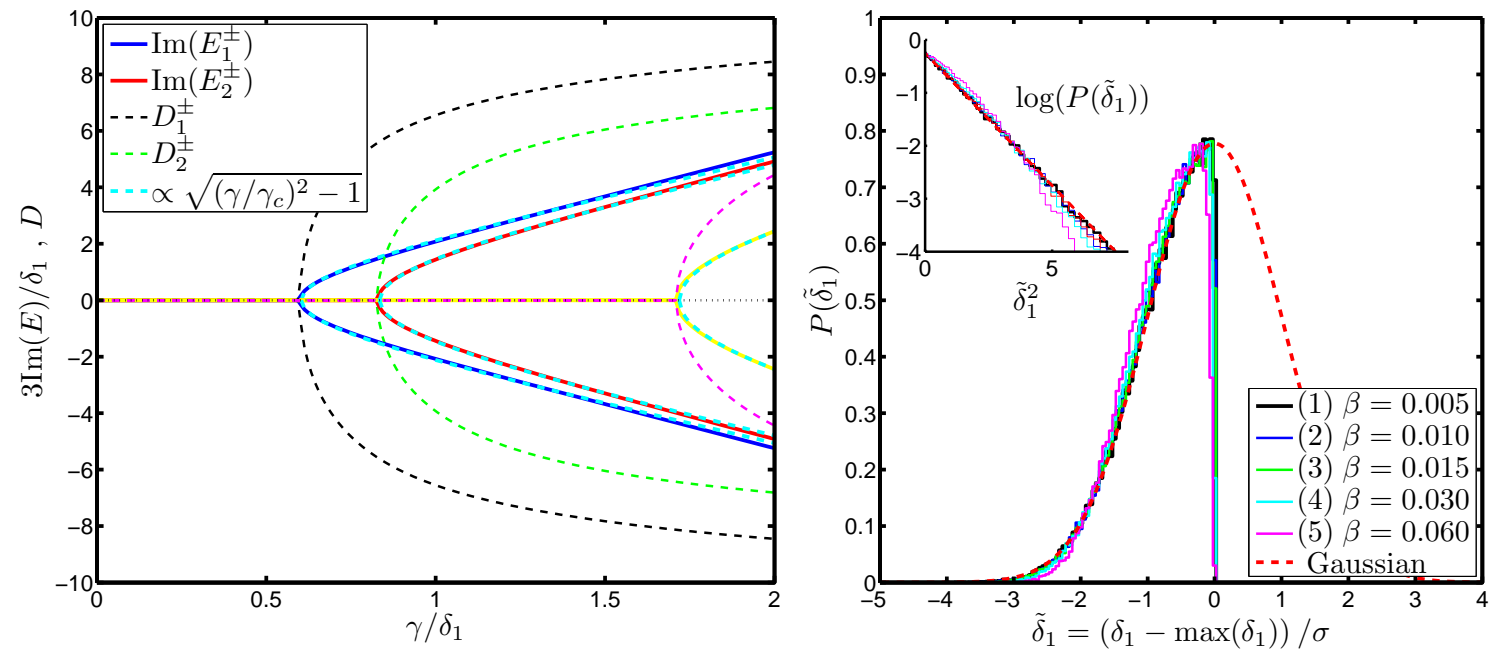

Abbildung 4.7.: Bifurkationsszenario des Dipolmoments und Verteilungsfunktion der kleinsten Energieaufspaltung im Fall schwacher Unordnung. (a) Bifurkationen für das Dipolmoment $D$ und für den Imaginärteil der Energieniveaus $\operatorname{Im}[E]$ für eine $\mathcal{P} \mathcal{T}$-symmetrische Kette mit $\gamma=$ const. und aus einem Intervall $[-\beta / 2, \beta / 2]$ gleichverteilter Zufallszahlen gezogener $\beta_{n}$ mit $N / \xi \ll 1$ (Fall "schwacher" Unordnung). Die ersten drei Bifurkationen (zugehörige Energieaufspaltungen $\delta_{1}, \delta_{2}$ und $\delta_{3}$ ) sind dargestellt. Die zyanfarbenen gestrichelten Linien geben das Quadratwurzelverhalten der Bifurkationen am jeweiligen Verzweigungspunkt wieder (siehe Text).

(b) Verteilungsfunktion $\mathrm{P}(\tilde{\Delta})$ der reskalierten ersten Energieaufspaltung $\widetilde{\Delta} \equiv \Delta \delta / \sigma$ (mit $\sigma$ als Standardabweichung) für 5 verschiedene Werte von $\beta$ mit jeweils $10^{7}$ Realisierungen. Die Verteilungsfunktion hat eine obere Grenze bei $\widetilde{\Delta}=0$. Zum Vergleich ist eine Gauß-Verteilung mit dargestellt (rote gestrichelte Linie). Die Unterabbildung zeigt die gleiche Verteilungsfunktion $\mathrm{P}(\widetilde{\Delta})$ in halb-logarithmischer Darstellung über $\widetilde{\Delta}^{2}$.

der kleinsten Energieniveauabstände mit $\delta^{(1)}=\Delta_{\min } \pm \Delta \delta_{1}$ bezeichnen wollen. Die Lösungen des ungestörten Falls sind Wellenfunktionen

$$
\psi_{j}(n)=\sqrt{\frac{2}{2 N+1}} \sin \left(\frac{n \pi j}{2 N+1}\right)
$$

mit $n \in[-N, N], N \in \mathbb{N}^{+}$. Mit der Bedingung $\beta_{n}=\beta_{-n}$ ergibt sich für die Korrekturen erster und zweiter Ordnung an der unteren Bandkante

$$
\Delta \delta^{u b k}=2 \cdot \frac{2}{2 N+1} \sum_{n=1}^{N} \beta_{n}\left[\sin ^{2}\left(2 \cdot \frac{[n+N] \pi}{2 N+1}\right)-\sin ^{2}\left(\frac{[n+N] \pi}{2 N+1}\right)\right]
$$

Mit den Additionstheoremen $\left.\sin ^{2}(\alpha)=[1-\cos (2 \alpha)]\right) / 2$ und $\cos (\alpha) \cos (\beta)=-2 \sin \left(\frac{\alpha+\beta}{2}\right) \sin \left(\frac{\alpha-\beta}{2}\right)$ ergibt sich

$$
\Delta \delta^{u b k}=+\frac{4}{2 N+1} \sum_{n=1}^{N} A_{n} \beta_{n}
$$

mit $A_{n}=\sin \left(3 \frac{[n+N] \pi}{2 N+1}\right) \sin \left(\frac{[n+N] \pi}{2 N+1}\right)$. Wir finden analog für die obere Bandkante

$$
\Delta \delta^{o b k}=-\frac{4}{2 N+1} \sum_{n=1}^{N} A_{n} \beta_{n}
$$




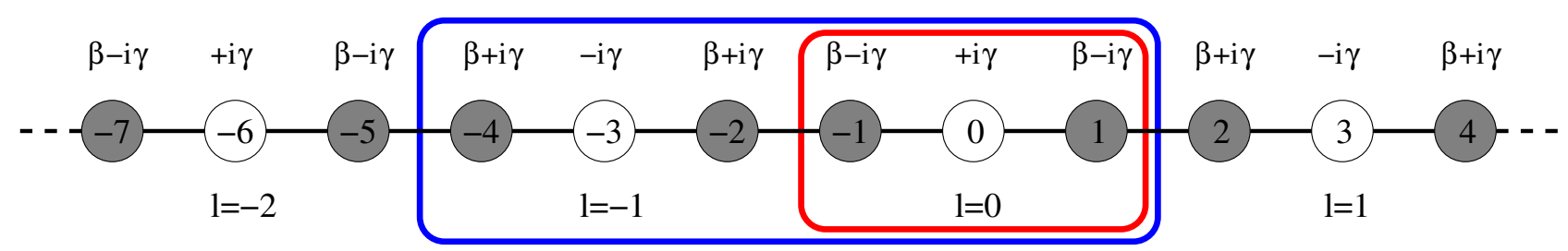

Abbildung 4.8.: Schematische Darstellung einer periodischen Kette mit den Potenzialbedingungen (4.45). Blau umrandet: Einheitszelle des komplexen periodischen Potenzials $(\gamma \neq 0)$. Rot umrandet: Einheitszelle des reellen periodischen Potenzials $(\gamma=0)$.

d.h. verkleinert sich der Energieniveauabstand an der unteren Bandkante, so vergrößert er sich in gleichem Maße an der oberen Bandkante und umgekehrt. Es gilt $\Delta \delta^{u b k}=-\Delta \delta^{o b k}$, und so kann wegen

$$
\delta^{(1)}=\Delta_{\min }-\max \left\{\Delta \delta^{u b k}, \Delta \delta^{o b k}\right\}=\Delta_{\min }-\left|\delta^{u b k}\right|
$$

immer eine Verkleinerung des kleinsten Energieniveauabstands $\delta^{(1)}$ gefolgert werden. Wenn $\beta_{n}$ einer Gauß-Verteilung folgt $\left(\beta_{n} \in\left[-\frac{\beta}{2}, \frac{\beta}{2}\right]\right.$ ), so folgt für $\mathrm{P}(\delta)$ eine halbe Gauß-Verteilung (Gauß-Verteilung mit scharfer oberer Grenze am Symmetriezentrum der Verteilung) für $\delta<\Delta_{\text {min. }}$. Für eine große Anzahl von Realisierungen sichert der zentrale Grenzwertsatz das Ergebnis einer Gauß-Verteilung für gutartige Verteilungsfunktionen, beispielsweise für aus einer Gleichverteilung gezogene $\beta_{n}$ [290]. Das geschilderte Verhalten wird durch einen Vergleich mit unseren numerischen Untersuchungen exzellent bestätigt.

\subsubsection{Periodische $\mathcal{P} \mathcal{T}$-symmetrische Potenziale}

Die Frage der $\mathcal{P} \mathcal{T}$-Symmetriebrechung in $\mathcal{P} \mathcal{T}$-symmetrischen periodischen Potenzialen wurde in [250] anhand des Potenzials $V(x)=4\left[\cos ^{2}(x)+i V_{0} \sin (2 x)\right]$ behandelt ${ }^{8}$. Hier wurde ein kritischer Wert $V_{0}=\frac{1}{2}$ gefunden, wobei die Anwesenheit des reellen Anteils des Potenzials $A \cos ^{2}(x)$ mit $A \neq 0$ von essenzieller Bedeutung ist. Fehlt der reelle Term - dies wurde in [242] für Potenziale der Form $V(x)=i V_{0} \sin ^{2 N+1}(x)$ untersucht - weisen diese Potenziale kein reelles Spektrum auf, unabhängig vom Wert $V_{0}$. Andere Beispiele für Potenziale mit vollständig komplexem Spektrum findet man in [245].

In periodischen Systemen endlicher Größe findet man jedoch lokalisierte Zustände an den Systemgrenzen (Oberflächenzustände) ${ }^{9}$ [291, 292]. Diese Zustände stellen sich als entscheidend für die Berechnung des kritischen $\mathcal{P} \mathcal{T}$-Schwellwerts heraus. Wir wollen die Bedeutung der Oberflächenzustände für die Symmetriebrechung anhand des folgenden periodischen (tight-binding) Potenzials veranschaulichen. Als Einheitszelle wählen wir 3 Gitterpunkte, deren periodisches Potenzial

$$
\varepsilon_{3 l}=0 ; \varepsilon_{3 l \pm 1}=\beta \pm i \gamma
$$

mit $l=0, \pm 1, \pm 2, \ldots, \pm \frac{N}{3}, \mathbb{N}^{+} \ni N \mid 3$ beträgt (siehe Abb. 4.8). Für die halb-unendliche Kette $0 \leq l \leq \frac{N}{3}$ im Fall $\gamma=0$ lässt sich das Problem der Oberflächenzustände exakt lösen. In diesem Fall lauten die

8 Das Potenzial $V(x)=A\left[\cos ^{2}(x)+i V_{0} \sin (2 x)\right]$ dient als theoretisches Modell einer möglichen optischen Realisierung. Wir werden im Abschnitt 4.4 genauer darauf eingehen.

9 Oberflächenzustände heißen historisch bedingt Shockley-Zustände als direkte Lösungen der Schrödinger-Gleichung, bzw. Tamm-Zustände als Lösungen von tight-binding Problemen. 
Schrödinger-Gleichungen für die Einheitszelle $l$

$$
\begin{aligned}
-\psi_{3 l+1}-\psi_{3 l-1} & =E \psi_{3 l} \\
-\psi_{3 l+2}-\psi_{3 l}+\beta \psi_{3 l+1} & =E \psi_{3 l+1} \\
-\psi_{3 l}-\psi_{3 l-2}+\beta \psi_{3 l-1} & =E \psi_{3 l-1} .
\end{aligned}
$$

Mit dem Ansatz für die gesuchten Oberflächenzustände

$$
\begin{aligned}
\psi_{3 l} & =A_{0} e^{-3 k l} \\
\psi_{3 l+1} & =A_{1} e^{-k(3 l+1)} \\
\psi_{3 l-1} & =A_{-1} e^{-k(3 l-1)}
\end{aligned}
$$

wobei $\operatorname{Re}[k]>0$ gelten muss, liefern die Stetigkeitsbedingungen an (4.46a), (4.46b) und (4.46c) die Koeffizientendeterminante für die Amplituden

$$
\left|\begin{array}{ccc}
E & e^{-k} & e^{k} \\
e^{k} & E-\beta & e^{-k} \\
e^{-k} & e^{k} & E-\beta
\end{array}\right|=0
$$

die Null gesetzt eine kubische Bestimmungsgleichung

$$
E\left[(E-\beta)^{2}-3\right]+2 \beta+2 \cosh (3 k)=0
$$

für die Energie liefert. Nach Substitution von $y \equiv E-\beta=e^{-3 k}$ finden wir eine quartische Gleichung in $y$, die sich als Produkt zweier quadratischer Gleichungen schreiben lässt

$$
\left(y^{2}-1\right)\left(y^{2}-\beta y-1\right)=0
$$

Nach Rücksubstitution von $y$ finden wir als eindeutige Lösung

$$
E=\frac{1}{2}\left(\beta+\sqrt{4+\beta^{2}}\right)
$$

für die Energie eines Oberflächenzustands, wobei die anderen drei Lösungen negative oder triviale (d.h. $\operatorname{Re}[k] \leq 0)$ inverse Lokalisierungslängen $k$ liefern, das aber im Widerspruch zum Ansatz (4.47) mit Re $[k]>0$ steht. Somit folgt für die Abklingrate

$$
\begin{aligned}
k & =-\frac{1}{3} \log [E-\beta] \\
& =-\frac{1}{3} \log \left[\frac{1}{2}\left(\sqrt{4+\beta^{2}}-\beta\right)\right] .
\end{aligned}
$$

Für die Amplituden aus Gleichung (4.47) finden wir

$$
A_{1}=0 ; A_{0}= \pm \frac{1}{\kappa} e^{5 k / 2}, A_{-1}=\mp \frac{1}{\kappa} e^{k / 2}
$$




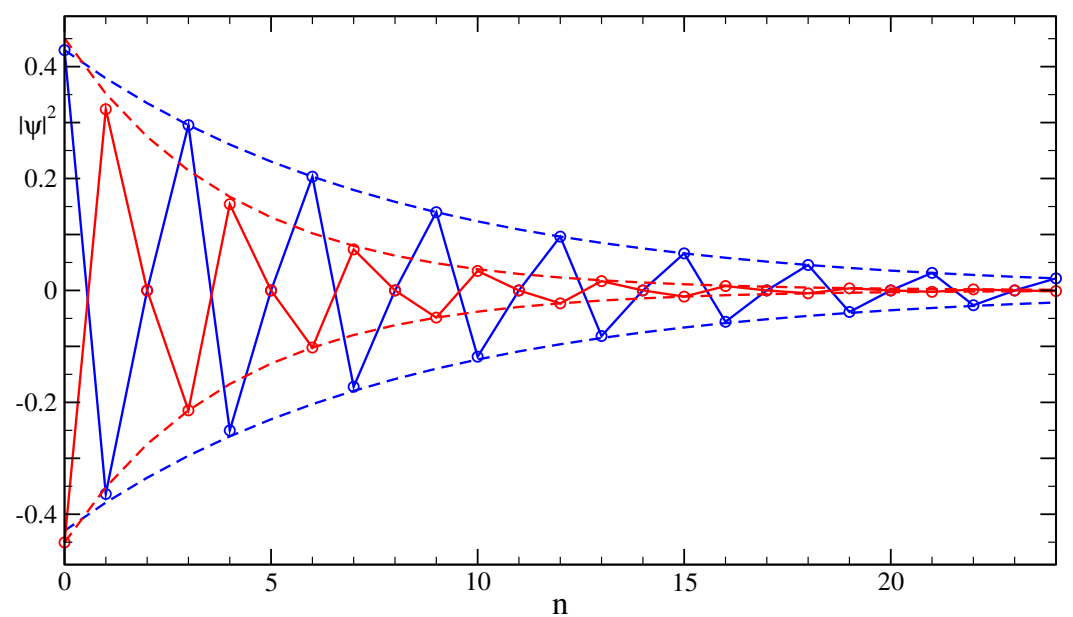

(a)

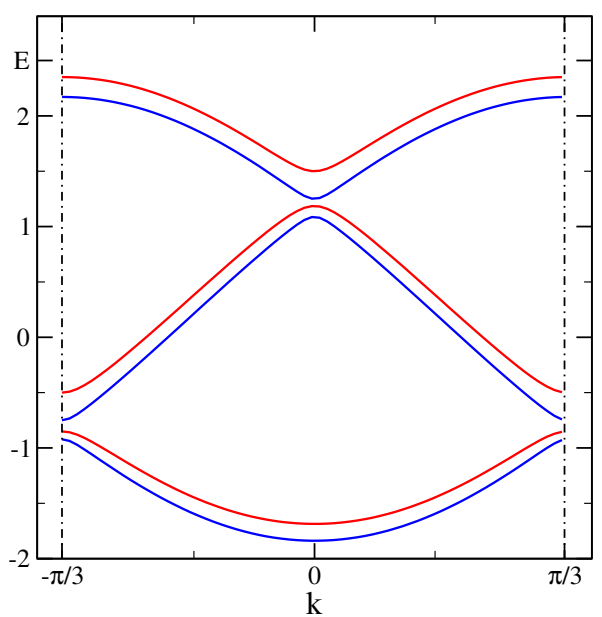

(b)

Abbildung 4.9.: Oberflächenzustände und Energiespektrum für die halb-unendliche periodische lineare Kette. (a) Zwei Oberflächenzustände für die halb-unendliche Kette für ein periodisches Potenzial $[\beta, 0, \beta, \beta, 0, \beta, \ldots)$ an ihrem linken Ende und in unmittelbarer Umgebung (siehe dazu auch Abb. 4.8). Die durchgezogene blaue Linie repräsentiert einen Oberflächenzustand für $\beta=\frac{1}{4}$, die durchgezogene rote Linie einen anderen mit $\beta=\frac{1}{2}$. Die gestrichelten Linien dienen dem Vergleich mit stetigem exponentiellen Abklingen. (b) Das Energiespektrum der zugehörigen Oberflächenzustände für die unendliche Kette (mit gleichem Potenzial $[\beta, 0, \beta]$ in der Einheitszelle) wartet mit zwei Bandlücken der Breite $\Delta=\frac{2}{3} \beta$ im Zentrum $k=0$ und an der Bandkante $|k|=\frac{\pi}{3}$ der ersten Brillouin-Zone $\left(k \in\left[-\frac{\pi}{3}, \frac{\pi}{3}\right]\right)$ auf, die durch gestrichelten Linien begrenzt wird. Die blauen Linien repräsentieren Energiebänder für $\beta=\frac{1}{4}$, die roten Linien die für $\beta=\frac{1}{2}$. Für detaillierte Erläuterungen sei auf den Text verwiesen.

wobei $\kappa=\sqrt{1+e^{3 k}(1+2[\cosh (k)+\cosh (3 k)])}$ den Normierungsfaktor für die Oberflächenzustände definiert. Hier kann o.B.d.A die Amplitude $A_{1}=0$ gewählt werden, da die Schrödinger-Gleichungen (4.46a), (4.46b) und (4.46c) gelten. Als Beispiel sind zwei Oberflächenzustände für eine halb-unendliche Kette für $\beta=1 / 2$ und $\beta=1 / 4$ in Abbildung 4.9a dargestellt. Die Berechnung der Eigenenergien der Oberflächenzustände für die endliche Kette (zwei Enden) verläuft analog. Im Kontinuums-Limes $(N \rightarrow \infty)$ mit $\gamma=0$ weist dann das Spektrum zwei Bandlücken auf, deren Größe $2 \beta / 3$ beträgt, wenn $\beta \ll 1$ gilt (siehe Abb. 4.9b). Die Existenz dieser Bandlücken legt eine gewisse Robustheit der $\mathcal{P} \mathcal{T}$-symmetrischen Phase nahe (wie in [250]), solange $\gamma \ll \beta$ gilt. Durch die Oberflächenzustände ist dies aber nicht der Fall. ${ }^{10}$ Für $\gamma=0$ existiert ein Paar von Oberflächenzuständen, exponentiell abfallend, mit den Scheitelpunkten am Ort $\pm N$. Für große, aber endliche $N$ haben diese Zustände einen Überlapp nahe des Zentrums der Kette $(n=0)$ und bilden ein Doublet mit einer exponentiell kleinen Energieaufspaltung $\Delta \delta$. In Analogie zur Berechnung des kritischen $\mathcal{P} \mathcal{T}$-Wertes im Unterabschnitt 4.2.1 wird schon ein exponentiell kleines $\gamma \simeq e^{-\beta N}$ die $\mathcal{P} \mathcal{T}$-Symmetrie brechen. Deshalb gilt auch im physikalischen Limes $N \rightarrow \infty$ für den kritischen Parameter $\gamma_{\mathcal{P} \mathcal{T}} \rightarrow 0$ aufgrund der Existenz der Oberflächenzustände. Hiermit wurde also gezeigt, dass sich Oberflächenzustände ebenfalls destruktiv auf die Stabilität der $\mathcal{P} \mathcal{T}$-symmetrischen Phase auswirken.

10 Oberflächenzustände wurden in [250] nicht behandelt. Dass deren Betrachtung jedoch zwingend erforderlich ist, belegen diese Ausführungen. 


\section{Zusammenfassung und Ausblick}

In diesem Abschnitt haben wir Konsequenzen diskutiert, die bei der Realisierung von ausgedehnten eindimensionalen $\mathcal{P} \mathcal{T}$-symmetrischen Systemen auftreten. Dabei stellte sich heraus, dass sowohl schwache Unordnung als auch Systemgrenzen bzw. Ränder zum Zusammenbrechen der $\mathcal{P} \mathcal{T}$-Symmetrie führen, die somit physikalische Realisierungen erschweren oder sogar ausschließen können. Als Grund hierfür fanden wir die Ausbildung von lokalisierten Zuständen mit einer exponentiell kleinen Energieaufspaltung der zugehörigen Eigenwerte. Hierbei muss jedoch die gebrochene $\mathcal{P} \mathcal{T}$-Symmetrie nicht zwangsläufig den Transport in diesen Systemen beeinflussen, da es eventuell eine große Entfernung (proportional zum Inversen des größten imaginären Energieeigenwerts) braucht, damit die lokalisierten Zustände den gerichteten Transport stören bzw. im System dominieren können.

Das Wissen um die charakteristische Aufspaltung der lokalisierten Zustände in Doublets, wobei jedes Doublet sich aus zwei lokalisierten Zuständen mit zueinander komplex-konjugierten Eigenwerten zusammensetzt, die ihrerseits langreichweitige Korrelationen implizieren, kann jedoch auch ausgenutzt werden. Durch starke Kopplung der zugehörigen Gitterpunkte bzw. Verringerung des Abstands, bildet jedes Doublet damit einen Dimer, dessen kleinstmögliche Realisierung aus zwei benachbarten Gitterpunkten besteht. Die Auswirkungen der Ausbildung von lokalisierten Zuständen bleibt somit auf den Dimer beschränkt. Nun werden viele solcher Dimere (beispielsweise in kettenförmigen Strukturen) schwach gekoppelt. Da die Dimere untereinander nicht wechselwirken, kann sich das Gesamtsystem stabil in Bezug auf Unordnung und gegenüber Systemgrenzen verhalten. Diese fundamentalen Untersuchungen sind Gegenstand des folgenden Abschnitts. 


\subsection{Strukturen mit lokaler $\mathcal{P}_{D} \mathcal{T}$-Symmetrie in quasi-1D}

Im vorhergehenden Abschnitt wurde die Lokalisierung als Ursache spontaner $\mathcal{P} \mathcal{T}$-Symmetriebrechung untersucht. Dabei stellte sich heraus, dass die $\mathcal{P} \mathcal{T}$-symmetrische Phase extrem instabil gegenüber der Anwesenheit von Fehlstellen bzw. Unordnung oder Oberflächen- bzw. Randeffekten reagiert [3]. Es gibt daher viele physikalische Systeme, die in ihrer (experimentellen) Realisierung trotz $\mathcal{P} \mathcal{T}-$ symmetrischem Potenzial keinen reellen Bereich im Eigenenergiespektrum aufweisen. Dieser Abschnitt dient dazu, einen möglichen Ausweg aus der Situation ausgedehnter lokalisierter Zustände aufzuzeigen. Dazu werden wir Konfigurationen entwickeln, die trotz Lokalisierung über eine stabile pseudohermitesche Phase verfügen.

\subsubsection{Der einfache Dimer}

Wir wollen eine neue Klasse von makroskopischen $\mathcal{P} \mathcal{T}$-symmetrischen Hamilton-Operatoren vorstellen, deren Spektrum rein reell in einem großen Parameterbereich ist, selbst in Anwesenheit eines (reellen oder komplexwertigen) Unordnungspotenzials und lokalisierten Zuständen. Das verbindende Element dieser Gruppe von Systemen ist eine Art Baustein, den wir als Dimer bezeichnen (siehe Abb. 4.10). Jeder dieser Dimere genügt der $\mathcal{P} \mathcal{T}$-Symmetrie und kann als ein Paar von Gitterpunkten mit den zugehörigen Energien (bzw. Potenzialen) $\left\{\varepsilon_{n}, \varepsilon_{n}^{*}\right\}$, $\epsilon_{n}=\beta_{n}+i \gamma_{n}$ mit $\beta_{n}, \gamma_{n} \in \mathbb{R}$ dargestellt werden, wobei $n$ den Gitterplatz nummeriert. Das jeweilige System ist dann eine irgendwie geartete Kopplung beliebig vieler Dimere. Für die Wahl von $n>1$ genügt das gesamte Dimer-System im Allgemeinen nicht der $\mathcal{P} \mathcal{T}$ Symmetriebedingung $(4.33)^{11}$. Auf der anderen Seite genügt jeder Dimer für sich betrachtet der $\mathcal{P} \mathcal{T}$-Symmetriebedingung bezüglich seines eigenen Symmetriezentrums. Diese Eigenschaft wollen wir

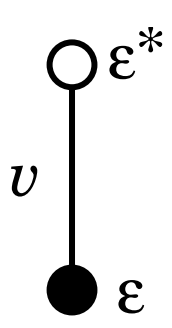

Abbildung 4.10.: Ein Dimer besteht aus zwei gekoppelten Gitterpunkten mit den Potenzialen $\varepsilon=\beta+i \gamma$ bzw. $\varepsilon^{*}=\beta-i \gamma$ mit der Kopplungsamplitude $v$. als lokale $\mathcal{P} \mathcal{T}$-Symmetriebedingung bezeichnen und dafür die Nomenklatur $\mathcal{P}_{D} \mathcal{T}$-symmetrisch reservieren. Die Hauptaussage dieses Abschnitts ist die Robustheit des reellen Spektrums für Systeme mit solcher $\mathcal{P}_{D} \mathcal{T}$-Symmetrie [3].

Im Folgenden wollen wir uns auf experimentell relevante, also einfache Realisierungen der DimerKonfiguration als Dimerkette beschränken, obwohl sich natürlich auch für andere Realisierungen mit Dimeren gleichwertige Aussagen finden lassen. Eine mögliche Realisierung in der Optik ist in Abbildung 4.11 dargestellt. Diese besteht in der Anordnung von gekoppelten Lichtwellenleitern aus angemessen dimensionierten Brechungsindexprofilen mit verstärkender bzw. abschwächender Wirkung. Eine experimentelle Untersuchung des einfachen Dimers aus Abbildung 4.10 wurde kürzlich in [255] realisiert. Zur mathematischen Beschreibung des einfachen Dimers mit analytischen Lösungen für Eigenwerte und Eigenvektoren sei auf Anhang $\mathrm{H}$ verwiesen. Die in diesem Abschnitt betrachteten HamiltonOperatoren für die zwei gekoppelten Dimere und die Dimerkette sind pseudo-hermitesch, da sie sich aus Blockmatrizen zusammensetzen lassen, die selbst pseudo-hermitesch sind. Diese Aussage gilt allerdings nur in der exakt $\mathcal{P} \mathcal{T}$-symmetrischen Phase.

11 Solch eine "globale" $\mathcal{P} \mathcal{T}$-Symmetriebedingung würde eine komplizierte präzise Bedingung an die $\varepsilon_{n}$ stellen. 


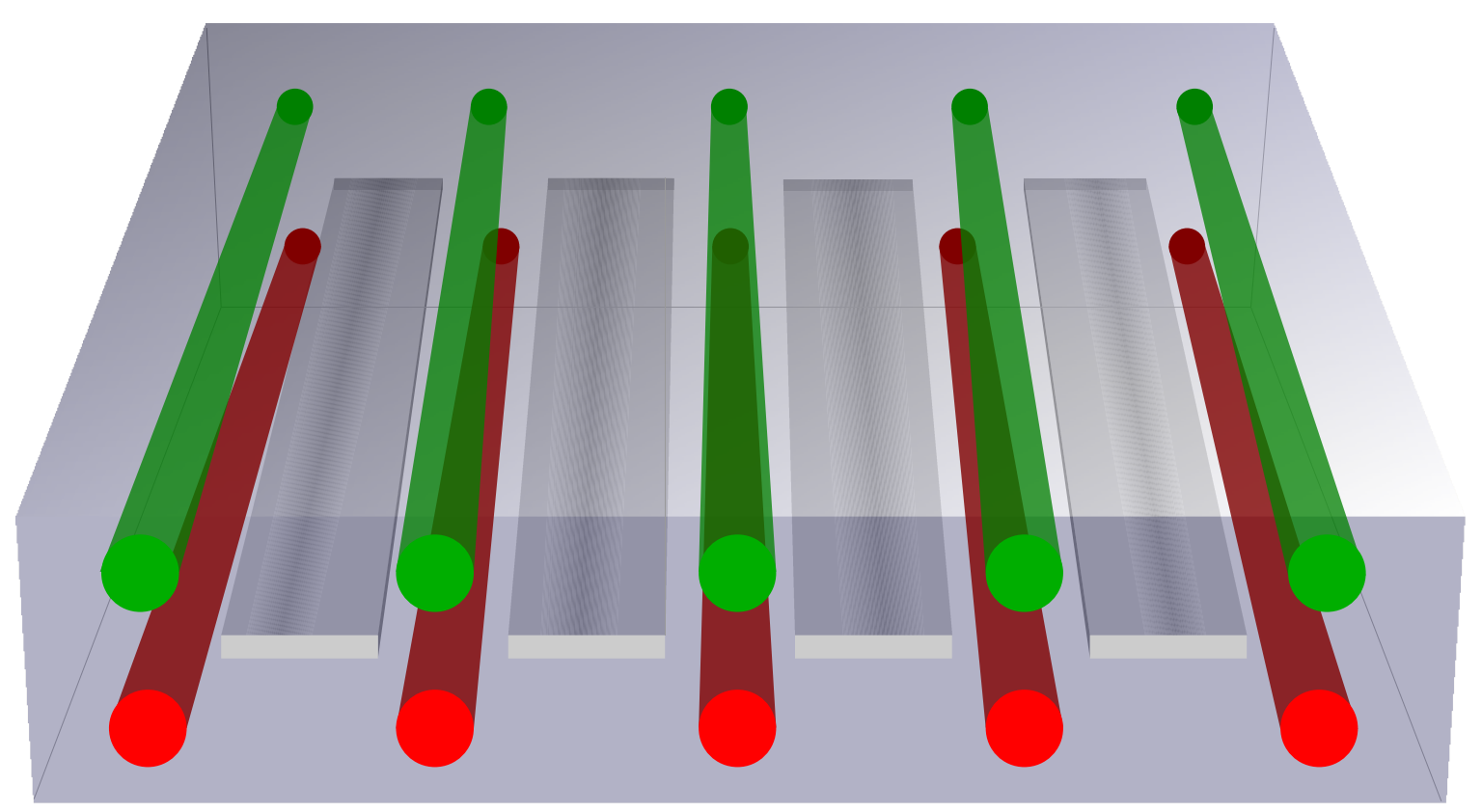

Abbildung 4.11.: Vorschlag zur experimentellen Realisierung einer Dimerkette durch Anordnung von gekoppelten optischen Fasern mit Verstärkung (grün) und Verlust (rot) im Brechungsindexprofil (Erklärung im Text). Die Maximalamplituden der jeweiligen Kopplung sind über den horizontalen bzw. vertikalen Abstand der Fasern definiert. Die flachen quaderförmigen Objekte (grau) repräsentieren Spiegel, die ein diagonales Koppeln der Fasern untereinander vermeiden. Diese sind jedoch für die Funktionsweise des gesamten optischen Bauelements nicht essenziell.

\subsubsection{Zwei gekoppelte Dimere}

Es erweist sich als besonders aufschlussreich, mit dem exakt lösbaren System von zwei gekoppelten Dimeren zu beginnen (siehe Abb. 4.12). Der Hamilton-Operator kann in diesem Fall durch

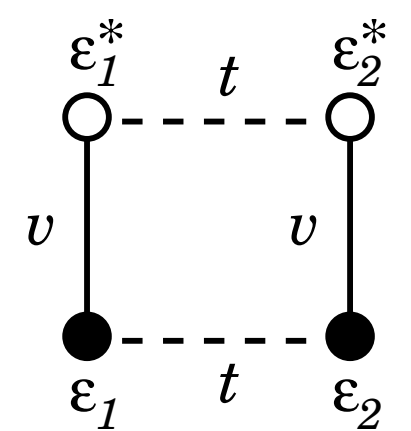

Abbildung 4.12.: Schematische Darstellung von zwei gekoppelten Dimeren $D_{1}, D_{2}$ mit den Gitterpotenzialen $\left\{\varepsilon_{1}, \varepsilon_{1}^{*}\right\}$ und $\left\{\varepsilon_{2}, \varepsilon_{2}^{*}\right\}$, Inter-Dimer Kopplung $t$ (gestrichelte Linien) und Intra-Dimer Kopplung $v$ (durchgezogene Linien). eine $4 \times 4$-Blockmatrix beschrieben werden (der Index 2 kennzeichnet die Anzahl der gekoppelten Dimere)

$$
\mathcal{H}_{2}=\left(\begin{array}{cc}
h_{1} & T \\
T & h_{2}
\end{array}\right) \text {, }
$$

mit den Unter- bzw. Blockmatrizen

$$
\begin{gathered}
h_{n}=\left(\begin{array}{cc}
\varepsilon_{n} & v \\
v & \varepsilon_{n}^{*}
\end{array}\right) \text { und } \\
T=\left(\begin{array}{ll}
t & 0 \\
0 & t
\end{array}\right),
\end{gathered}
$$

wobei $h_{n}$ den Hamilton-Operator des $n$-ten Dimers $(n=1,2)$ beschreibt. Die beiden Blockmatrizen $T$ definieren die Kopplung zwischen den beiden Dimeren. Das Modell besitzt damit insgesamt die zwei Kopplungskonstanten $t$ und $v$. Die Inter-Dimer Kopplung $t$ (dargestellt durch die horizontalen gestrichelten Linien in Abb. 4.12) kann sich dabei von der Intra-Dimer Kopplung $v$ (durchgezogene vertikale Linien in Abb. 4.12) durchaus unterscheiden. 
Die Eigenwerte der isolierten Dimere der Diagonal-Blockmatrizen $h_{n}$ aus Gleichung (4.55) lauten

$$
\lambda_{ \pm}^{(n)}=\beta_{n} \pm \sqrt{v^{2}-\gamma_{n}^{2}},(n=1,2)
$$

wobei die $\beta_{n}$ bzw. $\gamma_{n}$ die reell- bzw. komplexwertigen Gitterstellenpotenziale bezeichnen, vereinigt in $\varepsilon_{n}=\beta_{n}+i \gamma_{n}$ (siehe auch Anhang H). Vorerst wollen wir keine Abhängigkeiten zwischen $\varepsilon_{1}$ und $\varepsilon_{2}$ voraussetzen. Später wollen wir diese sogar als voneinander unabhängige Zufallsvariablen auffassen. Im Prinzip können auch die Kopplungskonstanten $t, v$ als Zufallsvariablen behandelt werden. Dieser Fall ist zwar von Interesse ${ }^{12}$, soll aber vorerst nicht verfolgt werden und wir setzen $t$ und $v$ daher als konstant an. Wir wählen, ohne die Allgemeinheit zu beschränken, für die Intra-Dimer Kopplung $v=1$ und legen damit die Energieskala fest. Für den Fall $\beta_{1}=\beta_{2}=0$ lauten die Eigenwerte ${ }^{13}$

$$
E_{\alpha}= \pm \frac{1}{\sqrt{2}}\left[2\left(1+t^{2}\right)-\gamma_{1}^{2}-\gamma_{2}^{2} \pm \sqrt{4 t^{2}\left[4-\left(\gamma_{1}+\gamma_{2}\right)^{2}\right]+\left(\gamma_{1}^{2}-\gamma_{2}^{2}\right)^{2}}\right]^{1 / 2}
$$

für $\alpha=1,2,3$, 4. Setzen wir in Gleichung (4.58) $t=0$, so reduziert sich das Problem auf das zweier ungekoppelter Dimere (also Gleichung (4.57) mit $\beta_{n}=0$ ). Solange $\left|\gamma_{n}\right| \leq 1$ gilt, sind die Eigenwerte $\lambda_{ \pm}^{(n)}$ reell. Ein endliches $t$ kann dann zu komplexen Eigenwerten führen. Wir wollen eine einfache Rechnung angeben, um dies zu verdeutlichen. Wir setzen o.B.d.A $\gamma_{1}>\gamma_{2}$ voraus, fest gewählt. Wir finden, dass alle Eigenwerte $E_{\alpha}$ reell sind, solange eine der zwei Bedingungen

$$
\left(1+\gamma_{1}\right)\left(1-\gamma_{2}\right) \leq t^{2} \quad \text { oder } \quad\left(1-\gamma_{1}\right)\left(1+\gamma_{2}\right) \geq t^{2}
$$

gilt. Interessanterweise bedeutet das, sollte beispielsweise $\gamma_{1}>1$ gelten - also einer der separaten Dimere für sich genommen komplexe Eigenwerte besitzen - so kann die Kopplung der einzelnen Dimere dazu führen, dass das Spektrum der gekoppelten Dimere trotzdem reell bleibt. Beispielsweise erhält man für $\gamma_{1}=0, \gamma_{2}=2$ aus Gleichung (4.58) $E_{\alpha}= \pm \sqrt{t^{2}+1-2 \pm 2}$, also reelle Eigenwerte für $t^{2}>3$.

Als Nächstes wollen wir das Phasendiagramm des Ensembles der zwei gekoppelten Dimere mit zufälligen Gitterstellenpotenzialen $\varepsilon_{n}=\beta_{n}+i \gamma_{n}$ untersuchen. Dabei nehmen wir an, dass $\beta_{n}$ und $\gamma_{n}$ unabhängige Zufallsvariablen sind, die aus einer Gleichverteilung gezogen werden, also $\beta_{n} \in[-\beta, \beta]$ und $\gamma_{n} \in[-\gamma, \gamma]$ mit $\beta, \gamma \in \mathbb{R}^{+}$. Dann verbleiben wir mit der Wahl von 3 Parametern $(\beta ; \gamma ; t)$, die jeweils Einfluss auf die (exakte oder gebrochene) $\mathcal{P}_{D} \mathcal{T}$-Phase nehmen können. In Abbildung 4.13 ist der Logarithmus des Maximums der Imaginärteile der Eigenwerte $E_{\alpha}$ des Ensembles gekoppelter zufälliger Dimere farbkodiert dargestellt. Blaue Flächen bedeuten hierbei exakte $\mathcal{P}_{D} \mathcal{T}$-symmetrische Phasen (nur reelle Eigenwerte), während rote Flächen gebrochene $\mathcal{P}_{D} \mathcal{T}$-Symmetrie bedeuten (komplexe Eigenwerte). Für $\beta<1$ findet man zwei Domänen der exakten $\mathcal{P}_{D} \mathcal{T}$-symmetrischen Phase. Die Domäne für $t>1$ ist unabhängig von $\beta$, während die erste Domäne für $0<t<1$ für $\beta>1$ gegen Null strebt (die Trivialfälle $t=0 \wedge 0 \leq \gamma \leq 1$ sowie $\gamma=0 \wedge 0 \leq t \leq 1$ sind hiervon ausgenommen). Später werden wir zeigen, dass viele Eigenschaften des Ensembles zweier gekoppelter zufälliger Dimere

12 Dies wäre auch in optischen Realisierungen der Fall, jedoch würden $(t ; v)$ gewissen Phasenbeziehungen unterliegen. Mit einer speziellen unitären Transformation können $(t ; v)$ aber auf konstante Werte transformiert werden (siehe Anhang I).

13 Auch für $\left\{\beta_{1}=0, \beta_{2} \neq 0\right\}$ oder $\left\{\beta_{1} \neq 0, \beta_{2}=0\right\}$ oder $\left\{\beta_{1} \neq 0, \beta_{2} \neq 0\right\}$ lassen sich analytische Lösungen finden. Da diese jedoch eher länglich sind und überdies nicht zusätzlich zum theoretischen Verständnis beitragen, wurde hier auf diese vollständige Angabe verzichtet. 

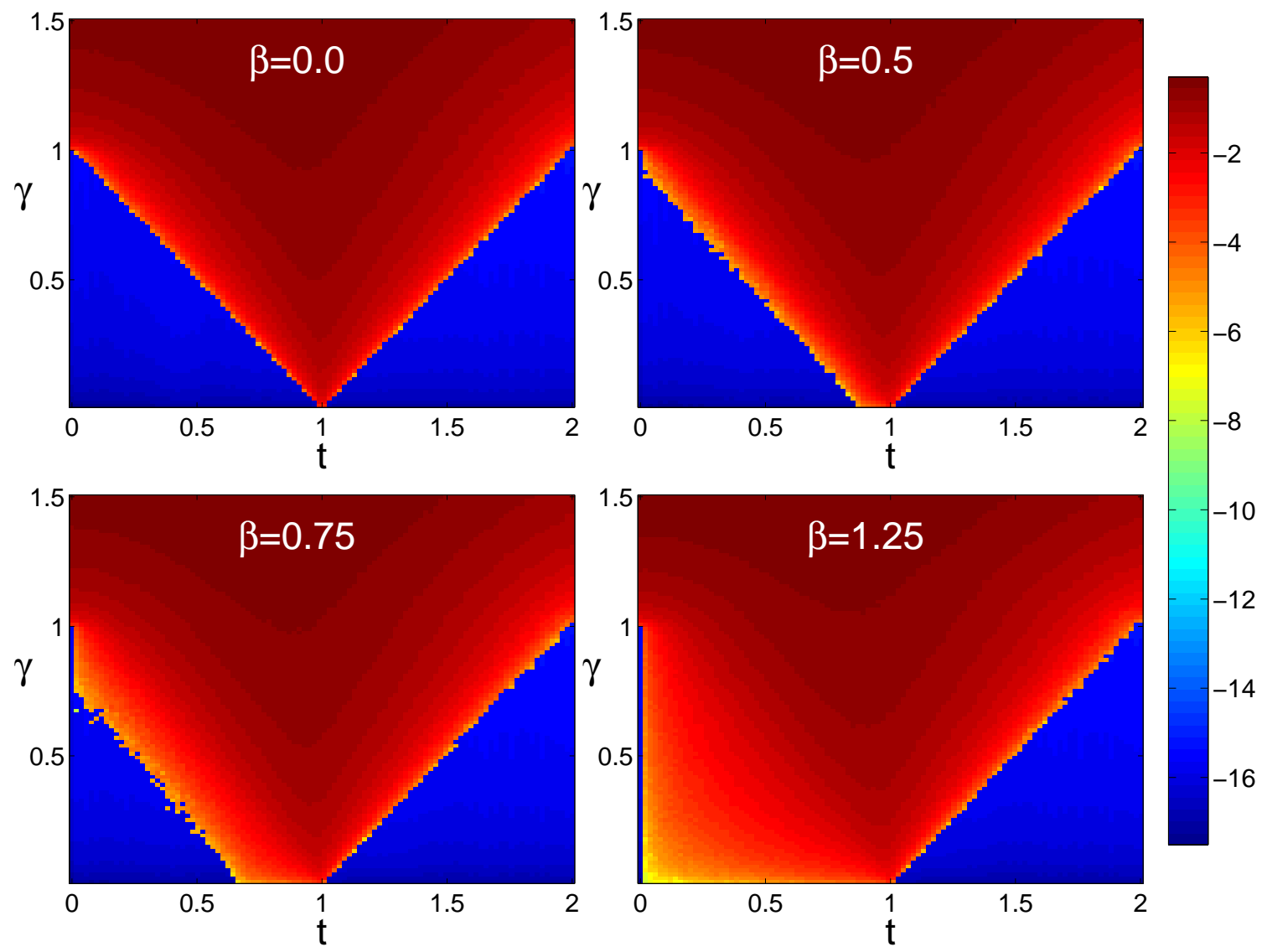

Abbildung 4.13.: Farbkodierte grafische Darstellung von $\log [\max \{\operatorname{Im}[E]\}]$ für das Ensemble zweier gekoppelter zufälliger Dimere für den Parameterraum $(t ; \beta ; \gamma)$ mit $t, \beta, \gamma \geq 0$. Die Restriktion auf den ersten Quadranten des $t$ - $\gamma$-Diagramms ist wegen der doppelten Achsensymmetrie [ $\gamma=0)$ - und $(t=0)$-Achsen] gewählt worden. Mehr als $10^{6}$ Realisierungen von Hamilton-Matrizen $\mathcal{H}_{N=2}$ aus Gleichung (4.54) wurden diagonalisiert. Die blauen Flächen (jeweils zwei Domänen) bedeuten reelle Eigenwerte $(\max \{\operatorname{Im}[E]\}=0$, bei einer numerischen Genauigkeit von $\approx 10^{-15}$ ), während die rot gefärbten Bereiche imaginäre Eigenwerte repräsentieren (siehe auch nebenstehende Legende der Potenzen zur dekadischen Basis). In den blau gefärbten Bereichen ist die $\mathcal{P}_{D} \mathcal{T}-$ symmetrische Phase exakt. Für Werte von $t>1$ ist diese Phase unabhängig von der Wahl von $\beta$. Die erste Domäne $(0<t<1)$ verkleinert sich mit der Zunahme von $\beta$ und verschwindet bei Werten von $\beta \geq 1$. Die trivialen Ausnahmen bilden hierbei die beiden Linien $(t=0 ; 0 \leq \gamma \leq 1)$ und $(\gamma=0 ; 0 \leq t \leq 1)$.

zumindest qualitativ herangezogen werden können, um die Stabilität der $\mathcal{P}_{D} \mathcal{T}$-symmetrischen Phase von längeren Dimerketten $(N>2)$ zu erklären. Daher ist es hilfreich, ein theoretisches Verständnis für die Stabilität der Phase des Ensembles zweier gekoppelter zufälliger Dimere im Bezug auf den Parameterraum $(\beta ; \gamma ; t)$ zu erlangen. Wir wollen insbesondere die untere Grenze der exakten $\mathcal{P}_{D} \mathcal{T}-$ symmetrischen Phase einer genaueren Analyse unterziehen. Für den Fall $t<1$ finden wir, dass die Konfiguration in der $\varepsilon_{1}=\beta+i \gamma$ und $\varepsilon_{2}=-(\beta+i \gamma)$ gelten, als erstes die $\mathcal{P}_{D} \mathcal{T}$-symmetrische Phase zerstören, während im Fall $t>1$ diese $\mathcal{P}_{D} \mathcal{T}$-Phase durch die Konfiguration $\varepsilon_{1}=i \gamma$ und $\varepsilon_{2}=-i \gamma$ gebrochen wird. Mit den zusätzlichen Annahmen $t, \gamma>0$ und $\gamma \leq 1$ erhalten wir ein reelles Spektrum ${ }^{14}$

14 Das Phasendiagramm $(\gamma, t)$ ist bi-achsensymmetrisch zu $\gamma=0$ und $t=0$. 
für

$$
\gamma \leq \gamma_{c}, \quad \text { mit } \quad \gamma_{c}=\left\{\begin{array}{cl}
-t+\sqrt{1-\beta^{2}} & 0<t<1 \\
t-1 & t \geq 1
\end{array}\right.
$$

Gleichung (4.60) liefert eine sehr gute Übereinstimmung mit den numerischen Untersuchungen aus Abbildung 4.13. An dieser Stelle sei bemerkt, dass die Konfiguration, die für $t>1$ die $\mathcal{P}_{D} \mathcal{T}$-Symmetrie bricht, auch gleichzeitig die $\mathcal{P} \mathcal{T}$-symmetrische Konfiguration ist, d.h. also für den Fall, dass $\varepsilon_{1}=$ $\varepsilon_{2}^{*}$ gilt. In diesem Fall ist die global $\mathcal{P} \mathcal{T}$-symmetrische Ausgangskonfiguration sogar die instabilste Konfiguration.

\subsubsection{Die Quasi 1D-Dimerkette}

Mit dem Wissen über das spektrale Verhalten des Ensembles der zwei gekoppelten zufälligen Dimere können wir uns nun der Quasi-1D Kette gekoppelter Dimere zuwenden (siehe Abb. 4.14). Solch eine

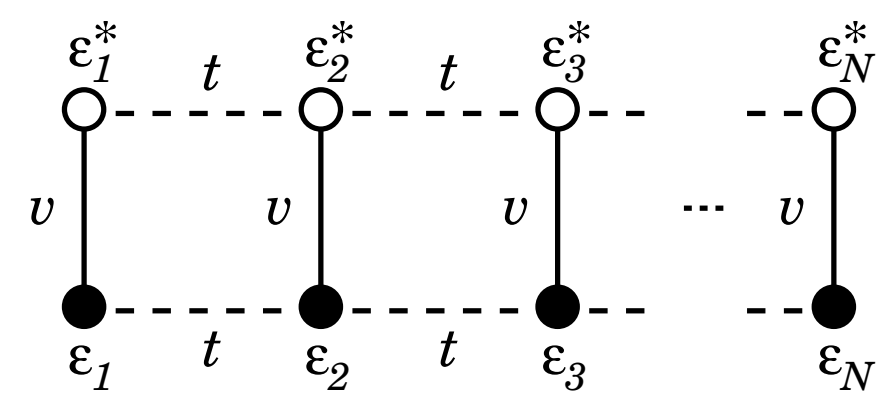

Abbildung 4.14.: Schematische Abbildung einer Dimerkette der Länge $N$ mit horizontaler Kopplungsamplitude $t$ und vertikaler Kopplungsamplitude $v$. Diese Kette genügt nicht der (globalen) $\mathcal{P} \mathcal{T}-$ Symmetriebedingung, jedoch der (lokalen) $\mathcal{P}_{D} \mathcal{T}$-Symmetriebedingung.

Kette der Länge $N$ (also $2 N$ Gitterpunkte) kann mittels des Hamilton-Operators

$$
\mathcal{H}_{N}=\left(\begin{array}{ccccccc}
h_{1} & T & 0 & \cdots & 0 & 0 & 0 \\
T & h_{2} & T & 0 & \ldots & 0 & 0 \\
0 & T & h_{3} & T & 0 & \cdots & 0 \\
\vdots & \ddots & \ddots & \ddots & \ddots & \ddots & \vdots \\
0 & \cdots & 0 & T & h_{N-2} & T & 0 \\
0 & 0 & \cdots & 0 & T & h_{N-1} & T \\
0 & 0 & 0 & \cdots & 0 & T & h_{N}
\end{array}\right)
$$

dargestellt werden. Jeder Eintrag in Matrix (4.61) entspricht einer $2 \times 2$-Blockmatrix, wie in Gleichung (4.55) definiert. Wir wollen mit dem Fall einer unendlich langen periodischen Kette aus $N \rightarrow \infty$ identischen Dimeren beginnen, wobei das jeweilige Dimerpotenzial $\varepsilon=\beta+i \gamma$ unabhängig vom Gitterplatz bzw. Ort $n$ ist und damit für den Hamilton-Operator aus Gleichung (4.61) $h \equiv h_{i}, i=1 \ldots N$ gilt. Die 
Wellenfunktion, die die Schrödinger-Gleichung $H \psi=E \psi$ löst, setzen wir mit

$$
\vec{\psi}=\left(\ldots, \psi_{-1}, \psi_{0}, \psi_{1}, \ldots\right)^{T}
$$

an, wobei $\psi_{n}=\left(\alpha_{n}^{(1)}, \alpha_{n}^{(2)}\right)^{T}$ die Wellenfunktion eines Dimers am Ort $n$ beschreibt. Die SchrödingerGleichung kann also in die Form

$$
T \psi_{n+1}+T \psi_{n-1}+h \psi_{n}=E \psi_{n}
$$

überführt werden. Aus dem Bloch-Theorem folgt sofort, dass die Wellenfunktion $\psi$ für eine periodische Kette faktorisiert werden kann. Als Ansatz wählen wir also

$$
\psi_{n}=\left(\begin{array}{c}
\alpha^{(1)} \\
\alpha^{(2)}
\end{array}\right) e^{i k n},
$$

wobei die Amplituden $\left(\alpha^{(1)}, \alpha^{(2)}\right)^{T}$ unabhängig vom Ort $n$ sind, und in Verlaufsrichtung der Kette ebene Wellen angesetzt werden können. Das System von Schrödinger-Gleichungen (4.63) vereinfacht sich also zu den folgenden zwei Amplituden-Gleichungen

$$
\begin{aligned}
& \left(\varepsilon+E_{k}-E\right) \alpha^{(1)}+v \alpha^{(2)}=0 \\
& v \alpha^{(1)}+\left(\varepsilon^{*}+E_{k}-E\right) \alpha^{(2)}=0
\end{aligned}
$$

mit der Dispersionsrelation

$$
E_{k}=2 t \cos (k)
$$

Als Lösungen für die beiden Eigenwerte finden wir

$$
E_{ \pm}=E_{k}+\beta \pm \sqrt{v^{2}-\gamma^{2}}
$$

d.h. das Spektrum ist reell, solange $|\gamma| \leq|v|$ gilt. Wir erhalten also exakt die gleiche Bedingung für reelle Eigenwerte wie in Unterabschnitt 4.3.2 für den isolierten Dimer.

An dieser Stelle sei erwähnt, dass auch für den Fall der idealen halb-unendlichen periodischen Dimerkette mit zugehörigem Potenzial ${ }^{15}$

$$
\varepsilon_{j}=\left\{\begin{array}{ll}
\beta+i \gamma & j \mid(3 l \pm 1) \\
0+i \gamma & j \mid(3 l)
\end{array},\right.
$$

wobei $l \in \mathbb{N}$ die Einheitszellen des Potenzials nummeriert, die gleiche Bedingung für ein reelles Spektrum gestellt werden kann, d.h. $E_{ \pm} \in \mathbb{R} \Longleftrightarrow|\gamma| \leq|v|$. Für die Eigenwerte ergibt sich nach analoger Rechnung ebenfalls Gleichung (4.67), jedoch mit der Dispersionsrelation

$$
E_{k}=t e^{3 k} \quad \text { und } \quad k=-\frac{1}{3} \operatorname{arcsinh}\left(\frac{\beta}{2 t}\right)
$$

$\overline{15 \text { Die Periodizität des Realteils des Potenzials }}\{\beta, 0, \beta, \beta, 0, \beta, \ldots\}$ bedingt eine Bandlücke - eine notwendige Voraussetzung für das Auftreten von Oberflächenzuständen. 
als Abklingrate. Für eine Dimer-Fehlstelle in einer perfekten unendlichen Dimerkette mit zugehörigem Potenzial

$$
\varepsilon_{j}= \begin{cases}0 & j \neq 0 \\ \beta+i \gamma & j=0\end{cases}
$$

finden wir ebenfalls Gleichung (4.67), jedoch mit der Dispersionsrelation

$$
E_{k}=2 t e^{k}, \quad \text { mit } \quad k= \begin{cases}-\operatorname{arcsinh}\left(\frac{\beta}{2 t}\right) & \text { für } \gamma \approx 0 \\ -\operatorname{arcsinh}\left(\left[\beta \mp v \pm \sqrt{v^{2}-\gamma^{2}}\right] /(2 t)\right) & \text { für } \gamma \neq 0\end{cases}
$$

als Abklingrate. Für die Eigenwerte ergibt sich also insgesamt

$$
E_{ \pm}=\sqrt{q_{ \pm}} \pm v \quad \text { mit } \quad q_{ \pm}=4 t^{2}+\left(\beta \pm v \mp \sqrt{v^{2}-\gamma^{2}}\right)^{2}
$$

also die gleiche Bedingung $|\gamma| \leq|v|$ für reelle Eigenwerte. Damit ist auch gezeigt, dass sowohl lokalisierte Zustände (verursacht durch Fehlstellen), als auch existente Oberflächenzustände (siehe weiter oben) die Stabilität der $\mathcal{P}_{D} \mathcal{T}$-symmetrischen Phase nicht beeinflusst. Dieses Stabilitätsverhalten unterscheidet sich stark von dem Ergebnis der Stabilitätsanalyse der $\mathcal{P} \mathcal{T}$-symmetrischen Phase aus Abschnitt 4.2, wo eben genau das Auftreten oben aufgezählter Zustände die $\mathcal{P} \mathcal{T}$-symmetrische Phase zerstörten. Numerisch zeigte sich sogar, dass für $\beta_{n}$ als Zufallsgröße und $\gamma_{n}=\gamma=$ const die gleiche Bedingung $|\gamma| \leq|v|$ für reelle Eigenwerte gilt.

Als Nächstes wollen wir uns dem endlichen System der Dimerkette mit der Länge $N$ mit zufällig verteilten Energien $\varepsilon_{n}=\beta_{n}+i \gamma_{n}$ zuwenden. Hier setzen wir ebenfalls voraus, dass die Zufallsgrößen $\beta_{n}$ und $\gamma_{n}$ unabhängige reelle Zufallsvariablen sind, die aus gleichverteilten Intervallen $\beta_{n} \in[-\beta, \beta]$ respektive $\gamma_{n} \in[-\gamma, \gamma]$ mit $\beta, \gamma \in \mathbb{R}^{+}$gezogen werden (siehe dazu auch Unterabschnitt 4.3.2). Bevor wir uns allerdings der Analyse des numerisch ermittelten Phasendiagramms (analog zum Phasendiagramm Abb. 4.13) zuwenden, wollen wir darauf hinweisen, dass es eine Basis gibt, in der alle Matrixelemente von $\mathcal{H}_{N}$ reell sind. Die unitäre Matrix $J$, die diese Transformation leistet, ist die Block-Diagonalmatrix

$$
J=\left(\begin{array}{cccc}
J_{2} & & & 0 \\
& J_{2} & & \\
& & \ddots & \\
0 & & & J_{2}
\end{array}\right) \text { mit } \quad J_{2} \equiv \frac{1}{\sqrt{2}}\left(\mathbb{1}_{2}+i \sigma_{x}\right)
$$

wobei $\mathbb{1}_{2}$ die $2 \times 2$-Einheitsmatrix und $\sigma_{x}$ die erste Pauli-Matrix

$$
\mathbb{1}_{2} \equiv\left(\begin{array}{cc}
1 & 0 \\
0 & 1
\end{array}\right) \quad \text { und } \quad \sigma_{x} \equiv\left(\begin{array}{cc}
0 & 1 \\
1 & 0
\end{array}\right)
$$

definieren. Da in diesem Fall die Säkulargleichung $\left|J^{-1}\left[E \mathbb{1}_{N}-\mathcal{H}_{N}\right] J\right|=0$ reellwertig ist, können die Eigenwerte von $J^{-1} \mathcal{H}_{N} J$ entweder alle reell sein, oder aber gemischt als reelle Wertepaare und in komplex-konjugierten Paaren auftreten ( $N$ gekoppelte Dimere entsprechen dem charakteristischem Polynom der Ordnung $2 N$ ). Dies gilt dann auch für die ursprüngliche Hamilton-Matrix $\mathcal{H}_{N}$, da die unitäre Transformation $J^{-1} \mathcal{H}_{N} J$ die Eigenwerte von $\mathcal{H}_{N}$ erhält. Für den allgemeinen Fall mit komplex- 
wertigen Kopplungskonstanten lässt sich ebenfalls eine unitäre Transformation $\Omega$ finden. Die spezielle Form der Hamilton-Matrix (4.61) lässt eine solche Transformation zu, so dass die Verwendung reellwertiger Kopplungskonstanten $t, v$ gerechtfertigt ist. Detaillierte Ausführungen hierzu findet der interessierte Leser im Anhang I.

Im Falle des Ensembles gekoppelter Dimere mit zufälligem Potenzial gilt der Hamilton-Operator (4.61), für den wir die Domänen des reellen Spektrums im Parameterraum $(t ; \beta ; \gamma)$ bestimmen wollen. Die Größe der untersuchten Dimerketten betrug bis zu 60 Dimeren (also Matrixdiagonalisierungen von Matrizen mit rank $\left(\mathcal{H}_{60}\right)=120$ ), wobei mehr als $10^{6}$ Realisierungen mit verschiedenen Unordnungspotenzialen generiert worden sind. ${ }^{16}$ Aus jeder Realisierung identifizierten wir dann den größten Imaginärteil ${ }^{17}$ aller Eigenwerte $\max \{\operatorname{Im}[E]\}$. Das so entstandene Phasendiagramm ist in Abbildung 4.15 wiedergegeben, analog zum Phasendiagramm im Falle des Ensembles zweier gekoppelter zufälliger Dimere, jedoch mit dem Unterschied, das für $\beta=0$ nur eine Domäne existiert. Mit Zunahme von $\beta$ verkleinert sich der Bereich reeller Eigenwerte (in der Abb. blau dargestellt), um schließlich für $\beta \geq 1$ vollständig zu verschwinden. Die exakte $\mathcal{P}_{D} \mathcal{T}$-symmetrische Phase ist dann nur noch für den Trivialfall $t=0$ (ungekoppelte Dimere) und $0 \leq \gamma \leq \gamma_{c}=1 \equiv v$ existent. Qualitativ ist dieses Verhalten in Analogie zum Ensemble zweier gekoppelter zufälliger Dimere (siehe Abb. 4.13 zum Vergleich), auch wenn der Bereich der $\mathcal{P}_{D} \mathcal{T}$-symmetrischen Phase "schmaler" ist und für wachsende $\beta$ "schneller" verschwindet.

Im Gegensatz zum $\mathcal{P} \mathcal{T}$-symmetrischen 1D-Anderson Modell aus Abschnitt 4.2 weist das System aus gekoppelten lokal $\mathcal{P} \mathcal{T}$-symmetrischen Dimeren selbst in Gegenwart von Unordnung eine stabile pseudo-hermitesche Phase auf. Die Ursache der $\mathcal{P} \mathcal{T}$-Symmetriebrechung in global $\mathcal{P} \mathcal{T}$-symmetrischen Systemen ist die Überlagerung zweier zum gleichen Eigenwert gehörender lokalisierter Eigenzustände (symmetrischer und anti-symmetrischer Zustand bilden ein Doublet) nahe der Systemgrenzen bzw. an räumlich symmetrischen Fehlstellen. Diese Paare bilden effektive langreichweitige Dimere mit einer exponentiell kleinen Kopplung v und daher existiert ein exponentiell kleines kritisches $\gamma_{c}$ (siehe Gleichung (H.3)). Im lokal $\mathcal{P}_{D} \mathcal{T}$-symmetrischen System sind diese symmetrischen Paare stark gekoppelt (kleiner Abstand der Dimere). Dies führt zu der Stabilität des Eigenwertspektrums und somit letztlich auch zu einer robusten $\mathcal{P}_{D} \mathcal{T}$-symmetrischen Phase. Wir geben nun ein heuristisches Argument dafür an, dass zumindest zu einem qualitativen Verständnis des Phasendiagramms aus Abbildung 4.15 in der $t$ - $\gamma$-Ebene beiträgt. Für den einfachen Fall $\beta=0$ finden wir im Limes für $t \rightarrow 0-$ also im Fall $N$ ungekoppelter Dimere - den kritischen Wert $\gamma_{c}(v=1)=1$ (im Phasendiagramm nach Abb. 4.15 ist dies der Phasenübergang auf der $\gamma$-Achse). Auf der anderen Seite finden wir für $\gamma=0$ und ein endliches $t$ zwei Energiebänder, zentriert bei $E= \pm v$ (hier $v=1$ ), jedes mit der Breite $4 t$. Durch Zuwachs von $t$ nimmt die Bandlücke $\Delta$ zwischen den beiden Bändern wie $\Delta(t)=2-4 t$ ab. An der Stelle $t_{c}=\frac{1}{2}$ verschwindet diese Bandlücke und die beiden Energieniveaus der "inneren" Bandkanten würden sich kreuzen. An dieser Stelle ist bereits ein infinitesimal kleines $\gamma$ ausreichend, um die $\mathcal{P}_{D} \mathcal{T}$-Symmetrie $\mathrm{zu}$ brechen, woraus ein komplexes Eigenwertpaar resultiert [3] (hier $t=\frac{1}{2}, \gamma=0$ in Abb. 4.15, siehe auch Abb. 4.16a). Da die Abnahme der Bandlücke $\Delta$ linear in $t$ ist, finden wir auch einen linearen

16 Die Realisierungen beschränken sich vorerst auf die Zufallsgrößen $\beta_{n}$ und $\gamma_{n}$. Weiter unten findet man Anmerkungen über die Wahl anderer Zufallsgrößen, beispielsweise die der Kopplungskonstanten $t$ oder $v$.

17 Im eigentlichen Sinne ist der betragsmäßig größte Imaginärteil gemeint, jedoch treten - wie oben bereits erwähnt die Eigenwerte in komplex-konjugierten Paaren auf. 

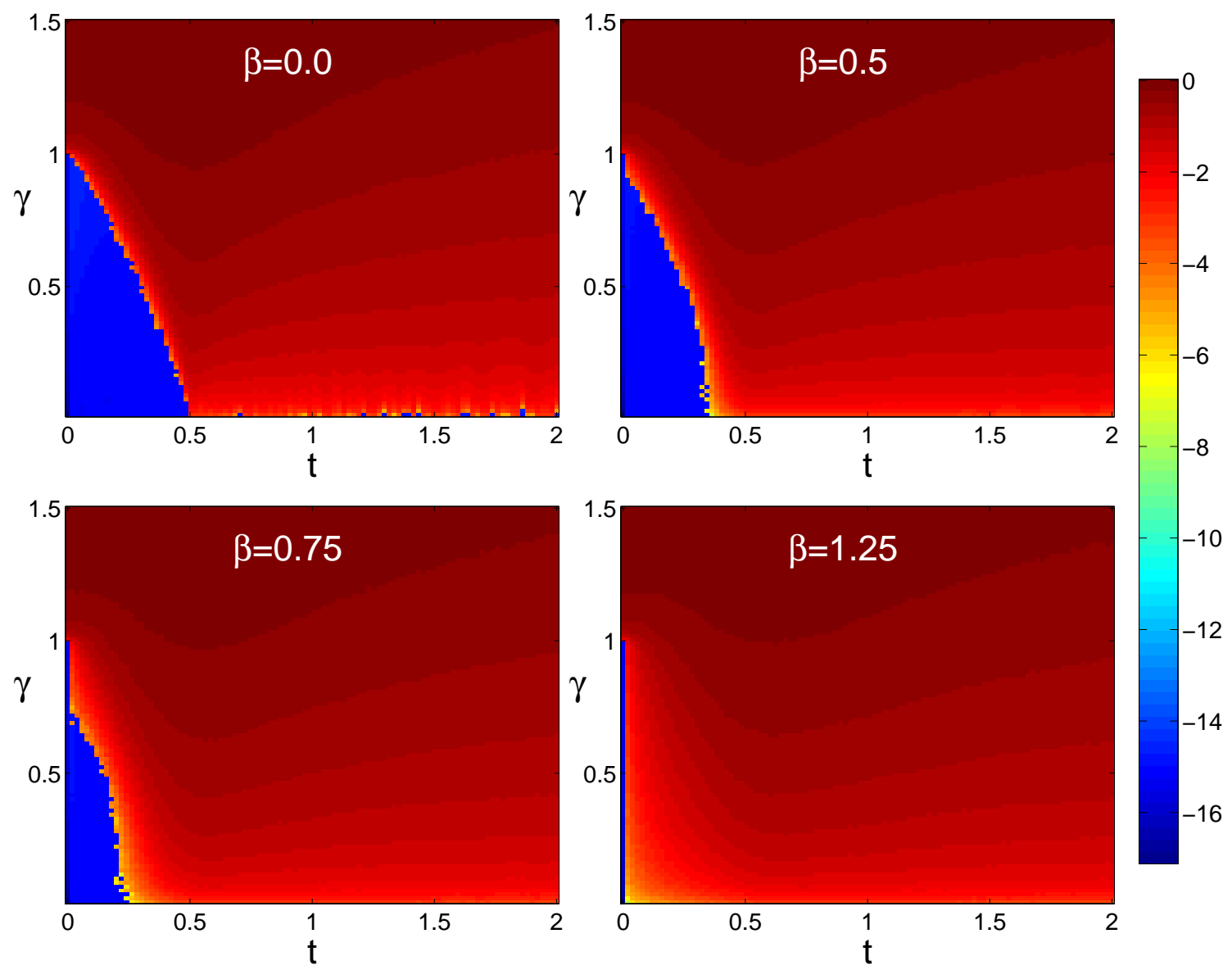

Abbildung 4.15.: Farbkodierte grafische Darstellung von $\log [\max \{\operatorname{Im}[E]\}]$ für das Ensemble aus $N=40$ gekoppelter zufälliger Dimere für den Parameterraum $(t ; \beta ; \gamma)$ mit $t, \beta, \gamma \geq 0$. Die Restriktion auf den ersten Quadranten des $t$ - $\gamma$-Diagramms ist wegen der doppelten Achsensymmetrie $[(\gamma=0)-$ und $(t=0)-$ Achsen $]$ gewählt worden. Mehr als $10^{6}$ Realisierungen von Hamilton-Matrizen $\mathcal{H}_{N=40}$ aus Gleichung (4.61) wurden diagonalisiert. Die blauen Flächen bedeuten reelle Eigenwerte $\left(\max \{\operatorname{Im}[E]\}=0\right.$, mit numerischer Genauigkeit von $\left.\approx 10^{-15}\right)$, während die rot gefärbten Bereiche imaginäre Eigenwerte repräsentieren (siehe auch nebenstehende Legende der Potenzen zur dekadischen Basis). In den blau gefärbten Bereichen ist die $\mathcal{P}_{D} \mathcal{T}$-symmetrische Phase exakt. Die Domäne reeller Eigenwerte $(0<t<1)$ verkleinert sich mit der Zunahme von $\beta$ und verschwindet bei Werten von $\beta \geq 1$. Die trivialen Ausnahmen bilden hierbei die beiden Linien $(t=0 ; 0 \leq \gamma \leq 1)$ und $(\gamma=0 ; 0 \leq t \leq 1)$.

Zusammenhang für den kritischen Parameter $\gamma_{\mathrm{c}}$ mit

$$
\gamma_{\mathrm{c}}(\beta=0)=1-2 t
$$

für den Grenzübergang der exakten $\mathcal{P}_{D} \mathcal{T}$-symmetrischen Phase für $\beta=0$, wie eine Interpolation zwischen den zwei Phasenraumpunkten $(t=0, \gamma=1)$ und $\left(t=\frac{1}{2}, \gamma=0\right)$ bestätigt. Ein Vergleich mit den numerischen Daten (aus Abb. 4.15 für $\beta=0$ ) zeigt eine gute Übereinstimmung mit den angestellten Überlegungen.

Mit wachsendem Unordnungsparameter $\beta$ wird diese $N$-fache Entartung der vorerst ungekoppelten $(t=0)$ Dimere angehoben, wobei sich bei gleichzeitiger Vergrößerung von $t$ die Breite der beiden Energiebänder $\Delta E_{1,2}$ ebenfalls vergrößert, was zu einer noch schnelleren und nicht mehr linearen Abnahme der Bandlücke $\Delta$ führt (siehe Abb. 4.16b). Dies erklärt auch den Umstand, dass die exakte $\mathcal{P}_{D} \mathcal{T}-$ symmetrischen Phase des Ensembles gekoppelter zufälliger Dimere mit wachsendem $t$ schneller abfällt 


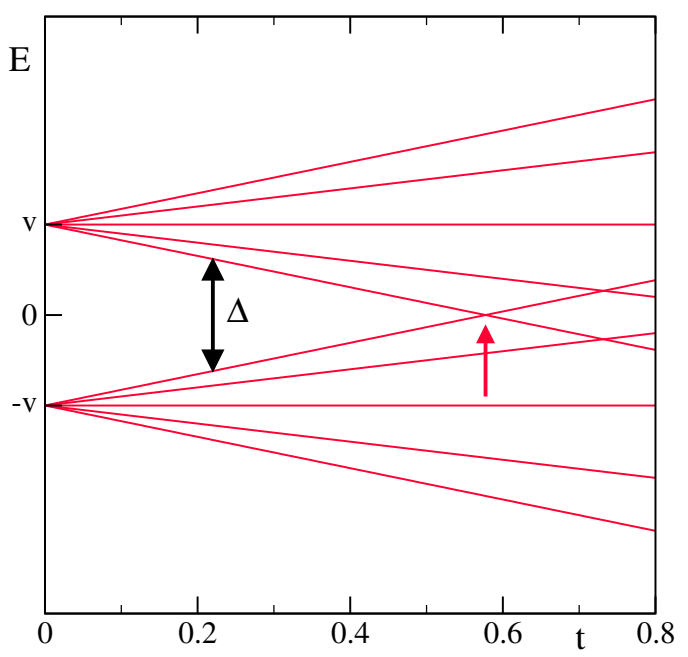

(a)

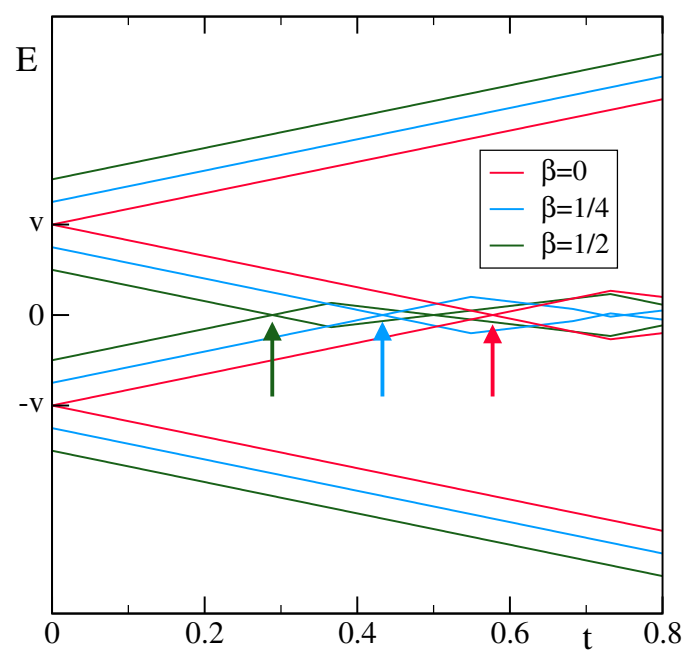

(b)

Abbildung 4.16.: Energiebänder und Einhüllende der Dimerkette $\mathcal{H}_{5}$ (5 gekoppelte identische Dimere, entsprechend Gleichung (4.61) mit $\epsilon=\beta+i \gamma$ ) zur Veranschaulichung der Abnahme der Bandlücke $\Delta$ mit wachsender Inter-Dimer-Kopplung $t$ und wachsendem Unordnungsparameter $\beta$. Die analytischen Lösungen für die Eigenwerte lauten $E_{1,2}=\beta \pm \alpha, E_{3,4,5,6}=\beta \pm t \pm \alpha$ und $E_{7,8,9,10}=\beta \pm \sqrt{3 t^{2} \pm 2 t \sqrt{3} \alpha+\alpha^{2}}$ mit $\alpha=\sqrt{v^{2}-\gamma^{2}}$. (a) Eigenwerte für $\beta=\gamma=0$. Der rote Pfeil markiert das Verschwinden der eingezeichneten Bandlücke $\Delta$ mit wachsendem $t$, d.h. nahe $t \rightarrow t_{\mathrm{c}} \equiv v / \sqrt{3}$ genügt ein infinitesimal kleines $\gamma$, um die $\mathcal{P}_{D} \mathcal{T}$-Symmetrie zu brechen $\left(\lim _{t \rightarrow t_{\mathrm{c}}} \gamma=0\right)$. (b) Einhüllende der Eigenwerte für $\gamma=0$ und verschiedene $\beta$-Werte. Die farbigen Pfeile markieren das Szenario einer schnelleren Abnahme der Bandlücke $\Delta_{\beta}$ mit $t_{\mathrm{c}}=(v-\beta) / \sqrt{3}$, da sich die Energiebänder mit wachsendem $\beta$ verbreitern und so die Bandlücke $\Delta_{\beta}$ verkleinern. Dieses Verhalten ändert sich qualitativ nicht durch Einführung von Unordnung in $\beta$ bzw. $\gamma$ (siehe Text).

als für das Ensemble zweier gekoppelter zufälliger Dimere mit zweifacher Entartung im Energieband. Dabei ist für eine Dimerkette der Länge $N$ die Abhängigkeit der Bandlücke $\Delta_{\beta}(t)$ ein komplizierter funktionaler Zusammenhang, der mit den Nullstellen der Eigenwert-Polynome $E_{n}(t, v, \beta) N$-ter Ordnung beschreibbar ist, wobei die beiden entscheidenden Nullstellen von den Eigenwerten $E_{i_{1}}$ und $E_{i_{2}}$ der inneren Bandkanten $i_{1}$ und $i_{2}$ gebildet werden (siehe z.B. Lösungen der Dimerkette $N=5$ für $\beta=$ const in der Bildunterschrift zu Abb. 4.16). Da die $\beta_{n}$ zufällig gewählt sind, lassen sich für Dimer-Ketten $N>4$ keine analytischen Lösungen mehr angeben, wie der Satz von Abel [293] beweist.

\section{Zusammenfassung und Ausblick}

Jede experimentelle Realisierung eines optischen oder elektronischen Bauteils ist dem Einfluss von Fehl- bzw. Störstellen (Rauschen) und Rand- bzw. Oberflächeneffekten unterworfen. Wir gingen deshalb der Frage nach, in wie weit die Realisierung von $\mathcal{P} \mathcal{T}$-symmetrischen Systemen dadurch behindert wird. Durch Einführung eines Dimers, der selbst eine stabile $\mathcal{P} \mathcal{T}$-symmetrische Phase besitzt, konnten wir zeigen, dass durch Bildung von Ketten aus solchen einzelnen Dimeren eine exakte $\mathcal{P}_{D} \mathcal{T}-$ symmetrische Phase resultiert, die stabil unter dem Einfluss von Fehl- bzw. Störstellen (Rauschen) und Rand- bzw. Oberflächeneffekten ist. Eine lokale $\mathcal{P}_{D} \mathcal{T}$-Symmetrie ist zudem experimentell viel leichter zu realisieren als eine globale $\mathcal{P} \mathcal{T}$-Symmetrie (siehe Abb. 4.11). Außerdem wurde gezeigt, dass Dimerketten unabhängig von Rand- bzw. Oberflächeneffekten sind. ${ }^{18}$

18 In einer experimentellen Realisierung muss die $\mathcal{P}_{D} \mathcal{T}$ - bzw. $\mathcal{P} \mathcal{T}$-Symmetriebrechung nicht notwendigerweise eine Verschlechterung der Transporteigenschaften oder sogar den Zusammenbruch des Transports bedeuten, denn es muss 


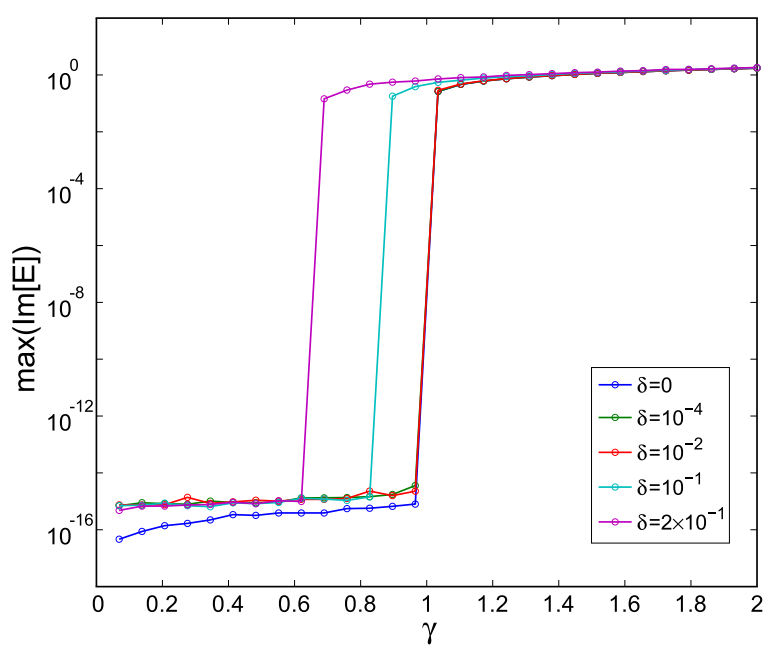

(a)

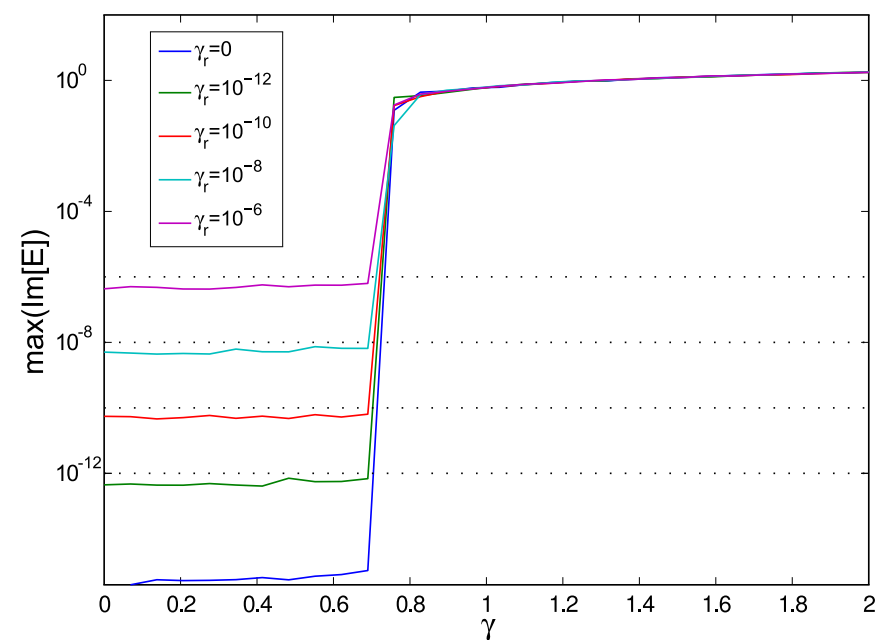

(b)

Abbildung 4.17.: Verschiedene Variationen der Unordnungsparameter einer aus $N=40$ Dimeren bestehenden Kette. (a) Variationen in $t$ (Inter-Dimer-Kopplung), $v$ (Intra-Dimer-Kopplung) und $u$ (diagonale Inter-DimerKopplung). Hier bezeichnet $\delta$ das Maximum der aus einem Intervall mit gleichverteilten Zufallszahlen gezogenen Unordnung. Wir betrachten zufällig parallel und diagonal und zusätzlich noch unterschiedlich stark intern gekoppelte Dimere $(v ; t ; u)=\left(v_{0} ; t_{0} ; u_{0}\right)+\delta([-1,1] ;[-1,1] ;[-1,1])$ mit $v_{0}=1$ und $t_{0}=u_{0}=0$. Selbst für starke Variation $(\delta=0.2)$ bleibt der Phasenübergang erhalten, wird jedoch nur linear proportional zur Störung $\delta \mathrm{zu}$ kleineren $\gamma$ verschoben. (b) Variationen in $\gamma$ (Imaginäres Dimer-Potenzial). Hier wurde eine schwache asymmetrische Unordnung zum imaginären Potenzial der einzelnen Dimere hinzugefügt $\gamma=\gamma_{0}+\gamma_{\mathrm{r}}([-1,1])$, wobei $\gamma_{\mathrm{r}}$ das Maximum der aus einem Intervall mit gleichverteilten Zufallszahlen gezogenen Unordnung darstellt. Dieses System ist sogar nicht mehr $\mathcal{P}_{D} \mathcal{T}$-symmetrisch. Die Grafik lässt aber erkennen, dass der Phasenübergangspunkt $\gamma_{\mathrm{c}}$ erhalten bleibt und das $\max \{\operatorname{Im}[E]\}$ nur linear proportional zur zusätzlichen Störung $\gamma_{\mathrm{r}}$ angehoben wird. Zum besseren Erkennen der Plateaus wurden die $\gamma_{\mathrm{r}}=$ const. ebenfalls dargestellt (gepunktete Linien).

Unser Modell kann in verschiedene Richtungen erweitert werden. So seien hier als Beispiele die zusätzliche kreuzweise Kopplung $u$ benachbarter Dimere angeführt (das entspräche der Entnahme der Spiegel aus der vorgeschlagenen experimentellen Realisierung in Abb. 4.11 bzw. Einführung der zusätzlichen Kopplung $u$ zwischen $\varepsilon_{i}$ und $\varepsilon_{i+1}^{*}$ respektive $\varepsilon_{i}^{*}$ und $\varepsilon_{i+1}$ in Abb. 4.14, siehe dazu auch Anhang I auf Seite 153) oder zufälliges Rauschen in den Kopplungskonstanten $t$ und $v$ (siehe Abb. 4.17a). Ebenfalls denkbar ist eine Störung der lokalen $\mathcal{P}_{D} \mathcal{T}$-Symmetrie der einzelnen Dimere einer Kette, was einer asymmetrischen Unordnung in $\gamma$ entspricht (siehe Abb. 4.17b). Obwohl hier die $\mathcal{P}_{D} \mathcal{T}$-Symmetrie bereits von Beginn an gebrochen ist, zeigt sich, das die deutliche Ausprägung des Phasenübergangs der pseudo-hermiteschen Phase erhalten bleibt (siehe 4.17b; man beachte die logarithmische Skala). Damit wird eine physikalische Realisierung überhaupt erst möglich.

Die Ausbildung von Plateaus im Phasendiagramm der pseudo-hermiteschen Phase vor dem kritischen Wert $\gamma_{c}$ - dargestellt durch unterschiedliche Stärken des Rauschlevels - zeigt, dass in einer experimentellen Realisierung dieser Rauschlevel nur klein genug sein muss, um die gewünschte Genauigkeit zu erzielen.

erst eine verhältnismäßig lange Strecke (proportional zum Inversen des größten Imaginärteils aller Eigenwerte des Systems) zurückgelegt werden, bevor der zu übertragende Lichtstrahl oder das Wellenpaket von den lokalisierten Moden beeinflusst wird. 


\subsection{Streuformalismus in lokal $\mathcal{P} \mathcal{T}$-symmetrischen Systemen}

Bisher mit der paraxialen Näherung untersuchte Systeme entsprachen unendlich ausgedehnten oder endlichen Systemen ohne Rückstreuung an deren Enden oder Fehlstellen. In diesem Abschnitt widmen wir uns dem realistischen Fall von offenen Systemen mit Rückstreuung, verursacht durch lokale $\mathcal{P} \mathcal{T}$ symmetrische Potenziale. Diese wollen wir am Beispiel eines bestimmten lokal $\mathcal{P} \mathcal{T}$-symmetrischen Potenzials mit der Schrödinger-Gleichung im Hinblick auf ihre Transporteigenschaften untersuchen, wobei jedoch - wie wir in den beiden vorangegangenen Kapiteln gesehen haben - diese Betrachtungen auch für Systeme gelten, die durch die optische Wellengleichung beschrieben werden können.

Über die Auswirkungen von $\mathcal{P} \mathcal{T}$-Symmetrie in Systemen mit Rückstreuung auf den Transport gibt es bereits einige theoretische Arbeiten (z.B. [294, 295]), jedoch wurden bisher keine Transporteigenschaften in ausgedehnten Systemen mit lokaler $\mathcal{P} \mathcal{T}$-Symmetrie behandelt. Für diesbezügliche Untersuchungen wird der Streuformalismus der in Kapitel 1 entwickelten Methode auf Systeme mit lokaler $\mathcal{P} \mathcal{T}$-Symmetrie übertragen.

\subsubsection{Systeme mit abgeschlossenen Rändern}

Als vorbereitende Untersuchung wollen wir uns zunächst den geschlossenen Systemen zuwenden. Die hier entwickelten theoretischen Konzepte werden dann später in geeigneter Form auf offene Systeme übertragen.

In zwei Dimensionen lautet der Hamilton-Operator eines geschlossenen Systems, in Analogie zur Gleichung (4.3), wie folgt

$$
\mathcal{H}=\mathcal{H}_{P}+V_{0}(x, y)
$$

wobei $\mathcal{H}_{P}$ den Hamilton-Operator des (perfekten) Gitters darstellt und $V_{0}(x, y) \in \mathbb{C} \times \mathbb{C}$ das komplexe zweidimensionale Potenzial des Gitters repräsentiert. Dieses Potenzial soll der lokalen $\mathcal{P} \mathcal{T}$-Symmetrie genügen, so dass folgende Bedingungen an das Potenzial gestellt werden

$$
\begin{aligned}
V_{0}(x, y) & =V_{0}(x, \cdot) \\
V_{0}(x) & =V_{0}^{*}(-x) .
\end{aligned}
$$

Lokal bedeutet in diesem Zusammenhang, dass nur die $\mathcal{P} \mathcal{T}$-Symmetriebedingung bezüglich der $x$ Richtung erfüllt ist. Für den Hamilton-Operator (4.75) in Matrix-Form schreiben wir

$$
[\mathcal{H}]_{i j}= \begin{cases}z \cdot t+V_{0}(x) & i=j \\ -t & i, j \text { nächste Nachbarn } \\ 0 & \text { sonst }\end{cases}
$$

als effektiven Hamilton-Operator mit nächster Nachbarwechselwirkung, wobei $z$ die Anzahl der nächsten Nachbarn bezeichnet und

$$
t=\frac{\hbar}{2 m a^{2}} \stackrel{(\text { A.1) }}{=} \frac{1}{2 a^{2}}
$$




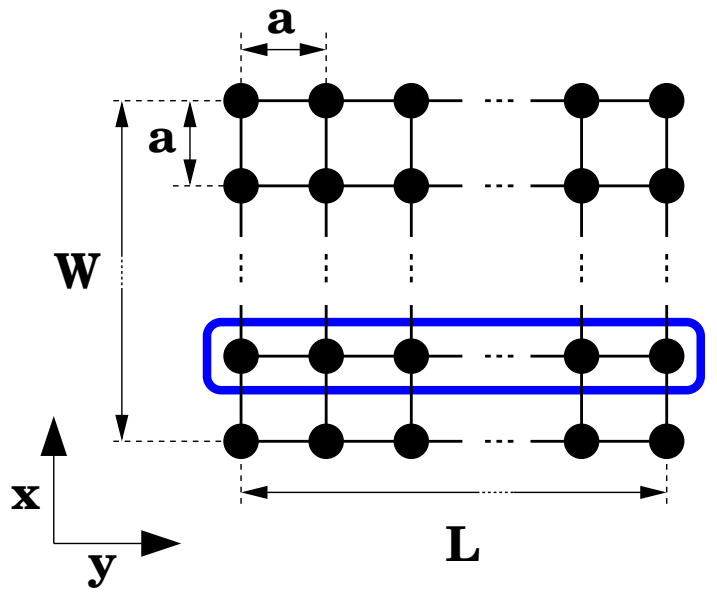

(a)

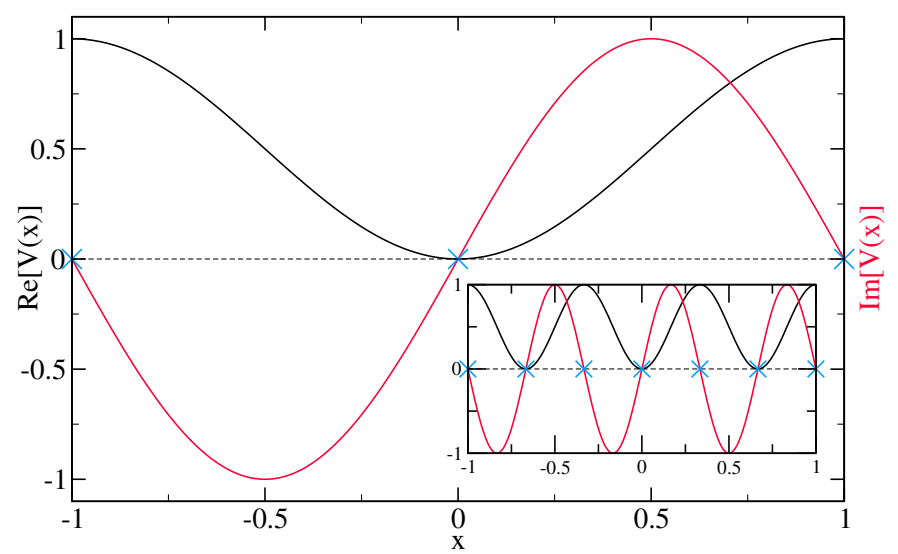

(b)

Abbildung 4.18.: Stützstellenapproximation periodischer Potenziale auf einem Quadratgitter. (a) Quadratgitter des geschlossenen zweidimensionalen Systems der Breite $W$ und Länge $L$. Die Gitterkonstante $a$ ist für $x$ - und $y$-Richtung gleich groß. Die Gitterstelle $P=\{x, y\}$ hat das Potenzial $V_{0}(x, y)$. Der blau umrandete Bereich stellt eine lineare Kette der Länge $L$ dar. (b) Profil des Realteils (schwarz) und des Imaginärteils (rot) des Potenzials $V_{0}(x)=A\left[1-\cos ^{2} C \frac{\pi x}{w}+i B \sin C \frac{2 \pi x}{w}\right]$ mit $A=B=C=1$. Nicht beitragende Stützstellen zum Imaginärteil sind durch blaue Kreuze markiert, hier ist der Imaginärteil gleich Null. Mit Zunahme von $C \in \mathbb{N}$ um $n \in \mathbb{N}$ entfallen $2 n$ weitere Stützstellen. Die eingebettete Abbildung zeigt das Profil für $A=B=1, C=3$. Die Anzahl der Stützstellen muss dementsprechend angepasst werden.

die Hüpf- bzw. Austauschenergie zweier horizontal oder vertikal benachbarter Gitterpunkte angibt. Wir beschränken uns hier auf äquidistante Ketten bzw. Gitter mit Gitterkonstante $a$, so dass für eine lineare Kette $z=2$ und für ein Quadratgitter $z=4$ gilt (siehe Abb. 4.18a auf dieser Seite). Der Parameter $a=\frac{1}{N_{W}+1}$ bezeichnet die Gitterkonstante, wobei $N_{W}$ die Anzahl der Gitterpunkte in $x$-Richtung angibt. Die Randpunkte des in $x$-Richtung ausgedehnten Gitters sind mit Definition von $a$ im numerischen Sinne keine "echten" Randpunkte, da diese durch die Dirichlet-Randbedingungen $\partial \psi_{\mathrm{R}}=0$ nicht in der Simulation enthalten sein müssen, folglich alle $N_{W}$ Punkte der $x$-Richtung zur Approximation der Wellenfunktion $\widetilde{\psi}_{\mathrm{R}}$ beitragen. ${ }^{19}$ Weiterhin sei angemerkt, dass voneinander verschiedene linke und rechte Eigenvektoren existieren, da $\mathcal{H}$ nicht hermitesch ist (siehe Anhang J), so dass die Bestimmung eines vollständigen Eigensystems den numerischen Aufwand mit Berechnung der linken Eigenvektoren $\widetilde{\psi}_{\text {L verdoppelt. }}{ }^{20}$

Wir wollen uns hier auf die Untersuchung von Potenzialen der Art

$$
V_{0}(x, y)=V_{0}(x)=A\left[1-\cos ^{2} C \frac{\pi x}{W}+i B \sin C \frac{2 \pi x}{W}\right]
$$

die in [250] für den rückstreuungsfreien Fall betrachtet wurden, beschränken. Weiter unten werden wir durch die Einbeziehung von Unordnung dieses Modell um Fluktuationen im Real- und Imaginärteil erweitern. Der Parameter $C \in \mathbb{N}^{+}$definiert die Anzahl der "Kanäle" in $x$-Richtung (der Realteil des Potenzials entspricht dem Realteil des Brechungsindexes, siehe Glg.(4.13) auf Seite 94), $B \in \mathbb{R}$ definiert die Stärke des Imaginärteils des Potenzials und $A \in \mathbb{R}$ seine maximale reelle Amplitude, d.h. $\pm A B$ entspricht der maximalen Amplitude des Imaginärteils, wobei für dieses Potenzial die $y$-Achse als

19 Die Randpunkte der Wellenfunktion sind bekannt und bilden zur inneren Lösung hinzugefügt eine vollständige Lösung $\left(\psi_{\mathrm{L}}, \psi_{\mathrm{R}}\right)^{T}=\left(\widetilde{\psi}_{\mathrm{L}}, \widetilde{\psi}_{\mathrm{R}}\right)^{T} \oplus\left(\partial \psi_{\mathrm{L}}, \partial \psi_{\mathrm{R}}\right)^{T}$

20 Linke und rechte Eigenwerte sind hingegen äquivalent, siehe dazu auch Anhang J. 
fiktive Zeitachse verstanden werden kann (siehe auch Ausführungen Abschnitt 4.1.2 auf Seite 93). Dieses Potenzial ist lokal $\mathcal{P} \mathcal{T}$-symmetrisch in $x$-Richtung $\left(\mathcal{P}_{x} \mathcal{T}\right.$-symmetrisch). Das Profil des Realund Imaginärteils ist in Abb. 4.18b auf der vorherigen Seite wiedergegeben. Zu der Diskretisierung von Potenzialen der Art nach Gleichung (4.79) sei angemerkt, dass die Wahl der Stützstellen eine große Rolle spielt. So müssen vor allem bei Mehrkanalsimulationen genügend Stützstellen vorhanden sein (vgl. eingebettete Abb. in 4.18b). ${ }^{21}$

Durch Erweiterung von Gleichung (4.79) durch Störterme im Real- und Imaginärteil

$$
V_{d}(x, y)=A\left[\left(1-\cos ^{2} C \frac{\pi x}{W}+d_{r} f(x, y)\right)+i B\left(\sin C \frac{2 \pi x}{W}+d_{i} f(x, y)\right)\right]
$$

mit $d_{r} \in \mathbb{R}$ als reellem und $d_{i} \in \mathbb{R}$ als imaginärem Unordnungsparameter und der "Zufallsfunktion" $f:(x, y) \mapsto[-1,1]$, die die Werte $x \in[0, W]$ und $y \in[0, L]$ zufällig gleichverteilt in das Intervall $[-1,1]$ abbildet, können wir verschiedene Realisierungen von Unordnung generieren und untersuchen. Ein Ordnungsparameter zur Bestimmung des kritischen Parameters $B=B_{\mathrm{c}}$, der die exakte $\mathcal{P} \mathcal{T}$-Phase von der gebrochenen $\mathcal{P} \mathcal{T}$-Phase separiert kann - für geschlossene Systeme sowohl mit als auch ohne Unordnung - über den Imaginärteil der Eigenwerte definiert werden und lautet

$$
q_{\mathrm{c}}(B)=\frac{1}{N} \sum_{k}\left|\operatorname{Im}\left(\lambda_{k}\right)\right|
$$

Die Ableitung $\frac{d q_{\mathrm{c}}}{d B}$ zeigt dann ein Maximum am Phasenübergang $B=B_{\mathrm{c}}$ (siehe Abb. 4.20a). Im Allgemeinen gilt $\sum_{k} \lambda_{k}=\operatorname{Spur}(\mathcal{H}) \in \mathbb{R}$, da die Eigenwerte in zueinander komplex konjugierten Paaren auftreten. Daher muss in Gleichung (4.81) über die Betragsfunktion des Imaginärteils der Eigenwerte summiert werden. Prinzipiell ist es möglich, den kritischen Parameter $q_{\mathrm{c}}$ über

$$
q_{\mathrm{c}}^{\max }(B)=\max _{k}\left\{\operatorname{Im}\left(\lambda_{k}\right)\right\}
$$

zu definieren (vergleiche eingebettete Abb. in 4.20a), da mit wachsendem $B$ die Eigenfunktionen des zugehörigen ersten in Erscheinung tretenden Eigenwertpaares, dessen Eigenwerte zueinander komplex konjugiert sind, die spontane Symmetriebrechung verursachen, wie in Abschnitt 4.2 gezeigt wurde. Diese Definition bleibt aber auf geschlossene Systeme beschränkt, da offene Systeme selbst für $B=0$ bereits über ein komplexes Eigenwertspektrum verfügen. Wir werden im nächsten Unterabschnitt genauer darauf eingehen.

Das numerisch bestimmte und in Abbildung 4.19a dargestellte Spektrum beweist die Existenz eines exzeptionellen Punktes ${ }^{22}$ bei $B_{\mathrm{c}} \approx 11.281$. Für das geschlossene System ohne Unordnung bedeutet

21 Die Anzahl der äquidistanten Stützstellen $N_{W}$ sollte ungleich $2 C+1$ sein, da sonst ausnahmslos die Nullstellen des Imaginärteils approximiert werden. Bei der Wahl von $N_{W}=4 C+1$ Stützstellen werden sowohl alle Nullstellen als auch alle Extrema bei der Approximation des Imaginärteils berücksichtigt. Vom numerischen Standpunkt aus sollte $N_{W}>4 C+1$ so gewählt werden, dass alle Maxima bzw. Minima, Nullstellen und zusätzliche Stützstellen enthalten sind. Dies gelingt beispielsweise mit der Stützstellenanzahl $N_{W}=2^{n} C+1$ für $n>2$. Die Diskretisierung des Realteils ist dagegen unkritisch.

22 Als exzeptionelle Punkte (exceptional points), auch Ausnahmepunkte genannt, werden Singularitäten bezeichnet, die bei der Verzweigung von reellen Eigenwerten in die komplexe Ebene durch Abstoßung der Energieniveaus auftreten. Dies darf nicht mit der "üblichen" Entartung von Energieniveaus, wie sie bei hermiteschen Operatoren auftreten, verwechselt werden, da die Verzweigung der Eigenwerte in die komplexe Ebene bei gleichem Eigenwert-Realteil auch auf die Eigenfunktionen zutrifft - ein Verhalten, das aus der Verwendung nicht-hermitescher Operatoren resultiert. Der umfassende mathematische Hintergrund kann in [296, 297] nachgelesen werden. 


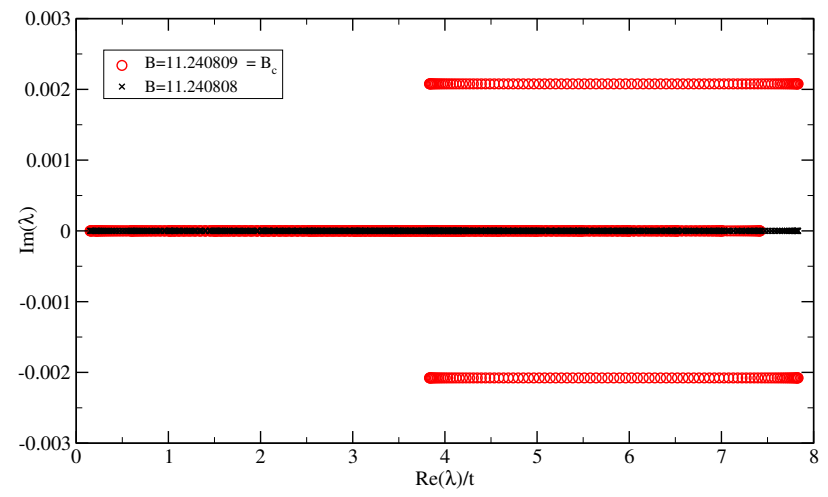

(a)

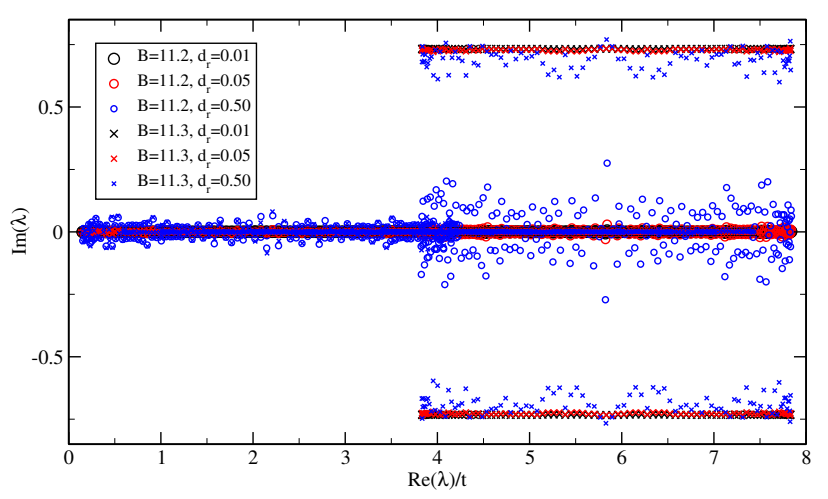

(b)

Abbildung 4.19.: Phasendiagramme der Eigenwerte des geschlossenen $11 \times 97-$ Systems mit Potenzial nach Gleichung (4.80) für $A=C=1$ (a) ohne Unordnung $\left(d_{r}=d_{i}=0\right)$. Beim kritischen Wert $B_{\mathrm{c}}=11.240809$ erscheinen imaginäre Eigenwerte $(N=2 W=2 \cdot 97)$ bei $\operatorname{Im}(\lambda) \approx \pm 0.002$ als "3-Level-System". (b) mit reeller Unordnung (jeweils eine Realisierung für $d_{r}=\{0.01,0.05,0.50\}$ ). Die Fluktuationen im Realteil des Potenzials stören die Energiebänder, deren Struktur aber im Wesentlichen erhalten bleibt.

dies das Erscheinen konjugiert komplexer Eigenwerte in einem "3-Level-System", das selbst unter der Einwirkung von verhältnismäßig großer reeller und komplexer Unordnung im Wesentlichen erhalten bleibt (siehe Abb. 4.19b).

Die Abweichung von der Hermitizität von $\mathcal{H}$ mit wachsendem Parameter $B$ wirkt sich auf eine Verletzung der Orthogonalitätsbedingung $\left\langle L_{k} \mid L_{l}\right\rangle=\delta_{k l}$ bzw. $\left\langle R_{k} \mid R_{l}\right\rangle=\delta_{k l}$ für linke und rechte Eigenvektoren aus. Dieser Sachverhalt kann zur Definition eines Ordnungsparameters benutzt werden (im historischen Zusammenhang mit optischen Lasern heißen diese Komponenten auch PetermannFaktoren, siehe [298, 299, 300]). Dabei gilt für den allgemeinen Petermann-Faktor [301]

$$
\kappa_{k l}=\frac{\left\langle L_{k} \mid L_{l}\right\rangle\left\langle R_{k} \mid R_{l}\right\rangle}{\left\|\left\langle L_{k} \mid R_{l}\right\rangle\right\|^{2}}
$$

Für bi-orthonormale Eigenvektoren sind die Petermann-Faktoren allein mit den Überlappmatrixelementen

$$
K_{k l}=\left\langle L_{k} \mid L_{l}\right\rangle\left\langle R_{k} \mid R_{l}\right\rangle
$$

beschreibbar, da für die Norm $\left\|\left\langle L_{k} \mid R_{l}\right\rangle\right\|=1$ gilt. ${ }^{23}$ Die Vollständigkeit impliziert $\sum_{k} K_{k l}=1$ (siehe [302]). Der mittlere diagonale Petermann-Faktor

$$
\bar{K}=\left\langle\frac{1}{N} \sum_{k} K_{k k}\right\rangle_{\mathcal{H}}
$$

definiert somit ein Maß für die Hermitizität von $\mathcal{H}$. Sind die Eigenfunktionen orthogonal, so gehen diese aus einer unitären Transformation hervor und der Petermann-Faktor ist 1. Abweichungen der Orthogonalität der Eigenvektoren vergrößern den Petermann-Faktor [303] und lassen ihn an Ausnahmepunkten des Eigenwertspektrums divergieren [304, 305]. Der besseren Darstellung wegen sind die Ableitung des Ordnungsparameters $\frac{d q_{\mathrm{C}}}{d B}$ und der inverse mittlere diagonale Petermann-Faktor $\frac{1}{\bar{K}}$ in Abbildung 4.20 gegenüber gestellt. Letzterer ist auch der physikalischen Bedeutung wegen erwähnt, da 


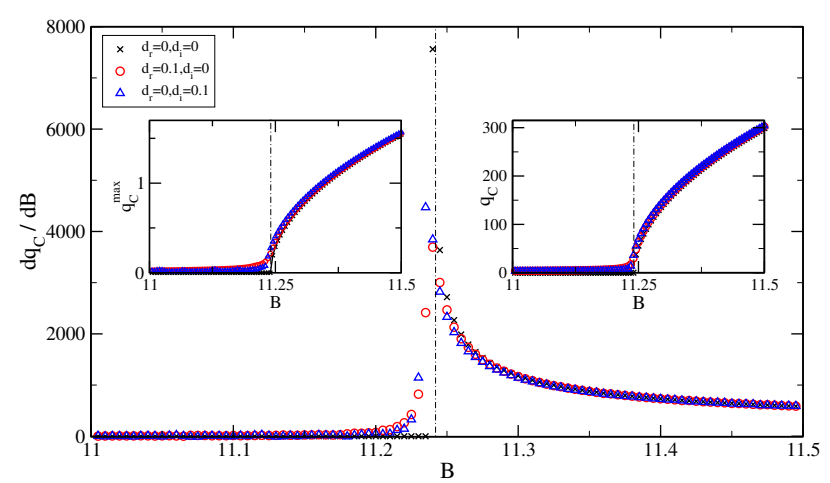

(a)

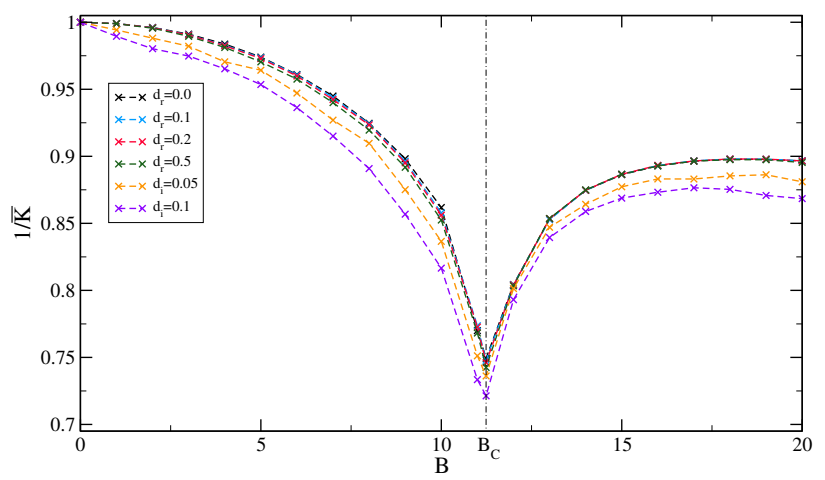

(b)

Abbildung 4.20.: Gegenüberstellung der kritischen Parameter für die Eigenwerte bzw. Eigenvektoren des geschlossenen $11 \times 97$-Systems mit Potenzial nach Gleichung (4.80) für $A=C=1$ in Abhängigkeit von $B$. Der kritische Wert $B_{\mathrm{c}}=11.240809$ kennzeichnet den Phasenübergang für beide kritischen Größen. (a) $\frac{d q_{\mathrm{c}}}{d B}$ für verschiedene Realisierungen von Unordnung und ohne Unordnung. Die eingebetteten Abbildungen zeigen den kritischen Parameter definiert über die Maximumsfunktion (links $q_{\mathrm{c}}^{\max }$ ) und über die Summe der Betragsfunktionen des Imaginärteils der Eigenwerte (rechts $q_{\mathrm{c}}$ ). (b) Invertierter mittlerer diagonaler Petermann-Faktor $1 / \bar{K}$ für verschiedene Realisierungen von reeller und imaginärer Unordnung sowie ohne Unordnung.

in zeitabhängigen Systemen die Eigenvektoren sensitiv auf Fluktuationen (z.B. des Brechungsindexes) reagieren, während die Eigenwerte die Langzeitdynamik beschreiben. In unserem Fall des zeitunabhängigen Systems spiegeln Eigenvektoren und Eigenwerte die Eigenschaften der Langzeitdynamik $t \rightarrow \infty$ wider. Im Folgenden sollen diese Erkenntnisse auf offene Systeme übertragen werden.

\subsubsection{Systeme mit offenen Rändern}

Um Transportphänomene betrachten zu können, müssen wir das Modell mit externen Anschlüssen versehen. Durch diese Leiter können wir Wellen(pakete) in die Probe senden und Größen wie die Streumatrix, elektrische Leitfähigkeit, Transmission und Reflexion für die Ein- und Ausgänge berechnen. Die mathematische Beschreibung eines Systems mit (teilweise) offenen Rändern wird durch die Einführung einer retardierten Selbstenergie $\Sigma^{\mathrm{R}}$ nach Gleichung (1.28) auf Seite 15 möglich, so dass wir den Hamilton-Operator $\mathcal{H}$ aus Gleichung (4.75) zu

$$
\mathcal{H}=\mathcal{H}_{P}+\Sigma^{\mathrm{R}}+V_{0}(x, y)
$$

erweitern können, wobei

$$
\Sigma^{\mathrm{R}}=-t \sum_{p, m} \chi_{m}\left(p_{i}\right) \exp \left(i k_{m} a\right) \chi_{m}\left(p_{j}\right)
$$

den Selbstenergieterm in Matrixschreibweise repräsentiert (siehe auch Kapitel 1). Das Potenzial $V_{0}(x, y)$ bzw. $V_{d}(x, y)$ ist aus den Gleichungen (4.79) bzw. (4.80) bereits bekannt. Die reellwertigen Funktionen $\chi_{m}\left(p_{j}\right)$ sind die transversalen Eigenmoden $m$ des halb-unendlich ausgedehnten Leiters $p$ an der Stelle $j$, wobei sich im Leiter selbst diese Transversalmoden in $y$-Richtung als ebene Wellen ausbreiten (zur Diskretisierung siehe Abb. 4.21). Der Wellenvektor $k_{m}$ lässt sich aus der Dispersionsrelation des 


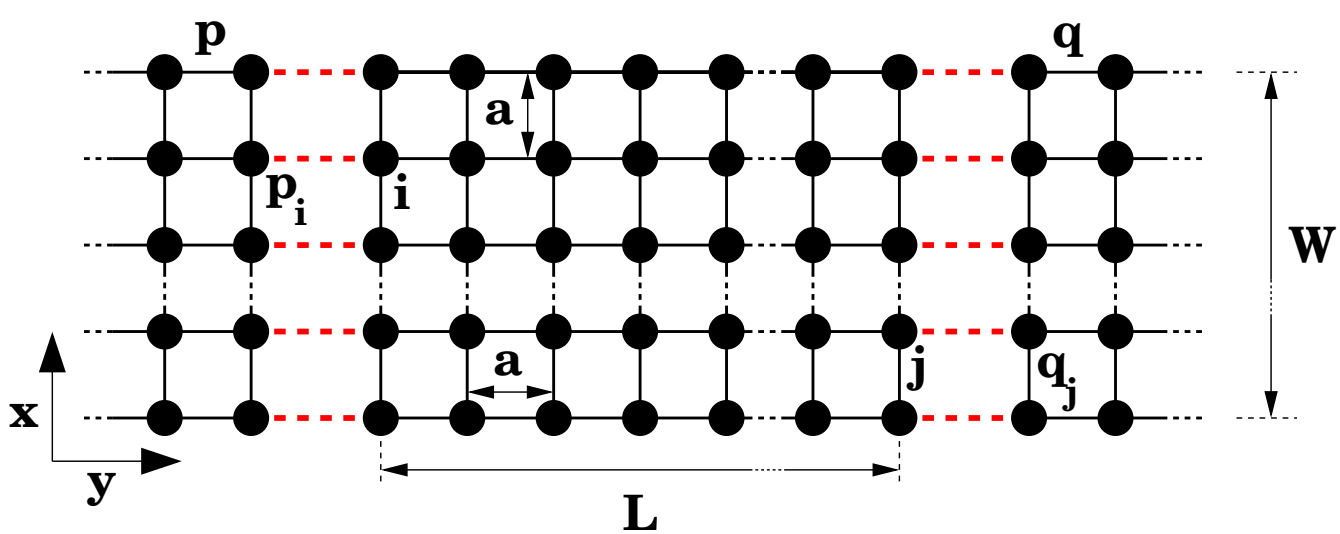

Abbildung 4.21.: Offenes diskretes System mit zwei externen Anschlüssen bzw. Leitern $p$ und $q$ in Transportrichtung. Die gestrichelten roten Linien repräsentieren Kopplungen zwischen den Punkten des Leiters $i$ bzw. $j$ und den Punkten der externen Anschlussleiter $p_{i}$ bzw. $q_{j}$, welche als ideale Leiter halb-unendlich ausgedehnt sind, vergleiche auch Abbildung 1.4 auf Seite 16.

zweidimensionalen Gitters berechnen. Hierbei gilt für die Gesamtenergie

$$
\begin{aligned}
E & =E_{m, x}+E_{m, y} \\
& =E_{m, x}+2 t\left[1-\cos \left(a k_{m}\right)\right],
\end{aligned}
$$

die als Summe der Eigenenergien der transversalen Moden $E_{m, x}$ und der Energien dieser Moden in Ausbreitungsrichtung $E_{m, y}$ mit der Dispersionsrelation für ein diskretes äquidistantes Gitter darstellbar ist. Für den Wellenvektor der ebenen Wellen in Ausbreitungsrichtung können wir also schreiben

$$
k_{m}=\frac{1}{a} \arccos \left[1-\frac{E-E_{m, x}}{2 t}\right] .
$$

Somit hängt die retardierte Selbstenergie $\Sigma^{\mathrm{R}}(E)$ nur von der Energie $E$ der einfallenden Welle ab, welche auch die Anzahl der aktivierten Moden $M(E)$ vorgibt. Daher muss zu jeder einfallenden Energie $E$ das Eigenspektrum des Hamilton-Operators aus Gleichung (4.86) berechnet werden. Für die numerische Bestimmung des kritischen Parameters $B_{\mathrm{c}}$ mit mittleren diagonalen Petermann-Faktoren $\bar{K}(B)$ bedeutet dies einen großen rechnerischen Aufwand und enormen Speicherbedarf, denn zu jeder einfallenden Energie $E$ müssen sowohl linke als auch rechte Eigenvektoren bestimmt werden. Wir wollen uns daher auf die Berechnung der Eigenwertspektren der retardierten Lösungen vermittels Ordnungsparameter $\widetilde{q}_{\mathrm{c}}(B)$ für offene Systeme analog zu Gleichung (4.81) beschränken, wobei die dafür benötigten numerischen Simulationen analog zu Kapitel 1 erfolgen, jedoch hier für ein komplexwertiges Potenzial durchgeführt wurden. Für die ausstehende Definition des Ordnungsparameters $\widetilde{q}_{\mathrm{c}}(B)$ müssen noch einige Voraussetzungen geklärt werden, da sich die Berechnung des Eigenwertspektrums für offene Systeme stark von der für geschlossene Systeme unterscheidet. Bei offenen Systemen sind die Eigenwerte von Beginn an komplexwertig, selbst für ein rein reelles Potenzial $V_{0}(B=0)$. Zur Verdeutlichung seien hier die Eigenwerte des in $\pm y$-Richtung geöffneten Systems aus den letzten beiden Unterabschnitten beispielhaft für die vier einfallenden ebenen Wellen mit den Energien $E=\{t, 2 t, 4 t, 6 t\}$ in Abbildung 4.22 dargestellt. Für "kleine" bzw. "große" eingehende Energien sind viele Eigenwerte reell, da die zugehörigen Energiebänder inaktiv sind. Für $E<4 t$ können die hoch-energetischen Moden nicht aktiv sein, während für $E>4 t$ die nieder-energetischen Moden inaktiv sind. Grund dafür ist die Energieer- 


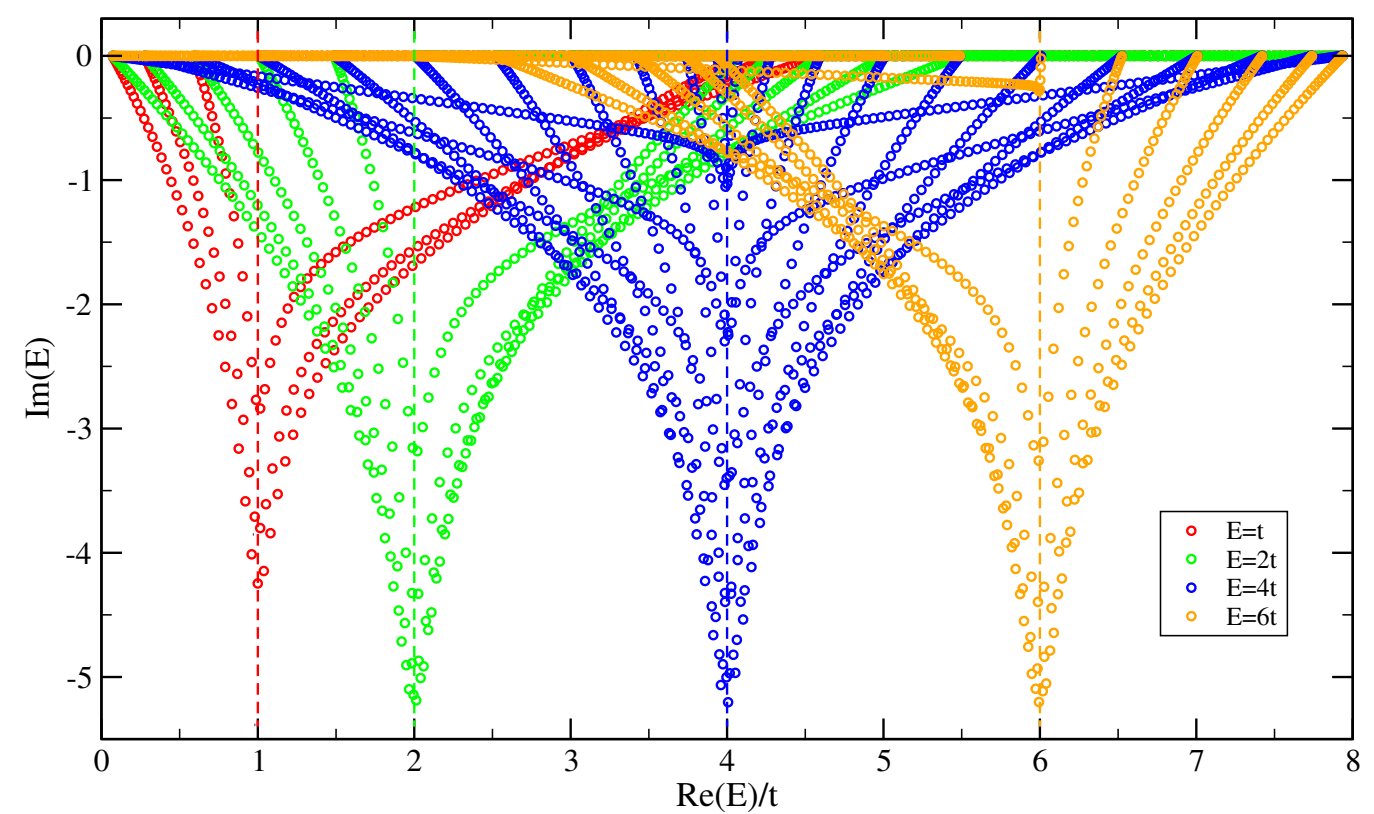

Abbildung 4.22.: Eigenwerte des nach links und rechts geöffneten $11 \times 97$-Systems für das Potenzial $V_{0}(x, y)$ nach Gleichung (4.80) mit $A=C=1$ und $B=0$ mit $t=72$ für einfallende ebene Wellen mit den Energien $E=\{t, 2 t, 4 t, 6 t\}$ (farbige gestrichelte Linien). Die einfallenden Wellen sind wegen der Dispersionsrelation nach Glg. (4.88) auf das Intervall $(0,8 t)$ beschränkt. Die Anzahl der imaginären Bänder für die jeweilige Energie ist äquivalent zur Anzahl der aktiven transversalen Eigenmoden der einfallenden ebenen Wellen (siehe Text).

haltung, denn nur für positive $k_{m}$ aus Gleichung (4.89) existieren überhaupt einfallende ebene Wellen in $y$-Richtung. Die Anzahl der imaginären Energiebänder in Abbildung 4.22 ist äquivalent zu der Anzahl der zum Energietransport in $y$-Richtung beitragenden ebenen Wellen. Die Berücksichtigung der Eigenschaften des Eigenwertspektrums von $\mathcal{H}$ ermöglicht uns eine Definition des Ordnungsparameters für offene Systeme

$$
\widetilde{q}_{\mathrm{c}}(B, E)=\frac{1}{N^{+}} \sum_{i}\left(\operatorname{Im}^{+}\left[\varepsilon_{i}(B, E)\right]-\operatorname{Im}^{+}\left[\varepsilon_{i}(B=0, E)\right]\right)
$$

als gewichtete Summe aller positiven Imaginärteile des Eigenwertspektrums abzüglich der positiven Imaginärteile des Eigenwertspektrums des offenen Systems für $B=0$ (rein reelles Potenzial) ${ }^{24}$, wobei $N^{+}$die Anzahl der positiven Imaginärteile der Eigenwerte bezeichnet. Mit der Definition über das Energieensemblemittel

$$
\widetilde{q}_{\mathrm{c}}(B)=\left\langle\widetilde{q}_{\mathrm{c}}(B, E)\right\rangle_{E}
$$

kann diese Definition energieunabhängig formuliert werden. In Abbildung 4.23a auf der nächsten Seite ist die Bestimmung des kritischen Parameters für das offene $11 \times 97$-System mit Potenzial $V_{0}(x, y)$ bzw. $V_{d}(x, y)$ mit $A=C=1$ beispielhaft dargestellt. Der kritische Punkt $B_{\mathrm{c}}$ ist in Ermangelung einer bestehenden Theorie als Schnittpunkt zweier Ausgleichsgraden bestimmt worden. Er separiert die beiden Phasen propagierender und absorbierender Wellen voneinander.

24 zur Eliminierung des komplexen Spektrums, das durch die Selbstenergie $\Sigma^{\mathrm{R}}$ verursacht wird 


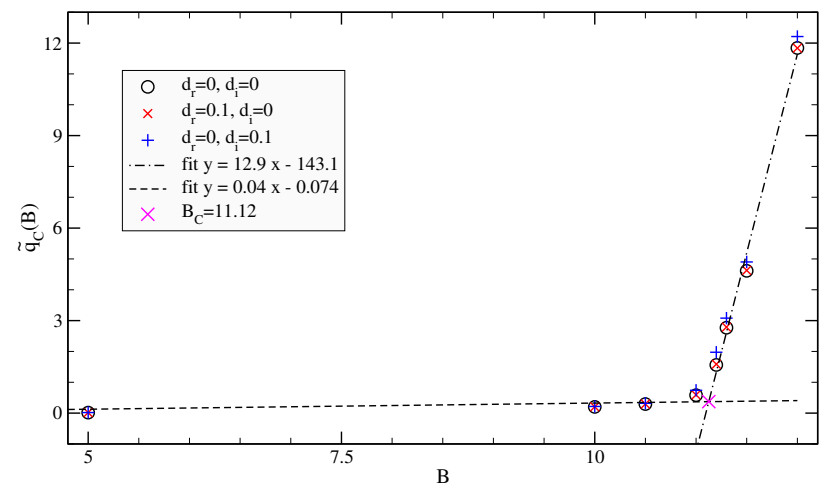

(a)

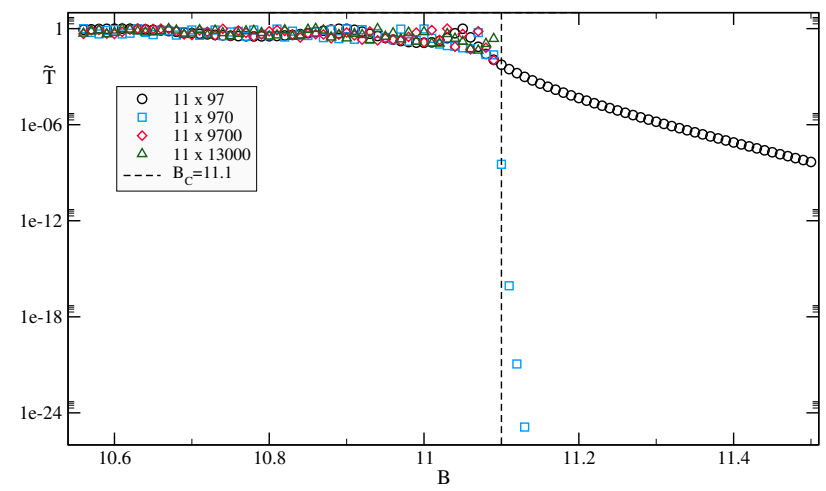

(b)

Abbildung 4.23.: Vergleich des kritischen Parameters $B_{\mathrm{c}}$ mit der Transmissionswahrscheinlichkeit $\widetilde{T}$. (a) Kritischer Parameter und approximative Bestimmung des kritischen Parameters $B_{\mathrm{c}}=11.12$ für das offene $11 \times 97$-System mit Potenzial $V_{0}(x, y)$ bzw. $V_{d}(x, y)$ mit $A=C=1$ und $t=72$. Über 200 verschiedene eingehenden Energien wurde gemittelt. Im Falle rein reeller bzw. rein imaginärer Unordnung wurde zusätzlich jeweils über 20 verschiedene Realisierungen von Unordnung gemittelt. (b) Kritischer Parameter bestimmt durch die Transmissionskoeffizienten $T$ mittels Streuformalismus (siehe Text) für gleiches Potenzial mit unterschiedlichen Längen (fiktive Zeiten) für $E \approx t / 7$. Der Transport ist durch den Phasenübergang bei $B_{\mathrm{c}} \approx 11.1$ klar begrenzt.

\section{Ausblick}

Mit dem in Kapitel 1 ausführlich beschriebenen Streumatrixformalismus kann der Phasenübergang $B=B_{\text {c }}$ ebenfalls bestimmt werden. Der große Vorteil liegt hier in der numerischen Berechnung der Transportkoeffizienten, da hier die Eigenvektoren bzw. Streuzustände des Systems nicht gespeichert werden müssen, sondern nur die Streumatrixelemente $s_{m n}^{p q}$ zu jedem Punkt des Parameterraums $\{E, B\}$ aus den Greenschen Funktionen zu bestimmen sind. Aus der Streumatrix lassen sich die Transmissionsund Reflexionskoeffizienten

$$
T_{p}=\sum_{q \neq p} T_{p \leftarrow q}=\sum_{q \neq p} \sum_{m, n}\left|s_{m n}^{p q}\right|^{2}
$$

und

$$
R_{p}=R_{p \leftarrow p}=\sum_{m, n}\left|s_{m n}^{p p}\right|^{2}
$$

einfach berechnen. Die Indices $p$ bzw. $q$ nummerieren die externen Anschlussleiter (Kanäle), die Subindices $m(p)$ bzw. $n(q)$ die Moden der Kanäle $p$ bzw. $q$. In konservativen Systemen ist die Summe der Transportkoeffizienten gleich der Anzahl der eingehenden Moden im jeweiligen Kanal. Es gilt also für den Kanal $p$ die Energieerhaltung $M_{p}=T_{p}+R_{p}$, eine direkte Folgerung aus der Eigenschaft der $S_{-}$ Matrix der Einzelmoden det $s^{p q}=1$. Mit Einführung des freien Parameters $B$ des Potenzials $V_{0}(x, y)$ verhält sich das System dissipativ, daher müssen die Transmissionskoeffizienten mittels $\widetilde{T}=\frac{T_{p}}{T_{p}+R_{p}}$ normiert werden. Für das behandelte Beispielsystem ist der Zusammenbruch des Transports für unterschiedliche Systemlängen (fiktive Zeiten) in Abbildung 4.23b dargestellt. Wir finden $B_{\mathrm{c}} \approx 11.1$ für $E \approx t / 7$ in der gleichen Größenordnung wie für die direkte Bestimmung mit Diagonalisierung der Hamilton-Matrix.

In zukünftigen Betrachtungen könnten die Petermann-Faktoren des offenen Systems [306] über die Streufunktionen $\psi$ und $\varphi$ der retardierten und avancierten Greenschen Funktionen $G^{\mathrm{R}}$ und $G^{\mathrm{A}}$ analog 
zu Gleichung (4.83) definiert werden

$$
\widetilde{\kappa}_{k l}=\frac{\left\langle\varphi_{k} \mid \varphi_{l}\right\rangle\left\langle\psi_{k} \mid \psi_{l}\right\rangle}{\left\|\left\langle\varphi_{k} \mid \psi_{l}\right\rangle\right\|^{2}}
$$

und zur vergleichenden Analyse verwendet werden.

Die in diesem Abschnitt gewonnenen Ergebnisse können bei der Klassifikation von Metamaterialien helfen, da die $\mu$ - $\varepsilon$-Phasendiagramme von optischen Strukturen mit periodischen Bereichen der Absorption und Anregung, d.h. lokaler $\mathcal{P} \mathcal{T}$-Symmetrie, bisher weitestgehend unverstanden sind.

\section{Zusammenfassung}

Um auch den Einfluss von Rückstreuung in lokal $\mathcal{P} \mathcal{T}$-symmetrischen Systemen zu berücksichtigen, wurde der Streuformalismus auf diese Systemklasse übertragen. Hierzu wurden diskrete HamiltonOperatoren zu einem lokal $\mathcal{P} \mathcal{T}$-symmetrischen Potenzial mit der Schrödinger-Gleichung untersucht. Dies bedeutet aber keine Einschränkung, denn alle Ausführungen gelten auch für Systeme, die sich mit der optischen Wellengleichung beschreiben lassen. Wir begannen mit der Analyse von geschlossenen lokal $\mathcal{P} \mathcal{T}$-symmetrischen Systemen und leiteten zur Klassifizierung der pseudo-hermiteschen Phase einen kritischen Parameter ab, der mit den diagonalen Petermann-Faktoren aus der Optik verglichen wurde. Die positive Übereinstimmung dieser beiden Größen erlaubte die Erweiterung des kritischen Parameters auf offene lokal $\mathcal{P} \mathcal{T}$-symmetrische Systeme, wodurch dann ein Vergleich mit den Transportkoeffizienten des Streuformalismus möglich wurde. Unsere Ergebnisse diesbezüglich deuten ebenfalls auf eine positive Übereinstimmung des kritischen Parameters der aus der direkten Diagonalisierung gewonnenen Eigenwerte mit den Transportkoeffizienten des Streuformalismus zur Klassifizierung der pseudo-hermiteschen Phase hin. Der deutliche Vorteil der hier vorgestellten Methode liegt in der effizienten und stabilen Simulation und der Flexibilität der theoretischen Untersuchung von $\mathcal{P} \mathcal{T}$-symmetrischen Strukturen aktiver optischer Metamaterialien, die experimentellen Realisierungen vorausgehen können und technische Anwendungen versprechen. 


\section{Zusammenfassung}

Es wurde eine effiziente und stabile numerische Methode vorgestellt, die eine optimierte Simulation offener quantenmechanischer und wellenoptischer Systeme ermöglicht. Auf Basis von Greenschen Funktionen und mithilfe des Landauer-Büttiker-Formalismus werden Streuwellenfunktionen, $S$-Matrizen und makroskopische Größen, wie Strom und Spannung vorhersagt. Der numerische Vorteil liegt in ihrer hohen Geschwindigkeit bei gleichzeitig geringem Speicherbedarf begründet, da durch eine geschickte Transformation auf Matrixinversion verzichtet werden kann. Durch die Überführung des Inversionsproblems auf die Problemstellung der Lösung eines spärlich besetzten linearen Gleichungssystems wird es möglich, feinere Gitterapproximationen zu wählen und damit auch eine deutlich höhere Anzahl von Moden in numerische Untersuchungen mit einzubeziehen. Der hier entwickelte Streuformalismus wurde in Kapitel 1 am Beispiel des ballistischen Gleichrichters verifiziert und damit eine bestehende semiklassische Theorie bekräftigt. Dabei konnten sowohl der Gleichrichtungseffekt, als auch der in [21] postulierte Vorzeichenwechsel der Gleichrichtungsspannung bestätigt werden.

Dieser Streuformalismus wurde zunächst genutzt, um optische Wellenleiter mit komplexer Dynamik, die in Kapitel 2 und Kapitel 3 vorgestellt wurden, zu studieren.

In Kapitel 2 wurde eine Konstruktionsmethode vorgeschlagen, um frequenz- bzw. energieabhängige Schalter und Strahlenteiler, Mikrolaser, Multi-Kavitätsresonatoren, Multi-Strahlenteiler und deren Kombinationen aus Wellenleitern mit gemischtem Phasenraum realisieren zu können. Dazu können sowohl elektronische als auch optisch transparente Materialien verwendet werden. Anhand eines Kosinusbillards, das als Prototyp für Billards mit gemischtem Phasenraum diente, wurde demonstriert, wie der Vergleich von klassischen mit quantenmechanischen Phasenräumen - genauer von transienten Poincaré-Abbildungen mit Husimi-Verteilungen - bei der Identifizierung von quasi-gebundenen Zuständen helfen und offene Systeme mit obigen Eigenschaften untersucht werden können. Es wurde gezeigt, dass man den Effekt des dynamischen Tunnelns dazu ausnutzen kann, resonante Moden in einem chaotischen Wellenleiter anzuregen, die die Rolle eines angekoppelten Resonators übernehmen. Die Untersuchung des Wellenleiters mit einer Strahlendynamik erlaubte dann die Konstruktion eines effektiven Einmodenlasers, die als realistische Anwendung eines Mikrolasers hoher Güte gilt.

In Kapitel 3 wurden Müllerzellen der Retina als biologische Wellenleiter hinsichtlich ihrer lichtleitenden Eigenschaften untersucht. Ein wichtiges Resultat mit erheblichen Auswirkungen auf das Streulicht im Auge ist, dass der Endfuß der Müllerzellen mit seiner ausgeprägten Trichterform die Funktion eines optischen Kollimators erfüllt. Des Weiteren konnten experimentelle Ergebnisse zur Wellenleitung der Müllerzelle in einem homogenem Medium quantitativ in den Simulationen reproduziert werden und damit die winkelaufgelösten Transmissions- und Reflexionsspektren in Abhängigkeit vom Einfallswinkel bestimmt werden. Dies erlaubte uns zu untersuchen, in wie weit die Wellenleitereigenschaften der 
Müllerzelle erhalten bleiben, wenn sie - wie in ihrer natürlichen Umgebung der Retina - in ein Medium mit fluktuierendem Brechungsindex eingebettet ist. Hierzu wurde die extrazelluläre Umgebung der Müllerzelle durch ein korreliertes Gaußsches Zufallsfeld modelliert. Es zeigte sich, dass der lichtwellenleitende Charakter der Müllerzelle erhalten bleibt, obwohl die Größe der Fluktuationen größer ist als die Differenz der mittleren Brechungsindices und das Müllerzellen auch im Auge als Lichtwellenleiter funktionieren.

Zellstrukturbedingte Deformationen und die Lage des Zellkerns fanden bei der Bestimmung der winkelabhängigen Transmissions- und Reflexionsspektren ebenfalls Berücksichtigung. Die quantitative Bestimmung der numerischen Apertur bzw. des $V$-Parameters mit Methoden der Faseroptik wurden mit den numerisch bestimmten Transmissionskoeffizienten verglichen. Eine positive Übereinstimmung erlaubte nicht nur die Bestätigung verschiedener Lichtwellenleitereigenschaften der Müllerzelle, sondern darüber hinaus auch die Formulierung eines vereinfachten rotationssymmetrischen Augenmodells, das eine Untersuchung der Streulichtverteilung auf der Retina zulässt. Dazu wurden die Anteile des direkt auf die Retina einfallenden Lichts mit denen des Streulichts 1. Ordnung verglichen. Überraschenderweise ergab sich trotz Vernachlässigung der Photorezeptorverteilung ein deutlich erhöhtes Signal-Rausch-Verhältnis in der Nähe der optischen Achse. Ein Ergebnis, das nur aus der geometrischen Beschaffenheit des Auges und der Müllerzellen resultiert und insbesondere für die Ausbildung einer fovealen Struktur spricht. Ein direkter Vergleich der natürlichen Konfiguration der invertierten Retina mit der fiktiven Konfiguration, in der die Photorezeptoren auf der Oberfläche der Retina angeordnet und somit keine Müllerzellen vorhanden wären, ergab ein in weiten Teilen der Retina deutlich erhöhtes Signal-Rausch-Verhältnis für den Fall der natürlichen Konfiguration, wobei das Modell in der Nähe der Fovea, dem Zentrum des Scharfsehens, ein um den Faktor 25 erhöhtes Signal-RauschVerhältnis voraussagt.

Im letzten Teil der Arbeit wurde der Streuformalismus auf aktive optische Systeme übertragen, die aus räumlich speziell angeordneten Bereichen von verstärkenden (gain) und verlustbehafteten (loss) Materialien bestehen, wobei in Metamaterialien die Verstärkung den Verlust ausgleicht.

In Kapitel 4 wurden erstmals Konsequenzen diskutiert, die sich bei der Realisierung von eindimensionalen $\mathcal{P} \mathcal{T}$-symmetrischen Strukturen mit periodischer Anregung und Absorption durch den Einfluss von Lokalisierung und Unordnung ergeben. Dabei stellte sich heraus, dass sowohl schwache Unordnung, als auch Systemgrenzen prinzipielle Szenarien für das Zusammenbrechen der $\mathcal{P} \mathcal{T}$-Symmetrie darstellen, die jedwede physikalische Realisierung erschweren oder sogar unmöglich machen. Wie analytisch untersucht wurde, bilden Paare von lokalisierten Zuständen mit exponentiell kleinen Energieaufspaltungen der zugehörigen Eigenwerte die Ursache dieses Phänomens.

Jedoch ermöglichte nun das Verständnis der $\mathcal{P} \mathcal{T}$-Symmetriebrechung durch Lokalisierung die Gestaltung einer Konfiguration, die trotz Unordnung und Lokalisierung eine stabile pseudo-hermitesche Phase zeigt. Diese besteht aus kettenartigen Verbindungen einzelner, als Dimer bezeichneter Elemente. Es zeigte sich, dass diese so geschaffenen Strukturen eine stabile (lokale) $\mathcal{P}_{\mathrm{D}} \mathcal{T}$-symmetrische Phase besitzen, die sich stabil unter dem Einfluss von Störstellen und Oberflächen- bzw. Randeffekten verhalten und darüber hinaus viel leichter realisieren lassen, als dies bei global $\mathcal{P} \mathcal{T}$-symmetrischen Systemen der Fall ist. Selbst bei symmetriebrechender Unordnung im Imaginärteil des Brechungsindex zeigt sich, dass die pseudo-hermitesche Phase nur von dem Rauschlevel begrenzt ist und nicht proportional zum 
absoluten Verlust oder Gewinn ist. Damit sind solche Systeme für technische Realisierungen hervorragend geeignet.

Um auch den Einfluss von Rückstreuung in $\mathcal{P} \mathcal{T}$-symmetrischen Systemen untersuchen zu können, wurde der Streuformalismus auf diese Klasse von Systemen übertragen. Dazu wurde zunächst ein kritischer Parameter für $\mathcal{P} \mathcal{T}$-Symmetriebrechung in geschlossenen Systemen definiert, der zu seiner Berechnung nur das Eigenwertspektrum des Systems benötigt und auf optische Systeme übertragbar ist. Durch Vergleich dieses kritischen Parameters mit dem Petermann-Faktor der theoretischen Optik, der ein Maß für die Nicht-Orthogonalität der Eigenfunktionen des Systems angibt, konnte die Verwendung des kritischen Parameters zur Klassifizierung des Phasenübergangs gerechtfertigt werden. Mit der Definitionserweiterung des kritischen Parameters auf offene Systeme wurde ein Vergleich mit den Transmissionskoeffizienten des Streuformalismus zulässig. Erste zu verzeichnende Ergebnisse deuten auf eine positive Übereinstimmung der aus der direkten Diagonalisierung gewonnenen kritischen Parameter sowie der Transmissionskoeffizienten des Streuformalismus hin. Damit ist eine Methode entwickelt worden, die es erlaubt, experimentell und technisch interessante $\mathcal{P} \mathcal{T}$-symmetrische Strukturen von optischen Metamaterialien und anderen aktiven optischen Systemen effizient zu simulieren und theoretisch zu untersuchen. 

Anhang 



\section{A. Dimensionslose Einheiten}

Die Verwendung von Naturkonstanten wie im Internationalen Einheitensystem (SI) definiert ist zwar physikalisch gesehen von großem praktischen Vorteil, im Rahmen numerischer Untersuchungen aber meist nicht zweckdienlich, da dies der numerischen Genauigkeit unzuträglich ist. Die Wahl eines Einheitensystems ist Konvention. So ist das in dieser Arbeit verwandte System von atomaren Einheiten durch die Wahl der folgenden dimensionslosen und auf Eins gesetzten Naturkonstanten

$$
\hbar=1, m_{\mathrm{e}}=1, e=1
$$

definiert. Aus Sicht der linearen Algebra kann auch ein anderer beliebiger Satz linear unabhängiger Basisgrößen gewählt werden (z.B. natürliche Einheiten). Wir wollen uns auf die Wahl der oben genannten physikalischen Größen - also Drehimpuls, Elektronenmasse und Elementarladung - beschränken, wobei dann noch jeweils eine der folgenden Einheiten aus Länge $[l]$, Zeit $[t]$, oder elektrischer Stromstärke $[I]$ frei gewählt werden kann. Bei jedem Basiswechsel von SI Einheiten zum System atomarer Einheiten (und umgekehrt) treten dimensionslose Umrechnungsfaktoren ${ }^{1}$ auf, die im Folgenden behandelt werden.

Das Plancksche Wirkungsquantum $\hbar$, die Ruhemasse des Elektrons $m_{\mathrm{e}}$ und die Elektronen-Elementarladung $e$ sind im Internationalen Einheitensystem definiert als ${ }^{2}$

$$
\begin{aligned}
\hbar & =(1.055571628 \pm 0.000000053) \cdot 10^{-34} \mathrm{Js} \\
m_{\mathrm{e}} & =(9.10938215 \pm 0.00000045) \cdot 10^{-31} \mathrm{~kg} \\
e & =(1.602176487 \pm 0.000000040) \cdot 10^{-19} \mathrm{C} .
\end{aligned}
$$

Die effektive Masse von gebundenen Elektronen im GaAs-Kristall ist $m_{0}=0.065 m_{\mathrm{e}}$ und stellt die verwandte Masseneinheit dar.

Mit $\mathrm{J}=\mathrm{V}$ As und $\mathrm{V}=\mathrm{m}^{2} \mathrm{~kg} \mathrm{~s}^{-3} \mathrm{~A}^{-1}$ können die Einheiten von $\hbar$ als

$$
\frac{[t]^{2}[I][l]^{2}[m]}{[t]^{3}[I]}=\frac{[l]^{2}[m]}{[t]} .
$$

geschrieben werden.

1 Eigentlich werden hier Vektorräume durch Basiswechel-Matrizen ineinander überführt. Eine mathematische Übersicht findet man in [307], eine grundlegende Betrachtung in den ersten beiden Kapiteln von [308].

2 SI Einheiten und Standardungenauigkeiten aus [309, 310]. Für eine ausführliche Darstellung siehe auch [311]. 
Durch die Einführung dimensionsloser Größen (diese Variablen sind im Folgenden durch eine "Tilde" gekennzeichnet)

$$
\begin{aligned}
t & =\widetilde{t} \tau_{0} \\
l & =\widetilde{l} a_{0} \\
m & =\widetilde{m} m_{0} \\
I & =\widetilde{I} I_{0}
\end{aligned}
$$

können die Gleichungen (A.2) und (A.4) mittels der zwei Gleichungen

$$
\begin{aligned}
& \hbar=\widetilde{\hbar} \frac{a_{0}^{2} m_{0}}{\tau_{0}} \\
& e=\widetilde{e} I_{0} \tau_{0}
\end{aligned}
$$

geschrieben werden. Da im natürlichen Einheitensystem die dimensionslosen Größen $\widetilde{\hbar}=1, \widetilde{m}=1$ und $\widetilde{e}=1$ bereits gewählt sind, kann entweder $\tau_{0}$ oder $a_{0}$ oder $I_{0}$ frei gewählt werden.

In dieser Arbeit wurde (in den numerischen Berechnungen und Simulationen) in Einheiten von $a_{0}$ gemessen, der jeweiligen typischen Längenskala des zu betrachtenden Systems. Die Konstante $a_{0}$ steht also beispielsweise für den Bohrschen Atomradius oder die Gitterkonstante eines Halbleiterkristalls. Die Verwendung dieser Längenskala führt zu Reskalierungen der Zeit

$$
\tau_{0}=\frac{a_{0}^{2} m_{0}}{\hbar}
$$

und der Einheit der Energie

$$
[E]=\frac{[m]\left[a_{0}\right]^{2}}{[t]^{2}},
$$

so dass für die reskalierte Energie als dimensionslose Größe folgt

$$
\widetilde{E}=E \frac{\tau_{0}^{2}}{a_{0}^{2} m_{0}}=E \frac{a_{0}^{2} m_{0}}{\hbar^{2}}
$$

Bei bekannter Fermi-Wellenlänge

$$
\lambda_{\mathrm{F}}=\widetilde{\lambda}_{\mathrm{F}} a_{0}
$$

und gegebener Gitterkonstante $a_{0}$ skaliert die Energie $^{3}$ also wie

$$
\widetilde{E}=\frac{\hbar^{2} k_{F}^{2}}{2 m_{0}} \frac{a_{0}^{2} m_{0}}{\hbar^{2}}=\frac{4 \pi^{2} \hbar^{2}}{2 m_{0} \lambda_{\mathrm{F}}^{2}} \frac{a_{0}^{2} m_{0}}{\hbar^{2}}=\frac{2 \pi^{2}}{\widetilde{\lambda}_{\mathrm{F}}^{2}} \simeq \frac{19.74}{\widetilde{\lambda}_{\mathrm{F}}^{2}}
$$

wobei $\tilde{\lambda}_{\mathrm{F}}=\frac{\lambda_{\mathrm{F}}}{a_{0}}$ die mit der Gitterkonstante skalierte Fermi-Wellenlänge repräsentiert.

3 Hier ist die freie Energie der Elektronen des zweidimensionalen Gitters gemeint, das häufig auch die Bezeichnung elektrochemisches Potenzial trägt. 


\section{B. Hamilton-Operatoren stark gebundener Elektronen}

Für die numerische Berechnung experimentell zugänglicher Größen, wie beispielsweise die Transmissionskoeffizienten des Landauer-Büttiker-Formalismus, wäre es wünschenswert, auf eine Methode zurückgreifen zu können, die einfach zu handhaben und dennoch universell genug ist, um sie für verschiedene Problemstellungen einsetzen zu können. Die Methode stark gebundener Elektronen (tightbinding-method) in Verbindung mit dem Greens-Formalismus stellt eine solche Möglichkeit bereit, wobei durch Vorgabe einer Gitterkonstanten $a$ das System quasi beliebig genau (nur begrenzt durch die Rechenzeit und den Speicherbedarf) an den Kontinuumsgrenzwert approximiert werden kann. Für Gitterkonstanten, die größer als die Fermi-Wellenlänge $\lambda_{\mathrm{F}}$ des Systems sind, bilden die Atome mit der Umgebung zum Radius a eine Art "Mittelwert" für Größen, wie z.B. die Aufenthaltswahrscheinlichkeit der Wellenfunktionen, welcher dann durch einen einzelnen Gitterplatz repräsentiert wird. Deshalb sollte die Gitterkonstante $a$ in der Größenordnung der Fermi-Wellenlänge $\lambda_{\mathrm{F}}$ gewählt werden.

Die Methode geht von Ein-Elektronen-Wellenfunktionen aus, die stark an den Atomen des Festkörpers lokalisiert sind. Damit wird vor allem die Superposition von Wellenfunktionen möglich, die dieses Modell so einfach und flexibel gestaltet. Der Hamilton-Operator des Gesamtsystems

$$
\mathcal{H}=\mathcal{H}_{0} \oplus \mathcal{H}_{\mathrm{el}}
$$

besteht aus dem Hamilton-Operator $\mathcal{H}_{0}$ der freien Elektronen und dem Hamilton-Operator $\mathcal{H}_{\text {el }}$, der den Einfluss des elektrischen Potenzials $V_{\text {el }}$ beschreibt, das von den Atomen gebildet wird. Für in dieser Arbeit verwandte Systeme können wir uns bei der Diskretisierung des Hamilton-Operators in Ortsdarstellung

$$
\mathcal{H}(\mathbf{r})=\frac{1}{2}(i \nabla+\mathbf{A}(r))^{2}+V_{\mathrm{el}}(\mathbf{r})
$$

mit äußerem Magnetfeld $\mathbf{B}(\mathbf{r})=\nabla \times \mathbf{A}(\mathbf{r})$ auf die zweidimensionale Formulierung beschränken, wobei der Ort

$$
\mathbf{r}=(x, y)^{\mathrm{T}}=(n a, m a)^{\mathrm{T}}
$$

mit $m, n \in \mathbb{Z}$ die Gitterplätze nummeriert. Die Näherung des Nablaoperators, häufig auch Methode der finiten Differenzen genannt

$$
\nabla f=\frac{1}{a}\left(\begin{array}{c}
f\left(\left[n+\frac{1}{2}\right] a\right)-f\left(\left[n-\frac{1}{2}\right] a\right) \\
f\left(\left[m+\frac{1}{2}\right] a\right)-f\left(\left[m-\frac{1}{2}\right] a\right)
\end{array}\right)
$$


führt auf die Darstellung des diskreten Hamilton-Operators [312]

$$
\begin{aligned}
\mathcal{H}= & -\sum_{n, m} \varepsilon_{n m}|n, m\rangle\langle n, m| \\
& +\sum_{n, m}\left[t_{n m}|n+1, m\rangle\left\langle n, m\left|+t_{n m}\right| n, m+1\right\rangle\langle n, m|+\text { h.c. }\right]
\end{aligned}
$$

mit der Gitterplatzenergie $\varepsilon_{n m}=V_{n m}+4 t$ und elektrischem Potenzial $V_{n m}=V(a n, a m)$ und den Austauschenergien $t_{n m}=t \exp \left(-i a \mathbf{A}\left[\mathbf{r}_{n}-\mathbf{r}_{m}\right]\right)$ mit $t=\frac{1}{2 a^{2}}$, wobei die Darstellung $|n, m\rangle$ lokalisierte Zustände am Ort $(n a, m a)$ bezeichnet und $\{|n, m\rangle\}$ eine orthogonale Menge bildet. Für den magnetfeldfreien Fall $(B=0)$ finden wir dann den Bandstrukturhamiltonian in der Darstellung der zweiten Quantisierung

$$
\mathcal{H}=-\sum_{i} \varepsilon_{i} c_{i}^{+} c_{i}-t \sum_{i, j} c_{i}^{+} c_{j}+\text { h.c. }
$$

mit den Kommutatorrelationen

$$
\begin{aligned}
{\left[c_{i}, c_{j}^{+}\right] } & =\delta_{i j} \\
{\left[c_{i}^{+}, c_{j}^{+}\right]=\left[c_{i}, c_{j}\right] } & =0
\end{aligned}
$$

aus den Bewegungsgleichungen der Erzeugungsoperatoren $c_{i}^{+}$und Vernichtungsoperatoren $c_{i}$ auf einem Quadratgitter mit Gitterkonstante $a$, wobei die Gitterpunkte mit $i$ und $j$ nummeriert werden. Theoretische Abhandlungen über Hamilton-Operatoren mit stark gebundenen Elektronen (tight-binding-hamiltonians) sind in den Arbeiten von Slater et al. [313] und Goringe et al. [314] zu finden. Einen Überblick über Rechenmethoden mit solchen Hamiltonians kann in den Proceedings of MRS [315] nachgelesen werden. Moderne experimentelle Realisierungen von Fermi-Hubbard-Modellen ${ }^{1}$ als übergeordnete Klasse der tight-binding-Hamiltonians durch Fermi-Quanten-Gase in optischen Gittern können in Esslinger [316] studiert werden.

1 Im Hubbard-Modell wird der Hamilton-Operator durch den zusätzlichen Term $U \sum_{i} c_{i, \uparrow}^{+} c_{i, \uparrow} c_{i, \downarrow}^{+} c_{i, \downarrow}$ erweitert, der die Elektron-Elektron Wechselwirkung berücksichtigt, wobei die Erzeugungs- und Vernichtungsoperatoren einen zusätzlichen Index $\sigma=\uparrow, \downarrow$ erhalten, der der Spinabhängigkeit des Systems Rechnung trägt. 


\section{Transversale Moden}

Analog zu den transversal-elektrischen oder den transversal-magnetischen Zuständen bzw. Moden in elektromagnetischen Wellenleitern bilden sich in schmalen (idealisiert halb-unendlichen) elektrischen 2D-Leitern transversale Moden aus, die diskrete Eigenenergien annehmen und deren Eigenfunktionen ein orthogonales System bilden. Der allgemeine Hamilton-Operator nach Gleichung (B.2) mit einem zur $y$-Achse, die entlang des Leiterquerschnitts verläuft, spiegelsymmetrischen Potenzial $V(y)$, welches häufig durch eine harmonische Näherung

$$
V(y)=\frac{1}{2} \omega_{0}^{2} y^{2}
$$

approximiert wird [16], kann mit der Schrödinger-Gleichung

$$
\left[E_{\mathrm{s}}+\frac{1}{2}(i \nabla+\mathbf{A})^{2}+V(y)\right] \psi(x, y)=E \psi(x, y)
$$

ausgedrückt werden, wobei $\mathbf{A}$ das Vektorpotenzial zum Magnetfeld $\mathbf{B}=\nabla \times \mathbf{A}$ und die $x$-Achse die Ausbreitungsrichtung der propagierenden Welle bezeichnet. Die Energie $E_{\mathrm{s}}$ ist bei tiefen Temperaturen die konstante Energie des untersten Bandes, die als niedrigste Eigenenergie aus der Beschränkung auf die $(x-y)$-Ebene aufgrund des Kontaktpotenzials $U(z)$ resultiert (siehe Unterkapitel 1.1). Für die Wellenfunktion liefert der Separationsansatz

$$
\psi(x, y)=\phi(x) \chi(y)
$$

wegen der großen längsgerichteten Ausdehnung des Systems und der Unabhängigkeit des Potenzials $V(y)$ bezüglich der $x$-Komponente in erster Näherung ebene Wellen

$$
\phi(x)=\frac{1}{\sqrt{L}} \exp \left(i k_{x} x\right)
$$

so dass wir für die Schrödinger-Gleichung der transversalen Moden (C.2) für $B=0 \mathrm{zu}$

$$
\left[E_{\mathrm{s}}+\frac{1}{2}\left(k^{2}+p_{y}^{2}+\omega_{0}^{2} y^{2}\right)\right] \chi(y)=E \chi(y)
$$

vereinfachen können. Als Lösungen finden wir die bekannten Eigenfunktionen des Harmonischen Oszillators (siehe Abb. C.1a)

$$
\chi_{n}(y)=\sqrt[4]{\frac{\omega_{0}}{\pi}} \sqrt{\frac{1}{2^{n} n !}} \exp \left(-\frac{\omega_{0} y^{2}}{2}\right) H_{n}\left(\sqrt{\omega_{0}} y\right),
$$




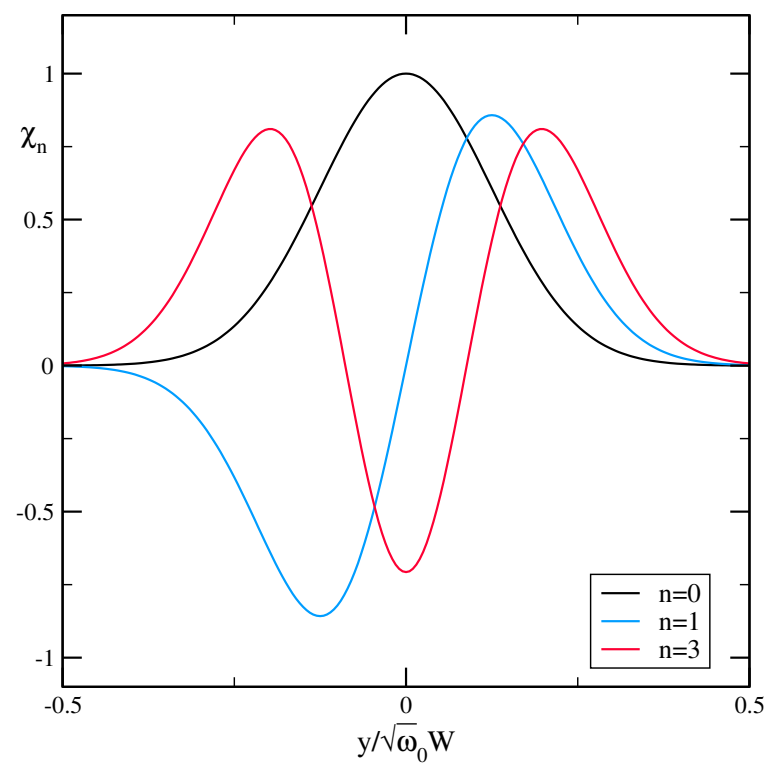

(a)

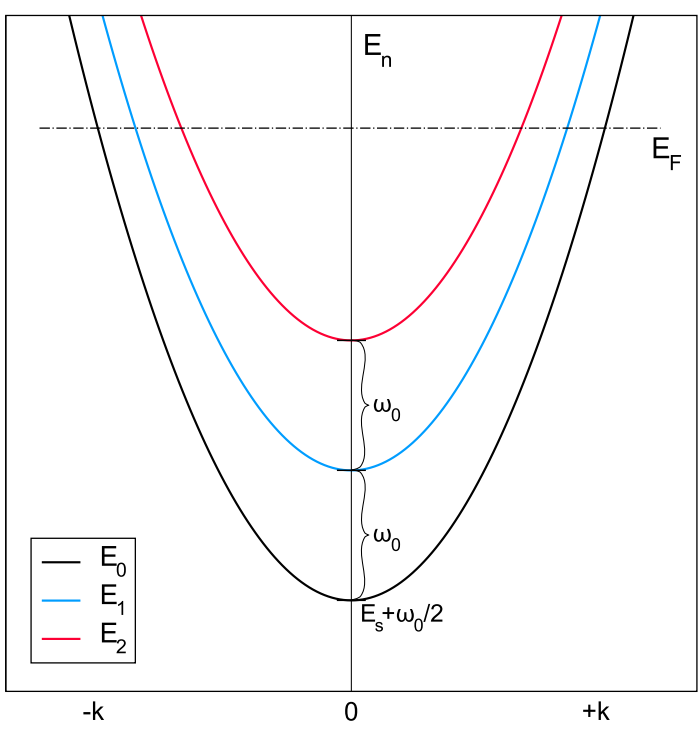

(b)

Abbildung C.1.: (a) Eigenfunktionen und (b) Eigenenergien des idealen Leiters in der harmonischen Näherung. Dargestellt sind die ersten drei Eigenmoden $\chi_{m}(y)$ bzw. Eigenenergien $E\left(k, \omega_{0}\right)$ der ersten drei elektrischen Subbänder.

wobei die explizite Darstellung der Hermite-Polynome

$$
H_{n}(y)=(-1)^{n} \sum_{l+2 m=n} \frac{n !}{l ! m !}(-1)^{l+m}(2 y)^{l}
$$

mit $l, m \in \mathbb{N}$ lautet, die mit der als Fà̀ di Bruno Formel ${ }^{1}$ bekannten höheren Kettenregel aus ihrer erzeugenden Funktion

$$
H_{n}(y)=(-1)^{n} e^{y^{2}} \frac{d^{n}}{d y^{n}} e^{-y^{2}}
$$

abgeleitet werden kann. Die Orthogonalitätseigenschaft der Hermite-Polynome

$$
\int e^{-y^{2}} H_{n}(y) H_{m}(y) d y=2^{n} n ! \sqrt{\pi} \delta_{n m}
$$

überträgt sich auf die Eigenfunktionen $\chi(y)$ und es gilt die Orthogonalitätsrelation

$$
\int \chi_{n}(y) \chi_{m}(y) d y=\delta_{n m}
$$

Für die Energieeigenwerte gibt der Ausdruck

$$
E_{n}=E_{\mathrm{s}}+\frac{k^{2}}{2}+\left(n+\frac{1}{2}\right) \omega_{0}
$$

die Bandstruktur eines perfekten Leiters in 2D wieder (siehe Abb. C.1b).

1 Diese Formel sollte eigentlich Tiburce Abadie Formel heißen, der die höhere Kettenregel bereits 5 Jahre früher entdeckte und auch veröffentlichte. Siehe dazu [317]. 


\section{Phasenraummethoden}

\section{D.1. Quantenmechanische Verallgemeinerung der klassischen Phasenraumdichte}

In der klassischen Physik können die Trajektorien im Phasenraum dazu benutzt werden, reguläre Bereiche des Phasenraums von chaotischen Bereichen zu unterscheiden. Dieser Abschnitt dient der Einführung von quantenmechanischen Phasenraumdichten, um einen Zugang zur Untersuchung von Lokalisierungsphänomenen in der Quantenmechanik zu ermöglichen. Gleichzeitig wird damit eine Vergleichsmöglichkeit mit dem Phasenraum der klassischen Physik geschaffen.

\section{D.1.1. Zustandsbeschreibungen in der Quantentheorie}

Die Definition eines quantenmechanischen Phasenraums - angelehnt an die klassische Beschreibung des Phasenraums - ist aufgrund des Welle-Teilchen-Dualismus nicht direkt möglich. Ein einzelnes Teilchen kann also keinem exakten Punkt im Phasenraum zugeordnet werden, d.h. der Ort und der Impuls eines Quantenobjektes ist nicht gleichzeitig und exakt bestimmbar. Die Genauigkeit der Lokalisierung des Teilchens ist durch die Heisenbergsche Unschärferelation [318]

$$
\Delta x \Delta k \geq \frac{1}{2}
$$

limitiert. ${ }^{1}$ Die Unschärferelation lässt sich noch verallgemeinern. Demnach gilt die Aussage über die Unmöglichkeit der gleichzeitigen und exakten Bestimmung zweier Messgrößen $a$ und $b$ eines Quantenobjekts, wenn deren Produkt die Dimension einer Wirkung hat. Sind also $a$ und $b$ zueinander konjugierte Variablen, so gilt für das Produkt ihrer Standard-Streuungen

$$
\Delta a \Delta b \approx n \geq \frac{1}{2}
$$

wobei $n$ für einen systemabhängigen Parameter des betrachteten Zustands steht. Handelt es sich um einfache Potenziale, wie z.B. das Kastenpotenzial, das Coulomb-Potenzial oder das Potenzial des harmonischen Oszillators, dann ist $n$ die Quantenzahl des betrachteten reinen Zustands. Sonst legt $n=\frac{1}{2}$ eine untere Schranke für das Produkt der Streuungen für jedes Paar kanonischer Variablen fest [319]. So ist z.B. die gleichzeitige Messung des Ortes und des Impulses mit dieser minimalen Unschärfe besetzt. In allen weiteren Betrachtungen wollen wir uns aber auf das kanonische Variablenpaar $(x, k)$ beschränken.

1 In dieser Arbeit gilt $\hbar=1$, siehe Anhang A. 


\section{D.1.2. Wellenfunktionen und Dichtefunktionen}

Mit der komplexen Wellenfunktion eines Zustands $\psi(x)$ im Ortsraum ist auch die Darstellung des Zustands $\phi(k)$ im Impulsraum über die Fourier-Transformation bezüglich der konjugierten Variablen $x$ und $k$ über

$$
\phi(k)=\int \psi\left(x^{\prime}\right) e^{-i k x^{\prime}} d x^{\prime}
$$

festgelegt. Die zugehörige Rücktransformation

$$
\psi(x)=\int \phi\left(k^{\prime}\right) e^{i k^{\prime} x} d k^{\prime}
$$

liefert dann den Ortszustand im Falle eines gegebenen Impulszustands. So sind stark lokalisierte Wellenfunktionen des Ortsraums im Impulsraum nicht lokalisiert und umgekehrt. Es sei angemerkt, dass sich Gleichung (D.1) als Eigenschaft der Fourier-Transformation herleiten lässt (siehe [319]).

In Analogie zur klassischen Phasenraumdichte werden in der Quantenmechanik Dichtekerne eingeführt [320]. Wir wollen uns hier auf die Angabe des Ortsdichtekerns

$$
\rho\left(x, x^{\prime}\right)=\psi(x) \psi^{*}\left(x^{\prime}\right)
$$

und des Impulsdichtekerns

$$
\sigma\left(k, k^{\prime}\right)=\phi(k) \phi^{*}\left(k^{\prime}\right)
$$

beschränken. ${ }^{2}$ Die beiden Dichtekerne sind durch die Fourier-Transformationen (D.3a) und (D.3b) ineinander überführbar. Durch die Wahl von $x=x^{\prime}$ geht der Ortsdichtekern (D.4a) in die rein reellwertige positive Ortsverteilung

$$
\rho(x)=|\psi(x)|^{2} \quad
$$

durch Wahl von $k=k^{\prime}$ geht der Impulsdichtekern (D.4b) in die rein reellwertige positive Impulsverteilung über

$$
\sigma(k)=|\phi(k)|^{2}
$$

\section{D.1.3. Verteilungsfunktionen des Phasenraums}

\section{D.1.3.1. Die Wigner-Funktion}

Die Wigner-Funktion [321, 322], auch Wigner-Weyl-Transformation oder Wigner-Ville-Verteilung genannt, ist eine Quasi-Wahrscheinlichkeitsverteilung. Ihre Ortsdarstellung ergibt sich durch Wahl von $\rho(x+y, x-y)$ und einer Fourier-Transformation bezüglich $y=\frac{x^{\prime}}{2}$

$$
\begin{aligned}
W(x, y) & =2 \int \exp (2 i k y) \rho(x-y, x+y) d y \\
& =\int \exp \left(i k x^{\prime}\right) \psi\left(x-\frac{x^{\prime}}{2}\right) \psi^{*}\left(x+\frac{x^{\prime}}{2}\right) d x^{\prime}
\end{aligned}
$$

2 Diese Ausführungen sind auch mithilfe der Operatordarstellung bzw. mit Dichtematrizen möglich. Mit dem Dichteoperator $\hat{\rho}_{i j}=|i\rangle\langle j|$ lässt sich der Mittelwert einer Observable $A$ als $\langle A\rangle=\operatorname{Spur}(\hat{\rho} A)$ schreiben. 
und ihre Impulsdarstellung durch Wahl von $\sigma(k-y, k+y)$ und einer Fourier-Transformation bezüglich $y=\frac{k^{\prime}}{2}$

$$
\begin{aligned}
W(x, k) & =\frac{1}{\pi} \int \exp (i y x) \sigma(k-y, k+y) d y \\
& =\frac{1}{2 \pi} \int \exp \left(i k^{\prime} x\right) \phi\left(k+\frac{k^{\prime}}{2}\right) \phi^{*}\left(k-\frac{k^{\prime}}{2}\right) d k^{\prime} .
\end{aligned}
$$

Sie nimmt für nicht-klassische Zustände negative Werte an und kann dazu benutzt werden, genau solche Zustände aufzufinden. Die Wigner-Verteilung hat noch andere interessante Eigenschaften. So liefert beispielsweise eine Integration von $W(x, y)$ über den Ortsraum die Impulsverteilung $\sigma(k)$, eine Integration von $W(x, y)$ über den Impulsraum die Ortsdichte $\rho(x)$. Obwohl man die Wigner-Verteilung nicht direkt als Phasenraumdichte deuten kann, ist sie hier aufgrund ihrer historischen Bedeutung für die Quantenmechanik und die Anwendung in vielen Gebieten der Physik der Vollständigkeit halber angegeben.

\section{D.1.3.2. Die Husimi-Funktion}

Die positive Husimi-Funktion, auch Husimi-Q-Funktion oder Husimi-Verteilung genannt, ist definiert als echte Wahrscheinlichkeit [118], findet also direkt als quantenmechanische Phasenraumdichte Verwendung. Die minimale Verschmierung mit einem Gaußschen Wellenpaket, d.h. mit einem Gaußartigen Quantenzustand, verhindert dabei, dass Zustände im Phasenraum stärker lokalisiert sein können, als dies von der Unschärferelation (D.1) zugelassen wird

$$
H(x, k)=\left|\frac{1}{\sqrt[4]{2 \pi \sigma^{2}}} \int \exp \left(-\frac{\left(x-x^{\prime}\right)^{2}}{4 \sigma^{2}}-i k x^{\prime}\right) \psi\left(x^{\prime}\right) d x^{\prime}\right|^{2}
$$

Die Varianz $\sigma^{2}=\frac{\pi}{2 \sqrt{2 E}}$ der Gauß-Funktion ergibt sich dabei als direkte Konsequenz aus der Heisenbergschen Unschärferelation (D.1) unter Beachtung der Phasenraumsymmetrie. Die Husimi-Funktion lässt sich durch eine direkte Messung bestimmen [323]. Der Vollständigkeit halber sind die Transformationen der Wigner-Funktion in die Husimi-Funktion mittels Faltung mit einer Gauß-Funktion

$$
H(x, k)=\frac{1}{2 \pi} \int \exp \left(-\frac{\left(x-x^{\prime}\right)^{2}}{2 \sigma^{2}}-2 \sigma^{2}\left(k-k^{\prime}\right)\right) W\left(x^{\prime}, k^{\prime}\right) d x^{\prime} d k^{\prime}
$$

und umgekehrt

$$
W(x, k)=\frac{1}{4 \pi^{2}} \int\left\{\int \exp \left(\frac{\xi^{2}}{8 \sigma^{2}}+\frac{\sigma^{2} \kappa^{2}}{2}-i \kappa\left(x-x^{\prime}\right)-i \xi\left(k-k^{\prime}\right)\right) H\left(x^{\prime}, k^{\prime}\right) d x^{\prime} d k^{\prime}\right\} d \xi d \kappa
$$

angegeben. Diese Transformationen sind exakt.

\section{D.1.3.3. Andere Verteilungsfunktionen}

Eine weitere Quasi-Verteilungsfunktion, mit der klassische Zustände von nicht-klassischen Zuständen unterschieden werden können, ist die Glauber-Sudarshan-Verteilung [324, 325]. Sie hat ihren Ursprung in der Quantenoptik und nutzt die Übervollständigkeit von kohärenten Photonenzuständen 
aus. Die Glauber-Sudarshan-Verteilung kann für optische Fock-Zustände oder für gequetschte, d.h. in der Amplitude oder in der Phase lokalisierte Zustände, hochgradig singulär werden. Es gibt weitere Quasi-Wahrscheinlichkeitsverteilungen, wie die Kirkwood-Verteilung aus der Quantenstatistik [326], die generalisierte P-Verteilung oder die positive P-Verteilung für komplexe Probleme der Quantenoptik [327]. Alle aufgezählten Verteilungen lassen sich aber durch spezielle Klassen von bilinearen oder quadratischen Transformationen ineinander überführen [328].

Eine Übersicht der oben genannten quantenmechanischen Phasenraum-Verteilungsfunktionen mit Fokus auf ihre charakteristischen Eigenschaften und den Relationen zwischen den Verteilungen, sowie einigen einfachen Anwendungen findet man im Physics Report von H.-W. Lee [329]. 


\section{E. Zur Dichteverteilung der Photorezeptoren im menschlichen Auge}

Die Dichteverteilung der Photorezeptoren ist eine Funktion der retinalen Exzentrizität. Bei den Zapfen ist die Photorezeptorart (S, M oder L als Bezeichnung für short, middle oder long im Bezug auf die Wellenlänge) eine weitere bestimmende Größe. Es existiert bis heute keine geschlossene Theorie, die die Verteilungen in analytischer Weise zu quantifizieren vermag. Qualitative Untersuchen gibt es jedoch viele (siehe z.B. [162, 164, 165, 166, 330]). Gleichwohl finden sich in der Literatur Bemühungen, die Dichteverteilung der Photorezeptoren zu approximieren. Diesem Versuch soll hier gefolgt werden.

\section{E.1. Zapfen-Photorezeptordichte}

Im Falle der approximativen Bestimmung der Dichteverteilung $[160,161]$ für die Zapfen-Photorezeptoren fand die modifizierte Lorentz-Ausgleichsrechnung

$$
p_{\text {Zapfen }}\left(e_{\mathrm{r}}\right)=\frac{a_{0}}{\left(\left|e_{\mathrm{r}}\right|+a_{1}\right)^{2}}+a_{2}
$$

Verwendung, wobei $e_{\mathrm{r}}$ die retinale Exzentrizität (medialer Abstand von der Fovea) darstellt und die Parameter

$$
a_{0}=23263.5, a_{1}=0.346373, \text { und } a_{2}=3500
$$

aus Abbildung 3.3 auf Seite 58 bestimmt worden. Der Parameter $a_{2}$ ist dabei das Minimum der experimentell bestimmten Verteilung, wobei $p_{\text {Zapfen }}\left(e_{\mathrm{r}}=0\right) \approx \frac{a_{0}}{a_{1}^{2}}$ das Maximum der Dichteverteilung darstellt. Durch Einsetzen der experimentell bestimmten Extremwerte besitzt die Approximationsfunktion (E.1) noch einen freien Parameter $a_{1}$. Die erhöhte Konzentration der Zapfen in Foveanähe und ihr Vorkommen in geringerer, schwach sinkender Anzahl im Bereich der Retina außerhalb der Fovea wird hier durch die modifizierte Lorentz-Verteilung, die weder Mittelwert noch Varianz besitzt, gut wiedergegeben.

\section{E.2. Stäbchen-Photorezeptordichte}

Für die Approximation der Dichteverteilung der Stäbchen wurde [162, 163] folgend eine Regression mit einem Polynom 6. Grades durchgeführt

$$
p_{\text {Stäbchen }}\left(e_{\mathrm{r}}\right)=\sum_{n=0}^{6} a_{n} e_{\mathrm{r}}^{n} .
$$

Die numerisch bestimmten Parameter der biquadratisch-stabilen Regression können der Tabelle E.1 auf der nächsten Seite entnommen werden. 


\begin{tabular}{|lc||c|c|c|r|r|r|c|}
\hline & & $a_{0}$ & $a_{1}$ & \multicolumn{1}{c|}{$a_{2}$} & \multicolumn{1}{c|}{$a_{3}$} & \multicolumn{1}{c|}{$a_{4}$} & \multicolumn{1}{c|}{$a_{5}$} & $a_{6}$ \\
\hline \hline nasal & $\left(e_{\mathrm{r}}<0\right)$ & -9338.2 & -98487 & -25692 & -3445.8 & -253.35 & -9.4523 & -0.13931 \\
\hline temporal & $\left(e_{\mathrm{r}}>0\right)$ & -8506.8 & 100550 & -26212 & 3406.7 & -248.41 & 9.5841 & -0.15227 \\
\hline
\end{tabular}

Tabelle E.1.: Parameter der biquadratisch-stabilen Regression mit Polynom 6. Grades zur Approximation der Stäbchen-Dichteverteilung in gesunder ausgewachsener menschlicher Retina. 


\section{F. Retinale Verschaltung}

Abgesehen von der differenzierten Verteilung der Zapfen- und Stäbchen in der Retina (vgl. Unterabschnitt 3.1.2) unterscheiden sich die Photorezeptoren auch in ihrer neuronalen Verschaltung. Dies hat einen großen Einfluss auf das Sehen. Im optischen Nerv findet man ca. 1.2 $10^{6}$ Einzelfasern [331], jedoch gibt es mehr als $1.3 \cdot 10^{8}$ Photorezeptoren in der Netzhaut. Für diese so genannte Konvergenz über zwei Größenordnungen sind Verschaltungen auf neuronaler Ebene notwendig, die sich auf die 5 Klassen Neuronen der Retina (Photorezeptoren, Bipolarzellen, Horizontalzellen, Amakrinzellen und Ganglienzellen) beziehen.

Schaltungstechnisch lassen sich zwei Gruppen von retinalen Verschaltungsarten angeben:

1. vertikale Verschaltungen von den Photorezeptoren über Bipolarzellen zu den Ganglienzellen

2. lateral konvergente Verschaltungen

a) von den Photorezeptoren über die Horizontalzellen wieder zu den Photorezeptoren

b) von den Bipolarzellen über Amakrinzellen zu den Ganglienzellen.

Funktionstechnisch lassen sich zwei Gruppen von Verschaltungen ableiten: die neuronalen Verschaltungen für photopisches und für skotopisches Sehen. Diese beiden Gruppen sollen im Folgenden kurz vorgestellt werden.

Das photopische Sehen wird von den Zapfen-Photorezeptoren gestützt, die jeweils mit zwei Bipolarzellen "ON" und "OFF" über eine invertierende und eine nichtinvertierende Synapse, von den Bipolarzellen weiter mit jeweils einer Ganglienzelle zusammengeschaltet sind (Verschaltungstyp 1). Die Kennzeichnung "ON" und "OFF" bezieht sich auf die rezeptiven Felder, denen sowohl Bipolarzellen, als auch Ganglienzellen angehören. Diese Felder werden durch die Horizontalzellen (Verschaltungstyp 2a) und Amakrinzellen (Verschaltungstyp 2b) ausgebildet. Durch die erhöhte Konvergenz der Ganglienzellen sind die rezeptiven Felder größer und können zwischen $10 \mu \mathrm{m}$ in der Fovea und mehr als $300 \mu \mathrm{m}$ in der Retinaperipherie durchmessen [332]. Bei einem rezeptiven Feld mit "ON"-Zentrum handelt es sich um ein konzentrisches Areal auf der Netzhaut, deren Zentrum bei Beleuchtung eine Erhöhung der Ganglienzellaktivität und deren Abdunkelung in der Peripherie eine Hemmung der Ganglienzellaktivität hervorruft. Beim entsprechenden rezeptiven Feld mit "OFF"-Zentrum ist es umgekehrt.

Das skotopische Sehen wird von den Stäbchen getragen, die ebenfalls konvergent mit StäbchenBipolarzellen verschaltet sind (Verschaltungstyp 1). Bis zu 15 Stäbchen-Photorezeptoren sind so mit einer Stäbchen-Bipolarzelle verbunden. Diese ist wiederum über Amakrinzellen mit dem System des photopischen Sehens vernetzt (Verschaltungstyp 2b). Das von den Stäbchen getragene skotopische System kann demnach als Untergruppe des photopischen Systems angesehen werden [333]. Beide vorgestellten minimalen Grundsysteme - Zapfen-Photorezeptor, "ON"- und "OFF"-Bipolarzelle, "ON"und "OFF"-Ganglienzelle auf der einen und Stäbchen-Photorezeptoren, Stäbchen-Bipolarzelle und 
Amakrinzelle auf der anderen Seite - bilden zusammen mit einer Müllerzelle eine säulenartige Einheitszelle. In der menschlichen Retina existieren etwa $10^{7}$ solcher Einheitszellen [156] (siehe Abb. 3.2). Für die Übertragung der aufgenommenen und zum Teil verarbeiteten Bildinformation dienen die Nervenfasern der Ganglienzellen, die zum optischen Nerv gebündelt werden. Da die Retina des linken und des rechten Auges jeweils vertikal in zwei funktionale Hälften unterteilt ist, wechseln bei vielen Wirbeltieren die nasal gelegenen Sehnervenfasern am Sehnervenkreuz (chiasma opticum) auf die gegenüberliegende Seite. Der Sehnerv der korrespondierenden Netzhauthälften (Gesichtsfelder) läuft weiter zum linken bzw. rechten Kniehöcker (corpus geniculatum laterale) des Zwischenhirns und von dort zum primären Sehrinde (visueller Cortex, V1). Für eine detaillierte Beschreibung der Sehbahn, also der neuronalen Verschaltung von den Photorezeptoren bis zu den Neuronen der primären Sehrinde, sei auf die Literatur verwiesen [334, 335]. 


\section{G. Einfache Fehlstelle in perfekter unendlicher Kette}

Die Voraussetzung zur Untersuchung von lokalisierten Zuständen in 1D Multi-Fehlstellensystemen (Systeme mit Unordnung) ist fundiertes Wissen über die einfache Fehlstelle, eingebettet in eine sonst perfekte Kette, wie in Abbildung G.1 schematisch dargestellt.

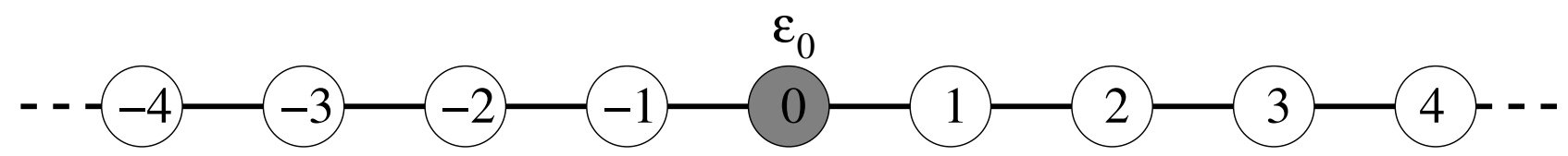

Abbildung G.1.: Schematische Darstellung einer (unendlichen) linearen Kette mit einer (reellwertigen) Fehlstelle $\varepsilon_{0}=-\beta$ bei $n=0$.

Die Schrödinger-Gleichung für dieses System kann mit

$$
-\psi_{n+1}-\psi_{n-1}=\left(E-\varepsilon_{n}\right) \psi_{n}
$$

angegeben werden. Als Ansatz für eine lokalisierte Wellenfunktion können wir

$$
\psi_{n}= \begin{cases}A e^{-k n} & n \geq 0 \\ A e^{k n} & n \leq 0\end{cases}
$$

schreiben, unter der Voraussetzung, dass $\operatorname{Re}[k]>0$ für exponentiell abfallende Lösungen gilt. Für das Potenzial der Gitterpunkte setzen wir an die Stelle $n=0$ einen Potenzialtopf

$$
\varepsilon_{n}= \begin{cases}-\beta & n=0, \beta>0 \\ 0 & \text { sonst }\end{cases}
$$

da wir im Falle einer positiven Potenzialstufe $\varepsilon_{0}=\beta$ eine stark oszillierende Wellenfunktionen erhalten würden. Diese andere Lösung wollen wir hier jedoch nicht betrachten. Die Stetigkeitsbedingung an die Wellenfunktion im Punkt $n=0$ aus (G.2) liefert $\psi_{0}=A \equiv$ const, und für die Energie aus der Schrödinger-Gleichung (G.1) an der Stelle $n \neq 0$ muss also

$$
E=-e^{k}-e^{-k} \equiv-2 \cosh (k)
$$

gelten. Das Fehlstellenpotenzial $\varepsilon_{0}$ aus der Schrödinger-Gleichung (G.1) an der Stelle $n=0$ kann als

$$
\varepsilon_{0}=E-2 e^{-k} \equiv-2 \sinh (k)
$$

geschrieben werden. Ersetzen wir den Term $e^{-k}$ in Gleichung (G.4) durch $\frac{E-\varepsilon_{0}}{2}$ aus Gleichung (G.5), 
so erhalten wir für die inverse Lokalisierungslänge

$$
k=\ln 2-\ln \left(\varepsilon_{0}-E\right) \equiv \ln \left[\frac{1}{2}\left(\beta+\sqrt{4+\beta^{2}}\right)\right],
$$

und wegen $\operatorname{Re}[k]>0$ als einzige Lösung für die Eigenenergie des gebundenen Zustands

$$
E_{\text {geb }}=-\sqrt{4+\varepsilon_{0}^{2}}=-\sqrt{4+\beta^{2}} .
$$

Wegen $\beta>0$ liegt dieser Eigenwert somit unterhalb des Energiebands $E \in[-2,2]$ der perfekten Kette

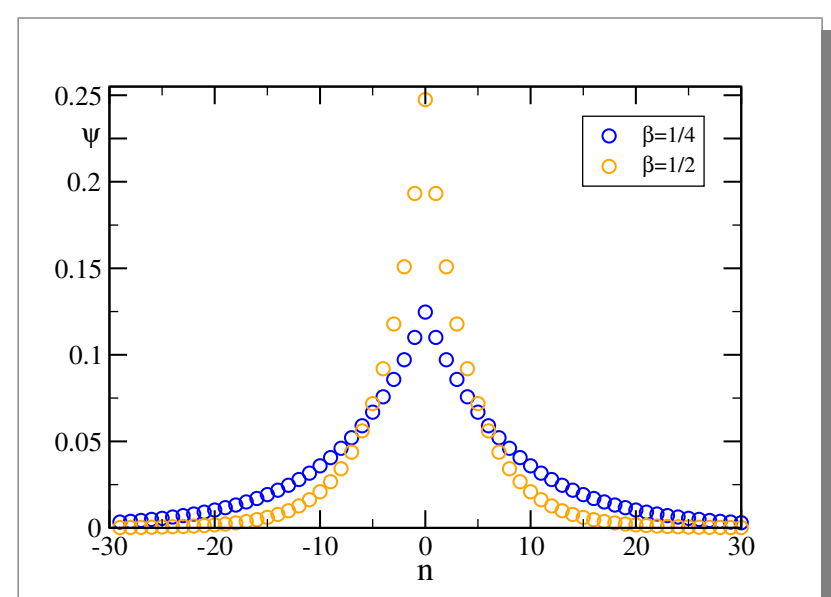

Abbildung G.2.: Lokalisierte Wellenfunktionen für eine unendliche lineare Kette im Intervall $[-30,30]$ mit einer reellwertigen Fehlstelle $\beta$ am Ort $n=0$ für verschiedene Werte $\beta=\frac{1}{4}$ (blau) und $\beta=\frac{1}{2}$ (orange).

ohne Potenzial. ${ }^{1}$ Zusammen mit

$$
\int_{0}^{\infty}\left|\psi_{n}\right|^{2} d n \equiv 1
$$

als Normierungsbedingung ergibt sich für die Wellenfunktion des gebundenen Zustands

$$
\psi_{n}= \begin{cases}k e^{k n} & n \leq 0 \\ k e^{-k n} & n \geq 0\end{cases}
$$

mit der inversen Lokalisierungslänge $k$ aus Gleichung (G.6). Damit wächst auch die maximale Amplitude $A=k$ der Wellenfunktion linear mit $\beta$. Als Beispiel für lokalisierte Wellenfunktionen der Art aus Gleichung (G.8) sind in Abbildung G.2 für eine perfekte unendliche lineare Kette mit reellwertigen Fehlstellen am Ort $n=0$ für $\beta=1 / 4$ bzw. $\beta=1 / 2$ in einer Umgebung des Lokalisierungszentrums $U=[-30,30]$ um diese Fehlstellen angegeben.

Eine ausführliche Darstellung der Theorie lokalisierter Zustände und ihrer Auswirkungen auf den Transport bzw. Diffusion findet man in der Orginalarbeit von P. W. Anderson [287]. Für Lichttransmission siehe Arbeiten [336, 337] und dort angegebene Referenzen. Universelle Eigenschaften lokalisierter Zustände findet man in [338].

1 Dies gilt für die Darstellung der Dispersionsrelation in Hückel-Näherung $E_{n}=U_{0}+2 t \cos \left(\frac{n \pi}{N+1}\right)$, wobei hier für das Potenzial des Untergrunds $U_{0}=0$ und für den Kopplungsterm $t=1$ angenommen wurden. Das abgeschlossene Intervall $[-2,2]$ ergibt sich dann im Kontinuumslimes $N \rightarrow \infty$, siehe auch Abb. 4.6 auf Seite 103. 


\section{H. Der einfache Dimer}

Wir wollen die Eigenwerte und Eigenvektoren eines Dimers analytisch bestimmen. Der HamiltonOperator für einen Dimer (siehe Abb. 4.10) in Matrixform lautet

$$
\mathcal{H}_{1}=\left(\begin{array}{cc}
\varepsilon & v \\
v & \varepsilon^{*}
\end{array}\right)
$$

mit $\varepsilon=\beta+i \gamma$, wobei $\beta$ bzw. $\gamma$ den rein reell- bzw. komplexwertigen Anteil am Gitterstellenpotenzial $\varepsilon$ bezeichnen. Unter $v \neq 0$ verstehen wir die Gitterkopplungsamplitude des Dimers. Der Fall ungekoppelter Gitterstellen $v=0$ stellt keinen Dimer dar und soll ausgeschlossen werden. Die Eigenwerte ergeben sich aus der Säkulargleichung

$$
\left|\mathcal{H}_{1}-\lambda \mathbb{1}\right|=\beta^{2}+\gamma^{2}-v^{2}-2 \lambda \beta+\lambda^{2}=0
$$

wobei $\mathbb{1}$ die Einheitsmatrix mit dem Rang 2 bezeichnet, zu

$$
\lambda_{1,2}=\beta \pm \sqrt{v^{2}-\gamma^{2}} .
$$

Wir erhalten also ein rein reelles Spektrum, solange $|\gamma| \leq|v|$ gilt, während im Fall $|\gamma|>\gamma_{\mathrm{c}} \equiv v$ beide Eigenwerte in die komplexe Ebene verzweigen und der Realteil konstant bei $\beta$ liegt (siehe Abb. H.1). Aus $\left(\mathcal{H}_{1}-\lambda \mathbb{1}\right) \vec{\psi}_{\mathrm{R}}=0$ ergibt sich für die beiden Eigenvektoren

$$
\vec{\psi}_{\mathrm{R}}^{(1,2)}=\frac{1}{\sqrt{2}}\left(\begin{array}{c}
\frac{i \gamma \pm \sqrt{v^{2}-\gamma^{2}}}{v} \\
1
\end{array}\right)
$$

wobei hier die transponierten Linkseigenvektoren mit den Rechtseigenvektoren identisch übereinstimmen. Dies gilt wegen

$$
\vec{\psi}_{\mathrm{L}}^{T} A=\lambda \vec{\psi}_{\mathrm{L}}^{T}=\left(A^{T} \vec{\psi}_{\mathrm{R}}\right)^{T},
$$

mit einer invertierbaren Matrix $A \in \mathbb{C}_{n \times n}$ und den zugehörigen Links- und Rechteigenvektoren $\vec{\psi}_{\mathrm{L}}, \vec{\psi}_{\mathrm{R}}$ zum Eigenwert $\lambda$ (siehe auch Gleichung (J.3) auf Seite 159). Damit sind also die Linkseigenvektoren von $A$ identisch mit den Rechtseigenvektoren der transponierten Matrix $A^{T}$.

Wenn $A$ symmetrisch ist, also $\forall i, j: a_{i j}=a_{j i} \Longleftrightarrow A=A^{T}$ gilt, dann gilt auch $\vec{\psi}_{\mathrm{L}}^{T}=\vec{\psi}_{\mathrm{R}}$ (dies trifft in der Verallgemeinerung auf hermitesche Matrizen jedoch nicht zu). Da in unserem Fall für die transponierte Hamiltonmatrix $\mathcal{H}_{1}^{T}=\mathcal{H}_{1}$ gilt, $\mathcal{H}_{1}$ also symmetrisch ist, sind die Rechtseigenvektoren (H.4) gleich den transponierten Linkseigenvektoren von $\mathcal{H}_{1}$. Weiterhin sind die Eigenvektoren unabhängig von der Wahl von $\beta$, welches nur Einfluss auf die Lage des Energiebands nimmt (siehe Abbildung H.1). Für $|v| \leq|\gamma|$ sind die Komponenten der Eigenvektoren entweder rein reell oder rein imaginär. 

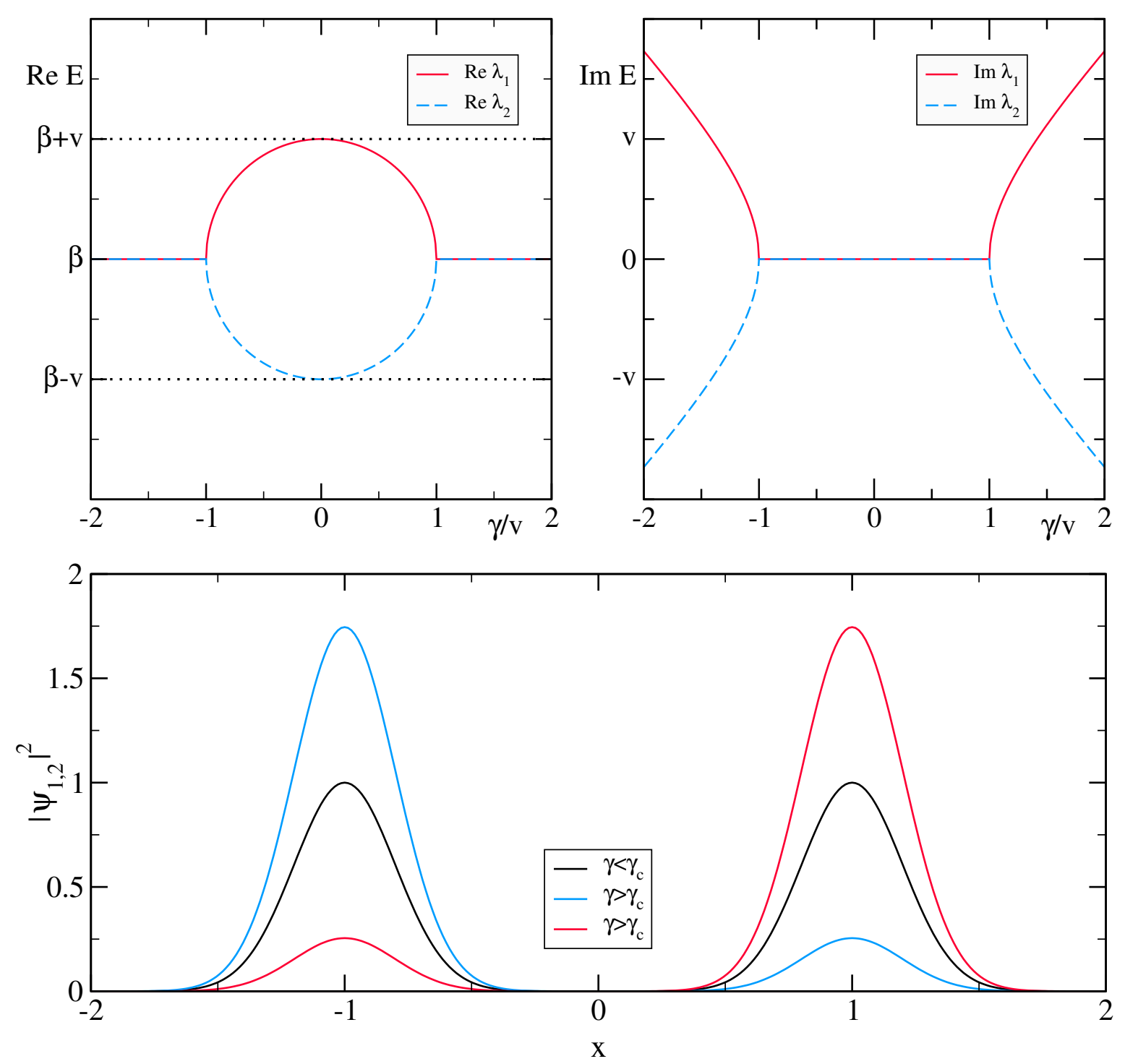

Abbildung H.1.: Eigenwertspektrum mit den Eigenwerten $\lambda_{1,2}=\beta \pm \sqrt{v^{2}-\gamma^{2}}$ und Eigenfunktionen eines Dimers. Oben links: Realteil der Eigenwerte. Oben rechts: Imaginärteil der Eigenwerte. Die gepunkteten Linien $\beta \pm v$ begrenzen das reelle Spektrum bzw. sind selbst Eigenwerte für $\gamma=0$. Beide Eigenwerte sind für $|\gamma| \leq|v|$ reell. Im Fall $|\gamma|>\gamma_{\mathrm{c}} \equiv v$ verzweigen sie in die komplexe Ebene. Die rot durchgezogenen Linien stellen den Eigenwert $\lambda_{1}$, die blau gestrichelten Linien den Eigenwert $\lambda_{2}$ dar. Das Spektrum ist symmetrisch zur $(\gamma=0)-$ und $(E=0)$-Achse. Unten: Absolutwertquadrat der Eigenfunktionen $\left|\psi_{1,2}(x)\right|^{2}$ (mit Gaußfunktionen $\mu= \pm 1$, $\sigma=0.25$ gefaltet und nach Glg. (J.7) normiert). In schwarz: Symmetrische Eigenfunktionen $\psi_{1,2}$ für $\gamma<\gamma_{\mathrm{c}}$. Für den Fall $\gamma>\gamma_{c}$ wird die Symmetrie der Eigenfunktionen aufgehoben (blau und rot). 


\section{Unitäre Transformationen von Hamiltonmatrizen linear gekoppelter Dimere}

\section{I.1. Der allgemeine Fall $N$ linear gekoppelter verschiedener komplexer Dimere}

Nach der Definition von pseudo-hermiteschen Operatoren $\mathcal{H}$ wird die Existenz eines positiv-definiten Operators $\eta$ vorausgesetzt, so dass die Bedingung $\mathcal{H}^{\dagger}=\eta \mathcal{H} \eta^{-1}$ erfüllt ist. Wie im Abschnitt 4.3.3 auf Seite 113 bereits erwähnt, lässt sich für den Hamilton-Operator einer Kette aus $N$ lokal $\mathcal{P} \mathcal{T}$ symmetrischen Dimeren eine unitäre Transformation $J_{N}$ finden, so dass die transformierte Hamiltonmatrix $J_{N}^{-1} \mathcal{H}_{N} J_{N}$ nur reelle Matrixelemente besitzt. Hier wird nun eine allgemeine unitäre Transformation $\Omega_{N}$ vorgeschlagen, die im Falle komplexwertiger Kopplungskonstanten ebenfalls eine reelle Matrix $\Omega_{N}^{-1} \mathcal{H}_{N} \Omega_{N}$ erzeugt, ebenfalls vorausgesetzt, dass wir uns in der pseudo-hermiteschen Phase befinden. Komplexe Kopplungskonstanten entstehen durch Phasenbeziehungen bei der Intra- und Inter-Dimerkopplung. Daher müssen die Blockmatrizen (4.55) und (4.56) wie folgt modifiziert bzw. verallgemeinert werden

$$
D_{n}+V_{n}=h_{n}=\left(\begin{array}{cc}
\beta_{n}+i \gamma_{n} & v_{n}-i w_{n} \\
v_{n}+i w_{n} & \beta_{n}-i \gamma_{n}
\end{array}\right) \quad \text { und } \quad T_{n}=\left(\begin{array}{cc}
t_{n}+i u_{n} & 0 \\
0 & t_{n}-i u_{n}
\end{array}\right)
$$

wobei $D_{N}$ die Diagonalmatrix von $h_{n}$ definiert und die Intra-Dimer-Kopplungsmatrix $V_{n}$ die Nebendiagonalelemente von $h_{n}$ enthält. Wir behandeln den allgemeinen Fall $\forall n: \beta_{n}, \gamma_{n}, v_{n}, w_{n}, t_{n}, u_{n} \in \mathbb{R}$, in dem alle Kopplungskonstanten und Gitterstellenpotenziale "zufällige" komplexe Werte annehmen dürfen, mit der Einschränkung, dass das Potenzial des Dimers an sich, ebenso wie seine Kopplung innehalb (Intra-Dimer-Kopplung) und untereinander (Inter-Dimer-Kopplung) der lokalen $\mathcal{P} \mathcal{T}$-Symmetriebedingung unterworfen sind. Der Verzicht auf diese Voraussetzung führt zu komplexen Eigenwerten von $\mathcal{H}_{2}$ für beliebige von Null verschiedene Werte $\gamma_{n}, w_{n}$ oder $u_{n}$, und daher gibt es auch keine unitäre Transformation, die die Untermatrizen (I.1) in reelle Matrizen zu transformieren vermag. Weiterhin behandeln wir aus Gründen der Allgemeingültigkeit beliebige komplexwertige Kopplungskoeffizienten, die sich, dem physikalischen Verständnis entsprechend, in Euler-Darstellung (exponentielle Schreibweise) auch als Phasenbeziehungen $v_{n}+i w_{n}=v_{0, n} e^{i \phi_{n}}$ bzw. $t_{n}+i u_{n}=t_{0, n} e^{i \vartheta_{n}}$ darstellen lassen. Mit den Blockmatrizen aus (I.1) definieren wir den Hamilton-Operator $\mathcal{H}_{N}$ in Matrixform für Ketten von $N$ gekoppelten verschiedenen Dimeren, analog zu Gleichung (4.61), als

$$
\mathcal{H}_{N}=\left(\begin{array}{ccccccc}
h_{1} & T_{1} & 0 & \ldots & 0 & 0 & 0 \\
T_{1} & h_{2} & T_{2} & 0 & \ldots & 0 & 0 \\
0 & T_{2} & h_{3} & T_{3} & 0 & \ldots & 0 \\
\vdots & \ddots & \ddots & \ddots & \ddots & \ddots & \vdots \\
0 & \ldots & 0 & T_{N-3} & h_{N-2} & T_{N-2} & 0 \\
0 & 0 & \ldots & 0 & T_{N-2} & h_{N-1} & T_{N-1} \\
0 & 0 & 0 & \ldots & 0 & T_{N-1} & h_{N}
\end{array}\right) .
$$


Ohne Beschränkung der Allgemeinheit nehmen wir an, dass die Länge $N$ der Dimerkette in einer Potenz von zwei darstellbar ist $N=2^{n}, n \in \mathbb{N}^{+} .{ }^{1}$ Wir definieren eine Transformationsmatrix

$$
\Omega_{N}=\left(\bigotimes_{j=1}^{N-1} K_{2}\right) \otimes J_{2}=\underbrace{K_{2} \otimes K_{2} \otimes \cdots \otimes K_{2}}_{(N-1) \text {-mal }} \otimes J_{2}
$$

wobei

$$
J_{2}=\frac{1}{\sqrt{2}}\left(\begin{array}{cc}
1 & i \\
i & 1
\end{array}\right) \quad \text { und } \quad K_{2}=\left(\begin{array}{cc}
i & 0 \\
i & -i
\end{array}\right)
$$

unitäre $2 \times 2$-Matrizen definieren $\left(J_{2}^{-1}=J_{2}^{*}, K_{2}^{-1}=K_{2}^{*}\right.$, $\left.\operatorname{det} J_{2}=\operatorname{det} K_{2}=1\right)$ und " $\otimes$ " das KroneckerProdukt bezeichnet. Es folgen die Definition des Kronecker-Produkts sowie drei seiner wichtigen Eigenschaften, die uns den Beweis führen lassen, das $\Omega_{N}$ eine unitäre und abbildungstreue Matrix ist.

Definition Ist $A$ eine $\mathbb{C}_{m \times n}$-Matrix und $B$ eine $\mathbb{C}_{p \times q}$-Matrix, so ist das Kronecker-Produkt " $\otimes$ " definiert als

$$
C=A \otimes B=\left\{a_{i j} \cdot B\right\}=\left(\begin{array}{ccc}
a_{11} B & \cdots & a_{1 n} B \\
\vdots & \ddots & \vdots \\
a_{m 1} B & \cdots & a_{m n} B
\end{array}\right)
$$

Die Matrix $C$ hat damit die Dimension $(m p) \times(n q)$.

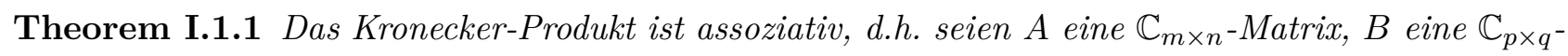
Matrix und $C$ eine $\mathbb{C}_{r \times s}-$ Matrix. Dann gilt

$$
(A \otimes B) \otimes C=A \otimes(B \otimes C)
$$

Zum Beweis dieser Aussage siehe [339].

Theorem I.1.2 Seien $A$ eine invertierbare $\mathbb{C}_{m \times m}$-Matrix und $B$ eine invertierbare $\mathbb{C}_{n \times n}$-Matrix. Dann gilt

1. $(A \otimes B)^{-1}=A^{-1} \otimes B^{-1}$

2. $(A \otimes B)^{*}=A^{*} \otimes B^{*}$

3. $\operatorname{det}(A \otimes B)=(\operatorname{det} A)^{n} \cdot(\operatorname{det} B)^{m}$.

Zum Beweis dieser Aussagen siehe [340, 341].

Theorem I.1.3 Das Kronecker-Produkt einer unitären $(m \times m)$-Matrix A mit einer unitären $(n \times n)$ Matrix $B$ ist eine unitäre $(m n \times m n)$-Matrix $C=A \otimes B$.

\section{Beweis}

$$
C^{-1}=(A \otimes B)^{-1}=A^{-1} \otimes B^{-1}=A^{*} \otimes B^{*}=(A \otimes B)^{*}=C^{*} .
$$

1 Für Längen $M<N=2^{n}, M \nmid 2^{m}, m<n$ muss $\mathcal{H}_{N}$ für die "überzähligen Dimere" mit $(N-M) 2 \times 2$-Einheitsmatrizen auf der Diagonalen "aufgefüllt" werden. Die Kopplungsmatrizen für $N \geq i>M$ sind dann $T_{i}=V_{i}=0 \mathrm{zu}$ wählen. 
Es sind die folgenden Eigenschaften von $\Omega_{N}$ zu zeigen

1. $\Omega_{N}^{-1}=\Omega_{N}^{*}$ (Unitarität)

2. $\operatorname{det} \Omega_{N}=1$ (Abbildungstreue)

Beweis $\mathrm{Zu}(1)$ :

$$
\begin{aligned}
& \Omega_{N}^{-1}=\left(\begin{array}{l}
K_{2} \otimes K_{2} \otimes \cdots \otimes K_{2} \otimes J_{2}
\end{array}\right)^{-1} \\
& =K_{2}^{-1} \otimes K_{2}^{-1} \otimes \cdots \otimes K_{2}^{-1} \otimes J_{2}^{-1} \\
& =K_{2}^{*} \otimes K_{2}^{*} \otimes \cdots \otimes K_{2}^{*} \otimes J_{2}^{*} \\
& =\left(K_{2} \otimes K_{2} \otimes \cdots \otimes K_{2} \otimes J_{2}\right)^{*}=\Omega_{N}^{*} .
\end{aligned}
$$

Beweis $\mathrm{Zu}(2)$ :

$$
\begin{aligned}
\operatorname{det} \Omega_{N} & =\operatorname{det}\left(K_{2} \otimes K_{2} \otimes \cdots \otimes K_{2} \otimes J_{2}\right) \\
& =\left(\operatorname{det} K_{2}\right)^{2(N-1)}\left(\operatorname{det} J_{2}\right)^{2} \\
& =1^{2 N-2+2}=1
\end{aligned}
$$

Damit ist $\Omega_{N} \in \mathrm{SU}(2 N)$ und erhält bei der unitären Transformation $\Omega_{N}^{-1} \mathcal{H}_{N} \Omega_{N}$ die Eigenwerte von $\mathcal{H}_{N}$. Weiterhin ist das charakteristische Polynom reeller Natur, wonach die Eigenwerte von $\mathcal{H}_{N}$ entweder alle reell sind oder in Paaren aus reellen und Paaren aus konjugiert komplexen Eigenwerten auftreten. Zum Beweis dieser Aussagen für Ähnlichkeitsrelationen siehe [342, 343]. Nicht-hermitesche Matrizen vom Lie-algebraischen Typ werden ausführlich in [344] behandelt.

\section{I.2. Zwei gekoppelte Dimere mit komplexen Kopplungskonstanten}

Als Anwendungsbeispiel und zum tieferen Verständnis der Dimerkette mit $N=2$ wollen wir uns mit dem allgemeinen Fall zweier gekoppelter Dimere befassen. Die allgemeine Hamiltonmatrix für zwei gekoppelte Dimere mit komplexen Kopplungskoeffizienten, wobei alle vorkommenden Größen voneinander unabhängig gewählt sind, lautet

$$
\mathcal{H}_{2}=\left(\begin{array}{cccc}
\beta_{1}+i \gamma_{1} & v_{1}-i w_{1} & t+i u & 0 \\
v_{1}+i w_{1} & \beta_{1}-i \gamma_{1} & 0 & t-i u \\
t-i u & 0 & \beta_{2}+i \gamma_{2} & v_{2}-i w_{2} \\
0 & t+i u & v_{2}+i w_{2} & \beta_{2}-i \gamma_{2}
\end{array}\right)
$$

Mit der unitären Transformation $\Omega_{2}=K_{2} \oplus J_{2}$ nach Gleichung (I.3) finden wir

$$
\mathcal{H}_{2}^{(\Omega)} \equiv \Omega_{2}^{-1} \mathcal{H}_{2} \Omega_{2}=\left(\begin{array}{cccc}
t+w_{1}+\beta_{1} & u+v_{1}-\gamma_{1} & -t & -u \\
-u+v_{1}+\gamma_{1} & t-w_{1}+\beta_{1} & u & -t \\
\Delta \beta+\Delta w & \Delta v-\Delta \gamma & -t+w_{2}+\beta_{2} & -u+v_{2}-\gamma_{2} \\
\Delta v+\Delta \gamma & \Delta \beta-\Delta w & u+v_{2}+\gamma_{2} & -t-w_{2}+\beta_{2}
\end{array}\right),
$$

mit $\Delta \beta=\beta_{1}-\beta_{2}, \Delta \gamma=\gamma_{1}-\gamma_{2}, \Delta w=w_{1}-w_{2}$, und $\Delta v=v_{1}-v_{2} . \mathcal{H}_{2}^{(\Omega)}$ besitzt nur reelle Matrixelemente. 
Damit ist die Säkulargleichung

$$
P(\lambda)=\operatorname{det}\left(\mathcal{H}_{2}-\lambda \mathbb{E}_{2}\right)=0
$$

ebenfalls reell und es gilt für die Eigenwerte bzw. Nullstellen, dass sie entweder reell sind oder in komplex konjugierten Paaren auftreten, d.h. es gilt folgendes

Theorem I.2.1 Sei $P_{N}(z)=\sum_{k=0}^{N} a_{k} z^{k}$ ein Polynom in $z \in \mathbb{C}$ mit reellen Koeffizienten $a_{k} \in \mathbb{R}$ mit $k=0, \ldots, N, N \in \mathbb{N}$. Dann gilt für alle $z \in \mathbb{C}$

$$
P_{N}(z)^{*}=P_{N}\left(z^{*}\right)
$$

\section{Beweis}

$$
P_{N}(z)^{*}=\sum_{k=0}^{N}\left(a_{k} z^{k}\right)^{*}=\sum_{k=0}^{N} a_{k}\left(z^{*}\right)^{k}=P_{N}\left(z^{*}\right) .
$$

Für die Säkulargleichung (I.8) gilt nach Gleichung (I.9) Theorem I.2.1

$$
P_{N}(\lambda)=P_{N}\left(\lambda^{*}\right)=0
$$

Das bedeutet, dass sowohl $\lambda$ als auch $\lambda^{*}$ Eigenwert ist, woraus sich schlussfolgern lässt, dass die Eigenwerte $\lambda$ entweder reell sind oder in Paaren aus zueinander komplex konjugierten Werten vorliegen.

\section{I.3. Zwei gekoppelte identische Dimere mit komplexen Kopplungskonstanten}

Wir wollen uns erneut dem Beispiel aus Abschnitt I.2 zuwenden, diesmal allerdings mit der zusätzlichen Einschränkung, dass es sich um zwei gekoppelte identische Dimere handeln soll. Wir werden nun

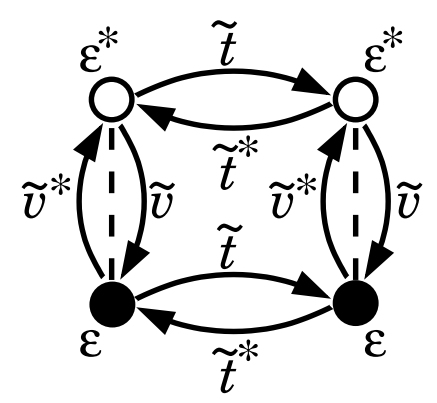

Abbildung I.1.: Zwei Dimere (durch gestrichelte Linien gekennzeichnet) mit komplexwertiger Inter-Dimerkopplung $\tilde{t}=t+$ $i u$ und Intra-Dimerkopplung $\tilde{v}=v+i w$. zeigen, dass eine Hintereinanderschaltung von zwei speziellen unitären Transformationen die Hamiltonmatrix zweier gekoppelter identischer Dimere mit komplexen Kopplungskonstanten in die Hamiltonmatrix zweier verschiedener Dimere mit reellen Kopplungskonstanten aus Gleichung (4.54) überführt. Die Hamiltonmatrix nach Abbildung I.1 lautet

$$
\mathcal{H}_{2}=\left(\begin{array}{cccc}
\beta+i \gamma & v-i w & t+i u & 0 \\
v+i w & \beta-i \gamma & 0 & t+i u \\
t-i u & 0 & \beta+i \gamma & v-i w \\
0 & t-i u & v+i w & \beta-i \gamma
\end{array}\right)
$$

Mit der Definition der unitären Transformation $P_{2}=J_{2} \otimes J_{2}$, also ${ }^{2}$

2 Diese unitäre Transformation kann analog zu Gleichung (I.3) erweitert werden. In diesem Fall transformiert $P_{N}=\bigotimes_{j=1}^{N} J_{2}$ eine Hamiltonmatrix für $N$ gekoppelte identische Dimere in eine reelle Matrix mit $2 \times 2-$ DiagonalBlockmatrizen abseits der Hauptdiagonalen. 


$$
P_{2}=\frac{1}{2}\left(\begin{array}{rrrr}
1 & i & i & -1 \\
i & 1 & -1 & i \\
i & -1 & 1 & i \\
-1 & i & i & 1
\end{array}\right)
$$

können wir die komplexen Einträge der Untermatrizen $T_{n}$ der Inter-Dimer-Kopplungen eliminieren

$$
P_{2}^{-1} \mathcal{H}_{2} P_{2}=\left(\begin{array}{cccc}
\beta-u+w & v-\gamma & t & 0 \\
v+\gamma & \beta-u-w & 0 & t \\
t & 0 & \beta+u+w & v-\gamma \\
0 & t & v+\gamma & \beta+u-w
\end{array}\right)
$$

Diese Matrix ist reell, d.h. es gelten die gleichen Aussagen über das charakteristische Polynom und seine Eigenwerte wie in Kapitel (I.1) und (I.2). Mit einer zweiten unitären Transformation

$$
L_{2}=\left(\begin{array}{cccc}
-\sqrt{\frac{v}{v+\gamma}} & \frac{\sqrt{\left(w^{2}-\gamma^{2}\right)}-w}{\sqrt{v(v+\gamma)}} & 0 & 0 \\
0 & -\sqrt{\frac{v+\gamma}{v}} & 0 & 0 \\
0 & 0 & -\sqrt{\frac{v}{v+\gamma}} & \frac{\sqrt{\left(w^{2}-\gamma^{2}\right)}-w}{\sqrt{v(v+\gamma)}} \\
0 & 0 & 0 & -\sqrt{\frac{v+\gamma}{v}}
\end{array}\right)
$$

rotieren wir die Untermatrizen $h_{n}$ mit den Intra-Dimer Kopplungen, so dass die asymmetrischen Einträge auf den Nebendiagonalen der $2 \times 2$-Blöcke $V_{n}$ von (I.13) verschwinden

$$
\mathcal{H}_{2}^{(P L)}=L_{2}^{-1} P_{2}^{-1} \mathcal{H}_{2} P_{2} L_{2}=\left(\begin{array}{cccc}
\beta-u+i \kappa & v & t & 0 \\
v & \beta-u-i \kappa & 0 & t \\
t & 0 & \beta+u+i \kappa & v \\
0 & t & v & \beta+u-i \kappa
\end{array}\right)
$$

wobei $\kappa=\sqrt{\gamma^{2}-w^{2}}$ die Funktion des ursprünglichen Imaginärteils $\gamma$ des Potentials übernimmt. Zusammen mit den Ersetzungen $\beta_{1}=\beta-u$ und $\beta_{2}=\beta+u$ können wir die Hamiltonmatrix $\mathcal{H}_{2}^{(P L)}$ auf die Form der Hamiltonmatrix $\mathcal{H}_{2}$ unseres Modells aus Gleichung (4.54) zurückführen

$$
\mathcal{H}_{2}^{(P L)}=\left(\begin{array}{cccc}
\beta_{1}+i \kappa & v & t & 0 \\
v & \beta_{1}-i \kappa & 0 & t \\
t & 0 & \beta_{2}+i \kappa & v \\
0 & t & v & \beta_{2}-i \kappa
\end{array}\right)=\mathcal{H}_{2}^{(\mathrm{Gl} .4 .54)}
$$

Mit Fluktuationen (Unordnung) in $\beta_{i}$ (und $\gamma_{i}$ ) sind wir also in der Lage, die komplexen Kopplungskonstanten identisch zu ersetzen. Dabei sei nochmals darauf hingewiesen, dass unitäre Transformationen die Eigenwerte erhalten.

Damit wurde gezeigt, dass sich spontan auftretende Phasenbeziehungen in den Kopplungskonstanten mithilfe von speziellen unitären Transformationen zu Null transformieren lassen. Die Wahl von rein reellen Kopplungskonstanten ist demnach durchaus gerechtfertigt. 



\section{J. Eigenwerte und Eigenvektoren nicht-hermitescher Matrizen}

Nicht-hermitesche Matrizen besitzen im Allgemeinen voneinander verschiedene linke und rechte Eigenvektoren. Für viele physikalische Problemstellungen ist es ausreichend, die rechten Eigenvektoren zu bestimmen. Für andere, wie zum Beispiel die Berechnung der Petermann-Faktoren (siehe Gleichung (4.84) auf Seite 123 in Abschnitt 4.4), müssen ebenfalls die linken Eigenvektoren berechnet werden. ${ }^{1}$ Für die jeweiligen zugehörigen Eigenwerte (linke oder rechte) gilt deren Äquivalenz. Für die Eigenvektoren gilt dies im Allgemeinen nicht [346]. Dieses Kapitel dient daher der Ableitung einer Bi-orthogonalitätsbedingung. Dazu beginnen wir mit folgender Betrachtung:

Sei $\mathcal{H}$ ein nicht-hermitescher Hamilton-Operator der zeitunabhängigen Schrödinger-Gleichung

$$
\mathcal{H} \vec{\psi}_{\mathrm{R}, i}=\lambda_{\mathrm{R}, i} \vec{\psi}_{\mathrm{R}, i}
$$

wobei $\vec{\psi}_{\mathrm{R}, i}$ der $i$-te rechte Eigenvektor und $\lambda_{\mathrm{R}, i}$ der zugehörige rechte Eigenwert der quadratischen Matrix $\mathcal{H} \in \mathbb{C}_{N \times N}$ ist. Wegen der Orthogonalitätsbedingung $\left(\mathcal{H}-\lambda_{\mathrm{R}, i} \mathbb{1}\right) \vec{\psi}_{\mathrm{R}, i}=0$ muss die Säkulargleichung

$$
\operatorname{det}\left(\mathcal{H}-\lambda_{\mathrm{R}, i} \mathbb{1}\right)=0
$$

erfüllt sein. Für den $j$-ten linken Eigenvektor $\vec{\psi}_{\mathrm{L}, j}$ mit zugehörigem Eigenwert $\lambda_{\mathrm{L}, j}$ schreiben wir analog

$$
\begin{aligned}
\vec{\psi}_{\mathrm{L}, j} \mathcal{H}=\lambda_{\mathrm{L}, j} \vec{\psi}_{\mathrm{L}, j} & \Longleftrightarrow\left(\vec{\psi}_{\mathrm{L}, j} \mathcal{H}\right)^{T}=\lambda_{\mathrm{L}, j} \vec{\psi}_{\mathrm{L}, j}^{T} \\
& \Longleftrightarrow \mathcal{H}^{T} \vec{\psi}_{\mathrm{L}, j}^{T}=\lambda_{\mathrm{L}, j} \vec{\psi}_{\mathrm{L}, j}^{T}
\end{aligned}
$$

wobei wir die Rechenregeln für transponierte Matrizen verwendet haben. Wegen der Orthogonalitätsbedingung $\left(\mathcal{H}^{T}-\lambda_{\mathrm{L}, i} \mathbb{1}\right) \vec{\psi}_{\mathrm{L}, i}^{T}=0$ für die linken Eigenvektoren muss die Säkulargleichung

$$
\begin{aligned}
\operatorname{det}\left(\mathcal{H}^{T}-\lambda_{\mathrm{L}, j} \mathbb{1}\right)=0 & \Longleftrightarrow \operatorname{det}\left(\mathcal{H}-\lambda_{\mathrm{L}, j} \mathbb{1}\right)^{T}=0 \\
& \Longleftrightarrow \operatorname{det}\left(\mathcal{H}-\lambda_{\mathrm{L}, j} \mathbb{1}\right)=0
\end{aligned}
$$

erfüllt sein. Der letzte Schritt folgt hierbei aus der Rechenregel für die Determinante einer transponierten Matrix $\operatorname{det}\left(A^{T}\right)=\operatorname{det}(A)$. Ein Vergleich mit Gleichung (J.2) für gleichgesetzte Indices liefert

$$
\forall i=j: \lambda_{\mathrm{R}, i}=\lambda_{\mathrm{L}, j}
$$

d.h. für den $i$-ten rechten und linken Eigenvektor sind zugehöriger $i$-ter rechter und linker Eigenwert identisch. Eine Projektion von $\vec{\psi}_{\mathrm{L}, j}$ von links auf Gleichung (J.1) und Projektion von $\vec{\psi}_{\mathrm{R}, i}$ von rechts auf

1 Numerisch kann das Problem der Berechnung von linken und rechten Eigenvektoren nichtsymmetrischer quadratischer Matrizen aus $\mathbb{C}_{N \times N}$ mit der Schur-Faktorisierung gelöst werden. Dies ist beispielsweise in der LAPACK-Routine zgeev realisiert [64]. Für eine Übersicht numerischer Methoden siehe z.B. [345]. 
Gleichung (J.3) und die Subtraktion der entstandenen zwei Gleichungen voneinander liefert folgende notwendige Bedingung in Bra-Ket-Schreibweise

$$
\left(\lambda_{\mathrm{R}, i}-\lambda_{\mathrm{L}, j}\right)\left\langle\psi_{\mathrm{L}, j} \mid \psi_{\mathrm{R}, i}\right\rangle=0
$$

die zusammen mit Identität (J.5) die Definition einer Bi-Orthogonalitätsbedingung

$$
\left\langle\psi_{\mathrm{L}, j} \mid \psi_{\mathrm{R}, i}\right\rangle=\delta_{i j}
$$

zulässt und zu einer bi-orthogonalen Menge von linken und rechten Eigenvektoren mit zugehörigen Eigenwerten führt [347]. Erfüllen die linken und rechten Eigenvektoren zusätzlich die hinreichende Bedingung

$$
\sum_{j}\left|\psi_{\mathrm{L}, j}\right\rangle\left\langle\psi_{\mathrm{R}, j}\right|=\mathbb{1}
$$

so heißen die Eigenvektoren bi-orthonormal. Dies ist z.B. für Vektoren $\vec{\psi}_{\mathrm{L}}^{T}, \vec{\psi}_{\mathrm{R}}$ des gleichen separablen Hilbertraums $\mathbb{H}$ erfüllt. In diesem Fall ist Bedingung (J.6) bikonditional [348]. 


\section{Literaturverzeichnis}

[1] O. Bendix and J. A. Méndez-Bermúdez, Design of switches and beam splitters by use of chaotic cavities, Opt. Lett. 30(11), 1396-1398 (Jun 2005).

[2] O. Bendix, J. A. Méndez-Bermúdez, G. A. Luna-Acosta, U. Kuhl, and H. J. Stöckmann, Design of beam splitters and microlasers using chaotic waveguides, Microelectron. J. 36(3-6), 285-288 (Mar 2005), Low Dimensional Structures and Devices Conference.

[3] O. Bendix, R. Fleischmann, T. Kottos, and B. Shapiro, Exponentially Fragile $\mathcal{P} \mathcal{T}$-Symmetry in Lattices with Localized Eigenmodes, Phys. Rev. Lett. 103(3), 030402 (Jul 2009).

[4] O. Bendix, R. Fleischmann, T. Kottos, and B. Shapiro, Optical Structures With Local $\mathcal{P} \mathcal{T}$-symmetry, J. Phys. A: Math. Theor. 43(26), 265305 (Jul 2010).

[5] O. Bendix, R. Fleischmann, and T. Geisel, Reduction of scattering light by Retina Müller cells in vertebrate eyes, in preparation (2011).

[6] A. B. Fowler, F. F. Fang, W. E. Howard, and P. J. Stiles, Magneto-Oscillatory Conductance in Silicon Surfaces, Phys. Rev. Lett. 16(20), 901-903 (May 1966).

[7] R. A. Webb and S. Washburn, Quantum Interference Fluctuations in Disordered Metals, Phys. Today 41(12), 46-53 (1988).

[8] B. J. van Wees, H. van Houten, C. W. J. Beenakker, J. G. Williamson, L. P. Kouwenhoven, D. van der Marel, and C. T. Foxon, Quantized conductance of point contacts in a two-dimensional electron gas, Phys. Rev. Lett. 60(9), 848-850 (Feb 1988).

[9] D. A. Wharam, T. J. Thornton, R. Newbury, M. Pepper, H. Ahmed, J. E. F. Frost, D. G. Hasko, D. C. Peacock, D. A. Ritchie, and G. A. C. Jones, One-dimensional transport and the quantisation of the ballistic resistance, J. Phys. C 21(8), L209 (Mar 1988).

[10] K. v. Klitzing, G. Dorda, and M. Pepper, New Method for High-Accuracy Determination of the Fine-Structure Constant Based on Quantized Hall Resistance, Phys. Rev. Lett. 45(6), 494-497 (Aug. 1980).

[11] E. Abrahams, P. W. Anderson, D. C. Licciardello, and T. V. Ramakrishnan, Scaling Theory of Localization: Absence of Quantum Diffusion in Two Dimensions, Phys. Rev. Lett. 42(10), 673-676 (Mar 1979).

[12] B. L. Altshuler and P. A. Lee, Disordered Electronic Systems, Phys. Today 41(12), 36-44 (Dec 1988).

[13] C. P. Umbach, S. Washburn, R. B. Laibowitz, and R. A. Webb, Magnetoresistance of small, quasi-one-dimensional, normal-metal rings and lines, Phys. Rev. B 30(7), 4048-4051 (Oct 1984).

[14] B. L. Altshuler, Fluctuations in the extrinsic conductivity of disordered conductors, JETP Letters 41(12), 648 (Dec 1985).

[15] P. A. Lee and A. D. Stone, Universal Conductance Fluctuations in Metals, Phys. Rev. Lett. 55(15), 1622-1625 (Oct 1985).

[16] C. W. J. Beenakker and H. van Houten, Quantum Transport in Semiconductor Nanostructures, Solid State Phys. 44, 1-111 (1991).

[17] E. N. Economou, Green's Functions in Quantum Physics, Springer Berlin Heidelberg, Nov 2009.

[18] R. Landauer, Electrical resistance of disordered one-dimensional lattices, Philos. Mag. 21(172), 863-867 (Apr 1970).

[19] M. Büttiker, Symmetry of electrical conduction, IBM J. Res. Dev. 32(3), 317-334 (May 1988).

[20] A. M. Song, A. Lorke, A. Kriele, J. P. Kotthaus, W. Wegscheider, and M. Bichler, Nonlinear Electron Transport in an Asymmetric Microjunction: A Ballistic Rectifier, Phys. Rev. Lett. 80(17), 3831-3834 (Apr 1998). 
[21] R. Fleischmann and T. Geisel, Mesoscopic Rectifiers based on Ballistic Transport, Phys. Rev. Lett. 89(1), 016804 (Jun 2002).

[22] Y. Yamamoto and R. E. Slusher, Optical Processes in Microcavities, Phys. Today 46(6), 66-73 (Jun 1993).

[23] C. Gmachl, F. Capasso, E. E. Narimanov, J. U. Nöckel, A. D. Stone, J. Faist, D. L. Sivco, and A. Y. Cho, High-Power Directional Emission from Microlasers with Chaotic Resonators, Science 280(5369), 1556-1564 (Jun 1998).

[24] J. A. Méndez-Bermúdez, G. A. Luna-Acosta, P. Šeba, and K. N. Pichugin, Understanding quantum scattering properties in terms of purely classical dynamics: Two-dimensional open chaotic billiards, Phys. Rev. E 66(4), 046207 (Oct 2002).

[25] J. A. Méndez-Bermúdez, P. Luna-Acosta, G. A. Šeba, and K. N. Pichugin, Chaotic waveguide-based resonators for microlasers, Phys. Rev. B 67(16), 161104 (Apr 2003).

[26] W. T. Arkin, Trends in lasers and electro-optics research, Nova Science Pub Inc, Jan 2006.

[27] J. M. Enoch, F. L. Tobey, and H. E. Bedell, Vertebrate photoreceptor optics, volume 23 of Springer Series in Optical Sciences, Springer-Verlag, Berlin; New York, 1981.

[28] V. Lakshminarayanan, Waveguiding in retinal photoreceptors: and overview, Proc. SPIE 3211(1), 182-192 (1998).

[29] W. S. Stiles and B. H. Crawford, The Luminous Efficiency of Rays Entering the Eye Pupil at Different Points, Proc. R. Soc. Lond. B Biol. Sci. 112(778), 428-450 (Mar 1933).

[30] K. Franze, J. Grosche, S. N. Skatchkov, S. Schinkinger, C. Foja, D. Schild, O. Uckermann, K. Travis, A. Reichenbach, and J. Guck, Müller cells are living optical fibers in the vertebrate retina, Proc. Natl. Acad. Sci. U.S.A. 104(20), 8287-8292 (May 2007).

[31] J. Pendry, Introduction to Focus Issue: Negative Refraction and Metamaterials, Opt. Express 11(7), 639-639 (Apr 2003).

[32] V. G. Veselago, The electrodynamics of substances with simultaneously negative values of $\varepsilon$ and $\mu$, Sov. Phys. Usp. 10(4), 509 (Jan-Feb 1968).

[33] D. R. Smith, W. J. Padilla, D. C. Vier, S. C. Nemat-Nasser, and S. Schultz, Composite Medium with Simultaneously Negative Permeability and Permittivity, Phys. Rev. Lett. 84(18), 4184- (May 2000).

[34] W. Cai, U. K. Chettiar, A. V. Kildishev, and V. M. Shalaev, Optical cloaking with metamaterials, Nat. Photon. 1(4), 224-227 (Apr 2007).

[35] X. Zhang and Z. Liu, Superlenses to overcome the diffraction limit, Nat. Mater. 7(6), 435-441 (Jun 2008).

[36] Y. G. Ma, S. Sahebdivan, C. K. Ong, T. Tyc, and U. Leonhardt, Evidence for subwavelength imaging with positive refraction, New Journal of Physics 13(3), 033016- (Mar 2011).

[37] C. M. Bender and S. Boettcher, Real Spectra in Non-Hermitian Hamiltonians Having $\mathcal{P} \mathcal{T}$ Symmetry, Phys. Rev. Lett. 80(24), 5243-5246 (Jun 1998).

[38] A. Mostafazadeh, Pseudo-Hermiticity versus $\mathcal{P} \mathcal{T}$ symmetry: The necessary condition for the reality of the spectrum of a non-Hermitian Hamiltonian, J. Math. Phys. 43(1), 205-214 (Jan 2002).

[39] A. Mostafazadeh, Pseudo-Hermiticity versus $\mathcal{P} \mathcal{T}$-symmetry II. A complete characterization of non-Hermitian Hamiltonians with a real spectrum, J. Math. Phys. 43(5), 2814-2816 (May 2002).

[40] A. Mostafazadeh, Pseudo-Hermiticity versus $\mathcal{P} \mathcal{T}$-symmetry III: Equivalence of pseudo-Hermiticity and the presence of antilinear symmetries, J. Math. Phys. 43(8), 3944-3951 (Aug 2002).

[41] J. Mehra and H. Rechenberg, The Creation of Wave Mechanics; Early Response and Applications 1925-1926, Part 2, volume 5 of Historical Development of Quantum Theory, Springer, 2000.

[42] P. Reimann, M. Grifoni, and P. Hänggi, Quantum Ratchets, Phys. Rev. Lett. 79(1), 10-13 (Jul 1997).

[43] H. Linke, W. Sheng, A. Löfgren, H. Xu, P. Omling, and P. E. Lindelof, A quantum dot ratchet: Experiment and theory, Europhys. Lett. 44(3), 341 (Nov 1998).

[44] H. Linke, T. Humphrey, P. Lindelof, A. Löfgren, R. Newbury, P. Omling, A. Sushkov, R. Taylor, and H. Xu, Quantum ratchets and quantum heat pumps, Appl. Phys. A: Mater. Sci. Process. 75(2), 237-246 (Aug 2002). 
[45] K. Hieke and M. Ulfward, Nonlinear operation of the Y-branch switch: Ballistic switching mode at room temperature, Phys. Rev. B 62(24), 16727- (Dec 2000).

[46] H. Q. Xu, Electrical properties of three-terminal ballistic junctions, Appl. Phys. Lett. 78(14), $2064-2066$ (Apr 2001).

[47] A. M. Song, Formalism of nonlinear transport in mesoscopic conductors, Phys. Rev. B 59(15), 9806-9809 (Apr 1999).

[48] A. M. Song, Electron ratchet effect in semiconductor devices and artificial materials with broken centrosymmetry, Appl. Phys. A: Mater. Sci. Process. 75(2), 229-235 (Aug 2002).

[49] A. Löfgren, I. Shorubalko, P. Omling, and A. M. Song, Quantum behavior in nanoscale ballistic rectifiers and artificial materials, Phys. Rev. B 67(19), 195309 (May 2003).

[50] M. Büttiker and D. Sánchez, Comment on "Mesoscopic Rectifiers Based on Ballistic Transport", Phys. Rev. Lett. 90(11), 119701 (Mar 2003).

[51] T. Geisel and R. Fleischmann, Geisel and Fleischmann Reply, Phys. Rev. Lett. 90(11), 119702 (Mar 2003$).$

[52] S. de Haan, A. Lorke, J. P. Kotthaus, W. Wegscheider, and M. Bichler, Rectification in Mesoscopic Systems with Broken Symmetry: Quasiclassical Ballistic Versus Classical Transport, Phys. Rev. Lett. 92(5), 056806 (Feb 2004 ).

[53] S. de Haan, A. Lorke, J. P. Kotthaus, M. Bichler, and W. Wegscheider, Quantized transport in ballistic rectifiers: sign reversal and step-like output, Physica E 21(2-4), 916-920 (Mar 2004).

[54] S. Datta, Electronic Transport in Mesoscopic Systems, Cambridge University Press, 1997.

[55] R. Dingle, H. L. Störmer, A. C. Gossard, and W. Wiegmann, Electron mobilities in modulation-doped semiconductor heterojunction superlattices, Appl. Phys. Lett. 33(7), 665-667 (Oct 1978).

[56] E. H. Hwang and S. Das Sarma, Limit to two-dimensional mobility in modulation-doped GaAs quantum structures: How to achieve a mobility of 100 million, Phys. Rev. B 77(23), 235437-1-6 (Jun 2008).

[57] E. L. Shangina, K. V. Smirnov, D. V. Morozov, V. V. Kovalyuk, G. N. Goltsman, A. A. Verevkin, A. I. Toropov, and P. Mauskopf, Concentration dependence of energy relaxation time in AlGaAs/GaAs heterojunctions: direct measurements, Semicond. Sci. Technol. 26(2), 025013 (Feb 2011).

[58] H. B. G. Casimir, On Onsager's Principle of Microscopic Reversibility, Rev. Mod. Phys. 17(2-3), 343-350 (Apr 1945).

[59] R. Feynman, R. Leighton, and M. Sands, The Feynman Lectures on Physics: Quantum mechanics, The Feynman Lectures on Physics, Pearson/Addison-Wesley, 2nd edition, 1963.

[60] S. Datta, Nanoscale device modeling: the Green's function method, Superlattice Microst. 28(4), 253-278 (Oct 2000).

[61] D. S. Fisher and P. A. Lee, Relation between conductivity and transmission matrix, Phys. Rev. B 23(12), 6851-6854 (Jun 1981).

[62] L. D. Landau, E. M. Lifschitz, and P. Ziesche, Lehrbuch der theoretischen Physik: Quantenmechanik, Harri Deutsch, 2007.

[63] T. A. Davis, UMFPACK User Guide, volume 5.5.1, Dept. of Computer and Information Science and Engineering, Jan 2011.

[64] E. Anderson, Z. Bai, C. Bischof, J. Demmel, J. Dongarra, J. Du Croz, A. Greenbaum, S. Hammarling, A. McKenney, S. Ostrouchov, and D. Sorensen, LAPACK's user's guide, Society for Industrial and Applied Mathematics, Philadelphia, PA, USA, 3rd edition, Jan 1999.

[65] T. A. Davis, Direct methods for sparse linear systems, volume 2, Society for Industrial Mathematics, 2006.

[66] S. Rotter, J.-Z. Tang, L. Wirtz, J. Trost, and J. Burgdörfer, Modular recursive Green's function method for ballistic quantum transport, Phys. Rev. B 62(3), 1950-1960 (Jul 2000).

[67] W. Nolting, Grundkurs Theoretische Physik 7: Viel-Teilchen-Theorie, Springer, 7th edition, 2009.

[68] E. N. Economou and R. John, Green's Functions in Quantum Physics, Springer, 3rd edition, June 2006. 
[69] K. Richter, Semiclassical Theory of Mesoscopic Quantum Systems, Springer tracts in modern physics 161, 1-221 (2000), Habilitationsschrift Oktober 1997.

[70] H. Xu, Theory of nonlinear ballistic transport in quasi-one-dimensional constrictions, Phys. Rev. B 47(23), 15630-15637 (Jun 1993).

[71] A. Jacobsen, I. Shorubalko, L. Maag, U. Sennhauser, and K. Ensslin, Rectification in three-terminal graphene junctions, Appl. Phys. Lett. 97(3), 032110-3 (Jul 2010).

[72] M. V. Costache and S. O. Valenzuela, Experimental Spin Ratchet, Science 330(6011), 1645-1648 (Dec 2010).

[73] P. F. Bagwell and T. P. Orlando, Landauer's conductance formula and its generalization to finite voltages, Phys. Rev. B 40(3), 1456-1464 (Jul 1989).

[74] S. Datta, Quantum Transport: Atom to Transistor, Cambridge University Press, 3rd edition, 2007.

[75] G. D. Mahan, Quantum transport equation for electric and magnetic fields, Phys. Rep. 145(5), 251-318 (Jan 1987).

[76] F. A. Buot, Mesoscopic physics and nanoelectronics: nanoscience and nanotechnology, Phys. Rep. 234(2-3), 73-174 (Nov 1993).

[77] S. Luryi, J. Xu, and A. Zaslavsky, Future Trends In Microelectronics, John Wiley \& Sons, 2010.

[78] J. S. Foresi, P. R. Villeneuve, J. Ferrera, E. R. Thoen, G. Steinmeyer, S. Fan, J. D. Joannopoulos, L. C. Kimerling, H. I. Smith, and E. P. Ippen, Photonic-bandgap microcavities in optical waveguides, Nature 390(6656), 143-145 (Nov 1997).

[79] C. Walther, G. Scalari, M. I. Amanti, M. Beck, and J. Faist, Microcavity Laser Oscillating in a Circuit-Based Resonator, Science 327(5972), 1495-1497 (Mar 2010).

[80] E. M. Purcell, Spontaneous Emission Probabilities at Radio Frequencies, Phys. Rev. 69(11-12), 681 (Jun 1946).

[81] K. J. Vahala, Optical microcavities, Nature 424(6950), 839-846 (Aug 2003).

[82] S. Haroche and D. Kleppner, Cavity quantum electrodynamics, Phys. Today 42(1), 24-30 (Jan 1989).

[83] L. Maleki, Introduction to the Issue on Microresonators, IEEE J. Sel. Topics in Quantum Electron. 12(1), 1-2 (Jan/Feb 2006).

[84] M. S. Ünlü, A. Ozbay, B. B. Goldberg, and N. F. van Hulst, Introduction to the Issue on Nanophotonics, IEEE J. Sel. Topics in Quantum Electron. 12(6), 1069-1071 (Nov 2006).

[85] J. F. Mulligan, Who were Fabry and Pérot?, Am. J. Phys. 66(9), 797-802 (Sep 1998).

[86] R. Sauleau, Fabry-Perot Resonators, John Wiley \& Sons, Inc., 2005.

[87] H. Lohmeyer, K. Sebald, C. Kruse, R. Kroger, J. Gutowski, D. Hommel, J. Wiersig, N. Baer, and F. Jahnke, Confined optical modes in monolithic II-VI pillar microcavities, Appl. Phys. Lett. 88(5), 051101-3 (Jan 2006).

[88] S. Reitzenstein and A. Forchel, Quantum dot micropillars, J. Phys. D: Appl. Phys. 43(3), 033001- (Jan 2010).

[89] H. Lohmeyer, Optischer Einschluss, Lasing und Purcell-Effekt in Mikrosäulenkavitäten auf Basis breitlückiger Halbleiter, Mensch \& Buch, 1st edition, Jun 2008.

[90] B. Gayral, J. M. Gerard, A. Lemaitre, C. Dupuis, L. Manin, and J. L. Pelouard, High-Q wet-etched GaAs microdisks containing InAs quantum boxes, Appl. Phys. Lett. 75(13), 1908-1910 (Sep 1999).

[91] T. J. Kippenberg, S. M. Spillane, and K. J. Vahala, Demonstration of ultra-high-Q small mode volume toroid microcavities on a chip, Appl. Phys. Lett. 85(25), 6113-6115 (Dec 2004).

[92] O. Painter, J. Vučkovič, and A. Scherer, Defect modes of a two-dimensional photonic crystal in an optically thin dielectric slab, J. Opt. Soc. Am. B 16(2), 275-285 (Feb 1999).

[93] P. Snee, Y. Chan, D. Nocera, and M. Bawendi, Whispering-Gallery-Mode Lasing from a Semiconductor Nanocrystal/Microsphere Resonator Composite, Adv. Mater. 17(9), 1131-1136 (May 2005).

[94] A. N. Oraevsky, Whispering-gallery waves, Quantum Electronics 32(5), 377-400 (May 2002).

[95] D. K. Armani, T. J. Kippenberg, S. M. Spillane, and K. J. Vahala, Ultra-high-Q toroid microcavity on a chip, Nature 421(6926), 925-928 (Feb 2003). 
[96] J. U. Nöckel and A. D. Stone, Ray and wave chaos in asymmetric resonant optical cavities, Nature 385(6611), 45-47 (Jan 1997).

[97] C. G. B. Garrett, W. Kaiser, and W. L. Bond, Stimulated Emission into Optical Whispering Modes of Spheres, Phys. Rev. 124(6), 1807-(Dec 1961).

[98] H. Guggenheim, Growth of Single-Crystal Calcium Fluoride with Rare-Earth Impurities, J. Appl. Phys. 32(7), 1337-1338 (Jul 1961).

[99] A. Rosenberger, J. Rezac, S. Koterba, and S. Bates, Temperature tuning of optical whispering-gallery modes as a sensitive probe of thermal interaction between a fused-silica microsphere and the ambient air, in Lasers and Electro-Optics, 2002. CLEO '02. Technical Digest. Summaries of papers presented at the Conference on Lasers and Electro-Optics. Conference Edition (IEEE Cat. No.02CH37337), pages 217-218, 2002.

[100] K. M. Ho, C. T. Chan, and C. M. Soukoulis, Existence of a photonic gap in periodic dielectric structures, Phys. Rev. Lett. 65(25), 3152-3155 (Dec 1990).

[101] F. Garcia-Santamaria, C. Lopez, F. Meseguer, F. Lopez-Tejeira, J. Sanchez-Dehesa, and H. T. Miyazaki, Opal-like photonic crystal with diamond lattice, Appl. Phys. Lett. 79(15), 2309-2311 (Oct 2001).

[102] S. Miller, A. Chynoweth, and I. Kaminow, Optical fiber telecommunications, volume 1, Academic Press, Mar 1979.

[103] W. Burns and A. Milton, Mode conversion in planar-dielectric separating waveguides, IEEE J. Quantum. Electron. 11(1), 32-39 (Jan 1975).

[104] H. Yajima, Coupled mode analysis of dielectric planar branching waveguides, IEEE J. Quantum. Electron. 14(10), 749-755 (Oct 1978).

[105] W. E. Martin, A new waveguide switch/modulator for integrated optics, Appl. Phys. Lett. 26(10), 562-564 (May 1975).

[106] Q. Xu, B. Schmidt, S. Pradhan, and M. Lipson, Micrometre-scale silicon electro-optic modulator, Nature 435(7040), 325-327 (May 2005).

[107] M. V. Berry, Regular and irregular motion, AIP Conf. Proc. 46(1), 16-120 (Sep 1978).

[108] T. Geisel, G. Radons, and J. Rubner, Kolmogorov-Arnol'd-Moser Barriers in the Quantum Dynamics of Chaotic Systems, Phys. Rev. Lett. 57(23), 2883-2886 (Dec 1986).

[109] G. B. Akguc and L. E. Reichl, Conductance and Statistical Properties of Chaotic and Integrable Electron Waveguides, J. Stat. Phys. 98(3), 813-834 (Feb 2000).

[110] A. Bäcker, A. Manze, B. Huckestein, and R. Ketzmerick, Isolated resonances in conductance fluctuations and hierarchical states, Phys. Rev. E 66(1), 016211 (Jul 2002).

[111] J. A. Méndez-Bermúdez, G. A. Luna-Acosta, and F. M. Izrailev, From chaos to disorder in quasi-1D billiards with corrugated surfaces, Physica E 22(4), 881-899 (May 2004).

[112] S. Smale, Differentiable dynamical systems, Bull. Amer. Math. Soc. 73, 747-817 (Nov 1967).

[113] J. Guckenheimer and P. Holmes, Nonlinear Oscillations, Dynamical Systems, and Bifurcations of Vector Fields, Applied Mathematical Sciences, Springer-Verlag, New York, 1983.

[114] A. J. Lichtenberg and M. A. Lieberman, Regular and Chaotic Dynamics, volume 38 of Applied Mathematical Sciences, Springer-Verlag New York, Inc., 2nd edition, Jun 1992.

[115] B. Rückerl and C. Jung, Scaling properties of a scattering system with an incomplete horseshoe, J. Phys. A: Math. Gen. 27(1), 55-77 (Jan 1994).

[116] A. B. Katok, Smooth ergodic theory and its applications: proceedings of the AMS Summer Research Institute on Smooth Ergodic Theory and Its Applications, volume 69, AMS Bookstore, 2001.

[117] M. Büttiker, Absence of backscattering in the quantum Hall effect in multiprobe conductors, Phys. Rev. B 38(14), 9375-9389 (Nov 1988).

[118] K. Husimi, Some Formal Properties of the Density Matrix, Proc. Phys. Math. Soc. Japan 22(4), 264-314 (Apr 1940). 
[119] M. J. Davis and E. J. Heller, Quantum dynamical tunneling in bound states, J. Chem. Phys. 75(1), $246-254$ (Jul 1981).

[120] G. A. Luna-Acosta, J. A. Méndez-Bermúdez, P. Scaroneba, and K. N. Pichugin, Classical versus quantum structure of the scattering probability matrix: Chaotic waveguides, Phys. Rev. E 65(4), 046605- (Mar 2002).

[121] H.-J. Stöckmann, Quantenchaos zum Anfassen - das Mikrowellenbillard, Phys. Blätter 53(2), 121 (Feb 1997).

[122] H.-D. Gräf, H. L. Harney, H. Lengeler, C. H. Lewenkopf, C. Rangacharyulu, A. Richter, P. Schardt, and H. A. Weidenmüller, Distribution of eigenmodes in a superconducting stadium billiard with chaotic dynamics, Phys. Rev. Lett. 69(9), 1296-1299 (Aug 1992).

[123] H.-J. Stöckmann, Quantum Chaos: An Introduction, Cambridge University Press, 2nd edition, Oct 1999, Hardcover.

[124] U. Kuhl, E. Persson, M. Barth, and H.-J. Stöckmann, Mixing of wavefunctions in rectangular microwave billiards, Eur. Phys. J. B 17(2), 253-259 (Sep 2000).

[125] Y.-H. Kim, M. Barth, H.-J. Stöckmann, and J. P. Bird, Wave function scarring in open quantum dots: A microwavebilliard analog study, Phys. Rev. B 65(16), 165317 (Apr 2002).

[126] H. Ishio and K. Nakamura, Quantum Transport in Open Billiards: Dependence on Degree of Opening, J. Phys. Soc. Jpn. 61(8), 2649-2651 (Aug 1992).

[127] P. J. Price, Quasi-bound states and resonances in heterostructures, Microelectr. J. 30(10), 925-934 (Oct 1999).

[128] E. J. Heller, Bound-State Eigenfunctions of Classically Chaotic Hamiltonian Systems: Scars of Periodic Orbits, Phys. Rev. Lett. 53(16), 1515-1518 (Oct 1984).

[129] S. W. McDonald and A. N. Kaufman, Wave chaos in the stadium: Statistical properties of short-wave solutions of the Helmholtz equation, Phys. Rev. A 37(8), 3067- (Apr 1988).

[130] J. Méndez-Bermúdez, G. Luna-Acosta, and P. Šeba, Directional Emission from Microlasers with Open Chaotic Resonators, Phys. Status Solidi B 230(2), 385-389 (Apr 2002).

[131] J. A. Mendez-Bermudez, C. Tapia-Ignacio, and G. A. Luna-Acosta, Multidirectional tunable wave resonators, in Proc. SPIE, volume 7499, pages 74991A-6, Guadalajara, Jalisco, Mexico, Sep 2009, SPIE.

[132] H. L. F. v. Helmholtz, Populäre wissenschaftliche Vorträge, Number 1-2 in Populäre wissenschaftliche Vorträge, F. Vieweg, 1865.

[133] I. Solovei, M. Kreysing, C. Lanctôt, S. Kösem, L. Peichl, T. Cremer, J. Guck, and B. Joffe, Nuclear Architecture of Rod Photoreceptor Cells Adapts to Vision in Mammalian Evolution, Cell 137(2), 356-368 (Apr 2009).

[134] C. W. J. Beenakker and H. van Houten, Billiard model of a ballistic multiprobe conductor, Phys. Rev. Lett. 63(17), 1857-1860 (Oct 1989).

[135] L. W. Molenkamp, A. A. M. Staring, C. W. J. Beenakker, R. Eppenga, C. E. Timmering, J. G. Williamson, C. J. P. M. Harmans, and C. T. Foxon, Electron-beam collimation with a quantum point contact, Phys. Rev. B 41(2), 1274-1277 (Jan 1990).

[136] M. Rammerstorfer, Lebewesen und Design: Eine Einführung, Books on Demand GmbH, 2010.

[137] R. L. K. Virchow, Gesammelte Abhandlungen zur Wissenschaftlichen Medicin, Meidinger, 1856.

[138] R. L. K. Virchow, Über das granulierte Aussehen der Wandungen der Gehirnventrikel, Allg. Z. Psychiat. 3(2), 242-250 (Apr 1846).

[139] O. F. K. Deiters, Untersuchungen über Gehirn und Rückenmark des Menschen und der Säugethiere, Friedrich Vieweg, Braunschweig, 1865.

[140] G. G. Somjen, Nervenkitt: Notes on the history of the concept of neuroglia, Glia 1(1), 2-9 (Jan/Feb 1988).

[141] Y.-B. Lu, K. Franze, G. Seifert, C. Steinhäuser, F. Kirchhoff, H. Wolburg, J. Guck, P. Janmey, E.-Q. Wei, J. Käs, and A. Reichenbach, Viscoelastic properties of individual glial cells and neurons in the CNS, Proc. Natl. Acad. Sci. USA 103(47), 17759-17764 (Nov 2006).

[142] D. H. Hubel, Eye, Brain, and Vision, Number 22 in Scientific American Library, W. H. Freeman, 1995.

[143] P. M. Kiely, G. Smith, and L. G. Carney, The Mean Shape of the Human Cornea, J. Mod. Opt. 29, 1027-1040 (Aug 1982). 
[144] B. G. Soni, A. R. Philp, R. G. Foster, and B. E. Knox, Novel retinal photoreceptors, Nature 394(6688), 27-28 (Jul 1998).

[145] D. H. McDougal and P. D. Gamlin, The influence of intrinsically-photosensitive retinal ganglion cells on the spectral sensitivity and response dynamics of the human pupillary light reflex, Vision Res. 50(1), $72-87$ (Jan 2010).

[146] E. H. Hess and J. M. Polt, Pupil Size in Relation to Mental Activity during Simple Problem-Solving, Science 143(3611), 1190-1192 (Mar 1964).

[147] E. H. Hess and J. M. Polt, Pupil Size as Related to Interest Value of Visual Stimuli, Science 132(3423), 349-350 (Aug 1960).

[148] O. Friedrich, Physiologie - GK 1, Springer Medizin Verlag, 2007.

[149] E. R. Berman and M. Voaden, The vitreous body. In: Biochemistry of the Eye, Academic Press N.Y., 1970.

[150] M. Lich, Visuelle Navigation: Dynamik der Wahrnehmung von Eigenbewegung, PhD thesis, Philipps Universität Marburg, Nov 2010.

[151] G. L. Walls, The vertebrate eye and its adaptive radiation, Hafner publishing company, 1942.

[152] S. Polyak, Vertebrate Visual System: its origin, structure, and function and its manifestations in disease with an analysis of its role in the life of animals and in the origin of man, preceded by a historical review of investigations of the eye, and of the visual pathways and centers of the brain, University of Chicago Press, Sep 1957.

[153] R. L. Gregory and J. Cronly-Dillon, Evolution of the Eye and Visual System, volume 2 of Vision and visual dysfunction, Macmillan, 1991.

[154] B. B. Boycott, J. E. Dowling, and H. Kolb, Organization of the Primate Retina: Light Microscopy, Philos. Trans. R. Soc. Lond. B. Biol. Sci. 255(799), 109-184 (Mar 1969).

[155] A. Reichenbach, M. Ziegert, J. Schnitzer, S. Pritz-Hohmeier, P. Schaaf, W. Schober, and H. Schneider, Development of the rabbit retina. V. The question of [']columnar units', Dev. Brain Res. 79(1), 72-84 (May 1994).

[156] A. Reichenbach and S. R. Robinson, Phylogenetic constraints on retinal organisation and development, Prog. Retin. Eye Res. 15(1), 139-171 (Jan 1995).

[157] O. Strauss, The Retinal Pigment Epithelium in Visual Function, Physiol. Rev. 85(3), 845-881 (Jul 2005).

[158] N. Drasdo and C. W. Fowler, Non-linear projection of the retinal image in a wide-angle schematic eye, Br. J. Ophthalmol. 58(8), 709-714 (Aug 1974).

[159] C. A. Curcio, K. R. Sloan, R. E. Kalina, and A. E. Hendrickson, Human photoreceptor topography, J. Comp. Neurol. 292(4), 497-523 (Feb 1990).

[160] S. A. Burns, S. Wu, F. Delori, and A. E. Elsner, Direct measurement of human-cone photoreceptor alignment, J. Opt. Soc. Am. A 12(10), 2329-2338 (Oct 1995).

[161] T. Y. Chui, H. Song, and S. A. Burns, Adaptive-optics imaging of human cone photoreceptor distribution, J. Opt. Soc. Am. A 25(12), 3021-3029 (Dec 2008).

[162] D. B. Farber, J. G. Flannery, R. N. Lolley, and D. Bok, Distribution patterns of photoreceptors, protein, and cyclic nucleotides in the human retina, Invest. Ophthalmol. Vis. Sci. 26(11), 1558-68 (Nov 1985).

[163] V. J. Volbrecht, E. E. Shrago, B. E. Schefrin, and J. S. Werner, Spatial summation in human cone mechanisms from $0^{\circ}$ to $20^{\circ}$ in the superior retina, J. Opt. Soc. Am. A 17(3), 641-650 (Mar 2000).

[164] K. Wikler and P. Rakic, Distribution of photoreceptor subtypes in the retina of diurnal and nocturnal primates, J. Neurosci. 10(10), 3390-3401 (Oct 1990).

[165] B. L. Finlay, E. C. S. Franco, E. S. Yamada, J. C. Crowley, M. Parsons, J. A. P. C. Muniz, and L. C. L. Silveira, Number and topography of cones, rods and optic nerve axons in New and Old World primates, Vis. Neurosci. 25(03), 289-299 (May 2008).

[166] A. Roorda and D. R. Williams, The arrangement of the three cone classes in the living human eye, Nature 397(6719), 520-522 (Feb 1999). 
[167] N. Hart, J. Partridge, and I. Cuthill, Visual pigments, oil droplets and cone photoreceptor distribution in the european starling (Sturnus vulgaris), J. Exp. Biol. 201(9), 1433-46 (Sep 1998).

[168] G. Østerberg, Topography of the layer of rods and cones in the human retina, Nyt Nordisk Forlag, 1935.

[169] J. K. Bowmaker and H. J. Dartnall, Visual pigments of rods and cones in a human retina, J. Physiol. (Lond.) 298(1), 501-511 (Jan 1980).

[170] G. Wyszecki and W. S. Stiles, Color Science: Concepts and Methods, Quantitative Data and Formulae, Pure \& Applied Optics Series, John Wiley \& Sons Inc, 2nd edition, Sep 1982, Paperback reprint: Aug 2000, ISBN 9780471021063, 968p.

[171] A. Stockman, L. T. Sharpe, and C. Fach, The spectral sensitivity of the human short-wavelength sensitive cones derived from thresholds and color matches, Vision Res. 39(17), 2901-2927 (Aug 1999).

[172] A. Stockman and L. T. Sharpe, The spectral sensitivities of the middle- and long-wavelength-sensitive cones derived from measurements in observers of known genotype, Vision Res. 40(13), 1711-1737 (Jun 2000).

[173] F. Müller and U. B. Kaupp, Signaltransduktion in Sehzellen, Naturwissenschaften 85(2), 49-61 (Feb 1998).

[174] G. M. Cooper and R. E. Hausman, The Cell: A Molecular Approach, ASM Press and Sinauer Associates, Inc., 4th edition, 2007.

[175] E. Newman and A. Reichenbach, The Müller cell: a functional element of the retina, Trends Neurosci. 19(8), 307-312 (Apr 1996).

[176] E. A. Newman and K. R. Zahs, Modulation of Neuronal Activity by Glial Cells in the Retina, J. Neurosci. 18(11), 4022-4028 (Jun 1998).

[177] A. Bringmann, T. Pannicke, J. Grosche, M. Francke, P. Wiedemann, S. N. Skatchkov, N. N. Osborne, and A. Reichenbach, Müller cells in the healthy and diseased retina, Prog. Retin. Eye Res. 25(4), 397-424 (Jul 2006).

[178] A. Reichenbach and A. Bringmann, Müller Cells in the Healthy and Diseased Retina, Springer New York, Mar 2010.

[179] B. Miller, H. Miller, R. Patterson, and S. J. Ryan, Retinal Wound Healing: Cellular Activity at the Vitreoretinal Interface, Arch. Ophthalmol. 104(2), 281-285 (Feb 1986).

[180] S. L. Burmeister, D. Hartwig, G. A. Limb, C. Kremling, H. Hoerauf, M. Müller, and G. Geerling, Effect of Various Platelet Preparations on Retinal Müller Cells, Invest. Ophthalmol. Vis. Sci. 50(10), 4881-4886 (Oct 2009).

[181] E. Newman, D. Frambach, and L. Odette, Control of extracellular potassium levels by retinal glial cell $\mathrm{K}^{+}$ siphoning, Science 225(4667), 1174-1175 (Sep 1984).

[182] S. Poitry, C. Poitry-Yamate, J. Ueberfeld, P. R. MacLeish, and M. Tsacopoulos, Mechanisms of Glutamate Metabolic Signaling in Retinal Glial (Müller) Cells, J. Neurosci. 20(5), 1809-1821 (Mar 2000).

[183] B. S. Winkler, M. J. Arnold, M. A. Brassell, and D. G. Puro, Energy Metabolism in Human Retinal Müller Cells, Invest. Ophthalmol. Vis. Sci. 41(10), 3183-3190 (Sep 2000).

[184] C. L. Poitry-Yamate and M. Tsacopoulos, Glucose metabolism in freshly isolated Müller glial cells from a mammalian retina, J. Comp. Neurol. 320(2), 257-266 (Jun 1992).

[185] M. Wong-Riley, Energy metabolism of the visual system, Eye and Brain 2(1), 99-116 (Jul 2010).

[186] A. Reichenbach, J.-U. Stolzenburg, W. Eberhardt, T. Chao, D. Dettmer, and L. Hertz, What do retinal Müller (glial) cells do for their neuronal 'small siblings'?, J. Chem. Neuroanat. 6(4), 201-213 (Jul 1993).

[187] S. R. y Cajal, Die Retina der Wirbelthiere, Bergmann, 1894.

[188] S. R. y Cajal, The structure of the retina, C.C. Thomas, Springfield, Ill., 1972, First published in 1892 in the journal La Cellule.

[189] V. Sarthy and H. Ripps, The Retinal Müller Cell: Structure and Function, Perspectives in vis. res., Springer, 2002.

[190] M. Bass, C. DeCusatis, J. M. Enoch, J. Enoch, V. Lakshminarayanan, G. Li, C. MacDonald, V. N. Mahajan, V. Mahajan, and E. V. Stryland, Handbook of Optics, Third Edition Volume III: Vision and Vision Optics, volume 3, McGraw-Hill, 3rd edition, 2010. 
[191] V. C. Sundar, A. D. Yablon, J. L. Grazul, M. Ilan, and J. Aizenberg, Fibre-optical features of a glass sponge, Nature 424(6951), 899-900 (Aug. 2003).

[192] J. Aizenberg, J. C. Weaver, M. S. Thanawala, V. C. Sundar, D. E. Morse, and P. Fratzl, Skeleton of Euplectella sp.: Structural Hierarchy from the Nanoscale to the Macroscale, Science 309(5732), 275-278 (Jul 2005).

[193] S. V. Patwardhan, Biomimetic and bioinspired silica: recent developments and applications, Chem. Commun. 47(27), 7567-7582 (Apr 2011).

[194] V. G. Kravets and A. N. Grigorenko, Retinal light trapping in textured photovoltaic cells, Appl. Phys. Lett. 97(14), 143701-3 (Oct 2010).

[195] Y. Han, J. Shin, D. Kim, S. Park, Y. Park, and H. Sung, A Rigorous 2D Approximation Technique for 3D Waveguide Structures for BPM Calculations, ETRI Journal 25(6), 535-537 (Dec 2003).

[196] V. P. Tychinsky, I. N. Masalov, V. L. Pankov, and D. V. Ublinsky, Computerized phase microscope for investigation of submicron structures, Opt. Commun. 74(1-2), 37-40 (Dec 1989).

[197] J. Beuthan, O. Minet, J. Helfmann, M. Herrig, and G. Mueller, The spatial variation of the refractive index in biological cells, Phys. Med. Biol. 41(3), 369-382 (Mar 1996).

[198] R. Winston, The Visual Receptor as a Light Collector, Springer, New York, 1981.

[199] R. L. Sidman, The structure and concentration of solids in photoreceptor cells studied by refractometry and interference microscopy, J. Biophys. Biochem. Cytol. 3(1), 15-30 (Jan 1957).

[200] T. I. Chao, J. Grosche, B. Biedermann, M. Francke, T. Pannicke, W. Reichelt, M. Wulst, C. Muhle, S. PritzHohmeier, H. Kuhrt, F. Faude, W. Drommer, M. Kasper, E. Buse, and A. Reichenbach, Comparative studies on mammalian Muller (retinal glial) cells, J. Neurocytol. 26(7), 439-454 (Jul 1997).

[201] A. Yacoby and Y. Imry, Quantization of the conductance of ballistic point contacts beyond the adiabatic approximation, Phys. Rev. B 41(8), 5341-5350 (Mar 1990).

[202] A. Khintchine, Korrelationstheorie der stationären stochastischen Prozesse, Mathematische Annalen 109, 604-615 (Dec 1934).

[203] R. J. Adler and J. E. Taylor, Random Fields and Geometry, Springer Monographs in Mathematics, Springer Science+Business Media LLC, 2007.

[204] K. Bröking, Transport in Micro-Hallbars, Master's thesis, Georg-August-Universität Göttingen, Institut f $\tilde{A}^{1} / 4 \mathrm{r}$ Nichtlineare Dynamik der Georg-August-Universität zu Göttingen, 2006.

[205] J. E. Dowling, Organization of Vertebrate Retinas, Invest. Ophthalmol. Vis. Sci. 9(9), 655-680 (Sep 1970).

[206] X. Liang, A. Liu, C. Lim, T. Ayi, and P. Yap, Determining refractive index of single living cell using an integrated microchip, Sensor. Actuat. A 133(2), 349-354 (Feb 2007).

[207] A. M. Labin and E. N. Ribak, Retinal Glial Cells Enhance Human Vision Acuity, Phys. Rev. Lett. 104(15), 158102 (Apr 2010).

[208] J. J. Metzger, Branched Flow and Caustics in Two-Dimensional Random Potentials and Magnetic Fields, PhD thesis, Universität Göttingen, 2010.

[209] J. J. Metzger, R. Fleischmann, and T. Geisel, Universal Statistics of Branched Flows, Phys. Rev. Lett. 105(2), 0206011-0206014 (Jul 2010).

[210] J. Guck, R. Ananthakrishnan, T. J. Moon, C. C. Cunningham, and J. Käs, Optical Deformability of Soft Biological Dielectrics, Phys. Rev. Lett. 84(23), 5451-5454 (June 2000).

[211] J. Guck, R. Ananthakrishnan, H. Mahmood, T. J. Moon, C. C. Cunningham, and J. Käs, The Optical Stretcher: A Novel Laser Tool to Micromanipulate Cells, Biophys. J. 81(2), 767-784 (Aug 2001).

[212] A. Ashkin, Acceleration and Trapping of Particles by Radiation Pressure, Phys. Rev. Lett. 24(4), 156-159 (Jan 1970).

[213] S. Chu, J. E. Bjorkholm, A. Ashkin, and A. Cable, Experimental Observation of Optically Trapped Atoms, Phys. Rev. Lett. 57(3), 314-317 (Jul 1986). 
[214] Y. Harada and T. Asakura, Radiation forces on a dielectric sphere in the Rayleigh scattering regime, Opt. Commun. 124(5-6), 529-541 (Mar 1996).

[215] C. P. Technologies, Corning PureMode HI 1060 Photonic Fiber: Single-mode Component Fiber for High Performance Photonic Applications, Photonic Technologies, Corning Inc. P.O. Box 7429, Endicott, NY 13760 USA, 2001.

[216] M. Young, Mode-Field Diameter of Single-Mode Optical Fiber by Far-Field Scanning: Addendum, Appl. Opt. 37(36), 8361-8361 (Dec 1998).

[217] A. Ashkin, J. M. Dziedzic, J. E. Bjorkholm, and S. Chu, Observation of a single-beam gradient force optical trap for dielectric particles, Opt. Lett. 11(5), 288-290 (May 1986).

[218] B. E. A. Saleh and M. C. Teich, Fundamentals of photonics, John Wiley \& Sons Inc., 2nd edition, Apr 2007.

[219] C. L. Curl, C. J. Bellair, T. Harris, B. E. Allman, P. J. Harris, A. G. Stewart, A. Roberts, K. A. Nugent, and L. M. D. Delbridge, Refractive index measurement in viable cells using quantitative phase-amplitude microscopy and confocal microscopy, Cytometry 65A(1), 88-92 (May 2005).

[220] H.-L. Liou and N. A. Brennan, Anatomically accurate, finite model eye for optical modeling, J. Opt. Soc. Am. A 14(8), 1684-1695 (Aug 1997).

[221] M. S. d. Almeida and L. A. Carvalho, Different schematic eyes and their accuracy to the in vivo eye: a quantitative comparison study, Braz. J. Phys. 37, 378-387 (Jun 2007).

[222] S. Kasthurirangan and A. Glasser, Age related changes in the characteristics of the near pupil response, Vision Res. 46(8-9), 1393-1403 (Apr 2006).

[223] E. Taylor and A. Jennings, Calculation of total retinal area, British Journal of Ophthalmology 55(4), 262-265 (Apr 1971).

[224] D. A. Brinton and C. P. Wilkinson, Retinal Detachment: Principles and Practice, Ophthalmology Monographs, Oxford University Press, 3rd edition, 2009.

[225] M. Aggarwal, H. Hua, and N. Ahuja, On cosine-fourth and vignetting effects in real lenses, in Eighth IEEE International Conference on Computer Vision, volume 1, pages 472-479, 2001.

[226] W. J. Smith, Modern optical engineering: the design of optical systems, SPIE Press/McGraw-Hill professional engineering, McGraw Hill, 4th edition, 2008.

[227] C. Lau and H. A. Richter, Anatomie der Augen verschiedener Spezies, http://www.itemp.rwthaachen.de/retina/retina04.html, 2001.

[228] I. E. Loewenfeld, Pupillary changes related to age, Topics in neuro-ophthalmology, Herbert Stanley Thompson, editor, Williams \& Wilkins, 1979.

[229] A. Hendrickson, A morphological comparison of foveal development in man and monkey, Eye (Lond). 6(2), 136-144 (Jun 1992).

[230] J. W. Goodman, Introduction to Fourier Optics, Roberts \& Company Publishers, 3rd edition, 2005.

[231] P. Dutré, K. Bala, and P. Bekaert, Advanced Global Illumination, A. K. Peters, Ltd., Natick, MA, USA, 2nd edition, 2006.

[232] G. I. Pokrowski, Über die Abhängigkeit des Koeffizienten der diffusen Reflexion vom Einfallswinkel des Lichtes, Zeitschrift für Physik A: Hadrons and Nuclei 32(1), 563-568 (Dec 1925).

[233] M. Kreysing, L. Boyde, J. Guck, and K. J. Chalut, Physical insight into light scattering by photoreceptor cell nuclei, Opt. Lett. 35(15), 2639-2641 (Aug 2010).

[234] W. S. Stiles, The Luminous Efficiency of Monochromatic Rays Entering the Eye Pupil at Different Points and a New Colour Effect, Proc. R. Soc. Lond. B Biol. Sci. 123(830), 90-118 (Jun 1937).

[235] B. Vohnsen, On the spectral relation between the first and second Stiles-Crawford effect, J. Mod. Opt. 56(20), 2261-2271 (Jun 2009).

[236] W. Heisenberg, Schritte über Grenzen. Gesammelte Reden und Aufsätze., Piper, 1971. 
[237] A. Mostafazadeh and A. Batal, Physical aspects of pseudo-Hermitian and PT-symmetric quantum mechanics, J. Phys. A: Math. Gen. 37(48), 11645-11679 (Dec 2004).

[238] E. P. Wigner, Normal Form of Antiunitary Operators, J. Math. Phys. 1(5), 409-413 (Sep 1960).

[239] L. Solombrino, Weak pseudo-Hermiticity and antilinear commutant, J. Math. Phys. 43(11), 5439-5445 (Nov 2002).

[240] C. M. Bender, Making sense of non-Hermitian Hamiltonians, Rep. Prog. Phys. 70(6), 947-1018 (May 2007 ).

[241] C. M. Bender, S. Boettcher, and P. N. Meisinger, PT-symmetric quantum mechanics, J. Math. Phys. 40(5), 2201-2229 (May 1999).

[242] C. M. Bender, G. V. Dunne, and P. N. Meisinger, Complex periodic potentials with real band spectra, Phys. Lett. A 252(5), 272-276 (Mar 1999).

[243] C. M. Bender and J. Feinberg, Does the complex deformation of the Riemann equation exhibit shocks?, J. Phys. A: Math. Theor. 41(24), 244004 (8pp) (Jun 2008).

[244] C. M. Bender, J. Feinberg, D. W. Hook, and D. J. Weir, Chaotic systems in complex phase space, Pramana 73(3), 453-470 (Sep 2009).

[245] Z. H. Musslimani, K. G. Makris, R. El-Ganainy, and D. N. Christodoulides, Optical Solitons in $\mathcal{P} \mathcal{T}$ Periodic Potentials, Phys. Rev. Lett. 100(3), 030402 (Jan 2008).

[246] S. Klaiman, U. Günther, and N. Moiseyev, Visualization of Branch Points in $\mathcal{P} \mathcal{T}$-Symmetric Waveguides, Phys. Rev. Lett. 101(8), 080402 (Aug 2008).

[247] E. M. Graefe, U. Günther, H.-J. Korsch, and A. E. Niederle, A non-Hermitian $\mathcal{P} \mathcal{T}$ symmetric Bose-Hubbard model: eigenvalue rings from unfolding higher-order exceptional points, J. Phys. A: Math. Theor. 41(25), 255206 (26pp) (May 2008).

[248] M. Hiller, T. Kottos, and A. Ossipov, Bifurcations in resonance widths of an open Bose-Hubbard dimer, Phys. Rev. A 73(6), 063625 (Jun 2006).

[249] A. Guo, G. J. Salamo, D. Duchesne, R. Morandotti, M. Volatier-Ravat, V. Aimez, G. A. Siviloglou, and D. N. Christodoulides, Observation of $\mathcal{P} \mathcal{T}$-Symmetry Breaking in Complex Optical Potentials, Phys. Rev. Lett. 103(9), 093902 (Aug 2009).

[250] K. G. Makris, R. El-Ganainy, D. N. Christodoulides, and Z. Musslimani, Beam Dynamics in $\mathcal{P} \mathcal{T}$-Symmetric Optical Lattices, Phys. Rev. Lett. 100(10), 103904 (Mar 2008).

[251] R. El-Ganainy, K. G. Makris, D. N. Christodoulides, and Z. H. Musslimani, Theory of coupled optical $\mathcal{P} \mathcal{T}$ symmetric structures, Opt. Lett. 32(17), 2632-2634 (Aug 2007).

[252] A. Ruschhaupt, F. Delgado, and J. G. Muga, Physical realization of $\mathcal{P} \mathcal{T}$-symmetric potential scattering in a planar slab waveguide, J. Phys. A: Math. Gen. 38(9), L171-L176 (Mar 2005).

[253] M. V. Berry, Optical lattices with $\mathcal{P} \mathcal{T}$-symmetry are not transparent, J. Phys. A: Math. Theor. 41(24), 244007 (7pp) (Jun 2008).

[254] C. T. West, T. Kottos, and T. Prosen, PT-Symmetric Wave Chaos, Phys. Rev. Lett. 104(5), 054102 (Feb 2010$).$

[255] C. E. Rüter, K. G. Makris, R. El-Ganainy, D. N. Christodoulides, M. Segev, and D. Kip, Observation of parity-time symmetry in optics, Nat. Phys. 6(3), 192-195 (Mar 2010).

[256] Z. H. Musslimani, K. G. Makris, R. El-Ganainy, and D. N. Christodoulides, Analytical solutions to a class of nonlinear Schrödinger equations with $\mathcal{P} \mathcal{T}$-like potentials, J. Phys. A: Math. Theor. 41(24), 244019 (12pp) (Jun 2008).

[257] M. Timme, T. Geisel, and F. Wolf, Speed of synchronization in complex networks of neural oscillators: Analytic results based on Random Matrix Theory, Chaos 16(1), 015108 (Mar 2006).

[258] H. J. Sommers, A. Crisanti, H. Sompolinsky, and Y. Stein, Spectrum of Large Random Asymmetric Matrices, Phys. Rev. Lett. 60(19), 1895-1898 (May 1988).

[259] D. R. Nelson and N. M. Shnerb, Non-Hermitian localization and population biology, Phys. Rev. E 58(2), 1383-1403 (Aug 1998). 
[260] J. T. Chalker and Z. J. Wang, Diffusion in a Random Velocity Field: Spectral Properties of a Non-Hermitian Fokker-Planck Operator, Phys. Rev. Lett. 79(10), 1797-1800 (Sep 1997).

[261] N. Hatano and D. R. Nelson, Localization Transitions in Non-Hermitian Quantum Mechanics, Phys. Rev. Lett. 77(3), 570-573 (Jul 1996).

[262] Y. V. Fyodorov, T. Kottos, and H.-J. Stöckmann, Trends in quantum chaotic scattering, J. Phys. A: Math. Gen. 38(49), 10433-10878 (Dec 2005).

[263] J. Ginibre, Statistical Ensembles of Complex, Quaternion, and Real Matrices, J. Math. Phys. 6(3), 440-449 (Mar 1965).

[264] J. Feinberg, Non-Hermitian random matrix theory: summation of planar diagrams, the "single-ring" theorem and the disc-annulus phase transition, J. Phys. A: Math. Gen. 39(32), 10029-10056 (Jul 2006).

[265] I. Rotter, A non-Hermitian Hamilton operator and the physics of open quantum systems, J. Phys. A: Math. Theor. 42(15), 153001 (51pp) (Apr 2009).

[266] J. Okolowicz, M. Ploszajczak, and I. Rotter, Dynamics of quantum systems embedded in a continuum, Phys. Rep. 374(4-5), 271-383 (Feb 2003).

[267] Y. V. Fyodorov and H.-J. Sommers, Random matrices close to Hermitian or unitary: overview of methods and results, J. Phys. A: Math. Gen. 36(12), 3303-3347 (Mar 2003).

[268] K. Makris, R. El-Ganainy, D. Christodoulides, and Z. Musslimani, Optical solitons in $\mathcal{P} \mathcal{T}$ periodic potentials, in 2008 Quantum Electronics and Laser Science Conference (QELS), page 2 pp., Piscataway, NJ, USA, 2008 2008, IEEE, 2008 Quantum Electronics and Laser Science Conference (QELS), 4-9 May 2008, San Jose, CA, USA.

[269] M. V. Berry and D. H. J. O'Dell, Diffraction by volume gratings with imaginary potentials, J. Phys. A: Math. Gen. 31(8), 2093-2101 (Feb 1998).

[270] K. Kawano and T. Kitoh, Introduction to Optical Waveguide Analysis: Solving Maxwell's Equations and the Schrödinger Equation, Wiley, 2001.

[271] U. Peschel, R. Morandotti, J. M. Arnold, J. S. Aitchison, H. S. Eisenberg, Y. Silberberg, T. Pertsch, and F. Lederer, Optical discrete solitons in waveguide arrays. 2. Dynamic properties, J. Opt. Soc. Am. B 19(11), 2637-2644 (Nov 2002).

[272] D. Christodoulides, F. Lederer, and Y. Silberberg, Discretizing light behaviour in linear and nonlinear waveguide lattices, Nature 424, 817-823 (Aug 2003).

[273] S. Longhi, Optical Realization of Relativistic Non-Hermitian Quantum Mechanics, Phys. Rev. Lett. 105(1), 013903- (Jun 2010).

[274] T. Pertsch, T. Zentgraf, U. Peschel, A. Bräuer, and F. Lederer, Beam steering in waveguide arrays, Appl. Phys. Lett. 80(18), 3247-3249 (May 2002).

[275] T. Pertsch, T. Zentgraf, U. Peschel, A. Bräuer, and F. Lederer, Anomalous Refraction and Diffraction in Discrete Optical Systems, Phys. Rev. Lett. 88(9), 093901 (Feb 2002).

[276] T. Pertsch, U. Peschel, J. Kobelke, K. Schuster, H. Bartelt, S. Nolte, A. Tünnermann, and F. Lederer, Nonlinearity and Disorder in Fiber Arrays, Phys. Rev. Lett. 93(5), 053901 (Jul 2004).

[277] H. Bartelt, Influences of micro- and nanostructuring on light guiding (Invited Paper), in Proceedings of SPIE, edited by M. Voet, R. Willsch, W. Ecke, J. Jones, and B. Culshaw, volume 5855, pages 114-117, SPIE, 2005.

[278] J. Kobelke, K. Schuster, S. Grimm, D. Litzkendorf, J. Kirchhof, A. Schwuchow, H. Bartelt, and A. Gebhardt, Multicomponent glass microstructured fibers for nonlinear applications, Proc. SPIE 6990(1), 699005(11) (Apr 2008).

[279] A. Burkert, W. Triebel, U. Natura, and R. Martin, Microchannel formation in fused silica during ArF excimer laser irradiation, Phys. Chem. Glasses 48(48), 107-112 (Jun 2007).

[280] T. Heinzel, Mesoscopic electronics in solid state nanostructures, Physics textbook, Wiley-VCH, 2nd edition, 2007.

[281] W. Gambling, The rise and rise of optical fibers, IEEE J. Sel. Topics in Quantum Electron. 6(6), 1084-1093 (Nov 2000). 
[282] P. Russell, Photonic Crystal Fibers, Science 299(5605), 358-362 (Jan 2003).

[283] J. D. Joannopoulos, S. G. Johnson, and J. N. Winn, Photonic crystals: molding the flow of light, Princeton University Press, 2nd edition, 2008.

[284] G. P. Agrawal, Applications of Nonlinear Fiber Optics, Optics and Photonics Series, Academic Press, 2nd edition, Apr 2008.

[285] W. Cai and V. Shalaev, Optical Metamaterials: Fundamentals and Applications, Springer, 2009.

[286] C. M. Bender, Introduction to $\mathcal{P} \mathcal{T}$-Symmetric Quantum Theory, Contemp. Phys. 46(4), 277-292 (Jul 2005$).$

[287] P. W. Anderson, Absence of Diffusion in Certain Random Lattices, Phys. Rev. 109(5), 1492-1505 (Mar 1958).

[288] I. M. Lifshits, S. A. Gredeskul, and L. A. Pastur, Introduction to the Theory of Disordered Systems, Wiley, New York, Jul 1988, Russian original by M. Nauka, Moscow, 1982.

[289] B. L. Altshuler and V. N. Prigodin, Distribution of local density of states and NMR line shape in a one-dimensional disordered conductor, J. Exp. Theor. Phys. 68(1), 198-209 (Jan 1989).

[290] G. Pólya, Über den zentralen Grenzwertsatz der Wahrscheinlichkeitsrechnung und das Momentenproblem, Math. Z. 8, 171-181 (1920).

[291] I. E. Tamm, On the possible bound states of electrons on a crystal surface, Phys. Z. Sowjetunion 1, 733-746 (1932).

[292] W. Shockley, On the Surface States Associated with a Periodic Potential, Phys. Rev. 56(4), 317-323 (Aug 1939).

[293] N. H. Abel, Beweis der Unmöglichkeit, algebraische Gleichungen von höheren Graden als dem vierten allgemein aufzulösen., J. Reine Angew. Math. 1, 65-84 (1826).

[294] A. Mostafazadeh, Spectral Singularities of Complex Scattering Potentials and Infinite Reflection and Transmission Coefficients at Real Energies, Phys. Rev. Lett. 102(22), 220402 (Jun 2009).

[295] H. Schomerus, Quantum Noise and Self-Sustained Radiation of PT-Symmetric Systems, Phys. Rev. Lett. 104(23), 233601 (Jun 2010).

[296] T. Kato, Perturbation Theory for Linear Operators, Springer-Verlag New York, LLC, 2nd edition, 2008.

[297] W. D. Heiss, Exceptional points of non-Hermitian operators, J. Phys. A: Math. Gen. 37(6), 2455 (Jan 2004$).$

[298] K. Petermann, Calculated spontaneous emission factor for double-heterostructure injection lasers with gain-induced waveguiding, IEEE J. Quantum. Electron. QE-15(7), 566-570 (Jul 1979).

[299] A. E. Siegman, Excess spontaneous emission in non-Hermitian optical systems. I. Laser amplifiers, Phys. Rev. A 39(3), 1253-1263 (Feb 1989).

[300] A. E. Siegman, Excess spontaneous emission in non-Hermitian optical systems. II. Laser oscillators, Phys. Rev. A 39(3), 1264-1268 (Feb 1989).

[301] M. V. Berry, Mode degeneracies and the petermann excess-noise factor for unstable lasers, J. Mod. Opt. 50(1), 63-81 (Jan 2003).

[302] B. Mehlig and J. T. Chalker, Statistical properties of eigenvectors in non-Hermitian Gaussian random matrix ensembles, J. Math. Phys. 41(5), 3233-3256 (May 2000).

[303] G. H. C. New, The Origin of Excess Noise, J. Mod. Opt. 42(4), 799-810 (Apr 1995).

[304] W. D. Heiss, Repulsion of resonance states and exceptional points, Phys. Rev. E 61(1), 929-932 (Jan 2000$).$

[305] S.-Y. Lee, J.-W. Ryu, J.-B. Shim, S.-B. Lee, S. W. Kim, and K. An, Divergent Petermann factor of interacting resonances in a stadium-shaped microcavity, Phys. Rev. A 78(1), 015805 (Jul 2008).

[306] K. M. Frahm, H. Schomerus, M. Patra, and C. W. J. Beenakker, Large Petermann factor in chaotic cavities with many scattering channels, Europhys. Lett. 49(1), 48-54 (Jan 2000).

[307] A. Maksymowicz, Natural units via linear algebra, Am. J. Phys. 44(3), 295-297 (Mar 1976).

[308] C. M. Focken, Dimensional methods and their applications, Eduard Arnold \& Co. London, 1953.

[309] P. J. Mohr, B. N. Taylor, and D. B. Newell, CODATA recommended values of the fundamental physical constants: 2006, Rev. Mod. Phys. 80(2), 633-730 (Jun 2008). 
[310] P. J. Mohr, B. N. Taylor, and D. B. Newell, CODATA recommended values of the fundamental physical constants: 2006, J. Phys. Chem. Ref. Data 37(3), 1187-1284 (Jul 2008).

[311] P. L. of NIST, The NIST reference on Constants, Units, and Uncertainty, Internet: http://physics.nist.gov/cuu/index.html, 2009, Information at the foundation of modern science and technology from the Physics Laboratory of NIST.

[312] D. K. Ferry, S. M. Goodnick, and J. P. Bird, Transport in Nanostructures, Cambridge University Press, 2nd edition, 2009.

[313] J. C. Slater and G. F. Koster, Simplified LCAO Method for the Periodic Potential Problem, Phys. Rev. 94(6), 1498-1524 (Jun 1954).

[314] C. M. Goringe, D. R. Bowler, and E. Hernández, Tight-binding modelling of materials, Rep. Prog. Phys. 60(12), 1447-1512 (Dec 1997).

[315] P. E. A. Turchi, editor, Tight-binding approach to computational materials science, number 491 in Materials Research Society symposium proceedings, Warrendale, Pa., 1998, Materials Research Society.

[316] T. Esslinger, Fermi-Hubbard Physics with Atoms in an Optical Lattice, Annu. Rev. Cond. Mat. Phys. 1(1), 129-152 (Aug 2010).

[317] W. Johnson, The curious history of Faà di Bruno's formula, Am. Math. Mon. 109(3), 217-234 (Mar 2002).

[318] W. Heisenberg, Über den anschaulichen Inhalt der quantentheoretischen Kinematik und Mechanik, Z. Phys. A Hadron Nucl. 43(3), 172-198 (Mar 1927).

[319] E. H. Kennard, Zur Quantenmechanik einfacher Bewegungstypen, Z. Phys. A Hadron Nucl. 44(4), 326-352 (Jul 1927).

[320] H. Kümmel, Die Eigenschaften der quantentheoretischen Phasenraumdichte, Il Nuovo Cimento 3(5), 870-879 (May 1956).

[321] H. Weyl, Quantenmechanik und Gruppentheorie, Z. Phys. A Hadron Nucl. 46(1), 1-46 (Nov 1927).

[322] E. Wigner, On the Quantum Correction For Thermodynamic Equilibrium, Phys. Rev. 40(5), 749-759 (Jun 1932).

[323] S. Stenholm, Simultaneous measurement of conjugate variables, Ann. Phys. 218(2), 233-254 (Sep 1992).

[324] R. J. Glauber, Coherent and Incoherent States of the Radiation Field, Phys. Rev. 131(6), 2766-2788 (Sep 1963).

[325] E. C. G. Sudarshan, Equivalence of Semiclassical and Quantum Mechanical Descriptions of Statistical Light Beams, Phys. Rev. Lett. 10(7), 277-279 (Apr 1963).

[326] J. G. Kirkwood, Quantum Statistics of Almost Classical Assemblies, Phys. Rev. 44(1), 31-37 (Jul 1933 ).

[327] M. Hillery, R. F. O'Connell, M. O. Scully, and E. P. Wigner, Distribution functions in physics: Fundamentals, Phys. Rep. 106(3), 121-167 (Apr 1984).

[328] L. Cohen, Time-frequency distributions-a review, Proc. IEEE 77(7), 941-981 (Jul 1989).

[329] H.-W. Lee, Theory and application of the quantum phase-space distribution functions, Phys. Rep. 259(3), 147-211 (Aug 1995).

[330] A. Roorda and J. Neitz, Chromatic Topography of the Retina, J. Opt. Soc. Am. A 17(3), 495-496 (Mar 2000).

[331] J. B. Jonas, A. M. Schmidt, J. A. Müller-Bergh, U. M. Schlötzer-Schrehardt, and G. O. Naumann, Human optic nerve fiber count and optic disc size, Invest. Ophthalmol. Vis. Sci. 33(6), 2012-2018 (May 1992).

[332] G. K. V. Noorden and E. C. Campos, Binocular vision and ocular motility: theory and management of strabismus, Mosby, 6th edition, 2002.

[333] A. Reichenbach, Neuroglia - das andere zelluläre Element im Nervensystem: Die Müllersche Gliazelle, SocioMedico, 1999.

[334] R. W. Rodieck, Visual Pathways, Annu. Rev. Neurosci. 2(1), 193-225 (Mar 1979).

[335] F. Rowe, Visual fields via the visual pathway, Blackwell Pub., 2006.

[336] D. S. Wiersma, P. Bartolini, A. Lagendijk, and R. Righini, Localization of light in a disordered medium, Nature 390(6661), 671-673 (Dec 1997). 
[337] T. Schwartz, G. Bartal, S. Fishman, and M. Segev, Transport and Anderson localization in disordered twodimensional photonic lattices, Nature 446(7131), 52-55 (Mar 2007).

[338] J. J. Ludlam, S. N. Taraskin, S. R. Elliott, and D. A. Drabold, Universal features of localized eigenstates in disordered systems, J. Phys.: Condens. Matter 17(30), L321-L327 (Aug 2005).

[339] J. Brewer, Kronecker products and matrix calculus in system theory, IEEE T. Circuits. Syst. 25(9), $772-781$ (Sep 1978).

[340] P. A. Regalia and S. K. Mitra, Kronecker Products, Unitary Matrices and Signal Processing Applications, SIAM Review 31(4), 586-613 (Dec 1989).

[341] C. F. V. Loan, The ubiquitous Kronecker product, J. Comput. Appl. Math. 123(1-2), 85-100 (Nov 2000).

[342] J. H. Wilkinson, The algebraic eigenvalue problem, Numerical Mathematics and Scientific Computation, Oxford University Press, Apr 1988.

[343] W. Pfeifer, The Lie-Algebras su(N), an introduction, Birkhäuser, 2nd edition, Jan 2003.

[344] P. E. G. Assis and A. Fring, Non-Hermitian Hamiltonians of Lie algebraic type, J. Phys. A: Math. Theor. 42(1), 015203 (23pp) (Jan 2009).

[345] Y. V. Makarov and Z. Y. Dong, Eigenvalues and Eigenfunctions. Wiley Encyclopedia of Electrical and Electronics Engineering, volume 12, John Wiley \& Sons Inc, Dec 1999.

[346] R. B. Nelson, Simplified calculation of eigenvector derivatives, AIAA Journal 14(9), 1201-1205 (Sep 1976 ).

[347] J. Dieudonné, On biorthogonal systems, Mich. Math. J. 2(1), 7-20 (1953/54).

[348] Y. Sibuya, On biorthogonal systems, Michigan Math. J. 13(2), 165-168 (Jul 1966). 



\section{Danksagung}

Mein größter Dank gilt dem Kopf und Zentrum unserer Abteilung, Prof. Theo Geisel und seinem unermüdlichen Engagement, dem wir so eine exzellente und einzigartige Arbeitsatmosphäre verdanken. Für jedwede Art der Förderung inklusive der kritischen Beurteilung meiner wissenschaftlichen Projekte bin ich ihm zutiefst dankbar.

Ich danke Dr. Ragnar Fleischmann für seine fortwährende Unterstützung und seine unendliche Geduld, über den Fragen zu brüten, die bei der Entwicklung einer Doktorarbeit zwangsweise auftauchen. Sein wissenschaftlicher Spürsinn und sein Humor, soweit ich das einschätzen kann, verdienen mindestens Weltruhm.

Den anderen aktiven und ehemaligen Mitgliedern unserer mesoskopischen Arbeitsgruppe sind meines herzlichen Dankes versichert: Kai Broeking, Dr. Holger Hennig, Dr. Moritz Hiller, sowie Dr. Jakob Metzger, der zusätzlich ein verlässliches und unverzichtbares Mitglied im Geometrieklub war.

Ich danke den wissenschaftlichen Mitarbeitern der einzelnen Projekte, wobei ich vor allem Prof. Boris "PT" Shapiro nennen möchte. Seine wissenschaftliche Denk- und Arbeitsweise hat mich stets inspiriert, auch wenn er mich nicht immer überzeugen konnte, denn ich glaube weiterhin fest daran, dass der Mensch ein wenig Schlaf und ab und zu ein wenig Wochenende braucht. אני מרגיש עייף ומנומנם. Vielen Dank an Prof. Tsampikos Kottos für sein großes Herz und die klugen Ideen in den vielen vielen

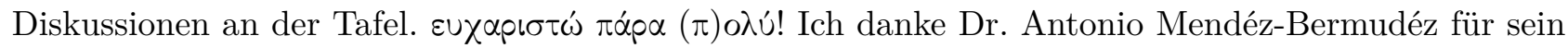
klassisches Verständnis der Physik und die interessanten Diskussionen über die erstaunlichen Kosinusbillards. ¡Muchas gracias por pasar este tiempo maravilloso conmigo! Für die Einsichten rund um die Müllerzellen und die wundervollen und lehrreichen Tage am Cavendish Laboratory möchte ich mich bei Prof. Jochen Guck, Dr. Christian Franze und Moritz Kreysing bedanken.

Unserer IT-Gruppe, namentlich Yorck-Fabian Beensen, Dr. Hecke Degering, Dr. Denny Fliegner, Matthias Mittner und vor allem Markus Schwamberger sei gedankt für die aufopfernden Bemühungen "das Ding am Laufen" zu halten, besonders während der Umzugsphase unseres Instituts und in den letzten Tagen vor der Abgabe meiner Arbeit. Auf der administrativen Seite bedanke ich mich herzlich bei Ayse Bolik, Tanja Gindele, Katharina Jeremias, Viktoryia Novak, Corinna Trautsch und Regina Wunderlich. Ihr macht einen tollen Job!

Aus Gründen der Zeitdilatation und Rezeptarmut wurde der FLC (fast lunch club) gegründet, der 
zwischenzeitlich ruhend nun hoffentlich bald wieder aufblüht und anderen Doktoranden das Leben so erleichtern möge, wie er es dereinst für mich getan hat. Danke an Dr. Georg Martius und Dr. Michael Monteforte! Es darf niemand vergessen werden: Prof. Dirk Brockmann, Dr. Markus Diesmann, Dr. Stephan Eule, Dr. Lars Hufnagel, Harold Gutch, Dr. Björn Naundorf, Dr. Tobias Niemann, Dr. Michael Schnabel und Prof. Marc Timme seien gedankt für die Diskussionen rund um Fragen zu arg speziellen Gebieten, nicht immer nur physikalischer Natur. Danke an Dr. Jan Nagler für alles Andere. Mein Dank gilt auch allen ehemaligen und derzeitigen Mitgliedern, die bisher nicht genannt worden sind. Ihr alle habt individuell zum fabelhaften Arbeitsklima unseres Instituts beigetragen.

Meinem Bürokollegen Dr. Lars Reichl danke ich für das nette Arbeitsklima und das stets offene Ohr für Probleme dies- und jenseits der Diss.

Über alle Maßen danke ich meiner Partnerin und Gefährtin Katja für ihren unerschütterlichen Glauben an mich, ihre aufopfernde Unterstützung und ihre bemerkenswerte Geduld mit mir.

Meinen engsten Freunden danke ich für ihre mentale Unterstützung in guten wie in schwierigen Zeiten.

Mein besonderer Dank gilt, obwohl am Ende genannt natürlich nicht zuletzt gedacht, meinen wundervollen Eltern, die mich in jeglicher Hinsicht immer unterstützen und auf deren Rückhalt ich mich immer verlassen kann. 


\section{Lebenslauf}

\section{Persönliche Daten}

Name:

Oliver Bendix

Geburtsdatum:

01.07.1973

Geburtsort:

Wernigerode

Staatsangehörigkeit:

deutsch

Familienstand:

ledig

\section{Schulausbildung}

07/1980-06/1990

Allgemeinbildende polytechnische Oberschule "Lenin" Wernigerode Abschlussprüfung mit Auszeichnung

07/1990-06/1992

Gerhart-Hauptmann-Gymnasium Wernigerode am Harz

Abschluss: Allgemeine Hochschulreife

\section{Zivildienst}

$10 / 1992-12 / 1993$

Altenpflegeheim "Stadtfeld" Wernigerode

\section{Studium}

04/1994-04/2001

Physik an der Technischen Universität Carolo Wilhelmina zu Braunschweig Abschluss: Diplom-Physiker

\section{Promotion}

$10 / 2001-$

Max-Planck-Institut für Dynamik und Selbstorganisation in Göttingen Institut für nichtlineare Dynamik der Georg-August-Universität zu Göttingen

$10 / 2004$ Immatrikuliert im Promotionsstudiengang Physik der Georg-AugustUniversität zu Göttingen 